\title{
NUMERICAL INVESTIGATIONS OF INSTABILITY AND TRANSITION IN ATTACHED AND SEPARATED SHEAR LAYERS
}

\author{
by \\ Joshua R. Brinkerhoff \\ A thesis submitted to the Faculty of Graduate and Postdoctoral Affairs \\ in partial fulfillment of the requirements for the degree of \\ Doctor of Philosophy \\ in \\ Aerospace Engineering \\ Carleton University \\ Ottawa, Ontario
}

(c) 2014 Joshua R. Brinkerhoff 
Dedicated to my family 


\section{Abstract}

This integrated thesis documents a series of five complementary numerical investigations aimed at understanding the flow instability and laminar-to-turbulent transition process in attached and separated shear layers. Direct numerical simulation was used in these studies to accurately resolve the spatial and temporal scales of the simulated flows. The first two investigations simulate the flow in an idealized computational domain that approximates the conditions on the suction surface of a low-pressure turbine (LPT) blade. The first study investigates the interaction of the Tollmien-Schlichting instability in the attached-flow region with the Kelvin-Helmholtz instability in the separated-flow region. The interaction produces packets of coherent vortices that facilitate transition to a fully-turbulent boundary layer. The effect of swept-blade conditions on the transition process is investigated in the second numerical study by sweeping the simulation domain by $45^{\circ}$. The resulting three-dimensional pressure field creates the potential for a crossflow-induced instability mode, but its effect on the transition process is minimal and transition follows the same mechanisms observed in the unswept configuration.

The characteristics of the coherent vortices that facilitate transition to turbulence in the first study are further investigated through a series of fundamental studies of an isolated turbulent spot that is triggered by a transverse jet. The first of these fundamental studies demonstrates the sensitivity of the formation and structure of the coherent vortices to the level of free-stream acceleration. A subsequent fundamental study identifies the regeneration process by which the turbulent spot grows laterally through the formation of packets of hairpin vortices along the spanwise edges of the spot and longitudinally by the increase in the spatial scale of the hairpin vortices. The final fundamental study investigates the effects of elevated free-stream turbulence levels and LPT-realistic pressure distributions on the transition

process. Under elevated turbulence conditions, bypass transition occurs as streamwise streaks 
form in the laminar boundary layer and roll-up into hairpin-shaped vortices via a KelvinHelmholtz instability mode that is accelerated by the free-stream turbulence. The wave-packet regeneration model identified under low free-stream turbulence is promoted by elevated freestream turbulence, increasing the growth-rate of turbulent spots under elevated turbulence conditions. 


\section{Preface}

This thesis is an integrated, article-based thesis as defined in Section 12.4 of the 2013-2014 Carleton University Graduate Calendar. It consists of the following peer-reviewed journal articles that are either published or currently under review:

1. Brinkerhoff, J.R. and Yaras, M.I. (2011), "Interaction of viscous and inviscid instability modes in separation-bubble transition," Physics of Fluids, 23, pp. 124102. (URL: http://dx.doi.org/10.1063/1.3666844)

2. Brinkerhoff, J.R. and Yaras, M.I. (2013), "Direct numerical simulations of transitional separation-bubble development in swept-blade flow conditions," ASME Journal of Turbomachinery, 135, pp. 041006. (URL: http://dx.doi.org/10.1115/1.4007528)

3. Brinkerhoff, J.R. and Yaras, M.I. (2012), "Direct Numerical Simulation of a Square Jet Ejected Transversely into an Accelerating, Laminar Main Flow," Flow, Turbulence and Combustion, 89, pp. 519-546. (URL: http://dx.doi.org/10.1007/s10494-012-9406-z)

4. Brinkerhoff, J.R. and Yaras, M.I. (2013), "Numerical investigation of the generation and growth of coherent flow structures in a triggered turbulent spot," submitted to the Journal of Fluid Mechanics.

5. Brinkerhoff, J.R. and Yaras, M.I. (2013), "Transition in a boundary layer subjected to favourable and adverse streamwise pressure gradients and elevated free-stream turbulence," submitted to the Journal of Fluid Mechanics.

The body of the thesis contains Chapters $1-3$, which comprise the general introduction of the thesis, a literature review, and a summary of the methodological approach. The bulk of 
the research in this thesis is contained in Chapters $4-8$, in which the above-listed papers are reproduced. Chapter 9 presents the conclusions of each paper and how they connect to the objectives of the thesis, summarizes the contributions of the research, and recommends avenues for future research.

Papers 1-3 are copyrighted material. Permission to reprint the papers as chapters in this thesis has been obtained from the publishers, as indicated by the letters accompanying the thesis manuscript. Readers who wish to cite from copyrighted sections of the thesis are instructed to cite the papers themselves as per the citations listed above. Papers 4 and 5 are in review by the Journal of Fluid Mechanics but are not currently copyrighted material. Readers who wish to cite the sections of the thesis in which these two papers are reprinted are instructed to cite the thesis manuscript.

Modifications have been made to the journal papers to accommodate the integrated-thesis format. To maintain consistency and coherency of the thesis, the papers are re-set from the journal-specific format into the thesis format. The lists of references at the end of each paper are moved into a single list of references that follows Chapter 9, and the citation format in each paper is changed to an author-year format. As some of the above papers are published in journals that do not include a nomenclature section, a single nomenclature defining all the symbols used in the thesis and appendices is included before Chapter 1. To improve the coherency of the research narrative, the introduction sections of the papers are shortened and the conclusions sections are moved to Chapter 9 and expanded to highlight how each paper contributes to the overall objectives of the thesis.

Joshua R. Brinkerhoff was involved in the conceptualization of the present research, conducted the numerical analyses, and prepared all manuscripts. Professor Metin I. Yaras provided supervision of the research and reviewed the manuscripts. 


\section{Acknowledgements}

In the fall of 2006, when I was in the fourth year of my undergraduate degree, I took an aerodynamics course with Professor Metin Yaras. Early in the term, he asked me what I wanted to do with my degree, and I immediately responded, "I want your job." From that time onwards, Professor Yaras has done his best to help me reach that goal. I gratefully acknowledge the encouragement, support, advice, and patient critique that he has given me throughout the course of the present work, and for giving me many opportunities to learn leadership, teaching skills, human resources and project management, and how to be thorough as a researcher.

This work was aided by the support of many people. I gratefully acknowledge the fellow members of Professor Yaras' research group: Harun Oria, Chuk Azih, Majed Piedra Abu Sharrar, Hristo Valtchanov, Aneesh John, Nikunj Dave, Umut Tinis, Shawn Reinink, Mustafa Mogri, Mustafa Siddiqui, and many others. These fellow students became friends as we studied and researched together. I gratefully acknowledge the contributions of my thesis proposal committee, especially Professors Stavros Tavoularis and Steen Sjolander, for providing direction early in my research. I would like to thank Professors Edgar Matida and Joanna Rocha for their encouragement at various critical junctures in my research, Neil McFadyen and Bruce Johnston for their technical support, and the administrative help of Christie Egbert, Irene Helder, Nancy Powell, and the late Marlene Groves, whose conversations and laughs I sorely miss.

There are many people whose support and friendship enabled me to survive this research. Sean McTavish is gratefully acknowledged for happily sharing his office with me for so many years, for the innumerable coffee excursions, for being a sounding board and reviewer for most of my research, and for being such a good friend. I acknowledge Farzad Taremi, Peter Klimas, Danny Knezevici, Gord MacIsaac, and many others who shared their expertise, 
coffee conversations, and lively discussions. I am indebted to the friends at my two spiritual homes, Sunnyside Wesleyan Church and Ottawa University Bible Fellowship. I thank Pastor Samuel and Rebecca Lee and their family for their continual prayer and love, and for their encouragement to enter graduate school in the first place.

Most of all, I am a debtor to my family who cheered me onwards throughout my undergraduate and graduate study. I thank my parents, my mother- and father-in-law, and my sisters for their encouragement and love. I thank my children Abigail, Rebekah, and Ruth for forgiving their daddy for being away so much, and for inspiring me to be the best father that I can be. And most of all, I thank my wife Dana. Dear, I cannot begin to express how thankful I am for all your love and support, and for all you have sacrificed to help me through this thesis. 


\section{Table of Contents}

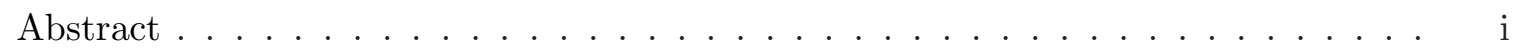

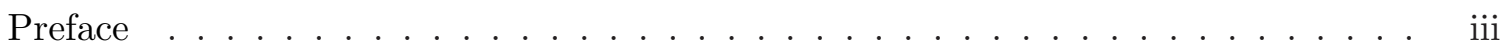

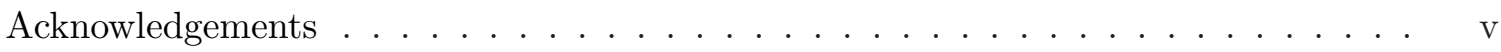

List of Tables . . . . . . . . . . . . . . . . . . . xiii

List of Figures . . . . . . . . . . . . . . . . xiv

List of Symbols . . . . . . . . . . . . . . . . . . xxi

1 Introduction $\quad 1$

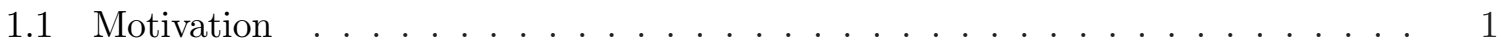

1.2 Thesis Objectives . . . . . . . . . . . . . . . . . 8

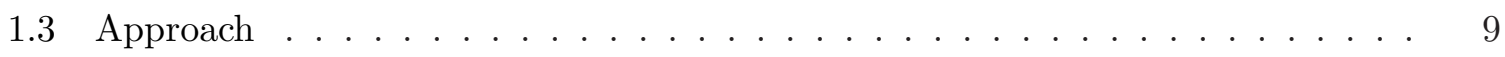

1.4 Outline of Thesis . . . . . . . . . . . . . . . 10

2 Literature Review $\quad 12$

2.1 Transition in Separated Shear Layers . . . . . . . . . . . . . . . . . . 17

2.1.1 Primary shear-layer instability . . . . . . . . . . . 17

2.1 .2 Secondary instabilities . . . . . . . . . . . . . . . 18

2.2 Transition in Attached Laminar Boundary Layers . . . . . . . . . . . . . . . . 22

2.2 .1 Linear growth stage . . . . . . . . . . . . . . . . 23

2.2.2 Non-linear growth and secondary instabilities . . . . . . . . . . . 24

2.2 .3 Break-down to turbulent flow . . . . . . . . . . . . . . 26

2.2.4 Hairpin-vortex regeneration mechanism . . . . . . . . . . . . 29 
2.2.5 Effect of elevated free-stream turbulence . . . . . . . . . . . . . . . 32

2.2.5.1 Formation of streamwise streaks . . . . . . . . . . . 33

2.2.5.2 Spatial characteristics of streamwise streaks . . . . . . . . 34

2.2.5.3 Instability of streamwise streaks . . . . . . . . . . . 35

2.2.5.4 Influence of free-stream flow conditions on bypass transition . 38

2.3 Instability of Three-Dimensional Boundary Layers . . . . . . . . . . . . . . . 41

2.3.1 Receptivity in crossflow instability . . . . . . . . . . . . . . . 44

2.3.2 Non-linear growth and secondary instabilities . . . . . . . . . . . 45

3 Computational Setup and Procedures $\quad 47$

3.1 Governing Equations . . . . . . . . . . . . . . . . . . . 47

3.2 Discretization . . . . . . . . . . . . . . . . . . . . . 49

3.3 Solution Methodology . . . . . . . . . . . . . . . . 52

3.4 Parallel Processing . . . . . . . . . . . . . . . . . . . 55

3.5 Computational Setup . . . . . . . . . . . . . . . 56

3.6 Data Reduction of Simulation Results . . . . . . . . . . . . . . . . . 59

3.6 .1 Averaging methods . . . . . . . . . . . . . . . . . 59

3.6 .2 Vortex identification . . . . . . . . . . . . . 61

3.6 .3 Spectral analysis . . . . . . . . . . . . . . . . 62

3.6.4 Turbulence integral length scale calculation . . . . . . . . . . . . 62

3.6 .5 Intermittency calculation . . . . . . . . . . . . 63

3.7 Linear Stability Analysis . . . . . . . . . . . . . . . . . . . . 63

4 Interaction of Viscous and Inviscid Instability Modes in Separation-Bubble $\begin{array}{ll}\text { Transition } & 66\end{array}$

4.1 Introduction . . . . . . . . . . . . . . . . . . 67

4.2 Numerical Method . . . . . . . . . . . . . . . . . . . . . . . 69

4.2.1 Computational domain and boundary conditions . . . . . . . . . 70

4.2 .2 Spatial grid . . . . . . . . . . . . . . . . . . . . . 72

4.2 .3 Solution method . . . . . . . . . . . . . . 74

4.3 Results and Discussion . . . . . . . . . . . . . . . . . . . 74 
4.3.1 Free-stream flow development . . . . . . . . . . . . . . 74

4.3.2 Instability development in the attached, laminar region . . . . . . . 77

4.3.3 Instability development in the separated region . . . . . . . . . . 83

4.3.4 Interaction of inviscid/viscous instabilities . . . . . . . . . . . 85

4.3.5 Wave packet development in the reattached turbulent boundary layer . 89

5 Interaction of Instability Modes in a Swept Separation Bubble Under Low $\begin{array}{ll}\text { Free-Stream Turbulence } & 93\end{array}$

5.1 Introduction . . . . . . . . . . . . . . . . . . 94

5.2 Numerical Method . . . . . . . . . . . . . . . . . . . 96

5.2.1 Computational domain and boundary conditions . . . . . . . . . 96

$5.2 .2 \quad$ Spatial grid . . . . . . . . . . . . . . . . 98

5.2 .3 Solution method . . . . . . . . . . . . . . . . . . . . 99

5.2 .4 Validation of numerical results . . . . . . . . . . . . . 101

5.3 Results and Discussion . . . . . . . . . . . . . . . . . 105

5.3.1 Free-stream flow development . . . . . . . . . . . . . . 105

5.3 .2 Boundary layer development f . . . . . . . . . . . . 108

5.3 .3 Instability and transition $\ldots \ldots \ldots \ldots \ldots \ldots \ldots \ldots$

6 Direct Numerical Simulation of a Square Jet Ejected Transversely into an Accelerating, Laminar Main Flow $\quad 120$

6.1 Introduction . . . . . . . . . . . . . . . . . . . 121

6.2 Numerical Method . . . . . . . . . . . . . . . . . . 124

6.2.1 Computational domain and boundary conditions . . . . . . . . . . 124

6.2 .2 Spatial grid . . . . . . . . . . . . . . . . . . 126

6.2 .3 Solution method . . . . . . . . . . . . . . 128

6.2 .4 Validation of numerical results . . . . . . . . . . . . . . . 129

6.3 Results and Discussion . . . . . . . . . . . . . . . . . . . 134

6.3 .1 Development of the main and jet flows . . . . . . . . . . . 134

6.3 .2 Interaction of the main flow and the jet . . . . . . . . . . . 139

6.3.2.1 Time-averaged flowfield . . . . . . . . . . . . . . . 139 
7 Numerical Investigation of the Generation and Growth of Coherent Flow Structures in a Triggered Turbulent Spot 159

7.1 Introduction . . . . . . . . . . . . . . . . . . . 160

7.2 Numerical Method . . . . . . . . . . . . . . . . . . 165

7.2 .1 Computational domain . . . . . . . . . . . . 166

7.2 .2 Base flow and turbulent spot initialization . . . . . . . . . 167

7.2 .3 Spatial grid . . . . . . . . . . . . . . . . 169

7.2 .4 Solution method . . . . . . . . . . . . . . . 171

7.2 .5 Validation of numerical approach . . . . . . . . . . . . 173

7.3 Results and Discussion . . . . . . . . . . . . . . . 177

7.3 .1 Laminar baseflow . . . . . . . . . . . . . . . . . . 177

7.3.2 Formation of the turbulent spot . . . . . . . . . . 178

7.3.3 Defining the laminar/turbulent interface . . . . . . . . . . . . . 182

7.3.4 Growth of the turbulent spot . . . . . . . . . . . . 185

7.3.4.1 Regeneration of hairpin vortices within the interior of the spot 187

7.3.4.2 Development of the leading-edge region of the spot . . . . . . 192

7.3.4.3 Regeneration of wave packets along the spanwise edges of the spot ........................ 194

7.3.4.4 Formation of the calmed region . . . . . . . . . . 198

7.3.5 Quantitative description of the spot growth mechanisms . . . . . . . 202

8 Numerical Investigation of Transition in a Boundary Layer Subjected to Favourable and Adverse Streamwise Pressure Gradients and Elevated FreeStream Turbulence $\quad 212$

8.1 Introduction . . . . . . . . . . . . . . . . . . . 213

8.2 Numerical Method . . . . . . . . . . . . . . . . . . . . . . . 219

8.2.1 Computational domain and boundary conditions . . . . . . . . 220

8.2 .2 Spatial grid . . . . . . . . . . . . . . . . . . 221

8.2 .3 Solution method . . . . . . . . . . . . . . . 223

8.2 .4 Validation of numerical approach . . . . . . . . . . . . . . 224 
8.3 Results and Discussion . . . . . . . . . . . . . . . . . 227

8.3.1 Free-stream flow development . . . . . . . . . . . . 228

8.3.2 Test-surface boundary-layer development f . . . . . . . . . . 235

8.3.3 Formation of boundary-layer streaks . . . . . . . . . . . . . 237

8.3.4 Visualisation of streak break-down and transition . . . . . . . . . . 247

9 Summary, Contributions and Recommendations $\quad 268$

9.1 Summary of Investigations . . . . . . . . . . . . . . . . 268

9.1.1 Effect of interacting viscous/inviscid instability modes in shear-layer transition . . . . . . . . . . . . . . . . 268

9.1.2 Effect of swept-blade conditions in shear-layer transition . . . . . . . . 270

9.1.3 Effect of free-stream acceleration on the development of a transverse jet and the resulting formation of coherent vortical flow structures . . . . . 272

9.1.4 Generation and growth of coherent flow structures in a turbulent spot . 274

9.1.5 Effect of elevated free-stream turbulence in shear-layer transition . . . . 278

9.2 Contributions . . . . . . . . . . . . . . . . . . . . . . . 280

9.3 Recommendations for Future Work . . . . . . . . . . . . . . . . 281

$\begin{array}{ll}\text { References } & 284\end{array}$

$\begin{array}{ll}\text { A Domain and Grid Coordinates } & 308\end{array}$

A.1 Description of the Grid Generation Software . . . . . . . . . . . . 308

A.1.1 Introduction . . . . . . . . . . . . . . . 308

A.1.2 Input text file format $\ldots \ldots \ldots \ldots . \ldots \ldots$

A.1.3 Software limitations . . . . . . . . . . . . . . . . 310

A.2 Domain and Grid Information . . . . . . . . . . . . . . 311

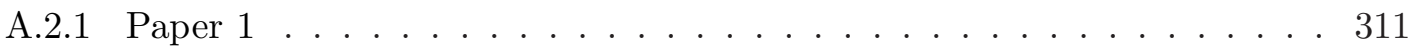

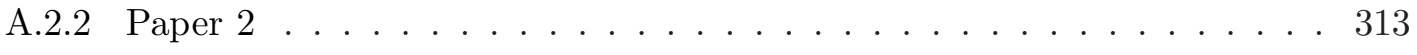

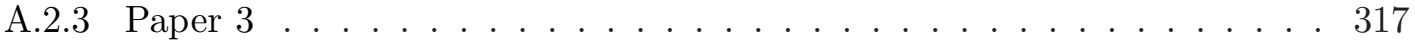

A.2.4 Paper $4 \ldots \ldots \ldots \ldots$

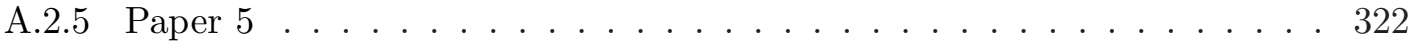


$\begin{array}{ll}\text { C ComPost Operator's Manual } & 331\end{array}$

C.1 Program structure . . . . . . . . . . . . . . . . . 331

C.2 File structure . . . . . . . . . . . . . . . . . . 332

C.3 Input files . . . . . . . . . . . . . . . . . . . . . . . . 338

C.3.1 Input file for the get_xyz program . . . . . . . . . . . . 338

C.3.2 Input file for the ComPost program . . . . . . . . . . . . . . 339

C.4 Procedures for using ComPost . . . . . . . . . . . . . . . . . . . . 343

C.4.1 Sorting structured-grid results . . . . . . . . . . . . . 343

C.4.2 Calculating temporal averages . . . . . . . . . . . . . . 344

C.4.3 Calculating spatial averages . . . . . . . . . . . . . . 345

C.4.4 Calculating integral parameters . . . . . . . . . . . . 345

C.4.5 Outputting velocity profiles . . . . . . . . . . . . 346

C.4.6 Calculating fluctuation quantities . . . . . . . . . . . . 347

C.4.7 Outputting time traces . . . . . . . . . . . . . . 348

C.4.8 Exporting Tecplot-formatted transient results . . . . . . . . . . . . 349

C.4.9 Calculating the vorticity field . . . . . . . . . . . . . . . 349

C.4.10 Calculating statistical turbulence quantities . . . . . . . . . . . 350

C.5 Spectral analysis . . . . . . . . . . . . . . . . . . . 350

C.5.1 Input file for the compute_FFT program . . . . . . . . . . . . . 351

D Permissions to Reproduce Copyrighted Material 353 


\section{List of Tables}

2.1 Instability Strouhal numbers in studies of free and separated shear layers . . . 19

3.1 Description of the computational platforms used in the present research . . . . 56

4.1 Flow conditions and numerical details of the current simulation . . . . . . . 75

5.1 Numerical details of the simulations used for validation of the numerical results 101

5.2 Flow conditions and numerical details of the separation-bubble simulations . . 106

6.1 Numerical details of the simulations used for validation of the numerical results. 130

6.2 Flow conditions and numerical details of the simulation. . . . . . . . . . . 135

6.3 Jet trajectory correlation coefficients. . . . . . . . . . . . . . . . 145

7.1 Number of nodes in the spatial grid . . . . . . . . . . . 171

7.2 Computational grids for the validation studies. . . . . . . . . . . . . 173

8.1 Flow conditions and numerical details of the current simulation . . . . . . . . 225

8.2 Computational grids for the validation studies. . . . . . . . . . . 226

A.1 Limitations of PatranMeshGenerator . . . . . . . . . . . 310 


\section{List of Figures}

1.1 Schematic illustrating the regions associated with instability modes on a typical LPT blade . . . . . . . . . . . . . . . . . . . . . 3

1.2 Roadmap to turbulence . . . . . . . . . . . . . . . . . . 4

1.3 Qualitative framework of the transition process in laminar shear layers . . . . . 6

2.1 Boundary layer developing on a flat surface subjected to a streamwise pressure distribution ............................. 14

2.2 Stability curves for spatial growth in a Blasius boundary layer . . . . . . . . . 16

2.3 (a) Typical pressure distribution on the suction-surface of an LPT blade. (b) Possible transition scenarios of a separated boundary layer. . . . . . . . . . 17

2.4 Disturbance growth in a vortex sheet through inviscid instability . . . . . . 18

2.5 Sketch of inviscid instability of a free shear layer . . . . . . . . . . . 20

2.6 Coherent structures in the separated shear layer f . . . . . . . . . . . 21

2.7 Schematic of the vortex loop structure observed in the separated shear layer under elevated free-stream turbulence . . . . . . . . . . . . . . 22

2.8 RMS-amplitude distribution of the streamwise disturbance velocity illustrating a typical eigenfunction in the linear-growth region . . . . . . . . . . . . 24

2.9 Smoke-wire visualizations of $\Lambda$-vortices, hairpin-vortices, and turbulent spots $\quad 25$

2.10 Schematic showing the wall-normal motions induced by a hairpin vortex . . . . 27

2.11 Schematic of the topology within a turbulent spot $\ldots \ldots \ldots$

2.12 Contours of normal vorticity illustrating the overhang region at the leading-edge of a turbulent spot . . . . . . . . . . . . . . 28

2.13 Hairpin-vortex regeneration mechanism proposed by Singer and Joslin (1994) • 30 
2.14 Hairpin-vortex regeneration mechanism proposed by Schröder and Kompenhans (2004) . . . . . . . . . . . . . . . . . . . . 31

2.15 Sketch of streak instability modes in the $(x-z)$ plane . . . . . . . . . 36

2.16 Streamwise streaks in the shear layer observed in an $x-z$ plane where $U \approx 1 / 2 U_{e}$, (a) streamwise fluctuations, (b) wall-normal fluctuations . . . . . . . . . 40

2.17 Three-dimensional boundary layer profile . . . . . . . . . . . . . . . . . 43

2.18 (a) Coordinate systems in a swept-blade flow (b) Profiles of the tangential and crossflow velocities in different orientations . . . . . . . . . . .

2.19 Locations of the amplitude maxima for the secondary instability modes of a stationary crossflow vortex . . . . . . . . . . . . . . 46

3.1 Two-dimensional representation of a finite volume as defined in ANSYS CFX .

3.2 Arrangement of sub-domain blocks in the computational grid for the $(a)$ separation bubble (Chapter 4) and (b) turbulent spot (Chapter 7) simulations .

4.1 Schematic of the computational domain . . . . . . . . . . . . . 71

4.2 Results of a companion DNS study assessing grid sensitivity in a zero-streamwiseacceleration turbulent boundary layer at $R e_{\theta}=500 \ldots \ldots$. . . . . . 73

4.3 Streamwise distribution of free-stream turbulence intensity $\ldots \ldots \ldots$. . . . 76

4.4 Streamwise distribution of time-averaged free-stream velocity . . . . . . . . 76

4.5 Streamwise distribution of time-averaged free-stream acceleration parameter . . 77

4.6 Frequency spectrum of the streamwise velocity in the attached laminar boundary

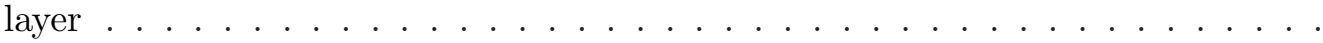

4.7 Streamwise variation of disturbance amplitude normalized by the reference velocity $U_{0} \ldots \ldots \ldots \ldots \ldots \ldots \ldots$

4.8 Streamwise variation of the disturbance growth rate obtained from linear

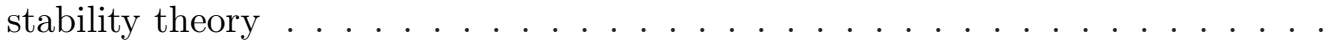

4.9 Iso-contours of wall-normal perturbation vorticity at three time values $(a)$ upstream of separation and $(b)$ downstream of separation $\ldots \ldots \ldots$. . . . . 82

4.10 Time-averaged characteristics of the separation bubble. Contours show stream-

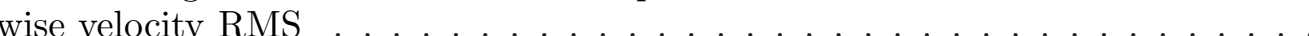

4.11 Roll-up of the separated shear layer observed through contours of spanwise vorticity in an $x-y$ plane in the separated shear layer . . . . . . . . .

4.12 Spanwise non-uniformity of the rolled-up separated shear layer observed through contours of spanwise vorticity in the $x-z$ plane at $y=\delta_{s}^{*} \ldots \ldots \ldots$ 
4.13 Transient break-down in the separated shear layer through iso-contours of the second invariant of the velocity gradient tensor, $Q \ldots \ldots . \ldots 8$

4.14 Streamwise distribution of the intermittency parameter $\gamma \ldots \ldots$. . . . . . 89

4.15 Growth of wave packets in the transitional shear layer seen through iso-contours of $Q$ coloured by the wall-normal height viewed from a (a) $x-y$ plane, $(b) x-z$ plane, and $(c)$ isometric projection $\ldots \ldots \ldots . \ldots \ldots$

5.1 Schematic of the computational domain . . . . . . . . . . . . . 97

5.2 Wall-normal node distribution . . . . . . . . . . . . . . . . . . . 100

5.3 Companion DNS study of a zero-pressure-gradient turbulent boundary layer at

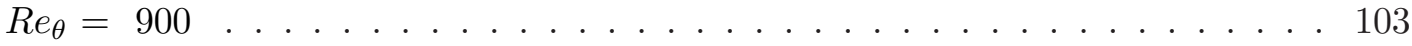

5.4 Companion DNS studies in a zero-pressure-gradient turbulent boundary layer

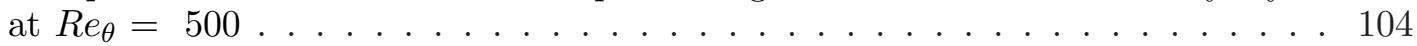

5.5 Streamwise distribution of the time-averaged free-stream velocity magnitude for the $\Lambda=0^{\circ}$ and $\Lambda=45^{\circ}$ cases . . . . . . . . . . . . 107

5.6 Axial distribution of the time-averaged free-stream acceleration parameter for the $\Lambda=0^{\circ}$ and $\Lambda=45^{\circ}$ cases $\ldots \ldots \ldots \ldots \ldots \ldots$

5.7 Axial distribution of the time-averaged spanwise pressure gradient and freestream flow angle for the $\Lambda=45^{\circ}$ case . . . . . . . . . . . . . 109

5.8 Axial variation of the crossflow velocity profile near the separation point for the $\Lambda=45^{\circ}$ case . . . . . . . . . . . . . . . . . . . 110

5.9 Axial distribution of the time-averaged displacement thickness for the $\Lambda=0^{\circ}$

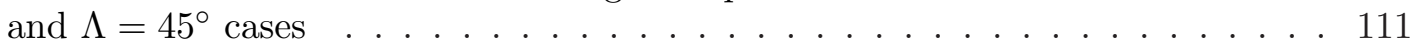

5.10 Axial distribution of the time-averaged momentum thickness for the $\Lambda=0^{\circ}$ and $\Lambda=45^{\circ}$ cases . . . . . . . . . . . . . . . . . 11

5.11 Axial distribution of the root-mean-square of the streamwise fluctuation velocity for the $\Lambda=0^{\circ}$ and $\Lambda=45^{\circ}$ cases $\ldots \ldots \ldots \ldots$

5.12 Time-averaged contours of $(a)$ tangential velocity at $z_{c} / L=0.00,(b)$ crossflow velocity at $z_{c} / L=0.00$, and $(c)$ crossflow velocity at $\left(x_{t}-x_{t s}\right) / L=0.19$ in the separated region of the $\Lambda=45^{\circ}$ case . . . . . . . . . . . 116

5.13 Periodic roll-up of the separated shear layer observed through contours of spanwise vorticity at $z / L=0.00$ for the $(a) \Lambda=0^{\circ}$ and $(b) \Lambda=45^{\circ}$ cases $\ldots 118$

5.14 Frequency spectra of the wall-normal fluctuation velocity at $z / L=0.00$ and at several streamwise locations in the separated-flow region for $(a) \Lambda=0^{\circ}$ and $(b)$ $\Lambda=45^{\circ}$ cases . . . . . . . . . . . . . . . . . . . . 119

6.1 Schematic of vortical structures in the flowfield of the transverse jet . . . . . 123 
6.2 Schematic of the computational domain . . . . . . . . . . . . . 125

6.3 Time history of the jet velocity observed at the center of the jet orifice . . . . 126

6.4 Wall-normal node distribution . . . . . . . . . . . . . . . . . . . 128

6.5 Companion DNS study of a zero-pressure-gradient turbulent boundary layer at

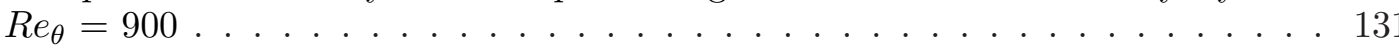

6.6 Companion DNS studies in a zero-pressure-gradient turbulent boundary layer

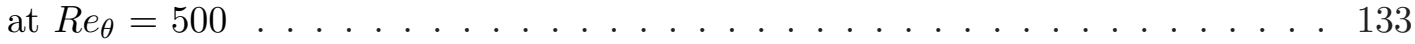

6.7 Streamwise distribution of acceleration parameter $(\eta)$, displacement thickness $\left(\delta^{*} / D\right)$, and free-stream velocity $\left(U / v_{j e t}\right)$ in the absence of the transverse jet . 136

6.8 Variation in the jet velocity components along the jet centerline . . . . . . . 137

6.9 Normalized root-mean-square of $y$-component of the jet velocity along $x / D=$ 0.0 and $z / D=0.0 \ldots \ldots \ldots \ldots \ldots \ldots$

6.10 Power spectral density of the normalized $v$-component velocity fluctuations at the orifice plane near the upwind side of the jet pipe $(x / D=-0.5)$, at the orifice center $(x / D=0.0)$, and near the downwind side of the jet pipe $(x / D=0.5)$.

6.11 Wall-normal development of the jet flowfield, shown through time-averaged streamlines and pressure coefficient contours . . . . . . . . . . . .

6.12 Time-averaged flowfield in a streamwise plane passing through the center of the transverse jet, visualized through streamlines and contours of vorticity

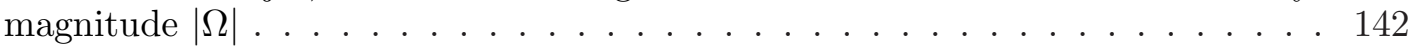

6.13 Magnified view of the near-field flow of the transverse jet . . . . . . . 143

6.14 Comparison of the simulated jet trajectory and that obtained from a scaling law in the form of Equation 6.3. . . . . . . . . . . . . . . . . 145

6.15 Transient development of the transverse jet shown through instantaneous streamlines and contours of $|U| / v_{j e t}$ in the streamwise plane located at $z / D=0.0147$

6.16 Instability of the upwind and downwind shear layers shown through $(a)$ instantaneous contours of vorticity magnitude and (b) Strouhal-number spectra at several wall-normal locations in the upwind and downwind shear layers . . . . . 149

6.17 Time trace of wall-normal velocity fluctuation at locations D3 and D4 from

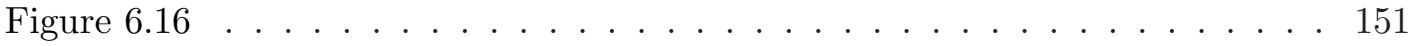

6.18 Vortical structures observed in the transverse jet during its initial transient development . . . . . . . . . . . . . . . . . . 153

6.19 Vortical structures observed in the transverse jet . . . . . . . . . . 156

7.1 Schematic of the hairpin-vortex regeneration model proposed by Singer and

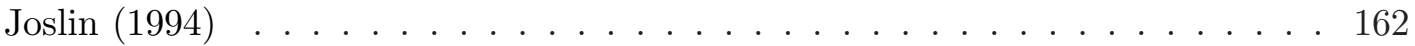


7.2 Schematic of the hairpin-vortex regeneration model proposed by Schröder and coworkers $(2004,2008) \ldots \ldots \ldots \ldots \ldots \ldots \ldots$

7.3 Schematic of the computational domains . . . . . . . . . . . . 167

7.4 Wall-normal node distribution . . . . . . . . . . . . . . . . . . 171

7.5 Validation DNS study of a zero-pressure-gradient turbulent boundary layer at

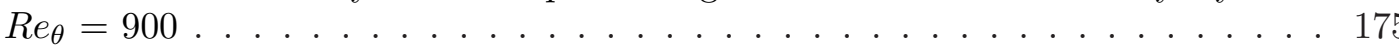

7.6 Validation DNS studies in a zero-pressure-gradient turbulent boundary layer at

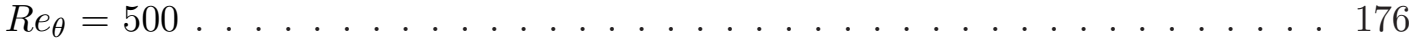

7.7 Streamwise distribution of the free-stream velocity, $U$, and streamwise acceleration parameter $K \ldots \ldots \ldots \ldots \ldots \ldots \ldots \ldots \ldots$

7.8 Streamwise distribution of the displacement thickness, $\delta^{*} / \delta_{0}^{*}$, and friction factor, $C_{f}$, of the undisturbed laminar boundary layer $\ldots \ldots \ldots \ldots$

7.9 Formation of the turbulent spot in Simulation-1 illustrated at $\tau=2,46$, and 90, visualized through iso-contours of $(a-c) \omega_{x}= \pm 1$ and $(d) Q=0.6$ (left) and instantaneous vorticity lines (right) . . . . . . . . . . 180

7.10 Schematic illustrating the formation of vortical structures in the early turbulent spot from the initial pair of streamwise vortices triggered by the transverse jet

7.11 Streamwise distribution of the spot spanwise dimension $\ldots \ldots \ldots$

7.12 Development of the turbulent spot at $\tau=107,255$, and 492, visualized through contours of $Q=0.6$ (left) and instantaneous vorticity lines (right) coloured according to the value of $y / \delta_{0}^{*} \ldots \ldots \ldots \ldots \ldots$

7.13 Schematic illustrating the lateral joining of a pair of hairpin vortices into a single hairpin vortex . . . . . . . . . . . . . . . 188

7.14 Magnified view of the development of a wave-packet between $\tau=255$ and 325, visualized through contours of $Q=0.6$ (left) and instantaneous vorticity lines

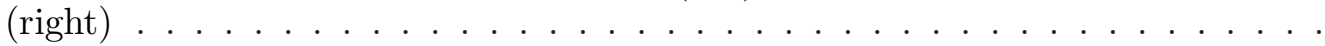

7.15 Schematic illustrating the regeneration of hairpin vortices from a parent hairpin vortex with $(a-d)$ widely-spaced and $(e-h)$ closely-spaced legs $\ldots \ldots \ldots$

7.16 Turbulent spot at $\tau=255$ and 492, visualized through contours of $Q=0.6$, viewed in the $-z$ direction . . . . . . . . . . . . . . . 193

7.17 Growth of the turbulent spot at $\tau=480,524$, and 568, visualized through iso-contours of $\left|\omega_{y}\right| \delta_{0}^{*} / U_{0}=0.095$ shaded by $y / \delta_{0}^{*}$ in the $-z$ half of the spot . .

7.18 Schematic illustrating the regeneration mechanism of wave packets along the spanwise edge of the turbulent spot . . . . . . . . . . . .

7.19 Contours of the streamwise perturbation velocity $u / U_{0}$ showing the development of streamwise streaks in the turbulent spot . . . . . . . . . . . . 199 
7.20 Formation of the calmed region upstream of the spot viewed through contours of $(a) u / U_{0}$ and $(b) \omega_{x} \delta_{0}^{*} / U_{0}$ in a $x-z$ plane at $y / \delta_{0}^{*}=2.0$ at $\tau=568 \ldots 201$

7.21 Contour levels of the budgets of the enstrophy transport equation in the $-z$ side of the spot at $\tau=568$ and $x / \delta_{0}^{*}=345 \ldots \ldots \ldots$. . . . . . . . 204

7.22 Contour levels of the components of the $P 1$ terms $(a)-(c)$ and $P 2$ terms $(d)-(f)$ in the $-z$ side of the spot at $\tau=568$ and $x / \delta_{0}^{*}=345 \ldots \ldots \ldots 20 \ldots$

7.23 Contour levels of the components of the $P 4$ terms in the $-z$ side of the spot at $\tau=568$ and $x / \delta_{0}^{*}=345 \ldots \ldots \ldots \ldots \ldots \ldots \ldots$

7.24 Vorticity and velocity fields in the $-z$ side of the spot at $\tau=568$ and $x / \delta_{0}^{*}=345207$

7.25 Schematic illustrating the enstrophy production mechanisms embodied in $(a)$ the $P 1$ term, $(b)-(c)$ the $P 2$ terms, and $(d)-(f)$ the $P 4$ terms $\ldots \ldots . . . .208$

7.26 Block diagram illustrating the regeneration cycles of turbulent enstrophy observed within the turbulent spot . . . . . . . . . . . . 210

7.27 Iso-contours of normalized turbulence kinetic energy production, $P^{+}=0.5$, at $\tau=480,524$, and 568 in the $-z$ half of the spot $\ldots \ldots \ldots 211$

8.1 Schematic of the computational domain . . . . . . . . . . . . 221

8.2 Wall-normal node distribution . . . . . . . . . . . . . . . . 223

8.3 Validation DNS study of a zero-pressure-gradient turbulent boundary layer at

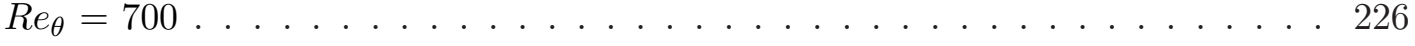

8.4 Streamwise distributions of free-stream velocity, $U / U_{0}$, and acceleration param-

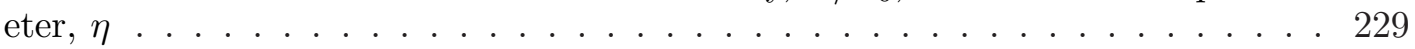

8.5 Contours of $U / U_{y z}$ in $y-z$ planes at $(a) x / L=-0.004$, (b) $x / L=0.121$,

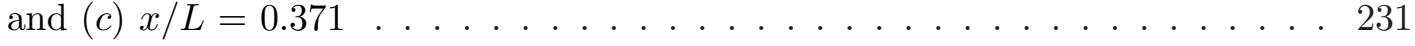

8.6 Streamwise decay of free-stream turbulence upstream of the test surface. Areaaveraged distribution of $(a)$ turbulence intensity, $T u,(b)$ normal Reynolds stresses, and $(c-d)$ integral length scales. Error bars indicate uncertainty in the spatial mean values . . . . . . . . . . . . . . . 232

8.7 Frequency spectrum of the streamwise velocity fluctuations at $x / L=-0.035 \quad$. 234

8.8 Streamwise decay of free-stream turbulence downstream of the test-surface

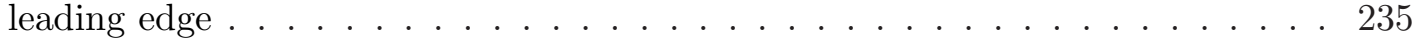

8.9 Streamwise distributions of $(a)$ displacement thickness $\left(\delta^{*} / L\right)$, momentum thickness $(\theta / L)$, and shape factor $(H) ;(b)$ friction factor $\left(C_{f} \times 10^{2}\right)$ and

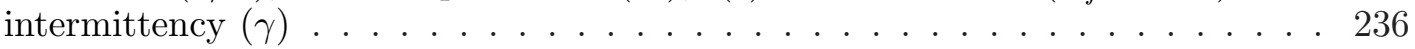

8.10 (a) Streamwise distribution of $u_{r m s}^{\prime}$ at $y / L=0.850 \times 10^{-3}$ and $y / L=10.77 \times$ $10^{-3}$. (b) Wall-normal profiles of $u_{r m s}^{\prime}$ in the laminar region . . . . . . . . 238 
8.11 Streamwise velocity fluctuation contours at $(a) y \approx 10 \delta^{*},(b) y \approx 3 \delta^{*}$,

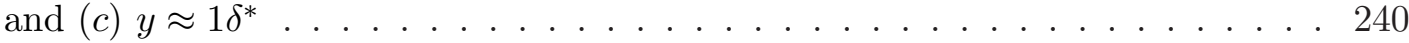

8.12 Temporal evolution of $u^{\prime}$ contours at $y / L=0.850 \times 10^{-3} \ldots \ldots \ldots$. . . . . 242

8.13 Streamwise distribution of the spanwise spacing of the low-speed $u$-velocity streaks 244

8.14 Streamwise development of the amplitude of the low-speed $u$-velocity streaks . 248

8.15 Temporal evolution of $(a) u^{\prime}$ and $(b) v^{\prime}$ contours at $y / L=0.850 \times 10^{-3} \ldots 249$

8.16 Transient break-down of a low-speed streak illustrated through iso-contours of (left) $u^{\prime} / U_{0}=-0.20$ and (right) $Q\left(L / U_{0}\right)^{2}=5000 \ldots \ldots \ldots \ldots$

8.17 Interaction of a free-stream vortical disturbance and a streamwise streak in the $z / L=-0.002$ plane . . . . . . . . . . . . . . . . 254

8.18 Temporal evolution of instantaneous spanwise shear through $\omega_{z}\left(L / U_{0}\right)$ contours in the $z / L=-0.002$ plane . . . . . . . . . . . . . . . 258

8.19 Enstrophy equation budgets in the $z / L=-0.002$ plane at $\tau=0.042 \ldots$. . . 260

8.20 Components of the $P 1$ terms $(a)-(d)$ and $P 2$ terms $(e)-(g)$ from Equation 8.5 in

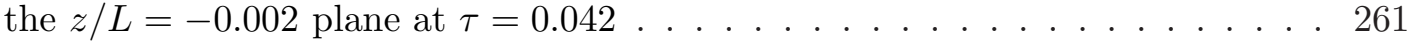

8.21 Components of the $P 4$ terms from Equation 8.5 in the $z / L=-0.002$ plane at

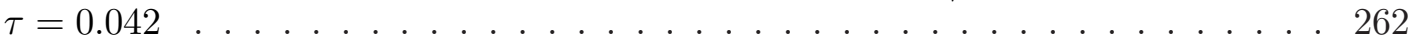

8.22 Contours of $(a) u^{\prime} / U_{0}$ and $(b) \omega_{y}^{\prime}\left(L / U_{0}\right)$ in the $x / L=0.625$ plane at $\tau=0.053263$

8.23 Schematic illustrating the enstrophy production mechanisms embodied in $(a)$ the $P 1$ term, $(b-c)$ the $P 2$ terms, and $(d)-(h)$ the $P 4$ terms $\ldots \ldots . . . . .265$

8.24 The block diagram of Brinkerhoff and Yaras (2013) illustrating the regeneration cycles of turbulent enstrophy within a turbulent spot, modified to include the effect of sweep motions induced by free-stream vortical disturbances . . . . . 266

B.1 Schematic illustrating the production of enstrophy via the P3 term in a boundary layer . . . . . . . . . . . . . . . . . . . . . . . . . 329

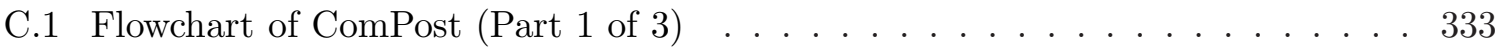

C.2 Flowchart of ComPost (Part 2 of 3$) \ldots \ldots \ldots \ldots \ldots$

C.3 Flowchart of ComPost (Part 3 of 3$) \ldots \ldots \ldots \ldots \ldots$ 


\section{List of Symbols}

\begin{tabular}{|c|c|}
\hline$a$ & Speed of sound \\
\hline$a_{i}$ & Coefficients of the coupled system of equations \\
\hline$a_{n}$ & Chebychev polynomial coefficient of order $n$ \\
\hline$b$ & Right side matrix in discrete system of equations \\
\hline$C_{f}$ & Friction factor $=0.5 \tau_{w} /\left(\rho U_{0}^{2}\right)$ \\
\hline$C_{p}$ & Pressure coefficient $=\frac{p-p_{r e f}}{\frac{1}{2} \rho U_{r e f}^{2}}$ \\
\hline$c$ & Disturbance wave phase speed \\
\hline & Redistribution coefficient \\
\hline & Eigenvalues of Orr-Sommerfeld equation \\
\hline$c_{0} \ldots c_{n}$ & Polynomial coefficients for ceiling height function \\
\hline$D$ & Rate of enstrophy diffusion \\
\hline & Transverse jet width \\
\hline$d$ & Bar width of turbulence generating grid \\
\hline$d_{i p}$ & Discrete redistribution coefficient \\
\hline$F$ & Dimensionless spatial growth eigenvalue $=\alpha c \nu / U_{0}^{2}$ \\
\hline$f$ & Redistribution coefficient \\
\hline & Frequency \\
\hline$f_{M A}$ & Most amplified frequency \\
\hline$H$ & Shape factor $=\delta^{*} / \theta$ \\
\hline
\end{tabular}




\begin{tabular}{|c|c|}
\hline$H_{d}$ & Hydraulic diameter of the transverse jet \\
\hline$h_{0}$ & Stagnation enthalpy \\
\hline$i$ & Imaginary number $=\sqrt{-1}$ \\
\hline$K$ & Acceleration parameter $=\frac{\nu}{U_{e}^{2}} \frac{d U_{e}}{d x}$ \\
\hline$k^{2}$ & Wavenumber vector amplitude $=\alpha^{2}+\beta^{2}$ \\
\hline$k_{z}$ & Spanwise wavenumber $=2 \pi / \lambda_{z}$ \\
\hline$L$ & Test surface length \\
\hline & Stroke length of the transverse jet $=\approx v_{j e t} t_{j e t}$ \\
\hline$M$ & Mach number $=U / a$ \\
\hline$N_{n}$ & Shape function for node $n$ \\
\hline$N_{t}$ & Number of time steps \\
\hline$N_{x}$ & Number of nodes in the $x$ direction \\
\hline$N_{y}$ & Number of nodes in the $y$ direction \\
\hline$N_{z}$ & Number of nodes in the $z$ direction \\
\hline$n_{j}$ & Normal surface vector \\
\hline$P$ & Turbulence kinetic energy production rate \\
\hline$P 1$ & First enstrophy production term \\
\hline$P 2$ & Second enstrophy production term \\
\hline$P 3$ & Third enstrophy production term \\
\hline$P 4$ & Fourth enstrophy production term \\
\hline$p$ & Static pressure \\
\hline$Q$ & Second invariant of the velocity gradient tensor \\
\hline Q2 & Region where $u^{\prime}<0$ and $v^{\prime}>0$ \\
\hline Q4 & Region where $u^{\prime}>0$ and $v^{\prime}<0$ \\
\hline$R(\Delta t)$ & Normalized autocorrelation function \\
\hline $\mathscr{R}^{n}$ & Residual at node $n$ \\
\hline
\end{tabular}




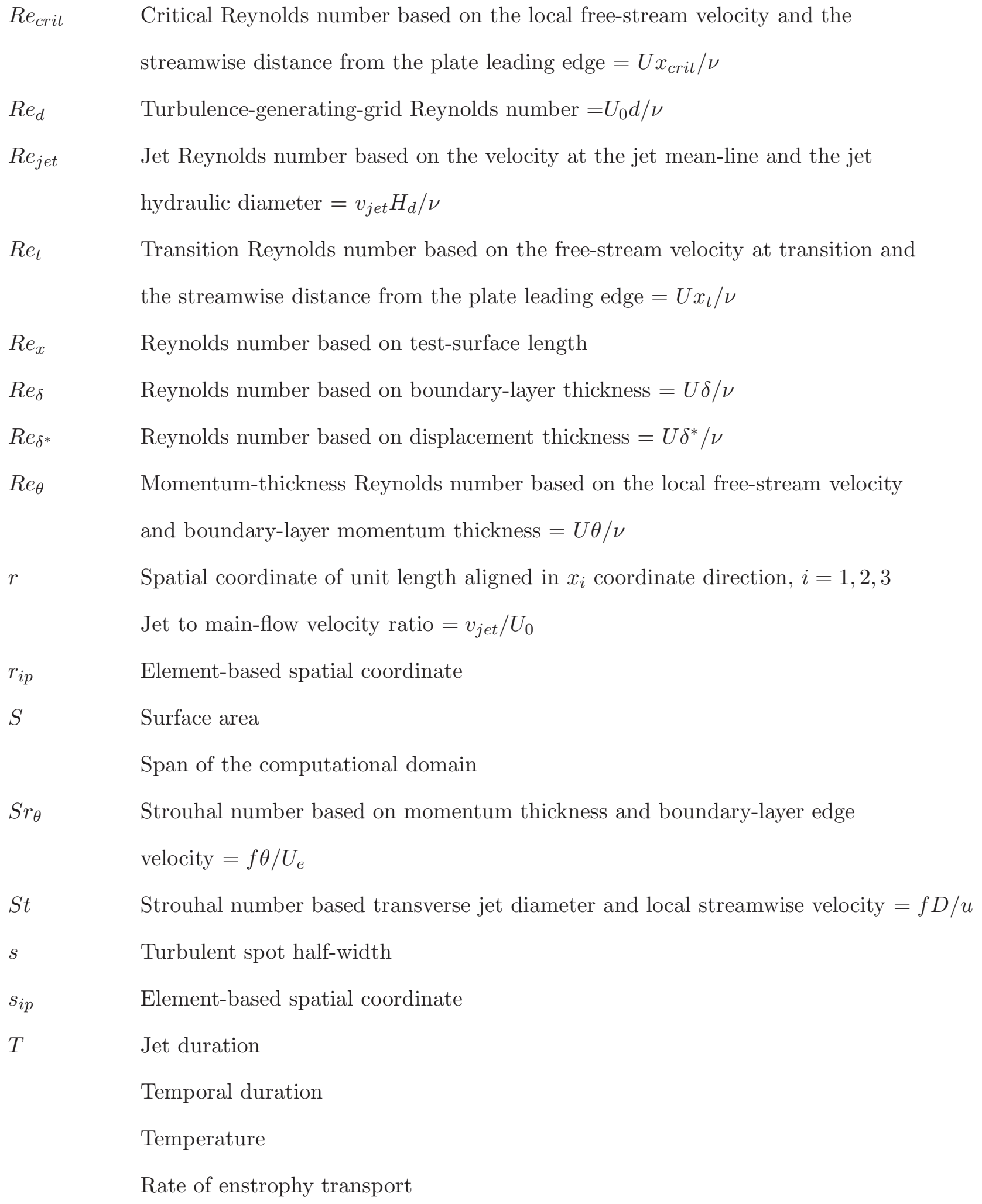




\begin{tabular}{|c|c|}
\hline$T_{n}$ & Chebyshev polynomial of order $n$ \\
\hline$T u$ & Turbulence intensity \\
\hline$t$ & Time \\
\hline$t_{i p}$ & Element-based spatial coordinate \\
\hline$t_{j e t}$ & Transverse jet duration \\
\hline$t_{\eta}$ & Kolmogorov time scale \\
\hline$U$ & Velocity magnitude $=\sqrt{u^{2}+v^{2}+w^{2}}$ \\
\hline$U_{0}$ & Reference velocity \\
\hline$U_{e}$ & Velocity magnitude at boundary-layer edge \\
\hline$U_{j}$ & Mean velocity vector \\
\hline$U_{u}$ & Local velocity at maximum height of coherent structures \\
\hline$U_{y z}$ & Streamwise velocity spatially-averaged in the local $y$ - $z$ plane \\
\hline$U_{\infty}$ & Free-stream velocity magnitude \\
\hline$u$ & Streamwise velocity component \\
\hline$u_{j}$ & Instantaneous velocity vector \\
\hline$u^{+}$ & Streamwise velocity component in wall units $=u / u_{\tau}$ \\
\hline$\hat{u}_{r m s, z}$ & Ensemble-averaged disturbance amplitude \\
\hline$u_{\text {streak }}^{\prime}$ & Mean streak amplitude \\
\hline$u_{\tau}$ & Friction velocity $=\sqrt{\tau_{w} / \rho}$ \\
\hline$V_{c}$ & Velocity component in the crossflow direction \\
\hline$V_{t}$ & Velocity component in the tangential direction \\
\hline $\mathscr{V}$ & Volume \\
\hline$v$ & Wall-normal velocity component \\
\hline$\hat{v}$ & Disturbance wave amplitude \\
\hline$v_{\text {jet }}$ & Wall-normal velocity of the transverse jet \\
\hline$w$ & Spanwise velocity component \\
\hline
\end{tabular}


Streamwise spatial coordinate

Tangential location of transition onset

Tangential location of reattachment

Tangential location of separation

Rate of enstrophy dissipation

Wall-normal spatial coordinate

Maximum height of coherent structures in the turbulent spot

Spanwise spatial coordinate

Coordinate normal to local inviscid streamline

Streamwise wave number $=2 \pi / \lambda_{x}$

Flow state parameter $=1$ for turbulent and 0 for laminar

Local flow angle

Spanwise wave number $=2 \pi / \lambda_{z}$

Intermittency $=1 / N \sum_{i-1}^{N} \beta_{i}$

Discrete outwards surface vector

Time step size

Streamwise grid spacing in wall units

Spanwise grid spacing in wall units

Spanwise streak width

Boundary-layer or shear-layer thickness

Reference boundary-layer thickness

Displacement thickness $=\int_{0}^{\delta}\left(1-\frac{u}{U_{e}}\right) d y$

Reference displacement thickness

Turbulence kinetic energy dissipation rate

Normalized spot streamwise coordinate $=\frac{\left(x-x_{t e}\right)}{\left(x_{l e}-x_{t e}\right)}$

Acceleration parameter $=\frac{\nu}{U_{e}^{2}} \frac{d U_{e}}{d x}$ 
Kolmogorov length scale

$\theta$

$\kappa$

$\Lambda$

$\Lambda_{x}$

$\Lambda_{x 0}$

$\Lambda_{x i}$

$\Lambda_{y}$

$\Lambda_{z}$

$\lambda$

$\lambda_{x}$

$\lambda_{z}$

$\lambda^{+}$

$\lambda_{\theta}$

$\mu$

$\nu$

$\xi$

$\rho$

$\rho^{0}$

$\tau$

$\tau_{j e t}$

$\tau_{w}$

$\phi$

$\phi\left(k_{z}\right)$
Momentum thickness $=\int_{0}^{\delta} \frac{u}{U_{e}}\left(1-\frac{u}{U_{e}}\right) d y$

Wavenumber $=2 \pi / \lambda$

von Karman constant

Sweep angle

Integral length scale of free-stream turbulence

Streamwise integral length scale of turbulence

Streamwise integral length scale of turbulence at leading edge

Integral length scale of turbulence in the $x_{i}$ coordinate direction, $i=1,2,3$

Wall-normal integral length scale of turbulence

Spanwise integral length scale of turbulence

Wavelength

Streamwise wavelength

Spanwise wavelength

Spanwise wavelength in wall units $=\lambda u^{*} / \nu$

Thwaites' pressure gradient parameter $=\frac{\theta^{2}}{\nu} \frac{d U_{e}}{d x}$

Dynamic viscosity

Kinematic viscosity

Enstrophy $=\overline{\omega_{j} \omega_{j}}$

Density

Initial density field

Dimensionless time

Normalized jet duration

Wall shear stress

Generic flow variable

Power spectral density as function of wavenumber $k_{z}$ 


$\begin{array}{ll}\phi(\omega) & \text { Power spectral density as function of frequency } \omega \\ \psi & \text { Generic flow variable } \\ \Omega & \text { Mean vorticity vector }=\nabla \times U_{j} \\ \Omega_{n} & n \text {-component of the mean vorticity vector } \\ \omega & \text { Frequency } \\ \omega_{j} & \text { Instantaneous vorticity vector }=\nabla \times u_{j}\end{array}$

\section{Subscripts}

0

avg Time average

crit Critical location

e Boundary-layer or shear-layer edge

le Turbulent spot leading edge

$i \quad$ Imaginary component of a complex number

ip Integration point

$i, j, k \quad$ Coordinate-direction identifier

$j \quad$ Index notation coordinate $j=1,2,3$

jet Jet-flow condition

$M A \quad$ Maximum amplification rate

nb Neighbouring nodes

r Reattachment location

Real component of a complex number

rms Root mean square

$s \quad$ Coordinate system aligned with inviscid streamline;

Separation location

Coordinate aligned with inviscid streamline 


$\begin{array}{ll}s p & \text { Suction peak location } \\ t & \text { Coordinate aligned with inviscid streamline } \\ t e & \text { Location of transition completion } \\ & \text { Turbulent spot trailing edge } \\ t s & \text { Location of transition onset } \\ w & \text { Wall shear } \\ x & \text { Component in } x \text { spatial direction } \\ y & \text { Component in } y \text { spatial direction } \\ z & \text { Component in } z \text { spatial direction }\end{array}$

\section{Superscripts}

$\begin{array}{ll} & \text { Fluctuation quantity } \\ & \text { Differentiation with respect to } y \\ + & \text { Quantity normalized using wall parameters }\end{array}$

\section{Mathematical Descriptions}

$\bar{\phi} \quad$ Time average of $\phi$

$\langle\phi\rangle_{z} \quad$ Spanwise average of $\phi$

$\widetilde{\phi} \quad$ Perturbation quantity $=\phi-\langle\phi\rangle_{z}$

\section{Acronyms}

2D Two dimensional

3D Three dimensional

AMD Advanced Micro Devices Corporation

ANSI American National Standards Institute

CFD Computational fluid dynamics

CPU Central processing unit

CVP Counter-rotating vortex pair

DCV Downwind corner vortex 


$\begin{array}{ll}\text { DNS } & \text { Direct numerical simulation } \\ \text { FFT } & \text { Fast Fourier transform } \\ \text { GB } & \text { Gigabyte } \\ \text { GGI } & \text { General grid interface } \\ \text { HV } & \text { Horseshoe vortex } \\ \text { K-H } & \text { Kelvin-Helmholtz } \\ \text { LPT } & \text { Low-pressure turbine } \\ \text { LST } & \text { Linear stability theory } \\ \text { MeTiS } & \text { Multilevel Graph Partitioning Algorithm } \\ \text { PSD } & \text { Power spectral density } \\ \text { PVM } & \text { Parallel virtual machine } \\ \text { RAM } & \text { Random access memory } \\ \text { RMS } & \text { Root-mean-square } \\ \text { SLV } & \text { Shear layer vortex } \\ \text { T-S } & \text { Tollmien-Schlichting } \\ \text { UCV } & \text { Upwind corner vortex } \\ \text { VL } & \text { Vortex loop }\end{array}$




\section{Chapter 1}

\section{Introduction}

\subsection{Motivation}

This thesis investigates laminar-to-turbulent transition in shear layers. To illustrate the importance of shear-layer transition in an engineering context, consider a gas turbine engine, a machine that plays a crucial role in aircraft propulsion and industrial power generation. When exhaust flows through the hot end of the gas turbine, shear layers form on the turbine blade surfaces. The state of these shear layers - whether they are laminar or turbulent, and whether they are attached to or separated from the blade surface-has a significant impact on the performance and efficiency of the gas turbine (e.g. Tani, 1964; Mayle, 1991; Dovgal et al., 1994; and Hodson and Howell, 2005). In a quest to improve the efficiency and costeffectiveness of gas turbine engines, manufacturers have sought to reduce the weight and size of the engine by reducing the number of blades and stages. To compensate, a higher aerodynamic loading must be applied to the remaining blades, increasing the likelihood that attached shear layers may separate from the suction surface of the turbine blade. Low-Reynolds-number

operation, which occurs when an aircraft is operating at low speed or high altitude, increases 
the likelihood that the shear layer will be laminar over a larger segment of the blade chord and thus more susceptible to flow separation (Mayle, 1991). Separation results in decreased flow turning and higher pressure losses, which in turn reduces the isentropic efficiency and power output of the turbine. However, if the laminar shear layer transitions to a turbulent state following separation from the blade surface, turbulent mixing energizes the fluid near the surface of the blade and may allow the separated shear layer to reattach as a turbulent boundary layer. The sequence of separation, transition, and turbulent reattachment forms a "separation bubble" on the blade surface when viewed in a time-averaged sense.

Transition on gas turbine blades highlights the importance for engineers to be able to reliably predict the location and rate of transition in attached or separated shear layers. Unfortunately, current transition models (e.g. Roberts and Yaras, 2005b) lack generality and thus suffer from a large uncertainty in their predictions. In order to improve such transition models, the physical mechanisms that contribute to transition in shear layers need to be further understood. This thesis aims to improve the current understanding of transition in shear layers through fundamental investigations of the multiple instability modes and freestream conditions that contribute to the transition process. The flow conditions prevailing on a low-pressure turbine (LPT) blade present an environment where multiple instability modes and free-stream conditions occur (e.g. Mayle, 1991; Michelassi et al., 2002). For this reason, the fundamental mechanisms investigated in the present research - while generally applicable beyond gas-turbine engines - are here placed in the context of the flow over a LPT blade.

Figure 1.1 illustrates the instability modes present in an exaggerated shear layer (boundary layer) with thickness $\delta$ developing on a LPT blade. Region I contains the instabilities associated with the attached shear layer upstream of separation. Downstream of separation, where the adverse streamwise pressure gradient causes flow reversal underneath the separated shear layer, Region II contains the instability mode associated with the inflectional shape of the velocity profile. Region III contains the instability modes occurring in the attached, reversed flow near 


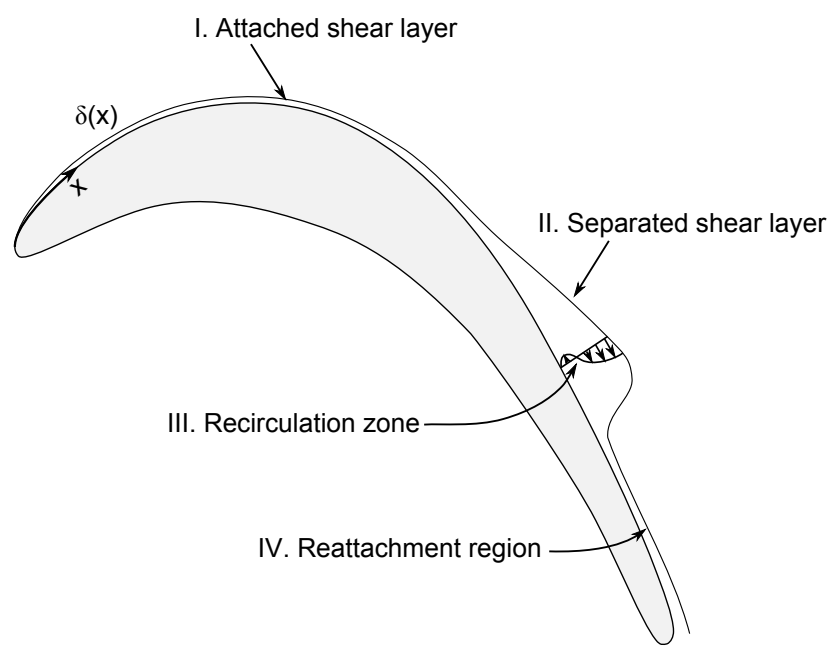

Figure 1.1: Schematic illustrating the regions associated with instability modes on a typical LPT blade.

the wall. Region IV is a highly-unsteady region where transition to turbulence is completed through the action of highly-three-dimensional vortical flow structures, the organization of which is similar to that of turbulent spots, which are well-studied in attached boundary layers (e.g. Wygnanski et al., 1976; Katz et al., 1990; Johnson, 2001).

Within the regions shown in Figure 1.1, there are several possible routes through which transition may occur depending on the level of environmental disturbances in the flow (e.g. Suder et al., 1988). Saric et al. (2002) proposed a "roadmap" to turbulence, reproduced in Figure 1.2, that illustrates the various transition mechanisms occurring in shear layers and their dependence on the level of environmental disturbances in the flow. Path A represents transition that occurs gradually through the linear growth of disturbances in the shear layer. As the disturbances amplify and convect downstream, secondary non-linear modes produce three-dimensional vortical flow structures that eventually break-down into regions of locally-turbulent flow. This so-called "natural" transition mechanism occurs in attached flows under mild streamwise pressure gradients and low free-stream disturbance levels. Path E represents transition in flows with very strong disturbances where the linear-growth stage is bypassed and the break-down to locally-turbulent flow occurs rapidly in the shear layer, 


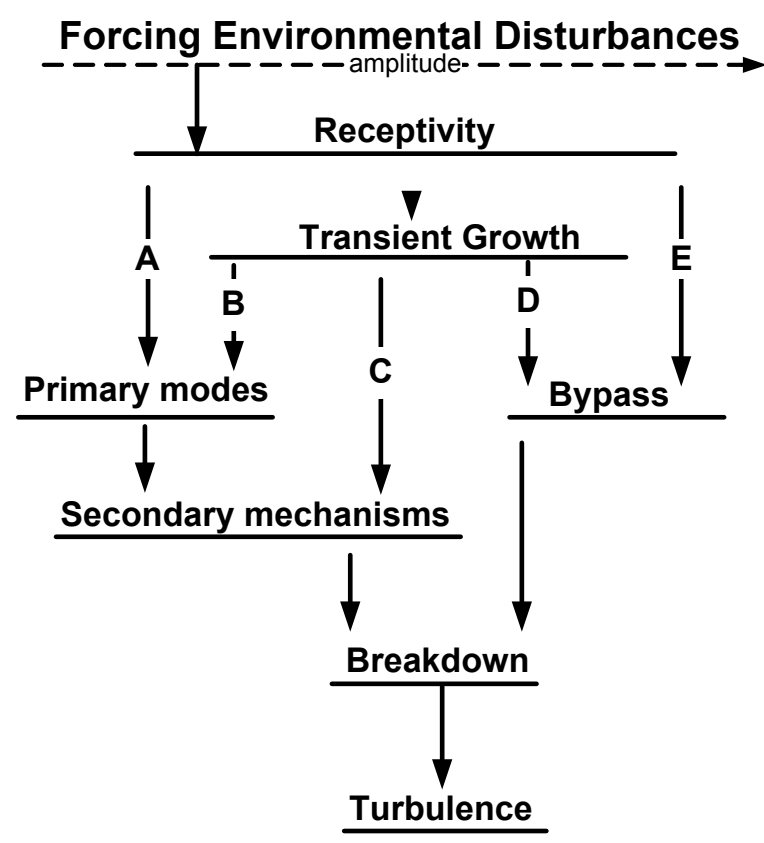

Figure 1.2: Roadmap to turbulence. Reproduced from Saric et al. (2002).

which Morkovin (1969) termed "bypass" transition. In between natural and bypass transition, transient growth mechanisms involve the interaction between instability waves and elongated streamwise structures produced, for example, by streamwise curvature (e.g. Hall, 1985), threedimensionality of the boundary layer (e.g. Saric et al., 2003), or the inclusion of free-stream turbulence into the boundary layer (e.g. Jacobs and Durbin, 2001).

Upon identifying the instability modes occurring on a LPT blade (as in Figure 1.1) and the influence of environmental disturbances on the transition process (as in Figure 1.2), a qualitative framework describing the overall transition process can be created, as illustrated in Figure 1.3. The framework, described in greater detail in Chapter 2, shows that transition from a laminar shear layer to turbulence begins with the creation of disturbances in the shear layer through a receptivity process, followed by the amplification of these disturbances via one or multiple instability modes to form a localized turbulent spot, and the growth of the spot into a fully-turbulent flow. Within this general framework, however, several questions remain, enclosed in ellipses in Figure 1.3: 
1. Do the instability modes in the attached and separated shear layers interact, and what effect may such an interaction have on the transition process?

2. How is the transition process modified if it occurs under a three-dimensional (3D) pressure field, and hence with a $3 \mathrm{D}$ velocity profile?

3. How does strong free-stream acceleration affect the formation of coherent vortical flow structures during transition?

4. What are the regeneration mechanisms that contribute to the growth of the turbulent spot?

5. What is the effect of elevated free-stream turbulence on the overall transition process?

Although numerous, ongoing attempts have been made to answer these questions, the contributions described in published literature to date contain only incremental answers that accumulatively provide only a partial understanding of the overall transition process.

The above questions indicate that there are gaps in our understanding of transition in shear layers. An awareness of these gaps is beginning to emerge in published literature. Regarding Question 1, Diwan and Ramesh (2009) published measurements which suggest that the inflectional instability of the separated shear layer originates in the viscous instability developed in the upstream attached boundary layer. Hain et al. (2009) found that the instability in the separated shear layer has almost the same most-unstable frequency as the attached boundary layer, which was also previously observed by Roberts and Yaras (2006), and that modifying the instability growth in the attached boundary layer also modifies the growth of the inflectional instability in the separated shear layer. McAuliffe and Yaras (2006) suggest that the correlation between the attached and separated instability modes may affect the vortical flow structures that periodically shed from the separated shear layer, and the subsequent interaction of these structures appears to have a significant role in the transition 


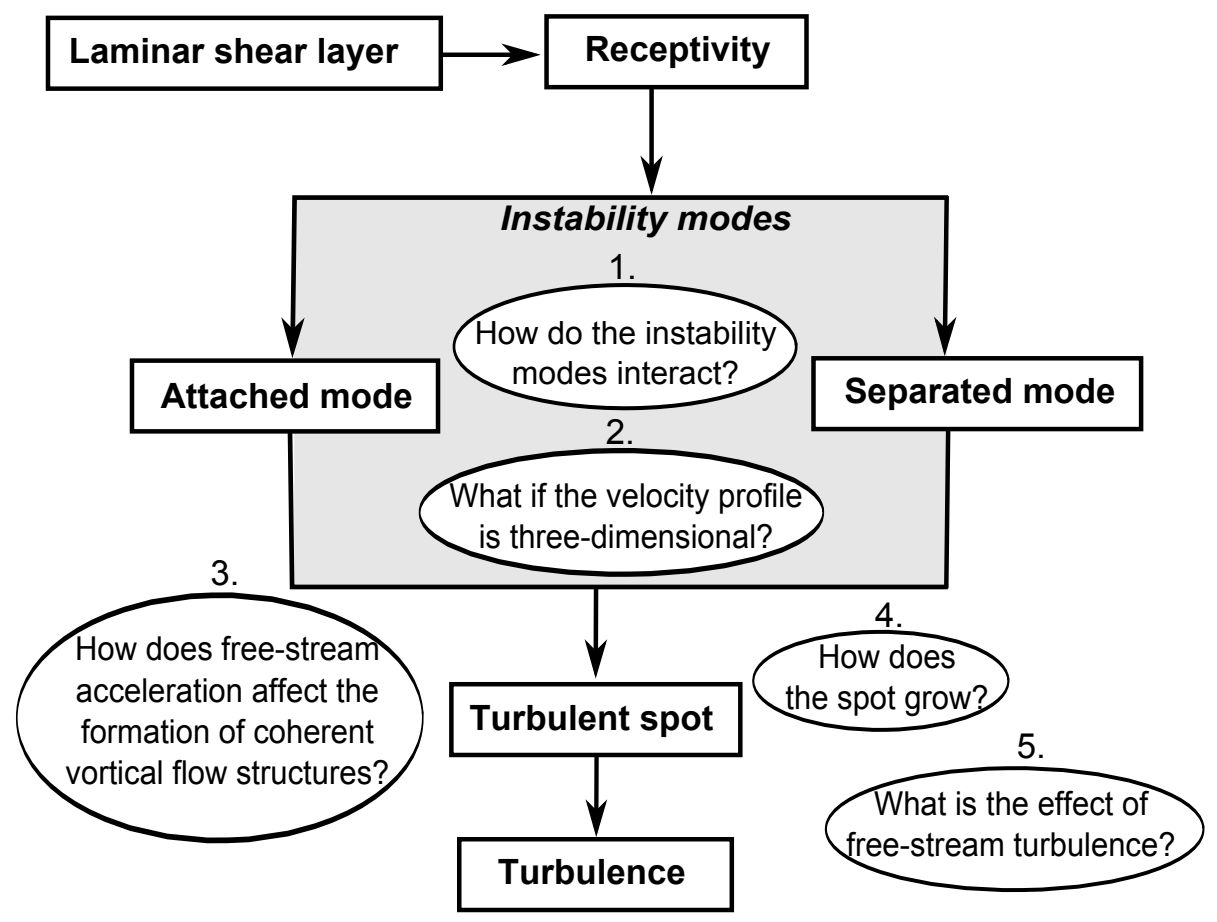

Figure 1.3: Qualitative framework of the transition process in laminar shear layers. Outstanding questions at each stage of the transition process, which are the focus of this thesis, are enclosed in ellipses. 
to turbulence.

Regarding Question 2, Pullan and Harvey (2007) note that a 3D pressure field is common on LPT blades due to the highly-flared hub and casing walls that occur in modern gas turbines, which produces an effective sweep angle in radially-stacked blades. Such a pressure field produces a velocity component in the boundary layer that is perpendicular to the local inviscid streamline. Although such flow three-dimensionality is known to generate additional instability modes (as described in Chapter 2), it is currently unclear how these extra modes affect the transition process.

Regarding Question 3, while the ability of strong free-stream acceleration to relaminarize an attached turbulent boundary layer at moderate Reynolds numbers has been understood since the work of Moretti and Kays (1965) and Narasimha and Sreenivasan (1979), the stabilizing effect of free-stream acceleration on the highly-amplified instability modes that result in the formation of coherent vortical structures during transition has not been thoroughly investigated. Yaras (2007) showed that a turbulent spot triggered by a jet of air ejected from a small hole in the test surface was able to grow to a significant size despite being subjected to a level of free-stream acceleration greater than that required for relaminarization. This suggests that the instability of the separated shear layers created by the jet as it pushes through the boundary layer overpowers the stabilizing effect of the free-stream acceleration. As such a scenario has been proposed for active flow control on turbine blades (e.g. Karagozian, 2010), the effect of free-stream acceleration on the shear-layer stability and the resulting formation of coherent vortical flow structures during the transition process warrants further investigation.

Regarding Question 4, the rate-of-production and rate-of-growth of turbulent spots following the saturation of instability modes are the basis for the majority of models that predict the transition rate (e.g. D'Ovidio et al., 2002; Roberts and Yaras, 2005b). However, the fundamental mechanisms driving the creation of coherent flow structures within the turbulent 
spot - the process that is primarily responsible for the spot's lateral growth (e.g. Strand and Goldstein, 2011) - are not completely understood. The two mechanisms proposed by Singer and Joslin (1994) and Schröder and Kompenhans (2004) (described in Chapter 2) are somewhat contradictory and fall short in explaining the various turbulent-spot topologies observed in published literature.

Regarding Question 5, the role of free-stream turbulence in altering the route to transition was shown earlier by the transition roadmap in Figure 1.2, but that roadmap was based primarily on flows with mild pressure gradients. In contrast, the flow in a typical turbine blade row will include strong streamwise pressure gradients that may alter the receptivity and instability processes involved in transition as well as modify the length scales, intensity, and isotropy of the free-stream turbulence. Although some recent studies have addressed the effect of favourable pressure gradients (e.g. Talamelli et al., 2002), adverse pressure gradients (e.g. Zaki and Durbin, 2006), and integral length scale (e.g. Brandt et al., 2004) on transition dominated by free-stream turbulence, the understanding of how free-stream turbulence modifies the receptivity and instability process in a realistic LPT environment is not complete.

\subsection{Thesis Objectives}

The studies referenced in the preceding section indicate that while there has been significant progress toward understanding the overall transition process, the detailed instability mechanisms, their interaction, and the influence of free-stream conditions are still not completely understood. The proposed research therefore aims to improve the understanding of the interaction of instability modes and free-stream conditions in shear-layer transition as encountered on a LPT blade. The following are the detailed objectives of the proposed research: 
1. Establish the extent of the interaction between the instability modes associated with the attached and separated portions of a laminar boundary layer and how this interaction affects the transition process.

2. Establish how three-dimensionality of the pressure field imposed onto the attached and separated shear layers by the free-stream flow influences the development of instability modes.

3. Establish how coherent vortical flow structures are created by an impulsively-activated transverse jet, and the affect of strong free-stream acceleration on the instabilities that result in the formation of said vortical flow structures.

4. Establish the internal structure and growth mechanism(s) of coherent vortical flow structures in a turbulent spot.

5. Establish how elevated free-stream turbulence influences the instability and transition processes that are observed under low free-stream turbulence conditions.

\subsection{Approach}

Transition encompasses a very wide range of temporal and spatial scales and is very sensitive to disturbances that may be artificially created by the measurement apparatus. The fine spatial resolution required for detailed analysis, especially in the early stages of transition, generally restricts the experimental measurements that can be obtained. To overcome this limitation, the approach taken by the present research consists of a series of computational fluid dynamics (CFD) simulations where the equations of motion are solved directly on a highlyrefined temporal and spatial grid without any turbulence modelling assumptions, referred to as direct numerical simulation (DNS). In addition to fine spatial and temporal resolution, this approach produces information of the flowfield in a temporally-synchronized form, which is 
helpful in analyzing how transient flow structures evolve in time. The main drawback of DNS is large computational expense, which is proportional to the flow Reynolds number raised to the third power (Pope, 2000). Fortunately, due to the rapid increase in the performance and affordability of computing power, DNS is becoming practical for low-Reynolds number flows, which are the focus of the current study. Validation of the computational approach is achieved through comparison with benchmark experimental and computational datasets available in published literature.

\subsection{Outline of Thesis}

This chapter introduced the motivation and objectives of the current research and outlined the approach that is used to fulfill these objectives. Chapter 2 reviews the literature relevant to shear-layer transition. Chapter 3 describes the computational approach, which includes a discussion of the governing equations, the method for discretizing the governing equations, and the method for solving the discretized system of equations. The data reduction procedures used to analyze the simulation results are also described in Chapter 3. In Chapters 4-8, the thesis objectives enumerated in Section 1.2 are addressed in sequence by reprinting the following journal papers:

1. Brinkerhoff, J.R. and Yaras, M.I. (2011), "Interaction of viscous and inviscid instability modes in separation-bubble transition," Physics of Fluids, 23, pp. 124102.

2. Brinkerhoff, J.R. and Yaras, M.I. (2013), "Direct numerical simulations of transitional separation-bubble development in swept-blade flow conditions," ASME Journal of Turbomachinery, 135, pp. 041006.

3. Brinkerhoff, J.R. and Yaras, M.I. (2012), "Direct Numerical Simulation of a Square Jet Ejected Transversely into an Accelerating, Laminar Main Flow," Flow, Turbulence and 
Combustion, 89, pp. 519-546.

4. Brinkerhoff, J.R. and Yaras, M.I. (2013), "Numerical investigation of the generation and growth of coherent flow structures in a triggered turbulent spot," submitted to the Journal of Fluid Mechanics.

5. Brinkerhoff, J.R. and Yaras, M.I. (2013), "Transition in a boundary layer subjected to favourable and adverse streamwise pressure gradients and elevated free-stream turbulence," submitted to the Journal of Fluid Mechanics.

Finally, the contributions of the present research are summarized in Chapter 9 along with conclusions and recommendations for future investigation. 


\section{Chapter 2}

\section{Literature Review}

This chapter provides a detailed survey of the literature pertaining to the stability of shear layers that develop under conditions that are typical of a low-pressure turbine (LPT) blade. Readers who are familiar with the stability of attached and separated shear layers under low and elevated free-stream turbulence levels may skip reading this chapter without affecting the continuity in reading the thesis manuscript; concise summaries of the literature are provided in the introduction sections of the journal papers reproduced in Chapters 4-8.

The suction surface of a LPT blade can be represented by a flat plate with an imposed pressure gradient, as shown in Figure 2.1. Such a simplification is reasonable because the convex curvature of a realistic LPT-blade suction surface has only a secondary effect on the boundary-layer development (e.g. Roberts, 2005). An exaggerated boundary layer is shown in Figure 2.1 to illustrate the effect of the applied pressure distribution on the development of the boundary layer in the different regions of the flow: the attached laminar region between the leading-edge and the suction peak, the attached inflectional region between the suction peak and the point of flow separation, the separated-flow region, and the turbulent region where the time-averaged flow appears to reattach to the plate surface. This chapter will review literature 
pertaining to the stability of the boundary layer in these regions of the flow.

Near the leading edge of the flat plate, the laminar boundary layer is beginning to grow and its spatial scales change rapidly, which makes it susceptible to the creation of small perturbations in the boundary layer by the inclusion of disturbances from the free-stream in a process referred to as receptivity (e.g., Morkovin, 1969; Goldstein, 1983; Davis, 1996). To determine the response of the perturbed laminar boundary layer, the disturbance can be modeled as a two-dimensional velocity wave aligned with the free-stream having the form

$$
v^{\prime}(x, y, t)=\hat{v}(y) e^{i(\alpha x-\alpha c t)},
$$

where $x$ and $y$ are the streamwise and wall-normal directions, respectively, $\hat{v}$ is the wave amplitude, $\alpha$ is the streamwise wave number, and $c$ is the wave phase speed. The amplitude of the disturbance $\hat{v}$ is governed by a fourth-order equation called the Orr-Sommerfeld equation:

$$
(U-c)\left(\hat{v}^{\prime \prime}-\alpha^{2} \hat{v}\right)-U^{\prime \prime} \hat{v}=-\frac{i}{\alpha R e_{\delta}}\left(\hat{v}^{i v}-2 \alpha^{2} \hat{v}^{\prime \prime}+\alpha^{4} \hat{v}\right)
$$

where $U$ is the free-stream velocity of the undisturbed flow, $R e_{\delta}=U \delta / \nu$ is the Reynolds number based on the local boundary-layer thickness $\delta$, and the prime denotes differentiation with respect to $y$. Equation 2.2 is a linearized form of the governing conservation equations applied to a sinusoidal disturbance, assuming that the flow remains parallel to the solid test surface. Gaster (1974) showed that this assumption is acceptable for analyzing the initial growth of disturbances in attached flows. For a full discussion of stability theory, the reader is referred to Schlichting (1968), Mack (1984), or White (2006).

Equation 2.2 shows that the response of a boundary layer after the creation of a disturbance is strongly influenced by the local Reynolds number. If the Reynolds number is infinite (i.e. the fluid viscosity is zero), the right-hand side of Equation 2.2 becomes zero, resulting in a stability mode referred to as inviscid instability. Rayleigh (1887) showed that for a boundary layer to 

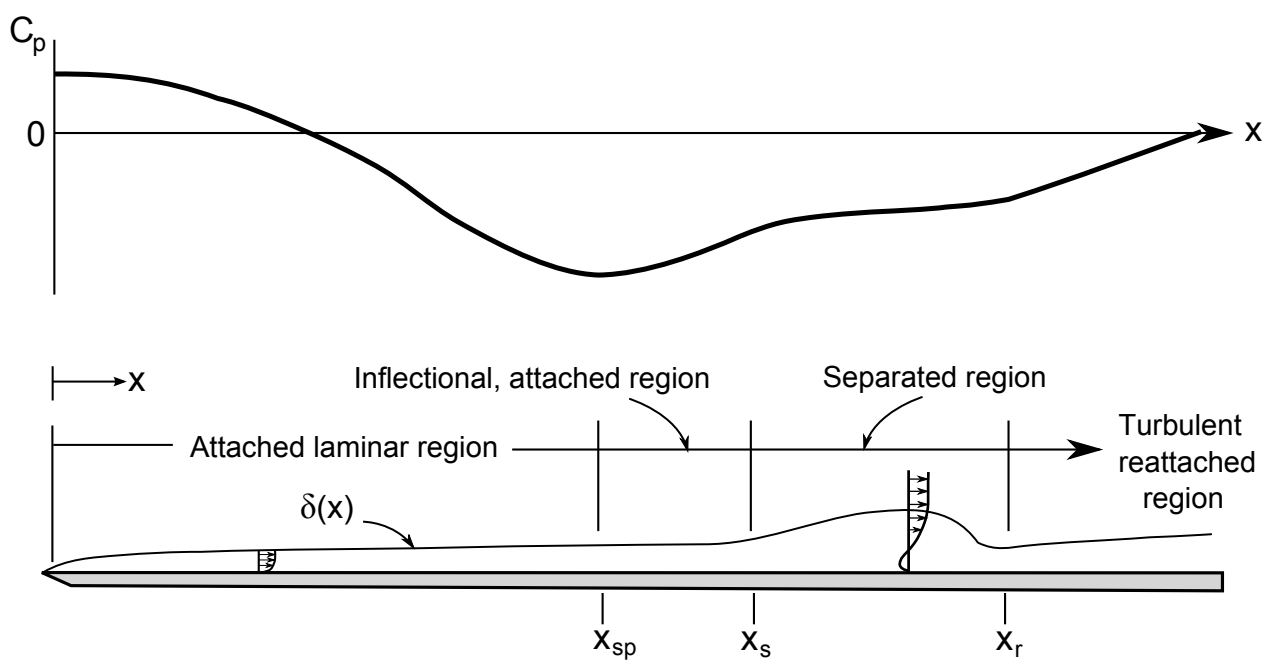

Figure 2.1: Boundary layer developing on a flat surface subjected to a streamwise pressure distribution.

be unstable through an inviscid instability, it must possess an inflectional velocity profile. However, the attached laminar boundary layer in Figure 2.1 does not possess an inflectional velocity profile up to the suction peak, so another instability mode must be present for a disturbance to grow. In a viscous fluid, the Reynolds number is finite and the right-hand side of Equation 2.2 is non-zero, which suggests that under certain flow conditions, viscous forces may destabilize the boundary layer to certain disturbance frequencies through a viscous instability. This is confirmed by solving for the eigenvalues of the Orr-Sommerfeld equation - the $\alpha$ and $c$ terms in Equation 2.2 - which determine if the disturbance will be damped (the boundary layer is viscous-stable) or grow (the boundary layer is viscous-unstable). The corresponding eigenfunctions determine the shape of the disturbance. The criterion for stability is determined by the sign of the imaginary component of the calculated eigenvalues; if $\alpha$ is assumed to be real and $c$ is complex, $c_{i}>0$ indicates that the disturbance will grow in time, while if $\alpha c$ is assumed to be real and $\alpha$ is complex, $\alpha_{i}<0$ indicates that the disturbance will grow in space, where the subscript $i$ indicates the imaginary component of the complex eigenvalue. Neutral solutions where there is no disturbance growth are important, as they divide the parameter space into stable and unstable regions. 
A typical stability curve that plots the dimensionless spatial-growth eigenvalue $F=\alpha c \nu / U_{o}^{2}$ $\left(\times 10^{6}\right)$ versus $R e$ for an attached laminar boundary layer is shown in Figure 2.2; the region inside the curves corresponds to flow conditions that are unstable through viscous instability. The oscillatory disturbances that grow due to viscous instability are referred to as Tollmien-Schlichting (T-S) waves. The growth of T-S waves is strongly-influenced by the Reynolds number, as shown in Figure 2.2, and the local streamwise pressure gradient, which modifies the wall-normal momentum distribution in the boundary layer in a manner that promotes disturbance growth for adverse pressure-gradients and impedes disturbance growth for favourable pressure gradients (Knapp and Roache, 1968; Saric and Nayfeh, 1975). Beyond these two primary factors, it has already been shown in Chapter 1 that environmental disturbances - including free-stream turbulence, surface roughness, and acoustic waves - also influence disturbance-growth, and may result in a different transition mechanism if the amplitude of environmental disturbances is sufficiently large (Saric et al., 2002). Figure 1.2 has already illustrated the various transition mechanisms and how they depend on the level of environmental disturbances in the flow. Path A represents transition that occurs gradually in a low-disturbance environment, referred to as "natural" transition, while path E represents the rapid break-down to turbulent flow that occurs in a high-disturbance environment, referred to by Morkovin (1993) as "bypass" transition. In between natural and bypass transition, transient growth mechanisms are affiliated with elongating streamwise structures in the boundary layer interacting with T-S waves, to be discussed in more detail shortly.

Returning to the boundary layer developing under an imposed streamwise pressure distribution, shown in Figure 2.1, as the boundary layer develops in the favourable pressure-gradient region upstream of the suction peak, disturbance growth is likely to be impeded due to the strong acceleration of the flow. However, once the flow enters the adverse pressure-gradient region downstream of the suction peak, the disturbances will be strongly amplified. Several transition scenarios are possible, shown in Figure 2.3: (A) the boundary-layer disturbances 


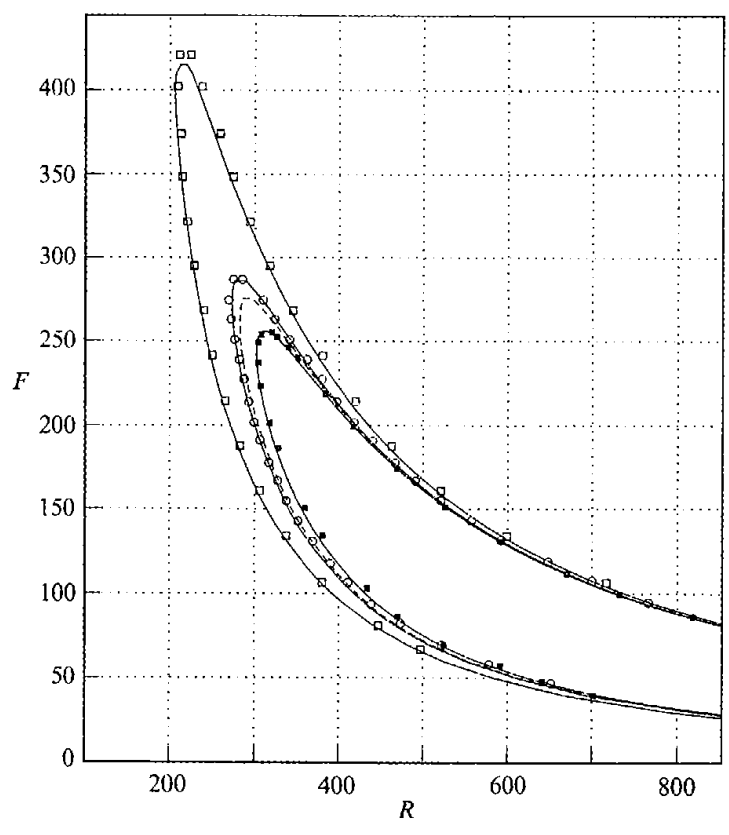

Figure 2.2: $\quad$ Stability curves for spatial growth in a Blasius boundary layer, reproduced from Bertolotti et al. (1992). The different symbols correspond to different measures of spatial growth, as described by Bertolotti et al. (1992).

grow slowly so that the boundary layer remains laminar past the suction peak and separates as a laminar shear layer, after which it transitions to turbulence; (B) the boundary-layer disturbances are mildly amplified downstream of the suction peak and so the boundary layer begins transition prior to separation, separating as a transitional shear layer; and $(\mathrm{C})$ the boundary-layer disturbances are strongly amplified downstream of the suction peak, causing complete transition to turbulence prior to separation. Although literature is available for all three scenarios, this thesis focuses primarily on scenario A and this chapter will therefore focus on the transition mechanisms occurring in laminar separated shear layers. For shear layers of this type, Rist and Maucher (2002) observed that two instability modes may lead to transition: an inviscid mode in the separated shear layer resulting from the inflectional shape of the local velocity profile, and a viscous mode in the reversed-flow region near the wall. The inviscid mode will be reviewed first, followed by a review of the viscous mode. 
(a)

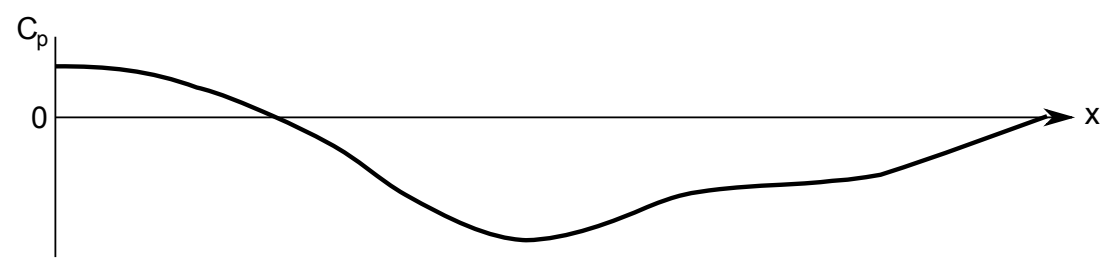

Scenario A Laminar separation

(b) Transitional separation

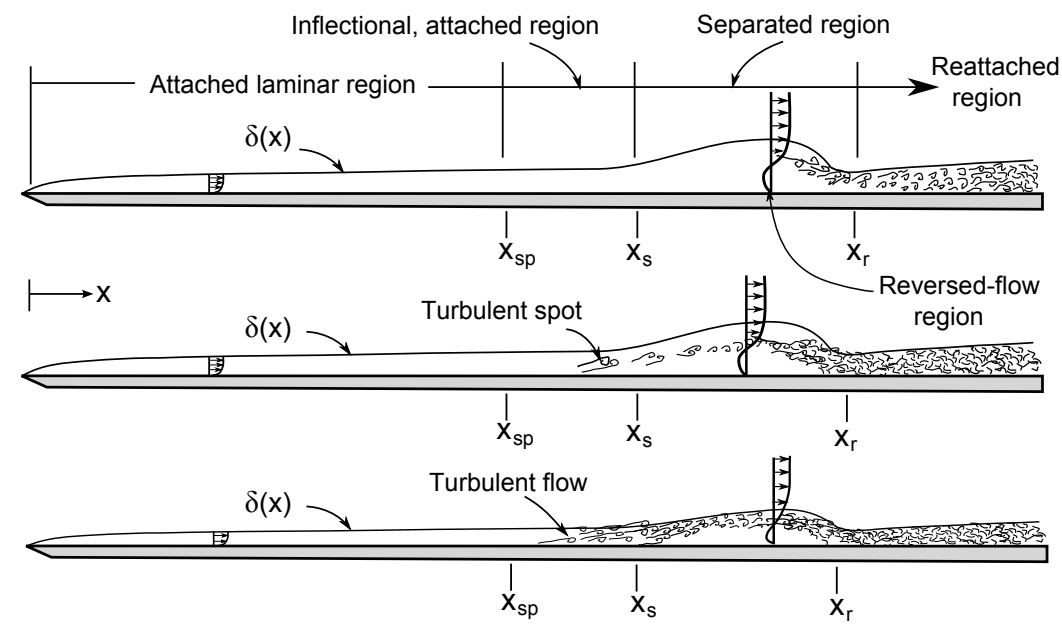

Figure 2.3: (a) Typical pressure distribution on the suction-surface of an LPT blade. (b) Possible transition scenarios of a separated boundary layer.

\subsection{Transition in Separated Shear Layers}

\subsubsection{Primary shear-layer instability}

When the boundary layer enters the adverse streamwise pressure gradient region downstream of the suction peak, an inflection point is created in the streamwise velocity profile. The presence of an inflection point makes the flow unstable to an inviscid instability mode. To explain the physical mechanism occurring in the inviscid mode, Drazin (2002) considered the simplified case of a free shear-layer approximated as a vortex sheet. Figure 2.4 shows the vortex sheet after it has been perturbed by a disturbance so that the vortex sheet resembles a sinusoid. The vorticity associated with the perturbed segments of the vortex sheet induces a velocity field that has a streamwise component, shown in Figure 2.4 by the hollow arrows. The induced streamwise velocities will carry vortex lines away from points like A and toward points like B, 


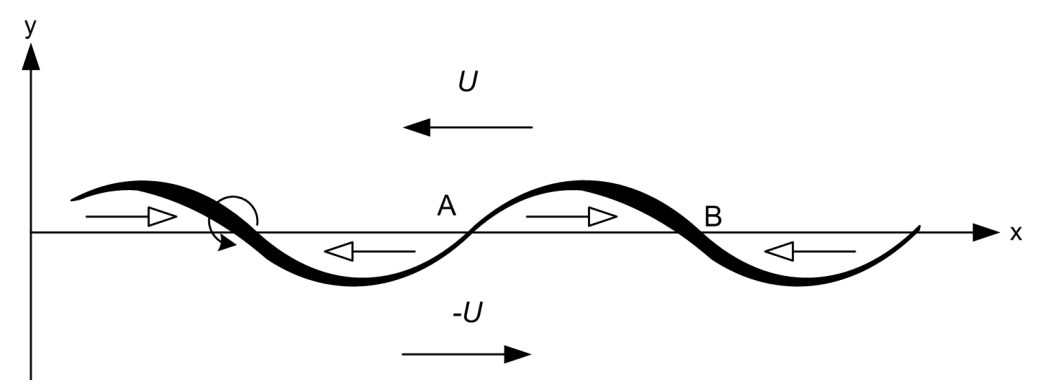

Figure 2.4: Disturbance growth in a vortex sheet through inviscid instability. Adapted from Drazin (2002).

resulting in a periodic streamwise grouping of spanwise vorticity, shown in Figure 2.4 by the varying width of the vortex sheet. Although the spanwise vorticity waves are similar to the T-S waves created through a viscous instability mode, the physical mechanism of the inviscid mode is fundamentally different. To distinguish this difference, the waves associated with the inviscid mode will be referred to as Kelvin-Helmholtz (K-H) waves. After the K-H waves are created, they induce streamwise-normal velocities that amplify the displacement of the vortex sheet, leading to spatial growth of the perturbation as the vortex sheet convects in the downstream direction. The frequency of the K-H waves growing in free and separated shear layers has been well-documented, generally expressed as a Strouhal number $\left(S r_{\theta}=f \theta_{s} / U_{e s}\right)$ based on conditions at separation for the latter case. The disturbance frequency that produces the highest amplification rate in a variety of free and separated shear layers is shown in Table 2.1. As the K-H waves convect downstream, their induced velocity field amplifies the perturbation of the separated shear layer until the shear layer rolls-up into discrete spanwise vortices that shed from the shear layer and convect downstream (Malkiel and Mayle, 1996; Spalart and Strelets, 2000).

\subsubsection{Secondary instabilities}

Following the initial roll-up and shedding of the shear layer, the shed spanwise vortices may become unstable through a sub-harmonic secondary mode of instability that develops 
Table 2.1: Instability Strouhal numbers in studies of free and separated shear layers

\begin{tabular}{lc}
\hline Investigators & $S_{\theta}$ \\
\hline McAuliffe and Yaras (2007b) & 0.011 \\
McAuliffe and Yaras (2005) & $0.008-0.016$ \\
Talan and Hourmouziadis (2002) & $0.010-0.014$ \\
Yang and Voke (2001) & $0.005-0.011$ \\
Muti-Lin and Pauley (1996) & $0.005-0.008$ \\
Ho and Huerre (1984) & 0.016 \\
\hline
\end{tabular}

at half the frequency of the primary shear-layer instability, illustrated in Figure 2.5. The secondary instability causes the primary vortices shedding from the separated shear layer to be alternatively shifted in the transverse direction. A vortex shifted into the high-velocity stream convects more rapidly than a vortex shifted into the low-velocity stream, and as the faster-moving vortex overtakes the previously-shed slower vortex, the mutually-induced vorticity fields causes a rotation of the two sequential vortices about each other (Winant and Browand, 1974), eventually resulting in merging of the two vortices. The new merged vortex train has approximately double the streamwise spacing of the original vortex train (Ho and Huerre, 1984), hence the vortex-merging process is identified as a sub-harmonic of the primary instability mode. Investigations of planar free shear layers (which resemble separated shear layers) have observed third and higher sub-harmonics and this instability-driven flow development is considered to be the dominant mechanism for transverse growth of free shear layers (Ho and Huerre, 1984). However, McAuliffe and Yaras (2007b) observe that this subharmonic instability is not a universal feature in all separated shear layers, but is limited to shear layers with lower Reynolds numbers $\left(R e_{\theta s}\right.$ of about 100).

Another secondary instability that may occur in separated shear layers is related to the spanwise non-uniformity that is frequently encountered in separated and free shear layers (Ho and Huerre, 1984; McAuliffe and Yaras, 2007b). The spanwise non-uniformity has been tied to the development of elongated, counter-rotating streamwise vortices (Pierrehumbert 


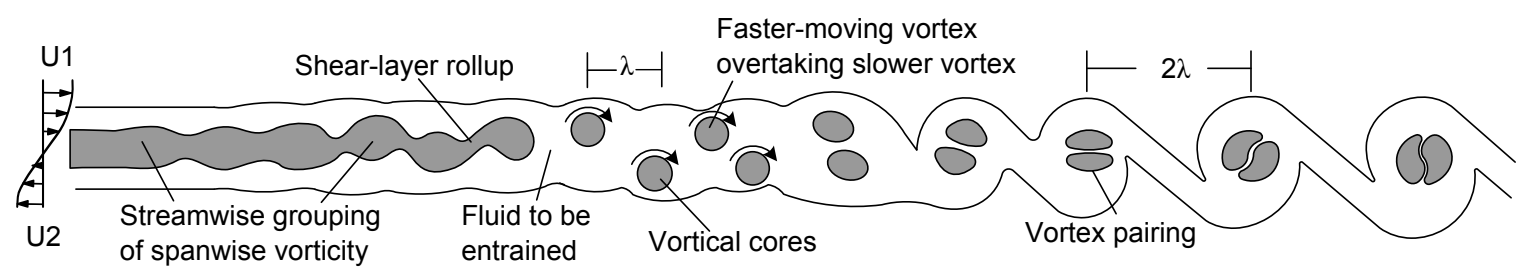

Figure 2.5: Sketch of sub-harmonic secondary instability of a free shear layer

and Widnall, 1982; Huang and Ho, 1990) which interact with the primary spanwise vortices produced by the K-H mechanism of instability. Malkiel and Mayle (1996) suggest that the interaction of the streamwise and spanwise vortices is responsible for generating small-scale turbulence near the core of the streamwise vortices, likely through the creation of coherent vortical structures in the separated shear layer. Watmuff (1999) and McAuliffe and Yaras (2007a) have observed hairpin-like structures in the separated shear layer-referred to by Watmuff (1999) as "vortex loops" — which provide a mechanism for the wall-normal exchange of streamwise momentum through the induced velocities of the vortex loops. Figure 2.6 shows the simulation results of McAuliffe and Yaras (2007b), illustrating the break-down of a separated shear layer under low free-stream turbulence conditions. Figure 2.6(a) shows iso-contours of the vorticity magnitude, showing a uniform shear layer up to about $x=0.60 \mathrm{~m}$, after which three-dimensional fluctuations are observed in the shear layer followed by a rapid break-down of the shear layer into smaller vortical scales. Figure 2.6(b) shows iso-contours of static pressure fluctuation, which reveals hairpin-like vortical structures in the separated shear layer.

In the simulation results shown in Figure 2.6(b), which were performed with low free-stream turbulence, the coherent structures appear to consist of a single vortex loop with its legs pointing upstream. In a subsequent simulation of McAuliffe and Yaras (2007a) with elevated free-stream turbulence, the coherent structure consisted of a wave packet with multiple vortex loops as sketched in Figure 2.7. The legs of the vortex loop at the leading-edge of the packet point upstream while the subsequent vortex loops point downstream as they curl around the column of low-momentum fluid ejected away from the wall by the induced velocity of the 

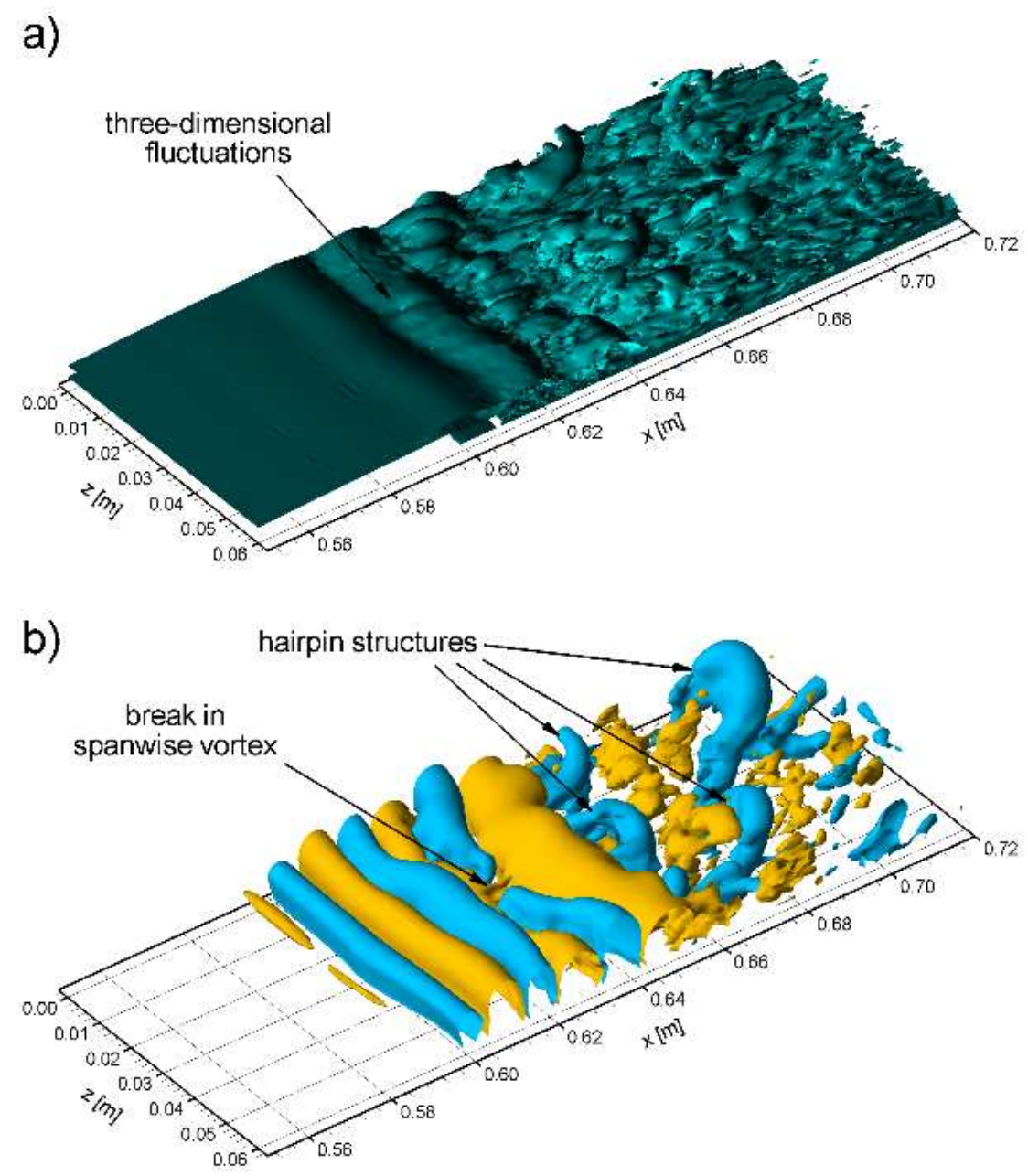

Figure 2.6: Coherent structures in the separated shear layer under low free-stream turbulence levels illustrated through $(a)$ vorticity iso-surfaces $\Omega=1500 \mathrm{~s}^{-1}$ and $(b)$ pressure fluctuation iso-surfaces $p^{\prime}=-1 \mathrm{~Pa}$ (blue), $p^{\prime}=+1 \mathrm{~Pa}$ (yellow). Adapted from McAuliffe (2007). 


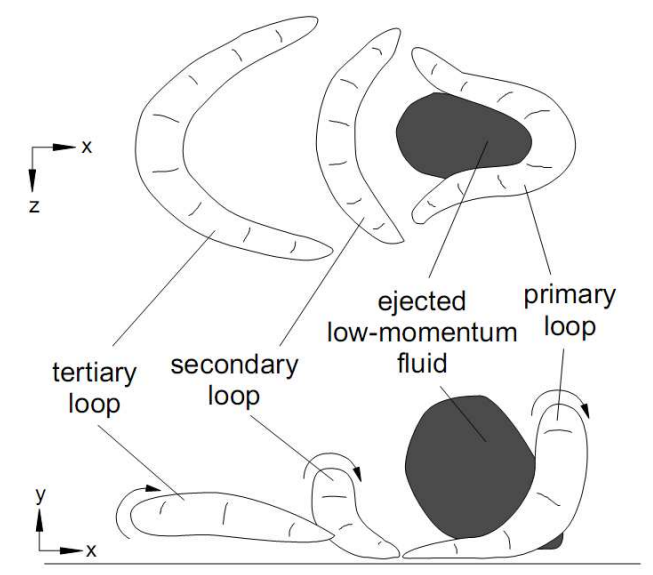

Figure 2.7: Schematic of the vortex loop structure observed in the separated shear layer under elevated free-stream turbulence. Reproduced from McAuliffe (2007).

primary loop. This topology approximately resembles the organization of hairpin vortices in the near-wall region of turbulent boundary layers (e.g., Adrian et al., 2000), except that the legs of vortex loops in attached turbulent boundary layers only point upstream. Further study is needed to determine if the wave-packet topology with an upstream-pointing vortex loop followed by multiple downstream-pointing vortex loops observed by McAuliffe and Yaras (2007a) in a separated shear layer under high free-stream turbulence also occurs in separated shear layers under low free-stream turbulence conditions.

\subsection{Transition in Attached Laminar Boundary Layers}

The preceding section reviewed the mechanisms for disturbance-growth in a separated shear layer. Rist and Maucher (2002) identified another instability mode in the reversed-flow region near the wall underneath the separated shear layer. Disturbance growth in this region occurs through a viscous mode as a result of the high rate of strain prevailing in the reversed-flow boundary layer. The viscous mode will dominate the transition process if the reversed-flow velocity is above a threshold value, identified by Alam and Sandham (2000) as approximately $15-20 \%$ of the local free-stream velocity. In such instances, the disturbance growth is expected 
to develop in a manner similar to an attached boundary layer, which generally occurs in three stages: (i) linear growth of T-S waves; (ii) non-linear growth through the formation of three-dimensional flow structures as a result of secondary instabilities; and (iii) breakdown into localized regions of turbulence, referred to as "turbulent spots," which grow and eventually merge to form a fully-turbulent boundary layer. The details of attached boundarylayer transition will be therefore be reviewed to provide a framework for understanding the growth of disturbances in the reversed-flow region.

\subsubsection{Linear growth stage}

Once the local Reynolds number exceeds a critical value, $R e_{\text {crit }}$, the boundary layer becomes viscous-unstable and disturbances amplify to form T-S waves, which grow as they convect downstream. The first experimental observation of this was achieved by Schubauer and Skramstad (1947). Numerous researchers refer to the initial growth of T-S waves as the "linear growth stage" because the disturbance amplitude grows linearly when plotted on logarithmic axes (Reed et al., 1996). It is worth reiterating that the T-S wave is the eigenfunction calculated from stability theory and its growth rate is determined by its eigenvalues. Figure 2.8 shows the typical shape of an unstable eigenfunction in the linear growth region by plotting the distribution of streamwise disturbance-velocity RMS in the boundary layer, as measured by Reynolds and Saric (1986). The maximum amplitude is observed near the wall $(y \approx 0.1 \delta)$ and a sharp zero occurs in the vicinity of the critical layer, where the phase speed of the T-S wave equals the speed of the local mean flow, indicating that the T-S wave propogates downstream at a fraction of the local boundary-layer edge velocity. From the shape of the disturbance profile, it is clear that the disturbance will cause spanwise vorticity to accumulate near the disturbance maximum. For a spatially-growing T-S wave, the streamwise wavelength remains constant (Kachanov, 1994), and thus to a stationary observer, the convecting T-S waves will appear as concentrated regions of spanwise vorticity ("vortex tubes") that propagate downstream in a 


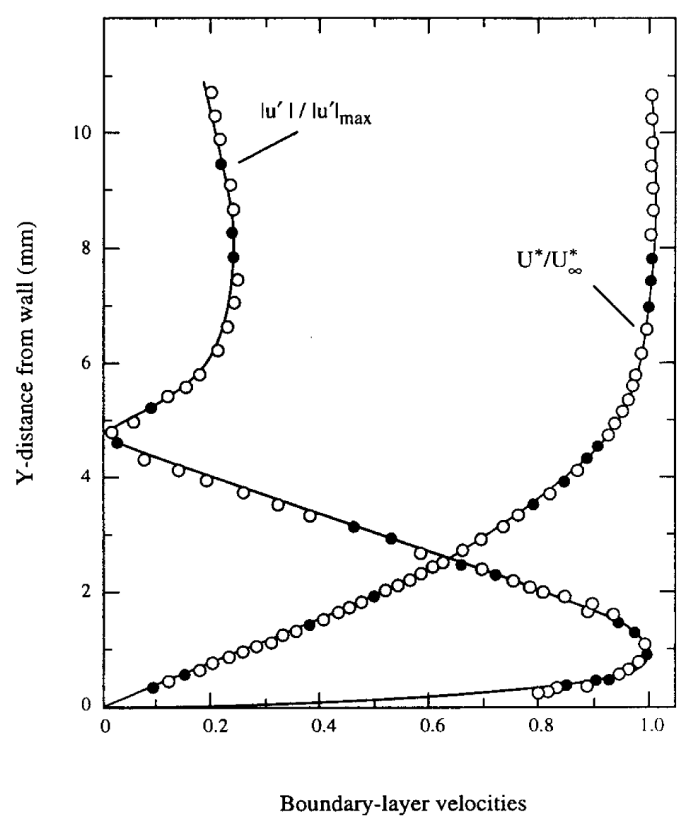

Figure 2.8: RMS-amplitude distribution of the streamwise disturbance velocity illustrating a typical eigenfunction in the linear-growth region. Comparison between linear stability theory (curves) and experiment (points). Reproduced from Reynolds and Saric (1986).

streamwise-periodic manner.

\subsubsection{Non-linear growth and secondary instabilities}

By the end of the linear growth region, the amplitudes of the T-S waves have increased substantially, up to about 1-2\% of the free-stream velocity (Asai and Nishioka, 1989; Kachanov, 1994). These T-S waves develop an instability which manifests itself as waviness in the spanwise direction. This instability of the T-S waves is referred to as a secondary instability, and early experimental observations were made by Klebanoff and Tidstrom (1959) and Klebanoff et al. (1962). As the amplitude of the waviness increases, the streamwise-oriented segments of the wavy vortex tubes induce a wall-normal velocity on each other that lifts the vortex tube away from the wall at the crests of the T-S waves and into the higher-velocity portion of the boundary layer. The non-uniform velocity field pulls the crest of the vortex tube downstream, stretching 


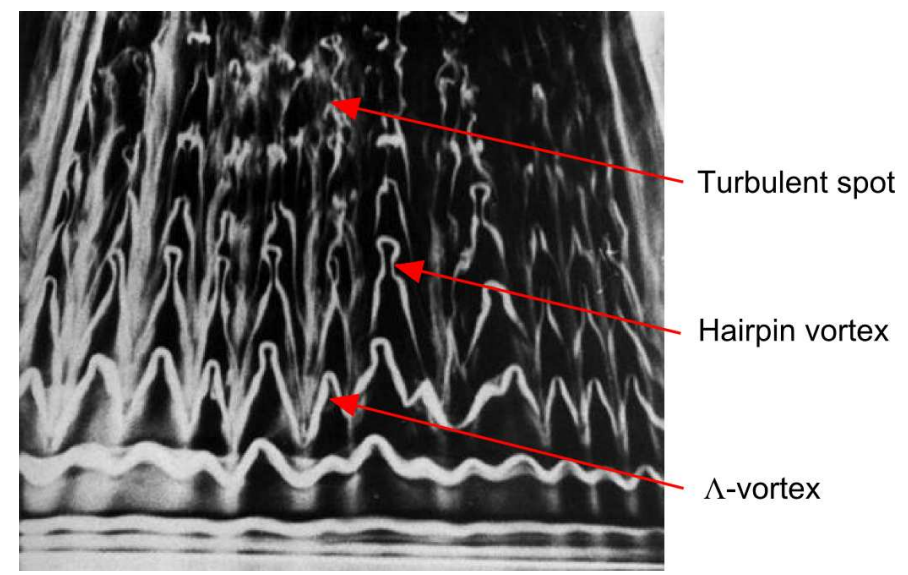

Figure 2.9: Smoke-wire visualizations of $\Lambda$-vortices, hairpin-vortices, and turbulent spots. Adapted from Perry et al. (1981).

the vortex tube into a $\Lambda$-shaped vortical structure, as shown in Figure 2.9 through a smokewire visualization of Perry et al. (1981). The $\Lambda$-vortex induces a velocity field that enhances wall-normal momentum exchange in its vicinity; in between the legs of the $\Lambda$-vortex, lowmomentum fluid is brought up from the wall, creating a low-momentum streak that is aligned in the streamwise direction (Hultgren and Gustavsson, 1981; Andersson et al., 2001). Between spanwise-adjacent $\Lambda$-vortices, high-momentum fluid is brought towards the wall, creating a high-momentum streak. Here, the terms "low" and "high" are relative to the local mean flow field; when the local mean flow field is subtracted from the instantaneous flow field, these streaks appear as regions of reversed- and forward-flow, respectively.

Considerable research has been conducted to understand the spatial characteristics of $\Lambda$-vortices. Saric and Thomas (1983) observed that the $\Lambda$-vortices remain aligned in the streamwise direction (peak following peak) when the fluctuation amplitude of the T-S waves exceeds about $1 \%$ of the free-stream velocity. In this scenario, the maximum amplification occurs when the spanwise spacing $\lambda_{z}$ of the $\Lambda$-vortices is approximately equal to the streamwise wavelength $\lambda_{x}$ of the T-S waves. Conversely, Corke and Mangano (1989) found a structure of staggered $\Lambda$-vortices (peak following valley) at lower T-S-wave amplitudes $(\approx 0.3 \%)$ with a large spanwise spacing $\left(\lambda_{z} \approx 1.5 \lambda_{x}\right)$, which Craik (1971) had predicted would occur when 
the T-S wave interacts with sub-harmonic oblique waves (that is, waves not aligned with the streamwise direction). From these studies, it is evident that the organization and spacing of the $\Lambda$-vortex is influenced by the amplitude of the T-S waves and the disturbance environment within the boundary layer.

As the $\Lambda$-vortices are stretched in the streamwise direction by the non-uniform streamwise flow in the boundary layer, the vorticity within the legs and crest of the $\Lambda$-vortex must amplify in order to conserve circulation. The strong vorticity at the head induces velocity towards the wall on the downstream side of the head and toward the free-stream on the upstream side, referred to as sweep and ejection events, respectively. These transient wall-normal motions produce highly-inflectional instantaneous velocity profiles that are inherently inviscid unstable. The destabilized flow further promotes the stretching of $\Lambda$-vortices which in turn results in more substantial wall-normal motions. The highly-stretched $\Lambda$-vortices are often referred to as "hairpin" vortices (e.g., Acarlar and Smith, 1987; Sandham and Kleiser, 1992; Suponitsky et al., 2005). Visualizations of hairpin vortices are shown in Figure 2.9.

\subsubsection{Break-down to turbulent flow}

The high levels of vorticity within the hairpin vortex produces regions of very high shear and induces wall-normal motions, shown schematically in Figure 2.10. Ejection events (where $u^{\prime}<0$ and $v^{\prime}>0$ ) brings low-momentum fluid away from the wall due to the induced velocity between the legs of the hairpin vortex loop. Sweep events (where $u^{\prime}>0$ and $v^{\prime}<0$ ) brings higher-momentum fluid toward the wall, also due to the induced velocity of the hairpin vortex loop, this time outside of the legs of the hairpin vortex where the induced velocity is toward the wall. The ejection and sweep events are also called Q2 and Q4 events, respectively, which refers to the quadrant of the motion in a $u^{\prime}-v^{\prime}$ coordinate system, shown in Figure 2.10. These wallnormal motions create instantaneous boundary-layer profiles that vary significantly in time 


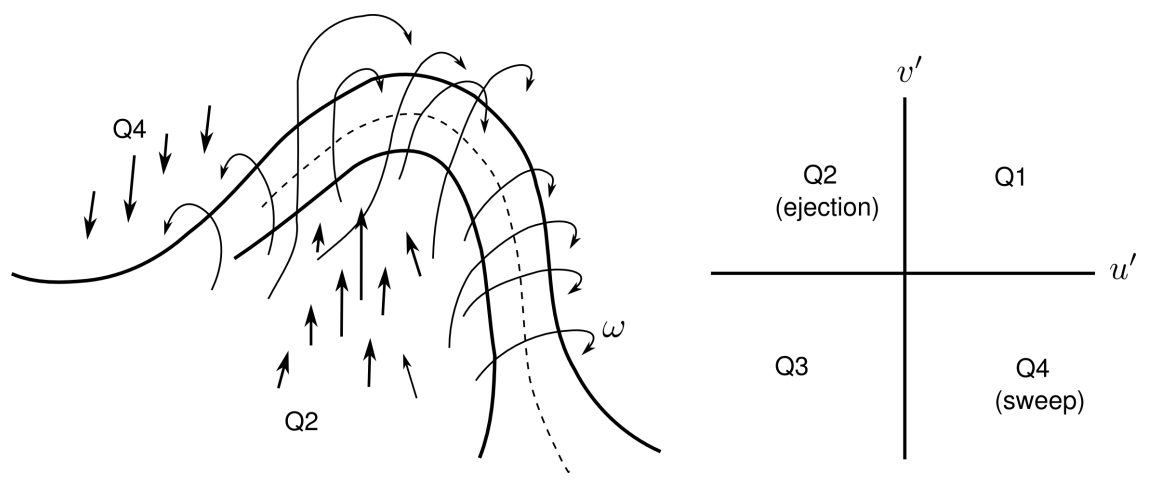

Figure 2.10: Schematic showing the wall-normal motions induced by a hairpin vortex. Reproduced from Robinson (1991).

and space, creating regions of high shear that become sites of localized instabilities leading to formation of even smaller vortical structures, which is typically identified as the onset of transition. These "turbulent spots" were first observed by Emmons (1951), and later by Tani and Hama (1953), Schubauer and Klebanoff (1956), and Hama et al. (1957).

Figure 2.11 illustrates the coherent structures occuring in a turbulent spot. The internal spot structure consists of an aggregation of hairpin vortices (Makita and Nishizawa, 2001; Schröder and Kompenhans, 2004) which produce longitudinal streaks as a result of the sweep and ejection process occuring between their legs. The spanwise spacing of the streaks observed in the spot is very similar to the boundary-layer streaks created by the $\Lambda$-vortices prior to transition (Hidalgo et al., 2006; Sabatino and Smith, 2008). Waviness of the streaks have been observed as a result of randomness in the location of the hairpin vortices; Yaras (2007) observed that the magnitude of this waviness is influenced by the free-stream conditions.

Early observations of spots in zero pressure-gradient boundary layers indicated that the spot leading- and trailing-edge celerities are approximately $0.88 U_{e}$ and $0.55 U_{e}$, respectively, where $U_{e}$ is the local boundary-layer edge velocity (Gostelow et al., 1996). The longitudinal growth of the spot occurs due to the growth and stretching of hairpin vortices; as they grow in wall-normal height, they extend into the faster-moving fluid away from the wall, which results 


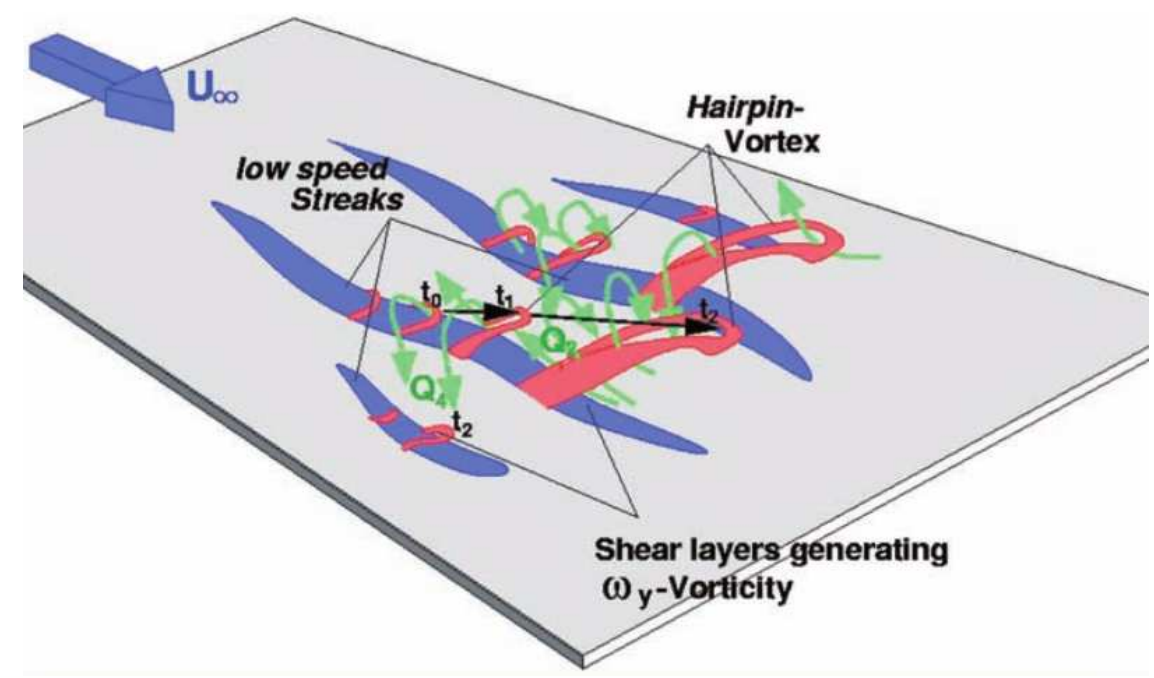

Figure 2.11: Schematic of the topology withing a turbulent spot. Reproduced from Schröder and Kompenhans (2004).

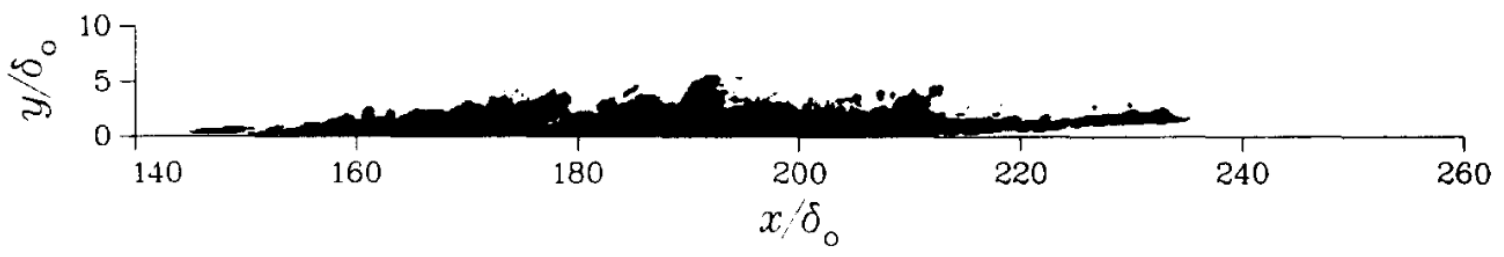

Figure 2.12: Contours of normal vorticity illustrating the overhang region at the leading-edge of a turbulent spot. Reproduced from Henningson et al. (1987).

in their being stretched in the streamwise direction. This produces an overhanging perturbed region above a laminar pocket at the leading edge of the spot; this is shown in the simulation of Henningson et al. (1987), illustrated in Figure 2.12. The elongated hairpin vortices produce instantaneous inflectional velocity profiles in the laminar flow pocket, which destabilizes it to rapidly-growing inviscid modes until it is entrained into the turbulent spot, resulting in longitudinal growth of the spot.

Wygnanski et al. (1976) observed that turbulent spots grow laterally at a half-angle of about $10^{\circ}$ which varies substantially with the streamwise pressure gradient and slightly with Reynolds number. Similar to the longitudinal growth mechanism, lateral growth is attributed to the destabilization of the laminar flow outboard of the spot by velocities induced by the 
hairpin vortices that lie along the spanwise extremities of the turbulent spot. Gad-El-Hak et al. (1981) consistently observed streamwise momentum-deficit streaks at the spanwise extremities

of the turbulent spots regardless of the number streaks occurring in the turbulent spot. The streamwise vorticity from the hairpin vortex that creates this streak destabilizes the laminar flow by inducing an inflectional spanwise velocity profile. Further observations by Tillmark (1995), Schröder and Kompenhans (2004), Chong and Zhong (2005), and Inasawa et al. (2007) provide support for this explanation of spanwise growth of the spot. Finally, as the enlarging spots merge with other spots, the whole width of the plate becomes populated with hairpin vortices, which effectively marks the completion of transition to turbulent flow.

Early studies of turbulent spots by Schubauer and Klebanoff (1956) observed a calmed region following the passing of the spot. This calmed region suggests that the local instantaneous boundary layer deviates from the undisturbed laminar flow in a manner that increases its stability. Computations performed by Johnson (2001) observed a vortex (what he calls a horseshoe vortex) at the trailing edge of the spot that brings high-momentum flow from the upper boundary layer toward the surface, producing a fuller and thus more stable velocity profile. Recent experiments by Sabatino and Smith (2008) agree, suggesting that hairpin packets at the trailing-edge of the spot produce an in-rush of high-momentum fluid from higher regions in the boundary layer to produce the calmed region.

\subsubsection{Hairpin-vortex regeneration mechanism}

Considering the important role of hairpin vortices in the growth of a turbulent spot, understanding the mechanisms by which these structures are created and grow within a turbulent spot is important. Through direct numerical simulation, Singer and Joslin (1994) studied the formation of hairpin vortices following the artificial-triggering of a primary hairpin vortex. Based on their results, they proposed the hairpin-vortex regeneration mechanism shown 


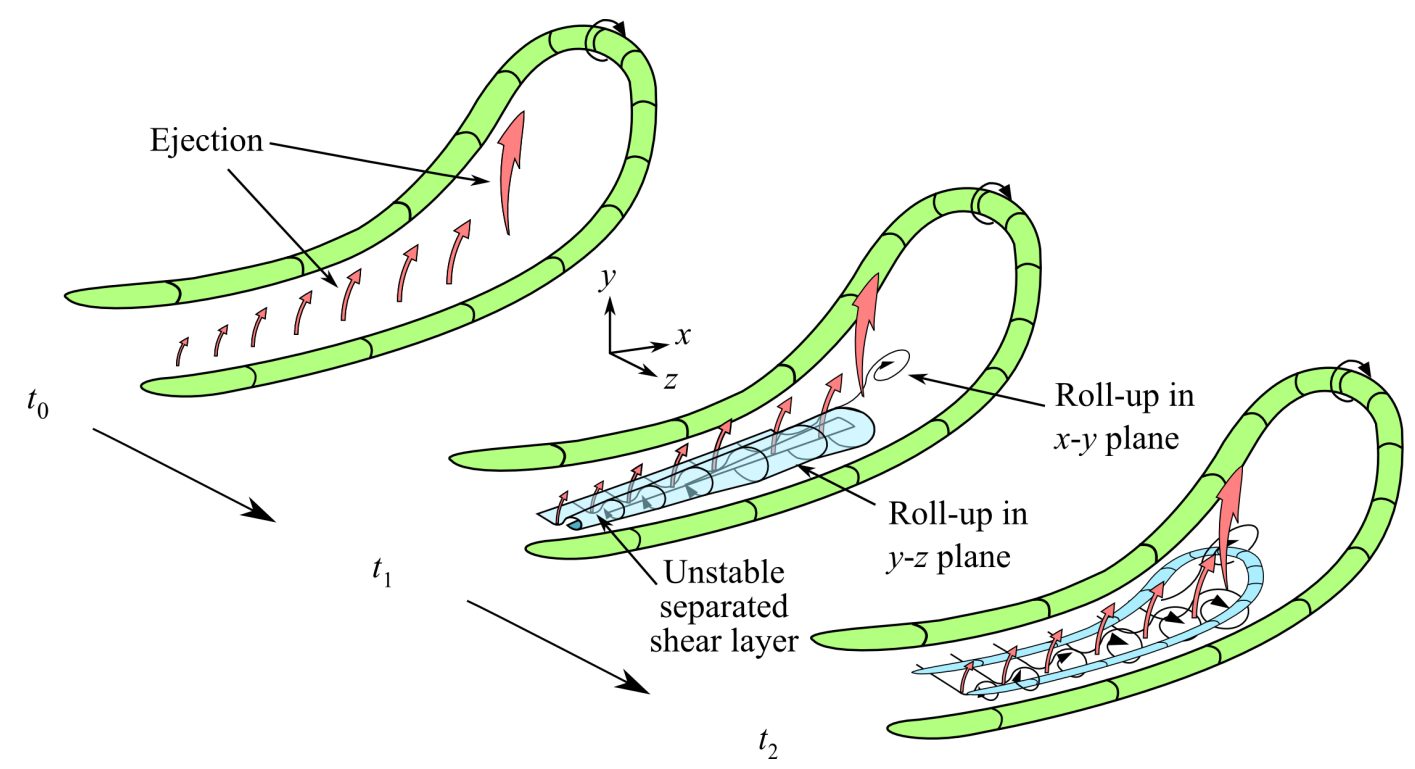

Figure 2.13: Hairpin-vortex regeneration mechanism proposed by Singer and Joslin (1994).

in Figure 2.13. An isolated hairpin vortex at time $t_{0}$ ejects low-momentum fluid between its legs, which creates transient regions of local adverse pressure gradient. By time $t_{1}$, the surrounding low-velocity fluid separates in response to the transient, adverse pressure-gradient regions. Separation was observed primarily in the wall-normal/spanwise $(y-z)$ plane and the unsteady separated shear layer rolls-up into streamwise-oriented vortices, forming the legs of a new hairpin vortex by $t_{2}$. A similar mechanism occurs in the streamwise/wall-normal ( $x$ y) plane, producing the spanwise-oriented head of the new hairpin vortex. This mechanism is qualitatively confirmed by visualization experiments of Guo et al. (2004) and the vortexregeneration model of Smith et al. (1991) that is based on complementary experimental and computational studies of fully-turbulent boundary layers.

However, recent tomographic particle-image-velocimetry experiments of triggered turbulent spots by Schröder and Kompenhans (2004) and Schröder et al. (2008) suggest a seemingly different regeneration mechanism for the hairpin vortices within a turbulent spot, as illustrated in Figure 2.14. Their results show that as the hairpin vortex grows, it generates increasinglystrong Q2 and Q4 events, which strengthen the high- and low-speed streaks surrounding 


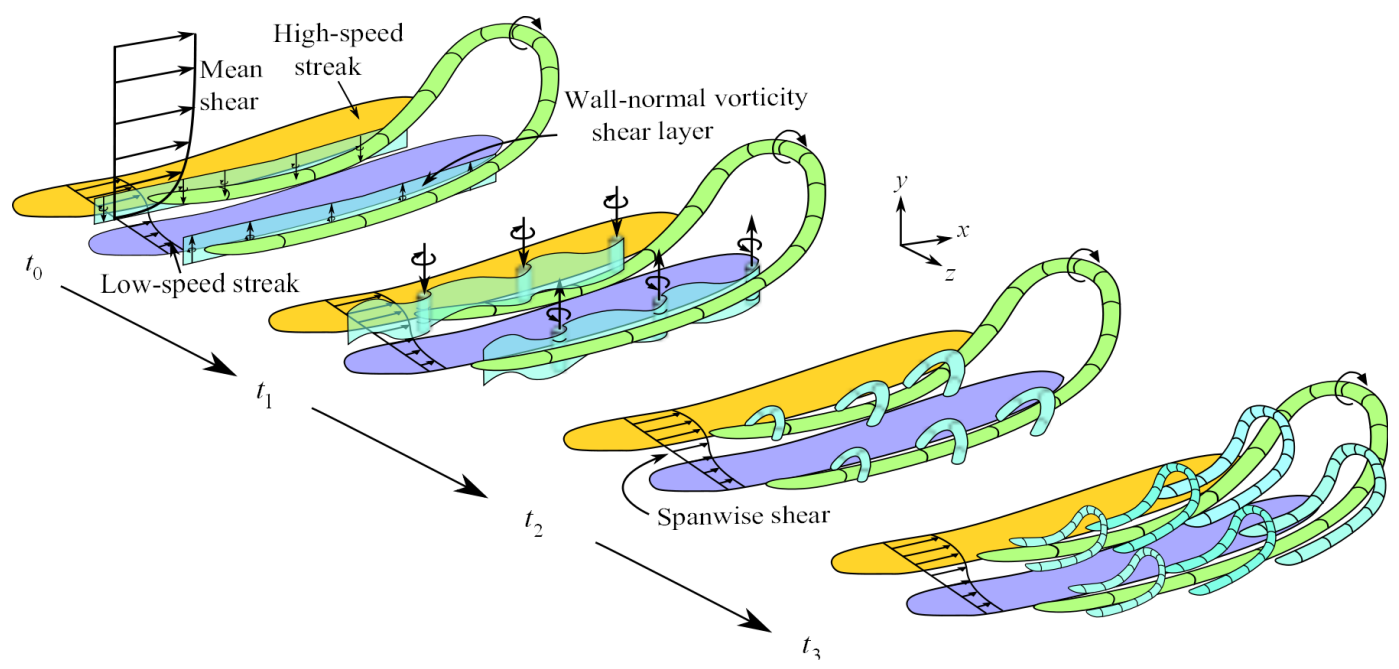

Figure 2.14: Hairpin-vortex regeneration mechanism proposed by Schröder and Kompenhans (2004).

the hairpin vortex. At $t_{0}$, a layer of wall-normal vorticity is generated between the highand low-speed streamwise streaks. This layer is likely unstable in an inviscid sense, and by $t_{1}$ the instability leads to a streamwise grouping of wall-normal vorticity into roughly vertically-oriented vortices at a set streamwise wavelength. The vertically-oriented vortices are themselves unstable, and by $t_{2}$ they quickly fold over to form arch-shaped structures that are then stretched in the streamwise direction by the mean shear to form hairpin vortices by $t_{3}$. As these new hairpin vortices grow, the cycle repeats itself. This mechanism is corroborated by the simulations performed by Krishnan and Sandham (2007) of a spot triggered by a localized blowing trip, who observed that the lateral spot spreading-rate is directly proportional to the strength of the wall-normal-vorticity shear layer.

While the regeneration mechanism proposed by Singer and Joslin (1994) (Figure 2.13) implies that a secondary hairpin vortex will develop primarily between the legs of the primary hairpin vortex, the mechanism proposed by Schröder and Kompenhans (2004) and Schröder et al. (2008) (Figure 2.14) implies that a secondary hairpin vortex will straddle a leg of the primary hairpin vortex. Published literature contains instances of turbulent spots with both kinds of hairpin-vortex topology, and so it is not clear what flow conditions will favour one 
mechanism over the other. Therefore, it is evident that a complete explanation for the growth mechanism of flow structures within a turbulent spot has not yet been achieved.

\subsubsection{Effect of elevated free-stream turbulence}

At this point in the review, the reader is referred back to the transition roadmap proposed by Saric et al. (1998), shown in Figure 1.2, which shows various transition mechanisms and their dependence on the amplitude of environmental disturbances in the flow. The LPT environment is generally full of disturbances, including free-stream turbulence, surface roughness, acoustic waves, and wakes from upstream bladerows (Mayle, 1991). A comprehensive review on the effects of each of these is beyond the scope of this review; the focus will be limited to how elevated free-stream turbulence modifies transition in attached boundary layers.

Path E in Figure 1.2 represents the bypass transition mechanism, where the linear and secondary-growth stages in natural transition are bypassed and a turbulent spot instantly develops following the inclusion of a disturbance into the boundary-layer (Morkovin, 1993). In between bypass and natural transition, Paths B-D represent transition through transient growth mechanisms, which are also referred to as non-modal growth, as the amplified disturbances are not eigenfunctions of the linearized stability equations. In this instability mode, multiple non-orthogonal stable modes interact and grow algebraically, which modulates the underlying boundary-layer flow in a manner that favours disturbance-growth (Landahl, 1980). The modulation grows in time, hence the name transient growth. Klebanoff (1971) observed that the fluctuations take the form of longitudinal streaks of high and low streamwise velocity near the wall and highlighted that increasing the level of free-stream turbulence increases the amplitude of the streaks. The streaks are often referred to as Klebanoff modes despite the fact that they are not represented by eigenfunctions of the linearized stability equations. 


\subsubsection{Formation of streamwise streaks}

The creation of streamwise streaks in boundary layers subjected to elevated free-stream turbulence is determined by two simultaneous processes: the inclusion of free-stream disturbances into the boundary layer and the growth of these disturbances within the boundary layer. The former process is severely hampered by the mean boundary-layer shear, which shelters the boundary layer from high-frequency vortical disturbances in the fast-moving free-stream fluid and allows only low-frequency disturbances to penetrate into the slower boundary layer fluid, a phenomenon referred to as "shear sheltering". To explain the shear-sheltering process, Jacobs and Durbin (1998) and Zaki and Durbin (2005) note that a complete eigenfunction basis of the Orr-Sommerfeld equations consists of a finite set of discrete modes and a continuous spectrum of modes. Because the eigenfunctions of the discrete modes tend exponentially to zero in the free-stream, free-stream disturbances are properly characterized by a superposition of continuous modes. Numerical solution of the Orr-Sommerfeld equation by Jacobs and Durbin (1998) shows that the eigenfunction of the continuous modes oscillate sinusoidally in the free-stream but are rapidly damped near the top of the boundary layer, and that the penetration depth of the continuous modes varies inversely with their frequency such that only low-frequency modes can penetrate into the shear layer. A direct numerical simulation (DNS) of bypass transition by Jacobs and Durbin (2001) illustrates the shear-sheltering effect by showing that nearly isotropic turbulence in the free-stream produces low-frequency, highlyelongated streaks within the laminar boundary layer. Hernon et al. (2007) measured the penetration depth of free-stream disturbances into a boundary layer by plotting the skewness function of the streamwise fluctuation velocity and found a similar inverse relationship to the disturbance frequency as Jacobs and Durbin (1998).

Once a disturbance has penetrated into the boundary layer, the form of the disturbance determines whether it will amplify or decay. A linear theory to predict the form of the most 
unstable disturbance - that is, the disturbance with maximal growth in a given time period, referred to as an optimal disturbance - via solution of the linearized Navier-Stokes equation is presented by Butler and Farrell (1992), Butler and Farrell (1993), Reddy et al. (1998), Andersson et al. (1999), and Luchini (2000). These studies demonstrate that the optimal disturbance in a boundary layer has the form of a pair of counter-rotating streamwise vortices with no streamwise dependence. Subsequent work by Cherubini et al. (2010) note that the previous analyses based on the linearized Navier-Stokes equations are deficient in that they assume that the optimal disturbance consists of a single wavenumber/frequency and non-linear effects are neglected. By applying a three-dimensional adjoint method and global eigenvalue model to the non-linear Navier-Stokes equations, Cherubini et al. (2010) determine that the global optimal disturbance consists of a packet of monochromatic waves that take the form of streamwise-modulated, counter-rotating streamwise vortices tilted in the upstream direction.

\subsubsection{Spatial characteristics of streamwise streaks}

The wall-normal motions induced by the optimal streamwise vortices generate streamwise streaks via the lift-up effect (Kline et al., 1967; Kim et al., 1971). Smith and Metzler (1983) studied the spatial structure of low-speed streaks in wall shear layers under zero streamwise pressure gradients through flow visualization of hydrogen bubbles created by a submerged wire in a water channel. The Reynolds number based on momentum thickness in their study was in the range $740 \leq R e_{\theta} \leq 5830$ and a non-dimensional streak spacing of $\overline{\lambda^{+}} \approx 100$ was observed throughout this range. In addition, a minimal variation in the probability distribution of the spacing was observed within the noted range of Reynolds numbers. Due to merging of low-speed streaks, the spanwise streak spacing increased linearly with wall-normal distance in the range $5<y^{+}<30$. Beyond $y^{+} \approx 30$, streak identification was more uncertain as the streak-merging process away from the wall became highly three-dimensional. Further work by Butler and Farrell (1993) and Rajaee et al. (1995) used optimal perturbation theory and 
correlation measurements of hot-wire data, respectively, to confirm the dimensionless streak spacing $\overline{\lambda^{+}} \approx 100$ and assign uncertainty bounds of \pm 20 .

Chernyshenko and Baig (2005) explained the mechanism for the selection of a certain streak spacing by considering a perturbation in the form of a streamwise vortex with fixed initial energy. Lift-up resulting from the wall-normal velocity component of the streamwise vortex creates fluctuations in the longitudinal velocity. For a perturbation with a small spanwise wavelength, viscosity causes rapid decay of the vortex and smoothing of the longitudinal fluctuations, leading to small streak amplitudes. Conversely, for a perturbation with a large spanwise wavelength, the majority of the kinetic energy of the streamwise vortex is contained in the spanwise velocity component due to the damping effect of the wall in the wall-normal direction. As a result, the wall-normal velocity component (and thus the lift-up effect) is diminished, again leading to small streak amplitudes. Chernyshenko and Baig (2005) concluded that the observed streak spacing represents a balance between viscous diffusion of smallwavelength perturbations and the diminishment of the wall-normal velocity component in large-wavelength perturbations.

\subsubsection{Instability of streamwise streaks}

Following the formation of streamwise streaks in the boundary layer, secondary instabilities of the streaks can occur and provide a route for break-down to turbulence. Swearingen and Blackwelder (1987) noticed that the highly-inflectional streamwise velocity profiles in the spanwise and wall-normal directions result in localized inviscid secondary instabilities, of which there are two modes: an asymmetric sinuous instability occurs as oscillations of the streamwise streaks in the streamwise/spanwise plane, while a symmetric varicose instability occurs as a periodic widening and narrowing of the streak (Andersson et al., 2001). These secondary modes are illustrated in Figure 2.15. Simulations of Elofsson et al. (1999) and Brandt and Henningson 


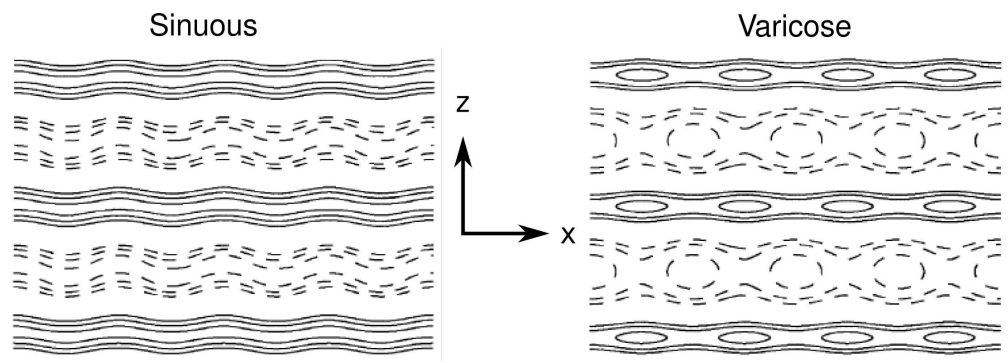

Figure 2.15: Sketch of streak instability modes in the $(x-z)$ plane. Solid lines represent lowspeed streaks while dashed lines represent high-speed streaks. Adapted from Andersson et al. (2001).

(2002) indicate that the streak amplitude determines whether secondary instabilities will occur.

For flow over a flat plate with zero streamwise pressure gradient, they observed the onset of a sinuous instability when the streak amplitude reached about $26 \%$ of the free-stream velocity. Varicose instability waves were observed to be more stable, with a critical streak amplitude of about $37 \%$ of the free-stream velocity. In addition to the streak amplitude, the streak width also affects the mode competition. Asai et al. (2002) performed detailed measurements of a single low-speed streak created by a small screen mounted normal to the wall, and symmetric and asymmetric modes were excited by external disturbances introduced through small holes in the screen. When the streak width is narrow, comparable to the shear-layer thickness, the streak is unstable to the asymmetric mode, while for wider streaks, the symmetric mode is preferred. Later experiments of spanwise-periodic streaks by Konishi and Asai (2004) in the same apparatus as Asai et al. (2002) note that when the spanwise streak spacing is greater than about 2.5 streak widths, both secondary modes are suppressed.

Brandt and de Lange (2008) showed that the interaction of high- and low-speed streaks determines how streaks affected by symmetric and asymmetric secondary instabilities break down. These authors performed a DNS of a laminar boundary layer with streaks with finite streamwise and spanwise wavelengths introduced at the inlet. When break down followed the growth of a symmetric secondary instability, a frontal collision was observed between an incoming high-speed streak and a low-speed streak, while in asymmetric break down, the 
high- and low-speed streaks were observed to slide along each other's sides. In both cases, a strong shear layer forms between the streaks and its instability produces coherent vortical flow structures that initiate the break down to smaller-scale turbulence. For the symmetric and asymmetric modes, the vortical flow structures consist of $\Lambda$-shaped vortices and a train of quasistreamwise vortices with alternating sense of rotation, respectively. In a DNS of asymmetric break down, Schlatter et al. (2008) observed that the coherent vortical flow structures occur not as isolated vortices, but in a wave packet consisting of 3-5 vortices straddling the low-speed streak, staggered in the streamwise direction.

The final stage of bypass transition occurs as the coherent vortical flow structures generated by the streak instabilities break down into small-scale turbulence. In cases where the streaks are unstable through a symmetric secondary mode, as in the DNS of Sandham and Kleiser (1992), the high-shear layers surrounding the legs of $\Lambda$-shaped vortices roll-up into hairpinshaped vortices. In cases where the streaks are unstable through an asymmetric secondary mode, as in the DNS of Schlatter et al. (2008), the amplitude of the spanwise oscillations of the quasi-streamwise vortices straddling the low-speed streaks increases to the point where the vortices straddling adjacent low-speed streaks actually connect, forming hairpin-shaped vortices that subsequently lead to the generation of smaller scales. In cases where secondary streak instabilities are not present due to low streak amplitudes or large streak spacing, such as the DNS results of Wu et al. (1999) and Jacobs and Durbin (2001), streak break-down occurs through a bursting process that develops as low-speed streaks move into the outer region of the boundary layer and interact with free-stream eddies, resulting in the creation of patches of irregular motion on the lifted low-speed streaks. The irregular patches differ from classic turbulent spots observed by Emmons (1951) in that they originate high in the boundary layer rather than near the wall. Linear stability analysis and DNS by Zaki and Durbin (2005) found that the coupling of free-stream turbulence to the lifted low-speed streaks is strongly influenced by the frequency of disturbances in the free-stream, and that a high-frequency free-stream mode 
is required to trigger transition of the low-speed streak.

Harmonics of the sinuous and varicose instability mechanism are also relevant to transition initiated in the low-speed streaks. The amplification of sub-harmonic modes is largely determined by the amplitude and spanwise spacing of the low-speed streak. Brandt and Henningson (2002) observed that harmonics of the sinuous instability wave can be more amplified than the fundamental modes. Asai et al. (2002) and Konishi and Asai (2004) artificially generated single and spanwise-periodic streaks, respectively, and observed that the fundamental sinuous mode is very sensitive to streak spacing while the sub-harmonic sinuous modes experience almost the same growth rate in spanwise-periodic streaks as in individual streaks. Additionally, in simulations where sinuous and varicose instabilities were not observed, such as in the simulations of Jacobs and Durbin (2001), the generation of turbulent patches was observed to be independent of the spanwise spacing of the low-speed streaks.

\subsubsection{Influence of free-stream flow conditions on bypass transition}

Whereas the studies reviewed in Section 2.2.5 were largely limited to bypass transition in flat-plate boundary layers, the flow in a typical turbomachinery blade row will include strong streamwise pressure gradients that may alter the receptivity and instability processes involved in bypass transition as well as modify the length scales and intensity of the freestream turbulence. A complete picture of bypass transition should therefore include the effects of streamwise pressure gradients and variations in the free-stream turbulence conditions. The effect of a favourable streamwise pressure gradient on the spanwise streak spacing was studied experimentally by Talamelli et al. (2002) and Blair (1992). These authors showed that although the absolute streak spacing decreases when the boundary layer is subjected to streamwise acceleration, when the streak spacing is scaled by the boundary-layer displacement thickness or viscous length scale, the non-dimensional spacing increases, implying enhanced stability of the 
boundary layer and a downstream shift in the transition-onset location. A DNS by McAuliffe and Yaras (2010) of a boundary layer subjected to a favourable-to-adverse streamwise pressure gradient typical of the low-pressure turbine in a gas-turbine engine found a preponderance for high-speed streaks in the accelerated region, which resulted in an overall increase in the streak width to the point where secondary streak instabilities were prevented despite observing sufficiently-high streak amplitudes. Litvinenko et al. (2005) performed experiments of symmetric secondary streak instabilities in zero and adverse pressure-gradient boundary layers, observing that the adverse pressure gradient amplifies the creation of $\Lambda$-vortices through the symmetric mode. Zaki and Durbin (2006) used linear stability calculations and DNS to highlight that the effect of adverse pressure gradients on bypass transition occurs primarily through increasing the receptivity of the boundary layer to free-stream vortical disturbances, thus enhancing the coupling of low-frequency disturbances to the boundary-layer fluid and strengthening the streamwise streaks.

If the boundary layer in which the streaks are embedded separates under the influence of a strong adverse streamwise pressure gradient, the streamwise streaks may also contribute to the growth of disturbances in the separated shear layer. Such a flow was simulated by McAuliffe and Yaras (2007b) to study the formation of a wave packet in a separated shear layer under elevated free-stream turbulence. Figure 2.16 reproduces the contours of streamwise and wallnormal velocity fluctuations observed by McAuliffe and Yaras (2007b) in a spanwise plane at a wall-normal distance where the mean velocity is approximately half the mean velocity at the boundary-layer edge. The strong spanwise variation of the streamwise velocity component indicates the presence of streaks in the attached portion of the boundary layer. As the streaks convect through the separated shear layer, McAuliffe and Yaras (2007b) observed a local inviscid instability of the shear generated by the streamwise streak, resulting in an abrupt roll-up of the shear layer into a sequence of hairpin-shaped vortices to form a turbulent spot. A planform view of the spot is visible in the wall-normal velocity fluctuation in Figure 2.16(b). 
Once the turbulent spot is initiated, its streamwise and spanwise growth occurs in a manner consistent with the description given in Section 2.2.3. Other studies by Watmuff (1999) and Wu et al. (1999) observed a similar roll-up of the shear layer vorticity due to an inviscid instability of the shear generated by the streamwise streak convecting through the shear layer.
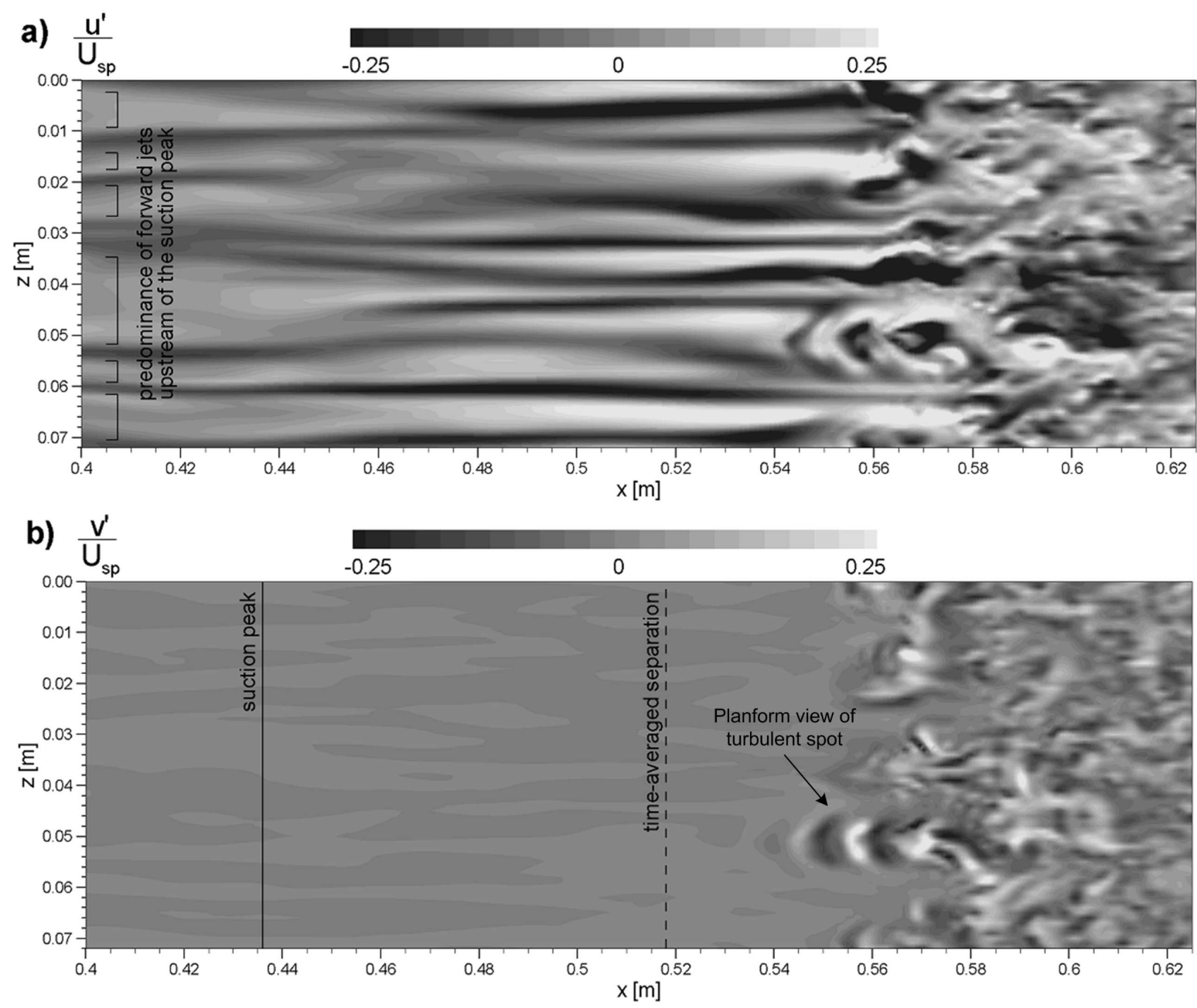

Figure 2.16: Streamwise streaks in the shear layer observed in an $x$ - $z$ plane where $\bar{U} \approx 1 / 2 \overline{U_{e}}$, (a) streamwise fluctuations, (b) wall-normal fluctuations. Adapted from McAuliffe and Yaras $(2007 b)$.

To assess the sensitivity of bypass transition to the turbulence length scales in the freestream, Brandt et al. (2004) performed a DNS of a transitional flat-plate boundary layer with synthetic turbulent inflow conditions consisting of a superposition of modes of the continuous 
spectrum of the linear stability equations (Orr-Sommerfeld equations). By varying the energy spectrum of the free-stream turbulence, they observed that the transition-onset location shifts to lower Reynolds numbers as the integral length scale is increased. Measurements of the intermittency — defined as the percentage of time a boundary layer is turbulent-by Jonás et al. (2000) observed the same trend, and that increasing the integral length scale increases the length of the transition region. However, their study did not discuss how the turbulence integral length scale affects the transition process. More recently, Nagarajan et al. (2007) and Ovchinnikov et al. (2008) demonstrated that the effect of the free-stream turbulence length scale on transition is strongly affected by the flow development along the test-surface leading edge. Their results demonstrate a complicated interplay between the free-stream turbulence integral length scale and the receptivity of the flow at the leading-edge that is qualitatively captured by the ratio of the integral length scale to the boundary-layer thickness at transition

onset. For the lower value of this ratio, transition occurs through the formation, instability, and break down of streamwise streaks, as described previously. However, for the higher value of this ratio, large, localized wavepackets formed far upstream of the earliest streamwise streaks. These wavepackets were shown to have significantly different properties than those formed by streak instability, which suggests that a threshold free-stream turbulence integral length scale may exist beyond which the sheltering effects of the mean shear are overcome and turbulent spots may be created by free-stream turbulent eddies directly without the development of streamwise streaks.

\subsection{Instability of Three-Dimensional Boundary Layers}

The preceding sections reviewed the stability of attached and separated boundary layers under conditions that are representative of a LPT blade. However, the preceding sections were limited to free-stream pressure gradients that developed in the streamwise direction only. In a 
realistic LPT environment, spanwise pressure gradients may occur, for example as the result of highly-flared hub and casing walls which yield an effective sweep angle in radially-stacked blades (e.g., Pullan and Harvey, 2007; Yaras, 2012). The spanwise pressure gradient results in the creation of a three-dimensional velocity profile in the boundary layer on the LPT blade. Three-dimensional boundary layers have been shown to demonstrate four types of instabilities (Saric et al., 2003): attachment-line, streamwise, centrifugal, and crossflow. Attachmentline instabilities are related to interactions between the boundary layer that develops just downstream of the leading-edge stagnation point and turbulent disturbances that propagate along the blade leading-edge, normally found in bodies with large leading-edge radii (Reed and Saric, 1989). Streamwise instability deals with the streamwise component of flow, and it follows the development of transition in two-dimensional boundary layers described in the preceding sections of this chapter. Centrifugal instabilities develop over concave surfaces and lead to the development of counter-rotating Görtler vortices. Crossflow instabilities are associated with an inflection point in the wall-normal profile of the spanwise velocity component. When a cross-stream pressure gradient is introduced to a boundary layer, the cross-stream pressure gradient creates a velocity component in the spanwise direction and thus streamlines at the boundary-layer edge develop curvature. Within the boundary layer, the streamwise velocity is reduced, so the pressure force can over-balance the centripetal acceleration of the boundarylayer fluid, creating a crossflow velocity component in the boundary layer (Saric et al., 2003). The resulting velocity distribution can be resolved into components parallel $\left(x_{t}\right)$ and normal $\left(z_{c}\right)$ to a streamline at the local boundary-layer edge. The shape of the resolved boundary layer is shown in Figure 2.17. The no-slip condition requires the crossflow velocity component to vanish at the wall, and since it must also vanish at the boundary-layer edge, there is an inflection point in the crossflow component of the boundary-layer profile. The inflectional shape of the crossflow velocity profile is the source of the crossflow instability mechanism.

Figure 2.18 illustrates a potential streamline along a swept blade with a coordinate system 


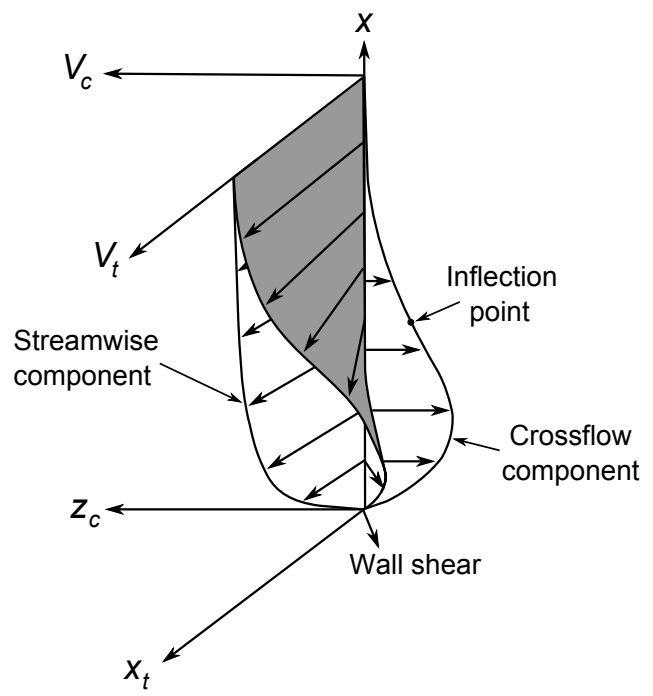

Figure 2.17: Three-dimensional boundary layer profile. Reproduced from Saric et al. (2003).

aligned with the tangential and crossflow components of the streamline. By varying the orientation of the coordinate system in which the boundary-layer velocity profile is viewed, a class of unstable boundary-layer profiles can be created, shown in Figure 2.18(b), each possessing unique stability characteristics. In particular, there is an orientation where the inflection point of the crossflow velocity component occurs at the point of zero crossflow velocity, as seen in the profile labeled $J$ in Figure 2.18(b). Gregory et al. (1955) found that this profile corresponds to a disturbance with zero wave velocity (i.e. a stationary vortex fixed relative to the test surface), while other orientations result in travelling waves. Therefore, unlike T-S instability, crossflow instabilities develop as both moving and stationary crossflow waves. The stationary crossflow wave occurs as counter-rotating vortices nearly aligned to the local inviscid streamlines. The spanwise and wall-normal motions that result from the stationary crossflow vortex cause disturbances in the streamwise flow, leading to regions of increased and reduced streamwise velocity that alternate across the span. The momentum excess and deficit can be visualized by subtracting the spanwise-averaged boundary-layer profile from the local boundary-layer profile at a given spanwise location. Nitschke-Kowsky and Bippes (1988) and Dagenhart and Saric (1999) and others have shown that the ratio of the wavelength of 


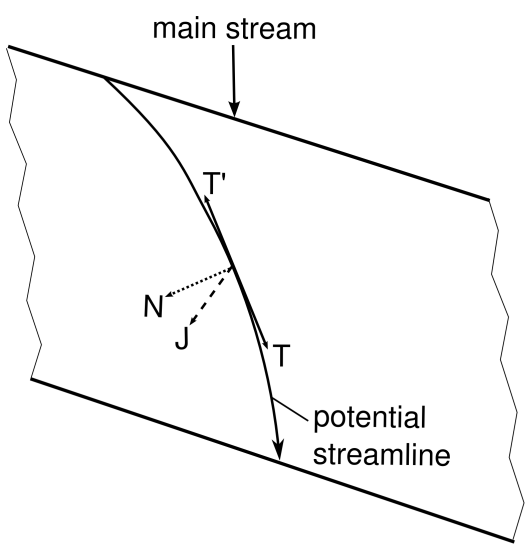

(a)

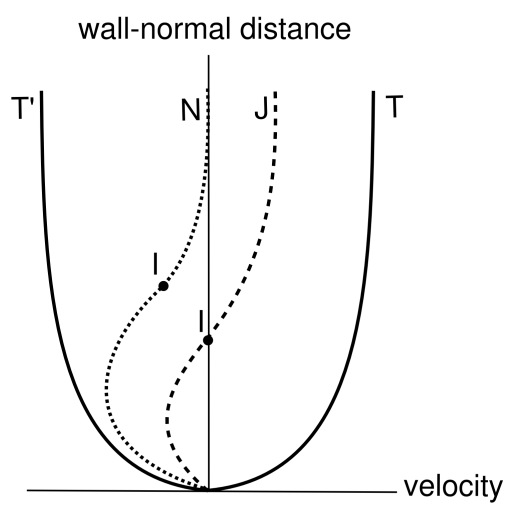

(b)

Figure 2.18: (a) Coordinate systems in a swept-blade flow (b) Profiles of the tangential and crossflow velocities in different orientations. Solid line denotes tangential component and dashed line denotes crossflow component. Adapted from Gregory et al. (1955).

the spanwise waviness $(\lambda)$ to boundary-layer thickness $(\delta)$ remains constant at approximately $\lambda / \delta=4.1-4.2$. Theoretical studies based on the inviscid stability equations, however, have severely over predicted the spanwise wavelength, although good agreement was achieved in calculating the crossflow vortex direction (Gregory et al., 1955). This suggests that although the inertial forces creating the inflectional instability of the boundary-layer are not affected by viscosity, the wavelength of this instability is Reynolds-number dependent.

\subsubsection{Receptivity in crossflow instability}

As described above, the crossflow instability exhibits both travelling and stationary disturbances. Although both are present, transition is normally dominated by one or the other, determined by the receptivity process. Deyhle and Bippes (1996) found that for a swept wing, travelling crossflow waves dominate when the free-stream turbulence level is greater than $0.15 \%$, and stationary crossflow waves dominate at lower turbulence levels. Since freestream turbulence in a typical turbomachinery environment is greater than $0.15 \%$, travelling crossflow waves would be expected to dominate the transition process. Radeztsky et al. (1999) 
observed that the crossflow disturbances are not sensitive to acoustic waves, even at amplitudes greater than $100 \mathrm{~dB}$, indicating that the dependence on turbulence is due only to the vortical components of free-stream turbulence (Saric et al., 2003). Work by Spalart (1993) confirmed that stationary crossflow waves should dominate in low-fluctuation environments and travelling crossflow waves should appear only in the presence of high free-stream fluctuation levels.

\subsubsection{Non-linear growth and secondary instabilities}

The type of crossflow disturbance (whether travelling or stationary) determines how the disturbance will propagate in the boundary layer. The growth of travelling waves is well-predicted by linear stability theory, but stationary waves result in distortion of the undisturbed flow which causes rapid onset of a secondary, non-linear disturbance growth and quick transition to turbulence (Bippes and Lerche, 1997; Bippes, 1999; Arnal et al., 1997; Kachanov, 1994). The location and frequency of the secondary instability is determined primarily by the shape of the inflectional boundary layer. Wasserman and Kloker (2002) observed three classes of secondary instabilities for stationary crossflow waves on a flat plate: ( $i$ ) mode I is a high-frequency mode associated with the local minimum of the spanwise gradient of the streamwise velocity; ( $i i)$ mode II is a high-frequency mode associated with the local maximum of the wall-normal gradient of the streamwise velocity; and (iii) mode III is a lowfrequency mode linked to the maximum of the spanwise gradient of the streamwise velocity. Modes I and II are referred to by Malik et al. (1999) as the ' $z$ ' and ' $y$ ' modes, respectively. Each mode exhibits a maximum amplitude in a particular region of the deformed boundary layer. As illustrated in Figure 2.19, for mode I this location is on the updraught side of the crossflow vortex, while for modes II and III this location is above and below the crossflow vortex, respectively. Wasserman and Kloker (2002) found that although mode III is observed to be activated earlier than modes I and II, the amplification rate of mode III is significantly smaller than the other modes, so transition is typically dominated by modes I and II. Since 
modes I and II are most amplified roughly in the middle of the boundary layer, the turbulent spot is created at this height, differing from a turbulent spot created from the streamwise amplification of T-S waves, which forms in the high-shear region near the wall. The creation and subsequent merging of these turbulent spots signifies the onset of turbulent flow.

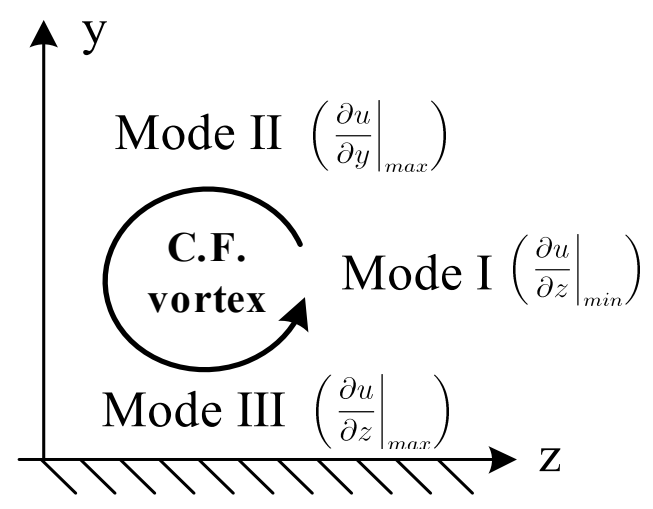

Figure 2.19: Locations of the amplitude maxima for the secondary instability modes of a stationary crossflow vortex. The symbol $u$ denotes the perturbation streamwise velocity. 


\section{Chapter 3}

\section{Computational Setup and Procedures}

This chapter documents the methodological approach used in the present research. Section 3.1 gives an overview of the governing equations. Sections 3.2-3.4 describe the discretization approach, solution methods, and computing platforms employed in the numerical simulations. Section 3.5 gives an overview of the setup of the computational domains and spatial grids used in the simulations. The data reduction procedures used in the analysis of the simulation results are presented in Section 3.6. Section 3.7 describes a linear Orr-Sommerfeld equation solver for computing the theoretical stability characteristics of the computed velocity fields.

\subsection{Governing Equations}

Direct numerical simulation is suitable for simulating transitional and turbulent flows because it can capture all the spatial and temporal scales of a flow without making modelling 
assumptions for the sub-grid turbulence. This requires solutions to the time-dependent mass and momentum conservation equations; the energy conservation equation is also required in the case of compressible flows. In the present research, the highest Mach number reached is $M \approx$ 0.03, which is sufficiently small for the flow to be considered an incompressible fluid. However, because the speed of sound varies according to $a^{2}=\partial p / \partial \rho$, assuming a constant density implies that the speed of sound is infinite and pressure disturbances will propagate at an infinite speed through the computational domain. For this reason, assuming an incompressible fluid leads to the result that any travelling waves in the flow will propagate at infinite velocity and thus will be improperly simulated. In some sections of the present research, the effect of travelling waves on the stability of the flow may be important, and so in these sections, the compressible form of the governing equations needs to be solved. Air behaving as an ideal gas with constant specific heats is the working fluid in the compressible simulations and air with constant properties at $25^{\circ} \mathrm{C}$ and $1 \mathrm{~atm}$ is the working fluid in the incompressible simulations.

The direct numerical simulations in the present research are performed using ANSYS CFX®), a commercial computational fluid dynamics (CFD) software suite. ANSYS CFX solves the the integral forms of the compressible mass, momentum, and energy conservation equations. These equations can be expressed using index notation as:

$$
\begin{gathered}
\frac{\partial}{\partial t} \int_{\mathscr{V}} \rho d \mathscr{V}+\int_{S} \rho u_{j} d n_{j}=0 \\
\frac{\partial}{\partial t} \int_{\mathscr{V}} \rho u_{j} d \mathscr{V}+\int_{S} \rho u_{i} u_{j} d n_{j}=-\int_{S} p d n_{j}+\int_{S} \mu\left(\frac{\partial u_{i}}{\partial x_{j}}+\frac{\partial u_{j}}{\partial x_{i}}\right) d n_{j} \\
\frac{\partial}{\partial t} \int_{\mathscr{V}} \rho h_{0} d \mathscr{V}-\frac{\partial}{\partial t} \int_{\mathscr{V}} p d \mathscr{V}+\int_{S} \rho h_{0} u_{j} d n_{j}=\int_{S} k \frac{\partial T}{\partial x_{i}} d n_{j}+\int_{S} \mu u_{i}\left(\frac{\partial u_{i}}{\partial x_{j}}+\frac{\partial u_{j}}{\partial x_{i}}\right) d n_{j}
\end{gathered}
$$

where the $S$ and $\mathscr{V}$ indicate integration over a surface and volume, respectively, and $n_{j}$ is the outward-pointing normal surface vector associated with each surface. 


\subsection{Discretization}

The governing equations defined in Equations 3.1, 3.2, and 3.3 are discretized spatially in ANSYS CFX through a finite-volume approach. A spatial grid is generated within the region to be simulated (the computational domain) with nodes that are appropriately distributed to resolve the local flow field. Finite volumes are generated for each of the nodes by connecting the centroids of the grid elements surrounding the node. An example is shown in Figure 3.1; the shaded region corresponds to the finite volume (which collapses to an area in 2D) surrounding the node that is constructed by connecting the centroids of the elements surrounding the node. The surface integrals in Equations 3.1-3.3 thus represent the summation of the mass, momentum, and energy fluxes across the boundaries surrounding the shaded finite volume. These surface integrals are discretized at integration points, denoted by the subscript $i p$, that are located on the boundaries of the finite volume, as shown in Figure 3.1. The surface integrals are thus quantified by summing the mass, momentum, and energy fluxes over all the integration points surrounding the finite volume. The resulting discrete form of Equations 3.1-3.3 are

$$
\begin{gathered}
\mathscr{V}\left(\frac{\rho-\rho^{0}}{\Delta t}\right)+\sum_{i p}\left(\rho u_{j} \Delta n_{j}\right)_{i p}=0 \\
\mathscr{V} \frac{\Delta\left(\rho u_{i}\right)}{\Delta t}+\sum_{i p}\left(\rho u_{j} \Delta n_{j}\right)_{i p}\left(u_{i}\right)_{i p}=\sum_{i p}\left(P \Delta n_{i}\right)_{i p}+\sum_{i p}\left[\mu\left(\frac{\partial u_{i}}{\partial x_{j}}+\frac{\partial u_{j}}{\partial x_{i}}\right) \Delta n_{j}\right]_{i p} \\
\mathscr{V} \frac{\Delta\left(\rho h_{o}\right)}{\Delta t}-\mathscr{V} \frac{\Delta(P)}{\Delta t}+\sum_{i p}\left(\rho u_{j} h_{0} \Delta n_{j}\right)_{i p}\left(u_{i}\right)_{i p}= \\
\sum_{i p}\left(k \frac{\partial T}{\partial x_{j}} \Delta n_{i}\right)_{i p}+\sum_{i p} u_{j}\left[\mu\left(\frac{\partial u_{i}}{\partial x_{j}}+\frac{\partial u_{j}}{\partial x_{i}}\right) \Delta n_{j}\right]_{i p}
\end{gathered}
$$

where $\mathscr{V}$ is the volume of the discrete control volume, $\Delta t$ is the discrete increment in time (for unsteady simulations), and $\Delta n_{j}$ is the discrete outward-pointing surface vector. 


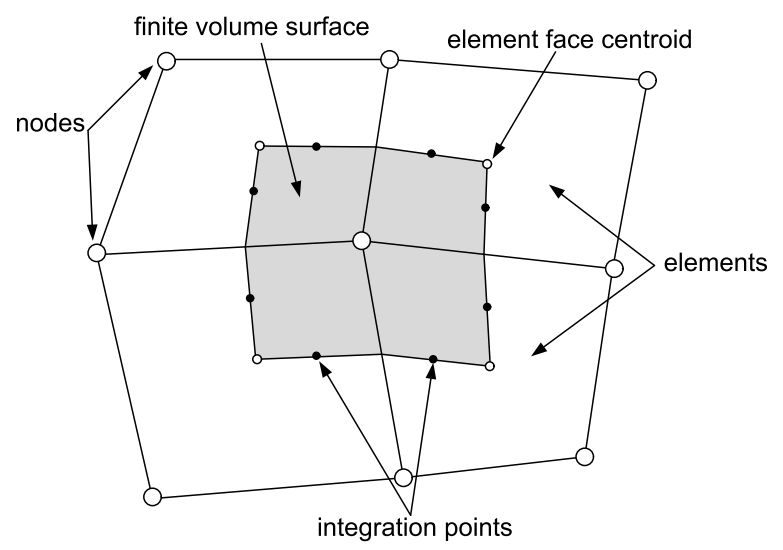

Figure 3.1: Two-dimensional representation of a finite volume as defined in ANSYS CFX. Adapted from McAuliffe (2007).

A pressure-velocity coupling is performed in order to evaluate the mass flux at the integration point, $\left.\rho u_{j}\right|_{i p}$. This coupling is implemented by expressing the velocity at an integration point as the average of the surrounding nodal values adjusted by a redistribution of pressure gradients and previous timestep velocities:

$$
\left.u_{j}\right|_{i p}=\left.\overline{u_{j}}\right|_{\text {nodes }}+f\left(\left.\frac{\partial p}{\partial x_{j}}\right|_{i p}-\left.\overline{\frac{\partial p}{\partial x_{j}}}\right|_{\text {nodes }}\right)-c \cdot f\left(\left.u_{j}\right|_{i p}-\left.\overline{u_{j}}\right|_{\text {nodes }}\right)_{t-\Delta t}
$$

where

$$
f=\frac{d_{i p}}{1-c \cdot d_{i p}}, \quad c=\frac{p}{\Delta t}, \text { and } d_{i p}=-\frac{\mathscr{V}_{\text {element }}}{\sum_{\text {element }}\left|\left(\rho u_{j} \Delta n_{j}\right)_{i p}\right|_{t-\Delta t}+8 \mu \frac{\left|\Delta n_{i p}\right|}{\Delta_{i p}}}
$$

In the denominator of $d_{i p}$, the summation of the absolute value of the mass flow rate is performed over all integration points within the element. The second term in the denominator is the magnitude of the local surface vector divided by the distance across the element, normal to the local surface, and passing through the integration point. The derivation of Equation 3.7 is provided in the ANSYS CFX Theory Guide (ANSYS, 2006).

A collocated-grid technique is used such that all the solution variables are stored at the 
grid nodes, corresponding to the centroids of each finite volume, which results in identical volumes for all transport equations. The values of the flow variables and their gradients at the integration points are interpolated from the centroid values using finite-element-based trilinear shape functions. The variation of any variable $\phi$ within an element is thus expressed as

$$
\phi=\sum_{n=1}^{N_{\text {node }}} N_{n} \phi_{n}
$$

where $N_{n}$ is the value of the shape function at node $n$ and $\phi_{n}$ is the value of $\phi$ at node $n$. The shape functions are linear functions of the parametric, element-based coordinates:

$$
\phi_{i p}=\sum_{n} N_{n}\left(r_{i p}, s_{i p}, t_{i p}\right) \phi_{n}
$$

where $\left(r_{i p}, s_{i p}, t_{i p}\right)$ represents the element-based coordinates of the integration points and the summation is performed over all the shape functions for the element under consideration. For the hexahedral finite volumes in the present study, the tri-linear shape functions are

$$
\begin{array}{llrl}
N_{1}(r, s, t) & =(1-r)(1-s)(1-t), & N_{2}(r, s, t) & =r(1-s)(1-t), \\
N_{3}(r, s, t) & =r s(1-t), & & N_{4}(r, s, t)=(1-r) s(1-t), \\
N_{5}(r, s, t) & =(1-r)(1-s) t, & & N_{6}(r, s, t)=r(1-s) t, \\
N_{7}(r, s, t) & =r s t, & & N_{8}(r, s, t)=(1-r) s t .
\end{array}
$$

For the advection terms, Equation 3.9 represents a centered-difference approximation, which has second-order accuracy, and is centered in space about the integration points. For the diffusion terms in Equations 3.5 and 3.6, the derivative of $\phi$ with respect to the coordinate direction $x_{i}$ at an integration point $i p$ is given by

$$
\left.\frac{\partial \phi}{\partial x_{i}}\right|_{i p}=\left.\sum_{n} \frac{\partial N_{n}}{\partial x_{i}}\right|_{i p} \phi_{n}
$$


The shape function derivatives are evaluated using a mapping procedure

$$
\left[\begin{array}{c}
\frac{\partial N_{n}}{\partial x} \\
\frac{\partial N_{n}}{\partial y} \\
\frac{\partial N_{n}}{\partial z}
\end{array}\right]=\left[\begin{array}{lll}
\frac{\partial x}{\partial r} & \frac{\partial y}{\partial r} & \frac{\partial z}{\partial r} \\
\frac{\partial x}{\partial s} & \frac{\partial y}{\partial s} & \frac{\partial z}{\partial s} \\
\frac{\partial x}{\partial t} & \frac{\partial y}{\partial t} & \frac{\partial z}{\partial t}
\end{array}\right]^{-1}\left[\begin{array}{c}
\frac{\partial N_{n}}{\partial r} \\
\frac{\partial N_{n}}{\partial s} \\
\frac{\partial N_{n}}{\partial t}
\end{array}\right]
$$

where $r, s$, and $t$ denote the local coordinate axes defined within each element. This discretization of the diffusion terms is also second-order accurate.

Temporal derivatives are approximated using a second-order Euler backwards difference scheme:

$$
\frac{\Delta\left(\rho u_{i}\right)}{\Delta t}=\frac{1}{\Delta t}\left[\frac{3}{2}\left(\rho u_{i}\right)^{t}-2\left(\rho u_{i}\right)^{t-\Delta t}+\frac{1}{2}\left(\rho u_{i}\right)^{t-2 \Delta t}\right]
$$

which makes use of the previous two time steps $t-\Delta t$ and $t-2 \Delta t$.

\subsection{Solution Methodology}

The discrete linearized forms of the governing equations (Equations 3.4-3.6) are a set of coupled equations and can be cast in matrix form as

$$
\sum_{n b_{i}} a_{i}^{n b} \phi=b_{i}
$$

where $\phi$ is the solution, $b$ the right hand side of the equation, $a$ the coefficients of the equation, $i$ is the identifying number of the finite volume in question and $n b$ represents the neighbouring nodes upon which the solution at $i$ depends plus the central coefficient multiplying the solution 
at the $i^{\text {th }}$ location. For the mass and momentum equations, $a, b$, and $\phi$ can be expressed as:

$$
a_{i}^{n b}=\left[\begin{array}{cccc}
a_{u u} & a_{u v} & a_{u w} & a_{u p} \\
a_{v u} & a_{v v} & a_{v w} & a_{v p} \\
a_{w u} & a_{w v} & a_{w w} & a_{w p} \\
a_{p u} & a_{p v} & a_{p w} & a_{p p}
\end{array}\right]_{i}, \quad \phi_{i}=\left[\begin{array}{c}
u \\
v \\
w \\
p
\end{array}\right]_{i}, \quad b_{i}=\left[\begin{array}{c}
b_{u} \\
b_{v} \\
b_{w} \\
b_{p}
\end{array}\right]_{i}
$$

The total enthalpy equation (Equation 3.6) is a scalar equation, so $a_{i}^{n b}, b_{i}$, and $\phi_{i}^{n b}$ are each single numbers. Scalar equations are solved in ANSYS CFX following the solution of the coupled mass-momentum system of equations.

Equation 3.15 illustrates that ANSYS CFX employs a coupled solver, which solves the mass and momentum equations as a single system, as opposed to a segregated solver, which solves the momentum equations first with a guessed pressure and then solves a pressure-correction equation. The advantages of the coupled treatment includes computational efficiency and a greater level of numerical stability, as the coupled system can be solved using a fully-implicit approach which avoids the need for judiciously-selected relaxation parameters for the variables (ANSYS, 2006). The primary disadvantage of the coupled solver is the large amount of memory needed to store all the coefficients.

The linearized system of equations can be written in general matrix form as:

$$
[A][\phi]=[b]
$$

This system is solved iteratively by ANSYS CFX using a multigrid-accelerated Incomplete Lower Upper (ILU) factorization method (ANSYS, 2006). In this method, an approximate initial solution $\left[\phi^{n}\right]$ is iteratively corrected by a correction term $\left[\phi^{\prime}\right]$ to give a corrected solution $\left[\phi^{n+1}\right]:$

$$
\left[\phi^{n+1}\right]=\left[\phi^{n}\right]+\left[\phi^{\prime}\right]
$$


Substituting Equation 3.17 into Equation 3.16 provides an expression for the solution of the correction term:

$$
[A]\left[\phi^{\prime}\right]=[b]-[A]\left[\phi^{n}\right]
$$

As the iterative solution converges to the solution of the discrete system of equations, the correction term tends towards zero. The difference between the actual value of the correction term and zero is defined as the residual, denoted $\mathscr{R}^{n}$ :

$$
\mathscr{R}^{n}=[A]\left[\phi^{\prime}\right]
$$

To assess an overall level of convergence and hence estimate the magnitude of the iterative error present in the solution, the residual at each node is normalized by ANSYS CFX using the relationship

$$
\widetilde{\mathscr{R}_{i}^{n}}=\frac{\mathscr{R}_{i}^{n}}{\eta_{i} \Delta \phi}
$$

where $\widetilde{\mathscr{R}_{i}^{n}}$ denotes the normalized residual at node $i$. The $\eta_{i}$ coefficient represents a scaling quantity based on the finite volume of the cell surrounding node $i$, and $\Delta \phi$ represents a measure of the variation of $\phi$ over all elements.

The iterations for reducing the residual within a given timestep are referred to as outer loops in ANSYS CFX (ANSYS, 2006). The outer iteration loops serve to converge the coefficients of the linearized governing equations and hence reduce the magnitude of the residual. Once the residual reaches a user-specified convergence criteria or a user-specified number of outer loops have been performed, the solver increments to the next timestep and the iterative solution is repeated with the updated flow information.

To accelerate convergence of the solution algorithm within each outer loop, an algebraic multigrid scheme is employed in the ANSYS CFX solver. In this technique, successively coarsened grids are solved in order to convect long-wavelength errors more rapidly through the 
computational domain, and the solution is then passed back up to finer grids. The multigrid scheme employed in ANSYS CFX is a W-type algebraic multigrid following the Additive Correction Method described by Hutchinson and Raithby (1986). The multigrid iterations within each outer loop are referred to as inner iteration loops; a single inner-loop iteration is performed per outer-loop iteration, and multiple outer-loop iterations are performed for each timestep. Within each inner-iteration loop, the multigrid technique passes the solution results to subsequently coarser grids (called a "restriction" operation) and a single solution sweep is performed at each coarsened grid level. The coarsening rate for a single restriction operation, defined as the ratio of fine-to-coarse mesh volumes for the restriction operation, is between 9 to 13, and restriction operations are stopped once the number of coarse grid volumes is below 20 . As the solution is interpolated back onto finer grids (called a "prolongation" operation), three solution sweeps are performed on the successively-finer grids. Previous studies by McAuliffe and Yaras (2006) indicate that a suitable level of convergence is obtained when the root-meansquare value of $\widetilde{\mathscr{R}_{i}^{n}}$ over all cells in the solution domain is less than $10^{-5}$, and this level of convergence can generally be attained within eight outer-loop iterations. As the simulation progresses towards a converged state, the equation residuals diminish asymptotically towards a

final residual of $O\left(10^{-6}\right)$ to $O\left(10^{-8}\right)$, depending on whether the equation under consideration is the mass, momentum, or energy conservation equation.

Further detailed information on the timestep sizes, simulation durations, and initialization procedures used in each of the simulations performed in this thesis are presented in the relevant sections of the journal papers reproduced in Chapters 4-8.

\subsection{Parallel Processing}

The large number of computational nodes required for performing DNS leads to large memory requirements and long simulation times. This problem is addressed through parallel 
Table 3.1: Description of the computational platforms used in the present research

\begin{tabular}{lcccc}
\hline Name & CPU type & Total cores & Memory type & Total RAM \\
\hline mcl & Intel Pentium 4 & 13 & Distributed & $52 \mathrm{~GB}$ \\
clusterq & Intel Xeon L5140 & 20 & Distributed & $80 \mathrm{~GB}$ \\
amd32-1 & AMD Opteron 6128 & 32 & Shared & $192 \mathrm{~GB}$ \\
amd32-2 & AMD Opteron 6128 & 32 & Shared & $192 \mathrm{~GB}$ \\
\hline
\end{tabular}

processing, where the computational domain is divided into smaller partitions and each partition is assigned to a separate processor. In the current research, partitioning is performed using an automated MeTiS partitioning method, which is an open-source partitioning package based on the Multilevel Graph Partitioning Algorithm (ANSYS, 2006). Several computational platforms are used in the present research; a brief description of each is given in Table 3.1. All platforms are located within the Department of Mechanical and Aerospace Engineering at Carleton University. It should be noted that the simulations that were executed on the amd32-1 and amd32-2 machines used only 24 of the available 32 CPU cores, as this was found to give the best computational efficiency on the shared-memory platforms. Communication between processors occurs through a Parallel Virtual Machine (PVM) interface.

\subsection{Computational Setup}

Because this thesis is written in an integrated-thesis format, this section describes only the general approach that was used for creating the computational domains and spatial grids employed in the simulations. The specific details of the computational domains, boundary conditions, and spatial grids used in each of the simulations performed in this thesis are presented in the relevant sections of the journal papers reproduced in Chapters 4-8.

Computational domains and spatial grids are created using a multi-block approach in which 


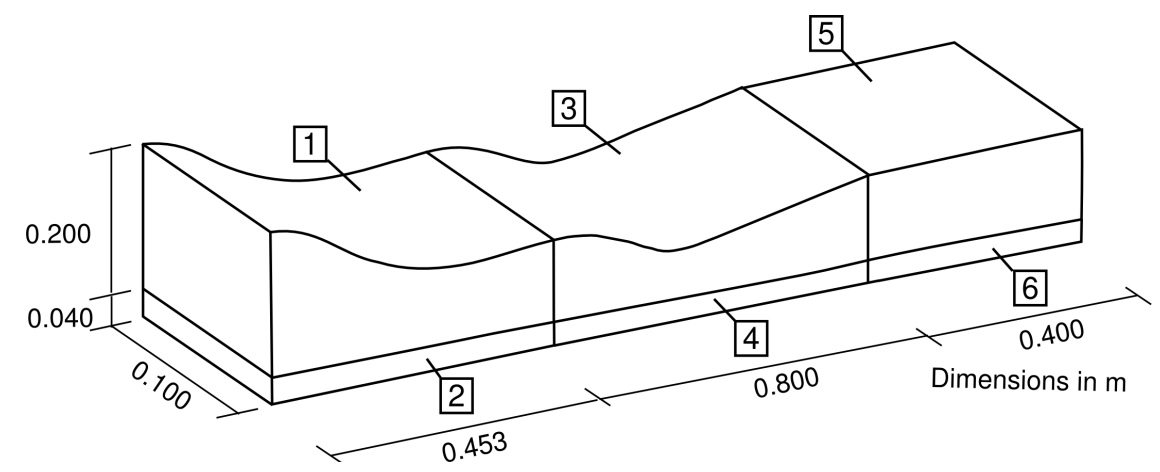

(a) Separation bubble domain (Chapter 4)

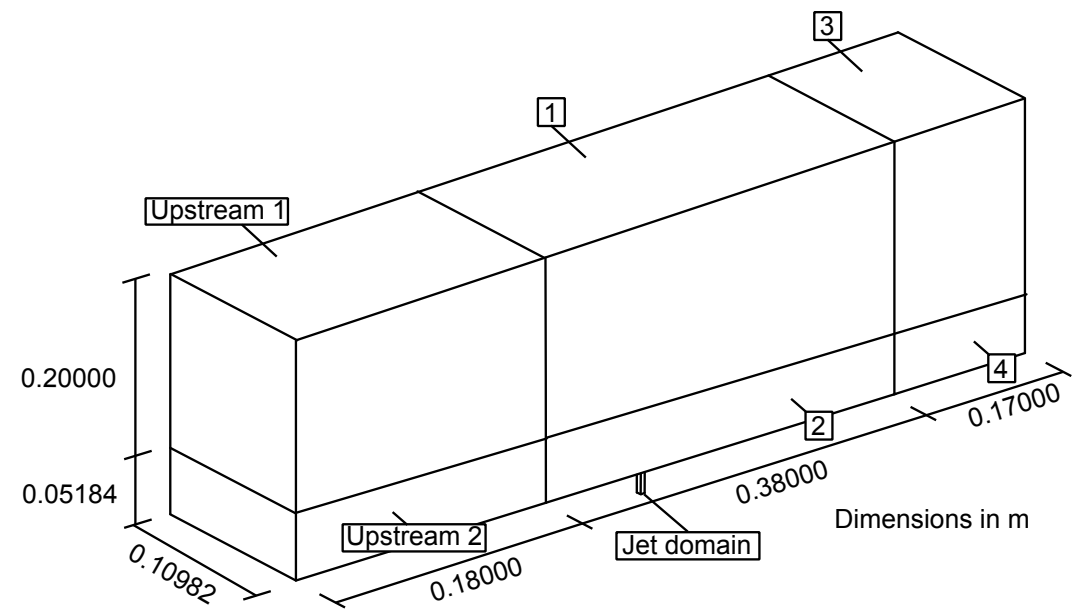

(b) Turbulent spot domain (Chapter 7)

Figure 3.2: Arrangement of sub-domain blocks in the computational grid for the $(a)$ separation bubble (Chapter 4) and (b) turbulent spot (Chapter 7) simulations.

the computational domain for a given simulation is formed from multiple hexahedral subdomains. Within each sub-domain, a structured grid is created for spatial discretization by the finite-volume method, as described in Section 3.2. Although ANSYS CFX uses an unstructured solver (ANSYS, 2006) and thus does not benefit from the performance improvement associated with using a structured grid, structured grids are used as they allow easier control of the grid quality and spatial resolution in regions where flow gradients are variable. The sub-domains are imported into the pre-processing module of ANSYS CFX and fluid-fluid interfaces between mating sub-domain interfaces are defined. As an illustration, Figure 3.2 shows the arrangement of the blocks in the computational domains used in Chapters 4 and 7 . 
When creating and combining the blocks, care was taken to ensure that the nodes on the boundaries of each block were coincident with the boundary nodes of the mating blocks. Although ANSYS CFX is capable of handling a mismatch in the boundary nodes between blocks, trial simulations using this functionality suffered from discontinuities in the spatial derivatives of the flow variables across the interface. As it is difficult to predict how these discontinuities will affect the solution, such a mismatch was generally avoided. One exception is the interface between blocks downstream of the region of interest, where the spatial resolution in the sub-domains downstream of the region of interest is purposely coarsened to damp out vortical disturbances before they reach the outlet boundary of the simulation. Such an approach improves the transmission of boundary information from the outlet boundary into the interior of the computational domain and improves the convergence rate of the iterative solver.

As shown in Figure 3.2, the shape of the upper-block surfaces are contoured to impose a desired pressure distribution onto the test surface. The shape of the upper-block surfaces are described by polynomials where the wall-normal height $(y)$ of the upper-block surface varies with the streamwise direction $(x)$ according to

$$
y=c_{0}+c_{1} x+c_{2} x^{2}+\ldots+c_{n} x^{n}
$$

where $n$ is the order of the polynomial. The polynomial coefficients for the upper-block surfaces, sub-domain sizes, boundary information, and grid information are recorded for all the simulated cases in Appendix A. 


\subsection{Data Reduction of Simulation Results}

\subsubsection{Averaging methods}

The data produced by the simulations are the three instantaneous components of velocity $(u, v, w)$ and the static pressure $(p)$. The spatial and temporal resolution of the simulations captures the length- and time-scales of the instability mechanisms occurring within the flow, permitting time-averaging of the simulation data to obtain time-averaged and fluctuation quantities. For a generic variable $\phi$, the time-average is given by

$$
\overline{\phi(x, y, z)}=\frac{1}{T} \int_{t=t_{0}}^{t=t_{0}+T} \phi(x, y, z, t) d t
$$

where the integration is taken over a time-period $T$. When discretized over a finite timestep $\Delta t$, the time-average becomes

$$
\overline{\phi(x, y, z)}=\frac{1}{N_{t}+1} \sum_{i=0}^{N_{t}} \phi\left(x, y, z, t_{0}+i \Delta t\right)
$$

where the summation is performed over the number of timesteps collected in each simulation, $N_{t}$. For the purposes of spectral analysis, it is convenient to collect $2^{N}$ timesteps, where $N$ is an integer; for the proposed simulations, $N=12$ is deemed adequate to capture the relevant statistical quantities of the flow.

Fluctuation correlations are also defined using a time-averaging method. For two generic variables $\phi$ and $\psi$, the discrete formulation of the fluctuation correlation is given by

$$
\overline{\phi^{\prime} \psi^{\prime}(x, y, z)}=\frac{1}{N_{t}+1} \sum_{i=0}^{N_{t}} \phi^{\prime} \cdot \psi^{\prime}
$$


where the instantaneous fluctuations $\phi^{\prime}$ and $\psi^{\prime}$ are given by

$$
\begin{gathered}
\phi^{\prime}=\phi\left(x, y, z, t_{0}+i \Delta t\right)-\overline{\phi(x, y, z)} \\
\psi^{\prime}=\psi\left(x, y, z, t_{0}+i \Delta t\right)-\overline{\psi(x, y, z)} .
\end{gathered}
$$

Similarily, the root-mean-square variables are calculated according to

$$
\phi_{r m s}=\sqrt{\frac{\sum_{i=0}^{N_{t}}\left[\phi\left(x, y, z, t_{0}+i \Delta t\right)-\overline{\phi(x, y, z)}\right]^{2}}{\left(N_{t}+1\right)}} .
$$

As described in Chapters 1 and 2, flow instabilities frequently form stationary high- and lowmomentum streaks in the boundary layer. When the streak amplitude is small in comparison to the mean velocity profile, these streaks are only visible when the mean boundary-layer profile is subtracted from the instantaneous flow field. As the streaks are stationary, and thus appear in the time-averaged flow field, subtracting the time-averaged flow field from the instantaneous flow field would also largely remove the streaks. Following the method of Dagenhart and Saric (1999), the streaks are visualized by removing the spanwise-averaged velocity profile from the instantaneous velocity field, producing the so-called "perturbation" velocity field, defined as:

$$
\widetilde{\phi}(x, y, z)=\phi(x, y, z)-\frac{1}{N_{z}+1} \sum_{k=0}^{N_{z}} \phi\left(x, y, z_{0}+k \Delta z\right)
$$

The second term on the right-hand-side of Equation 3.26 is the spanwise-averaged value of $\phi$. Analyzing the perturbation velocity field also enhances the visibility of vortical structures in the separated and transitional portions of the flow. 


\subsubsection{Vortex identification}

Transitional and turbulent shear flows are known to be dominated by spatially-coherent, temporally-evolving vortices, often referred to as coherent structures (Robinson, 1991). These coherent structures are responsible for numerous turbulence phenomena such as entrainment, mixing, and break-down to smaller turbulence scales during transition. In order to study the dynamics of coherent structures, the dynamically significant, large-scale vortical regions making up the coherent structures must first be identified. Jeong and Hussain (1995) reviews the definitions that have been suggested for eduction of coherent structures in numerical simulations, including low-pressure iso-surfaces, iso-surfaces of constant vorticity magnitude, regions of complex eigenvalues of the velocity gradient tensor, and regions containing a positive second-invariant of the velocity gradient tensor. Each of these definitions has been implemented, and the criteria of a positive second-invariant of the velocity gradient tensor was determined to best identify the coherent structures in the flow. Furthermore, clarity of the coherent structures is further improved by using the perturbation velocity gradient tensor $\nabla \widetilde{u_{j}}$. According to Chong et al. (1990), the second invariant of $\nabla \widetilde{u_{j}}$ is given by

$$
Q=\frac{1}{2}\left(\frac{\partial \widetilde{u}_{i}^{2}}{\partial x_{j}}-\frac{\partial \widetilde{u}_{i}}{\partial x_{j}} \frac{\partial \widetilde{u}_{j}}{\partial x_{i}}\right)
$$

where Einstein summation is implied over the indexed terms. For the calculation of the velocity gradients, a centered-difference formulation was used which accounts for non-uniform grid spacing. One-sided differences are used to compute gradients on boundary nodes, where the centered-difference method cannot be applied. For any spatial variable $x$ and field variable $\phi$, the first and second derivative of $\phi$ with respect to $x$ at node $i$ are given by:

$$
\left.\frac{\partial \phi}{\partial x}\right|_{i}=\frac{\phi_{i+1}\left(x_{i}-x_{i-1}\right)^{2}-\phi_{i-1}\left(x_{i+1}-x_{i}\right)^{2}+\phi_{i}\left[\left(x_{i+1}-x_{i}\right)^{2}-\left(x_{i}-x_{i-1}\right)^{2}\right]}{\left(x_{i+1}-x_{i}\right)\left(x_{i}-x_{i-1}\right)\left(x_{i+1}-x_{i-1}\right)}
$$




$$
\left.\frac{\partial^{2} \phi}{\partial x^{2}}\right|_{i}=\frac{2\left[\phi_{i+1}\left(x_{i}-x_{i-1}\right)-\phi_{i-1}\left(x_{i+1}-x_{i}\right)+\phi_{i}\left(x_{i+1}-x_{i-1}\right)\right]}{\left(x_{i+1}-x_{i}\right)\left(x_{i}-x_{i-1}\right)\left(x_{i+1}-x_{i-1}\right)} .
$$

These partial derivatives are also used in calculating the vorticity vector, $\Omega=\nabla \times U_{j}$, and the perturbation vorticity vector, $\widetilde{\Omega}=\nabla \times \widetilde{U_{j}}$.

\subsubsection{Spectral analysis}

Spectral analysis of the simulation results identifies which disturbance frequencies experience the greatest amplification rate. This so-called most-amplified frequency is associated with the instability mechanism which dominates at the point being analyzed. A fast Fourier transform (FFT) algorithm is used to transform the velocity and pressure time-traces to the frequency domain and calculate the power spectral density (PSD) distributions at various locations within the simulation results. An implementation of the open-source algorithm FFTW by Frigo and Johnson (2005) was used to compute the FFT of the simulation data.

\subsubsection{Turbulence integral length scale calculation}

The integral length scale defines the average size of the vortical structures in a turbulent flow that contain the most energy. Assuming that the scale of the energy-containing eddies change very little in the time it takes to convect the distance of the integral length scale (Taylor's frozen-eddy approximation), the length scale is given by

$$
\Lambda_{x}=\int_{0}^{\infty} R(\Delta t) d \Delta t
$$

where

$$
R_{x}(\Delta t)=\frac{\overline{u^{\prime}(t) u^{\prime}(t+\Delta t)}}{\overline{u^{\prime 2}}}
$$


is the normalized autocorrelation function. Equation 3.30 is used to calculate the length scales of the free-stream turbulence and the reattached turbulent boundary-layer in the proposed simulations. The length scales in the other coordinate directions are defined as in Equation 3.30 but with $v^{\prime}$ and $w^{\prime}$ used in the autocorrelation function for $\Lambda_{y}$ and $\Lambda_{z}$, respectively.

\subsubsection{Intermittency calculation}

Intermittency, defined as the fraction of time during which the flow at a given point is turbulent, is another important variable in analyzing transitional flows. The intermittency distribution is computed using an algorithm described by Volino et al. (2001) from data extracted at a constant wall-normal distance from the test surface. This algorithm analyzes the magnitude of the first- and second-derivative of the streamwise velocity time-trace $(d u / d t$ and $d^{2} u / d t^{2}$ ). A large value of $d u / d t$ indicates rapid fluctuation (i.e. a turbulent eddy), while the second-derivative is included to capture turbulence at the local maxima and minima where $d u / d t$ is 0 . If either of the derivatives exceeds a specified threshold at a given instant, a flow-state parameter $\beta$ is set to unity, otherwise it is set to zero. As suggested by Volino et al. (2001), the threshold for the first-derivative signal scales on the free-stream velocity, although a different scaling factor is used, as their scaling factor was developed for attachedflow transition. The threshold for the second-derivative signal is automatically adjusted by the algorithm so that the time-average of the $\beta$-distributions for the first- and second-derivatives are equal. The combined $\beta$-distribution is then low-pass filtered to avoid data "drop-outs" and the intermittency value $\gamma$ is determined by time-averaging the filtered $\beta$-distribution.

\subsection{Linear Stability Analysis}

As a complement to the simulation results based on the ANSYS CFX solver, linear stability analysis is performed by numerically solving the Orr-Sommerfeld equation to obtain the 
theoretical growth rates of temporally-growing disturbances within a mean velocity field $U(y)$. The perturbation is assumed to have the form given in Equation 2.1, with a real wavenumber $\alpha$ and a complex wave speed $c=c_{r}+i c_{i}$. As described in Chapter 2, for the perturbation to grow in time, the imaginary component of the complex wave speed must be positive. To obtain the complex wave speed, a hybrid spectral collocation method based on Chebyshev polynomials is used to calculate the eigenvalues of the Orr-Sommerfeld equation. For convenience, the Orr-Sommerfeld equation given in Equation 2.2 is rearranged as

$$
\left(U(y) k^{2}-U(y)^{\prime \prime}-\frac{k^{4}}{i \alpha R e}\right) \hat{v}+\left(U(y)-\frac{2 k^{2}}{i \alpha R e}\right) \hat{v}^{\prime \prime}-\frac{2 k^{2}}{i \alpha R e} \hat{v}^{i v}=c\left(\hat{v}^{\prime \prime}-k^{2} \hat{v}\right) .
$$

and the boundary conditions

$$
\hat{v}( \pm 1)=\hat{v}^{\prime}( \pm 1)=0
$$

are applied at the ends of the bounded domain $(y= \pm 1)$. The prime denotes differentiation with respect to the inhomogeneous wall-normal coordinate $y$, and $k^{2}=\alpha^{2}+\beta^{2}$ is the magnitude of the wavenumber vector; in this thesis, only one-dimensional disturbances are considered, so $\beta=0$ and $k=\alpha$.

Chebyshev polynomial series of the first kind are used to approximate the eigenfunction as

$$
\hat{v}(y)=\sum_{n=0}^{N} a_{n} T_{n}(y)
$$

and the derivatives of the eigenfunction are obtained by differentiating the above expression, yielding

$$
\begin{aligned}
\hat{v}^{\prime \prime} & =\sum_{n=0}^{N} a_{n} T_{n}^{\prime \prime}(y) \\
\hat{v}^{i v} & =\sum_{n=0}^{N} a_{n} T_{n}^{i v}(y)
\end{aligned}
$$


Substitution of these expansions into Equation 3.31 yields

$$
\begin{aligned}
& \left(U(y) k^{2}-U(y)^{\prime \prime}-\frac{k^{4}}{i \alpha R e}\right) \sum_{n=0}^{N} a_{n} T_{n}(y)+\left(U(y)+\frac{2 k^{2}}{i \alpha R e}\right) \sum_{n=0}^{N} a_{n} T_{n}^{\prime \prime}(y) \\
& -\frac{1}{i \alpha R e} \sum_{n=0}^{N} a_{n} T_{n}^{i v}(y)=c\left(\sum_{n=0}^{N} a_{n} T_{n}^{\prime \prime}(y)-k^{2} \sum_{n=0}^{N} a_{n} T_{n}(y)\right)
\end{aligned}
$$

The discretized boundary conditions are

$$
\begin{aligned}
\sum_{n=0}^{N} a_{n} T_{n}(1) & =0 \\
\sum_{n=0}^{N} a_{n} T_{n}^{\prime}(1) & =0 \\
\sum_{n=0}^{N} a_{n} T_{n}(-1) & =0 \\
\sum_{n=0}^{N} a_{n} T_{n}^{\prime}(-1) & =0
\end{aligned}
$$

Using these boundary conditions, Equation 3.36 can be written as a generalized eigenvalue problem of the form

$$
\mathbf{A} \mathbf{a}=c \mathbf{B} \mathbf{a}
$$

This system of equations is solved numerically using MATLABß to obtain the eigenvalues $c$. Further detail is provided by Schmid and Henningson (2000). 


\title{
Chapter 4
}

\section{Interaction of Viscous and Inviscid Instability Modes in}

\section{Separation-Bubble Transition}

\author{
Citation: Brinkerhoff, J.R. and Yaras, M.I. (2011), "Interaction of \\ viscous and inviscid instability modes in separation-bubble \\ transition," Physics of Fluids, 23, pp. 124102. DOI: \\ http://dx.doi.org/10.1063/1.3666844. Used with permission.
}




\subsection{Introduction}

A transitional separation bubble occurs when a laminar boundary layer separates in the presence of an adverse streamwise pressure gradient, undergoes laminar-to-turbulent transition through the amplification of disturbances within the flow, and then reattaches due to the enhanced wall-normal momentum exchange arising from turbulence. Transitional separation bubbles are frequently observed on the suction surface of highly-loaded airfoils, deteriorating the performance of the airfoil through reduced lift and increased drag (Mayle, 1991; Hodson and Howell, 2005). Numerous studies have been conducted to understand the effects of flow Reynolds number, streamwise pressure gradient, surface roughness, periodic free-stream unsteadiness, and vortical and acoustical disturbances on separation-bubble transition (Roberts and Yaras, 2005a; Malkiel and Mayle, 1996; Yang and Voke, 2001; Volino and Bohl, 2004). These studies have resulted in increasingly-refined engineering models that allow prediction of the transition onset location and transition rate in separated and attached boundary layers (e.g., Roberts and Yaras, 2005b). A clear understanding of the physics of the instability mechanisms that contribute to transition is a prerequisite to further improvements of such transition models.

In separation bubbles under low free-stream turbulence levels, the viscous TollmienSchlichting (T-S) and inviscid Kelvin-Helmholtz (K-H) instabilities have been shown to be the relevant instability modes leading to transition (Roberts and Yaras, 2006; McAuliffe and Yaras, 2006; Hatman and Wang, 1998; Marxen et al., 2004). The T-S instability develops primarily in the attached laminar region upstream of flow separation, forming two-dimensional disturbances known as T-S waves (Schlichting, 1968). As the T-S waves convect downstream in the attached boundary layer, they develop coherent three-dimensional vortices through a lift-up and vortex-stretching process which occurs as a result of the velocity field induced by the disturbed boundary-layer vorticity (Kachanov, 1994). The velocity field induced by 
these vortices ejects low-momentum fluid away from the wall and brings high-momentum fluid towards the wall, producing high- and low-momentum streaks aligned in the streamwise direction (e.g., Jacobs and Durbin, 2001). Further stretching of the three-dimensional vortical structures results in regions of high shear that undergo localized transition to turbulence (McAuliffe and Yaras, 2007b).

Upon two-dimensional separation of the boundary layer, an inviscid K-H instability develops due to the local highly-inflectional velocity profile. In this mode, perturbations of the separated shear layer induce a velocity field that results in a grouping of spanwise vorticity into two-dimensional, streamwise-periodic vortices that amplify and eventually shed from the separated shear layer in a time-periodic manner (Abdalla and Yang, 2004; MutiLin and Pauley, 1996; Talan and Hourmouziadis, 2002; Wissink and Rodi, 2006). Further downstream-depending on the value of the momentum-thickness Reynolds number at separation (McAuliffe and Yaras, 2007b) — an additional sub-harmonic instability may result in streamwise pairing of the shed vortices and a doubling of their streamwise spacing, similar to that observed in free shear layers (Ho and Huerre, 1984). Upon formation of the streamwise train of vortical structures, transition is typically observed to occur in regions of high shear between the shed spanwise vortices (McAuliffe and Yaras, 2007b; Jones et al., 2008). An alternative path to transition is provided by the reversed flow along the wall beneath the separated shear layer; viscous T-S instability of this wall shear layer may dominate the inviscid instability of the separated shear layer in instances where the reversed flow velocity exceeds about $20 \%$ of the local free-stream velocity (Hammond and Redekopp, 1998; Alam and Sandham, 2000; Rist and Maucher, 2002).

The location and rate of transition - and hence the shape and extent of the separation bubble - largely depend on which of the above-noted instability mechanisms dominates the transition process. Recent investigations have demonstrated the possibility of an interaction between the T-S and K-H instability modes. Simulations by Roberts and Yaras (2006) and 
McAuliffe and Yaras (2006) observed that the roll-up of the separated shear layer into vortical structures driven by the $\mathrm{K}-\mathrm{H}$ instability mechanism occurs at a frequency very close to the dominant T-S frequency of the upstream boundary layer. Hain et al. (2009) and Diwan and Ramesh (2009) also observed a similarity between the most-amplified frequency of the T-S waves in the upstream attached boundary layer and the $\mathrm{K}-\mathrm{H}$ instability in the separated boundary layer. Furthermore, several researchers have observed a spanwise non-uniformity of the shear layer following initial roll-up, attributed to the development of a secondary instability superimposed on the primary two-dimensional instability (Ho and Huerre, 1984; Huang and Ho, 1990; Malkiel and Mayle, 1996; Bao and Dallmann, 2004). It is plausible that this secondary instability is affected by the flow structures created in the upstream boundary layer under the influence of the viscous T-S instability mechanism. The present study was undertaken to examine the possible interactions between these instability mechanisms within the separated shear layer. A direct numerical simulation was performed of a flat-plate boundary layer developing under a streamwise pressure distribution and flow Reynolds number that is representative of the conditions of a low-pressure turbine blade in a gas-turbine engine. The results provide insight on the development of the viscous and inviscid instability modes, their mutual interaction within the separated shear layer, and how their interaction accelerates break-down to turbulence.

\subsection{Numerical Method}

A direct numerical simulation (DNS) was performed of a laminar boundary layer that separates and transitions to turbulent flow under a free-stream pressure distribution typical of the suction side of a low-pressure gas-turbine blade. ANSYS CFX $\AA$ (Version 11), a commercial computational fluid dynamics software package, was used to solve the time-varying governing equations using a finite-volume spatial discretization approach. Previous work by the 
authors' research group and several others in published literature have investigated separationbubble transition by numerically solving the incompressible form of the unsteady mass- and momentum-conservation equations (Ripley and Pauley, 1993; Na and Moin, 1998; Alam and Sandham, 2000; Yang and Voke, 2001; Michelassi et al., 2002; Rist and Augustin, 2006; Roberts and Yaras, 2006; McAuliffe and Yaras, 2006). The results of these computations show good agreement with experiments in terms of the shape and extent of the time-averaged separation bubble, the streamwise velocity fluctuation spectra, the location of transition onset, and the streamwise rate of transition. Given the emphasis of the present study on the interaction of viscous and inviscid instability mechanisms, the transient development of viscous instability in the attached boundary layer upstream of separation must be correctly simulated. This requires that pressure waves propagate at finite speeds through a proper treatment of the compressibility of the working fluid. In the present simulation, compressibility of the working fluid (air) was accounted for through a coupled solution of the energy equation with the mass and momentum equations, with the ideal-gas approximation providing closure for the thermodynamic state of the working fluid.

\subsubsection{Computational domain and boundary conditions}

The computational domain used in the present study, shown in Figure 4.1, consists of a contoured ceiling above a flat $800 \mathrm{~mm}$-long test surface. The inflow boundary is placed $453 \mathrm{~mm}$ upstream of the leading edge of the test surface and a free-slip surface is placed between the inflow boundary and the test-surface leading edge to allow the test-surface boundary layer to develop naturally starting from the leading edge. The test surface is represented by a noslip wall over which the boundary layer and separated region develop, and it is followed by a $420 \mathrm{~mm}$-long free-slip surface. The length of the downstream free-slip surface was chosen to place the outflow boundary a conservative distance from the region of interest, as the outflow

boundary condition available in ANSYS CFX ${ }^{\circledR}$ does not allow convective flow structures 


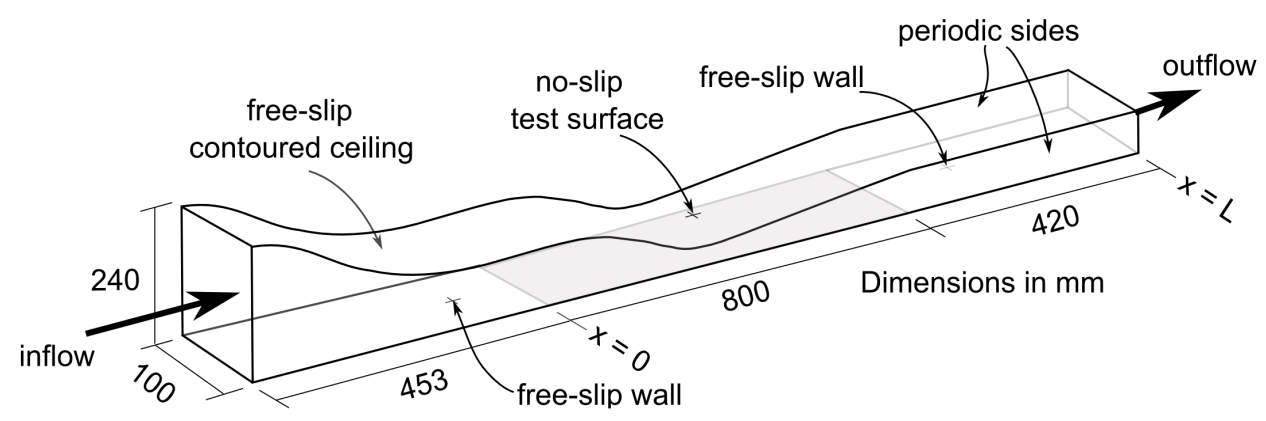

Figure 4.1: Schematic of the computational domain. Reprinted with the permission of the American Institute of Physics.

such as turbulent eddies to exit the domain through the boundary without distortion. A streamwise pressure distribution representative of the suction side of a low-pressure turbine blade was created on the test surface by contouring the free-slip ceiling of the computation domain. Spanwise periodicity was imposed along the sides of the computational domain, and a spanwise width of $S=100 \mathrm{~mm}$ was chosen to place the sides of the domain a conservative distance away from the region of interest, which is located near the midspan. A spatiallyuniform, time-invariant inlet velocity and a static pressure which remains fixed in an areaaveraged sense were specified for the inflow and outflow boundaries, respectively. An outflow boundary treatment similar to the non-reflective boundary proposed by Rudy and Strikwerda (1980) was implemented to reduce the artificial reflection of pressure waves from the outflow boundary back into the computational domain. The reference length $L=1220 \mathrm{~mm}$ is taken as the distance between the leading edge of the test plate and the outflow boundary of the computational domain. This length is consistent with the dimensions of the test surface in experimental studies of transition phenomena undertaken by the authors' research group at Carleton University (e.g., Yaras, 2001; Yaras, 2002; Roberts and Yaras, 2005a). 


\subsubsection{Spatial grid}

A structured grid consisting of hexahedral finite volumes was mapped to the domain described above. The region of interest above the no-slip wall was discretized with 491, 59, and 176 nodes in the streamwise, wall-normal, and spanwise directions, respectively; this region accounts for approximately $65 \%$ of the total grid nodes in the computational domain. The node distributions in the three coordinate directions were chosen to resolve the flow structures occurring from viscous instabilities in the attached laminar boundary layer, the instabilities and transitioning flow structures within the separated shear layer, and the turbulence scales in the reattached turbulent boundary layer. At $x / L=0.65$, which is well within the reattached turbulent boundary-layer, the $y^{+}$value of the first node above the test surface is about 0.8 and the corresponding values of $\Delta x^{+}$and $\Delta z^{+}$are 22 and 11, respectively. McAuliffe and Yaras (2007b) found a spatial grid with $\Delta x^{+}$ranging from 14 to 19 and $\Delta z^{+}=19$ to be adequate for performing DNS in transitioning separation bubbles in low-disturbance environments. A coarser spatial resolution is used in the free-slip regions upstream and downstream of the test surface and near the free-slip ceiling of the computational domain.

Although similar spatial resolutions have been successfully used by a number of other researchers of separation-bubble transition (e.g., Na and Moin, 1998; Alam and Sandham, 2000; Michelassi et al., 2002), a companion direct numerical simulation was performed to ascertain the sensitivity of the flowfield to the spatial grid. In this companion study, first a grid with an equivalent spatial resolution was used to simulate a zero-pressure-gradient turbulent boundary layer at a momentum-thickness Reynolds number of $R e_{\theta}=500$, chosen to match the Reynolds number of the reattached turbulent boundary layer downstream of the separation bubble in the current study. The companion study was then repeated with a refined spatial resolution of $\Delta x^{+}=\Delta z^{+}=3$. The simulation results for both grid resolutions compare favourably with the turbulent kinetic energy budgets computed by Spalart (1988) and turbulent velocity profile 

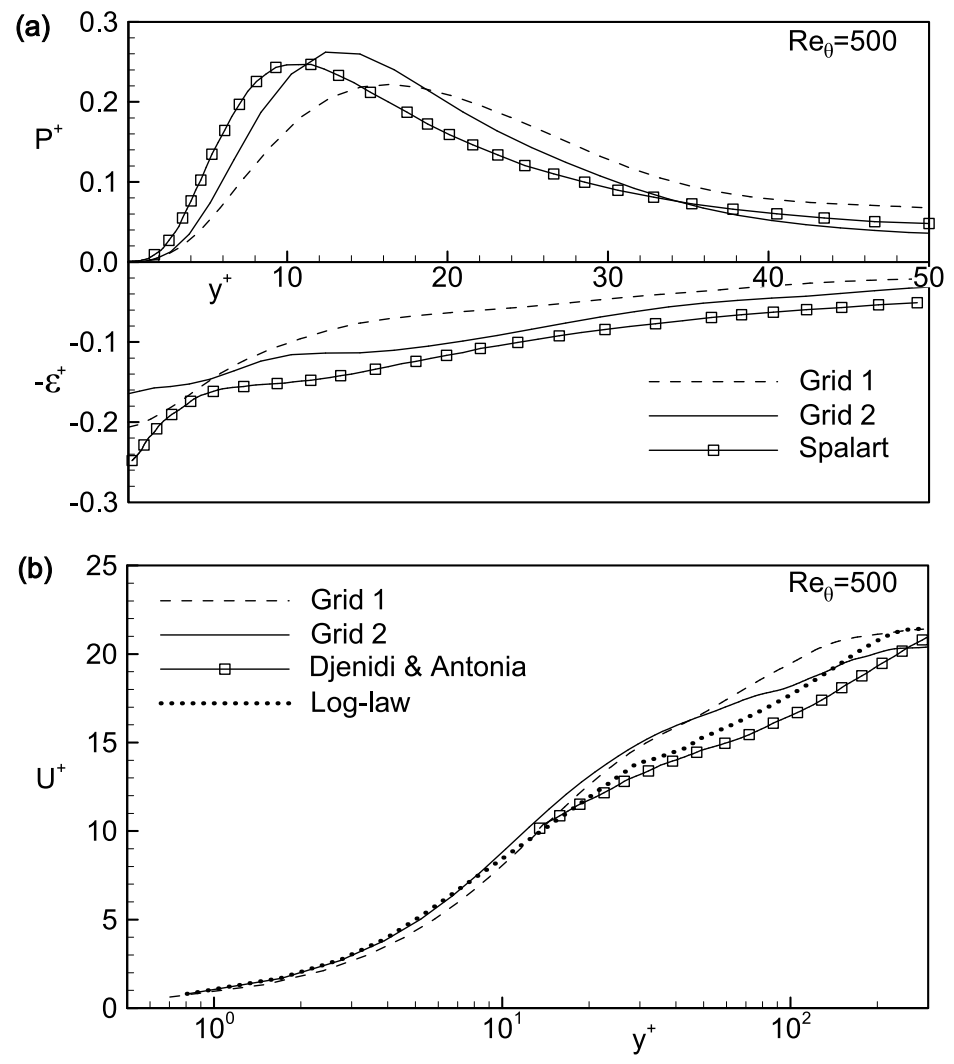

Figure 4.2: Results of a companion DNS study assessing grid sensitivity in a zero-streamwiseacceleration turbulent boundary layer at $R e_{\theta}=500$. Results from Grid $1\left(\Delta x^{+}=22\right.$, $\left.\Delta z^{+}=11\right)$ and Grid $2\left(\Delta x^{+}=\Delta z^{+}=3\right)$ are compared with $(a)$ numerical results of Spalart (1988) and (b) measurements of Djenidi and Antonia (1993). Reprinted with the permission of the American Institute of Physics.

measured by Djenidi and Antonia (1993), as shown in Figure 4.2. This provides evidence that the spatial grid in the current separation-bubble simulation is sufficient to adequately capture the flow development in the turbulent boundary layer downstream of the bubble. The sensitivity to the choice between these two spatial resolutions should be even further reduced in the transitional-flow region where the transient coherent flow structures tend to be of notably greater spatial scale than in the downstream reattached turbulent boundary layer. 


\subsubsection{Solution method}

Discretization of the governing equations is based on second-order central differencing and second-order Euler backward differencing for the spatial and temporal derivatives, respectively. To resolve the convection of pressure waves in a time-accurate manner, a temporal resolution was chosen such that a disturbance moving at the speed of sound would travel approximately one grid-cell per timestep, which produced a timestep size of $\Delta t=0.01 \mathrm{~ms}$ or $0.05 t^{+}$, where $t^{+}=\nu / u_{\tau}^{2}$ is the viscous timescale in the reattached turbulent boundary layer downstream of the separation bubble. Compared to the timestep size of $0.2 t^{+}$recommended by Choi and Moin (1994) for accurately computing turbulent flows, the timestep in the current study is conservatively sized. The discretized equations were converged through an algebraic multigrid scheme within eight inner-loop iterations per timestep, reducing the root-mean-square residual

of the governing equations by five orders of magnitude to less than $10^{-6}$. The simulation was initialized with a uniform velocity equal to the velocity specified at the inflow boundary, zero relative static pressure and a uniform temperature of $300 \mathrm{~K}$. Approximately 80,000 timesteps were required to reach a statistically steady state, after which 20,480 timesteps were computed for analysis. The simulation was partitioned and executed in parallel on 20 cores of five Intel $^{\mathrm{TM}}$ L5410 Xeon processors. Details of the simulation are given in Table 4.1.

\subsection{Results and Discussion}

\subsubsection{Free-stream flow development}

The present discussion of the simulation results focuses on the instability modes occurring in a boundary layer under low free-stream turbulence conditions and a streamwise pressure distribution that is representative of the suction side of a low-pressure axial turbine airfoil. The streamwise and spanwise directions are normalized by the reference length $(L=1220 \mathrm{~mm})$ 
Table 4.1: Flow conditions and numerical details of the current simulation. Reprinted with the permission of the American Institute of Physics.

\begin{tabular}{lc}
\hline Flow Reynolds number, $R e_{L}$ & 326,000 \\
Timestep size, $\Delta t$ & $0.01 \mathrm{~ms}$ \\
Number of grid nodes & $11.7 \times 10^{6}$ \\
Streamwise separation location, $x_{s} / L$ & 0.399 \\
Free-stream turbulence intensity at separation, $T u_{s}$ & $0.25 \%$ \\
Integral length scale of free-stream disturbances, $\Lambda_{s}$ & $18.1 \mathrm{~mm}$ \\
Momentum-thickness Reynolds number at separation, $R e_{\theta s}$ & 300 \\
Displacement thickness at separation, $\delta_{s}^{*}$ & $2.41 \mathrm{~mm}$ \\
Reference length, $L$ & $1220 \mathrm{~mm}$ \\
Reference velocity, $U_{0}$ & $4 \mathrm{~m} / \mathrm{s}$ \\
Reference temperature, $T_{0}$ & $300 \mathrm{~K}$ \\
Reference pressure, $P_{0}$ & $101.3 \mathrm{kPa}$ \\
Working fluid & $\mathrm{Air}$ \\
\hline
\end{tabular}

and the wall-normal direction is normalized by the displacement thickness at the point of flow separation $\left(\delta_{s}^{*}=2.41 \mathrm{~mm}\right)$. The reference velocity is $U_{0}=4 \mathrm{~m} / \mathrm{s}$, which corresponds to the velocity magnitude in the simulation results at $x / L=0$ and $y / \delta_{s}^{*}=15$. The Reynolds number based on the reference parameters is $R e_{L}=326,000$ and the reference free-stream Mach number is $U_{0} / a=0.012$.

Small free-stream disturbances required for the creation and growth of perturbations that lead to transition when the test-surface boundary layer reaches an unstable state are generated numerically through round-off errors during the iterative computations of the flow field. This method has also been used by other researchers as a disturbance source for computing natural transition (e.g., Spalart and Strelets, 2000). At the separation point, the integral length scale corresponding to these free-stream disturbances, computed using the frozen-eddy hypothesis, is $18.1 \mathrm{~mm}$ and the streamwise distribution of the resulting free-stream turbulence intensity is shown in Figure 4.3. The time-averaged streamwise velocity distribution at a wall-normal height of $15 \delta_{s}^{*}$ is shown in Figure 4.4. The streamwise variation of the time-averaged free-stream acceleration is shown in Figure 4.5 through a plot of the streamwise acceleration parameter, $\eta=\nu / U_{e}^{2} d U_{e} / d x$, where $U_{e}$ is the local boundary-layer edge velocity. The two dashed horizontal 


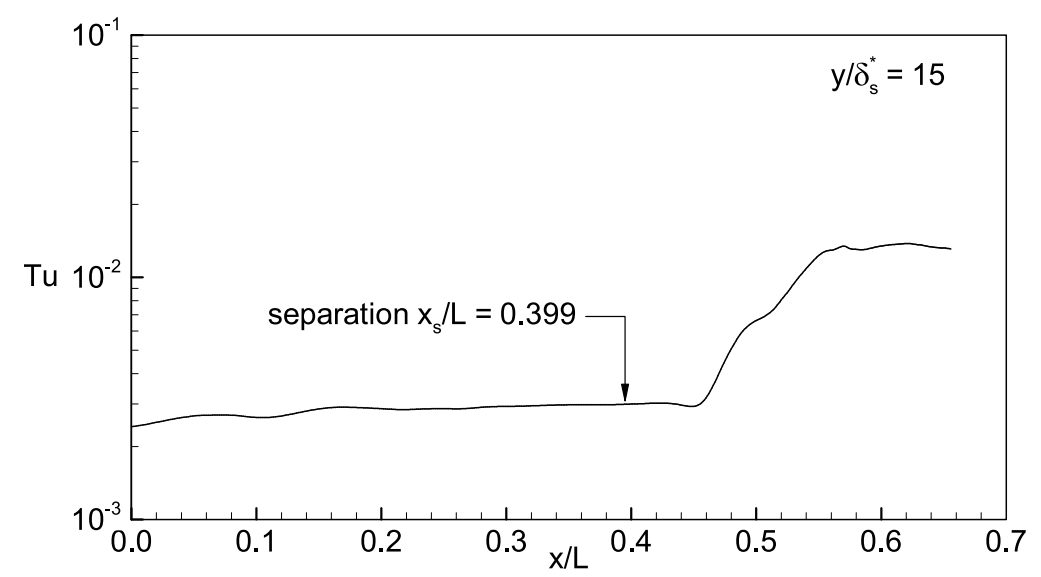

Figure 4.3: Streamwise distribution of free-stream turbulence intensity. Reprinted with the permission of the American Institute of Physics.

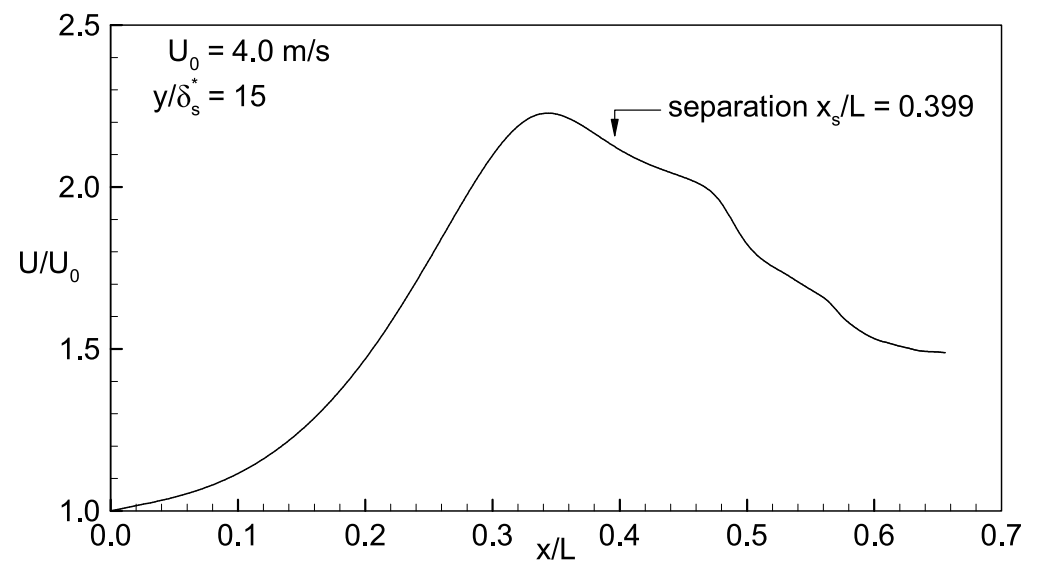

Figure 4.4: Streamwise distribution of time-averaged free-stream velocity. Reprinted with the permission of the American Institute of Physics.

lines represent the critical range of the acceleration parameter in which several researchers have observed that a turbulent boundary layer will begin to relaminarize (Jones and Launder, 1972; Moretti and Kays, 1965; Escudier et al., 1998). Figure 4.5 shows that the majority of the flow upstream of the suction peak is accelerated at a level well above the critical value for relaminarization. The growth of disturbances in this portion of the attached boundary layer is thus expected to be suppressed, as discussed in the following section. 


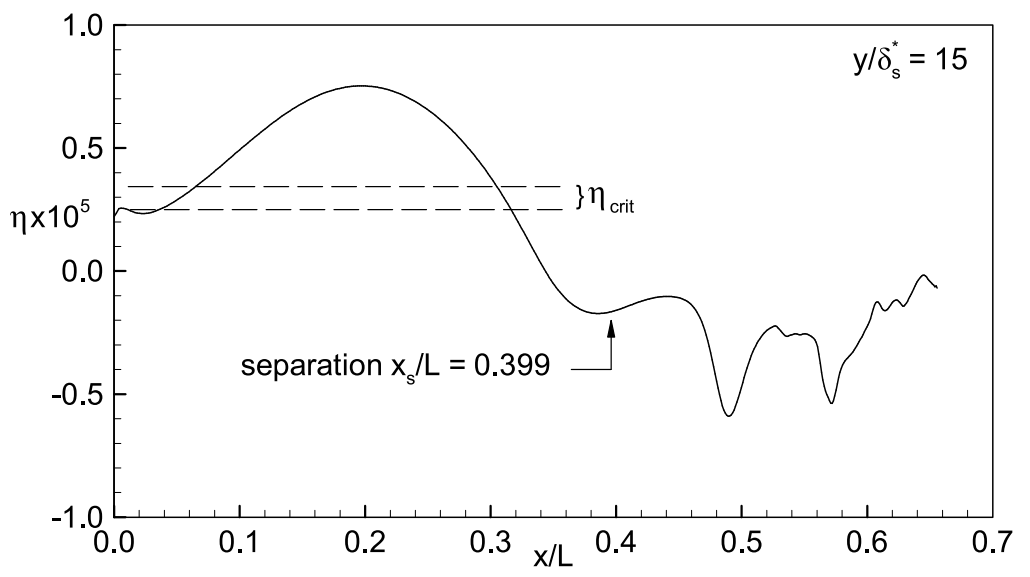

Figure 4.5: Streamwise distribution of time-averaged free-stream acceleration parameter. Reprinted with the permission of the American Institute of Physics.

\subsubsection{Instability development in the attached, laminar region}

Disturbance growth in the attached laminar boundary layer begins with the inclusion of environmental disturbances into the boundary layer through a receptivity process (Saric et al., 2002). In an unstable boundary layer, the disturbance is amplified and the accompanying velocity perturbation disturbs the boundary-layer flow in a manner that results in streamwise grouping of spanwise vorticity into streamwise-periodic waves that make up the well-known Tollmien-Schlichting (T-S) waves. As the T-S waves convect downstream in the attached boundary layer, they introduce a streamwise modulation of the flow that can be analyzed in the frequency spectrum of the streamwise velocity fluctuation at a location within the boundary layer. The frequency spectrum shown in Figure 4.6 indicates a peak at $137 \mathrm{~Hz}$. This value, denoted by $f_{M A}$, is the disturbance frequency that results in the highest amplification rate, and is somewhat larger than the value of $92 \mathrm{~Hz}$ suggested by the correlation of Walker (1989):

$$
f_{M A}=\frac{3.2 U_{e}^{2}}{2 \pi \nu R e_{\delta^{*}}^{3 / 2}} .
$$

A similar difference between the computed value of $f_{M A}$ and that predicted by Equation 4.1 was observed by McAuliffe and Yaras (2006). Moreover, the change in $f_{M A}$ observed in the 


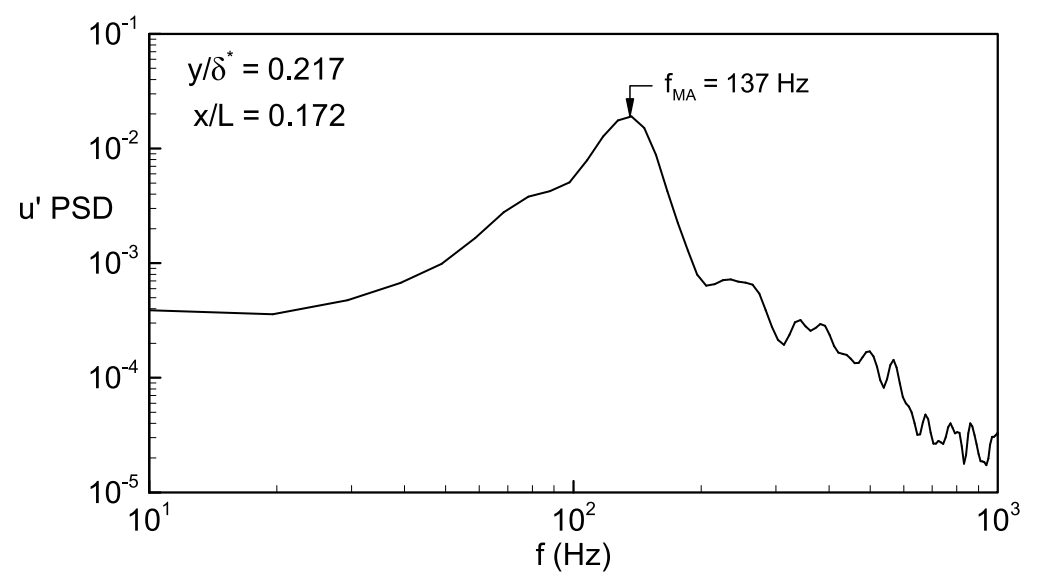

Figure 4.6: Frequency spectrum of the streamwise velocity in the attached laminar boundary layer. Reprinted with the permission of the American Institute of Physics.

simulations between the streamwise location of Figure 4.6 and the suction peak is about $2 \mathrm{~Hz}$; this trend agrees with Equation 4.1, which predicts an increase in $f_{M A}$ of about $3 \mathrm{~Hz}$ over this streamwise length.

The dominant frequency of T-S waves in the attached boundary layer $\left(f_{M A}=137 \mathrm{~Hz}\right)$ is noted to be very similar to the shedding frequency of rolled-up spanwise vortices from the downstream separated shear layer, described below. This similarity could be due to the attached-flow instability artificially locking onto the frequency of pressure oscillations propagating upstream from the separated shear layer. To determine if the attached-flow instability frequency is indeed being forced by the downstream separated-flow instability, a companion numerical study was performed with the adverse pressure gradient region downstream of the suction peak replaced by a nominally-zero streamwise pressure gradient region, which eliminated the separation bubble. Under these conditions, the dominant frequency and growth rate of T-S waves remained identical to those in the current case, suggesting that the attached-flow instability is not affected by the separated shear layer developing downstream.

To analyze the streamwise growth rate of disturbances in the attached boundary layer, the root-mean-square of the streamwise velocity fluctuation was calculated along a spanwise line 


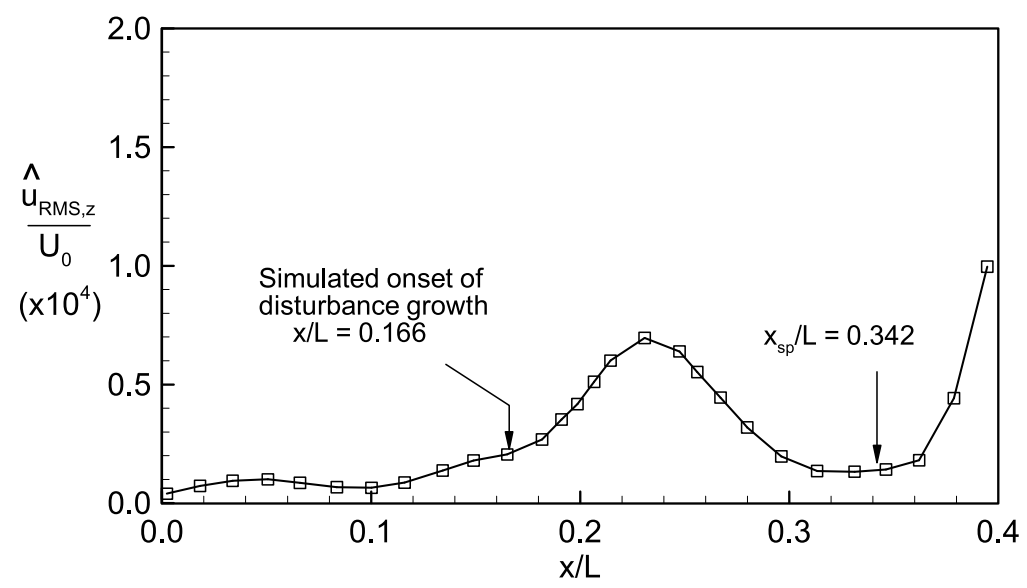

Figure 4.7: Streamwise variation of disturbance amplitude normalized by the reference velocity $U_{0}$. Reprinted with the permission of the American Institute of Physics.

at the wall-normal height where the power density was highest. This value was then averaged in time over 21 periods of the attached-flow instability (the reciprocal of $f_{M A}$ from Figure 4.6), producing what is effectively the ensemble-average of the disturbance amplitude, denoted by $\hat{u}_{R M S, z}$. The ensemble-averaging process was repeated at several streamwise locations between $x / L=0.166$ and the separation point to obtain the streamwise variation of the disturbance amplitude in the attached boundary layer. Figure 4.7, which plots the disturbance amplitude normalized by the reference velocity $U_{0}$, shows streamwise growth of the disturbance, although a decrease in the disturbance amplitude is observed in the highly-accelerated region between $x / L=0.230$ to 0.320 , suggesting that the boundary layer in that region is stabilized by the free-stream flow acceleration. Rapid growth in the disturbance amplitude is observed after the streamwise pressure gradient changes sign downstream of the suction-peak at $x_{s p} / L=0.342$.

To provide support for these computational results, the equations that govern the temporal growth of a disturbance were solved following linear stability theory, which assumes a disturbance in the form of a two-dimensional wave, $\psi(x, y, t)=\phi(y) e^{i(\alpha x-\alpha c t)}$, where $x$ and $y$ are the streamwise and wall-normal directions, respectively, $\phi$ is the wave amplitude, $\alpha=2 \pi / \lambda_{z}$ is the streamwise wave number, and $c$ is the wave phase speed. For a disturbance to grow in time, the imaginary component of the complex phase speed $c_{i}$ must be positive. A 
hybrid spectral collocation method based on Chebychev polynomials, described by Schmid and Henningson (2000), was used to calculate the eigenvalues of the Orr-Sommerfeld and Squire equations from the computed time-averaged velocity profile at a specified streamwise location. By varying the streamwise location where the eigenvalues were calculated, the streamwise distribution of the maximum growth of $c_{i} / U_{0}$ was obtained, as shown in Figure 4.8. The OrrSommerfeld and Squire equations assume that the flow is parallel to the surface, which is not satisfied downstream of separation.

The distribution shown in Figure 4.8 predicts that disturbances will begin to grow in the attached boundary layer near $x / L=0.100$, initially at a very low rate, switching to a notably higher rate of growth at $x / L=0.168$. This result is in favourable agreement with the simulation results where the most-upstream location for the observance of amplified disturbances is $x / L=0.166$. Downstream of $x / L=0.237$, the linear theory predicts a rapid increase in the growth rate, reaching a maximum at $x / L=0.298$, which is qualitatively observed in Figure 4.7, before reaching a minimum at the suction peak. In the adverse pressuregradient region downstream of the suction peak, the disturbance growth rate once again begins to increase, which is consistent with the trends observed in the simulation results in Figure 4.7. The reason for the predicted disturbance growth rate reaching a maximum at $x / L=0.298$ and a minimum at the suction peak is likely related to the high free-stream acceleration occurring in that region.

The T-S waves observed in the simulation and predicted by the linear stability analysis also introduce a spanwise modulation to the undisturbed boundary layer flow, which can be seen by analyzing the wavenumber spectrum of the streamwise velocity along a spanwise line at a constant wall-normal height. A peak in this spectrum was observed at $\lambda_{z}=4 \mathrm{~mm}$, which is suggested to correspond to the wavelength of the spanwise modulation of the flow resulting from T-S wave development. The peak is observed near the wall (approximately $0.2 \delta^{*}$ ) where the shear stress is highest. The spanwise wavelength of the T-S wave is normalized by wall units 


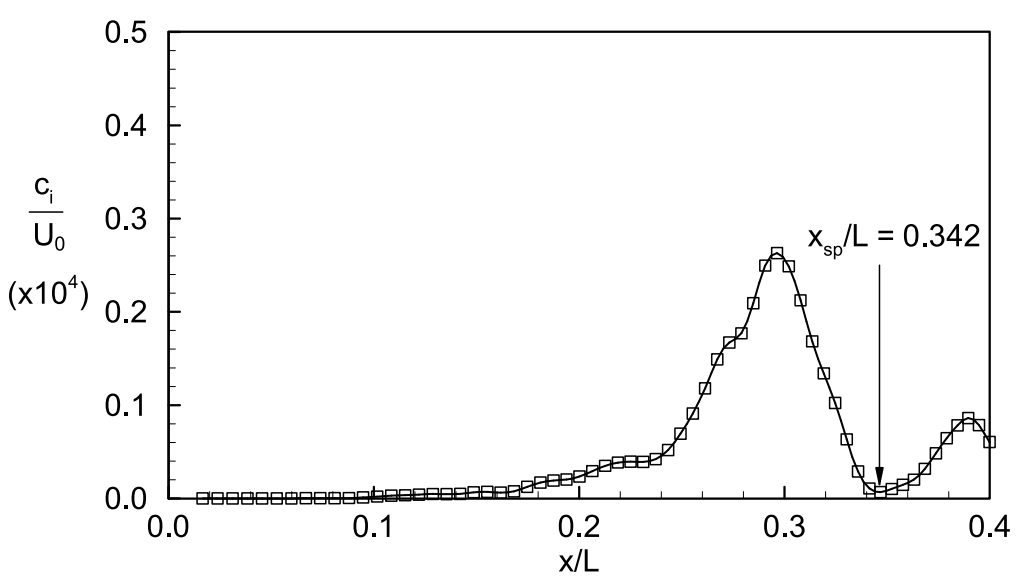

Figure 4.8: Streamwise variation of the disturbance growth rate obtained from linear stability theory. Reprinted with the permission of the American Institute of Physics.

calculated using the spanwise-averaged wall shear stress in the reattached turbulent boundary layer. This manner of normalization allows direct comparison of the spanwise wavelength of the T-S waves in the upstream boundary layer with the spanwise extent of coherent structures in the downstream turbulent boundary layer. When normalized in this manner, the spanwise wavelength of the T-S wave in the laminar attached portion of the boundary layer is about $\lambda_{z}^{+}=92$ wall units, which is very close to the typical spacing of streaks observed in equilibrium turbulent boundary layers (e.g., Smith and Metzler, 1983).

As the T-S waves amplify, segments of grouped spanwise vorticity are reoriented to establish a component in the streamwise direction, which then experience lift-up and stretching to produce $\Lambda$-shaped vortices. Visualization of the $\Lambda$-vortices is complicated by their very low amplitude in the current study and the masking effect of the mean vorticity in the boundary layer. It is helpful to remove the mean shear by subtracting the spanwise-averaged velocity profile from each streamwise location, which results in the perturbation velocity field, $\widetilde{u_{j}}$. The curl of this velocity field produces the perturbation vorticity field, $\widetilde{\omega_{j}}$. The lift-up and stretching associated with the $\Lambda$-vortex causes streamwise vorticity to develop a wall-normal component, and thus iso-contours of wall-normal perturbation vorticity provide visualization of the legs of the $\Lambda$-vortices. Figure 4.9 plots iso-contours of wall-normal perturbation vorticity 


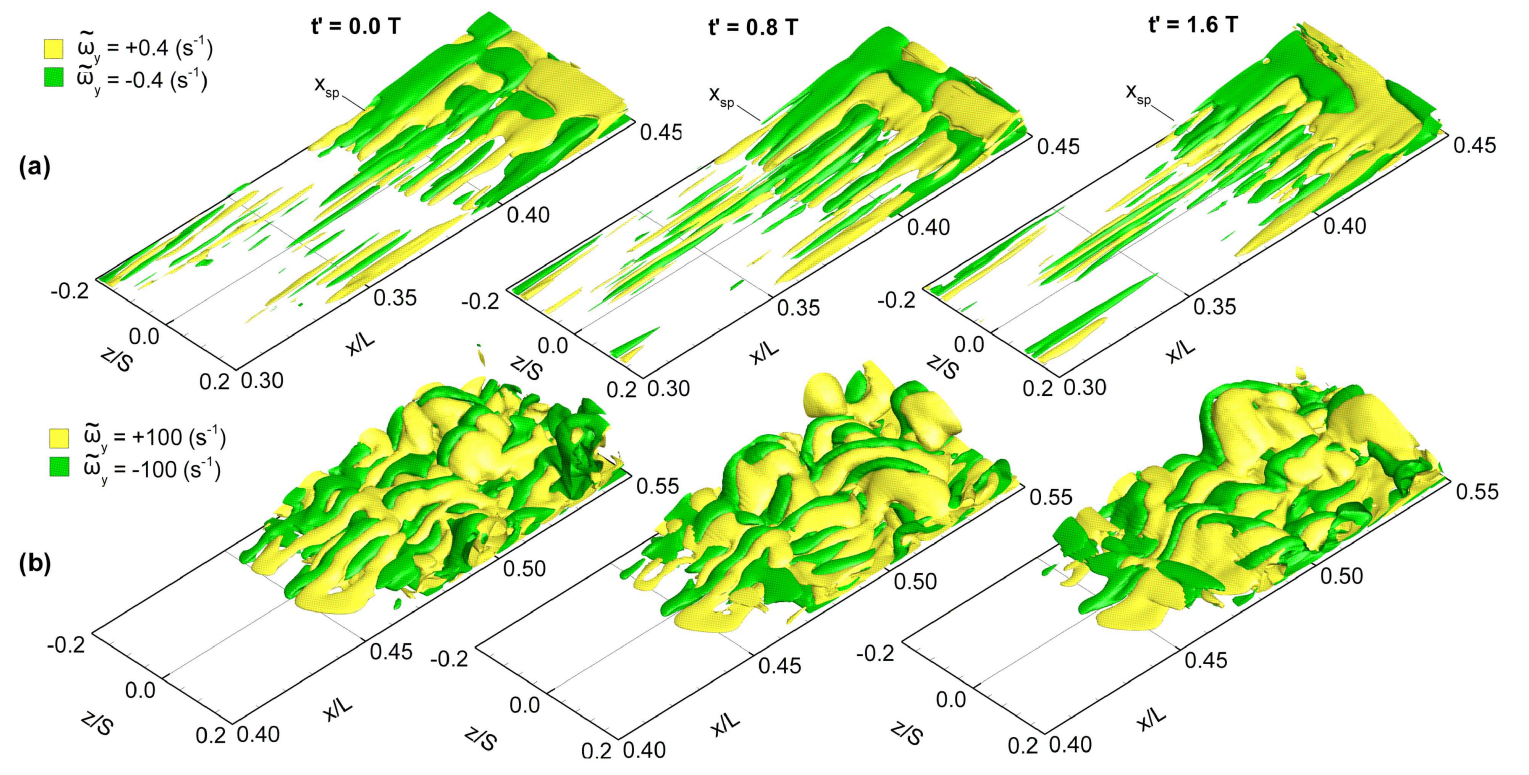

Figure 4.9: Iso-contours of wall-normal perturbation vorticity at three time values $(a)$ upstream of separation and $(b)$ downstream of separation. Reprinted with the permission of the American Institute of Physics.

in the attached and separated regions of the boundary layer at three instances in time. The upper set of plots in Figure 4.9(a) illustrate the transient development of streamwise-elongated vortices in the range $x / L=0.300$ to 0.450 . Time increments of $0.8 T$, where $T$ is the period of vortex-shedding from the separated shear layer, show that weak streamwise vortices with normal-components appear near the suction peak, close to the predicted location where the boundary layer becomes unstable, and convect downstream.

Between $x / L=0.350$ and 0.390 , the amplitude of the streamwise vortices remains relatively constant, but strong amplification begins as the streamwise vortices convect into the separated shear layer. The lower set of plots follows the amplified streamwise vortices as they develop in the range $x / L=0.400$ to 0.550 ; the contour levels are adjusted as a consequence of the higher strength of the vortical structures in this range. Whereas the streamwise vortices in the attached laminar boundary layer remained aligned in the streamwise direction, in the separated shear layer the streamwise vortices become less orderly as they interact with other vortical 
structures present in the separated region. When the spanwise spacing of the streamwise vortices is normalized by the friction velocity evaluated in the reattached turbulent boundary layer, the resulting spanwise spacing is $\lambda^{+}=\lambda u_{\tau} / \nu=85-95$. This result is independent of whether the streamwise vortices are located in the attached laminar boundary layer or separated shear layer. This spanwise spacing compares favourably with the spanwise spacing of streamwise vortices observed in the near-wall region of turbulent boundary layers (Smith and Metzler, 1983; Wu and Moin, 2009), in laminar boundary layers under elevated freestream turbulence (Butler and Farrell, 1993; Jacobs and Durbin, 2001), and in turbulent spots (Schumacher and Eckhardt, 2001; Schröder and Kompenhans, 2004). From these studies, the average spanwise spacing of streamwise vortices is $\lambda^{+} \approx 100$.

\subsubsection{Instability development in the separated region}

Using linear stability theory, Rist and Maucher (2002) showed that disturbance growth in a separated shear layer occurs primarily in two regions of the flow, and the instability associated with each region has different characteristics. The outer, separated portion of the shear layer is unstable via an inviscid Kelvin-Helmholtz mechanism due to the inflectional shape of the velocity profile, while the inner, reversed-flow region near the wall is susceptible to a viscous instability that may cause fluctuations to propagate upstream and potentially trigger an absolute instability. Rist and Maucher (2002) show that the shape of the velocity profile and the flow-reversal velocity determine which mode will dominate the break-down to turbulent flow. Alam and Sandham (2000) observed that the viscous instability dominates when the level of flow reversal exceeds approximately 15-20\% of the local free-stream velocity. To assess which instability mode is dominant in the current study, contours of the root-meansquare of the streamwise velocity fluctuation are plotted in Figure 4.10. Weak fluctuations are observed near the time-averaged location of maximum shear, indicating a predominance toward the outer-layer inviscid instability mode. This conclusion is expected, as the velocity of the 


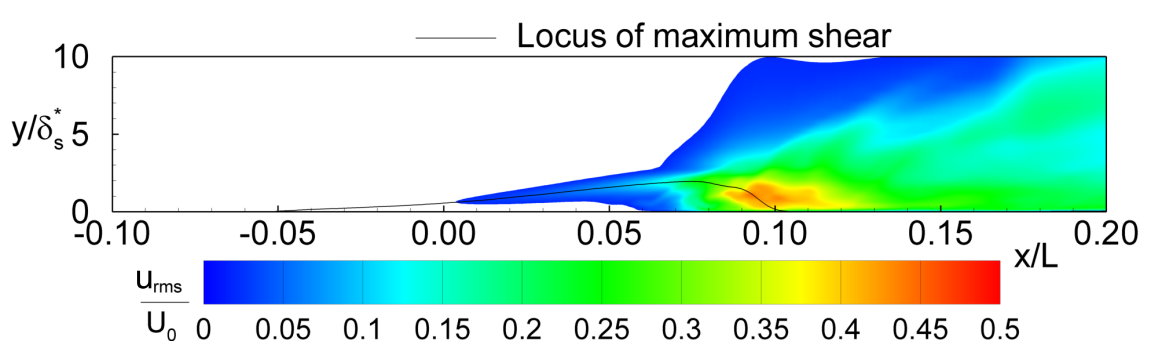

Figure 4.10: Time-averaged characteristics of the separation bubble. Contours show RMS of streamwise velocity fluctuations. Reprinted with the permission of the American Institute of Physics.

reversed flow in the current study remained less than $8 \%$ of the local free-stream velocity. For a separation bubble dominated by the inviscid instability mode, disturbances in the separated shear layer develop into streamwise-periodic waves of spanwise vorticity that amplify as they convect through the shear layer until they eventually roll-up and shed as distinct spanwiseoriented vortices. An example of the transient roll-up of vorticity from the separated shear layer is shown in Figure 4.11, which illustrates about one period of the dominant instability waves. The shedding frequency from the separated shear layer is conventially expressed as a Strouhal number based on the conditions at separation $\left(S r_{\theta s}=f \theta_{s} / U_{e s}\right.$, where $\theta_{s}$ and $U_{e s}$ are the momentum thickness and boundary-layer edge velocity at separation, respectively). In the current simulation, the dominant instability Strouhal number is 0.008, which compares favourably with the range of $S r_{\theta s}=0.005-0.016$ observed in several other experimental and computational studies of separated shear layers (Muti-Lin and Pauley, 1996; Yang and Voke, 2001; Talan and Hourmouziadis, 2002).

Smaller vortical scales are observed in the downstream portion of the separated shear layer, indicating the onset of secondary and possibly higher-order instabilities of different frequency and scale spectrum that lead to rapid localized growth of perturbations. A secondary instability in the form of streamwise pairing of the spanwise vortices observed by Wissink and Rodi (2002), McAuliffe and Yaras (2005) and others at low Reynolds numbers $\left(R e_{\theta s}\right.$ of about 100) is not observed in the current simulation results where $R e_{\theta s}$ is about 300 . In 
addition, although the primary instability of the separated shear layer creates spanwise vortices with theoretically infinite span, a spanwise non-uniformity of the spanwise vortices has been observed downstream of the separated shear layer. The spanwise non-uniformity is linked to the formation of streamwise vortices that interact with the shed spanwise vortices, accelerating break-down to small-scale turbulence (McAuliffe and Yaras, 2007b; Malkiel and Mayle, 1996). An example of the spanwise non-uniformity of the shed spanwise vortices is shown in Figure 4.12, which plots contours of spanwise vorticity in the $x$ - $z$ plane at a time corresponding to the final time frame in Figure 4.11. The wall-normal height of the $x-z$ plane in Figure 4.12 is $y=\delta_{s}^{*}$. In this plane, spanwise vorticity contours show a vortex that is relatively uniform in the spanwise direction at approximately $x / L=0.470$. About one streamwise wavelength downstream of this vortex, a previously-shed spanwise vortex at $x / L=0.490$ has begun to break into smaller-scale three-dimensional structures. By $x / L=0.510$, the shed spanwise structure has completely broken into small vortical structures.

\subsubsection{Interaction of inviscid/viscous instabilities}

The non-uniformity and eventual break-up of the spanwise vortices that shed from the separated shear layer, as shown in Figure 4.12, occurs at a spanwise wavelength that is approximately equal to the spanwise spacing of the streamwise vortices created by the viscous instability mode in the attached laminar boundary layer further upstream. This equivalence suggests that there may be an interaction between the viscous instability in the attached laminar region and the inviscid instability in the separated region. Malkiel and Mayle (1996) suggested that transition in a separation bubble may occur through such an interaction, but so far the nature of this interaction has not been clear. In the current study, however, the compressible simulation results demonstrate the transient development of naturally-developing T-S waves in the attached laminar boundary layer and their subsequent migration into the separated shear layer, which introduce streamwise vorticity. This study may therefore be in 

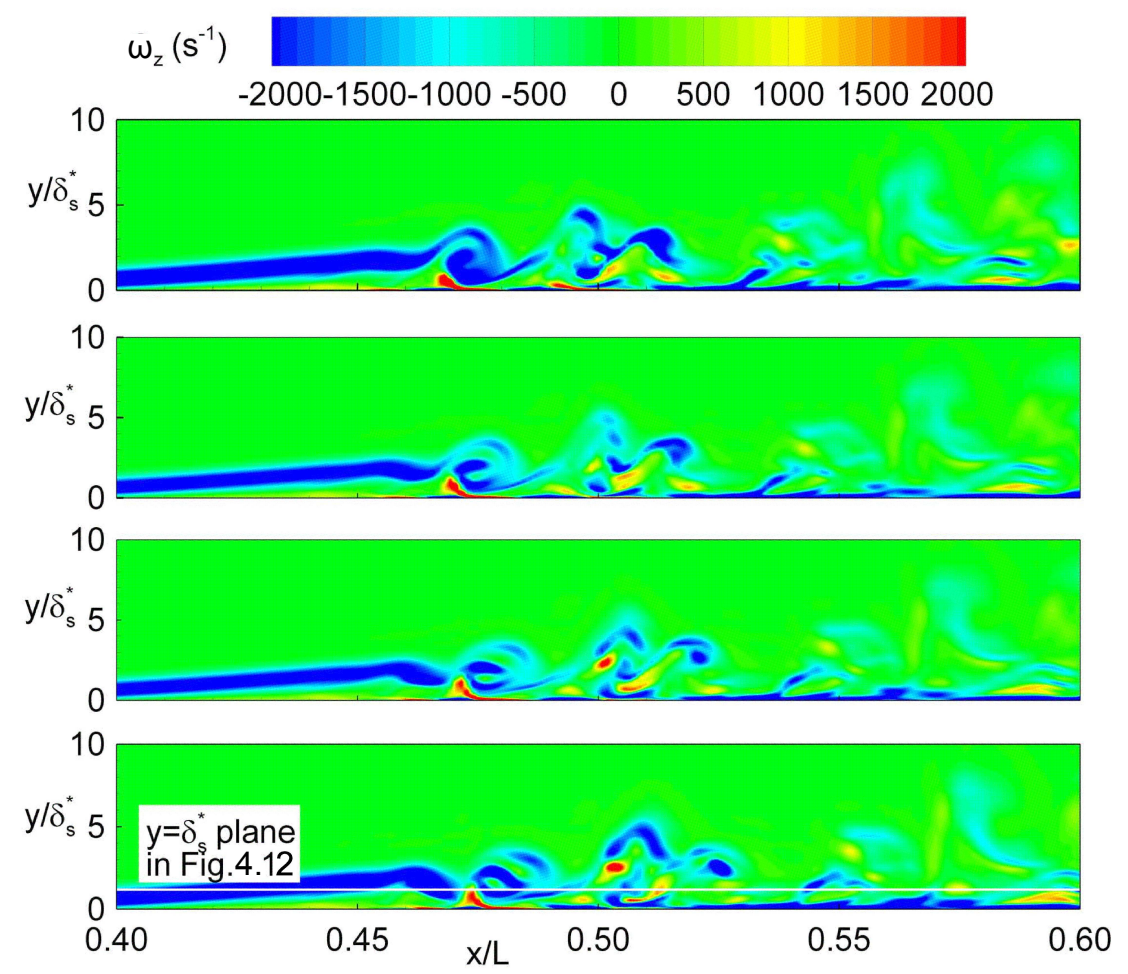

Figure 4.11: Roll-up of the separated shear layer observed through contours of spanwise vorticity in an $x-y$ plane in the separated shear layer. Reprinted with the permission of the American Institute of Physics.

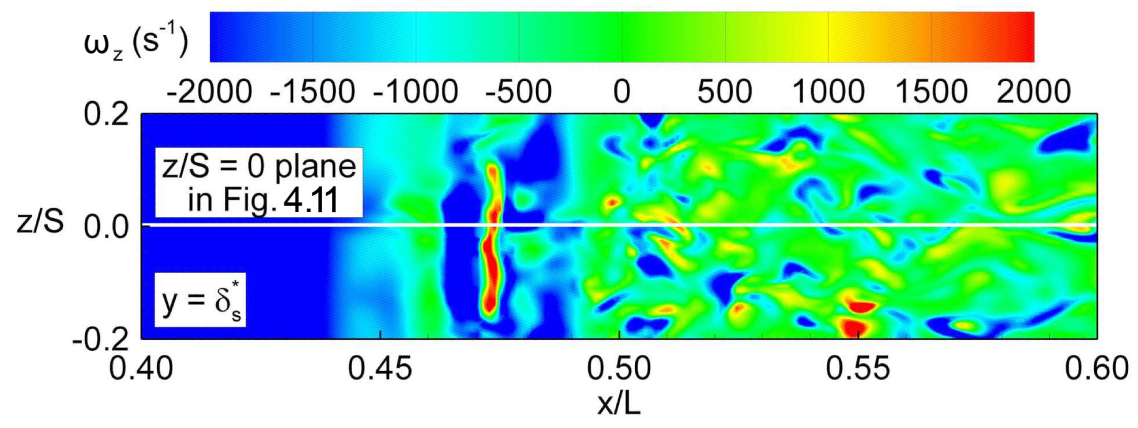

Figure 4.12: Spanwise non-uniformity of the rolled-up separated shear layer observed through contours of spanwise vorticity in the $x-z$ plane at $y=\delta_{s}^{*}$. Reprinted with the permission of the American Institute of Physics. 
a position to elucidate the nature of the interaction between the streamwise and spanwise vortices that are created by the viscous and inviscid instabilities, respectively.

Figure 4.13 shows coherent vortices in the separated shear layer of the present simulation through iso-contours of the second invariant of the velocity gradient $Q$, as defined by Chong et al. (1990). Three instances in time are separated by half the vortex-shedding period to illustrate the transient nature of the vortical interactions occurring within the separated shear layer. Structure A in Figure 4.13 is a spanwise vortex that has recently shed from the separated shear layer. Slightly below this structure, a hairpin vortex is present, labeled as Structure B. This hairpin vortex is created through the stretching of the T-S waves which were created through viscous instability in the attached laminar boundary layer. As the stretching T-S waves convect into the separated shear layer, the high shear amplifies the vortex stretching process to produce the hairpin vortices. The topology of the hairpin vortices observed in Figure 4.13 resembles the hairpin structures that have been observed in turbulent boundary layers (Wu and Moin, 2009). At $t^{\prime}=0.0$, the uniform spanwise vortex is slightly upstream of the hairpin vortex. The vorticity in the legs and head of the hairpin vortex brings low-momentum fluid from the wall higher into the shear layer through a column of low-momentum fluid ejected upwards between the legs of the hairpin vortex. When the spanwise structure encounters the low-speed column, a portion is retarded relative to the rest of the spanwise vortex, producing the spanwise-waviness of the spanwise vortex observed at $t^{\prime}=0.5 T$. As the spanwise waviness amplifies, the spanwise vorticity is reoriented in the streamwise direction and couples with the streamwise legs of the hairpin vortices. By $t^{\prime}=1.0 T$, the shed spanwise vortex has broken down into a smaller three-dimensional flow structure. At $x / L=0.450$, the next spanwise structure has arrived and the interaction will be repeated.

Figure 4.13 aids in illustrating the streamwise location in the separated shear layer where the interaction between the viscous and inviscid instabilities predominantly occurs. In the previous section, Figure $4.9 \mathrm{~b}$ shows that the development of streamwise vorticity due to 


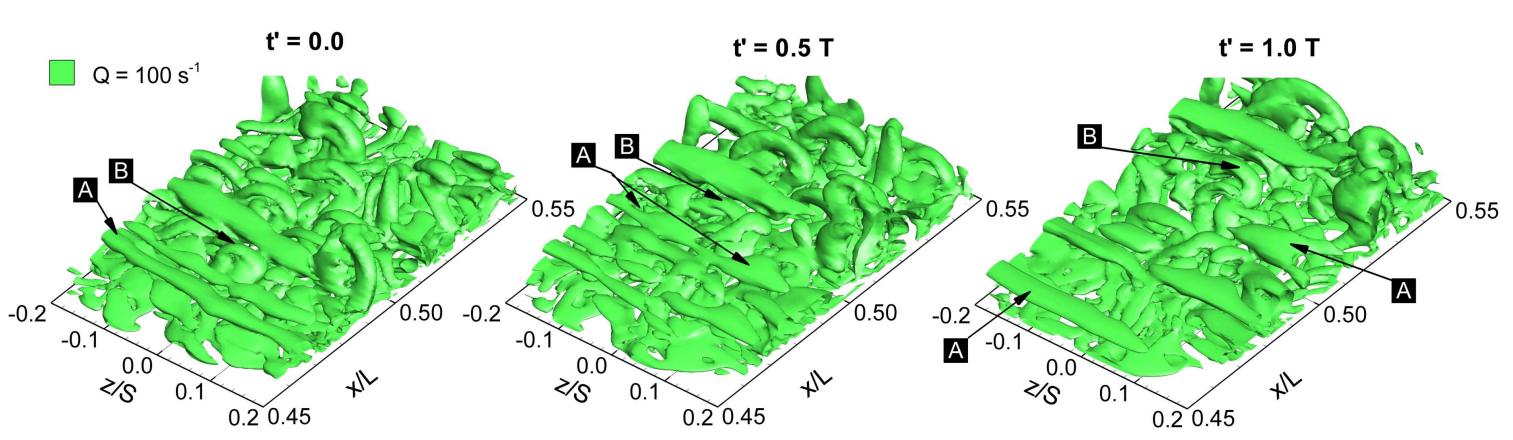

Figure 4.13: Transient break-down in the separated shear layer through iso-contours of the second invariant of the velocity gradient tensor, $Q$. Structure $\mathrm{A}$ is a spanwise vortex in the separated shear layer. Structure B corresponds to one of the hairpin vortices generated by the attached viscous instability. Reprinted with the permission of the American Institute of Physics.

the stretching and reorientation of T-S waves through viscous instability increases rapidly near $x / L=0.459$. In comparison, Structure A at $t^{\prime}=0.0$ in Figure 4.13 is located further downstream, near $x / L=0.469$. The observed spanwise uniformity of Structure A indicates that a substantial interaction between the viscous and inviscid instability has not yet occurred. Therefore it can be concluded that although a weak interaction between viscous and inviscid instabilities likely prevails throughout the separated region, the interaction does not yield a substantial reorientation of spanwise vorticity in the separated shear layer until the viscous instability has produced sufficiently-strong streamwise vorticity. As a result, the interaction between the viscous and inviscid instabilities is predominantly located between $x / L=0.470$ to 0.490 , which approximately coincides with the time-averaged locations of transition onset and transition completion. Figure 4.14, which plots the streamwise distribution of the intermittency parameter $(\gamma)$-defined as the fraction of time the local boundary layer contains velocity fluctuations typically associated with a turbulent state - shows that the timeaveraged transition onset and transition completion locations are at about $x / L=0.460$ and $x / L=0.480$, respectively. This correspondence between the time-averaged transition onset and completion locations and the region of interaction between the viscous and inviscid instability modes suggests that the interaction has a significant impact on the transition process. The 


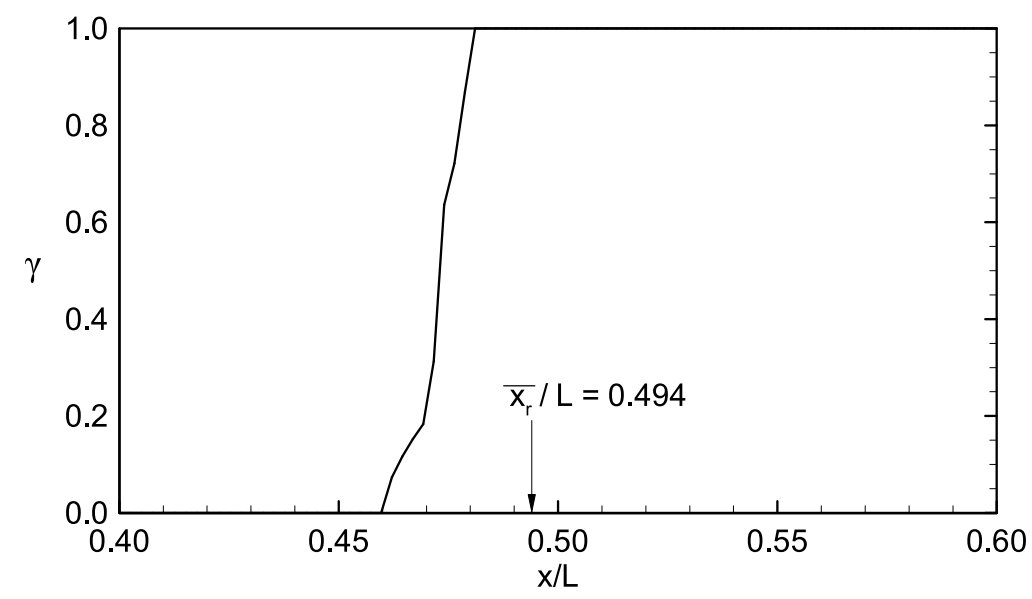

Figure 4.14: Streamwise distribution of the intermittency parameter $\gamma$. Reprinted with the permission of the American Institute of Physics.

time-averaged location where the separated flow reattaches as a turbulent boundary layer occurs at $\overline{x_{r}} / L=0.494$, resulting in a separation bubble with a time-averaged length of $\left(\overline{x_{r}}-x_{s}\right) / L=0.095$.

\subsubsection{Wave packet development in the reattached turbulent boundary layer}

In the studies of McAuliffe and Yaras (2007b) and Alam and Sandham (2000), the threedimensional vortices shed from the separated shear layer maintained their coherence well into the reattached turbulent boundary layer. A similar observation is made in Figure 4.15, which plots iso-contours of $Q=50,000 \mathrm{~s}^{-1}$ shaded by the wall-normal height in the transitional boundary layer. Six vortex-shedding events are labeled, and each event is called a "generation," with generation 1 being the youngest and generation 6 being the oldest. Following the terminology of McAuliffe and Yaras (2007a) and Watmuff (1999), the groups of vortical structures are referred to as "wave packets." According to the streamwise intermittency distribution shown in Figure 4.14, generations 3-6 occur in the reattached turbulent boundary layer, and so may be considered as representing young turbulence. A number of observations can be made of the wave packets: first, it is interesting to note in Figure 
4.15(a) that the streamwise spacing of the wave packets remains relatively constant throughout the reattached turbulent boundary layer, indicating the absence of a sub-harmonic streamwisepairing instability. Second, the spanwise location of the shed wave packets remains relatively constant between the sequence of generations. In Figure 4.15(b), very little deviation occurs between the centers of the wave packets (shown by the small circles) and the dashed axial line. Conversely, the wave packets observed by McAuliffe and Yaras (2007a) under elevated freestream turbulence were observed at random spanwise locations in the flow. Third, the topology of the wave packets consists of one or more hairpin-shaped vortex loops with upstream-pointing legs. In the first-generation wave packet, a single, primary vortex loop is observed very near the wall. By the second generation, the head of the primary vortex loop has been lifted to about twice the wall-normal height of the first-generation primary loop and - in some wave packetsa secondary upstream-pointing loop has formed. By the fourth generation, the wave packet has developed 3-4 vortex loops, all of which point upstream. McAuliffe and Yaras (2007a) suggest that the creation of multiple loops in the wave packet is the result of a sequence of localized inviscid instabilities initialized by the primary vortex loop. Ejection of fluid due to the first loop produces a local highly-inflectional velocity profile which is inviscid-unstable and perturbations will grow rapidly to form a second vortex loop. Ejection caused by the second loop will yield a third vortex loop, and so forth, resulting in the increasing number of vortex loops in the wave packets. The wave packets observed in the current dataset seem to support this explanation. Figure 4.15 shows that by the fifth and sixth generations, no new vortex loops are observed and the primary vortex (shown by the large red loops) has grown away from the wall, eventually reaching 3-4 times the boundary-layer thickness. The absence of further increase in the number of vortex loops may be explained by the stabilizing influence of the primary vortex loop on the flow field upstream of the wave packet. For example, the primary vortex of the fifth-generation wave packet would induce a downwash ahead of itself, thus preventing further streamwise growth of the sixth-generation wave packet through the creation of more vortex loops. 

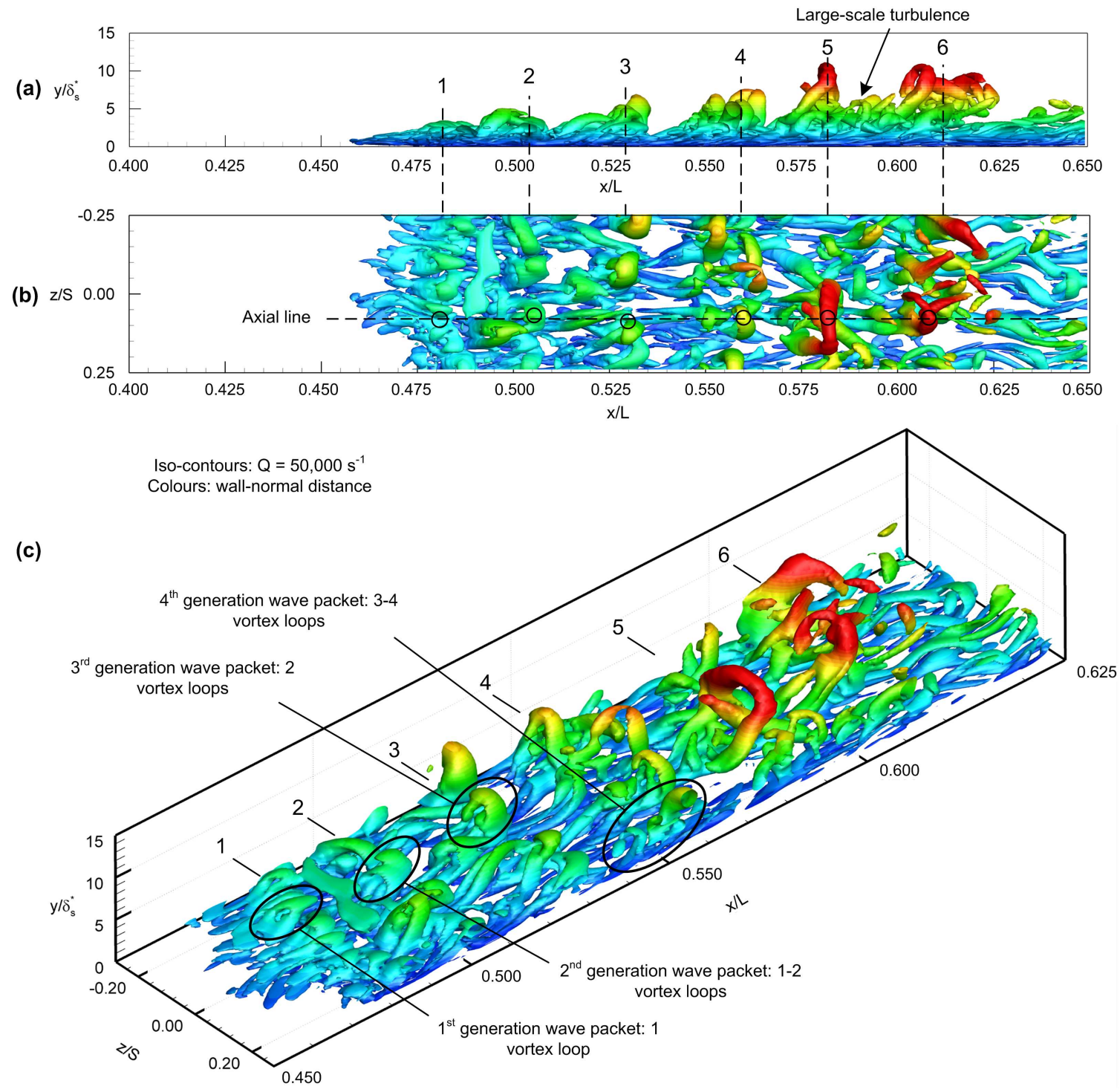

Figure 4.15: Growth of wave packets in the transitional boundary layer seen through isocontours of $Q$ coloured by the wall-normal height viewed from an $(a) x-y$ plane, $(b) x-z$ plane, and $(c)$ isometric projection. Reprinted with the permission of the American Institute of Physics. 
Finally, the topology of the wave packets observed in Figure 4.15 differs substantially from the wave packets McAuliffe and Yaras (2007a) observed under elevated free-stream turbulence in which the primary loop points upstream while the subsequent loops point downstream as they curl around the low-momentum fluid ejected by the primary loop. In the current study, only upstream-pointing vortex loops are observed in the wave packets. This difference may be because the wave packets observed by McAuliffe and Yaras (2007a) were located higher in the separated shear layer where they were less influenced by the retarding effect of wall shear, although this question warrants further investigation. 


\section{Chapter 5}

\section{Interaction of Instability Modes in a Swept Separation Bubble Under Low Free-Stream Turbulence}

Citation: Brinkerhoff, J.R. and Yaras, M.I. (2013), "Direct numerical simulations of transitional separation-bubble development in sweptblade flow conditions," ASME Journal of Turbomachinery, 135, pp. 041006. DOI: http://dx.doi.org/10.1115/1.4007528. Used with permission. 


\subsection{Introduction}

The trend towards increased aerodynamic loading in the design of low-pressure turbine (LPT) airfoils in aircraft gas turbine engines increases the likelihood of flow separation from the suction surface of the airfoil, deteriorating the performance of the airfoil through reduced lift and increased drag (Mayle, 1991; Hodson and Howell, 2005). If the separated flow undergoes laminar-to-turbulent transition, enhanced momentum exchange due to turbulence may allow the boundary layer to reattach, forming a transitional separation bubble. Numerous studies have been conducted to understand the effects of flow Reynolds number, streamwise pressure gradient, surface roughness, periodic free-stream unsteadiness, and environmental disturbances on separation-bubble transition (Yaras, 2012; Yang and Voke, 2001; Volino and Bohl, 2004), resulting in increasingly-refined engineering models that allow prediction of the transition onset location and transition rate in separated and attached boundary layers (e.g., Roberts and Yaras, 2005b). In addition, spanwise pressure gradients may occur in a realistic LPT environment as the result of highly-flared hub and casing walls that yield an effective sweep angle in radially-stacked blades (e.g., Pullan and Harvey, 2007). To verify the applicability of existing transition prediction models to such swept-blade configurations, the effect of pressurefield three-dimensionality on the instability and transition processes that develop in the laminar upstream boundary layer and the separated shear layer must be clearly understood.

When the separation bubble develops under a 3D pressure field, the spanwise pressure gradient causes the free-stream streamlines to curve to balance the spanwise pressure force. Within the boundary layer, the streamwise velocity is reduced, so the pressure force overbalances the centripetal acceleration of the boundary-layer fluid, creating a crossflow velocity component in the boundary layer (Saric et al., 2003). As the no-slip condition requires the crossflow velocity component to vanish at the wall, and since it must also vanish at the boundary-layer edge, there is an inflection point in the crossflow velocity profile, 
and the resulting inflectional instability is termed crossflow instability (Saric et al., 2003). Numerous analytical (e.g., Gregory et al., 1955) and experimental (e.g., Nitschke-Kowsky and Bippes, 1988; Deyhle and Bippes, 1996; Radeztsky et al., 1999; Bippes, 1999; Arnal et al., 1997; Kachanov, 1994) studies have found that crossflow instability manifests itself through the formation of stationary and traveling waves. The former dominate when the freestream turbulence level is lower than $0.15 \%$ and the latter at higher turbulence levels (Deyhle and Bippes, 1996). Stationary crossflow waves occur as counter-rotating vortices nearly aligned with the local inviscid streamline and with a spanwise wavelength of about four times the local boundary-layer thickness (Nitschke-Kowsky and Bippes, 1988).

Investigations of the effect of a 3D pressure field on separation-bubble transition began with the extensive hot-wire measurements of 2D and 3D separation bubbles by Horton (1968), who found that important flow parameters - including the bubble length - are independent of the sweep angle of the separation bubble if the chordwise flow conditions are kept constant. This observation is corroborated by direct numerical simulations (DNS) of separation bubbles with $0^{\circ}-45^{\circ}$ sweep angles by Hetsch and Rist (2009a), who also observe that the flow depends on chordwise conditions only. Additionally, the effect of crossflow instability on the growth of disturbances in the attached laminar boundary layer is observed to be minimal. Through linear stability analysis and DNS, Hetsch and Rist (2009b) found that above a sweep angle of $10^{\circ}-15^{\circ}$, the most unstable disturbance consists of oblique T-S waves that travel approximately aligned with the potential flow direction, and amplification of this disturbance increases only moderately with increasing sweep angle. In contrast, recent hot-wire experiments by Yaras (2012) that considered a separation bubble under a 3D pressure field observed that the redistribution of the free-stream flow by the 3D pressure field alters the dominant instability mode in the separated-flow region and causes a significant spanwise variation in the separationbubble structure. These studies indicate that a complete understanding of the effect of a 3D pressure field on separation-bubble transition has not yet been achieved. Therefore, a DNS 
study was performed of a boundary layer developing under a pressure distribution and flow Reynolds number that are representative of the conditions on the suction side of a typical LPT blade in a gas-turbine engine. Two blade sweep angles of $0^{\circ}$ and $45^{\circ}$ were simulated to provide insight on the effect of a 3D pressure field on the underlying instability modes in a transitional separation bubble.

\subsection{Numerical Method}

ANSYS CFX® (Version 12), a commercial computational fluid dynamics software package, was used to solve the time-varying governing equations using a finite-volume spatial discretization approach. The authors' research group and several other researchers have investigated separation-bubble transition by numerically solving the incompressible form of the unsteady mass- and momentum-conservation equations using similar algorithms (Ripley and Pauley, 1993; Michelassi et al., 2002; Rist and Augustin, 2006; McAuliffe and Yaras, 2006; Azih et al., 2012). The results of these computations show good agreement with experiments in terms of the shape and extent of the time-averaged separation bubble, the streamwise velocity fluctuation spectra, the location of transition onset, the streamwise rate of transition, the mean velocity profile, and the turbulence kinetic energy budgets. Validation of the numerical algorithm and the spatial and temporal resolutions is discussed below.

\subsubsection{Computational domain and boundary conditions}

The computational domain used in the present study, shown in Figure 5.1, consists of a contoured ceiling above a flat test surface of length $L=1220 \mathrm{~mm}$. The inflow boundary is placed $0.37 \mathrm{~L}$ upstream of the leading edge of the test surface and a free-slip surface is placed between the inflow boundary and the test-surface leading edge to allow the test-surface

boundary layer to develop naturally starting from the leading edge. The test surface is 


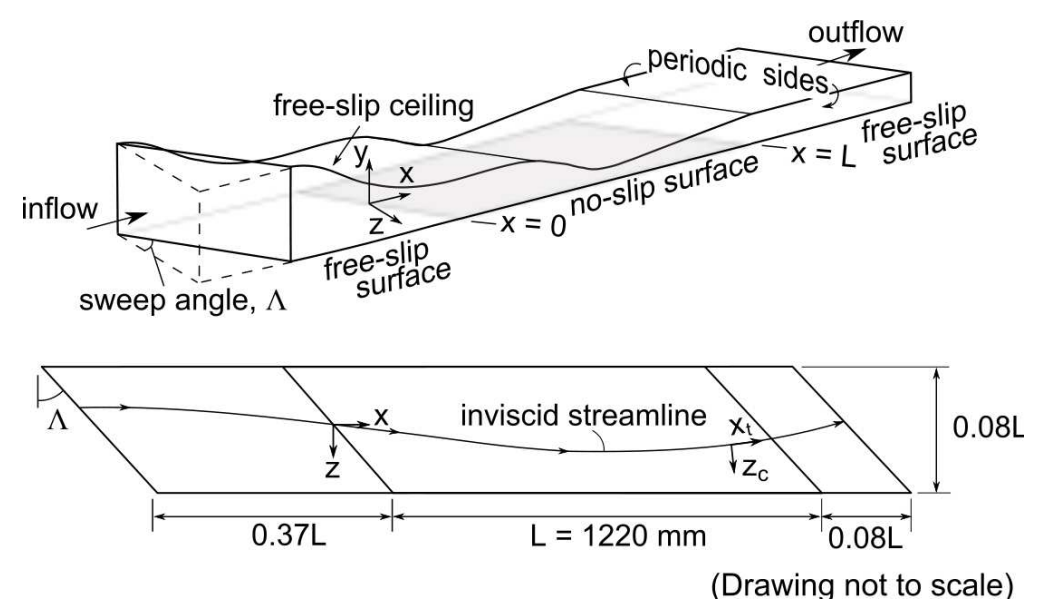

Figure 5.1: Schematic of the computational domain. Reprinted with the permission of the American Society of Mechanical Engineers.

represented by a no-slip wall over which the boundary layer and separated region develop, and it is followed by a $0.08 \mathrm{~L}$-long free-slip surface. The length of the downstream free-slip surface was chosen to place the outflow boundary a conservative distance from the region of interest, as the outflow boundary condition available in ANSYS CFX $\AA$ does not allow convective flow structures such as turbulent eddies to exit the domain through the boundary without distortion. A pressure distribution representative of the suction side of a low-pressure turbine blade was created on the test surface by contouring the free-slip ceiling of the computational domain. Spanwise periodicity was imposed along the sides of the computational domain, and a spanwise width of $0.08 \mathrm{~L}$ was chosen, which is close to eight times the spanwise wavelength of the largest-scale vortical structures occurring in the transitional separation bubble. This width has been shown to be sufficient for capturing the instability modes in the transitional separation bubble and reattached turbulent boundary layer (e.g., Abdalla and Yang, 2004; Alam and Sandham, 2000). A spatially-uniform, time-invariant inlet velocity and a static pressure that remains fixed in an area-averaged sense were specified for the inflow and outflow boundaries, respectively. The length of the test surface is consistent with the dimensions of the test surface in experimental studies of transition phenomena undertaken by the authors' research group at Carleton University (Yaras, 2001; Yaras, 2002; Roberts and Yaras, 2005a). 
The 3D pressure distribution is imposed in the simulations by sweeping the leading edge of the test surface by an angle of $\Lambda=45^{\circ}$. A simulation without a swept leading edge $\left(\Lambda=0^{\circ}\right)$ was also performed to provide a baseline dataset to aid in examining how a nominally two-dimensional separation bubble is modified by the 3D pressure distribution. As the $3 \mathrm{D}$ pressure distribution modifies the free-stream flow direction, and thus the streamwise pressure distribution imposed on the flow, the ceiling geometry in the unswept configuration was adjusted such that the streamwise pressure distributions are approximately equal in the unswept and swept configurations.

\subsubsection{Spatial grid}

A structured grid consisting of hexahedral finite volumes is mapped onto the computational domain shown in Figure 5.1. The finely resolved region near the test surface is populated by 580, 59, and 176 nodes in the streamwise, wall-normal and spanwise directions, respectively. To accurately simulate the flow in the current study using DNS, including the flow in the reattached turbulent region downstream of the separation bubble, the spatial and temporal grids must be sufficiently refined to capture the smallest spatial and temporal scales in the flow, which are on the order of the Kolmogorov length and time scales given by Eq. 5.1 and Eq. 5.2, respectively:

$$
\begin{aligned}
\eta & =\left(\frac{\nu^{3}}{\epsilon}\right)^{1 / 4} \\
t_{\eta} & =\left(\frac{\nu}{\epsilon}\right)^{1 / 2}
\end{aligned}
$$

where $\epsilon$ is the turbulence kinetic energy dissipation rate and $\nu$ is the kinematic viscosity. For wall-bounded turbulent shear layers, the Kolmogorov length scale is on the order of the viscous length scale, typically reaching a minimum value of $\epsilon^{+} \approx 2$ close to the no-slip boundary (e.g., Kim et al., 1971; Stanislas et al., 2008). Thus, based on the wall shear-stress occurring at 
an axial position well within the reattached turbulent boundary layer, $\left(x-x_{0}\right) / L=0.70$, where $x_{0}$ is the test-surface leading edge location, the nodes are distributed such that the average spacing yields values of $\Delta x^{+}=18$ and $\Delta z^{+}=9$ in the swept case and $\Delta x^{+}=22$ and $\Delta z^{+}=11$ in the unswept case, corresponding to approximately 4 to 10 times the Kolmogorov length scale at the target Reynolds number. The $y^{+}$value of the first node from the no-slip boundary is 0.5 and 0.8 for the grids in the swept and unswept cases, respectively. Close to the wall, $\left(0<y^{+}<30\right)$, the wall-normal node spacing is increased linearly from the value at the wall to a value that corresponds to the Kolmogorov length scale in the log-law region, which is approximated by:

$$
\eta^{+}=\left(\kappa y^{+}\right)^{1 / 4}
$$

where $\kappa=0.41$ is the von-Karman constant Stanislas et al. (2008). Above this wall-normal height, the node spacing is increased at a rate of $4 \%$ until $y / \theta_{s}=20$, where $\theta_{s}$ is the momentum thickness at the time-averaged separation location. The grid spacing remains constant in the range $20<y / \theta_{s}<42$, which approximately corresponds to the height of the largest-scale flow structures expected in the separated shear layer and reattached turbulent boundary layer, and is then increased at a rate of $12 \%$ until the free-slip ceiling is reached. This wall-normal node distribution at $\left(x-x_{0}\right) / L=0.70$ is shown in Figure 5.2.

\subsubsection{Solution method}

Discretization of the governing equations is based on second-order central differencing and implicit, second-order Euler backward differencing for the spatial and temporal derivatives, respectively. Although the Kolmogorov time scale in a wall-bounded turbulent boundary layer is on the order of the viscous time scale (Pope, 2000), Choi and Moin (1994) have shown that

to obtain accurate turbulence statistics with an implicit method, the computational timestep should be below $0.2 t^{+}$, where $t^{+}=\nu / u_{\tau}^{2}$ is the viscous time scale and $u_{\tau}$ is the friction 


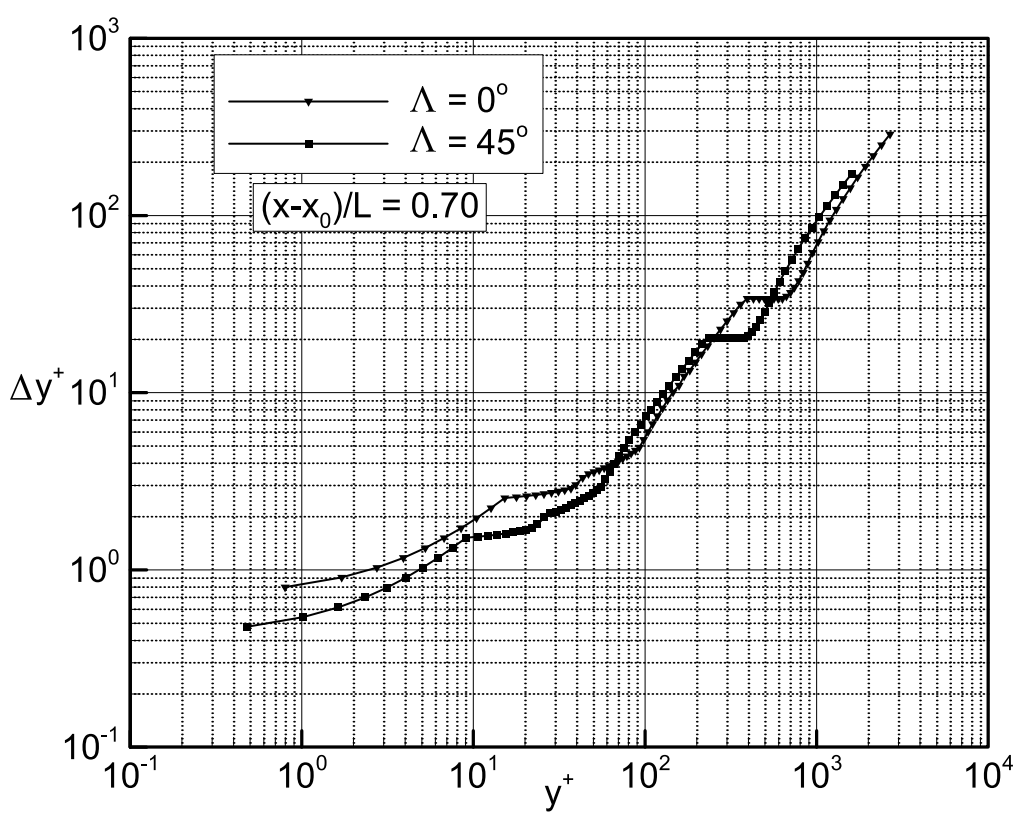

Figure 5.2: Wall-normal node distribution. Reprinted with the permission of the American Society of Mechanical Engineers.

velocity in the reattached turbulent boundary layer downstream of the separation bubble. A timestep size of $\Delta t=0.1 \mathrm{~ms}$ or $0.15 t^{+}$was therefore chosen, which corresponds to about $10 \%$ of the Kolmogorov time scale at the target Reynolds number. The discretized equations were converged through an algebraic multigrid scheme within eight inner-loop iterations per timestep, reducing the root-mean-square residual of the governing equations by five orders of magnitude to less than $10^{-6}$. The simulation was initialized with a uniform velocity equal to the velocity specified at the inflow boundary and zero relative static pressure. Approximately 20,000 timesteps were required to reach a statistically steady state, after which 2100 timesteps were computed for analysis. The simulation was partitioned and executed in parallel on 20 cores of five Intel ${ }^{\mathrm{TM}}$ L5410 Xeon processors. 
Table 5.1: Numerical details of the simulations used for validation of the numerical results. Reprinted with the permission of the American Society of Mechanical Engineers.

\begin{tabular}{lccc}
\hline Simulation & $\Delta x^{+}$ & $\Delta z^{+}$ & $R e_{\theta}$ \\
\hline Grid 1 & 3 & 3 & 900 \\
Grid 2 & 3 & 3 & 500 \\
Grid 3 & 22 & 11 & 500 \\
Spalart (1988) & 20 & 7 & 670,1410 \\
Wu and Moin (2009) & 6 & 11 & 900 \\
\hline
\end{tabular}

\subsubsection{Validation of numerical results}

Due to the lack of data in the published literature with flow conditions matching the present separation-bubble study, validation of the numerical algorithm and the spatial and temporal resolutions is accomplished by performing a series of companion DNS studies of a zero-pressure-gradient turbulent boundary layer and comparing the results of these simulations to statistical turbulence properties of corresponding turbulent boundary layers in literature. Details of these companion studies and the published numerical data used for validation are given in Table 5.1.

The first companion simulation (Grid 1) was performed at a Reynolds number of $\operatorname{Re}_{\theta}=900$ with a spatial resolution in the $x-z$ plane of $\Delta x^{+}=\Delta z^{+}=3$, which corresponds to about 1.5 times the Kolmogorov length scale at that Reynolds number. The $y^{+}$value of the first node from the no-slip boundary is 0.85 and the wall-normal node distribution closely follows the distribution shown in Figure 5.2. Figure 5.3 shows that the results achieve very good agreement compared with the turbulence kinetic energy production and dissipation rates and the root-mean-square streamwise velocity fluctuation $\left(u_{r m s}^{\prime+}\right)$ profiles (Figures $5.3(a)$ and $(b)$, respectively) computed by Spalart (1988), who used a spectral-method algorithm to simulate the boundary layer at a Reynolds number of $R e_{\theta}=1410$. Figure 5.3(c) also shows excellent agreement in the mean velocity profile for $y^{+}<40$ when compared to the DNS of Wu and Moin (2009) performed at $R e_{\theta}=900$, who used a finite-difference scheme with second-order 
accuracy in space and a mixed second- and third-order accuracy in time. Adequate agreement was also achieved in the outer part of the boundary layer, with a deviation of less than $10 \%$ in the mean velocity profile, which was likely due to the streamwise length of the computational domain used in the companion study being insufficient to fully capture the statistical properties of the larger-scale structures in the outer region of the boundary layer.

To isolate the effect of Reynolds number on the accuracy of the numerical results, a second DNS (Grid 2) was performed with the same spatial resolution as Grid 1 but at $R e_{\theta}=500$, which more-closely matches the Reynolds number of the present separation-bubble study. Finally, to ascertain the sensitivity of the results to the spatial grid, a third DNS (Grid 3) with a grid that matches the spatial resolution of the present separation-bubble study was performed at $R e_{\theta}=500$. The simulation results for Grids 2 and 3, shown in Figure 5.4, compare favourably with the turbulent kinetic energy budgets computed by Spalart (1988) at $R e_{\theta}=670$ and the mean velocity profile measured by Djenidi and Antonia (1993) at $R e_{\theta}=560$. Therefore, these validation studies provide strong evidence that the present study should accurately capture the flow development in the turbulent boundary layer downstream of the separation bubble and the transitioning flow in the bubble where the transient coherent flow structures tend to be of notably-greater spatial and temporal scale. The wall-normal node spacing and the spatial and temporal resolution of the present swept and unswept separation-bubble simulations closely match those of Grid 3. As per Figure 5.4, although the Grid-2 results are in slightly better agreement with the simulation data of Spalart (1988) and the experimental data of Djenidi and Antonia (1993) than the Grid-3 results, the resolution of Grid 3 was adopted for the present separation-bubble simulations to enable simulations with reasonable computing times while still ensuring that the flow physics are accurately captured. 

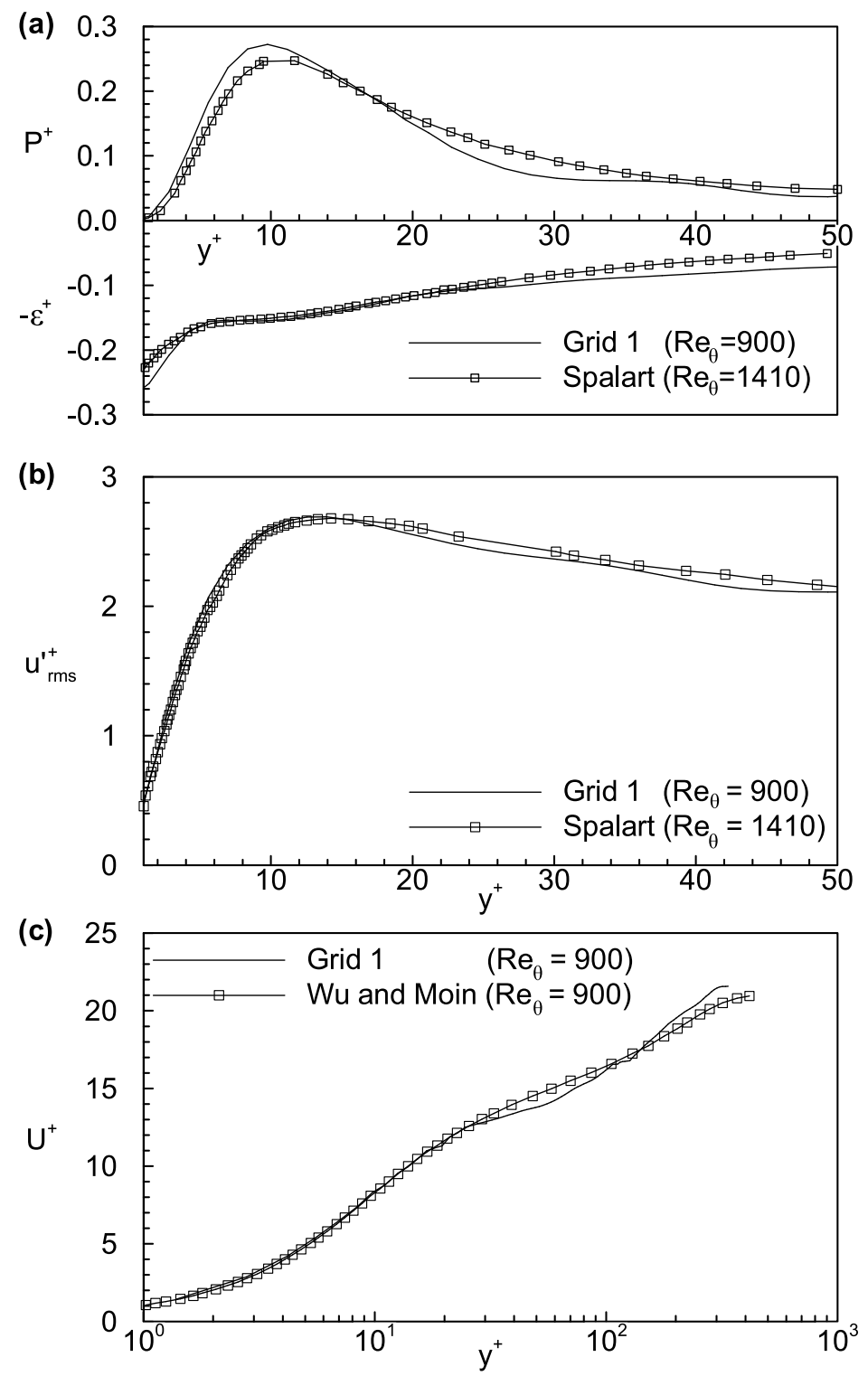

Figure 5.3: Companion DNS study of a zero-pressure-gradient turbulent boundary layer at $R e_{\theta}=900$. Results from Grid 1 are compared with the $(a)$ turbulence kinetic energy budgets and $(b)$ streamwise velocity fluctuation profiles of Spalart (1988) and (c) mean velocity profile of $\mathrm{Wu}$ and Moin (2009). Adapted from Azih et al. (2012). Reprinted with the permission of the American Society of Mechanical Engineers. 

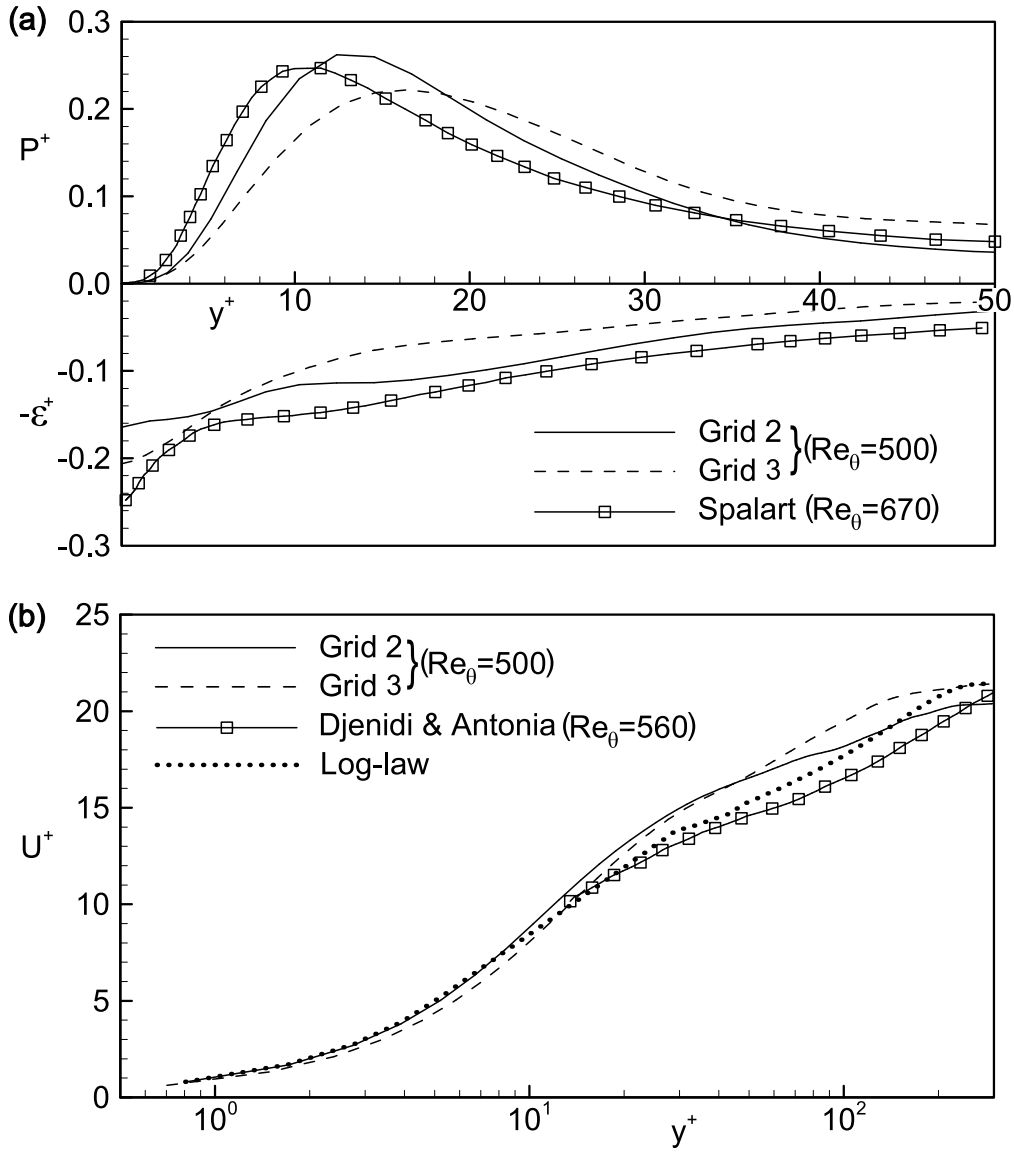

Figure 5.4: Companion DNS studies in a zero-pressure-gradient turbulent boundary layer at $R e_{\theta}=500$. Results from Grid 2 and Grid 3 are compared with the (a) turbulence kinetic energy budgets of Spalart (1988) and (b) mean velocity profile of Djenidi and Antonia (1993). Adapted from Brinkerhoff and Yaras (2011). Reprinted with the permission of the American Society of Mechanical Engineers. 


\subsection{Results and Discussion}

\subsubsection{Free-stream flow development}

The present discussion of the separation-bubble simulation results begins with a description of the free-stream flow development in the swept and unswept configurations. The flow development is examined at midspan $(z / L=0.00)$ in the unswept configuration and at three spanwise stations, located at $z / L=-0.03,0.00$, and 0.03 , in the swept configuration. Velocities are normalized by the reference velocity, $U_{0}=3.385 \mathrm{~m} / \mathrm{s}$, which corresponds to the velocity in the simulation results at the test-surface leading edge. Streamwise and spanwise lengths are normalized by the test-surface length $L=1220 \mathrm{~mm}$ and wall-normal lengths are normalized by the momentum thickness at the time-averaged separation point, $\theta_{s}$. The flow conditions based on these parameters are given in Table 5.2. The time-averaged separation and reattachment locations listed in Table 5.2 are defined as the furthest upstream and downstream locations where the time-averaged wall shear-stress is zero, respectively. The transition onset location is defined as the location where the streamwise distribution of the maximum velocity fluctuation correlation $\left(-\overline{u^{\prime} v^{\prime}}\right)$ deviates from an exponential trend.

The axial distribution of the free-stream velocity magnitude is plotted in Figure 5.5 at a wall-normal height of $31.63 \theta_{s}$. To clearly illustrate the differences in trends between the three spanwise stations, the axial distance is plotted as the distance from the swept testsurface leading edge, $x_{0}$. Figure 5.5 shows that when plotted this way, the free-stream velocity distributions at the three spanwise stations nearly overlap, indicating that the simulated test surface resembles a swept plate with infinite span. A trial-and-error approach was used to adjust the ceiling geometry in the unswept configuration to yield an axial velocity distribution matching the streamwise velocity distribution in the the swept configuration. Figure 5.5 shows that the resulting free-stream velocity distribution approximately matches the swept configuration, although slightly stronger flow acceleration is noted upstream of the suction peak 
Table 5.2: Flow conditions and numerical details of the separation-bubble simulations. Reprinted with the permission of the American Society of Mechanical Engineers.

\begin{tabular}{lcc}
\hline Configuration & Unswept & Swept \\
\hline Sweep angle, $\Lambda$ & $0^{\circ}$ & $45^{\circ}$ \\
Flow Reynolds number, $R e_{L}$ & 270,000 & 270,000 \\
Reference length, $L$ & $1220 \mathrm{~mm}$ & $1220 \mathrm{~mm}$ \\
Reference velocity, $U_{0}$ & $3.385 \mathrm{~m} / \mathrm{s}$ & $3.385 \mathrm{~m} / \mathrm{s}$ \\
Timestep size, $\Delta t$ & $0.10 \mathrm{~ms}$ & $0.10 \mathrm{~ms}$ \\
Number of grid nodes & $11.7 \times 10^{6}$ & $11.7 \times 10^{6}$ \\
Axial suction peak location, $\left(x_{s p}-x_{0}\right) / L$ & 0.363 & 0.356 \\
Axial separation location, $\left(x_{s}-x_{0}\right) / L$ & 0.487 & 0.457 \\
Displacement thickness at separation, $\delta_{s}^{*}$ & $3.30 \mathrm{~mm}$ & $3.12 \mathrm{~mm}$ \\
Momentum thickness at separation, $\theta_{s}$ & $0.94 \mathrm{~mm}$ & $0.98 \mathrm{~mm}$ \\
Momentum-thickness Reynolds number at separation, $R e_{\theta s}$ & 296 & 312 \\
Free-stream turbulence intensity at separation, $T u_{s}$ & $0.01 \%$ & $0.06 \%$ \\
Time-averaged tangential location of transition onset, $\left(x_{t t o}-x_{t s}\right) / L$ & 0.157 & 0.145 \\
Tangential length of time-averaged separation bubble, $\left(x_{t r}-x_{t s}\right) / L$ & 0.185 & 0.195 \\
\hline
\end{tabular}

and slightly weaker flow deceleration is noted downstream of the suction peak. Notwithstanding these differences, the suction peak and time-averaged separation location in the unswept configuration occur about $0.01 \mathrm{~L}$ and $0.03 \mathrm{~L}$ downstream of the corresponding locations in the swept configuration.

The axial variation of free-stream flow acceleration is shown in Figure 5.6 through a plot of the streamwise acceleration parameter, $\eta=\nu / U_{e}^{2} \partial U_{e} / \partial x$, where $U_{e}$ is the magnitude of the local boundary-layer edge velocity. Figure 5.6 shows that the majority of the flow upstream of the suction peak is accelerated at a level well above the range of $\eta_{\text {crit }}=2.5 \times 10^{-6}-3.5 \times$ $10^{-6}$. Above this critical range of acceleration parameter, several researchers have observed that turbulent boundary layers will begin to relaminarize (Jones and Launder, 1972; Escudier et al., 1998), and thus the growth of disturbances upstream of the suction peak is expected to be suppressed. The distributions for the swept and unswept configurations agree well, thus it is expected any differences observed in the development of the separation bubble between the swept and unswept configurations can be attributed to the development of the boundary layer before and after separation rather than differences in the free-stream flow development. 


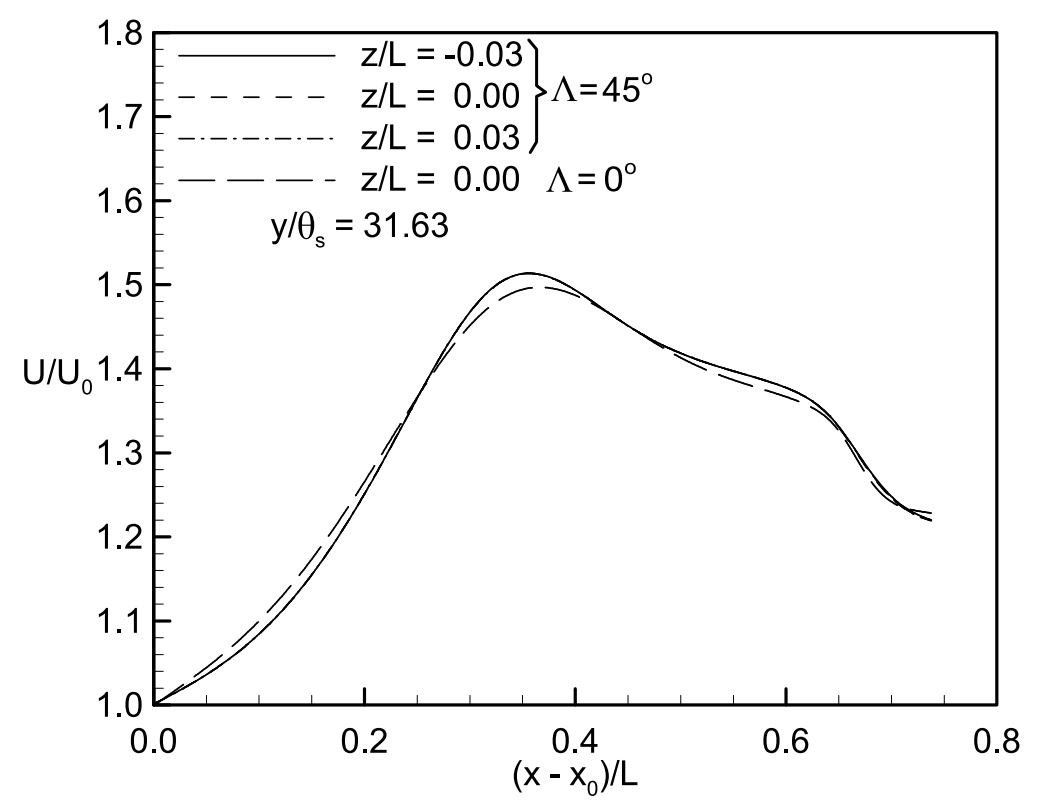

Figure 5.5: Streamwise distribution of the time-averaged free-stream velocity magnitude for the $\Lambda=0^{\circ}$ and $\Lambda=45^{\circ}$ cases. Reprinted with the permission of the American Society of Mechanical Engineers.

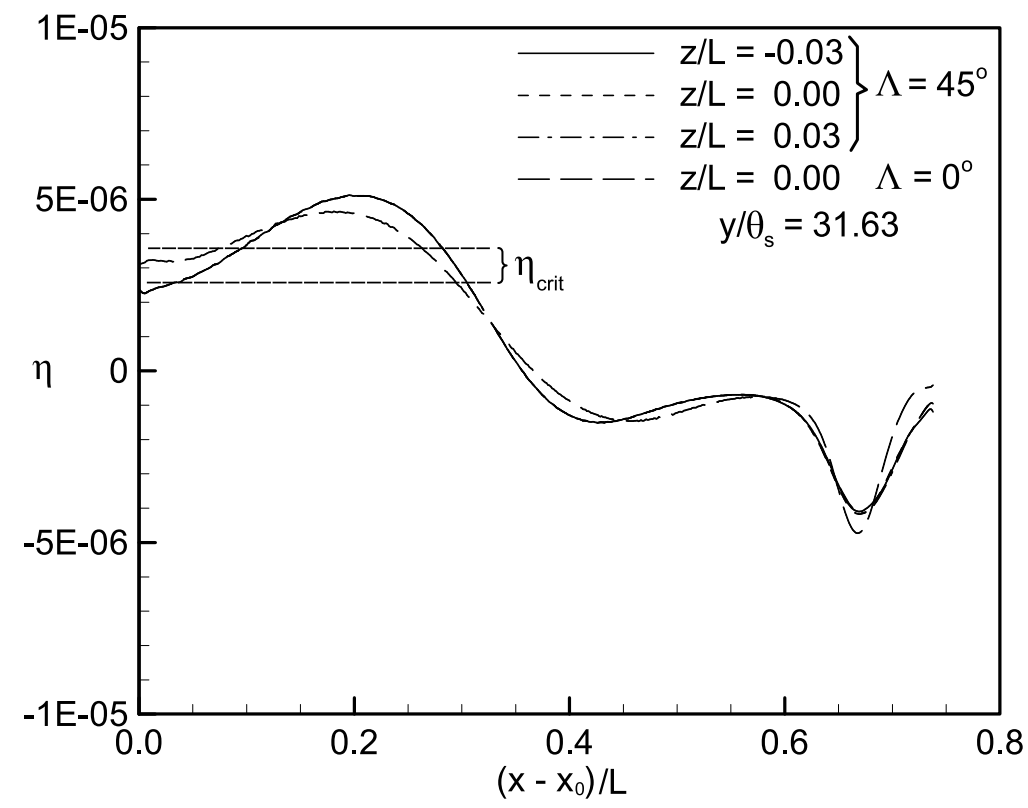

Figure 5.6: Axial distribution of the time-averaged free-stream acceleration parameter for the $\Lambda=0^{\circ}$ and $\Lambda=45^{\circ}$ cases. Reprinted with the permission of the American Society of Mechanical Engineers. 
The variation in the time-averaged spanwise pressure gradient in the swept configuration is shown in Figure 5.7 by plotting the spanwise derivative of the free-stream pressure coefficient, $\partial C_{p} / \partial z$, where $C_{p}=2 p /\left(\rho U_{0}^{2}\right)$ is the free-stream pressure coefficient. Noting that for a fixed sweep angle, an increase in the streamwise pressure gradient corresponds to an increase in the spanwise pressure gradient, the strong streamwise acceleration upstream of the suction peak also produces a strong spanwise pressure gradient in that region. As the spanwise pressure gradient acts on the free-stream flow, it induces turning of the flow in the $x-z$ plane. The streamwise variation in the free-stream flow angle, $\beta$, defined as the angle between the local inviscid streamline and the axial direction, is also shown in Figure 5.7. Notably, the flowturning distribution is observed to be nearly identical at all three spanwise stations. Between the test-surface leading-edge and the suction peak, the free-stream flow turns from $-4^{\circ}$ to $-20^{\circ}$. At the suction peak location, the spanwise pressure gradient equals zero, shown by the dashed line in the figure. As the flow passes this location, the spanwise pressure gradient changes sign and hence the direction of the spanwise pressure force switches direction. The flow begins to turn towards the $+z$ direction, resulting in the reduced flow angle observed in Figure 5.7 downstream of the suction peak.

\subsubsection{Boundary layer development}

The flow turning in the $x-z$ plane illustrated in Figure 5.7 develops as the free-stream flow in the swept case reacts to the spanwise pressure force. Within the boundary layer, the lower flow velocity causes the spanwise pressure force to remain unbalanced, which leads to the development of crossflow motion within the boundary layer that is directed perpendicular to the direction of the inviscid streamline at the boundary edge. The magnitude of the crossflow motion is determined by the local strength of the spanwise pressure gradient, the local boundary layer thickness, and the streamwise extent of the region over which the spanwise pressure force remains unbalanced. The magnitude of crossflow motion at midspan of the swept configuration 


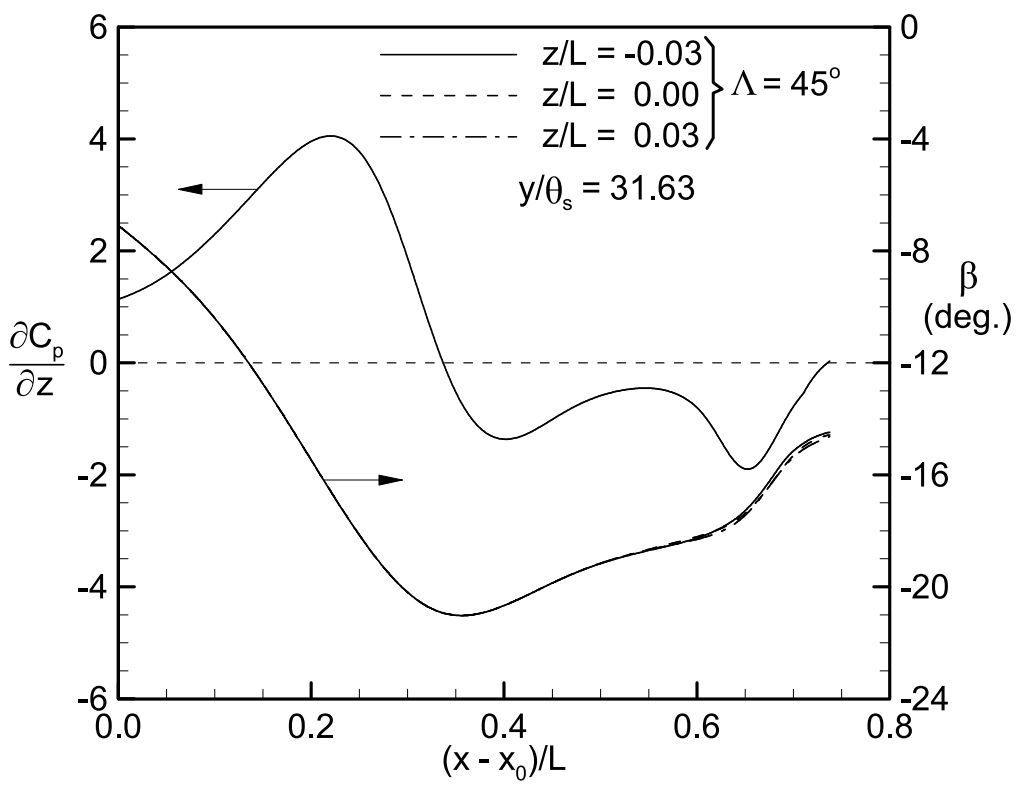

Figure 5.7: Axial distribution of the time-averaged spanwise pressure gradient and free-stream flow angle for the $\Lambda=45^{\circ}$ case. Reprinted with the permission of the American Society of Mechanical Engineers.

is shown in Figure 5.8 through plots of the crossflow velocity profiles at several axial stations along the test surface. In Figure 5.8, the crossflow direction is normal to the local inviscid streamline and the crossflow velocity component, denoted $V_{c}$, is normalized by the local velocity magnitude at the boundary layer edge, $U_{e}$. Profiles 1 and 2 are both located upstream of the suction peak location $\left(\left(x_{s p}-x_{0}\right) / L=0.356\right)$. The axial location of profile 1 corresponds to the location upstream of the suction peak where the crossflow velocity magnitude reaches a maximum. Figure 5.7 shows that this axial location approximately corresponds to the location of maximum spanwise pressure gradient. In profile 2 , the reduction in the strength of the spanwise pressure gradient has yielded reduced crossflow velocity in the boundary layer. Both profiles are inflectional with a single inflection point located $2 \theta_{s}-3 \theta_{s}$ above the test surface. The inflectional shape of the crossflow profiles creates an inviscid-unstable state, termed crossflow instability, which studies on swept wings have identified manifests itself in the form of stationary and traveling waves within the laminar boundary layer (e.g., Saric et al., 2003). In contrast to profiles 1-2, profiles 3-7 are located downstream of the suction peak, where the direction of the 


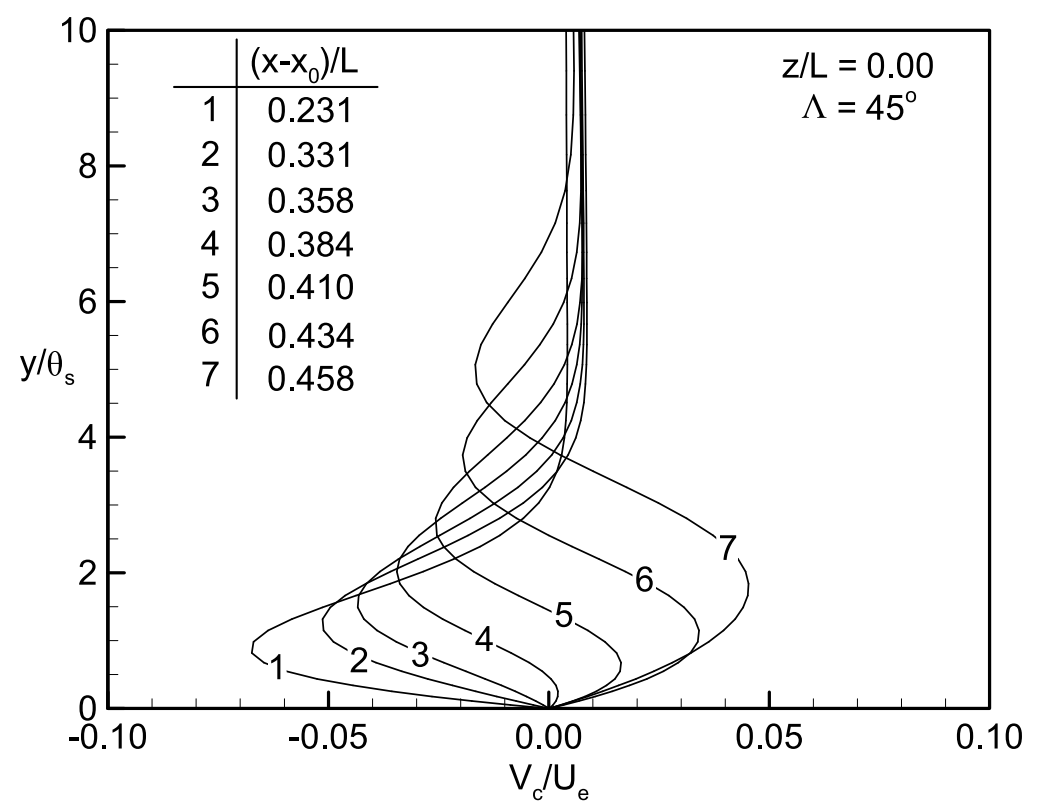

Figure 5.8: Axial variation of the crossflow velocity profile near the separation point for the $\Lambda=45^{\circ}$ case. Reprinted with the permission of the American Society of Mechanical Engineers.

spanwise pressure gradient has reversed. The low-momentum fluid near the wall reacts quickly to the sign change in spanwise pressure gradient by turning in the $+z$ direction, producing the $+z$ crossflow motion observed near the wall. The $-z$ crossflow motion further from the wall has higher momentum and thus reacts more slowly to the change in the spanwise pressure force. As a result, flow further from the wall retains its $-z$ crossflow momentum even as the magnitude of the $+z$ crossflow motion grows near the wall.

Development of the test-surface boundary layer in the swept and unswept configurations is shown in Figure 5.9 through plotting the axial variation in the displacement thickness $\delta^{*}$, normalized by the displacement thickness at the time-averaged separation location $\delta_{s}^{*}$ listed in Table 5.2. It should be noted that for the swept configuration, the displacement thickness is calculated on the basis of the tangential velocity profiles, which are aligned with the local inviscid streamline, rather than the axial velocity profiles. The displacement thickness distributions at the three spanwise stations in the swept configuration are nearly identical, and the distribution in the unswept configuration is also very similar up to about $\left(x-x_{0}\right) / L=0.40$, 


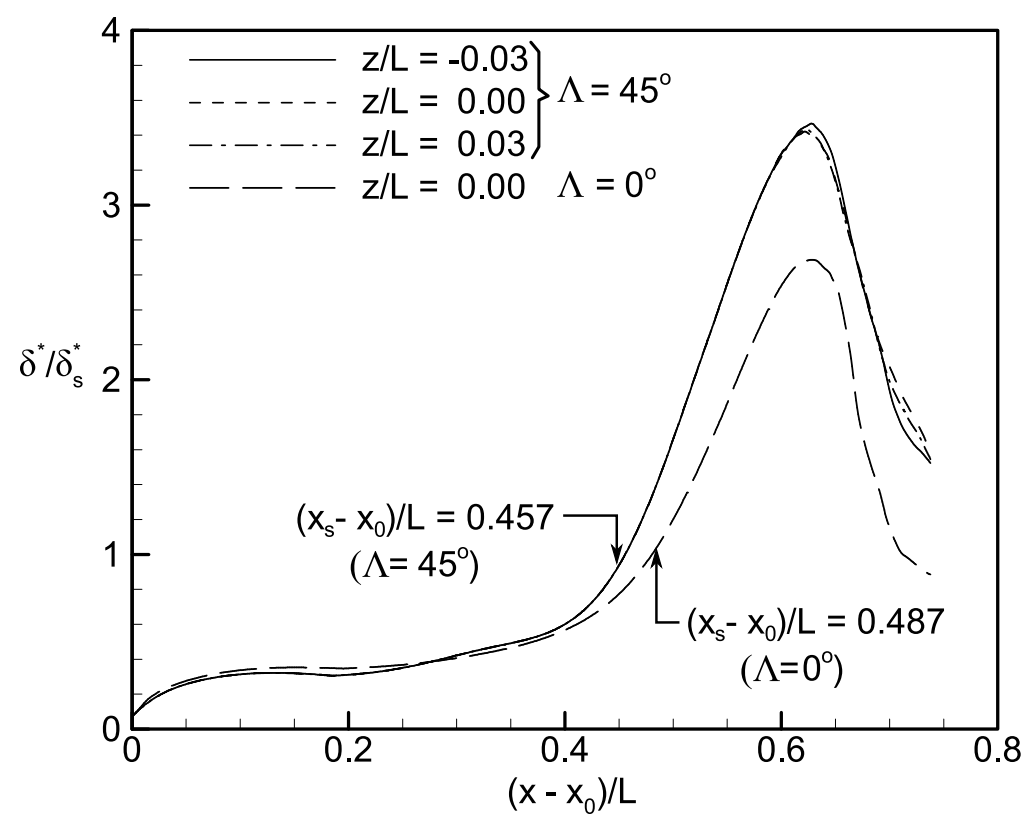

Figure 5.9: Axial distribution of the time-averaged displacement thickness for the $\Lambda=0^{\circ}$ and $\Lambda=45^{\circ}$ cases. Reprinted with the permission of the American Society of Mechanical Engineers.

suggesting that the presence of crossflow motion has a small impact on the displacement effect of the attached laminar boundary layer. Downstream of $\left(x-x_{0}\right) / L=0.40$, the rapid growth in the displacement thickness corresponds to the onset of boundary-layer separation and laminarto-turbulent transition within the separated shear layer.

Differences in the boundary-layer development between the swept and unswept configurations become more apparent in the axial variation of the tangential momentum-thickness $\theta$, shown in Figure 5.10. In the unswept case, the momentum thickness increases almost linearly between $\left(x-x_{0}\right) / L=0.20$ and 0.50 , while for the swept case, there is a rise from $\left(x-x_{0}\right) / L=0.20$ to about 0.33 , followed by a very gradual growth between $\left(x-x_{0}\right) / L=0.33$ and 0.45 and almost no growth between $\left(x-x_{0}\right) / L=0.45$ and about 0.53 . The variation in the momentum-thickness distribution in the swept configuration is attributed to the prevailing crossflow motion within the boundary layer, which results in a spanwise redistribution of mass and tangential momentum within the boundary layer. It should be noted that at the 


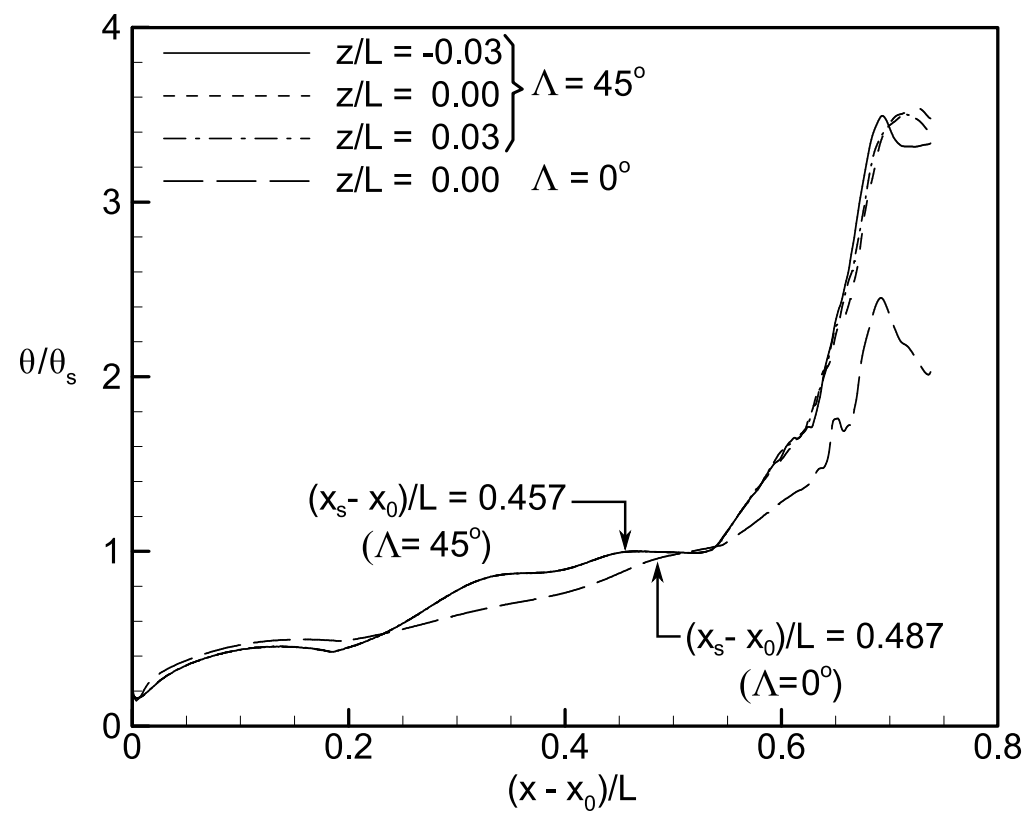

Figure 5.10: Axial distribution of the time-averaged momentum thickness for the $\Lambda=0^{\circ}$ and $\Lambda=45^{\circ}$ cases. Reprinted with the permission of the American Society of Mechanical Engineers.

time-averaged separation location $\left(\left(x_{s}-x_{0}\right) / L=0.487\right.$ and 0.457 for the unswept and swept configurations, respectively), the resulting tangential momentum thickness differs by about $4 \%$. Considering the strong correlation that has been documented between the location of transition onset and the Reynolds number based on the momentum-thickness at separation (e.g., Roberts and Yaras, 2005b), this difference likely translates into a difference in the growth of instabilities downstream of the time-averaged separation location.

\subsubsection{Instability and transition}

The inflectional crossflow velocity profiles present in the attached laminar boundary layer result in the formation of a crossflow instability mode that manifests itself as stationary and traveling disturbances. Studies on swept wings suggest that in cases where environmental disturbances correspond to a turbulence intensity less than $0.15 \%$, stationary waves in the form of vortices closely aligned with the inviscid streamline at the edge of the boundary layer 
tend to dominate the traveling waves, ultimately leading to transition to turbulence (e.g., Deyhle and Bippes, 1996). Table 5.2 shows that the turbulence intensity at the time-averaged separation location in the present study is well below this threshold, hence stationary crossflow vortices are expected to develop in the attached laminar region. Their presence should be detectable by examining the extent of distortion of the tangential velocity profile by wall-normal motions induced by these vortices. However, analysis of the perturbation tangential velocity field, obtained by subtracting the spanwise-averaged tangential velocity profile from the local tangential velocity profiles, does not reveal the pattered deformation suggestive of stationary vortical structures. Wavenumber spectra of the tangential and crossflow perturbation velocities in the crossflow direction near the suction peak at various wall-normal heights also do not reveal the presence of stationary waves in the attached laminar boundary layer. This is likely a result of the development of the stationary vortices being hindered by the strong favourable streamwise pressure gradient upstream of the suction peak.

The absence of stationary crossflow vortices in the attached laminar boundary layer suggests that crossflow instability may not play a significant role in the growth of disturbances leading up to transition, and the transition process is instead dominated by the $2 \mathrm{D}$ viscous and inviscid instability modes in the attached and separated regions, respectively. The growth of disturbances in the attached laminar boundary layer is shown in Figure 4.10 through plotting the axial distribution of the root-mean-square of the streamwise fluctuation velocity, $u_{r m s}^{\prime}$, at a wall-normal height of $y / \theta_{s}=1.664$, which approximately corresponds to the height where the disturbance growth-rate within the attached boundary layer is maximum. In the unswept configuration, amplification of boundary-layer disturbances commences near $\left(x-x_{0}\right) / L=0.20$ and linear growth via the T-S instability mode is observed until near the time-averaged separation location at $\left(x_{s}-x_{0}\right) / L=0.487$. Downstream of separation, a more strongly-amplified mode develops, suggestive of the onset of K-H instability. Disturbance growth saturates near $\left(x-x_{0}\right) / L=0.65$ which signifies the break-down of the flow to small- 


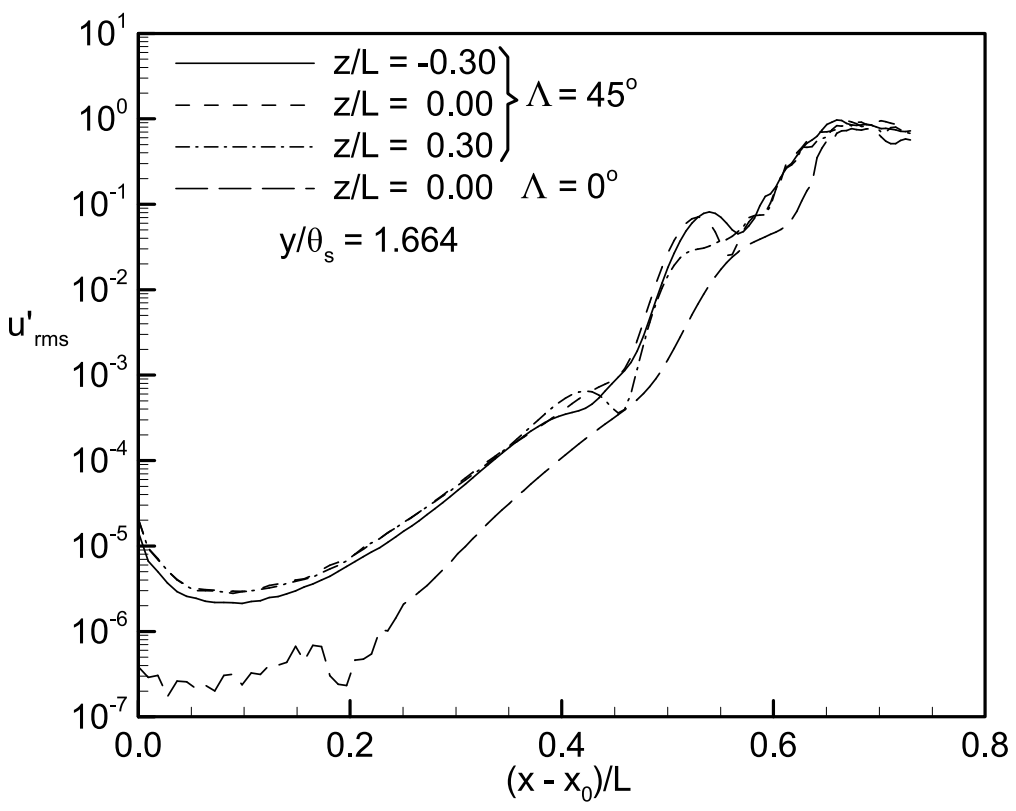

Figure 5.11: Axial distribution of the root-mean-square of the streamwise fluctuation velocity for the $\Lambda=0^{\circ}$ and $\Lambda=45^{\circ}$ cases. Reprinted with the permission of the American Society of Mechanical Engineers.

scale turbulence. In the swept configuration, the onset of T-S instability occurs slightly further upstream than the unswept configuration, but the disturbance growth rate in the attached laminar boundary layer is comparable to or even slightly lower than in the unswept configuration. This observation supports the earlier assertion that the crossflow instability mode does not seem to play a noticeable role in the growth of disturbances in the attached laminar boundary layer.

Figure 5.11 shows that downstream of the time-averaged separation location $\left(\left(x_{s}-\right.\right.$ $\left.x_{0}\right) / L=0.457$ for the swept configuration), the amplification rate of the $\mathrm{K}-\mathrm{H}$ mode in the swept configuration is slightly stronger than in the unswept configuration. The amplification rate of the $\mathrm{K}-\mathrm{H}$ instability mode is largely determined by the magnitude of the shear in the separated shear layer. In the unswept configuration, the shear occurs primarily in the $x-y$ plane because the separated flow is nominally two-dimensional. In the swept configuration, however, the nearly-stationary fluid beneath the separated shear layer reacts strongly to the spanwise 
pressure gradient and results in a substantial crossflow motion within the separation bubble. The shear created in the $z_{c}-y$ plane by the crossflow velocity component is also unstable to a K-H mode. The extent to which the K-H mode in the crossflow shear layer affects the overall growth rate of disturbances in the separated shear layer can be determined by assessing the relative level of shear in the tangential and crossflow shear layers. Figure 5.12 provides a qualitative comparison of the strengths of the tangential and crossflow shear layers in the swept configuration by plotting the time-averaged contours of the tangential and crossflow velocity components in the separated region. The orientation of the tangential and crossflow planes was obtained by assuming that the flow angle in the separated region remains constant at $\beta=-18^{\circ}$, estimated from Figure 5.7. In Figures $5.12(a)$ and $(b)$, the velocities are plotted in the tangential plane $z_{c} / L=0.00$, which is aligned with the local inviscid free-stream, while Figure 5.12(c) illustrates the crossflow shear layer in the crossflow plane $\left(x_{t}-x_{0}\right) / L=0.63$, which is normal to the local inviscid streamline. Velocities are normalized by the free-stream velocity magnitude at separation, $U_{s}$, and velocity contour lines are plotted in dimensionless increments of 0.2. The spacing of the contour lines illustrates the magnitude of the wallnormal gradient of the tangential and crossflow velocities and hence the relative strengths of their respective shear layers. Comparing Figures 5.12(a) and (b) reveals that the crossflow velocity component in the separation bubble is about an order of magnitude lower than the tangential velocity component, and comparing Figures 5.12(a) and (c) reveals that the crossflow shear layer is substantially weaker than the tangential shear layer. This suggests that while the crossflow motion contributes to the level of shear in the separated shear layer, this contribution is quite weak, and the resultant $\mathrm{K}-\mathrm{H}$ type instability in the separated shear layer is still primarily driven by the shear in the tangential motion. The slightly larger disturbance growth rate for the swept case noted in Figure 5.11 is therefore likely the result of a larger value of $R e_{\theta}$ at separation (Table 5.2) as elaborated below, and not due to a fundamental change in the development of the shear-layer instability. 

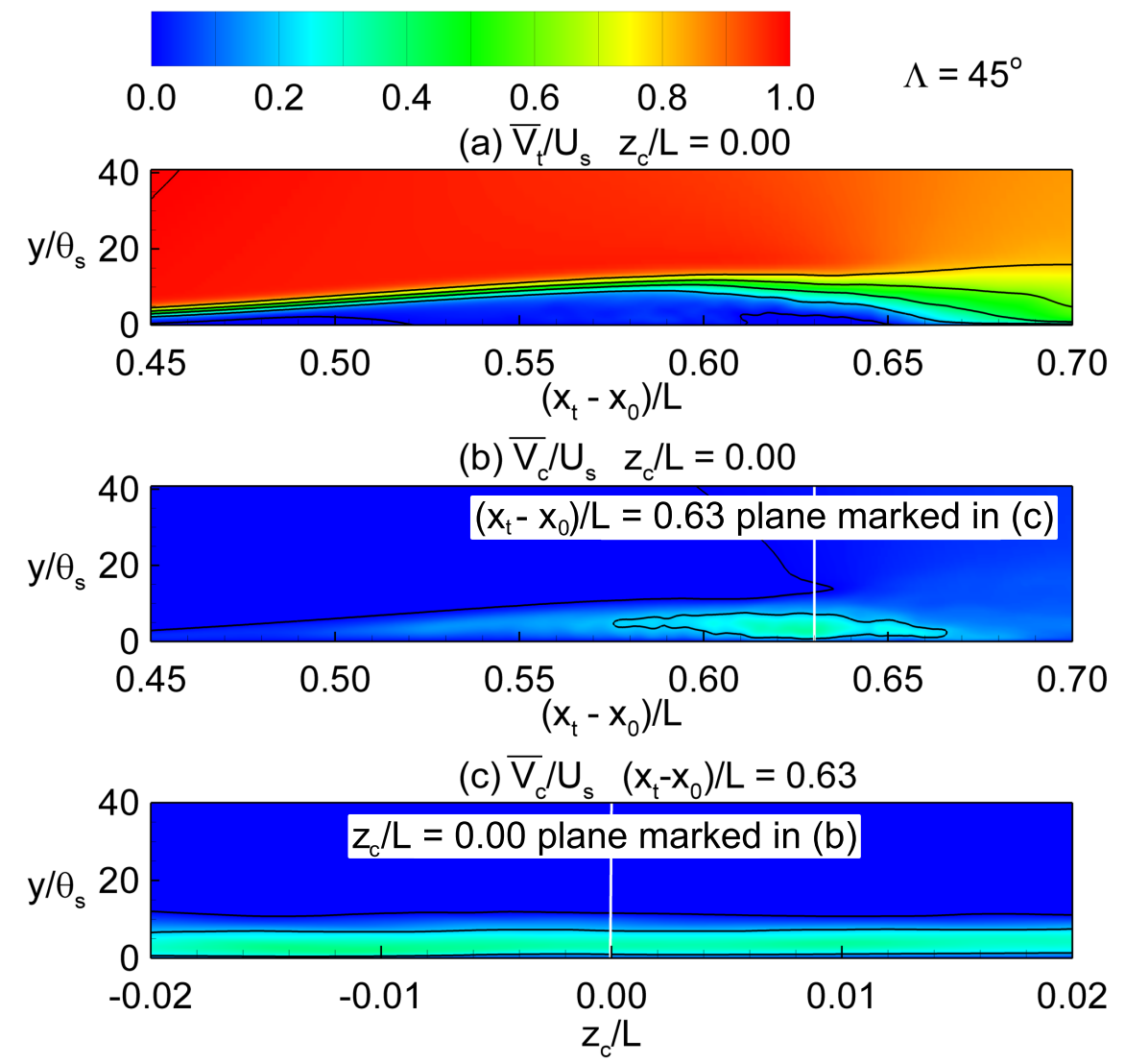

Figure 5.12: Time-averaged contours of $(a)$ tangential velocity at $z_{c} / L=0.00,(b)$ crossflow velocity at $z_{c} / L=0.00$, and $(c)$ crossflow velocity at $\left(x_{t}-x_{t s}\right) / L=0.19$ in the separated region of the $\Lambda=45^{\circ}$ case. Contour lines are plotted in dimensionless increments of 0.2. Reprinted with the permission of the American Society of Mechanical Engineers. 
An example of the growth of disturbances in the separated shear layer for the unswept and swept configurations is given in Figure 5.13(a) and $(b)$, respectively, which illustrates about one period of the dominant instability waves. Dimensionless time is denoted by $\tau=t U_{s} / \theta_{s}$, where $U_{s}$ is the time-averaged velocity magnitude in the free-stream at the time-averaged separation location. To ensure that the streamwise distances in Figure 5.13 are consistent between the unswept and swept configurations, the trajectory of the local inviscid streamline was followed, and the tangential distance of this trajectory relative to the separation location is plotted in Figure 5.13; in the unswept case, this corresponds to the axial length. The white lines in Figure 5.13 track the roll-up and shedding of a distinct spanwise vortex from the separated shear layer, and the line's slope gives a qualitative measure of the vortex-shedding frequency. It is readily observable that roll-up of the separated shear layer occurs earlier and at a higher frequency in the swept configuration than in the unswept configuration. This is consistent with the upstream shift in the transition onset location for the swept configuration shown in Table 5.2. It is notable that although transition onset occurs earlier in the swept configuration, the time-averaged separation bubble in the swept configuration is about $5 \%$ longer than in the unswept configuration (Table 5.2). The shear layer time-mean reattachment location is established by time averaging a highly unsteady flow field dominated by vortical structures shedding downstream as illustrated in Figure 5.13. Any small changes in the development of these vortical structures can potentially affect the time-mean reattachment location. Figure 5.13 shows that the vortical structures shed from the separated shear layer maintain coherence notably farther downstream in the swept configuration than in the unswept configuration, which translates into the observed downstream shift in the time-mean reattachment location. The most-amplified frequency of the K-H instability in the separated shear layer is examined quantitatively by comparing the frequency spectra of the wall-normal fluctuation velocity $v^{\prime}$ at several streamwise stations within the separated region, shown in Figure 5.14. The most amplified frequency of disturbances is noted to be about $15 \%$ higher for the swept configuration, and break-down to high-frequency smaller scale flow structures 

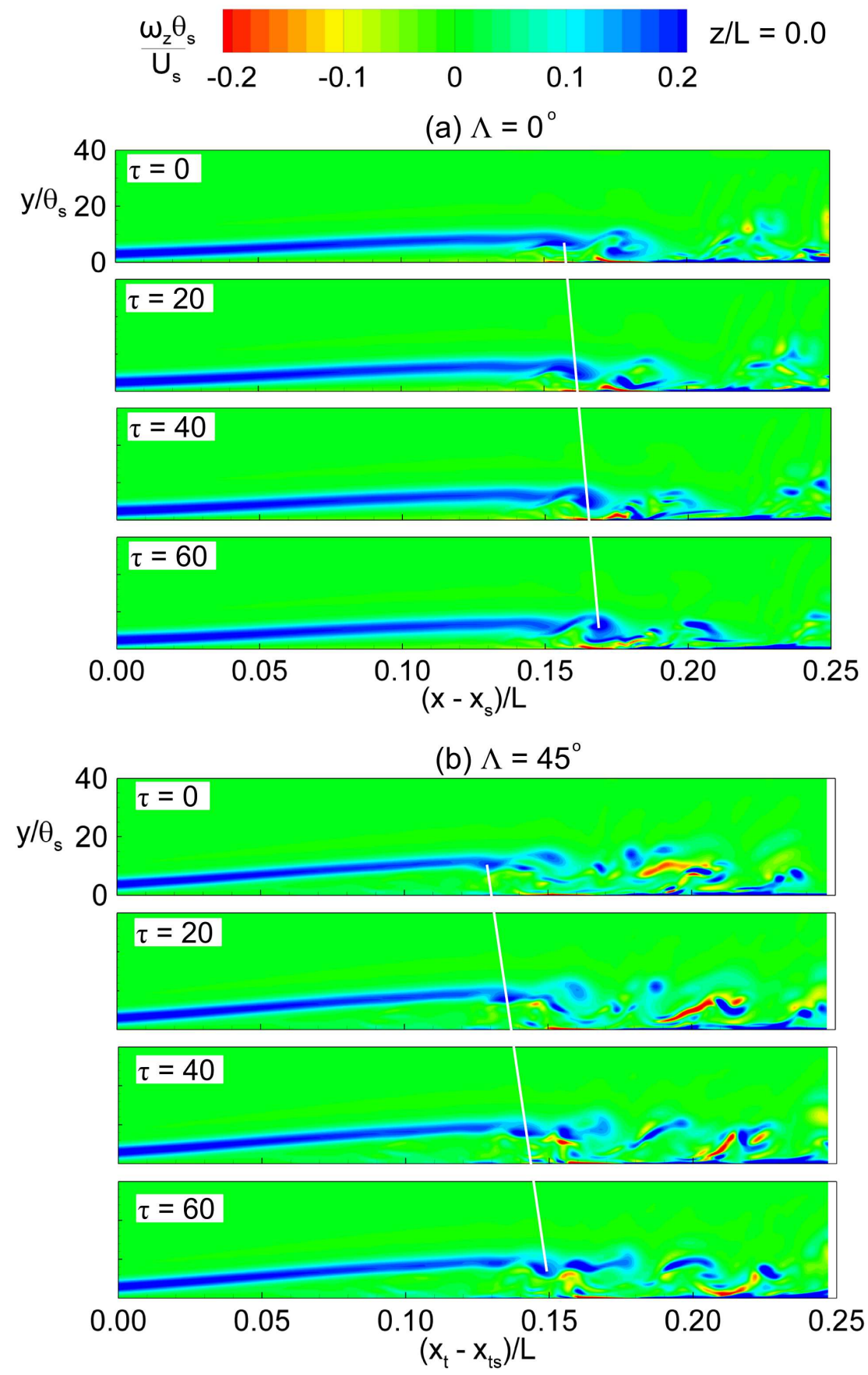

Figure 5.13: Periodic roll-up of the separated shear layer observed through contours of spanwise vorticity at $z / L=0.00$ for the $(a) \Lambda=0^{\circ}$ and $(b) \Lambda=45^{\circ}$ cases. The development of a shed spanwise vortex is traced by a white line. Reprinted with the permission of the American Society of Mechanical Engineers. 


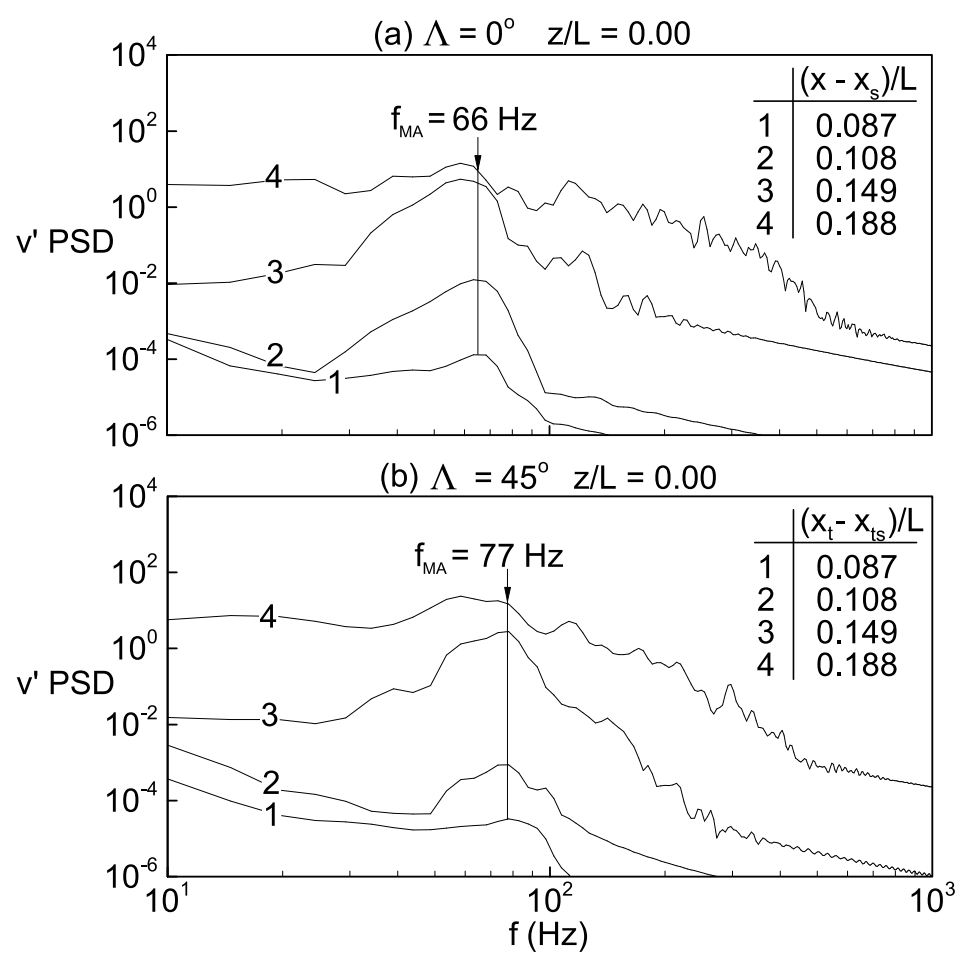

Figure 5.14: Frequency spectra of the wall-normal fluctuation velocity at $z / L=0.00$ and at several streamwise locations in the separated-flow region for $(a) \Lambda=0^{\circ}$ and $(b) \Lambda=45^{\circ}$ cases. Reprinted with the permission of the American Society of Mechanical Engineers.

(i.e. transition onset) occurs earlier in this configuration as seen through comparison of the swept and unswept frequency spectra at station 3 . Table 5.2 shows that $R e_{\theta s}$ in the swept configuration is greater by about $5 \%$ compared to the unswept configuration. The sensitivity of the development of the K-H instability mode to $R e_{\theta s}$ is well-established for free shear layers (e.g. Ho and Huerre, 1984), where an increase in $R e_{\theta s}$ is known to yield an increase in the shedding frequency from the shear layer. The increased growth rate and shedding frequency of the K-H instability mode observed in the swept configuration is therefore mostly the result of a higher value of momentum-thickness Reynolds number at separation. Thus, although the crossflow instability mode does not appear to have a noticeable effect on the instability development in the transitional separation bubble, the 3D pressure field does indirectly alter the transition process and hence separation bubble development by modifying the boundarylayer integral conditions at separation. 


\section{Chapter 6}

\section{Direct Numerical Simulation of a}

\section{Square Jet Ejected Transversely into}

\section{an Accelerating, Laminar Main Flow}

Citation: Brinkerhoff, J.R. and Yaras, M.I. (2012), "Direct Numerical Simulation of a Square Jet Ejected Transversely into an Accelerating, Laminar Main Flow," Flow, Turbulence and Combustion, 89, pp. 519-546. doi: http://dx.doi.org/10.1007/s10494-012-9406-z. Used with permission. 


\subsection{Introduction}

The flowfield of a jet ejected transversely into a laminar boundary layer has been the subject of extensive research for well over 60 years (Ruggeri et al., 1950; Keffer and Baines, 1963; McMahon et al., 1971). Early studies were aimed at understanding the dissipation of industrial pollutants from exhaust stacks and effluent plumes in rivers (Margason, 1993). More recently, transverse jets have been used in gas turbine engines to dilute high-temperature combustion products before they enter the turbine section and to modify the local air-to-fuel ratio and hence the production rate of emissions (Vermeulen et al., 1992; Bowman, 1992). Transverse jets also occur in film cooling and active flow-control of turbine blades, in thrust-vectoring of rocket engines, and during the transition from vertical to forward flight in vertical take-off and landing aircraft (e.g., Karagozian, 2010). The transverse jet achieves a high degree of near-field mixing due to the interaction of the jet fluid with the moving fluid in the free-stream and with the vorticity in the main-flow boundary layer. The extent of this interaction depends primarily on the jet-to-main-flow velocity ratio $r=v_{j e t} / U_{0}$ and the jet Reynolds number $R e_{j e t}=v_{j e t} D / \nu$. The majority of engineering applications of transverse jets involve velocity ratios greater than $r=1$, which results in a complex system of vortical structures in the flowfield surrounding the transverse jet (Karagozian, 2010). These vortical structures, sketched in Figure 6.1, can be categorized into four groups (e.g. Fric and Roshko, 1994): (i) shear-layer vortices; (ii) a counter-rotating vortex pair (CVP); (iii) a horseshoe vortex; and (iv) wake vortices.

As the jet is ejected from the orifice, the spatial profile of the exit velocity contains an inflection point due to the shear between the jet and main flow (Kelso et al., 1996; Kelso and Smits, 1995), which provides the conditions necessary for a Kelvin-Helmholtz instability to develop within the jet shear layer. This instability leads to the periodic accumulation of circumferential vorticity in the direction of the jet flow and eventually to the periodic roll-up of the jet shear-layer into toroidal vortex rings (Fric and Roshko, 1994; Megerian et al., 2007; de 
B. Alves et al., 2008). As the shear-layer vortices are convected upwards, their interaction with the main flow produces the CVP, which consists of a pair of counter-rotating vortices roughly aligned with the jet trajectory. This flow structure is arguably the most prominent structure produced by the transverse jet and it is repeatedly observed in the jet flowfield for a wide range of flow and geometric parameters (Keffer and Baines, 1963; Kamotani and Greber, 1972; Sau et al., 2004). Early researchers studying transverse jets thought that the CVP was caused by the impulse of the fluid ejected from the jet, which imparts a transverse force onto the main-flow fluid, causing it to roll-up into the CVP (e.g., Broadwell and Breidenthal, 1984). More recent investigations have corrected this view, showing that the CVP is created when the shear-layer vortices described above are folded in the presence of the main flow (Yuan et al., 1999; Kelso et al., 1996; Sau et al., 2004; Rivero et al., 2001; Lim et al., 2001; Cortelezzi and Karagozian, 2001). Because the presence of the CVP contributes significantly to the nature of the jet flowfield, particularly in enhancing the entrainment of main-flow fluid from the free-stream Haven and Kurosaka (1997), controlling its near-field formation can have a significant effect on the far-field flow patterns produced by the jet. The most common efforts towards controlling the development of the CVP have included pulsing the jet (Johari, 2006; Shapiro et al., 2006; Eroglu and Breidenthal, 2001; Narayanan et al., 2003), which affects the roll-up of the jet shear layer, and using different orifice shapes (Haven and Kurosaka, 1997; Liscinsky et al., 1996; Gutmark and Grinstein, 1999), which affects the shape of the jet shear layer.

The horseshoe vortex system is the result of the interaction between the main-flow boundary layer and the transverse jet (Kelso et al., 1996; Krothapalli et al., 1990). An adverse pressure gradient is created locally in the vicinity of the jet as main-flow streamlines stagnate or are entrained upwards into the jet flow. The separation and subsequent roll-up of the main-flow boundary layer upstream of the jet in response to this adverse pressure gradient produces a spanwise vortex, which is then convected and stretched around the sides of the jet in a 


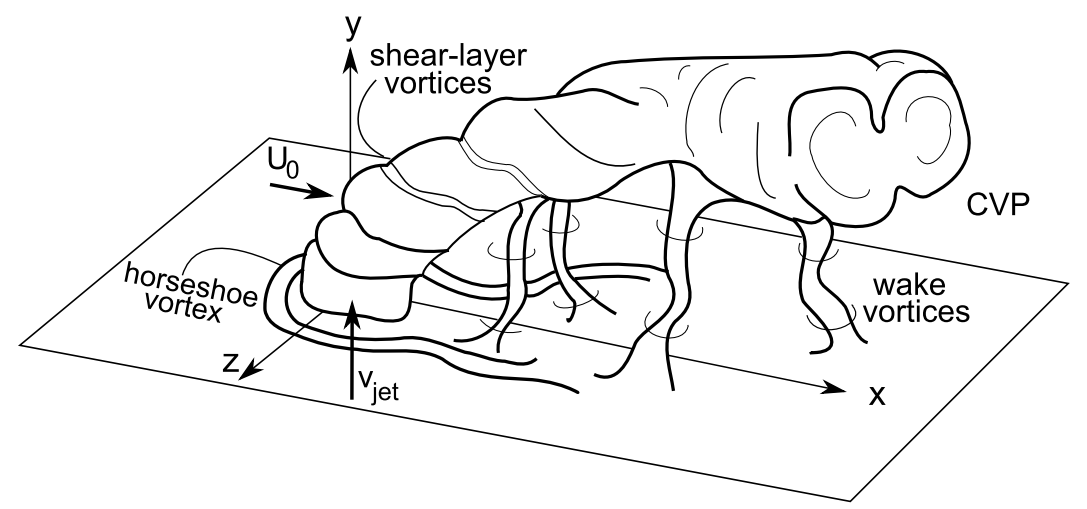

Figure 6.1: Schematic of vortical structures in the flowfield of the transverse jet. Adapted from Karagozian (2010). Reprinted with the permission of Springer Science and Business Media.

manner analogous to the horseshoe vortex created around a solid cylinder (Baker, 1979). Kelso and Smits (1995) observed that the horseshoe vortex system can become unsteady at higher Reynolds numbers and the unsteadiness can be transmitted into the wake of the jet, affecting the growth of structures downstream of the jet orifice, such as the wake vortices, which are roughly-vertical, tornado-like vortices that span from the jet trajectory to the wall. The opposite senses of rotation of the wake vortices caused early researchers to liken them to the vortex sheet behind a solid cylinder. However, Fric and Roshko (1994), Morton and Ibbetson (1996), and others pointed out that a transverse jet, unlike a cylinder, lacks a solid surface and so cannot create vorticity within the fluid. As a result, free-stream streamlines will close around the rear of the jet similar to potential flow, producing a local adverse pressure gradient as streamtubes diverge behind the jet. Fric and Roshko (1994) observed the separation of the main-flow boundary layer in response to this adverse pressure gradient, concluding that the subsequent reorientation and entrainment of the separated boundary-layer vorticity produces the wake vortices.

The flowfield produced by a transverse jet is evidently strongly connected to the development of the main-flow boundary layer and the jet shear-layer. Little attention has been given, however, to the effect of the main-flow free-stream conditions, particularly the effect of streamwise pressure gradients applied to the main flow. To address this question, a direct 
numerical simulation (DNS) was performed of a jet that is ejected transversely through a spatially-accelerating laminar boundary layer. A strongly-favourable streamwise pressure gradient was imposed on the boundary layer with the expectation that the resulting free-stream acceleration would stabilize the flat-plate boundary layer so that the structures resulting from the interaction of the jet and main flows would be more organized and thus easier to detect and study. The results provide insight on the transient formation of the vortical fluid structures that are created in this scenario and how they are affected by a strongly-favourable streamwise pressure gradient applied to the main flow.

\subsection{Numerical Method}

\subsubsection{Computational domain and boundary conditions}

The computational domain, shown in Figure 6.2, consists of a main-flow sub-domain containing a flat, no-slip test surface and a jet sub-domain comprised of a rectangular pipe. The jet sub-domain has dimensions of $D=2.2 \mathrm{~mm}$ and a wall-normal length of $10 D$, and the main-flow sub-domain has streamwise and spanwise dimensions of $250 D$ and $50 D$, respectively. This spanwise width was chosen to prevent the free-slip sides of the main-flow sub-domain from affecting the interaction of the jet and main flow. The wall-normal height of the main-flow sub-domain at the inflow boundary is $114.5 D$. A uniform, time-invariant velocity of $4 \mathrm{~m} / \mathrm{s}$ parallel to the test surface and a static pressure that remains fixed in an area-averaged sense are specified at the inflow and outflow boundaries of the main-flow sub-domain, respectively, and the sides and upper wall are specified as free-slip walls. The level of free-stream acceleration is set by sloping the upper wall towards the test surface by $5.6^{\circ}$. The orifice of the jet is located at the midspan of the test surface and $80 D$ from the test-surface leading edge. This distance allows the main-flow boundary layer to grow to a sufficient thickness to enhance the interaction between the vorticity in the main-flow boundary layer and that introduced by the jet. The 


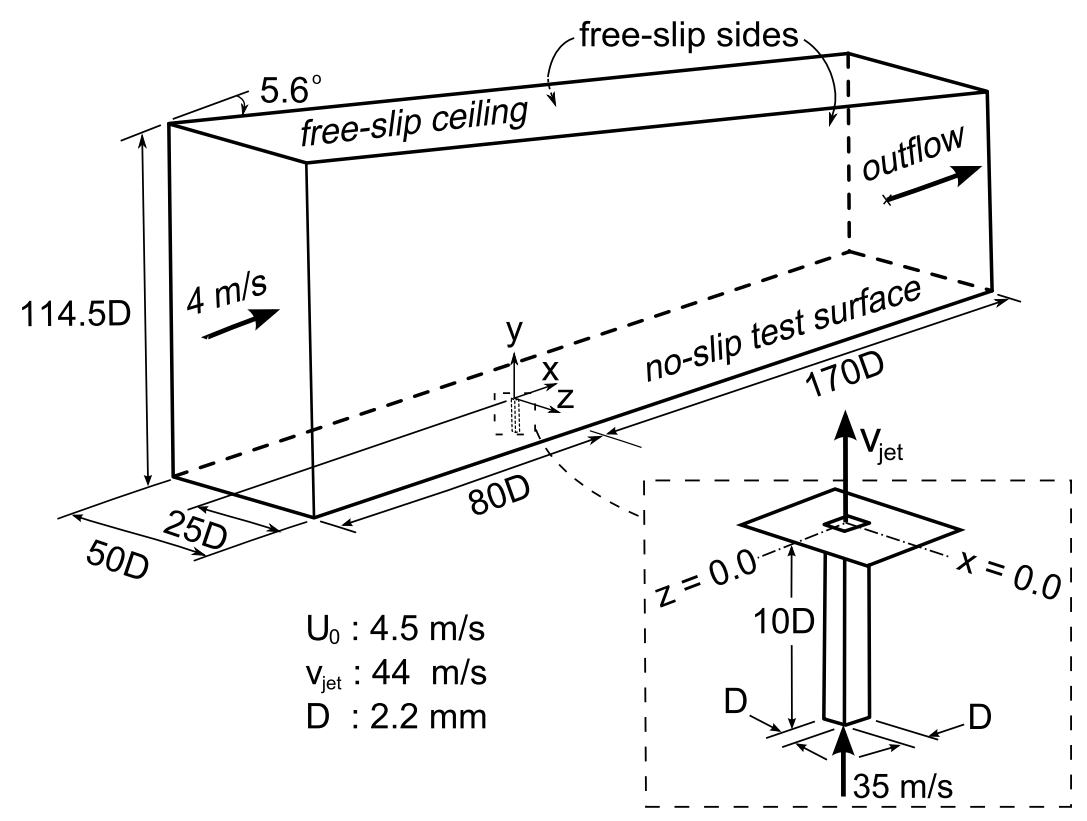

Figure 6.2: Schematic of the computational domain. A magnified view of the jet-pipe is shown in the dashed box. Reprinted with the permission of Springer Science and Business Media.

sides of the jet sub-domain are specified as no-slip walls to allow development of the boundary layer within the jet. A spatially-uniform velocity of $35 \mathrm{~m} / \mathrm{s}$ is applied at the inlet of the jet sub-domain for a duration of $t_{j e t}=8.65 \mathrm{~ms}$. Growth of the jet boundary layer along the walls of the jet sub-domain accelerates the core flow in the jet to a value of $v_{j e t}=44 \mathrm{~m} / \mathrm{s}$ at the center of the jet orifice, and the resulting jet velocity ratio is $r=v_{\text {jet }} / U_{0}=9.78$; the reference velocity $U_{0}=4.5 \mathrm{~m} / \mathrm{s}$ is defined as the free-stream velocity at a wall-normal height of $16 D$ above the center of the jet orifice when the jet is turned off.

Because the simulation captures the transient development of the jet flowfield as the jet is turned on and off, the simulated jet resembles a single period of a periodically-pulsed transverse jet (Shapiro et al., 2006). Pulsed jets have been created experimentally by pushing a column of fluid of length $L$ through an orifice of width $D$, and the resulting flowfield is largely determined by the ratio $L / D$ (called the stroke ratio) and the jet velocity ratio (Eroglu and Breidenthal, 2001; Johari, 2006; Sau and Mahesh, 2008). The analogous length of the fluid column ejected by the simulated jet is $L \approx v_{\text {jet }} t_{\text {jet }}$ and therefore the simulated stroke ratio, $L / D$, is equivalent 


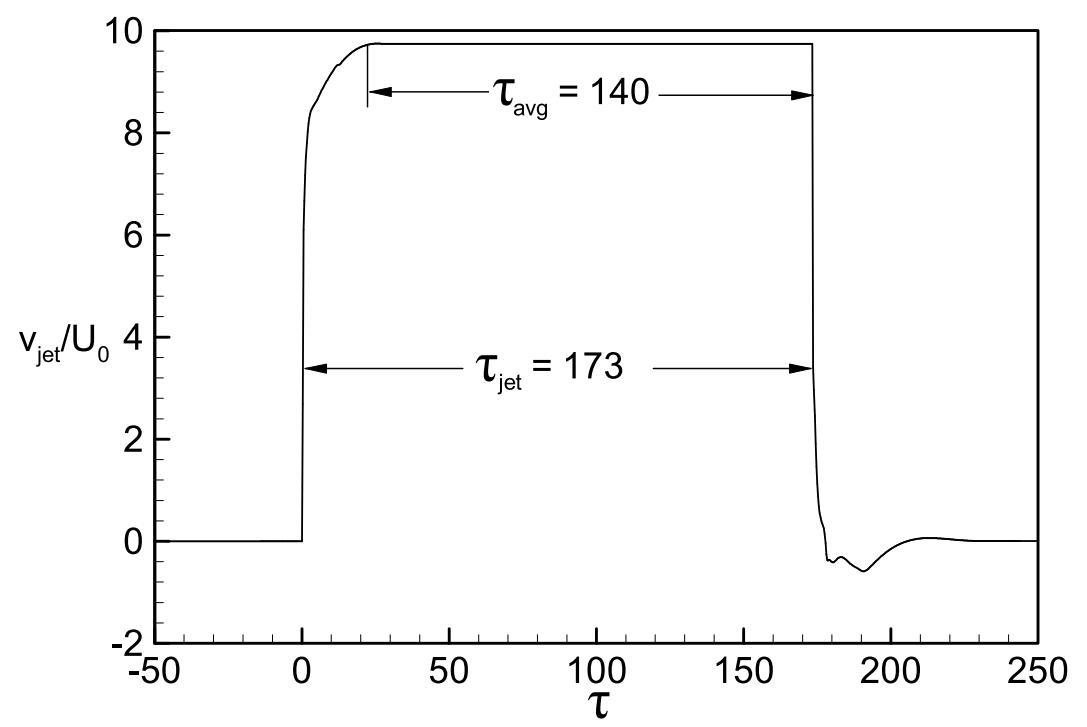

Figure 6.3: Time history of the jet velocity observed at the center of the jet orifice. Reprinted with the permission of Springer Science and Business Media.

to the normalized jet duration $\tau_{j e t}=t_{j e t} v_{j e t} / D$. Figure 6.3 shows the time history of the jet velocity observed in the simulation results at the center of the orifice and shows that the normalized duration of the simulated jet is $\tau_{j e t}=173$. Previous studies of pulsed transverse jets (e.g. Sau and Mahesh, 2008) have shown that for jet velocity ratios greater than $r=2$, the flowfield of a pulsed jet approaches that of a steady jet when the stroke ratio exceeds about $L / D=8$. Considering that the stroke ratio in the current study is substantially larger than this value, the simulated flowfield is likely comparable to the flowfield resulting from a steady jet with an equivalent jet velocity ratio.

\subsubsection{Spatial grid}

A structured grid consisting of hexahedral finite volumes was mapped to the test-surface and jet sub-domains described above. The node distributions were chosen to resolve the development of the main-flow boundary layer upstream of the jet, to capture the development of the jet-flow boundary layer in the jet-pipe, and to resolve the vortical flow structures that are 
created through the mixing of these two streams near the jet orifice. The spatial and temporal grids must therefore be sufficiently refined to capture the smallest spatial and temporal scales in the flow. As the size of these scales are not known a priori, it is reasonable to assume that they are of the order of the Kolmogorov length and time scales of a turbulent boundary layer at an equivalent Reynolds number based on the same test-surface length and main-flow velocity as the current study $\left(R e_{x}=112,000\right)$. For such a boundary layer, the Kolmogorov length scale, denoted $\eta$, is on the order of the viscous length scale, typically reaching a minimum value of $\eta^{+} \approx 2$ close to the no-slip boundary (Pope, 2000; Kim et al., 1971; Stanislas et al., 2008). Therefore, the main-flow sub-domain is discretized with 345 and 197 nodes in the streamwise and spanwise directions, distributed such that the node spacing yields values of $\Delta x^{+}=24$ and $\Delta z^{+}=10$, corresponding to approximately 4 to 10 times the Kolmogorov length scale at the present Reynolds number. Wall units are calculated from the wall shear-stress estimated from the local friction factor correlation for a fully-developed turbulent boundary layer proposed by Schlichting (1968):

$$
C_{f}=\left(2 \log _{10}\left(R e_{x}\right)-0.65\right)^{-2.3}
$$

where $C_{f}=0.5 \tau_{w} / \rho U_{0}^{2}$ is the local friction factor and $\tau_{w}$ is the wall shear stress. The wallnormal direction is discretized with 88 nodes, spaced such that the $y^{+}$value of the first node from the no-slip boundary is 0.8 . Close to the wall, $\left(0<y^{+}<30\right)$, the wall-normal node spacing is increased linearly from the value at the wall to a value that corresponds to the Kolmogorov length scale in the log-law region, which is approximated by:

$$
\eta^{+}=\left(\kappa y^{+}\right)^{1 / 4}
$$

where $\kappa=0.41$ is the von-Kármán constant (Pope, 2000; Stanislas et al., 2008). Above this wall-normal height, the node spacing is increased at a rate of $4 \%$ until $y^{+}=70$, remains constant in the range $70<y^{+}<140$, and is then increased at a rate of $10 \%$ until the free-slip 


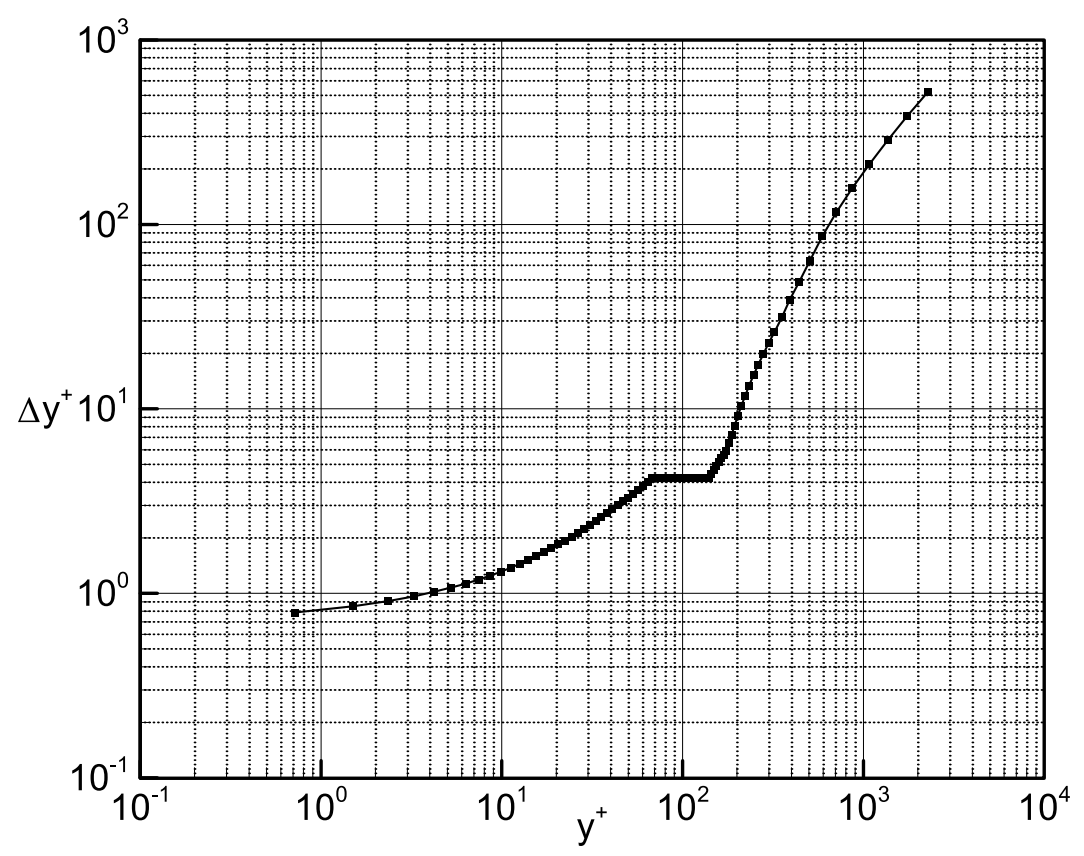

Figure 6.4: Wall-normal node distribution. Reprinted with the permission of Springer Science and Business Media.

ceiling is reached. This wall-normal node distribution is shown in Figure 6.4.

The jet sub-domain is discretized with 60 nodes in the directions normal to the jet flow ( $x$ and $z$ ), spaced to place at least 20 nodes in the jet boundary layer, while the direction parallel to the jet flow is discretized with 88 nodes that are distributed to provide the highest spatial resolution near the jet orifice. The spatial resolution near the orifice is refined to about $\Delta x^{+} \approx 8$ and $\Delta z^{+} \approx 3$ to account for the smaller length scales expected in the initially-triggered jet.

\subsubsection{Solution method}

ANSYS CFX @ (Version 12), a commercial computational fluid dynamics software package, was used to solve the incompressible form of the time-varying mass- and momentumconservation equations through a finite-volume approach. This software package has been used extensively by this research group to simulate the coherent vortical structures occurring in attached and separated boundary layers and the growth of these structures as the flow 
transitions to turbulence (Roberts and Yaras, 2006; Azih et al., 2012). Excellent agreement has been achieved between these simulations and experimentally-measured quantities such as the velocity fluctuation spectra, the location of transition onset, and the streamwise rate of transition. As a transverse jet possesses several of the dominant flow features present in a transitional boundary layer (namely, flow separation and recirculation, vortical structures undergoing rapid temporal and spatial growth, and strong shear layers), the agreement that was obtained in past simulations provides confidence that similar accuracy can be obtained using ANSYS CFX $(\mathbb{R})$ in the present study. Discretization of the governing equations is based on central differencing and second-order Euler backward differencing for the spatial and temporal derivatives, respectively. To resolve the transient interaction between the jet and the main-flow boundary layer, a temporal resolution is chosen so that the jet flow takes about 10 timesteps to penetrate a distance equivalent to the displacement thickness of the main-flow boundary layer at the jet orifice, resulting in a timestep size of $\Delta t=7 \times 10^{-6} \mathrm{~s}$. The discretized equations are converged through an algebraic multigrid scheme within eight inner-loop iterations per timestep, reducing the root-mean-square residual of the governing equations by five orders of magnitude to less than $10^{-6}$. The simulations were partitioned and executed in parallel on 20 Intel $^{\mathrm{TM}}$ L5410 Xeon processors. Approximately 20,000 timesteps are required for the laminar main-flow boundary layer to reach a steady state, after which the vertical jet is impulsively turned on for $8.65 \mathrm{~ms}$ - which corresponds to 1236 timesteps - and then impulsively turned off and the simulation is continued for 6400 more timesteps.

\subsubsection{Validation of numerical results}

Validation of the numerical results through comparison with experimental data is hindered by the lack of published experiments of transverse jets in accelerating main flows. Therefore, validation of the numerical algorithm and the spatial and temporal resolutions is accomplished by performing a series of companion DNS studies of a zero-pressure-gradient turbulent 
boundary layer and comparing the results of these simulations to statistical turbulence properties of corresponding turbulent boundary layers in literature. Although a zero-pressuregradient turbulent boundary layer differs from the accelerating, laminar main flow in the present study, such a validation provides strong evidence that the present study accurately captures the flow development in the transverse jet, particularly considering that the transient coherent flow structures in the present study are expected to be of notably-greater spatial and temporal scale than in a turbulent boundary layer. Details of the companion studies and the published numerical data used for validation are given in Table 6.1.

Table 6.1: Numerical details of the simulations used for validation of the numerical results. Reprinted with the permission of Springer Science and Business Media.

\begin{tabular}{lccc}
\hline Simulation & $\Delta \mathbf{x}^{+}$ & $\Delta \mathbf{z}^{+}$ & $\mathbf{R e}_{\theta}$ \\
\hline Grid 1 & 3 & 3 & 900 \\
Grid 2 & 3 & 3 & 500 \\
Grid 3 & 22 & 11 & 500 \\
Spalart (1988) & 20 & 7 & 670,1410 \\
Wu and Moin (2009) & 6 & 11 & 900 \\
\hline
\end{tabular}

The first companion simulation (Grid 1) was performed at a Reynolds number based on the boundary-layer momentum thickness and free-stream velocity of $R e_{\theta}=900$ with a spatial resolution in the $x-z$ plane of $\Delta x^{+}=\Delta z^{+}=3$, which corresponds to about 1.5 times the Kolmogorov length scale at that Reynolds number. The $y^{+}$value of the first node from the no-slip boundary is 0.85 and the wall-normal node distribution follows closely the distribution shown in Figure 6.4. Figure 6.5 shows that the results achieve very good agreement compared with the turbulence kinetic energy production and dissipation rates and the root-mean-square streamwise velocity fluctuation $\left(u_{r m s}^{\prime}\right)$ profiles (Figures $6.5(a)$ and $(b)$, respectively) computed by Spalart (1988), who used a spectral-method algorithm to simulate the boundary layer at a Reynolds number of $R e_{\theta}=1410$. Figure $6.5(c)$ also shows excellent agreement in the mean velocity profile for $y^{+}<40$ when compared to the DNS of Wu and Moin (2009) performed at $\operatorname{Re}_{\theta}=900$, who used a finite-difference scheme with second-order accuracy in space and 

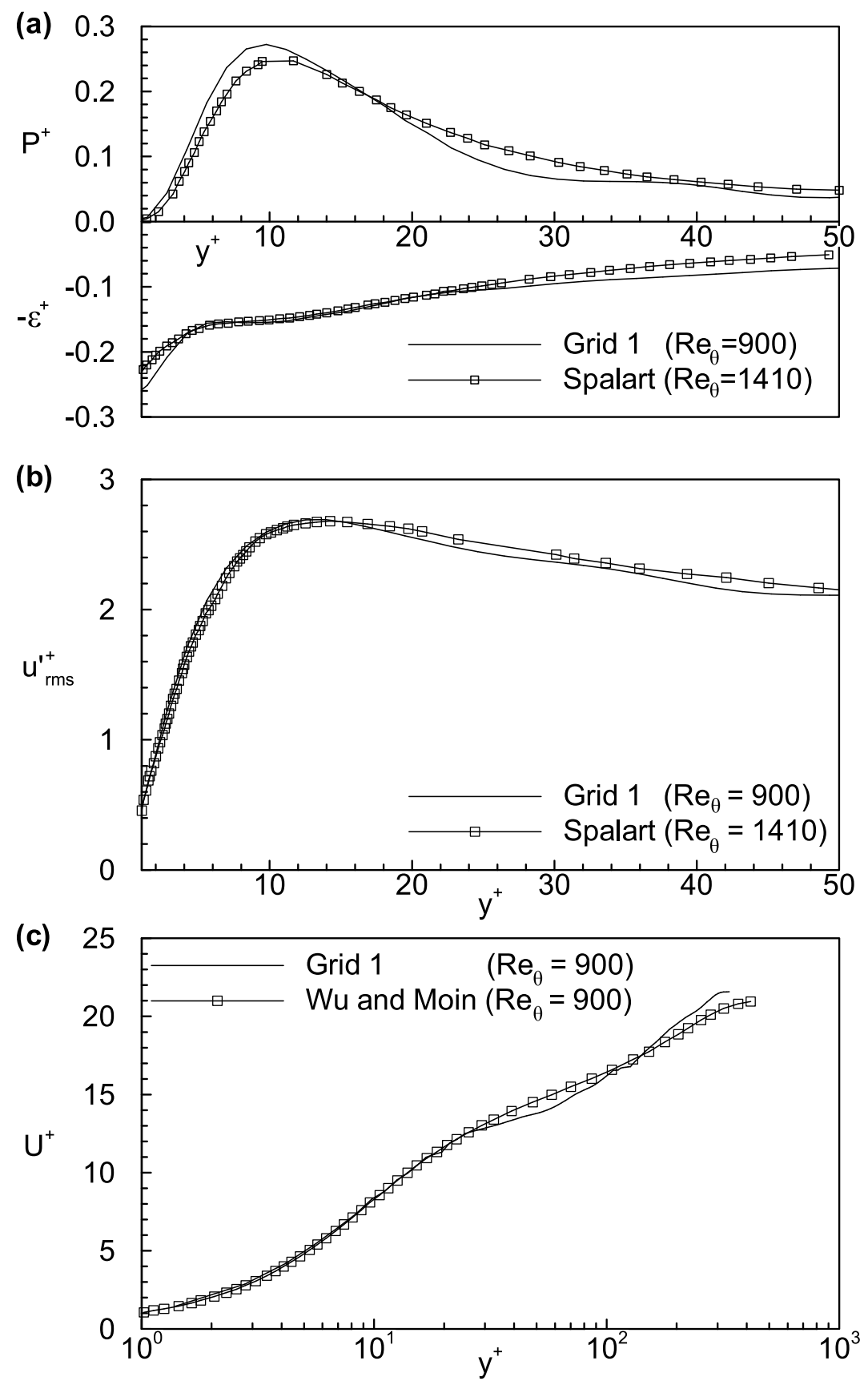

Figure 6.5: Companion DNS study of a zero-pressure-gradient turbulent boundary layer at $R e_{\theta}=900$. Results from Grid 1 are compared with the $(a)$ turbulence kinetic energy budgets and $(b)$ streamwise velocity fluctuation profiles of Spalart (1988) and (c) mean velocity profile of Wu and Moin (2009). Adapted from Azih et al. (2012). Reprinted with the permission of Springer Science and Business Media. 
a mixed second- and third-order accuracy in time. Adequate agreement was also achieved in the outer part of the boundary layer, with a deviation of less than $10 \%$ in the mean velocity profile, which was likely due to the streamwise length of the computational domain used in the companion study being insufficient to fully capture the statistical properties of the larger-scale structures in the outer region of the boundary layer.

To isolate the effect of Reynolds number on the accuracy of the numerical results, a second validation DNS (Grid 2) was performed with the same spatial resolution as Grid 1 but at $R e_{\theta}=500$, which is closer to the present study, in which the Reynolds number based on the average momentum thickness in the turbulent region triggered by the jet at its mostmature state in the simulation, averaged over the turbulent area, is $R e_{\theta}=332$. Finally, to ascertain the sensitivity of the results to the spatial grid, a third DNS (Grid 3) with a grid that closely matches the spatial resolution of the present study was performed at $R e_{\theta}=500$. The simulation results for Grids 2 and 3, shown in Figure 6.6, compare favourably with the turbulent kinetic energy budgets computed by Spalart (1988) at $R e_{\theta}=670$ and the mean velocity profile measured by Djenidi and Antonia (1993) at $R e_{\theta}=560$. Therefore, these validation studies provide strong evidence that the present study should accurately capture the flow development in the transverse jet where the transient coherent flow structures are expected to be of notably-greater spatial and temporal scale. The wall-normal node spacing and the spatial and temporal resolution of the present turbulent spot simulations closely match those of Grid 3. As per Figure 6.6, although the Grid-2 results are in slightly better agreement with the simulation data of Spalart (1988) and the experimental data of Djenidi and Antonia (1993) than the Grid-3 results, the resolution of Grid 3 was adopted for the present transverse jet study to enable simulations with reasonable computing times while still ensuring that the flow physics are accurately captured. 

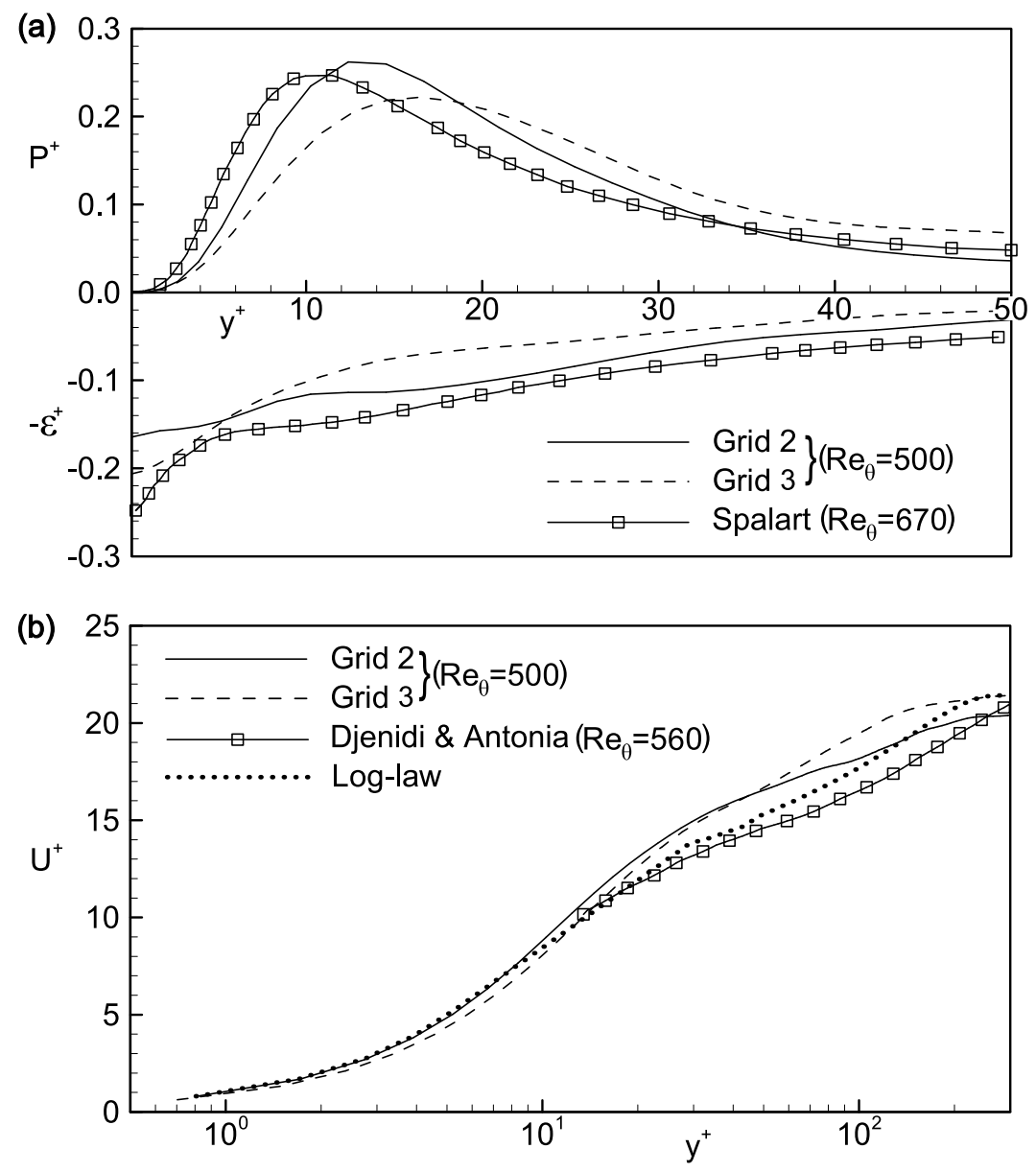

Figure 6.6: Companion DNS studies in a zero-pressure-gradient turbulent boundary layer at $R e_{\theta}=500$. Results from Grid 2 and Grid 3 are compared with the (a) turbulence kinetic energy budgets of Spalart (1988) and (b) mean velocity profile of Djenidi and Antonia (1993). Adapted from Brinkerhoff and Yaras (2011). Reprinted with the permission of Springer Science and Business Media. 


\subsection{Results and Discussion}

To enhance clarity in the present discussion of the simulation results, the directions "upwind" and "downwind" are defined with respect to the main-flow direction, while "upstream"

and "downstream" are defined with respect to the jet-flow direction. Spatial coordinates are normalized by the jet width, $D$, velocities are normalized by the jet velocity at the center of the orifice, $v_{j e t}$, and time is normalized by the ratio $D / v_{j e t}$; the normalized time is hereafter denoted by $\tau$. The values of these parameters are given in Table 6.2. The subscript "0" denotes main-flow conditions at the center of the orifice $(y / D=16$ for free-stream values) in the absence of the jet. The subscript "jet" denotes the jet-flow conditions at the center of the orifice once the jet has reached a steady velocity.

\subsubsection{Development of the main and jet flows}

The discussion of the simulation results begins with a description of the main flow in the absence of the jet and the steady features of the flow within the jet-pipe. A favourable streamwise pressure-gradient is imposed on the main-flow test surface by the sloped ceiling. Figure 6.7 plots the streamwise distribution of the velocity magnitude $\left(U / v_{j e t}\right)$ at a wallnormal height of $y / D=16$ in the absence of the jet. The magnitude of free-stream acceleration is also shown by plotting the distribution of the free-stream acceleration parameter, $\eta=\nu / U_{e}^{2} d U_{e} / d x$, where $U_{e}$ is the local boundary-layer edge velocity. The two horizontal lines in Figure 6.7 identify the critical range of the acceleration parameter, beyond which several researchers have observed that a turbulent boundary layer will begin to relaminarize (Jones and Launder, 1972; Moretti and Kays, 1965; Escudier et al., 1998). Figure 6.7 shows that the flow downwind of the location where the jet is produced experiences approximatelyconstant acceleration at a level that is well above the critical value for relaminarization. It was expected that by stabilizing the main-flow boundary layer to this extent, structures created 
Table 6.2: Flow conditions and numerical details of the simulation. Reprinted with the permission of Springer Science and Business Media.

\begin{tabular}{lc}
\hline Number of grid nodes & $10.7 \times 10^{6}$ \\
Jet width, $D$ & $2.20 \mathrm{~mm}$ \\
Jet velocity, $v_{\text {jet }}$ & $44.0 \mathrm{~m} / \mathrm{s}$ \\
Reference velocity, $U_{0}$ & $4.50 \mathrm{~m} / \mathrm{s}$ \\
Velocity ratio, $r=v_{\text {jet }} / U_{0}$ & 9.78 \\
Jet Reynolds number, $R e_{\text {jet }}=v_{\text {jet }} D / \nu$ & 6330 \\
Main-flow displacement thickness at jet orifice, $\delta_{0}^{*} / D$ & 0.63 \\
Normalized time step, $\Delta \tau=\Delta t /\left(D / v_{\text {jet }}\right)$ & 0.14 \\
Jet duration, $\tau_{j e t}$ & 173 \\
Kinematic viscosity, $\nu$ & $1.53 \times 10^{-5} \mathrm{~m}^{2} / \mathrm{s}$ \\
Density, $\rho$ & $1.225 \mathrm{~kg} / \mathrm{m}^{3}$ \\
\hline
\end{tabular}

by the interaction of the main-flow with the jet would be more organized and their transient development would occur more slowly and thus could be studied more precisely. Figure 6.7 also plots the steady boundary-layer displacement thickness that was allowed to reach a steadystate prior to activation of the jet. The boundary-layer displacement thickness downstream of the jet is approximately constant, indicating that the growth of the main-flow boundary layer is hindered by the strong streamwise acceleration.

Once the main flow has developed to a steady state, the vertical jet is impulsively turned on for a specified duration and then impulsively turned off. The time history of the jet (presented earlier in Figure 6.3) shows that an initial transient period lasting $\tau=33$ time units is required for the jet velocity at the center of the jet orifice to reach a steady value, after which the jet velocity ratio remained constant at $r=9.78$ for a period of $\tau_{\text {avg }}=140$. Here, the subscript "avg" indicates that this time period is used to calculate the time-averaged flowfield. The variation of the velocity components along the length of the jet pipe is shown in Figure 6.8. The increasing $v$-velocity component along the length of the jet occurs due to the reduction in the effective flow area that results from the growth of the jet boundary layer along the walls of the jet pipe. The increase in the $u$-velocity component near $y / D=0.0$ is due to the upstream influence of the non-uniform static pressure field that is projected onto the test surface by the 


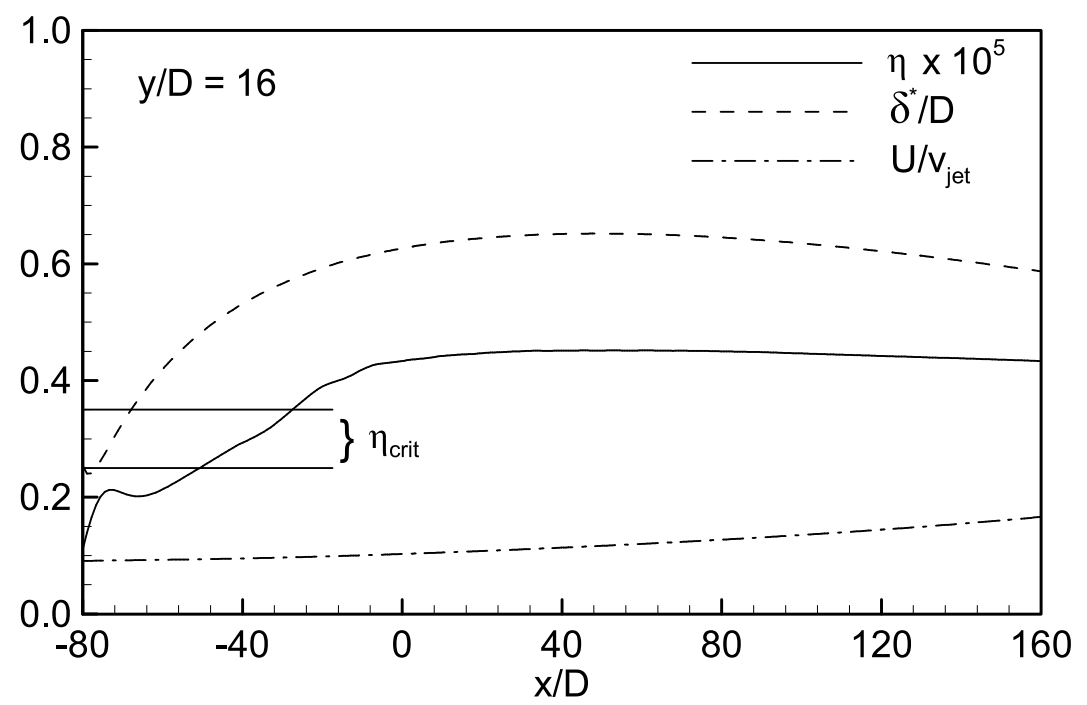

Figure 6.7: Streamwise distribution of acceleration parameter $(\eta)$, displacement thickness $\left(\delta^{*} / D\right)$, and free-stream velocity $\left(U / v_{j e t}\right)$ in the absence of the transverse jet. Reprinted with the permission of Springer Science and Business Media.

main flow as it travels around the jet: main-flow streamlines near the center of the test surface stagnate on the upwind side of the jet, creating a local high-pressure region that propagates into the jet pipe, pushing the jet fluid in the downwind (positive $u$ ) direction. Figure 6.8 shows that the upstream influence of this pressure field extends about one jet-width below the orifice, which confirms that the chosen jet-pipe length of $10 D$ with a uniformly-distributed axial inlet flow was conservatively sized.

To assess the unsteadiness of the flow within the jet pipe, the root-mean-square (RMS) of the $v$-component of the jet velocity is calculated over the time-period spanned by $\tau_{\text {avg }}$. Figure 6.9 plots this result normalized by $v_{j e t}$ at the orifice plane along a spanwise line at $x / D=0.0$ and a streamwise line at $z / D=0.0$. In both directions, the unsteadiness is highest within the jet boundary-layer due to the strong spatial gradient in the $v$-velocity component and the low momentum of the jet fluid. The results along the $x / D=0.0$ line indicate that the fluctuations have approximately the same amplitude on the left and right sides of the jet, while the results along the streamwise line $z / D=0.0$ show that the fluctuation amplitude 


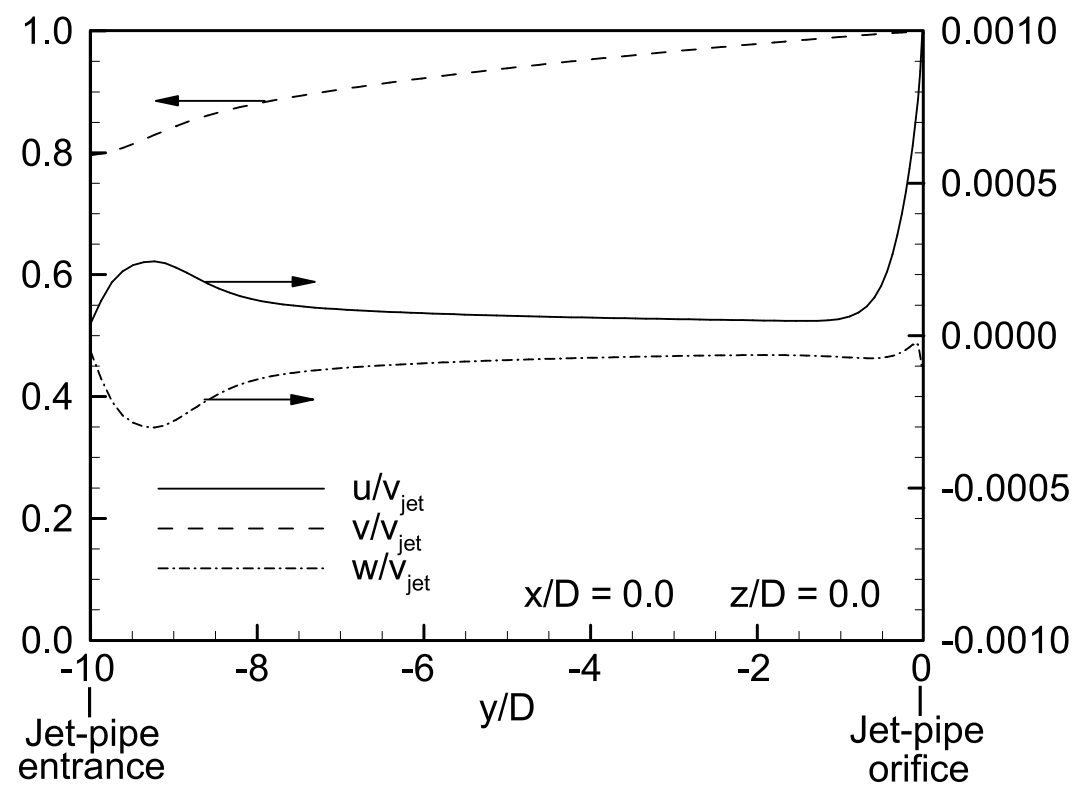

Figure 6.8: Variation in the jet velocity components along the jet centerline. Reprinted with the permission of Springer Science and Business Media.

is stronger on the downwind face of the jet than the upwind face. This trend agrees with the expectation that the near-wall flow downwind of the jet will be more unsteady due to the vortical structures that are created as the jet-flow reorients vorticity from the main-flow boundary layer. The fluctuations created by these vortical structures induce pressure pulses that propagate into the jet pipe, leading to the unsteadiness observed in Figure 6.9.

Figure 6.10 plots the power spectral density (PSD) of the $v$-velocity fluctuation along the $z / D=0.0$ line in Figure 6.9. The spectra is calculated near the upwind and downwind walls of the jet pipe and at the center of the jet orifice. The spectra for all three locations show a broad frequency spectrum, with the power at both near-wall locations $(x / D= \pm 0.5)$ being about four orders of magnitude larger than at the orifice center. It is notable that the power spectra at the upwind and downwind sides of the jet are very similar. In contrast, previous studies observed much stronger fluctuations in the near-wall flow on the upwind side, attributed to the horseshoe vortex system created on the test surface upwind of the jet, which couples with the flow in the jet pipe and produces periodic oscillations of the jet boundary layer on the upwind side of 


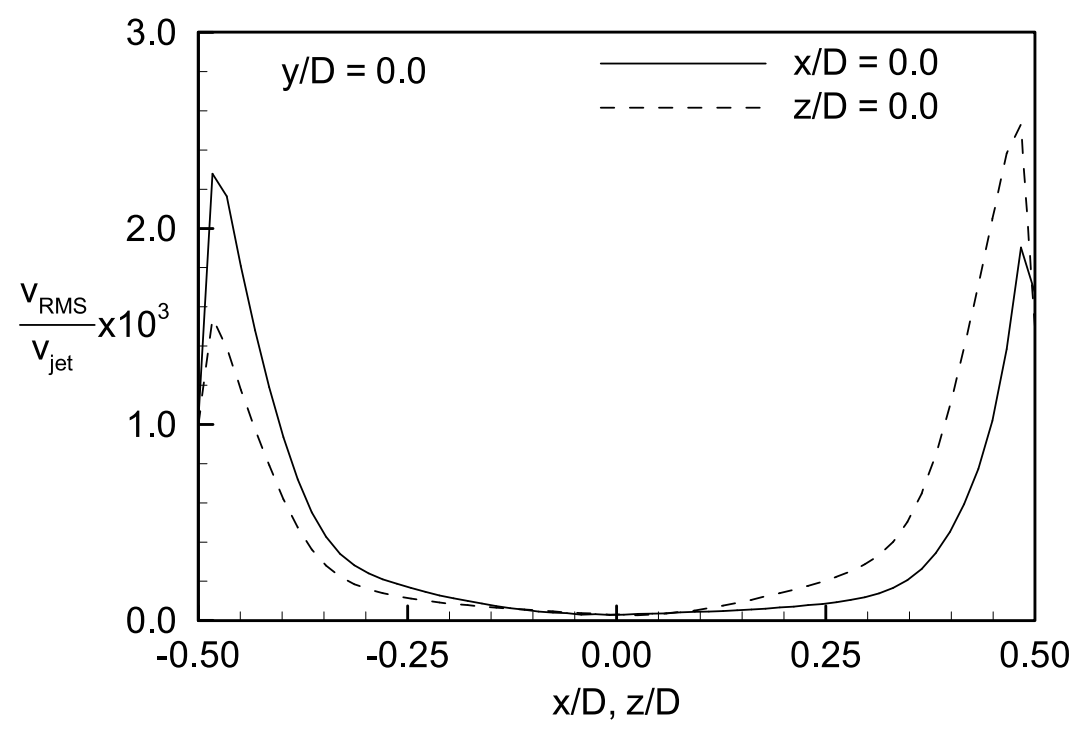

Figure 6.9: Normalized root-mean-square of $y$-component of the jet velocity along $x / D=0.0$ and $z / D=0.0$. Reprinted with the permission of Springer Science and Business Media.

the jet (Krothapalli et al., 1990; Kelso and Smits, 1995). Krothapalli et al. (1990) observed that for jet velocity ratios greater than 10 (which is comparable to the current study), the Strouhal number associated with these oscillations is approximately $S t_{0}=f D / U_{0}=0.10$, which translates to a frequency of about $200 \mathrm{~Hz}$ based on the conditions in the present study. This frequency is comparable to the lowest resolved frequency of approximately $240 \mathrm{~Hz}$ dictated by the duration of the present simulation. Recognizing that the horseshoe vortex is created by the separation of the main-flow boundary layer due to the local adverse pressure gradient that is created on the test surface upwind of the jet (Krothapalli et al., 1990; Kelso and Smits, 1995; Rivero et al., 2001; Sau et al., 2004), because the main-flow boundary layer is stabilized by the strong free-stream acceleration in the present instance, the degree of flow separation-and thus the strength of the horseshoe vortex - may be reduced. These observations may explain the similarity in the frequency spectra on the upwind and downwind sides of the jet. 


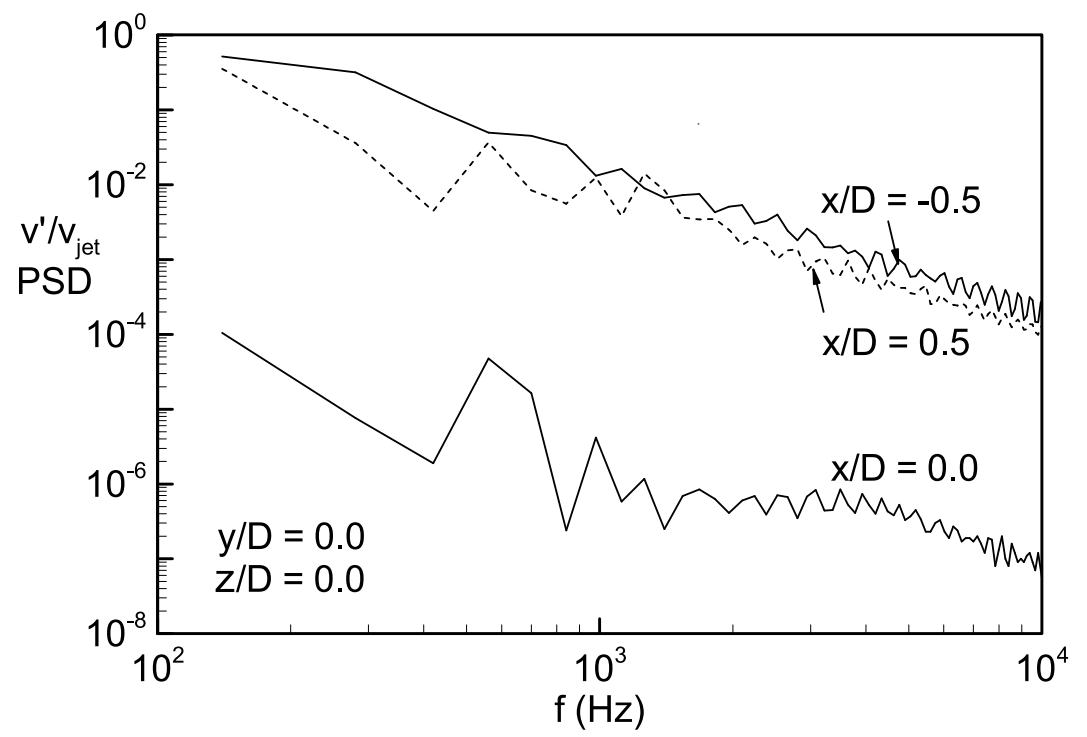

Figure 6.10: Power spectral density of the normalized $v$-component velocity fluctuations at the orifice plane near the upwind side of the jet pipe $(x / D=-0.5)$, at the orifice center $(x / D=0.0)$, and near the downwind side of the jet pipe $(x / D=0.5)$. Reprinted with the permission of Springer Science and Business Media.

\subsubsection{Interaction of the main flow and the jet}

\subsubsection{Time-averaged flowfield}

Understanding the origin of the unsteadiness observed in Figures 6.9 and 6.10 requires an analysis of the interaction between the jet and main flows. As the jet flow is ejected from the orifice, it penetrates through the main-flow boundary layer and into the free-stream. To the main-flow fluid in the free-stream, the transverse jet resembles a bluff body, with the important difference that the jet lacks a solid surface. Accordingly, as the main flow deflects around the jet, the local acceleration and deceleration of the free-stream fluid along the upwind and downwind sides of the jet creates pressure variations that deform the jet cross-section. Additionally, as net vorticity cannot be created within the flow, the main flow does not periodically shed from the jet and form a wake of vortical structures downstream as it typically would behind a solid bluff body (e.g., Fric and Roshko, 1994; Morton and Ibbetson, 1996). Rather, the free-stream 
fluid closes around the rear of the jet, strengthening the locally-adverse streamwise pressure gradient downwind of the jet. This sequence is shown in Figure 6.11 by plotting time-averaged streamlines and pressure coefficient contours $\left(\overline{C p}=2 \bar{p} / \rho v_{\text {jet }}^{2}\right)$ at several wall-normal planes within the main-flow boundary layer and free-stream. The projection of the jet orifice location is shown on the wall-normal planes by a black square. The $y / D=1.8$ plane, which is located well into the free-stream, shows streamlines deflecting around the sides of the jet and then closing tightly behind the rear of the jet, producing a non-uniform static pressure field as shown by the $C_{p}$ contours. As this non-uniform pressure field is projected onto the wall of the test surface, it promotes the separation of the main-flow boundary layer. This is clearly seen in the $y / D=0.023$ plane: streamlines near the midspan of the test surface deflect around the front of the jet and converge at two points near the upwind corners of the orifice, while streamlines further from the midspan deflect around the jet and converge at two points near the downwind corners of the orifice. The points to which the streamlines converge are the locations where the boundary layer separates from the wall and is entrained vertically into the transverse jet flow. Plane $y / D=0.167$ shows that further from the wall, the separated fluid joins vertically-oriented vortices that originate near the corners of the jet orifice. These vortices will be termed "corner vortices" and it will be shown later that they originate from the vorticity introduced from the jet boundary layer. Further away from the wall in plane $y / D=0.905$, which is located near the edge of the undisturbed main-flow boundary layer, the upwind corner vortices begin to bend downwind due to the increased momentum of the main flow. The sense of rotation of the upwind corner vortices is such that they induce a velocity on the downwind pair that brings the downwind pair closer together. The downwind pair are also elongated in the streamwise direction. By the $y / D=1.8$ plane, the downwind vortex pair appears to have merged with the upwind pair, evidenced by the single pair of lowpressure regions and rotating streamlines; it will be shown later that the corner vortices have not actually merged but the downwind pair undergoes a transient development that results in its apparent disappearance from the time-averaged flowfield. 

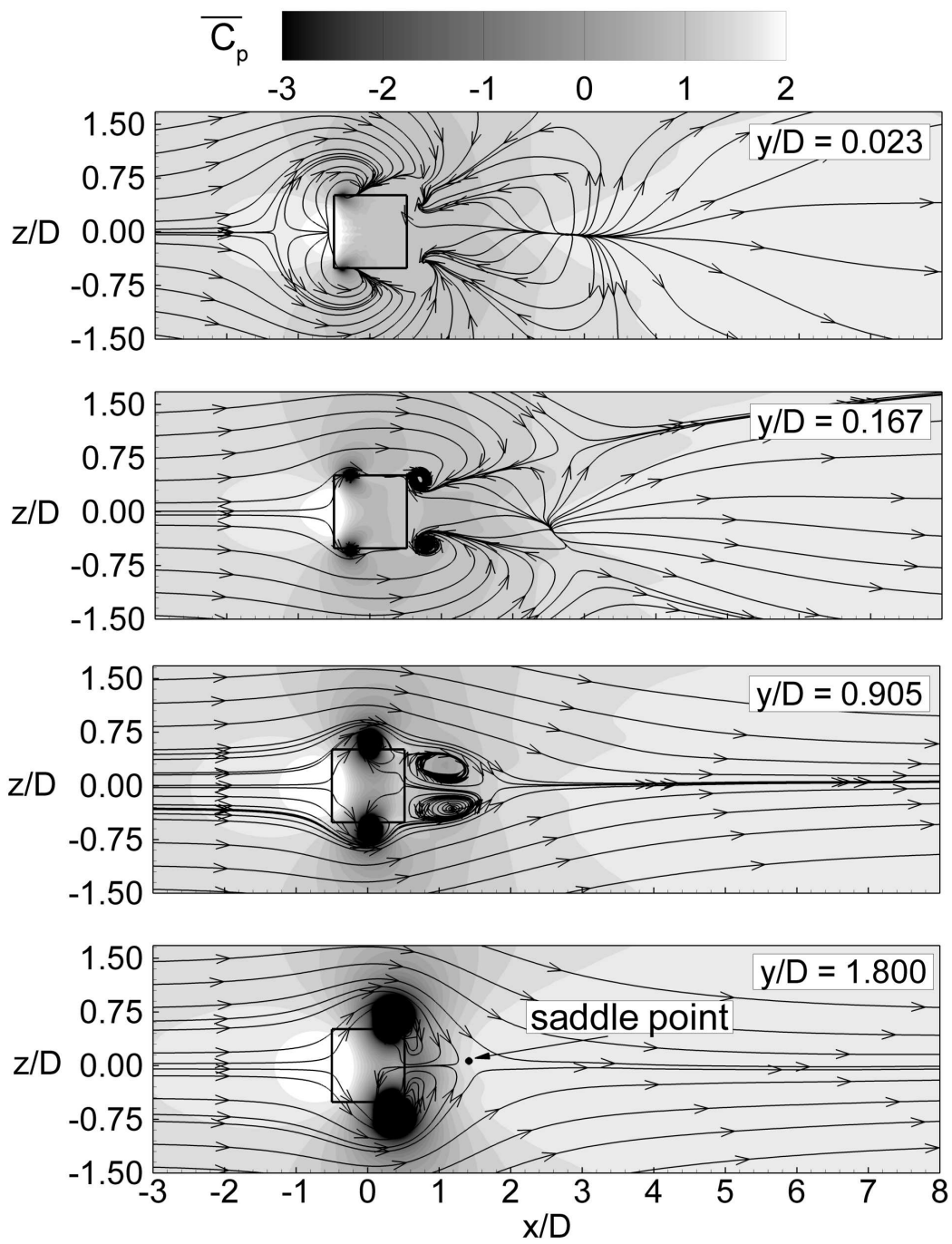

Figure 6.11: Wall-normal development of the jet flowfield, shown through time-averaged streamlines and pressure coefficient contours. Reprinted with the permission of Springer Science and Business Media. 


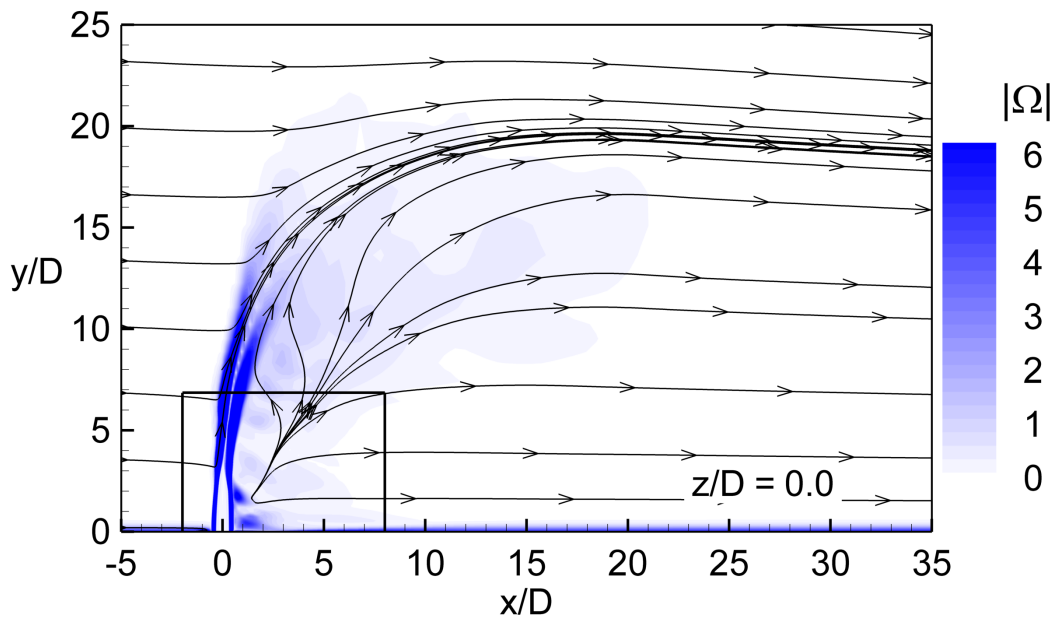

Figure 6.12: Time-averaged flowfield in a streamwise plane passing through the center of the transverse jet, visualized through streamlines and contours of normalized vorticity magnitude $|\Omega|$. The boxed region in the near-field of the jet is magnified in Figure 6.13. Reprinted with the permission of Springer Science and Business Media.

The overall outcome of the interaction between the jet and main flows can be seen through the mixing of the jet and main-flow fluid and in the trajectory of the ejected jet. Figure 6.12 plots the time-averaged flowfield in the streamwise plane $z / D=0.0$ which passes through the center of the orifice and is coplanar with the jet axis. The contours represent the time-averaged vorticity magnitude normalized by $v_{j e t} / D$, denoted $|\Omega|$. The far-field features of the flow are clearly visible: the jet penetrates into the main flow to a wall-normal height of slightly more than 20 jet widths, and mixing of the jet fluid with the main flow (observed through diffusion of jet vorticity) is completed within a similar streamwise distance downwind of the jet. The main flow streamlines on the upwind side of the jet either stagnate on the test surface if they are located near the wall or are entrained upwards into the jet. Similarly, the main flow downwind of the jet is entrained and reoriented upwards due to the separation of the main-flow boundary layer and growth of corner vortices, as described earlier. The boxed region in Figure 6.12 is magnified in Figure 6.13 to highlight the flow close to the jet. The contours of vorticity magnitude show that the main-flow boundary layer separates from the wall slightly upwind of the jet (at about $x / D=-1.5$ ) and rolls up into a small region of concentrated vorticity, labeled 


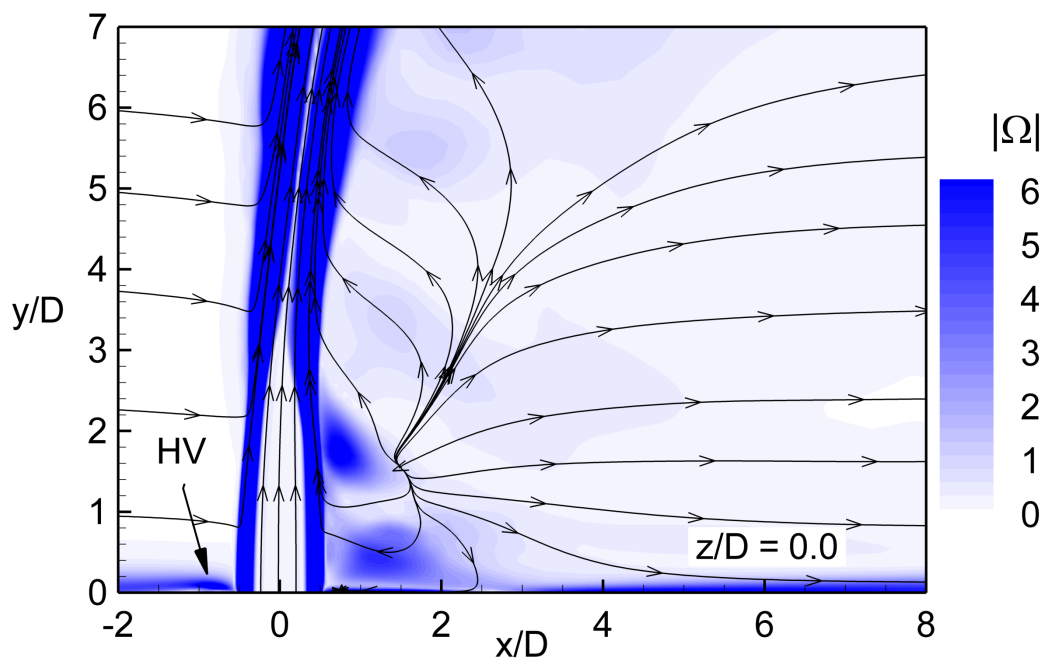

Figure 6.13: Magnified view of the near-field flow of the transverse jet. The field of view corresponds to the boxed region from Figure 6.12. Reprinted with the permission of Springer Science and Business Media.

HV in Figure 6.13. This structure is identified as a weak horseshoe vortex that forms due to the locally-adverse pressure gradient upwind of the jet. The darkly-coloured, vertically-oriented vorticity contours show the time-averaged evolution of the jet shear layer. In comparison to the main-flow boundary layer, the jet shear layer contains much higher shear and is substantially thicker, which may result in the jet shear layer dominating the interaction of the two shear layers. Downwind of the jet, the streamlines pointing upwind and towards the wall indicate that reversed flow exists for a substantial distance downwind of the jet and the main-flow boundary layer does not reattach in a time-averaged sense until about $x / D=5$. The two concentrated regions of vorticity that are located near $y / D=0.5$ and 2.0 and about one jet width downwind of the jet are structures that were likely created through the reorientation of the main-flow vorticity that would normally lie between $x / D=1.0$ and 4.0. Features such as these that remain visible in the time-averaged flow are assumed to have a long lifetime in comparison to the time-averaging period. Their physical significance becomes clear in the context of the transient development of the jet, which will be described shortly.

In several early studies of transverse jets, scaling laws for the jet trajectory-which is defined 
here as the locus of maximum jet velocity-were the primary objective (Ruggeri et al., 1950; Keffer and Baines, 1963; Margason, 1993). Although a universal scaling law is yet to be established, several studies have adopted the form

$$
\frac{y}{r D}=A r^{B}\left(\frac{x}{r D}\right)^{C}
$$

where $r=v_{\text {jet }} / U_{0}$ is the velocity ratio and the values of the constants from published literature are given in Table 6.3 (Margason, 1993; Muppidi and Mahesh, 2005; Karagozian, 2010). The studies from which Equation 6.3 was developed involved a main flow with a nominally zero streamwise pressure gradient. To analyze the effect of the strongly-favourable streamwise pressure gradient applied in the present study, a scaling law in the form given by Equation 6.3 was fitted to the time-averaged jet trajectory obtained from the simulation results. The coefficients were allowed to vary in order to obtain the best match to the simulated jet trajectory, and the resulting coefficients are shown in Table 6.3 next to the values found in published literature. Figure 6.14 plots the resulting scaling law along with the simulated trajectory, defined as the locus of maximum time-averaged velocity magnitude, showing that in the near-field of the jet, the form given by Equation 6.3 provides a reasonable match to the simulation results. It should be noted that the agreement observed in Figure 6.14 deteriorates in the far-field region downwind of the jet because the sloped ceiling in the present study also reorients the free-stream streamlines, pointing them slightly downwards; this trend is visible in the top-right corner of Figure 6.12. Nonetheless, by comparing the coefficients obtained in the current study to the values in published literature, only $A$ falls out of the range of published values. The relatively lower value of $A$ in the present study is consistent with the higher rate of streamwise acceleration of the free-stream. This result seems to suggest that a favourable streamwise pressure gradient does not fundamentally change the trajectory of the transverse jet. To obtain a more complete picture of the effect of a favourable streamwise pressure gradient on the flowfield, the transient development of the flow must be studied. 
Table 6.3: Jet trajectory correlation coefficients. Reprinted with the permission of Springer Science and Business Media.

\begin{tabular}{cc}
\hline Current study & Literature \\
\hline$A=0.98$ & $1.20<A<2.60$ \\
$B=0.30$ & $B=0.30$ \\
$C=0.32$ & $0.28<C<0.34$ \\
\hline
\end{tabular}

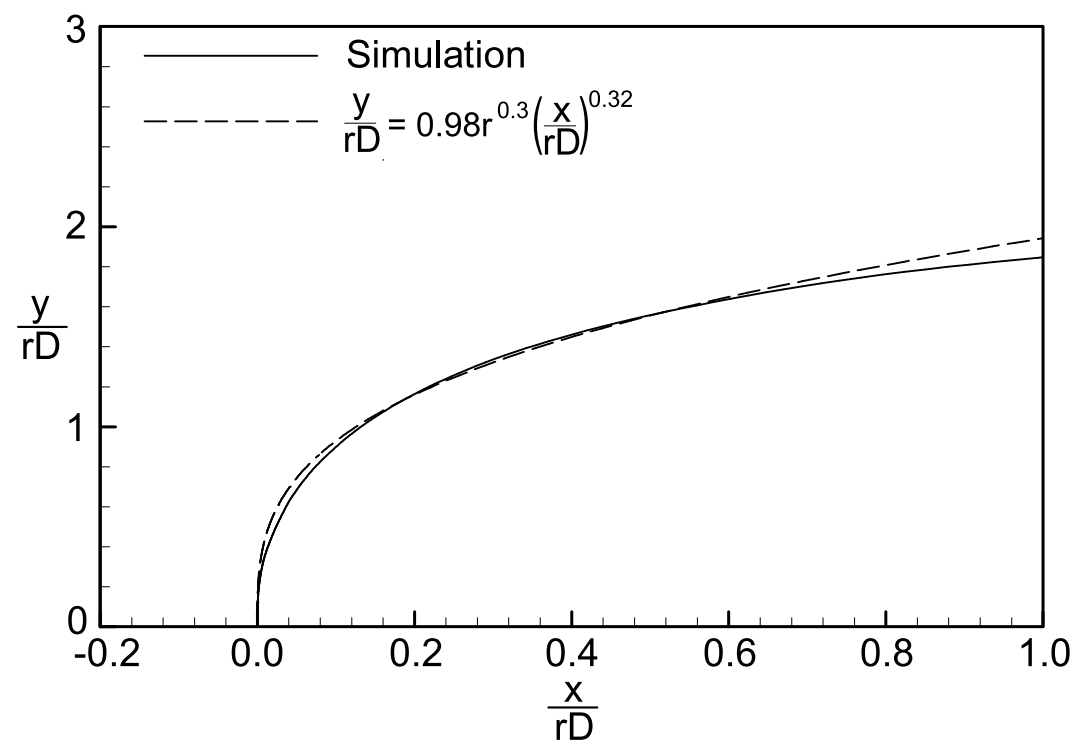

Figure 6.14: Comparison of the simulated jet trajectory and that obtained from a scaling law in the form of Equation 6.3. Reprinted with the permission of Springer Science and Business Media. 


\subsubsection{Transient flowfield}

Figure 6.15 plots streamlines and velocity magnitude contours at several instances in time on the same streamwise plane as Figures 6.12 and 6.13. The plots show that the jet trajectory continues to evolve throughout the lifetime of the jet and the far-field flow does not reach a statistically steady-state within the duration of the jet. To qualitatively assess the sensitivity of the time-averaged results to the time-averaging period, the time average was recalculated using only the data between $\tau=120$ and $\tau=172$, which is the period that shows the least change in the far-field flow. A scaling law in the form of Equation 6.3 was re-derived to fit the jet trajectory obtained from the modified time-averaged flow. Upon comparison to the original scaling law given in Table 6.3 , the $B$ and $C$ coefficients changed to $B^{\prime}=0.36$ and $C^{\prime}=0.38$, which are somewhat higher than the typical values given in the published literature.

Figure 6.15 provides insight on the initial transient development of the jet after it is impulsively activated. The flow at $\tau=1$ shows that as the jet leaves the jet pipe, the jet boundary layer that had developed within the pipe immediately rolls-up into a square-shaped vortex loop, shown by the counter-rotating spanwise vortices observed near the upwind and downwind edges of the orifice. Streamlines near the test surface show that the downwind side of the vortex loop induces a velocity on the jet flow that causes some of the jet streamlines to rotate around the vortex and flow downwind, which enhances the turning of the jet and the mixing of the jet fluid with the main-flow. Conversely, the upwind side of the vortex loop induces a velocity that causes some jet streamlines to rotate and flow upwind against the oncoming main flow. As these streamlines encounter the main flow moving in the opposite direction, the main-flow boundary layer separates and rolls up to form the horseshoe vortex, which is already visible in the near-wall streamlines upwind of the jet by $\tau=6$, labeled HV. The horseshoe vortex appears to remain stationary and does not appear to grow. In contrast, by $\tau=12$, the size of the vortex loop has increased and it has been tilted in the downwind 

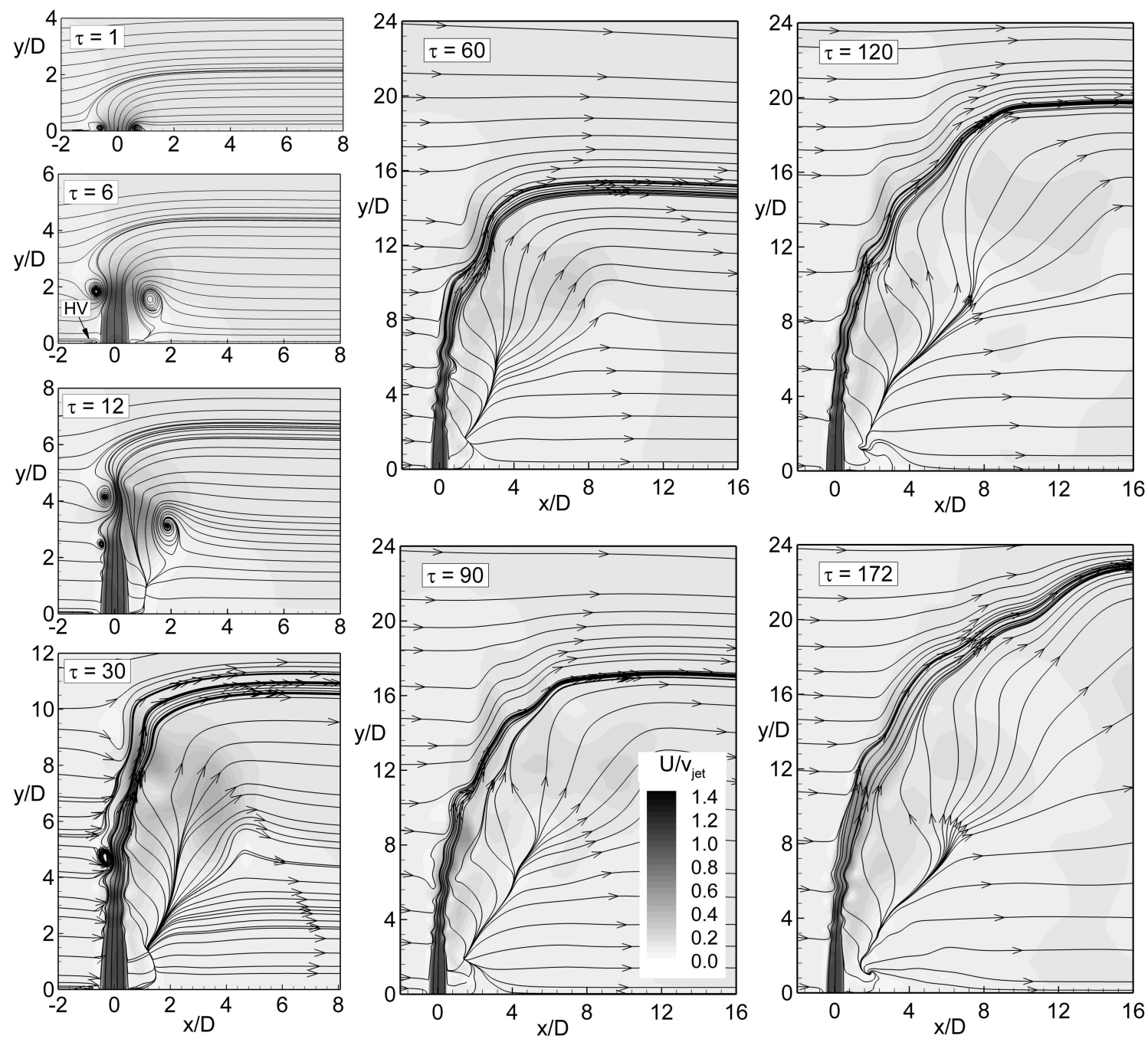

Figure 6.15: Transient development of the transverse jet shown through instantaneous streamlines and contours of $|U| / v_{\text {jet }}$ in the streamwise plane located at $z / D=0.0$. Reprinted with the permission of Springer Science and Business Media. 
direction. Sau and Mahesh (2008) suggest that the tilting is caused by the downwind side of the vortex loop entraining the main-flow fluid more effectively than the upwind side. They argued that this occurs because the adverse streamwise pressure gradient set up downwind of the jet aids in the entrainment process by driving the main flow in the wake towards the downwind side of the vortex loop. Figure 6.15 appears to agree with this explanation: large regions of reversed flow are observed downwind of the jet after about $\tau=6$, shown by the streamlines pointing upwind and away from the wall. Furthermore, by $\tau=12$, the downwind side of the vortex loop has developed a much larger cross-section than the upwind side and the streamlines show that it is being fed by fluid that was entrained upwards by the pressure field created around the jet. By $\tau=30$, the vortex loop appears to have been convected out of the near-field flow and it no longer participates in the interaction between the jet and main flow.

Figure 6.15 shows that the shear layer created at the interface between the jet and main flow becomes unstable and begins to waver at about $\tau=12$. Spanwise-oriented vortices are formed along the upwind edge of the jet and can be seen at $\tau=12$ and $\tau=30$. The wavering of the jet and the formation of shear-layer vortices continues throughout the lifetime of the jet; if streamlines were drawn at appropriate locations, the shear-layer vortices could be visualized after $\tau=30$. Numerous studies have linked these shear-layer vortices to a KelvinHelmholtz-like instability of the jet shear layer (Yuan et al., 1999; Kelso and Smits, 1995; Sau and Mahesh, 2010; Megerian et al., 2007). While the majority of literature deals with axisymmetric jets and the discussion is limited to the upwind shear layer of the jet, the square jet in the current study is non-axisymmetric and the downwind shear layer also possesses the conditions for instability. Roll-up of both shear layers is shown in Figure 6.16(a) through instantaneous contours of vorticity magnitude. Figure 6.16(b) plots the frequency spectra of $v^{\prime} / v_{\text {jet }}$ at the four points labeled in the upwind and downwind shear layers, the locations of which were chosen to capture the characteristic frequencies of the shear-layer instabilities and any sub-harmonics which may develop further into the main flow. Here, frequency is expressed 


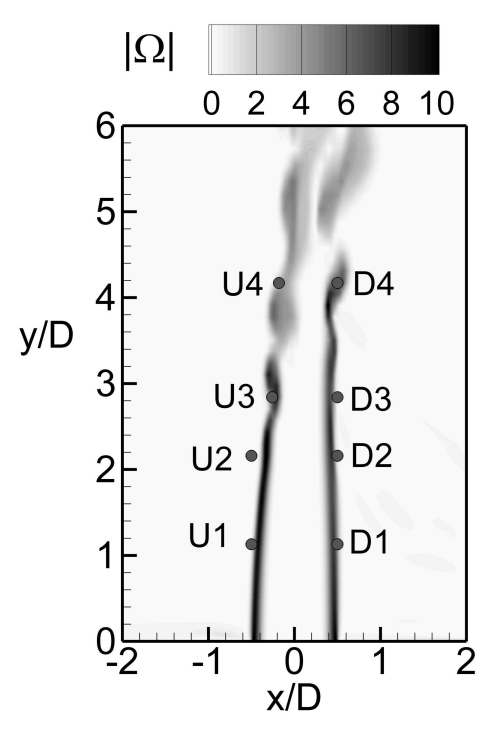

(a)

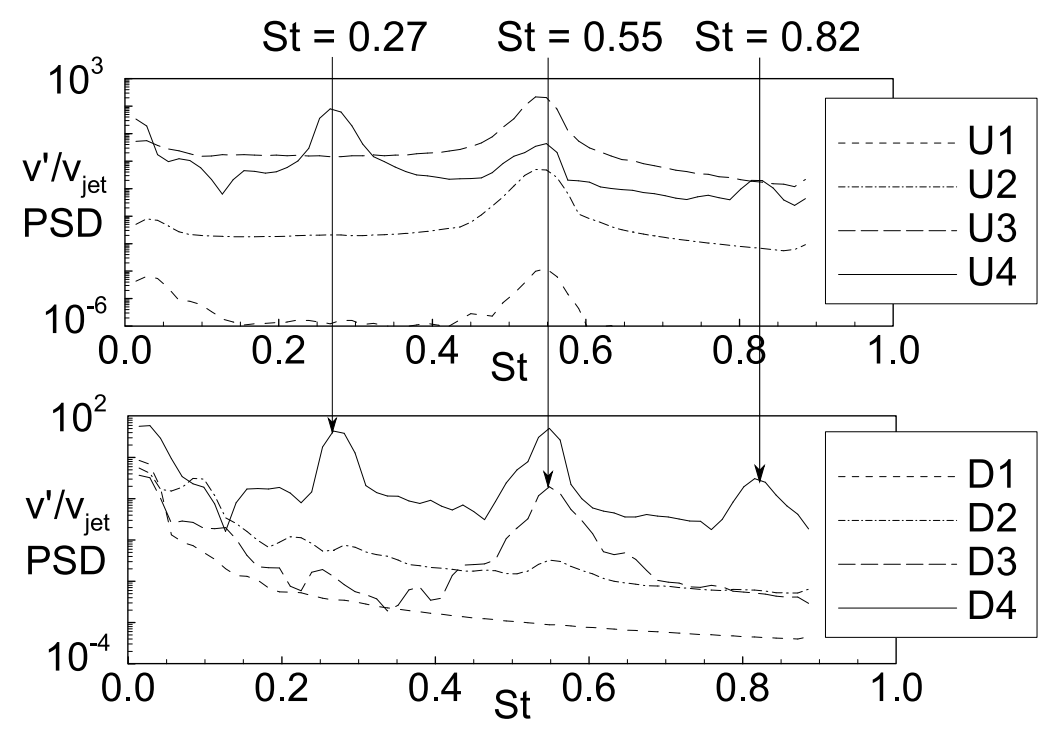

(b)

Figure 6.16: Instability of the upwind and downwind shear layers shown through $(a)$ instantaneous contours of vorticity magnitude at $\tau=90$ and (b) Strouhal-number spectra at several wall-normal locations in the upwind (U) and downwind (D) shear layers. Reprinted with the permission of Springer Science and Business Media.

as the jet Strouhal number, $S t=f D / v_{j e t}$. The spectra of the upwind shear layer shows that the growth of periodic disturbances has begun by point U1 with a distinct peak at $S t=0.55$. The disturbances grow steadily to point U3. At point U4, the characteristic frequency is less pronounced and other peaks at $S t=0.27$ and $S t=0.82$ become visible.

Downwind of the jet, vertical entrainment of the main-flow fluid increases the overall disturbance level in the downwind shear layer but also decreases the level of shear because the entrained fluid is moving roughly parallel and in the same direction as the shear layer. Because of the lower shear, the growth of periodic disturbances in the downwind shear layer is delayed until a very small peak appears at point D2 and then much more distinctly at point D3 at the same characteristic frequency as in the upwind shear layer $(S t=0.55)$. At point D4, peaks at $S t=0.27$ and $S t=0.82$ appear, as in the upwind shear layer. Noting that the high-frequency peak is much stronger in the downwind shear layer than in the upwind shear layer, and considering that the downwind shear-layer is closer to the rapidly-evolving 
vortices in the wake of the jet, the high-frequency peak is likely caused by fluctuations induced by these vortices, described in greater detail below. The origin of the $S t=0.27$ peak is less obvious; its value is close to half the characteristic frequency, suggesting that it may be a subharmonic occurring from a pairing of the shed shear-layer vortices. However, such a doubling is not observed in the transient evolution of the jet vortices. Instead, the time trace of $v^{\prime} / v_{\text {jet }}$ at points D3 and D4, shown in Figure 6.17, shows that the low-frequency component of the signal is produced by a periodic amplification of $v^{\prime}$ at point D4. Figure 6.17 also shows that the lowfrequency component of the signal is approximately in phase with the roll-up of the shear layer at the characteristic frequency. Observing from Figure 6.16a that the vortex-shedding events from the upwind and downwind shear layers are substantially out of phase, it is unlikely that the low-frequency component comes from an interaction between the upwind and downwind shear layers. The low-frequency peak is likely the result of a streamwise wavering of the shear layers in the plane of Figure 6.16a, which is the precursor to the pairing instability; such a wavering would periodically bring fluid with higher and lower vertical velocity near points U4 and D4, producing a time-signal similar to that observed in Figure 6.17. The $x$-direction gradient of the $v$-component of velocity is large at points $\mathrm{U} 4$ and D4, and even a small wavering of the shear layer would translate to notable magnitudes for the $v^{\prime}$ signal. Unfortunately, comparison of the observed frequencies with published values is difficult because of the large ranges observed in the published literature. A review by Megerian et al. (2007) tabulated Strouhal numbers based on the orifice diameter and jet centerline velocity ranging from 0.1 to 2.0 for a Reynolds numbers range of $R e_{j e t}=220-16,600$ and a velocity ratio range of $r=2-6$. Similarly, studies of free jets (e.g., Ho and Huerre, 1984) have shown that the most energetic disturbances occur near the end of the jet's potential core at Strouhal numbers based on the jet diameter and potential-core velocity of $S t=0.24-0.51$.

Returning to the developing jet flowfield shown in Figure 6.15, another observation can be made about the flow in the latter part of the jet's lifetime: vertically-oriented wake vortices 


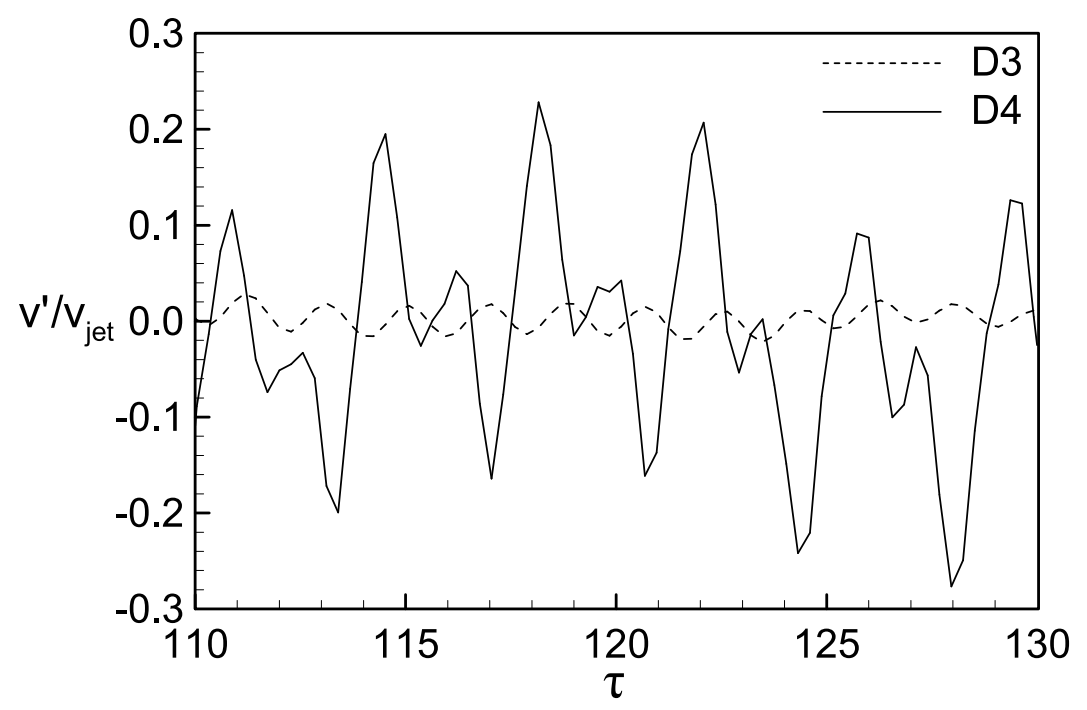

Figure 6.17: Time trace of wall-normal velocity fluctuation at locations D3 and D4 from Figure 6.16. Reprinted with the permission of Springer Science and Business Media.

that connect the jet trajectory with the wall boundary layer-which are a common feature in other studies of transverse jets (Fric and Roshko, 1994; Morton and Ibbetson, 1996; Ziefle and Kleiser, 2009) - appear to be absent in the current study. Instead, the streamline patterns after $\tau=60$ contain a nodal point near the wall, suggesting that the vortical structures downwind of the jet are confined to the near-wall region. This observation holds true even when the plane in Figure 6.15 is moved to different spanwise locations. The absence of wake vortices is a significant departure from the flowfield that is normally observed downwind of a transverse jet. Understanding the reason for this requires an in-depth study of the vortical structures in the near-field of the jet. Figures 6.18 and 6.19 show these vortical structures through iso-surface plots of the second-invariant of the velocity gradient tensor normalized by $v_{j e t} / D$, denoted by $Q$, at times that roughly correspond to the instances shown previously in Figure 6.15. The isosurfaces are coloured according to their wall-normal height in order to aid in discerning their relative proximity to the wall. For brevity in this section, the acronyms defined in Figure 6.18 will be used to refer to the coherent vortices. At $\tau=1$ the square vortex loop (VL) is ejected from the orifice and is convected upwards at a speed of about half the jet velocity due to its 
origin in the slower-moving fluid in the jet boundary-layer. By $\tau=4$, narrow upright vortices are created at the corners of the jet. These correspond to the upwind and downwind corner vortices (UCV and DCV, respectively) observed earlier in Figure 6.11. The UCV and DCV are created through the accumulation and reorientation of jet vorticity in the corners of the jet due to the velocities induced on the jet flow by the VL and the non-uniform pressure field surrounding the jet; Figure 6.11 shows a high-pressure region near the stagnation point at the middle of the jet, which would tend to drive fluid towards the lower-pressure corners. The velocity induced on the corner vortices by the VL pushes them inwards towards the highermomentum flow at the center of the jet so that by $\tau=6$, the UCV and DCV have been convected above the VL. Simultaneously, the UCV and DCV induce velocities on each other that brings the DCV pair closer while driving the UCV pair further apart. Since the vortex lines associated with these vortices cannot end in the fluid, by $\tau=9$ the top of the DCV pair are seen to connect to each other (as they are closely spaced) while the UCV pair (which are further apart) couple with the vorticity in the VL.

The shear-layer vortices (SLV) resulting from the instability of the jet shear layer become prominent at $\tau=9$. At the same time, the VL begins to tilt downwind, attributed earlier to the enhanced entrainment of main-flow fluid on the downwind side of the VL. At $\tau=12$, the VL induces a velocity on the legs of the first-shed SLV that pulls them upwards through the middle of the VL. Subsequently-shed SLVs are further away from the VL and are not influenced to the same extent, so their legs point downwards and connect to the upwind jet shear-layer. The downwards-pointing shape is consistent with the folding of the shear-layer vortices described by Kelso et al. (1996), Lim et al. (2001), and others. At $\tau=15$, the top of the UCV, the legs of the first SLV, and the downwind-tilted VL interact in a way that breaks-down the VL so that it is no longer visible by $\tau=27$. Several wavelengths of the SLV are also visible at $\tau=27$. The shedding frequency of the SLV approximated by visual inspection is $S t=0.52-0.57$, which confirms that the main peak in the frequency spectra plotted in Figure 6.16 is indeed the 


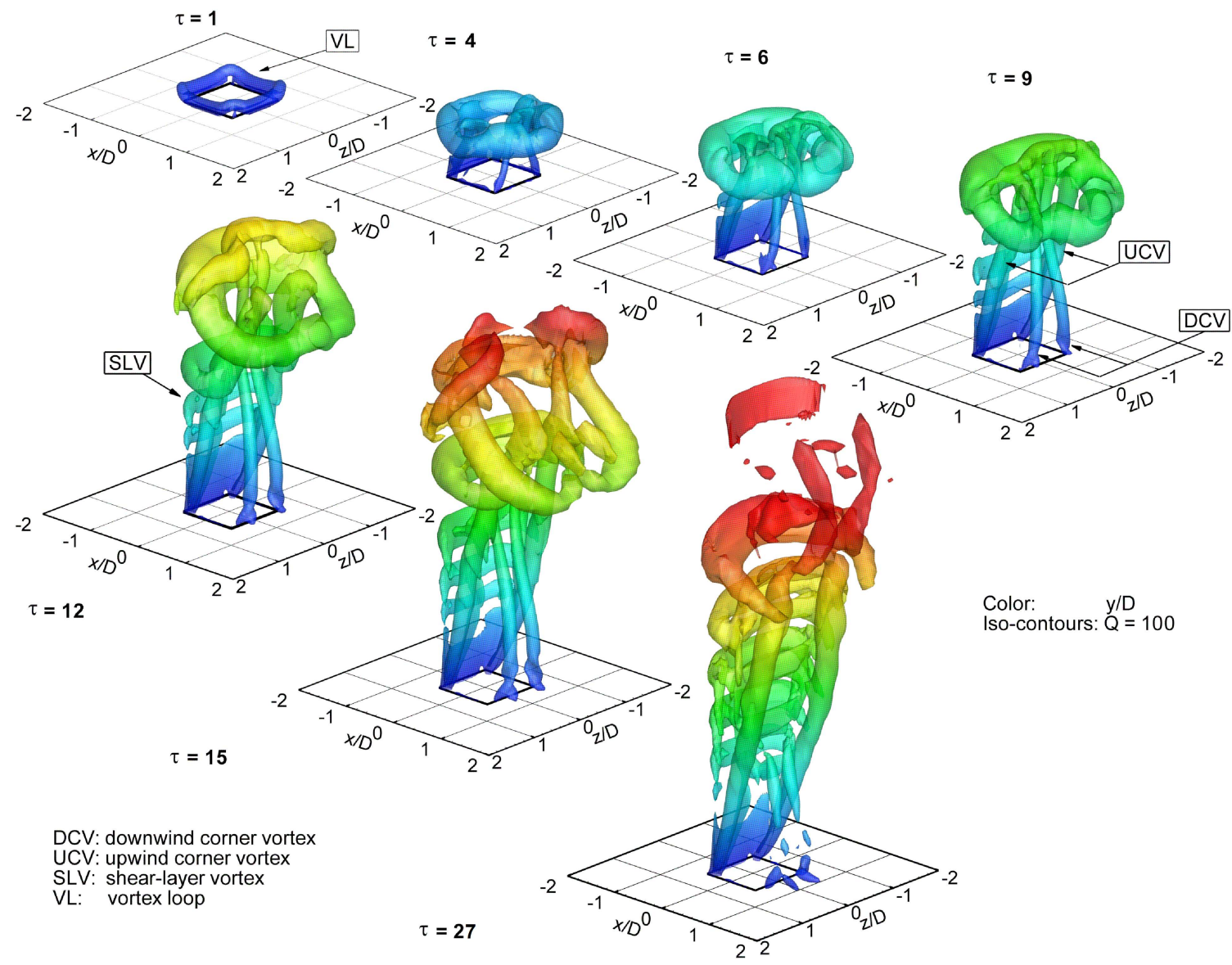

Figure 6.18: Vortical structures observed in the transverse jet during its initial transient development. Iso-surfaces are of $Q$ and the colouring is according to $y / D$. Reprinted with the permission of Springer Science and Business Media. 
characteristic frequency of the shear-layer instability. A pairing of the SLV is not observed at $\tau=27$ or at later times. As was discussed earlier, the absence of a distinct doubling in the wavelength of the SLV indicates that the low-frequency peak in Figure 6.16 is not the result of a sub-harmonic of the jet shear-layer instability.

At the same time as the SLV is developing, the UCV is convected in the vertical direction by the jet fluid and its cross-section increases as the induced velocity field entrains main-flow fluid that is brought into the rear of the jet by the adverse streamwise pressure gradient set up behind the jet. As the UCV stretches into the free-stream, it is tilted downwind by the main flow while still entraining main-flow fluid, becoming the streamwise-oriented counterrotating vortex pair (CVP) that is a common feature of transverse jets and is responsible for the majority of the mixing of the jet fluid with the main flow (Kamotani and Greber, 1972; Haven and Kurosaka, 1997).

The continued development of the vortical structures in the jet flowfield until near the end of the jet's lifetime is shown in Figure 6.19, which plots iso-surfaces of $Q$ as in Figure 6.18 but at a lower value in order to visualize the weaker structures downwind of the jet. The spatial location of the UCV remains quite steady throughout the jet lifetime, at least in the near-field regions shown in the plots. In contrast, the DCV appears to be highly unsteady. The plot at $\tau=30$ shows that a hairpin-shaped vortex loop is created downwind of the jet near the wall. This structure is created as follows: the velocity field induced by the DCV (labeled 1) causes the spanwise-oriented vorticity that is constantly being ejected from the downwind side of the orifice to be re-oriented in the streamwise direction. The reoriented streamwise vorticity then induces a velocity on the DCV that draws the bottoms of its right and left legs nearer to the orifice and to each other. Eventually, the legs of the DCV are brought close enough that they merge and connect into each other, forming a hairpin-shaped structure with its legs pointing in the downwind direction. The bending of the legs of the DCV is visible at $\tau=30$, the coupling of the legs of the DCV can be seen at $\tau=60$, and the growth and movement of the resulting 
hairpin structure is seen at $\tau=90$. The hairpin-shaped vortex remains embedded in the main-flow boundary layer and grows laterally and longitudinally as it convects downwind. At $\tau=90$, a second DCV (labeled 2) is seen forming from the vorticity that is being ejected from the jet and from the vorticity of the main-flow boundary layer behind the jet as it separates from the wall under the influence of the adverse pressure gradient. As the second-generation DCV grows, it undergoes the same process to produce a second hairpin-shaped vortex loop, which is visible in the near-wall region slightly upwind of the previously-created structure at $\tau=120$. The creation of the DCV and its subsequent evolution to form a hairpin structure repeats periodically until the fourth generation, after which the jet duration has completed and the jet flow turns off.

Figure 6.19 at $\tau=60$ shows that after the hairpin structure is created from the near-wall portion of the DCV, the two segments of the DCV that are located further away from the wall connect to each other. As it does so, it entrains main-flow fluid that is moving upwards in the rear of the jet. Since the top of the DCV is still connected to the downwind side of the jet shear layer, entrainment of vertically-moving fluid causes the now-connected legs of the DCV to rotate upwards, producing elongated structures angled at about $45^{\circ}$ to the vertical. This angle decreases (i.e. the structures become more horizontal) as the structures entrain more of the main-flow fluid while convecting away from the wall. Meanwhile, the induced velocity field produced by the elongated structures causes the leg on the $-z$ side to move towards the midspan and the leg on the $+z$ side to move underneath it so that the structures are approximately aligned one on top of the other in the vertical direction. As each generation of DCV breaks down to produce a hairpin-shaped structure, this process yielding the elongated, downwards-pointing vortices also repeats. The labels in Figure 6.19 distinguish the third- and fourth-generation structures as they are created and convected upwards in the rear of the jet.

At $\tau=172$, a DCV pair (labeled 4) is seen beginning to break down into a hairpin structure. Just above that, the structure labeled $4^{*}$ is a DCV that did not break-up into 


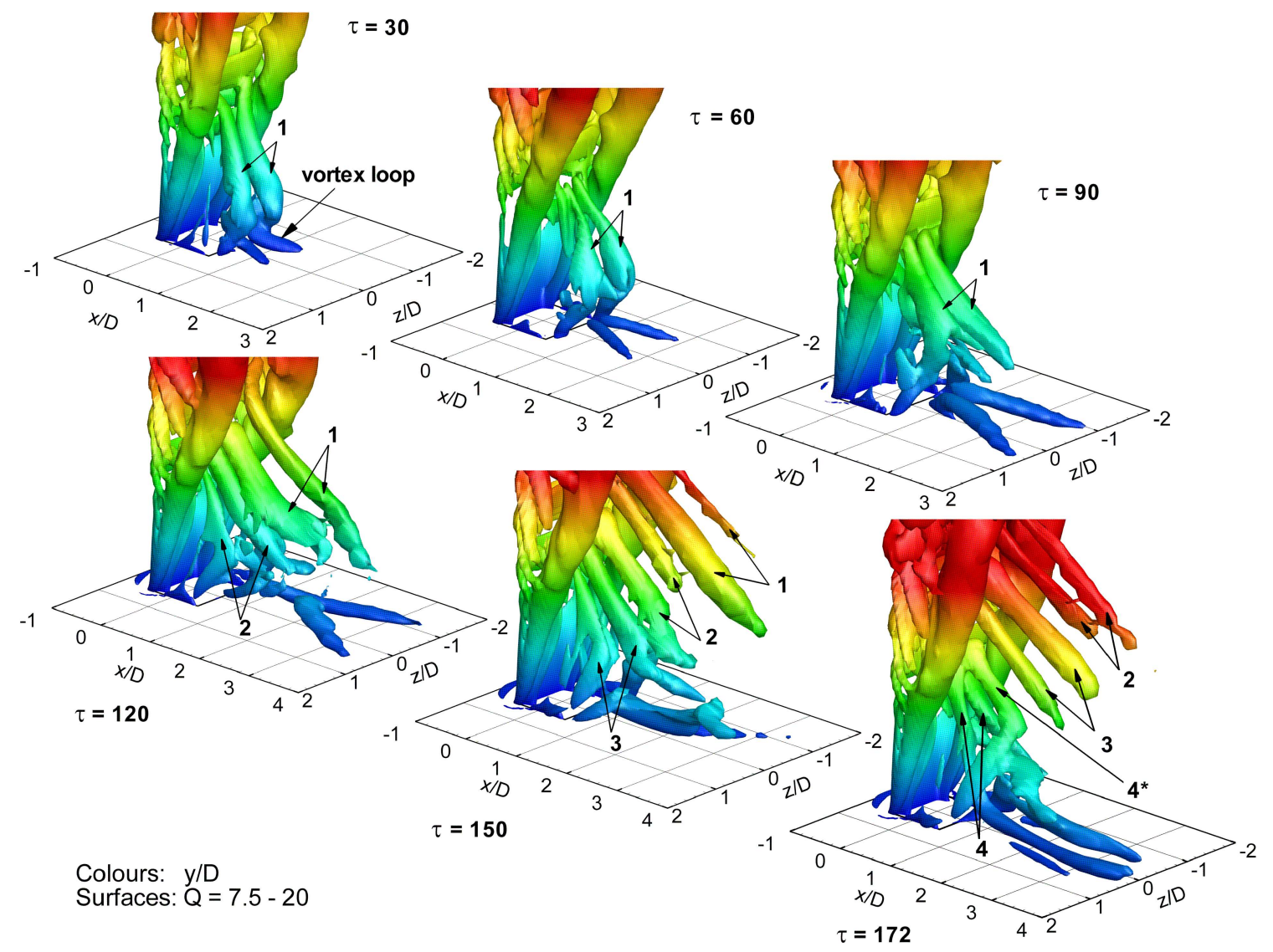

Figure 6.19: Vortical structures observed in the transverse jet. Iso-surfaces are of $Q$ and are coloured according to $y / D$. Reprinted with the permission of Springer Science and Business Media. 
a hairpin structure but remained as a single vortex with a roughly-vertical alignment. The further evolution of this structure in time cannot be studied because the jet flow was turned off shortly after $\tau=172$, but the structure initially appears similar to the upright wake vortices that are commonly observed in the wakes of transverse jets but absent in the current study. Fric and Roshko (1994) attribute wake vortices to the entrainment and reorientation of boundarylayer vorticity after the main-flow separates from the wall downwind of the jet due to the local adverse pressure gradient, which is similar to the explanation given above for the creation of the hairpin structures and downwards-pointing vortices observed in the current study. This suggests that the mechanism for the creation of wake vortices is present in the current study but the conditions are not suitable for full-sized wake vortices, like the structure labeled $4^{*}$, to be created. Again, Fric and Roshko (1994) observed that the spread in the Strouhal number of the wake vortex shedding was smallest and the spectral peak was most distinct at a velocity ratio of $r=4$; below this ratio, the jet is too close to the main-flow boundary layer and the wake vortices get mixed with other structures, while above this ratio the jet is more upright and entrainment of the separated main-flow boundary-layer vorticity into the free-stream behind the jet is more difficult. These observations suggest that the absence of wake vortices in the current study may be partially due to the relatively high velocity ratio in the current study $(r=9.78)$, but the favourable streamwise pressure gradient applied to the main flow is likely to play a role as well. free-stream acceleration not only stabilizes the vortical structures ejected from the jet but it also delays the separation of the main-flow boundary layer at the rear of the jet. By weakening separation, the flow conditions may no longer favour the creation of full-size wake vortices and instead produce the observed hairpin structures in the main-flow boundary layer and the elongated, downwards-point vortices in the free-stream.

Once the jet stops ejecting vorticity, the jet vortical structures in the main flow are swept downwind and interact with each other and the main flow so that they dissipate quite rapidly. The hairpin-like loops created in the boundary layer remain embedded in the main- 
flow boundary layer much longer and convect downstream at a fraction of the boundary-layer edge velocity. The mutual interaction and break-down of these structures to form a region of locally-turbulent flow — which appears quantitatively similar to a turbulent spot - is the subject of a subsequent paper. 


\section{Chapter 7}

Numerical Investigation of the

\section{Generation and Growth of Coherent}

\section{Flow Structures in a Triggered}

\section{Turbulent Spot}

Citation: $\quad$ Brinkerhoff, J.R. and Yaras, M.I. (2013), "Numerical investigation of the generation and growth of coherent flow structures in a triggered turbulent spot," submitted to the Journal of Fluid Mechanics. 


\subsection{Introduction}

Hairpin-like vortices have been identified as the dominant coherent flow structures in transitional and turbulent boundary layers and free shear layers (Robinson, 1991; Fiedler, 1988; Wu, 2010). To better understand the characteristics of these flows, it is important to study the creation and growth mechanisms of the hairpin vortices and the resultant wave packets containing multiples of these flow structures. A turbulent spot in isolation provides a suitable environment to study how a hairpin vortex forms in a shear layer and how the formation of one such flow structure may produce a local flow environment that promotes the creation of similar structures in sequence.

Turbulent spots are localized regions of turbulent flow within a laminar shear layer. A turbulent spot may be created by the selective amplification of disturbances in a boundary layer via the Tollmien-Schlichting instability mode, as in the first observations of turbulent spots by Emmons (1951), or be triggered artificially by, for example, localized roughness elements (Chong and Zhong, 2003), electric discharges (Wygnanski et al., 1976), or intermittent wall jets (Perry et al., 1981). Early experiments such as those by Schubauer and Klebanoff (1956) and Wygnanski et al. (1976) showed that sufficiently downstream of the location where a turbulent spot is induced, the spot characteristics, defined by mean quantities such as the streamwise convection velocity and the streamwise and spanwise spreading rates, are independent of the disturbance creating the spot. In the absence of a strong streamwise pressure gradient, a turbulent spot produced by a disturbance in the inner region of the boundary layer is shaped like a downstream-pointing arrowhead, the leading and trailing edges of which convect at approximately $88 \%$ and $55 \%$ of the local free-stream velocity, respectively (Gostelow et al., 1996). The leading edge of the spot typically appears as a turbulent region overhanging a laminar region near the wall, and the length of the overhanging region may be multiples of the local boundary-layer thickness (Wygnanski et al., 1976). A calmed region where the local 
flow exhibits increased stability relative to the local undisturbed boundary layer is typically observed in the wake of the turbulent spot (Schubauer and Klebanoff, 1956). Studies have shown that the size and shape of the spot, the leading- and trailing-edge celerities, and the size of the calmed region in the wake of the spot are highly sensitive to the streamwise pressure gradient (Katz et al., 1990; Sankaran and Antonia, 1988; D'Ovidio et al., 2001a; D'Ovidio et al., 2001b) and free-stream turbulence intensity (Yaras, 2007). Such studies have resulted in increasingly-refined engineering models that predict spot production and growth rates for a variety of free-stream conditions (Volino and Simon, 1995; Roberts and Yaras, 2005b), and are used in design optimization of advanced technology, such as the computational modelling of the transition region over highly-loaded axial gas-turbine blades (Gostelow et al., 1996). Further development of such models to improve the prediction accuracy for the location of transition onset and the length of the transition region requires a clearer understanding of the physical mechanisms responsible for the growth of turbulent spots.

Flow visualization experiments (Perry et al., 1981; Cantwell et al., 1978), hot-wire and particle-image-velocimetry measurements (Makita and Nishizawa, 2001; Chong and Zhong, 2005; Schröder and Kompenhans, 2004; Yaras, 2007; Schröder et al., 2008), and direct numerical simulation (DNS) (Henningson et al., 1987; Singer, 1996; Ovchinnikov et al., 2008; Strand and Goldstein, 2011) have identified streamwise-elongated, hairpin vortices to be the dominant coherent flow structures in the turbulent spot. The shape and organization of the hairpin vortices within the spot resemble those found in turbulent boundary layers, although the distribution of vortices in the spot is typically more orderly (Schröder et al., 2008). As the hairpin vortices grow in the wall-normal direction, they are stretched in the streamwise direction by the local mean shear. Hairpin vortices near the leading edge of the spot are stretched above a pocket of laminar fluid near the wall and produce instantaneous inflectional velocity profiles in the laminar flow pocket, which destabilizes it to rapidly-growing inviscid instability modes until this flow pocket is entrained into the turbulent spot (Henningson 


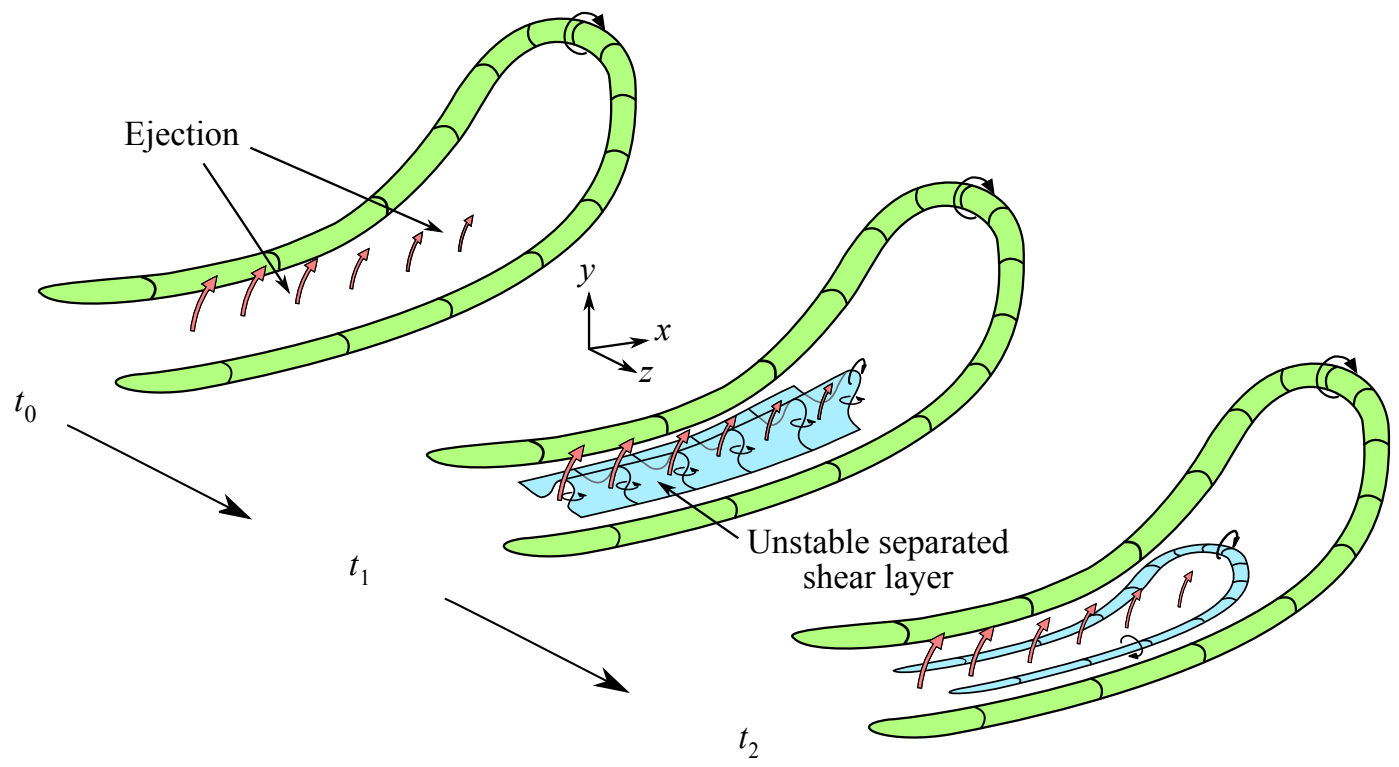

Figure 7.1: Schematic of the hairpin-vortex regeneration model proposed by Singer and Joslin (1994).

et al., 1987), thus contributing to the streamwise growth of the spot. Vortex stretching also enhances the lateral growth of the spot, because conservation of angular momentum requires vorticity in the legs of the hairpin vortices to increase as the vortices stretch in the streamwise direction. This high level of vorticity induces wall-normal velocities towards and away from the wall ("sweep" and "ejection", respectively) that create streamwise-aligned, high- and low-speed streaks within the turbulent spot. Gad-El-Hak et al. (1981) observed experimentally that the spanwise spacing of these streaks - typically about 100 wall units based on the average wall shear-stress within the turbulent spot - is approximately the same as in turbulent boundary layers (Perry et al., 1981; Cantwell et al., 1978), and that the spanwise edge of the spot is consistently terminated by a low-speed streak. Henningson and Alfredsson (1987) confirmed through linear stability analysis that the streamwise velocity profile along the spanwise edges of the spot affected by a low-speed streak is less stable than the undisturbed local laminar flow.

Considering the important role of hairpin vortices in the growth of a turbulent spot, 
understanding the mechanisms by which these structures are created and grow within a spot is important. Singer and Joslin (1994) studied the formation of hairpin vortices following the artificial-triggering of a primary hairpin vortex through DNS. The regeneration mechanism of hairpin vortices observed in their results is shown schematically in Figure 7.1. At time $t_{0}$, the wall-normal velocities induced by the legs of the primary hairpin vortex eject low-momentum fluid away from the wall between the legs of the primary vortex loop. Singer (1996) observed that the uplift of low-velocity fluid near the head of the primary vortex is weaker than near the legs, likely because the head of the primary hairpin vortex has experienced less streamwise stretching by the background shear than the legs and it resides in a region of lower background shear. Lift-up of the head of the primary vortex is a self-induced motion driven by the wallnormal velocities induced by the legs. By time $t_{1}$, the ejected low-momentum fluid causes the fluid near the wall to separate from the surface. The vorticity of the separated fluid, which was originally oriented in the spanwise direction, is reoriented in the wall-normal direction to form an unstable separated shear layer. By time $t_{2}$, the unstable separated shear layer rolls-up into streamwise- and spanwise-oriented vortices to produce the legs and head of a secondary hairpin vortex. These mechanisms are qualitatively confirmed by visualization experiments of Guo et al. (2004) and the vortex-regeneration model of Smith et al. (1991) that is based on complementary experimental and computational studies of fully-turbulent boundary layers.

Recent tomographic particle-image-velocimetry measurements of triggered turbulent spots by Schröder and Kompenhans (2004) and Schröder et al. (2008) suggest an alternative regeneration mechanism of the hairpin vortices within the spot, illustrated in Figure 7.2. Their results show that at time $t_{0}$, strong spanwise shear is created between the high- and low-speed streamwise streaks, producing shear layers of wall-normal vorticity. By time $t_{1}$, inviscid instability of this shear layer in the streamwise/spanwise $(x-y)$ plane leads to waviness in this plane that promotes streamwise grouping of wall-normal vorticity into coherent vortices oriented approximately in the wall-normal direction. By time $t_{2}$, self-induced motions cause 


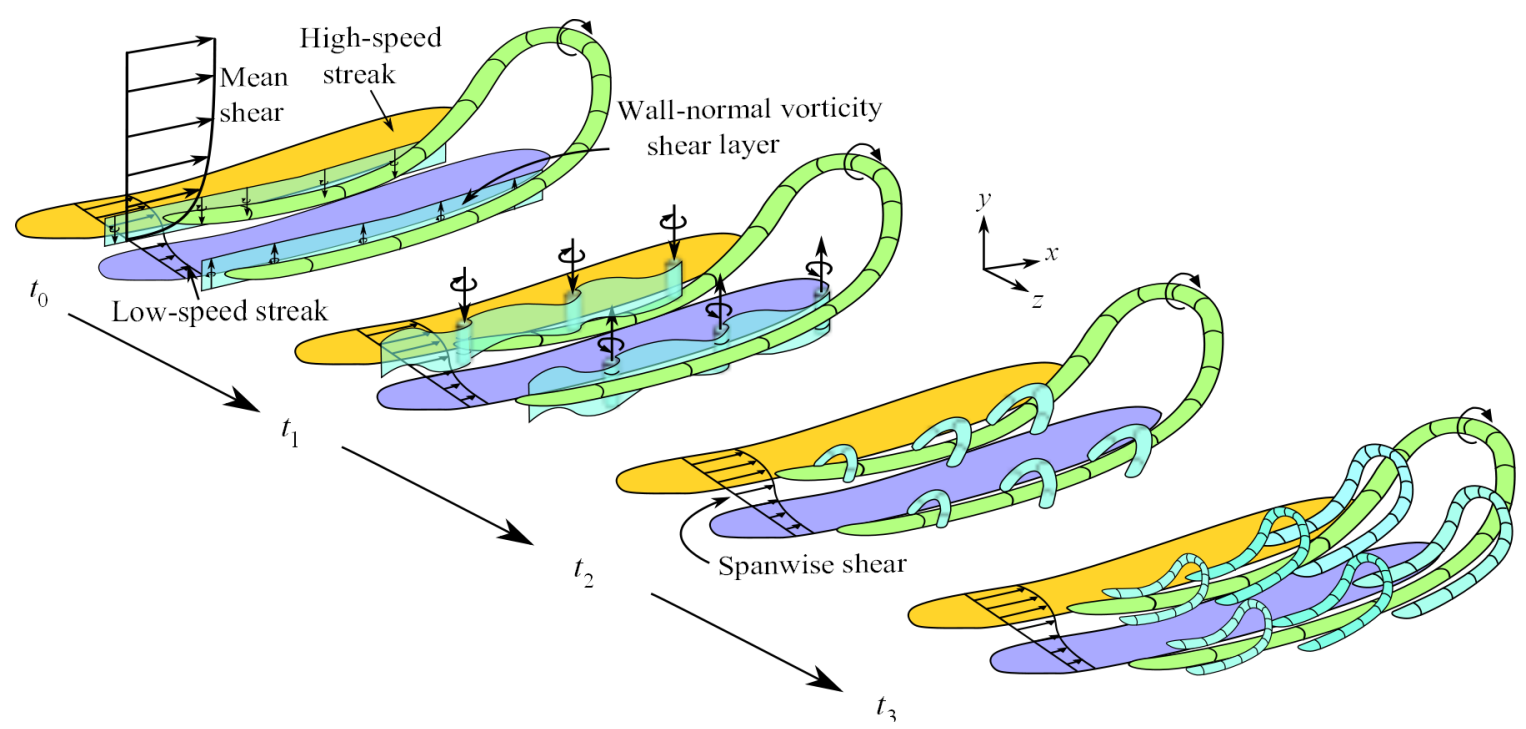

Figure 7.2: Schematic of the hairpin-vortex regeneration model proposed by Schröder and coworkers $(2004 ; 2008)$. For clarity, the high-speed streak that should appear on the $+z$ side of the primary hairpin vortex is omitted.

these vortices to fold over into arch-shaped structure that are then stretched in the streamwise direction to form secondary hairpin vortices by time $t_{3}$. This mechanism is corroborated by the DNS performed by Krishnan and Sandham (2007) of a spot triggered by a localized blowing trip, who observed that the lateral spot spreading-rate is directly proportional to the magnitude of vorticity in the shear layer between the high- and low-speed streaks.

While the regeneration mechanism proposed by Singer and Joslin (1994) implies that a secondary hairpin vortex will develop primarily between the legs of the primary hairpin vortex (Figure 7.1), the mechanism proposed by Schröder and coworkers (2004; 2008) implies that a secondary hairpin vortex will straddle a leg of the primary hairpin vortex (Figure 7.2). Furthermore, due to the variable spanwise width of the streamwise streaks, the spacing between the wall-normal-vorticity shear layers at $t_{0}$ of Figure 7.2 may vary. In cases when the wallnormal shear layers are in close proximity to each other, the wall-normal vortices at $t_{1}$ fold over towards the middle of the parent hairpin vortex and connect to each other, resulting in a secondary hairpin-vortex forming between the legs of the parent hairpin vortex, resembling 
the topology described by Singer and Joslin (1994). Published literature contains instances of turbulent spots with both kinds of hairpin-vortex topology, and it is not clear what flow conditions will favour one mechanism over the other. It is also not clear how either hairpinvortex topology would relate to the mechanism(s) of lateral growth of the spot.

It is evident that a complete explanation for the growth mechanisms of flow structures within the spot has not yet been achieved. To contribute to this understanding, the present study investigates the development of a turbulent spot that is artificially-triggered in a zeropressure-gradient laminar boundary layer by a pulsed wall-normal jet. The results of this numerical study provide a complete history of the growth of the turbulent spot, thus providing insight on the growth mechanisms of the coherent flow structures within the spot and how these mechanisms relate to the lateral and longitudinal growth of the spot.

\subsection{Numerical Method}

Accurately capturing the development of a turbulent spot in a numerical simulation requires spatial and temporal computational grids that are adequately refined to capture the spatial and temporal scales of the coherent flow structures within the spot, and a computational domain large enough to accommodate the growth of the spot into a mature turbulent region. In the interest of computational efficiency, instead of a single simulation, two smaller direct numerical simulations that are respectively optimized to capture the turbulent spot in its initial and later stages of development are performed to capture the full development history of the spot. Simulation-1 studies the initial formation of the coherent flow structures triggered in a zero-pressure-gradient laminar boundary layer by a pulsed transverse jet and their subsequent development into a young turbulent spot, while Simulation-2 studies the growth of this young turbulent spot into a mature turbulent region containing multiple instances of such vortical structures. 


\subsubsection{Computational domain}

Simulation-1 and -2 are performed using Domains 1 and 2, respectively, shown in Figure 7.3. Domain 1, shown in Figure 7.3(a), consists of a flat, no-slip test surface with streamwise and spanwise dimensions of $264 \delta_{0}^{*}$ and $76 \delta_{0}^{*}$, respectively, where the reference length scale, $\delta_{0}^{*}=1.44 \mathrm{~mm}$, is the displacement thickness of the undisturbed boundary layer in Domain 1 at the streamwise location where the turbulent spot is triggered. In both simulations, the working fluid is air at 1 atm and $25^{\circ} \mathrm{C}$. Triggering of the turbulent spot is accomplished by ejecting fluid from a rectangular jet sub-domain located at the midspan of the test surface and $124 \delta_{0}^{*}$ downstream of the inlet plane. The origin of the coordinate system in Domains 1 and 2 coincides with the center of the jet orifice. The side boundaries of the jet sub-domain are specified as no-slip walls with streamwise and spanwise dimensions of $1.53 \delta_{0}^{*}$ and a length of $15.2 \delta_{0}^{*}$. Domain 2, shown in Figure 7.3(b), has increased streamwise and spanwise dimensions of $486 \delta_{0}^{*}$ and $112 \delta_{0}^{*}$, respectively, to allow for the continued growth of the spot without artificial influence by the domain boundaries. The spanwise width of both domains is such that the free-slip side walls are more than $30 \delta_{0}^{*}$ away from the closest edge of the turbulent spot at its most mature state. Insensitivity to the spanwise domain width was confirmed by repeating Simulation-2 with the spanwise width increased by $50 \%$ to $168 \delta_{0}^{*}$, with no change observed in the spanwise-spreading rate of the spot. The wall-normal height of Domains 1 and 2 is chosen to allow the unconstrained development of the ejected jet above the test surface, as described by Brinkerhoff and Yaras (2012). To ensure a nominally-zero streamwise pressure gradient

along the test surface, the wall-normal height is adjusted in the streamwise direction following the displacement-thickness distribution of a Blasius boundary layer at the local flow Reynolds number. 
(a) Domain 1

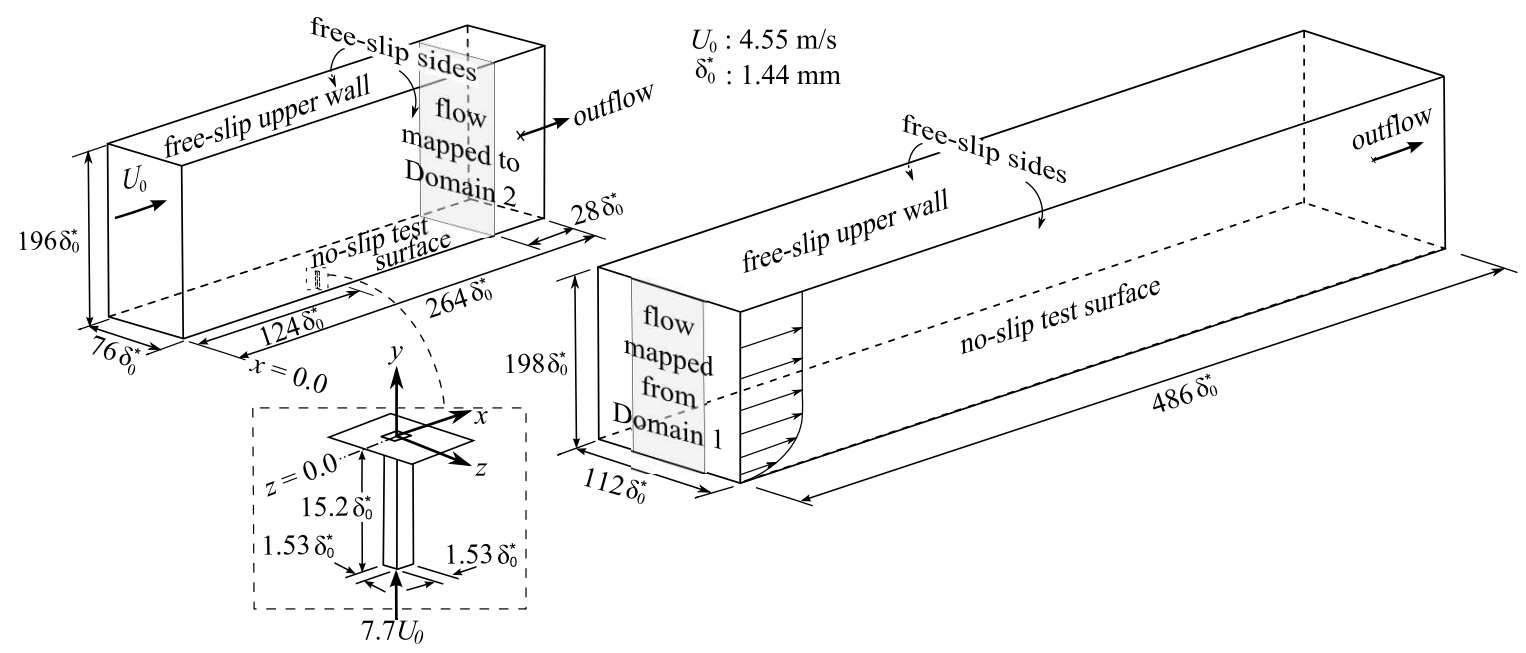

Figure 7.3: Schematic of the computational domains. A magnified view of the jet sub-domain is shown framed by dashed lines.

\subsubsection{Base flow and turbulent spot initialization}

A laminar boundary layer is developed on the test surface within Domain 1 by specifying a uniform inflow velocity and a uniform initial velocity field of $U_{0}=4.55 \mathrm{~m} / \mathrm{s}$ parallel to the test surface and computing to steady-state convergence. The no-slip condition on the test surface conflicts with the uniform velocity condition imposed at the inflow plane, resulting in a deviation between the computed velocity profile and the Blasius profile. A streamwise distance of about $50 \delta_{0}^{*}$ downstream of the inflow plane is required to recover the Blasius profile. The propagation of Tollmien-Schlichting waves in the boundary layer requires a proper treatment of the compressibility of the working fluid, and because the present simulations solve the incompressible form of the governing equations (Section 7.2.4), they are not able to capture the natural development of Tollmien-Schlichting waves. This inability does not adversely affect the formation of the turbulent spot because the local Reynolds number in Domain 1 remains below the critical Reynolds number, given by Schlichting (1968) as $R e_{\text {crit }}=U_{0} \delta^{*} / \nu=520$. A laminar boundary layer is also developed on the test surface of Domain 2 by matching the flow conditions at the inflow of Domain 2 to the undisturbed flow conditions at a spanwise/wall- 
normal plane located $28 \delta_{0}^{*}$ streamwise distance upstream of the outflow boundary of Domain 1. A zero relative static pressure that is time-independent in an area-averaged sense is specified at the outflow boundary of both domains. As a first-order discretization is used at the outflow boundaries, convective flow structures may be distorted as they exit the domain; the mapped plane in Domain 1 is located far enough upstream of the outflow boundary that the velocity field is not influenced by the presence of the outflow boundary.

Once a steady laminar boundary layer has developed in Domain 1, a turbulent spot is artificially-triggered by impulsively applying a spatially-uniform velocity of $7.7 U_{0}$ magnitude at the inlet to the jet sub-domain for a duration of $\tau_{j e t}=27$ time units, where $\tau=t U_{0} / \delta_{0}^{*}$ is the dimensionless time coordinate. The jet magnitude and duration were chosen to match a previous experimental investigation (Yaras, 2007). The instant that the jet is deactivated corresponds to $\tau=0$ and Simulation-1 is continued until $\tau=303$. Between $\tau=103$ and $\tau=303$, the instantaneous velocity information in the spanwise/wall-normal plane $28 \delta_{0}^{*}$ streamwise distance upstream of the outlet of Domain 1 is mapped to the inflow of Domain 2 as a transient inflow condition. The regions of Domain 2 on either side of the mapped data are specified with a steady, spanwise-uniform velocity profile that corresponds to the local undisturbed laminar boundary layer as established $28 \delta_{0}^{*}$ streamwise distance upstream of the outflow boundary in Domain 1. Insensitivity to the relative locations of the mapping plane and the outlet of Domain 1 was confirmed by repeating Simulation-1 with the outlet extended $28 \delta_{0}^{*}$ farther downstream. Within the mapped time interval, the triggered spot and the calmed region in its wake convect past the mapped plane and are introduced into Simulation-2 so that the complete history of the turbulent spot, from inception to maturity, is accurately captured in Simulation -1 and -2 . The latest time when the wake of the spot is observerable at the mapping plane in Simulation-1 occurs at $\tau=263$. The full recovery from the presence of the spot is therefore conservatively assumed to occur by $\tau=303$ so that a steady, undisturbed laminar velocity profile can be imposed at the inflow of Simulation-2 for $\tau>303$. Time integration is 
continued until $\tau=568$. The transition-length correlation of Narasimha (1985) indicates that natural transition beginning at the moment the jet was triggered would take approximately 350 time units to complete under the freestream conditions of the present study. This indicates that the turbulence observed in the spot for the majority of Simulation-2 corresponds to the turbulence that would be expected somewhat downstream of natural-transition completion, and thus the simulation duration is suitable to capture the full development of the spot.

\subsubsection{Spatial grid}

Structured grids consisting of hexahedral finite volumes are mapped to Domains 1 and 2. The spatial and temporal grids must be sufficiently refined to capture the smallest flow structures in the flow, which for turbulent flows are on the order of the Kolmogorov length and time scales. For wall-bounded turbulent shear layers, the Kolmogorov length scale is on the order of the viscous length scale, typically reaching a minimum value of $\eta^{+} \approx 2$ close to the no-slip boundary (e.g. Kim et al., 1971; Pope, 2000; Stanislas et al., 2008), where $\eta$ is the Kolmogorov length scale, the + superscript denotes normalization with the viscous length scale, $\nu / u_{\tau}, u_{\tau}=\sqrt{\tau_{w} / \rho}$ is the friction velocity, $\tau_{w}$ is the wall shear stress, and $\nu=1.53 \times 10^{-5} \mathrm{~m}^{2} / \mathrm{s}$ and $\rho=1.225 \mathrm{~kg} / \mathrm{m}^{3}$ are the kinematic viscosity and density of the working fluid, respectively. The friction velocity is calculated from the spatially-averaged friction factor computed over the footprint of the spot at the final timestep of Simulation-2. This friction velocity value agrees to within $1 \%$ of the value based on the wall shear stress estimated from the local friction factor correlation for a fully-developed turbulent boundary layer proposed by Schlichting (1968):

$$
C_{f}=\left(2 \log _{10}\left(R e_{L}\right)-0.65\right)^{-2.3}
$$

where $C_{f}=0.5 \tau_{w} / \rho U_{0}^{2}$ is the local friction factor and $R e_{L}=309,200$ is the Reynolds number based on $U_{0}$ and the effective streamwise length of the test surface, $L$. The location of the 
spot at the last time step of the simulation from the inlet of Domain 1 dictates the value of $L$; accounting for the mapping of flow information between Domain 1 and Domain 2, this length is $622 \delta_{0}^{*}$. Based on this value of the friction velocity, the nodes in Domains 1 and 2 are distributed to produce $\Delta x^{+}=20$ and $\Delta z^{+}=8$ in Domain 1 and $\Delta x^{+}=20$ and $\Delta z^{+}=7$ in Domain 2, corresponding to approximately 4 to 10 times the Kolmogorov length scale at the wall at the target Reynolds number. Similar spatial resolutions have been used by other researchers to study turbulent spots and turbulent boundary layers (e.g. Henningson et al., 1987; Spalart, 1988). The present choice is validated in Section 7.2.5. The spatial resolution of Domain 1 is refined in the vicinity of the jet orifice to about $\Delta x^{+} \approx 8$ and $\Delta z^{+} \approx 3$ to account for the smaller length scales in the initially-triggered spot. The $y^{+}$value of the nodes adjacent to the no-slip boundary within the spot is equal to or less than 0.7 . In the range of $0.7<y^{+}<30$, the wall-normal node spacing is increased linearly from the value adjacent to the wall to a value that corresponds to the Kolmogorov length scale in the log-law region, which is approximated by:

$$
\eta^{+}=\left(\kappa y^{+}\right)^{1 / 4}
$$

where $\kappa=0.41$ is the von-Karman constant (e.g. Pope, 2000; Stanislas et al., 2008). Beyond $y^{+}=30$, the node spacing is increased at a rate of $4 \%$ until $y / \delta_{0}^{*}=5.0$ is reached. The grid spacing remains constant in the range of $5.0<y / \delta_{0}^{*}<8.7$, with $y / \delta_{0}^{*}=8.7$ approximately corresponding to the height of the largest-scale flow structures expected in the triggered turbulent spot (e.g. Yaras, 2007), and is then increased at a rate of $10 \%$ until the free-slip ceiling of the domain is reached. This wall-normal node distribution is shown in Figure 7.4.

The jet sub-domain is discretized with 60 nodes in each of the mutually-perpendicular coordinate directions normal to the jet flow $(x$ and $z$ ), spaced to place at least 20 nodes in the wall boundary layer of the jet, and the rate at which the node spacing varies in the $x$ and $z$ directions is less than $10 \%$. The direction parallel to the jet flow $(y)$ is discretized with 88 nodes that are distributed to place 15 nodes within $1 \delta_{0}^{*}$ of the jet discharge plane. The 


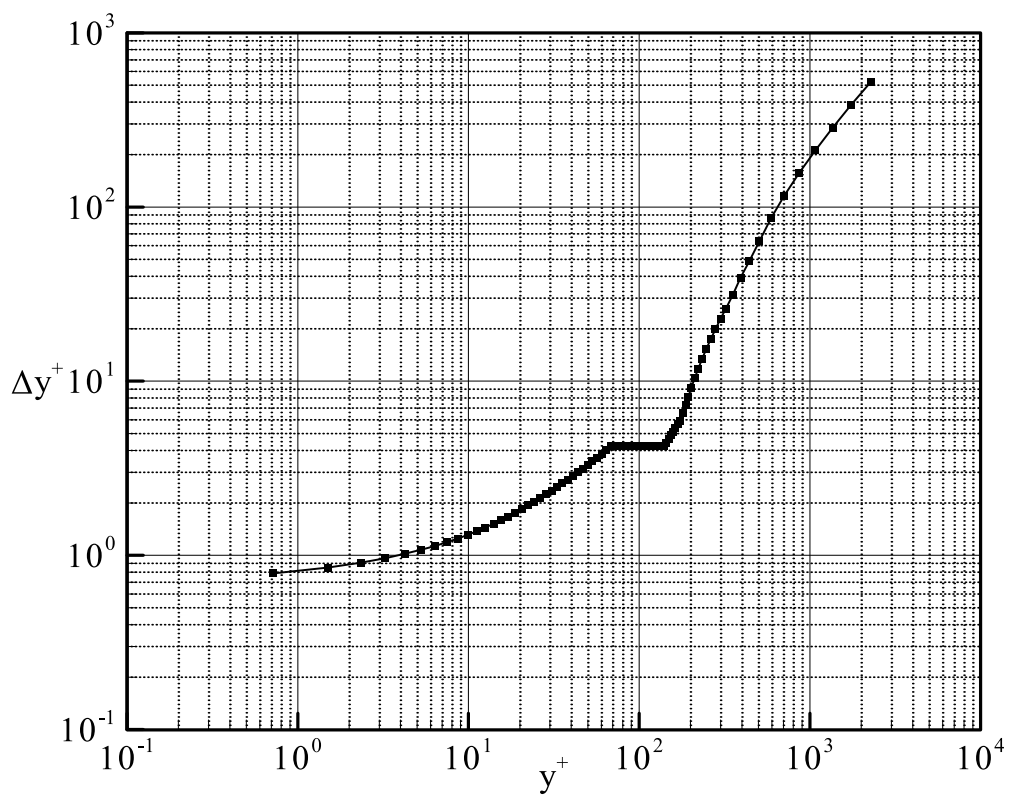

Figure 7.4: Wall-normal node distribution.

Table 7.1: Number of nodes in the spatial grid

\begin{tabular}{lccc}
\hline & $N_{x}$ & $N_{y}$ & $N_{z}$ \\
\hline Domain 1 & 345 & 82 & 197 \\
Jet sub-domain & 60 & 88 & 60 \\
Domain 2 & 456 & 82 & 266 \\
\hline
\end{tabular}

$y$-direction nodes are clustered so that the spacing at the jet discharge plane is $\Delta y^{+}=0.65$ and the spacing increases in the $-y$ direction at a rate of less than $10 \%$ until $\Delta y^{+}=4.0$, after which the node spacing remains constant. The number of nodes in the streamwise $(x)$, spanwise $(z)$, and wall-normal $(y)$ directions in each computational domain are recorded in Table .

\subsubsection{Solution method}

ANSYS CFX @ (Version 12), a commercial computational-fluid-dynamics software package, is used to solve the incompressible form of the time-varying mass and momentum equations through a finite-volume approach. This software package has been used extensively by the 
authors' research group to simulate transitional and turbulent boundary layers, producing excellent agreement with experimentally-measured parameters such as the velocity fluctuation spectra, turbulence kinetic energy production rate budgets, and mean velocity profile of turbulent boundary layers (McAuliffe and Yaras, 2010; Brinkerhoff and Yaras, 2011; Azih et al., 2012). Discretization of the governing equations is based on second-order central differencing and second-order Euler backward differencing for the spatial and temporal derivatives, respectively. In Simulation-1, a timestep size of $\Delta t=7 \times 10^{-6} \mathrm{~s}$ is chosen so that the pulsed-jet flow takes about 10 timesteps to penetrate a distance equivalent to the local displacement thickness of the undisturbed boundary layer at the jet discharge plane. In Simulation-2, a timestep size of $\Delta t=3.5 \times 10^{-5} \mathrm{~s}$ or $0.11 t^{+}$is used, where $t^{+}=\nu / u_{\tau}^{2}$ is the viscous timescale in the turbulent spot. Compared to the timestep size of $0.20 t^{+}$recommended by Choi and Moin (1994) for accurately computing turbulent flows, the temporal resolution in the current study is quite conservative.

Solution convergence of the discretized equations at each timestep is obtained with outer iteration loops serving to converge the coefficients of the linearized governing equations and inner iteration loops facilitating the solution of these equations through W-type algebraic multigrid cycles. Six grid levels are used in the multigrid cycle with one solution sweep performed at each grid level during the restriction passes and three solution sweeps performed at each grid level during the prolongation passes. The transient solution is obtained with one inner-loop iteration per outer-loop iteration, and multiple outer-loop iterations per timestep. For each timestep, the solution is declared as converged when the root-mean-square residuals of the governing equations are reduced by five orders of magnitude to less than $10^{-6}$. This is realized with up to eight outer-loop iterations per timestep. The simulations are partitioned and executed in parallel on 20 Intel $^{\mathrm{TM}} \mathrm{L} 5410$ Xeon processors. 
Table 7.2: Computational grids for the validation studies.

\begin{tabular}{lccc}
\hline Simulation & $\Delta x^{+}$ & $\Delta z^{+}$ & $R e_{\theta}$ \\
\hline Grid 1 & 3 & 3 & 900 \\
Grid 2 & 3 & 3 & 500 \\
Grid 3 & 22 & 11 & 500 \\
Spalart (1988) & 20 & 7 & 670,1410 \\
Wu and Moin (2009) & 6 & 11 & 900 \\
\hline
\end{tabular}

\subsubsection{Validation of numerical approach}

Validation of the numerical algorithm and the spatial and temporal resolutions was performed by Azih et al. (2012) and Brinkerhoff and Yaras (2011) by performing a series of companion DNS studies of a zero-pressure-gradient turbulent boundary layer and comparing the results of these simulations to statistical turbulence properties of corresponding turbulent boundary layers documented in literature. The spatial grids and flow Reynolds numbers of these studies are listed in Table 7.2. The dimensions of the computational domain for the companion simulations are 670, 1820, and 650 viscous units in the $x, y$, and $z$ directions, respectively, and is spatially-periodic in the $x$ and $z$ directions. The companion simulations are initialized with a spatially-uniform velocity of $1.6 \mathrm{~m} / \mathrm{s}$ and the boundary layer is developed in time until a momentum-thickness Reynolds number of $R e_{\theta}=900$ is reached. Transition is triggered at $R e_{\theta}=450$ through a mass source/sink pair that is located near the wall in the center of the domain. The trigger injects fluid into the domain at a wall-normal velocity of $0.16 \mathrm{~m} / \mathrm{s}$ for a duration of $4.5 t^{+}$.

The first validation simulation (Grid 1) was performed at a Reynolds number based on the boundary-layer momentum thickness of $R e_{\theta}=900$ with a spatial resolution in the $x-z$ plane of $\Delta x^{+}=\Delta z^{+}=3$, which corresponds to about 1.5 times the Kolmogorov length scale at this Reynolds number. The $y^{+}$value of the first node from the no-slip boundary is 0.85 and the wall-normal node distribution (normalized using wall scales) follows closely the distribution shown in Figure 7.4. Figure 7.5 shows that the results compare favourably 
to the turbulence kinetic energy production and dissipation rate budgets and the root-meansquare streamwise velocity fluctuation $\left(u_{r m s}^{\prime+}\right)$ profiles (Figures $7.5(a)$ and $(b)$, respectively) computed by Spalart (1988), who used an algorithm utilizing a spectral method for spatial discretization and a second-order-accurate hybrid Runge-Kutta/Crank-Nicholson scheme for temporal discretization to simulate the boundary layer at a Reynolds number of $\operatorname{Re}_{\theta}=1410$. Figure 7.5(c) also shows excellent agreement in the mean velocity profile for $y^{+}<40$ when compared to the DNS results of Wu and Moin (2009) corresponding to $R e_{\theta}=900$, who used a finite-difference scheme with second-order accuracy in space and mixed second- and thirdorder accuracy in time. Satisfactory agreement was also achieved in the outer part of the boundary layer, with a deviation of less than $10 \%$ in the mean velocity profile. This deviation is likely due to the streamwise length of the computational domain used in the validation study being insufficient to fully capture the statistical properties of the larger-scale structures in the outer region of the turbulent boundary layer; this restriction does not apply in the present turbulent spot simulations, as the largest-scale structures within the turbulent spot are obviously confined to within the boundaries of the computational domain.

To isolate the effect of Reynolds number on the accuracy of the numerical results, a second validation DNS (Grid 2) was performed with the same spatial resolution as Grid 1 but at $R e_{\theta}=500$, which is closer to the conditions of the present turbulent spot study, in which the Reynolds number based on the average momentum thickness in the spot at its most mature state is $R e_{\theta}=332$. Finally, to ascertain the sensitivity of the results to the spatial grid, a third DNS (Grid 3) with a grid that closely matches the spatial resolution of the present turbulent spot study was performed at $R e_{\theta}=500$. The simulation results for Grids 2 and 3, shown in Figure 7.6, compare reasonably well with the turbulence kinetic energy budgets computed by Spalart (1988) at $R e_{\theta}=670$ and the mean velocity profile measured by Djenidi and Antonia (1993) at $R e_{\theta}=560$. Based on these favourable comparisons, the present study should accurately capture the flow development in the triggered turbulent spot where the 
(a)
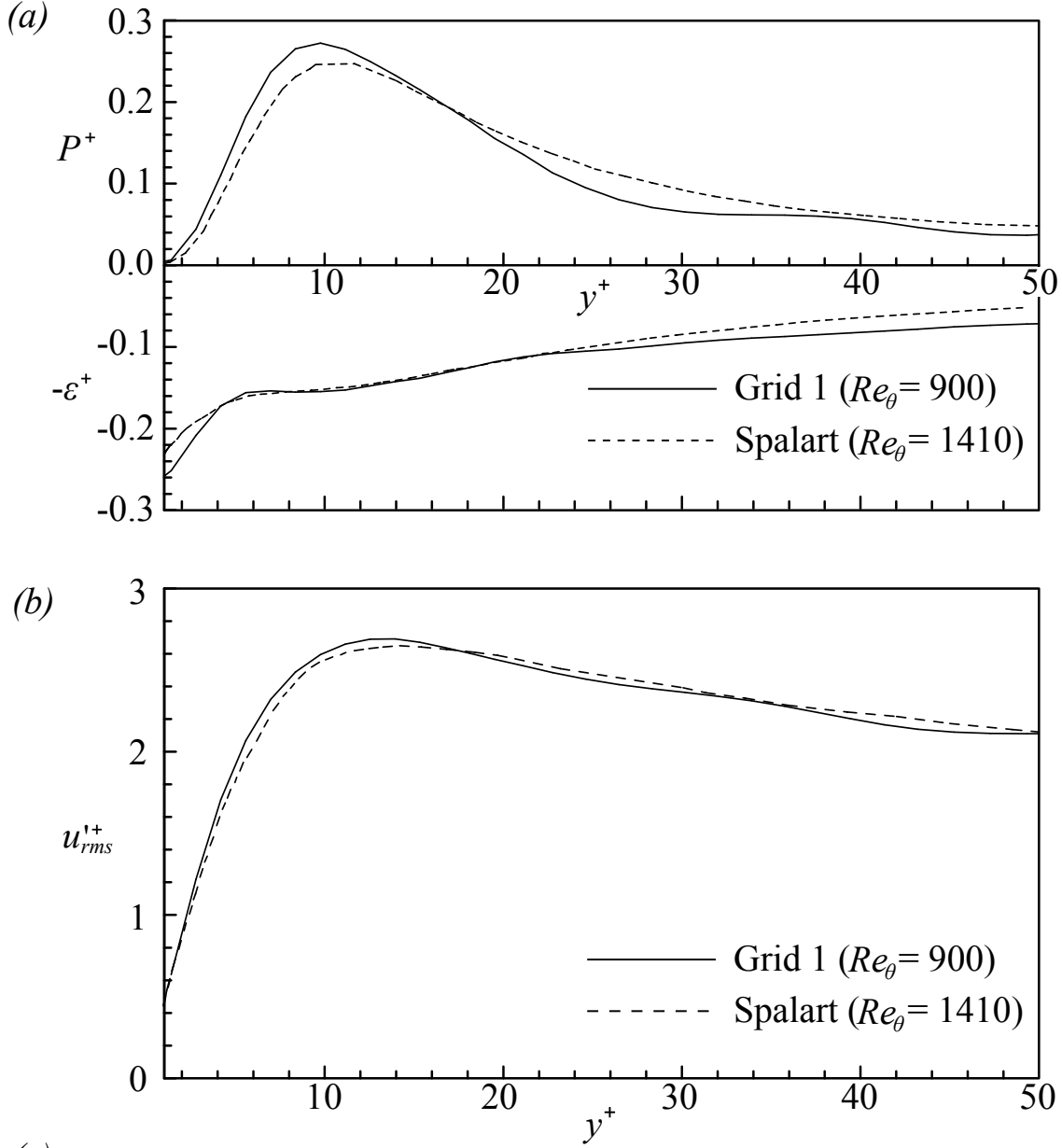

(c)

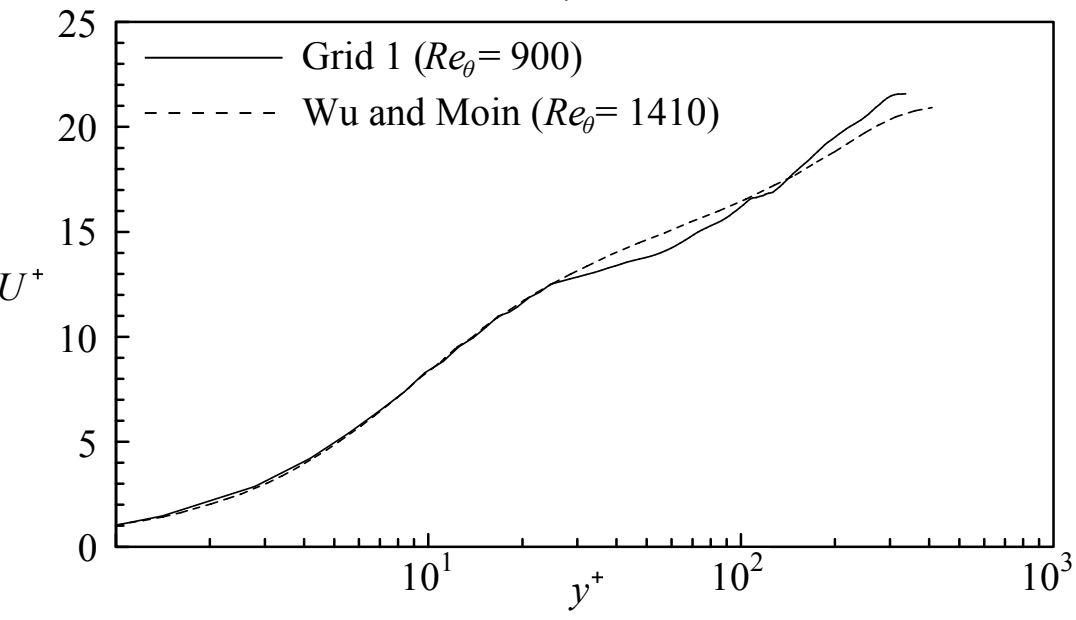

Figure 7.5: Validation DNS study of a zero-pressure-gradient turbulent boundary layer at $R e_{\theta}=900$. Results from Grid 1 are compared with the $(a)$ turbulence kinetic energy production and dissipation rate distributions and $(b)$ streamwise velocity fluctuation profiles of Spalart (1988) and (c) mean velocity profile of Wu and Moin (2009). Adapted from Azih et al. (2012) 


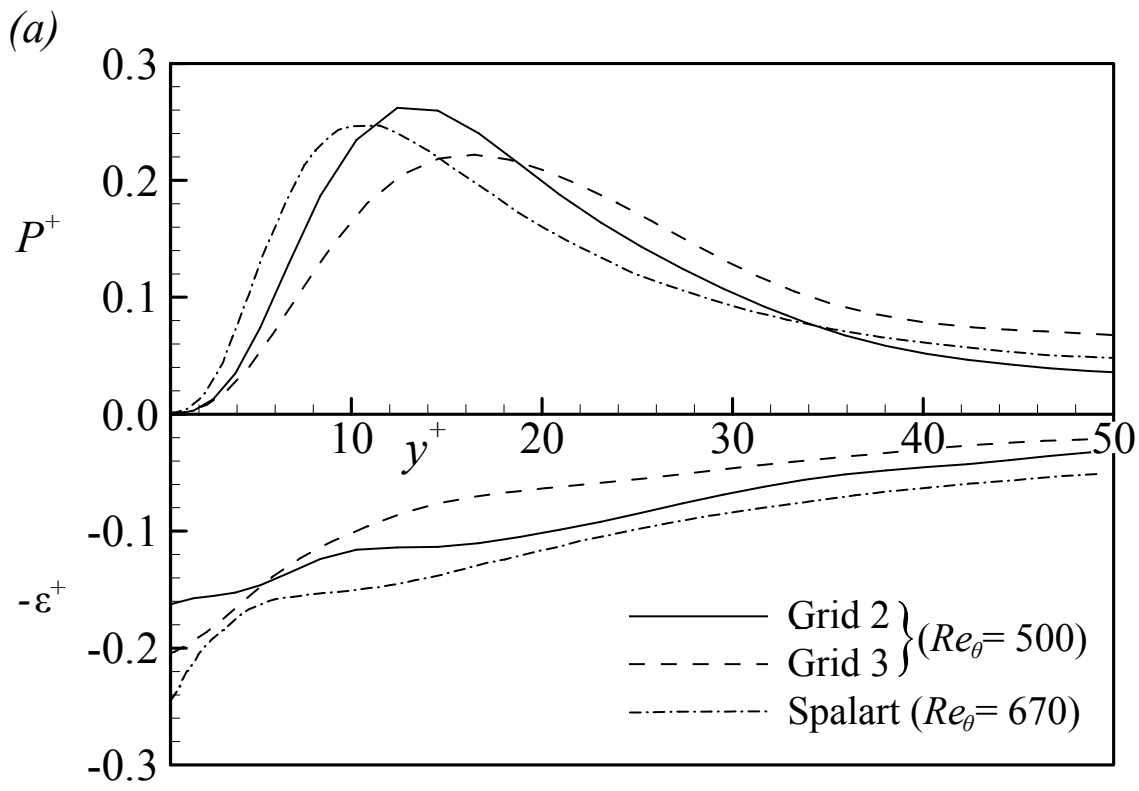

(b)

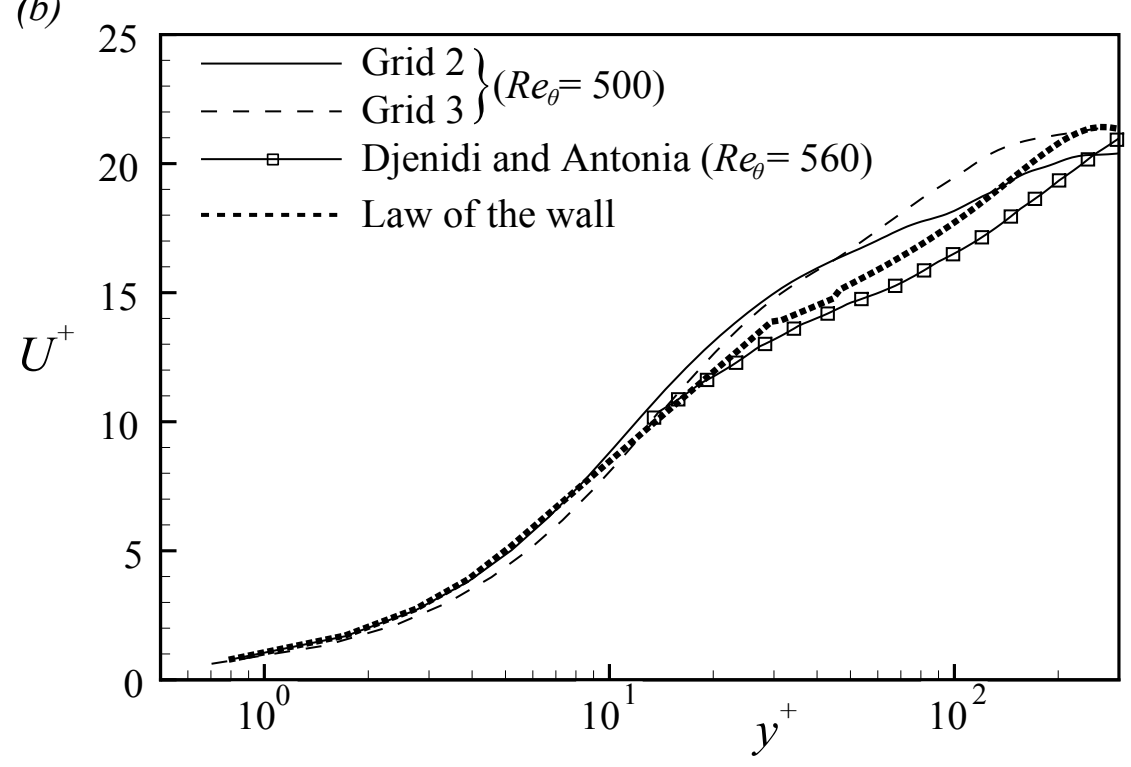

Figure 7.6: Validation DNS studies in a zero-pressure-gradient turbulent boundary layer at $R e_{\theta}=500$. Results from Grid 2 and Grid 3 are compared with the $(a)$ turbulence kinetic energy production and dissipation rate distributions of Spalart (1988) and (b) mean velocity profile of Djenidi and Antonia (1993). Adapted from Brinkerhoff and Yaras (2011). 
transient coherent flow structures tend to be of somewhat greater spatial and temporal scales than in a fully-turbulent boundary layer at comparable flow Reynolds numbers. The wallnormal node spacing and the spatial and temporal resolution of the present turbulent spot simulations closely match those of Grid 3. As per Figure 7.6, although the Grid-2 results are in slightly better agreement with the simulation data of Spalart (1988) and the experimental data of Djenidi and Antonia (1993) than the Grid-3 results, the resolution of Grid 3 was adopted for the present turbulent spot simulations to enable simulations with reasonable computing times while still ensuring that the flow physics are accurately captured.

\subsection{Results and Discussion}

\subsubsection{Laminar baseflow}

Figure 8.4 illustrates the free-stream conditions imposed on the laminar baseflow as it develops over the test surfaces of Simulation-1 and -2 . The arrow in Figure 8.4 at $x_{i n t} / \delta_{0}^{*}=112$ indicates the location where data is mapped between the two simulation domains. It is evident that the mapping has no effect on the free-stream flow development. Apart from short region near the leading and trailing edges of the test surface, the free-stream acceleration parameter $\left(K=\nu / U^{2} \partial U / \partial x\right)$ is close to zero, indicating that the laminar boundary layer develops in a way that closely resembles a zero-pressure-gradient boundary layer. The variations in $K$ near the leading and trailing edges correspond to peak variations of $U / U_{0}$ of less than 0.01 . Figure 7.8 illustrates the streamwise distribution of the displacement thickness and friction factor of the undisturbed laminar boundary layer. These parameters show a small discontinuity at the location of the mapped plane, $x_{i n t}$, indicating that the laminar boundary layer is slightly more sensitive to the mapping of flow information between simulation domains than the freestream flow, but the magnitudes of the discontinuities in the streamwise gradients of $\delta^{*}$ and $C_{f}$ are very small, and is unlikely to affect the development of the turbulent spot as it convects 


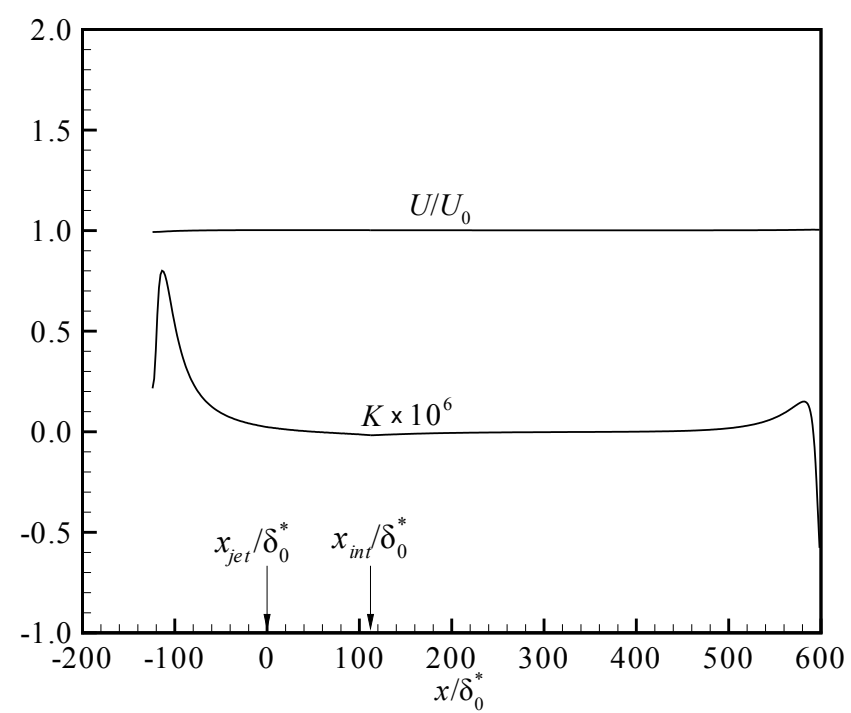

Figure 7.7: Streamwise distribution of the free-stream velocity, $U$, and streamwise acceleration parameter, $K$.

through the simulation domains. The maximum deviation between the computed values of $\delta^{*}$ and $C_{f}$ and the values given by the Blasius profile is $4 \%$ and $11 \%$ of the local values, respectively, excluding the regions near the inflow and outflow boundaries. The change in the streamwise-distribution slope of $K, \delta^{*}$, and $C_{f}$ that is observed near $x / \delta_{0}^{*}=600$ is due to the upstream influence of the outflow boundary. As such, the data beyond $x / \delta_{0}^{*}=500$ is discarded.

\subsubsection{Formation of the turbulent spot}

The growth of coherent flow structures within the triggered spot begins with the creation of vortices in the test-surface boundary layer by the triggering jet that resemble those occurring in the well-documented transverse-jet flow (e.g. Fric and Roshko, 1994). An earlier study by Brinkerhoff and Yaras (2012) showed that as the main-flow boundary layer convects around the jet, an adverse pressure gradient is created behind the jet that, when projected onto the test surface, causes the main-flow boundary layer to separate and roll into coherent streamwiseoriented vortices immediately downstream of the jet orifice. These vortices are the "seed" that 


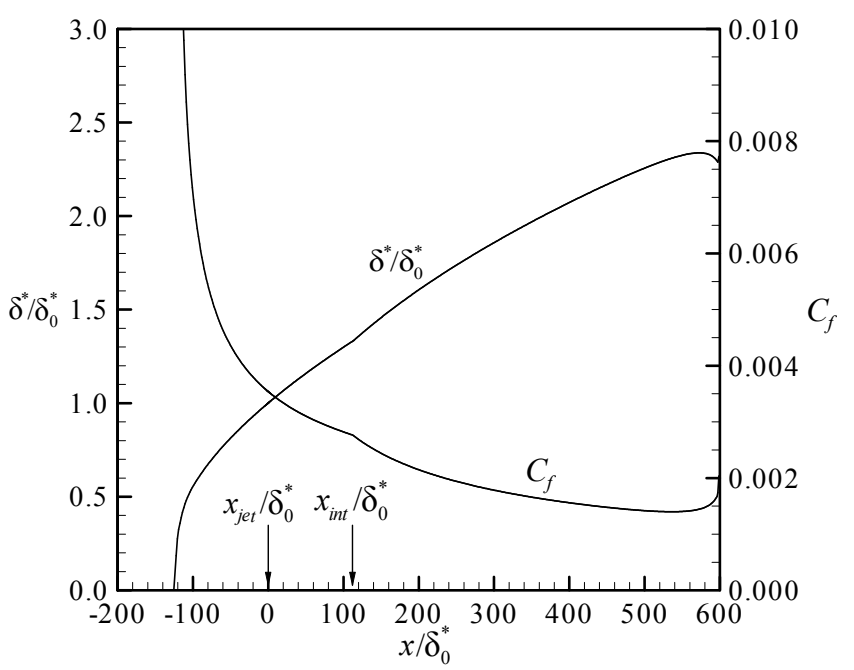

Figure 7.8: Streamwise distribution of the displacement thickness, $\delta^{*} / \delta_{0}^{*}$, and friction factor, $C_{f}$, of the undisturbed laminar boundary layer.

initiates the formation of the turbulent spot.

To illustrate the formation process, Figure 7.9 plots iso-contours of perturbation streamwise vorticity, $\omega_{x}$. The perturbation vorticity, $\omega$, is defined as the curl of the perturbation velocity, which is itself defined as the local instantaneous velocity at the same wall-normal location minus the velocity of the streamwise-local undisturbed laminar boundary layer. Instantaneous lines of vorticity that intersect the vortices are also plotted to aid in visualizing the development of the vorticity field. The location of each vorticity line is carefully chosen so that it intersects the corresponding vortical structure at the same relative location in each time instance. The iso-contours are normalized by $\left(U_{0} / \delta_{0}^{*}\right)^{2}$ and both the vorticity lines and iso-contours are coloured according to the value of $y / \delta_{0}^{*}$ to aid in comparing the relative heights of the vortical structures. At $\tau=2$, the pair of streamwise-oriented vortices created by the jet, labelled A, extend about $12 \delta_{0}^{*}$ downstream of the jet orifice (located at $x / \delta_{0}^{*}=0, z / \delta_{0}^{*}=0$ ). The small-scale vortices above the jet orifice are remnants of vortices created by the triggering jet, which dissipate by $\tau=10$. Because the A vortices are situated in the strain field associated with the laminar boundary layer, the vorticity field associated with the A vortices is stretched 
(a) $\tau=2 \quad \omega_{x}^{\prime}= \pm 1$

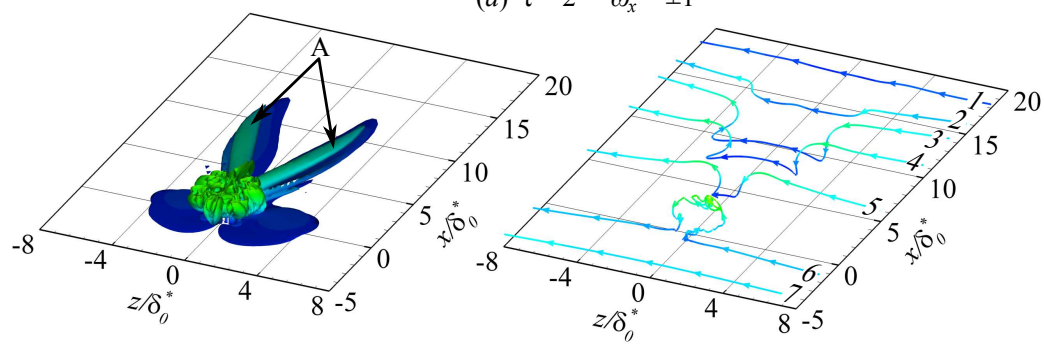

(b) $\tau=46 \quad \omega_{x}^{\prime}= \pm 1$

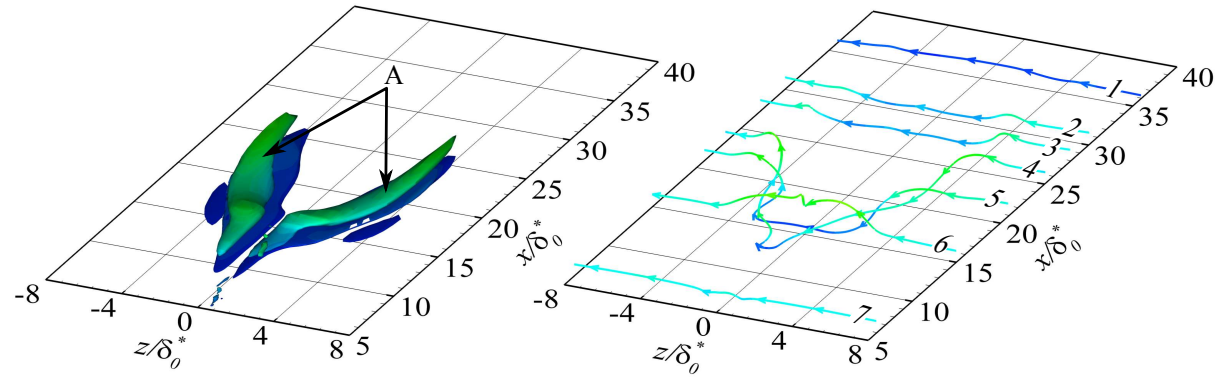

(c) $\tau=90 \quad \omega_{x}^{\prime}= \pm 1$

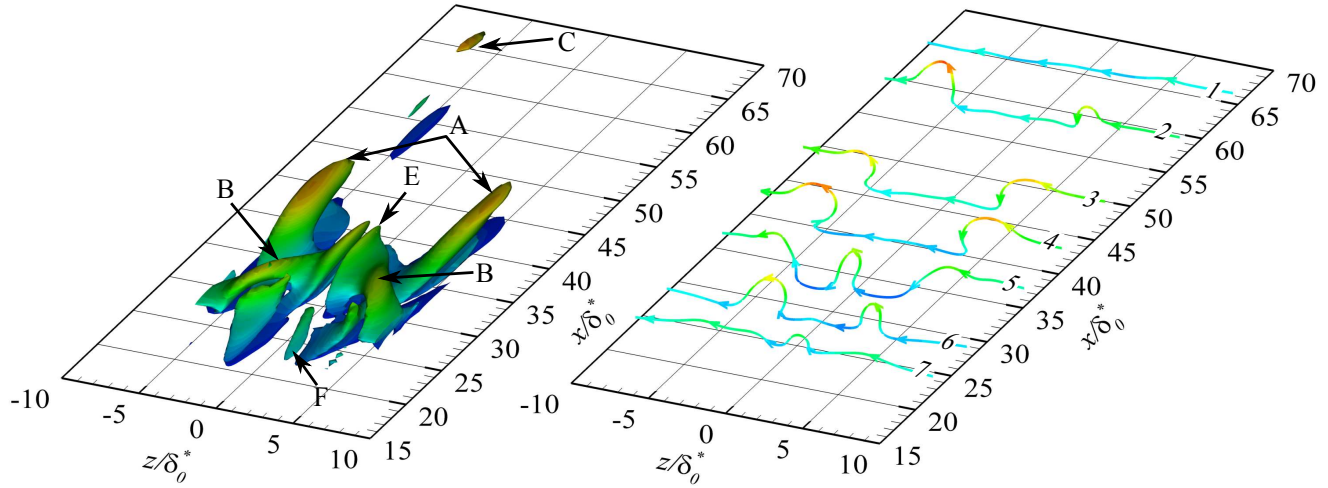

(d) $\tau=90 \quad Q=0.6$

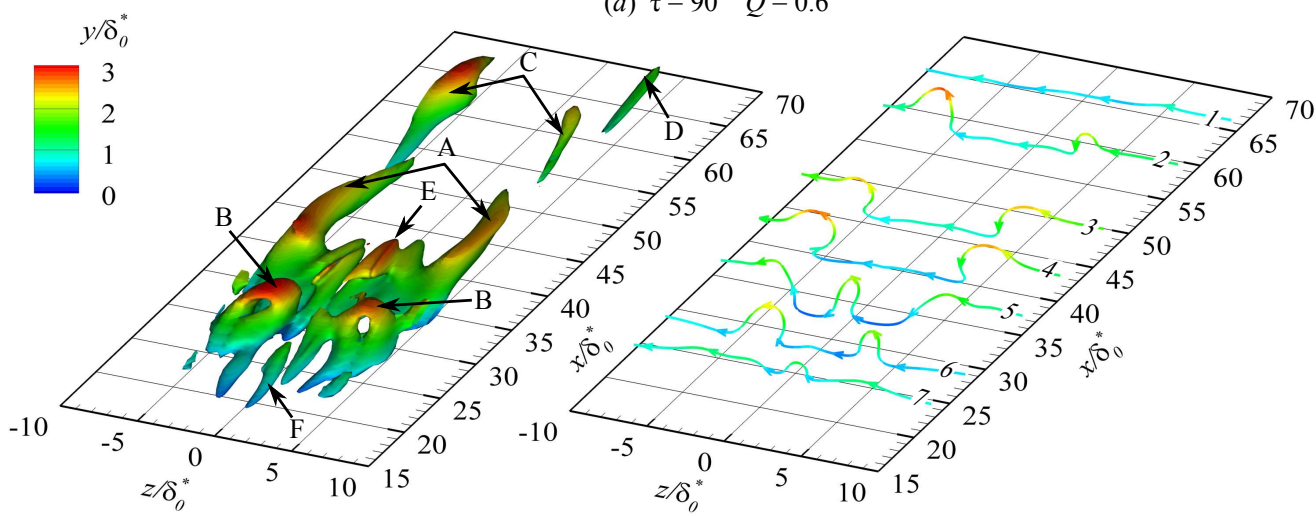

Figure 7.9: Formation of the turbulent spot in Simulation-1 illustrated at $\tau=2,46$, and 90, visualized through iso-contours of $(a-c) \omega_{x}= \pm 1$ and $(d) Q=0.6$ (left) and instantaneous vorticity lines (right). Iso-contours and vorticity lines are coloured according to the value of $y / \delta_{0}^{*}$. 
downstream so that by $\tau=46$, the A vortices have elongated to roughly twice their length at $\tau=2$. As a result, their peak magnitude increases to conserve angular momentum and thus the wall-normal motions induced by the A vortices become stronger and cause new generations of vortical structures to form. Figure $7.9(c)$ shows that by $\tau=90$, two distinct pairs of secondary vortical structures - labelled B and C-occur near the A vortices and other tertiary vortical structures-labelled D, E and F-occur near the secondary vortical structures. To more clearly illustrate the shape of the secondary and tertiary hairpin vortices, Figure $7.9(d)$ plots iso-contours of the second invariant of the velocity-gradient tensor, denoted $Q$, defined as

$$
Q=\frac{1}{2}\left({\frac{\partial u_{i}}{\partial x_{j}}}^{2}-\frac{\partial u_{i}}{\partial x_{j}} \frac{\partial u_{j}}{\partial x_{i}}\right)
$$

where Einstein summation is implied over the indexed terms. This property has been shown to improve the prominence of the hairpin vortices over the background vorticity (e.g. Jeong and Hussain, 1995). The $Q$ iso-contours reveal that the $\mathrm{B}$ vortices are hairpin vortices that straddle the $\mathrm{A}$ vortices, the $\mathrm{C}$ vortices are streamwise vortices downstream of the $\mathrm{A}$ vortices, and the tertiary vortical structures (D, E, and F) are vortices formed by the B and C vortices. By $\tau=103$ (not shown), the A vortices have been stretched in the streamwise direction to about three times their length and twice their spanwise extent at $\tau=2$.

The process by which the multiple vortical structures observed in Figure $7.9(d)$ form from the initial streamwise A vortices is discussed with the aid of the iso-contours and vorticity lines in Figure 7.9 and a simplified schematic shown in Figure 7.10. Because the turbulent spot is still in its infancy in Figure 7.9, the process is not wholly representative of what occurs when the spot has reached maturity, and the detailed regeneration mechanism of vortical structures within the mature turbulent spot is discussed in Section 7.3.4.1. Once the streamwise-aligned A vortices are triggered by the transverse jet, they induce downwash in the region between the A vortices and upwash in the region outboard of the A vortices (Figure 7.10(a)). These wall-normal motions convect the local boundary-layer vorticity closer to and farther away from 
the wall, respectively, as illustrated in Figure $7.9(a)$ by the vorticity lines labelled 2-5. As the A vortices are stretched in the downstream direction by the background strain field, the wallnormal motions they induce convect the boundary-layer vorticity outboard of the A vortices over and above the A vortices, as illustrated by the vorticity line labelled 6 in Figure $7.9(c)$. The portion of vorticity line 6 that is situated above A is simultaneously convected towards the wall by the downwash between the A vortices so that the vorticity field associated with vorticity line 6 develops a significant wall-normal component. Inviscid instability of this layer of wall-normal vorticity causes it to roll-up into a coherent B hairpin vortex (Figure 7.10(b)). A similar process occurs farther downstream to create the coherent C vortices. Figure $7.10(c)$ illustrates that as the B hairpin vortices are stretched by the background strain field, they elongate and are strengthened just like the A vortices. The downwash induced between the two B hairpin vortices convects the local boundary-layer vorticity towards the wall, as shown by vorticity line 5 in Figure $7.9(d)$, to form the coherent $\mathrm{E}$ hairpin vortex, while the upwash induced upstream of the B hairpin vortices convects the boundary-layer vorticity away from the wall, as shown by vorticity line 7 in Figure $7.9(d)$, and forms the coherent $\mathrm{F}$ hairpin vortex (Figure $7.10(d)$ ). Stretching by the background shear increases the size and strength of the secondary and tertiary vortical structures. Between $\tau=103$ and 303, the spot is mapped into Simulation-2, where its continued streamwise and spanwise growth can be analyzed in detail.

\subsubsection{Defining the laminar/turbulent interface}

Analyzing the growth rate of a turbulent spot requires a consistent and precise method to identify the boundary between the laminar and turbulent flow regions. In a recent DNS by Strand and Goldstein (2011), the spanwise edges of the turbulent region were identified as the farthest locations on each side of the spot centerline where the magnitude of the streamwise component of vorticity $\left|\omega_{x}\right|$ exceeds a threshold value. This flow variable was used because it is nominally zero in undisturbed laminar flow regions and reaches high values in turbulent flow 
(a) $\tau=2$

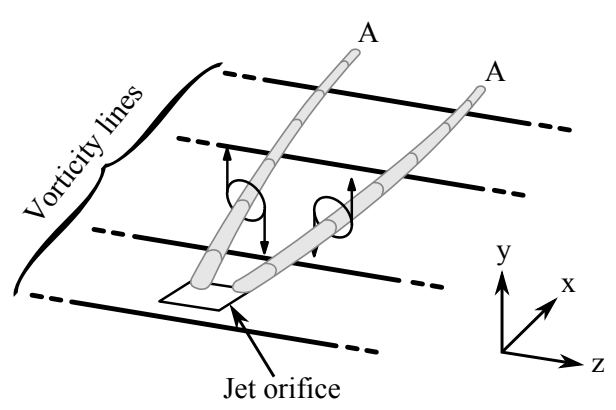

(c) $\tau=68$

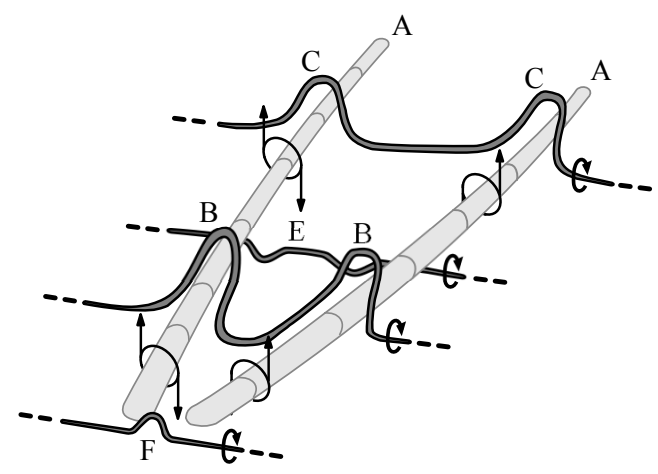

(b) $\tau=46$

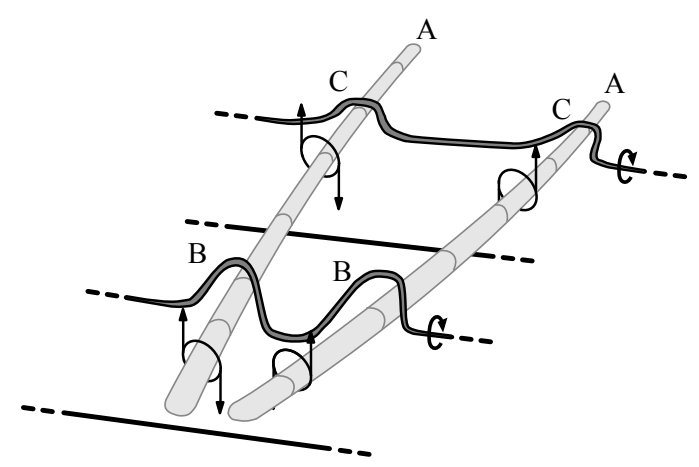

(d) $\tau=90$

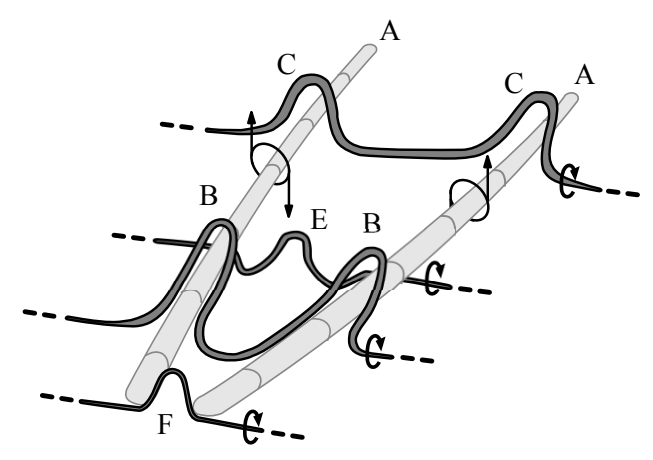

Figure 7.10: Schematic illustrating the formation of vortical structures in the early turbulent spot from the initial pair of streamwise vortices triggered by the transverse jet. 
regions. At each time interval, spot-edge locations using several threshold values of $\left|\omega_{x}\right|$ were averaged to give one location on either side of the turbulent spot, which were subsequently averaged to obtain the spot half-width. This method yields the maximum half-width of the spot at a particular instant; the widest location typically occurs about $20 \%-40 \%$ of the spot length downstream of the spot's trailing edge. This method was applied to the present numerical results with threshold values of $\left|\omega_{x}\right| \delta_{0}^{*} / U_{0}$ between 0.032 and 0.190 to produce the streamwise variation in the spot half-width, denoted $s$, that is plotted in Figure 7.11 with respect to the streamwise location of the spot's trailing edge, denoted by $x_{t e}$. To ascertain the sensitivity of the computed spot half-widths to the number of data points included in the averaging process, the method was repeated at selected instances in time using 4, 6, 8, 10, and 12 uniformly-spaced threshold values between 0.032 and 0.190 . The resulting uncertainty in the spot half-width, shown by the error bars in Figure 7.11, indicates that identification of the spot edge with this technique becomes more precise as the spot matures. The abscissa in Figure 7.11 is the streamwise location of the spot trailing edge, determined using the same threshold values of $\left|\omega_{x}\right| \delta_{0}^{*} / U_{0}$ as those used for locating the spanwise edges of the spot. In comparison to the data of Strand and Goldstein (2011), which is reproduced in Figure 7.11, the spot widths in the present study are consistently larger. Considering that the turbulent spot in the present study is triggered by a strong transverse jet, while the spot of Strand and Goldstein (2011) is triggered by a small roughness element, it is likely that the strong jet in the current study triggered a very rapid initial growth of the spot. Despite this offset, Figure 7.11 shows that the spot lateral growth rates in the current study agree quite favourably with the results of Strand and Goldstein (2011). The average spot lateral growth rate, $d s / d x$, and the spot spreading half-angle, defined as $\tan ^{-1}(d s / d x)$, are respectively 0.091 and $5.2^{\circ}$ in the current study, compared to 0.089 and $5.1^{\circ}$ found by Strand and Goldstein (2011). The trend observed in Figure 7.11 suggests that the turbulent spot lateral growth rate is constant starting at $x_{t e} / \delta_{0}^{*} \approx 118$, which corresponds to $\tau=255$. The time range over which the spot lateral growth rate can be assumed to have reached a constant value is thus $\tau=255-568$. This period 


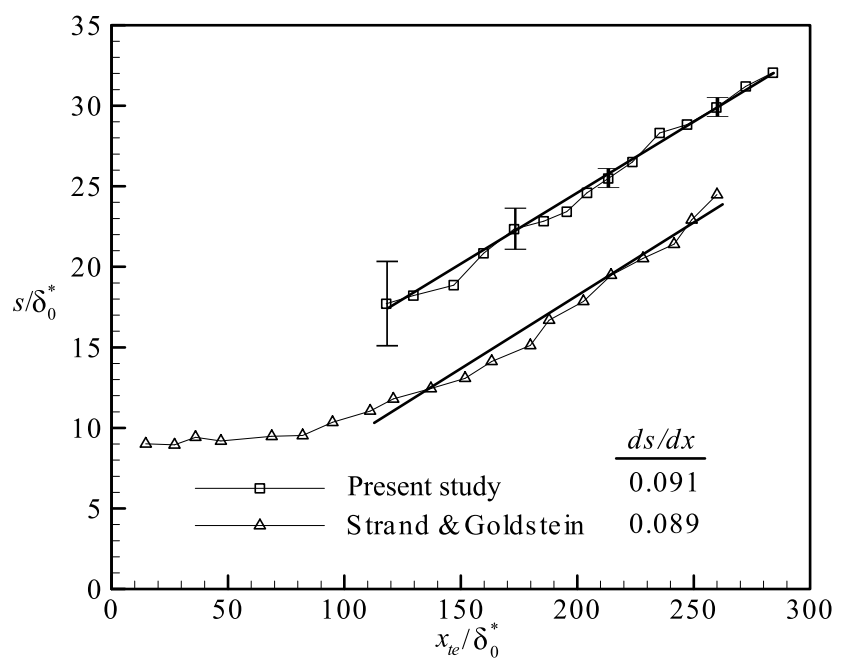

Figure 7.11: Streamwise distribution of the spot spanwise dimension.

of spot-growth is studied in detail in Section 7.3.4 to analyse the steady growth mechanisms within the turbulent spot.

\subsubsection{Growth of the turbulent spot}

Figure 7.12 illustrates the organization of vortical flow structures in the turbulent spot at $\tau=107,255$, and 492 by plotting iso-contours of $Q$ at the same iso-contour level as in Figure $7.9(d)$. Instantaneous vorticity lines are again shown, labelled according to the coherent vortical structures they intersect. As expected, the flow structures and vorticity lines have not changed appreciably between $\tau=90$ in Figure 7.9 and $\tau=107$ in Figure 7.12. Between $\tau=107$ and $\tau=255$, however, the coherent vortical structures within the spot develop a pattern that remains fairly consistent for the rest of the lifetime of the spot. Figure 7.12(b) and $(c)$ indicate that the pattern is as follows: numerous hairpin vortices of various spatial scales occur within the interior of the spot, such as those labelled B; larger-scale vortical structures occur near the leading edge of the spot, such as those labelled C, D, H, and G; and smaller-scale vortical structures occur near the trailing edge of the spot. Between $\tau=255$ and $\tau=492$, the growth of the spot occurs as follows: new hairpin vortices are generated within the interior of the 

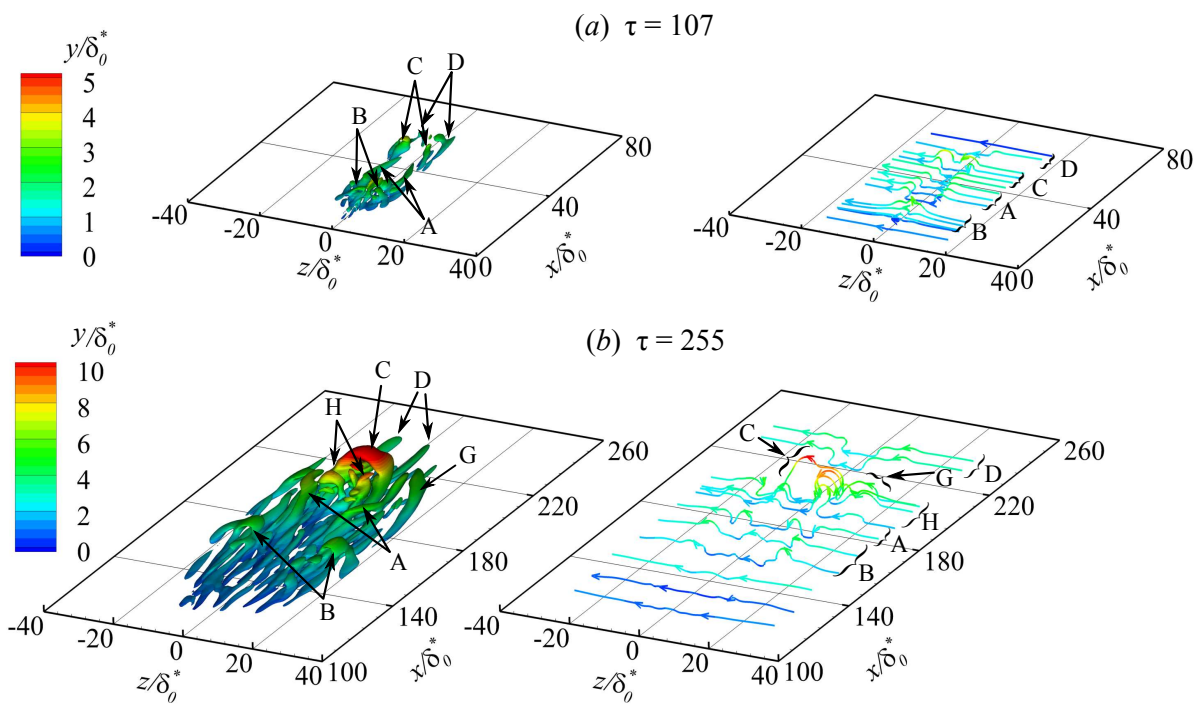

(c) $\tau=492$

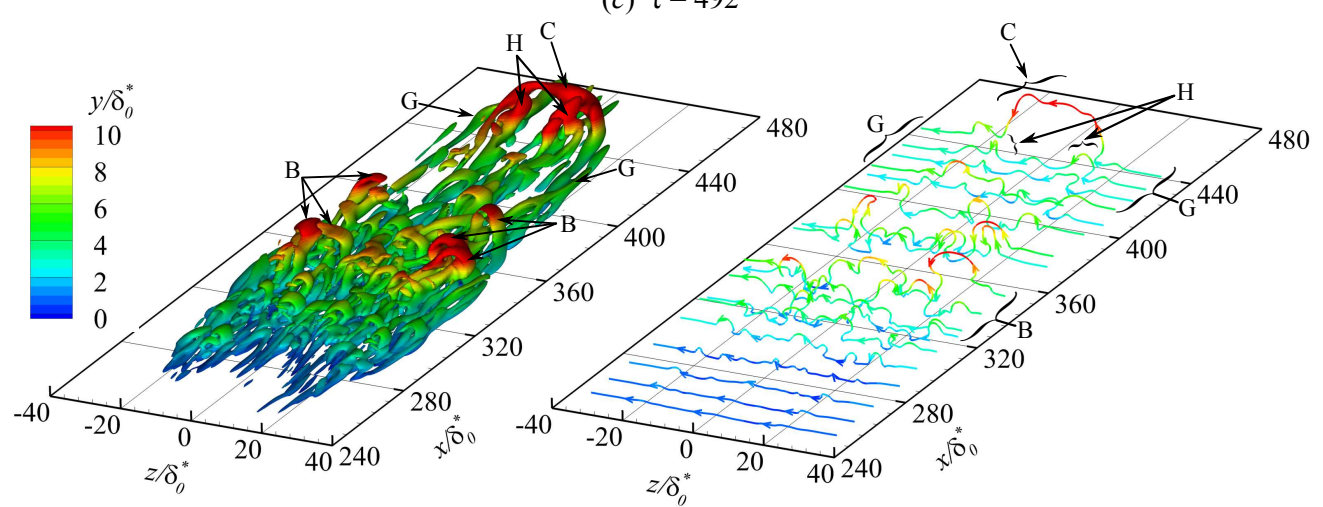

Figure 7.12: Development of the turbulent spot at $\tau=107,255$, and 492, visualized through contours of $Q=0.6$ (left) and instantaneous vorticity lines (right) coloured according to the value of $y / \delta_{0}^{*}$. The colour scale at $\tau=107$ is half of that at the other times. 
spot and form groups of two or three closely-spaced hairpin vortices called "wave packets"; the vortical structures near the leading edge of the spot are enlarged in their spatial scale; and new wave packets of hairpin vortices are created at the laminar/turbulent interface along the spanwise edges of the spot. In the following sections, the detailed development of the spot is analysed in the context of these growth mechanisms. Additionally, the low population of hairpin vortices observed in Figure 7.12(b) and $(c)$ upstream of the trailing edge of the spot implies the development of a "calmed" region in the wake of the spot; the development of this calmed region is discussed in Section 7.3.4.4.

\subsubsection{Regeneration of hairpin vortices within the interior of the spot}

Figure 7.12 shows numerous hairpin vortices of various scales forming within the spot, among which a few distinct examples are highlighted to demonstrate the hairpin-vortex regeneration mechanisms occurring within the spot. The pair of hairpin vortices labelled $\mathrm{C}$ at $\tau=107$ join into a single hairpin vortex centered at about $z / \delta_{0}^{*}=0$ by $\tau=255$. The joining process is illustrated in Figure 7.13 through a simplified schematic that is based on analysis of the simulation results at intermediate timesteps. As the spot convects downstream, the pair of $\mathrm{C}$ hairpin vortices identified in Figure 7.12 at $\tau=107$ are steadily lifted away from the wall through a self-induced lift-up effect (Figure 7.13(b)). On the basis of circulation conservation, the decreasing strength of the background strain rate away from the wall drives an increase in the lateral scale of the $\mathrm{C}$ vortices as they lift up (Figure 7.13(c)). The two $\mathrm{C}$ hairpin vortices grow increasingly closer to each other in the lateral direction and eventually join to form a single hairpin vortex by $\tau=255$ (Figure $7.13(d)$ ). The vorticity in the legs of C induce strong upwash motions between the legs so that two new hairpin vortices are observed starting at $\tau=255$, labelled $\mathrm{H}$. The vorticity lines in Figure 7.12 at $\tau=492$ indicate that the $\mathrm{H}$ hairpins straddle the legs of C. 
(a) $\tau=107$

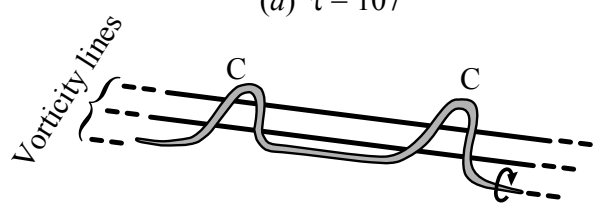

(c) $\tau=205$

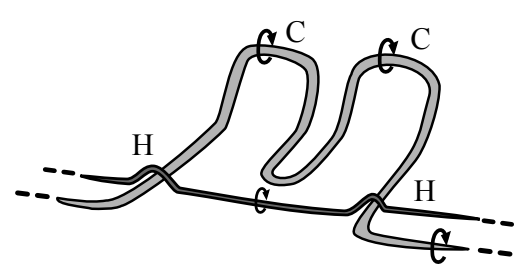

(b) $\tau=155$

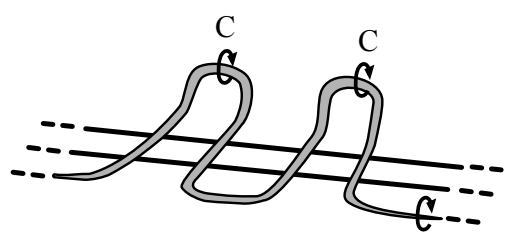

(d) $\tau=255$

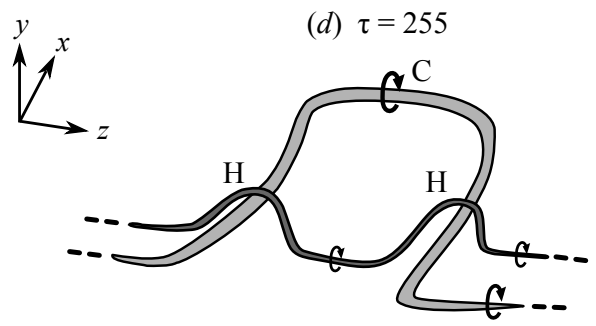

Figure 7.13: Schematic illustrating the lateral joining of a pair of hairpin vortices into a single hairpin vortex.

Several smaller-scale hairpin vortices also develop within the interior of the spot near the hairpin vortices labelled B at $\tau=255$. These smaller-scale hairpin vortices are organized into wave packets consisting of multiple (typically two or three) closely-spaced hairpin vortices that straddle one of the legs of the parent hairpin vortex, similar to the wave packets observed in turbulent boundary layers (e.g. Adrian et al., 2000). It is interesting to note that the wave packets at $\tau=255$ do not occur throughout the spot but develop only near large hairpin vortices like $\mathrm{B}$ and $\mathrm{C}$ that extend far away from the wall. This suggests that in order for a wave packet to form, the parent hairpin vortex must extend far enough into the free-stream fluid that it can be sufficiently stretched in the downstream direction. Under these conditions, the vorticity in the legs of the parent vortex increases to the point where it can induce strong upwash and downwash motions that in turn reorient the near-wall vorticity in the adjacent fluid to initiate the next generation of hairpin vortices.

The streamwise growth of wave packets occurs via the generation of multiple hairpin vortices from a single parent hairpin vortex. Figure 7.14 illustrates this process by plotting a magnified view of the wave packet that occurs near the B hairpin vortex on the $+z$ side of the spot. An arbitrary number of vorticity lines coloured according to the value of $y / \delta_{0}^{*}$ are also 

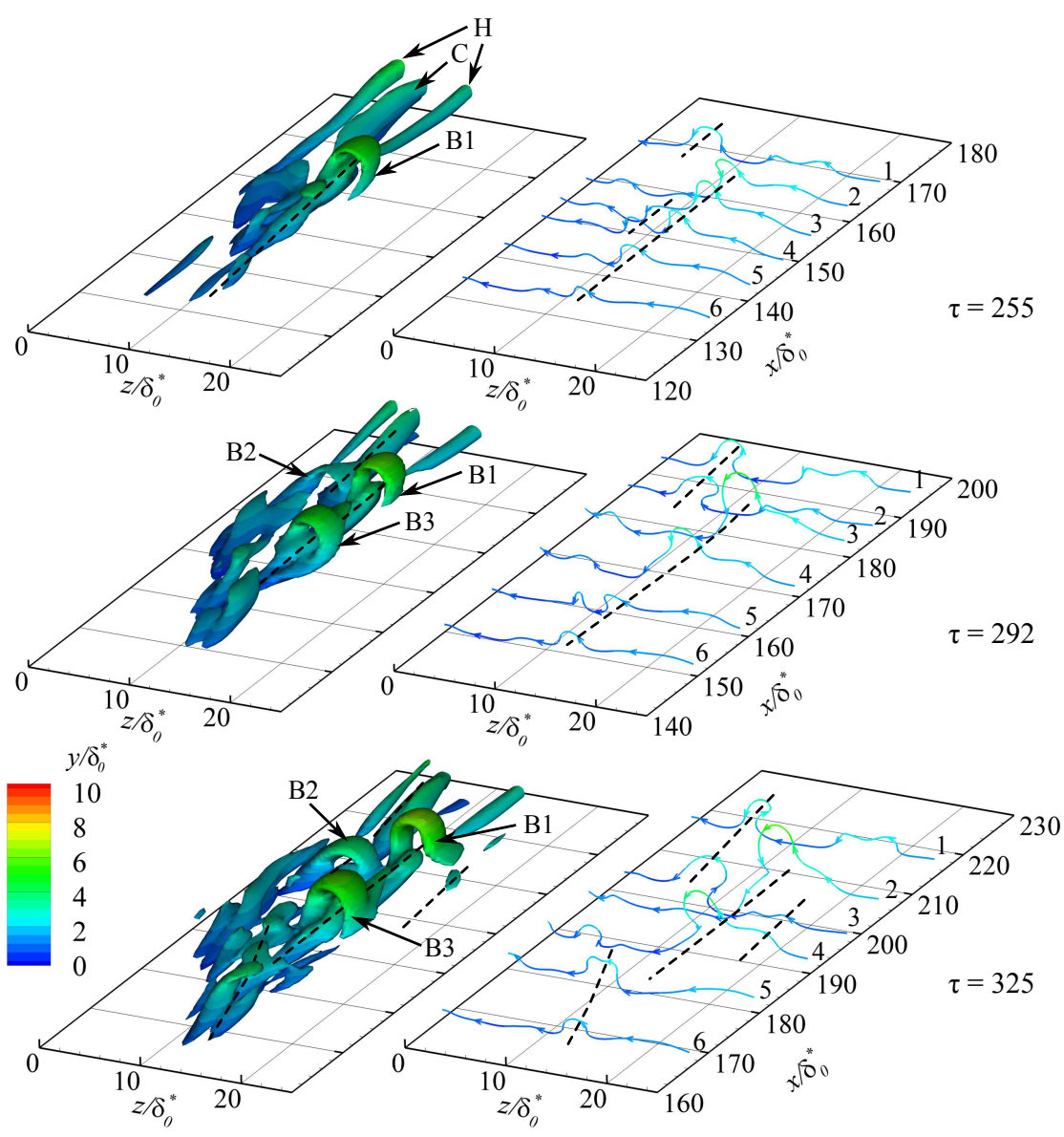

Figure 7.14: Magnified view of the development of a wave-packet between $\tau=255$ and 325, visualized through contours of $Q=0.6$ (left) and instantaneous vorticity lines (right). Isocontours and vorticity lines are coloured according to the value of $y / \delta_{0}^{*}$.

plotted to better illustrate the vortex dynamics. The primary hairpin vortex at $\tau=255$ is labelled B1. The streamwise vortices that lie downstream of B1 correspond to the legs of the C and $\mathrm{H}$ hairpin vortices. The inboard leg of B1, indicated with a long dashed line, corresponds to the streamwise-aligned sequence of perturbed vorticity lines labelled 2-6 in Figure 7.14 at $\tau=258$. Similarly, the perturbation in vorticity line 1 indicated by the short dashed line corresponds to the leg of the C hairpin vortex. By $\tau=292$, this perturbation has lengthened longitudinally to include vorticity line 2 and has amplified so that a coherent vortex resembling the head of a hairpin vortex is observed in the iso-contours, labelled B2. By $\tau=325$, B2 has grown farther from the wall into a clearly-defined hairpin vortex. Returning to $\tau=255$, a 
secondary perturbation of vorticity lines 3 and 4 is visible, indicated by the short dotted line. This secondary perturbation is created via a lift-up process whereby the stretching of the B1 vortex strengthens the vorticity field surrounding lines 3 and 4, which in turn induces the lift-up of the secondary perturbation, discussed further in Section 7.3.5. As the amplitude of the primary B1 vortex grows with time, the strengthening of the vorticity field surrounding lines 3 and 4 continues, strengthening the lift-up process so that the wall-normal height of the secondary perturbation also amplifies with time. By $\tau=292$, the perturbed vorticity field merges so that perturbed vorticity lines like line 4 in close proximity to each other coalesce to produce a secondary hairpin, labelled B3, which is clearly visible in the iso-contours. By $\tau=325$, B3 is nearly as tall as B1, and the stretching of B3 by the background shear amplifies the vorticity in its legs so that they begin to generate new perturbed vortex segments on the inboard side, as seen by the dashed line through vorticity lines 5 and 6 , and on the outboard side, as seen by the dashed line through vorticity lines 3 and 4 . Further timesteps reveal that the perturbation of vorticity lines 5 and 6 grows to produce a new wave-packet with multiple new hairpin vortices.

The regeneration process visible in Figure 7.14 can be summarized using the simplified schematics presented in Figure 7.15. The wall-normal motions induced by the legs of the parent hairpin vortex (B1 in Figure 7.14) reorients the surrounding boundary-layer spanwise vorticity in the wall-normal direction (Figure 7.15(a)). The reoriented vorticity is stretched in the streamwise direction by the background shear and the shear layer formed by the wallnormal component of vorticity rolls-up due to an inviscid instability in the $x$ - $z$ plane, leading to a streamwise grouping of wall-normal vorticity (Figure 7.15(b)). The roll-up forms one or more coherent secondary hairpin vortices that straddle a single leg of the parent hairpin vortex (Figure $7.15(c)$ ) and subsequently grow due to self-induced lift-up and stretching by the background shear (Figure 7.15(d)). This process is similar to the mechanism proposed by Schröder and Kompenhans (2004) shown in Figure 7.2. However, this is not the only hairpin- 
(a)

(b)

(c)

(d)

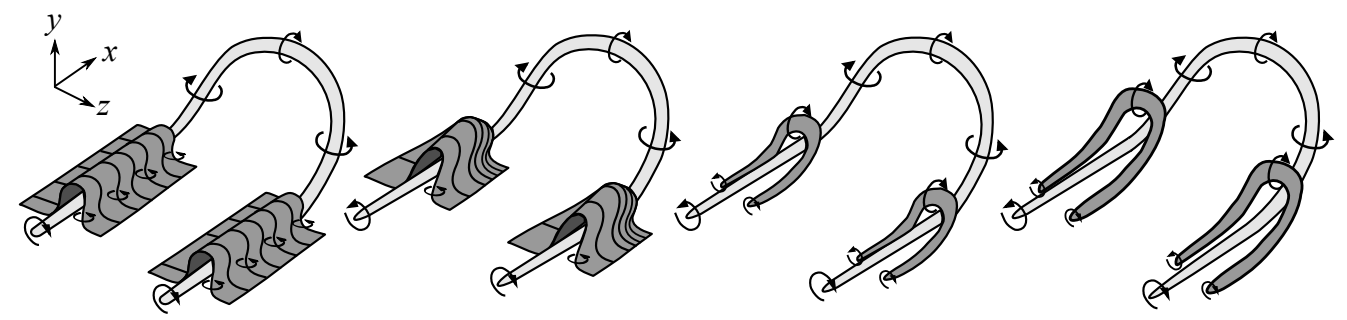

(e)

(f)

(g)

(h)
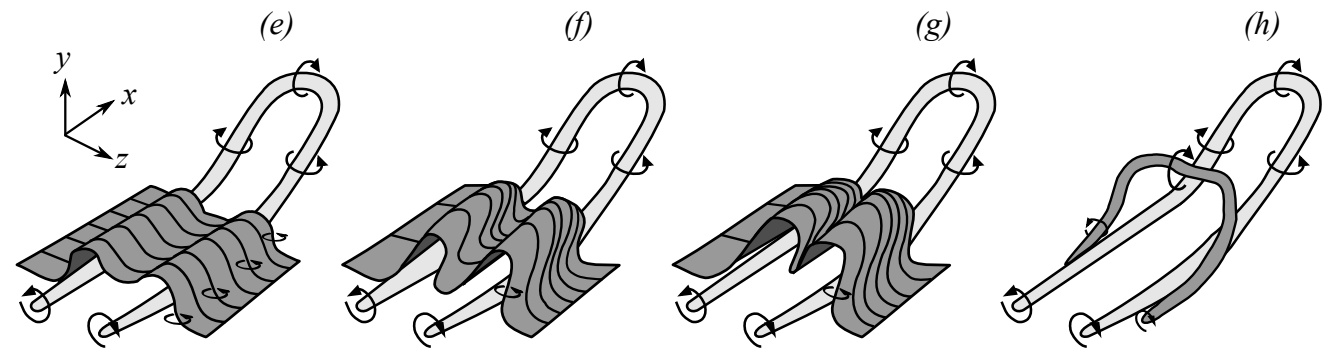

Figure 7.15: Schematic illustrating the regeneration of hairpin vortices from a parent hairpin vortex with $(a-d)$ widely-spaced and $(e-h)$ closely-spaced legs.

vortex regeneration mechanism observed in the present study. When the legs of the parent hairpin vortex are closely-spaced in the $z$ direction, as sketched in Figure 7.15(e), the regions of boundary-layer vorticity that are reoriented by the legs are also in close proximity to each other. As these regions of reoriented vorticity are stretched in the streamwise direction by the background shear, their spatial scales increase and they grow increasingly closer to each other (Figures $7.15(f)$ and $(g)$ ) until they eventually join and roll-up into a single hairpin vortex that straddles both legs of the parent hairpin vortex (Figure $7.15(h)$ ). The latter mechanism resembles the mechanism proposed by Singer and Joslin (1994) and shown in Figure 7.1. The mechanism depicted in Figure $7.15(a)-(d)$ is observed most often in the present study among the large-scale hairpin vortices near the leading and spanwise edges of the spot (such as vortices B in Figure 7.12), while the mechanism depicted in Figure 7.15(e)-( h) is observed only among the small-scale hairpin vortices that occur near the trailing-edge region of the spot. It should be stressed that despite the different outcomes for the widely- and closely-spaced parent hairpin vortices, it is the same fundamental physical process occurring in both. 


\subsubsection{Development of the leading-edge region of the spot}

The hairpin-vortex regeneration process described in Section 7.3.4.1 occurs at multiple locations within the interior of the turbulent spot so that by $\tau=492$, multiple generations of wave-packets are present. Figure $7.12(c)$ shows that the population of wave packets near the leading edge of the spot at $\tau=492$ is notably less dense than near the trailing edge. In contrast to structure B, structure C has not spawned a substantial number of smaller hairpins, and the streamwise-elongated structures adjacent to it, labelled G, have not deformed into hairpin vortices. The side view of the spot seen in Figure 7.16 shows that, especially at $\tau=492$, structure $\mathrm{C}$ is located a notable distance away from the wall where the prevailing background strain rates are much lower than closer to the wall. The $99 \%$ boundary-layer thickness of the undisturbed flow at $x / \delta_{0}^{*}=240$ is $4.42 \delta_{0}^{*}$, indicating that structure $\mathrm{C}$ reaches more than three times the local boundary-layer thickness above the wall. As a result, during this period of spot development, structure $\mathrm{C}$ undergoes a smaller degree of streamwise stretching than structures such as B that are located in closer vicinity of the wall. Furthermore, because the wall-normal gradients in the undisturbed streamwise velocity are greatly reduced (or are essentially non-existent) at this wall-normal distance, the background spanwise vorticity fieldthe deformation of which prompted the development of a series of new vortical structures around vortex B-would be absent for vortex C. Instead of spawning new hairpin vortices, Figures 7.12 and 7.16 indicate that the vortical structures near the leading edge of the spot increase their spatial scale as the spot matures. This occurs due to the entrainment of lowspeed fluid from the laminar region beneath the leading of the spot, as seen in Figure 7.16, discussed in more detail below.

To quantify the longitudinal growth rate of the spot, the trailing- and leading-edge celerities are compared to the local velocities at the corresponding wall-normal locations in the undisturbed flow. As there is no standard practice for obtaining the undisturbed local 


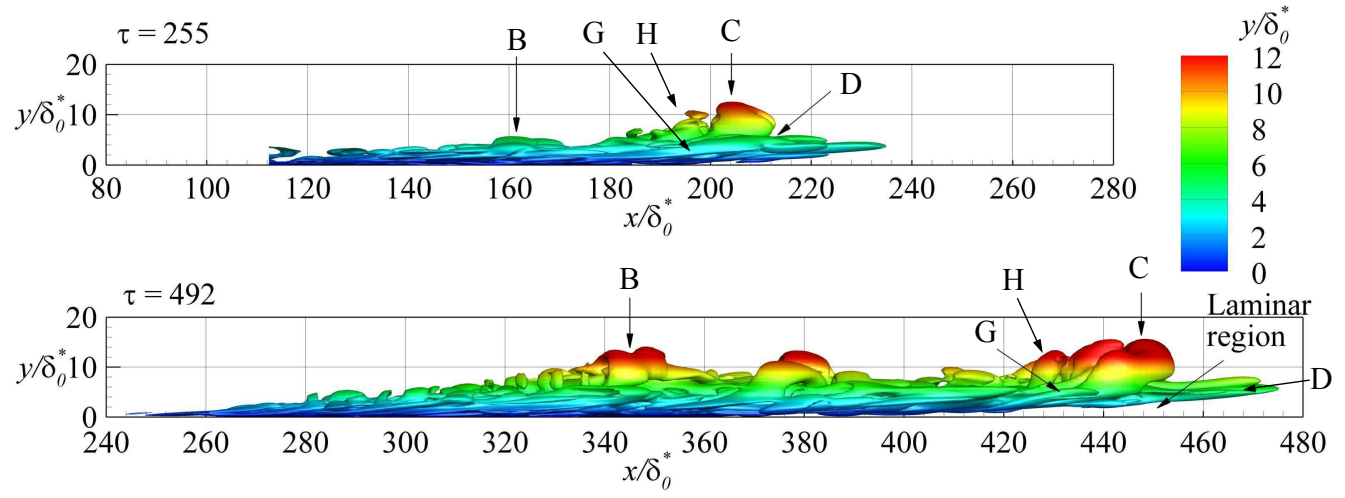

Figure 7.16: Turbulent spot at $\tau=255$ and 492, visualized through contours of $Q=0.6$, viewed in the $-z$ direction.

velocity, denoted $U_{u}$, it is presently estimated as

$$
U_{u}=\frac{1}{0.5 y_{\max }} \int_{0.25 y_{\max }}^{0.75 y_{\max }} U(y) d y
$$

where $y_{\max }$ is the maximum height of the coherent structures at the spot leading or trailing edge. The integration is performed at the trailing- and leading-edge locations for each streamwise station plotted in Figure 7.11, and these values are respectively averaged to yield mean $U_{u}$ values of $0.50 \pm 0.04 U_{0}$ and $0.98 \pm 0.02 U_{0}$ at the trailing- and leading-edge locations, respectively; the uncertainties indicate the sensitivity to the integration bounds in equation 7.4 over a range of 0.1 to $1.0 y_{\max }$. Relative to these values, the spot's trailing and leading edges, which are identified in the manner described in Section 7.3.3, move at an average speed of $0.04 \pm 0.04 U_{0}$ and $-0.14 \pm 0.02 U_{0}$, respectively. The corresponding leading- and trailing-edge celerities in the stationary frame of reference are $0.54 U_{0}$ and $0.84 U_{0}$, respectively, which agree favourably with the celerities stated in published literature for zero streamwise pressure gradients. These observations indicate that the streamwise movement of the spot trailing edge relative to the local undisturbed flow is essentially zero, while the leading edge of the spot moves at a slower rate than the local undisturbed flow.

The slower relative movement of the leading edge of the spot is caused by the entrainment 
of low-speed fluid from the laminar region. The mechanism for the entrainment of laminar fluid is observable in Figures 7.12 and 7.16. As the $\mathrm{C}$ hairpin vortex grows within the spot, the downwash motion induced ahead of it by the spanwise-oriented vorticity in its head is not spanwise-uniform, and thus initiates a reorientation of the spanwise vorticity downstream of the $\mathrm{C}$ hairpin vortex, producing the streamwise vortices labelled D. Figure 7.16 shows that the streamwise D vortices are very prominent in the spot leading edge and are located much nearer to the wall than the $\mathrm{C}$ hairpin vortex. As a result, their induced wall-normal motions are able to convect low-speed fluid into the spot leading edge (thus slowing down the leading edge relative to the local undisturbed flow) while also reorienting the spanwise vorticity in the laminar region into the wall-normal direction. The reoriented vorticity from the laminar region couples with the $\mathrm{C}$ or $\mathrm{H}$ hairpin vortices, which increases their overall circulation as noted from the spatial scales of the $\mathrm{H}$ and $\mathrm{C}$ hairpin vortices in Figure 7.16.

\subsubsection{Regeneration of wave packets along the spanwise edges of the spot}

In Figure 7.12, multiple wave packets are observed along the spanwise edges of the spot near the location where the spot reaches its maximum width, which suggests that these wave packets play a critical role in the lateral growth of the spot. To illustrate the process by which existing wave packets within the spot generate new packets of hairpin vortices along the spot's spanwise edge, Figure 7.17 plots iso-contours of $\left|\omega_{y}\right| \delta_{0}^{*} / U_{0}=0.095$ in the $-z$ half of the spot. Vorticity lines that intersect the heads of hairpin vortices within the wave packets are also plotted as an aid in connecting the coherent vortical flow structures to the surrounding vorticity field. The iso-contours are coloured according to the value of $y / \delta_{0}^{*}$ to show their relative heights and made semi-transparent to improve the visibility of the vorticity lines. The vortices that encompass the vorticity lines labelled 1 and 2 at $\tau=480$ correspond to hairpin vortices in the interior of the spot; the "head" of the hairpin vortex consists of primarily $z$ oriented vorticity and is thus not visible in the $\left|\omega_{y}\right|$ iso-contours. Vorticity line 1 is near the spot 

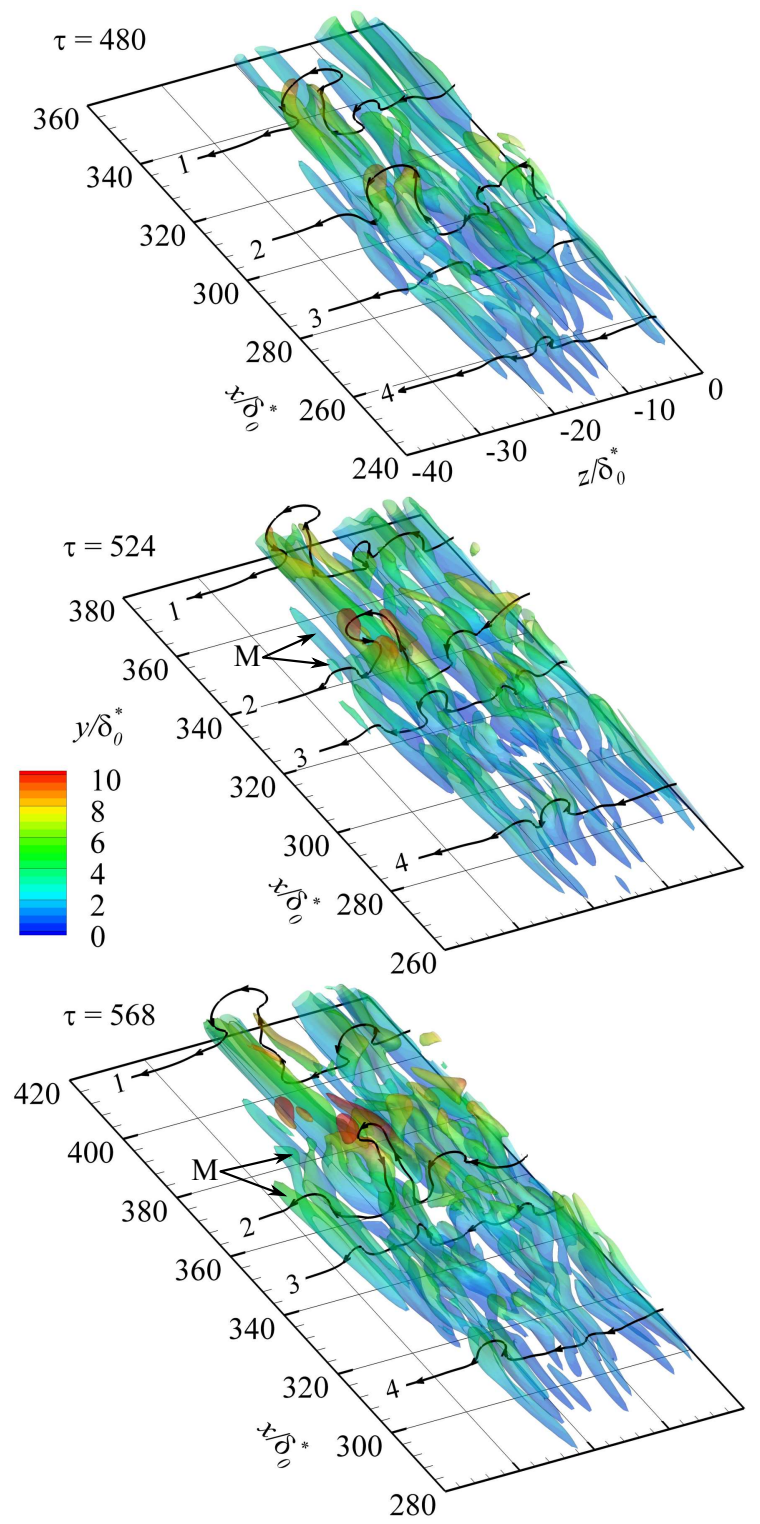

Figure 7.17: Growth of the turbulent spot at $\tau=480,524$, and 568, visualized through isocontours of $\left|\omega_{y}\right| \delta_{0}^{*} / U_{0}=0.095$ shaded by $y / \delta_{0}^{*}$ in the $-z$ half of the spot. Solid lines illustrate vorticity lines that intersect the coherent vortical structures. 

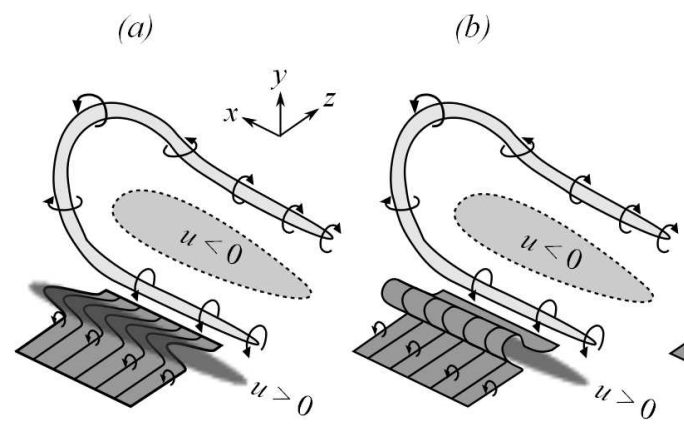

(c)

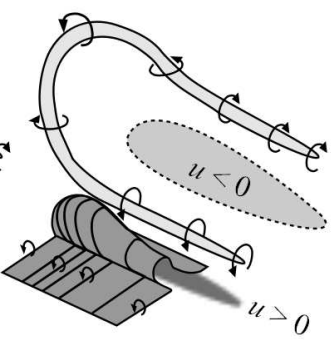

(d)

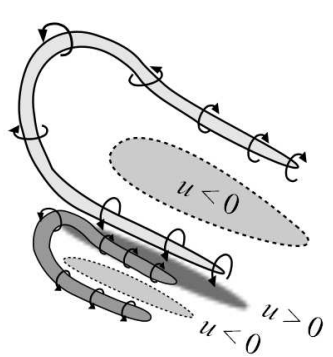

Figure 7.18: Schematic illustrating the regeneration mechanism of wave packets along the spanwise edge of the turbulent spot.

leading-edge, and as such it undergoes minimal temporal variation for the reasons discussed in Section 7.3.4.2. Vorticity line 2 is near the location of maximum spot width where the lateral growth rate of the spot is highest, and hence the vorticity field there undergoes a significant temporal evolution. At $\tau=524$, the hairpin vortex encompassing line 2 has been stretched in the streamwise direction by the mean shear and the stretched vortex induces a wall-normal perturbation on the outboard segment of this vorticity line. The iso-surfaces in the region of the perturbed vorticity line, labelled M, indicates that two streamwise-aligned regions of wallnormal vorticity are created, each one corresponding to the $-\omega_{y}$ and $+\omega_{y}$ regions indicated by the perturbed segments of vorticity line 2. By $\tau=568$, the vorticity field surrounding lines 2 and 3 has been significantly perturbed in the wall-normal direction as the M-labelled regions of wall-normal vorticity have lengthened and become stronger.

The regeneration process visible in Figure 7.17 can be summarized using the simplified schematics presented in Figure 7.18. As in Figure 7.15, the parent hairpin vortex induces upwash between its legs and downwash to the outside of its streamwise-oriented legs. The upwash ejects low-momentum fluid and forms a low-speed streak between the legs of the parent hairpin vortex, while the downwash brings fluid with high streamwise momentum into the boundary layer on the outboard side of the parent vortex (Figure 7.18(a)). Because the hairpin vortex is located at the laminar/turbulent interface, the downwash of high-momentum 
fluid on its outboard side stretches the background spanwise vorticity into the streamwise direction, as shown in Figure 7.18(a), introducing a streamwise component into the boundarylayer vorticity. The streamwise components of vorticity activate a lift-up of the stretched vorticity into the wall-normal direction (Figure 7.18(b)). As in Figure 7.15, Figure 7.18(c) shows that the wall-normal vorticity shear layers are unstable via an inviscid instability that produces a streamwise grouping of wall-normal vorticity and the eventual roll-up of the wallnormal vorticity shear layers into one or more discrete hairpin vortices (Figure 7.18(d)). As the secondary hairpin vortex is stretched by the background shear, it becomes stronger and begins to induce wall-normal motions that form a new series of high- and low-speed streaks along the spanwise edge of the turbulent spot. This sequence indicates that the formation of new wave packets along the spanwise edges of the spot follows the same fundamental processes as the creation of hairpin vortices within the spot interior described in Section 7.3.4.1.

The regeneration of wave packets along the spanwise edge of the spot can be linked to the temporal evolution of the velocity field at the same time instances shown in Figure 7.17 by analyzing the flow field at a constant streamwise location with respect to the spot trailingand leading-edges. This is realized through a streamwise coordinate referenced to the spot trailing-edge location and normalized by the spot length, defined as

$$
\zeta=\frac{\left(x-x_{t e}\right)}{\left(x_{l e}-x_{t e}\right)}
$$

where $x_{t e}$ and $x_{l e}$ are the streamwise locations of the trailing and leading edges of the spot, respectively. Figure 7.19 visualizes the streamwise streaks in $y-z$ planes at $\zeta=0.25,0.50$, and 0.75 through contours of $u / U_{0}$, where $u$ is the perturbation velocity defined earlier. Three simulation times $(\tau=391,480$, and 568) are shown to illustrate the development of the streamwise streaks as the spot develops. At all locations and times, the sides of the spot are consistently terminated by a low-speed streak. At $\zeta=0.25$, shown in Figure $7.19(a)$, the spanwise spacing of the interior streaks does not change significantly between the three 
simulation times, indicating that the spot's lateral growth cannot be attributed to lateral spreading of the streaks as they are fed by the induced wall-normal motions of the hairpin and streamwise vortices, although their sizes do vary somewhat. The outermost streaks, which correspond to the structure labelled $\mathrm{G}$ in Figure 7.12, are barely visible at $\tau=391$, become stronger and larger at $\tau=480$, and by $\tau=568$ have reached a size and strength that are comparable to those of the interior streaks. At $\tau=568$, a new streak is observed on the $-z$ side of the spot, labelled I, suggesting that the creation of new wave packets of hairpin vortices (and hence streaks) along the spanwise edges of the spot occurs with a temporal period close to $180 \tau$.

\subsubsection{Formation of the calmed region}

A ubiquitous property of turbulent spots since the early observations of Schubauer and Klebanoff (1956) has been the observation of a calmed region in the wake of the spot, upstream of the spot trailing edge, with this motion yielding a fuller streamwise velocity profile and thus enhanced stability. Johnson (2001) and Sabatino and Smith (2008) noted that the calmed region forms due to the wall-normal transfer of high-momentum fluid from higher in the boundary layer towards the wall. Johnson (2001) attributed this wall-normal momentum exchange to a spanwise-aligned vortex that lies on the laminar/turbulent interface at the spot trailing edge, while Sabatino and Smith (2008) attributed it to the sweep motions induced by the hairpin vortices that form near the trailing edge of the spot. As no spanwise-aligned vortex is observed near the spot trailing edge in the present numerical results (e.g. Figure 7.12), the mechanism proposed by Johnson (2001) is not supported by the present results. Furthermore, noting that the farthest-upstream sets of hairpin vortices are still at least $10 \%$ of the spot length downstream of the spot trailing edge (e.g. Figure 7.17) while the calmed region extends more than $20 \%$ of the spot length upstream of the spot trailing edge, it seems unlikely that the small hairpin vortices create such strong wall-normal motions that they are able to stabilize 

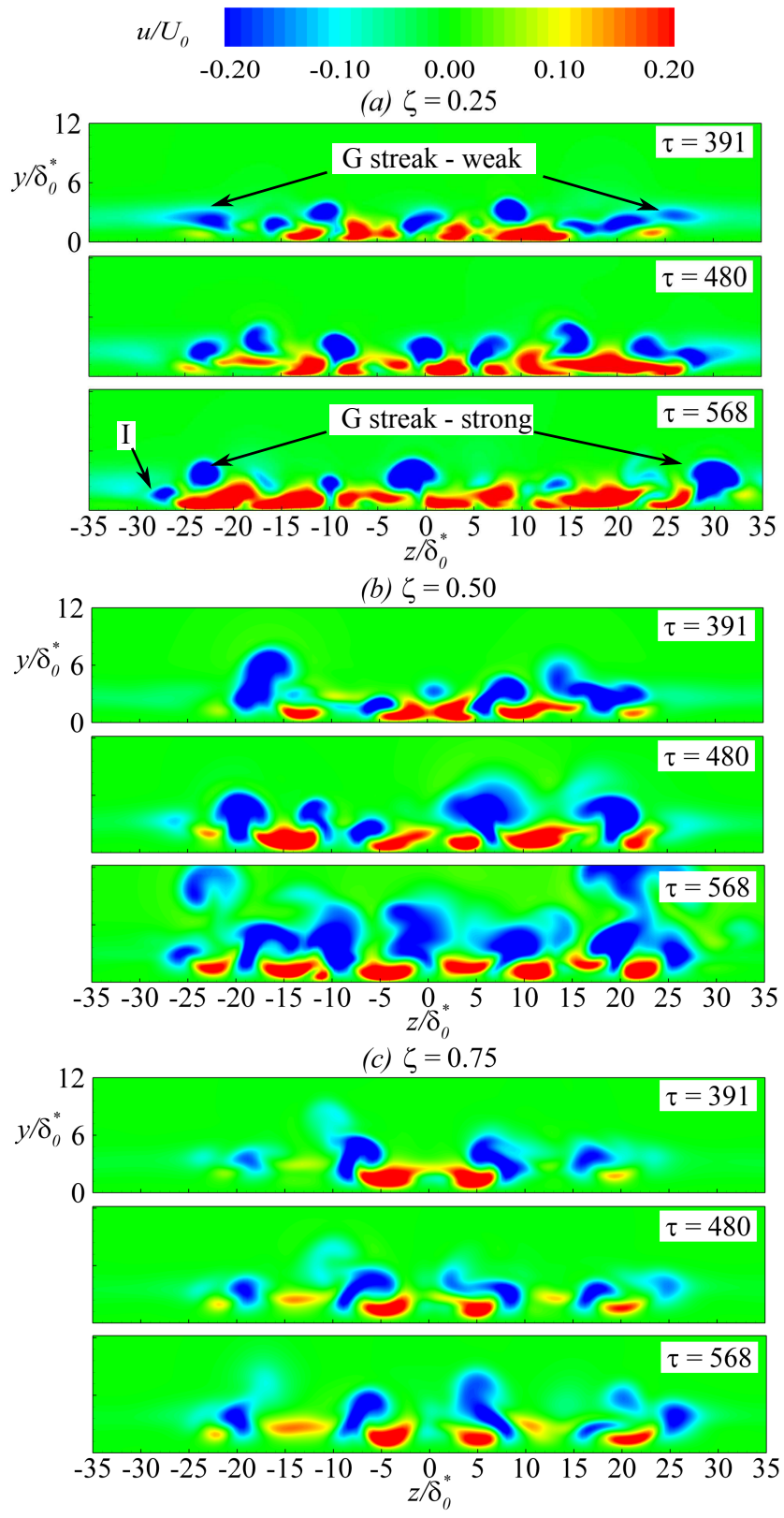

Figure 7.19: Contours of the streamwise perturbation velocity $u / U_{0}$ showing the development of streamwise streaks in the turbulent spot. Data is plotted in $y-z$ planes, located at $(a) \zeta=0.25$, (b) $\zeta=0.50$, and $(c) \zeta=0.75$. The simulation times correspond to $\tau=391,480$, and 568 . 
the flow so far upstream, as Sabatino and Smith (2008) propose. The present results therefore suggest that a different mechanism may be responsible for the formation of the calmed region.

To illustrate this mechanism, Figure 7.20 plots the instantaneous flowfield in the wake of the spot at $\tau=568$ in a $x-z$ plane located at $y / \delta_{0}^{*}=2.0$. Figure $7.20(a)$, which plots contours of the streamwise perturbation velocity, shows that the spot wake contains wide high-speed streaks in the boundary layer. These high-speed streaks account for the extra momentum that yields the fuller, more stable velocity profile within the calmed wake. The loci of upwash and downwash motions (Q2 and Q4, respectively) plotted in Figure 7.20(a) show that while regions of upwash are predominately confined to within the spot, large regions of downwash are observed in the calmed wake and coincide with the locations of the wide high-speed streaks, indicating that the streaks are indeed created by wall-normal momentum transfer from the high-speed fluid farther from the wall, as proposed by Johnson (2001) and Sabatino and Smith (2008), although apparently from a different origin than they propose. Figure 7.20(b) illustrates the origin of the downwash motions by plotting contours of streamwise perturbation vorticity, $\omega_{x}$, and lines of spanwise perturbation vorticity, $\omega_{z}=-0.063 U_{0} / \delta_{0}^{*}$ in the same $x-z$ plane as Figure $7.20(a)$. The contour lines of $\omega_{z}=-0.063 U_{0} / \delta_{0}^{*}$ show that the downwash is created by streamwisealigned "strips" of enhanced spanwise vorticity that extend from the spot into the wake and coincide directly with the locations of the high-speed streaks observed within the wake. The strips of enhanced spanwise vorticity are created inside the spot as a result of the hairpinvortex regeneration cycles that cyclically generate spanwise perturbation vorticity within the spot. As the spot convects downstream, the fluctuating spanwise vorticity embedded in the slower-moving fluid near the wall extends from the spot trailing edge into the wake, inducing downwash motions and producing a calmed region that grows in sync with the longitudinal growth of the spot. Viscous diffusion limits the effective streamwise length of the strips of enhanced spanwise vorticity, ensuring that the calmed region has a finite length. 


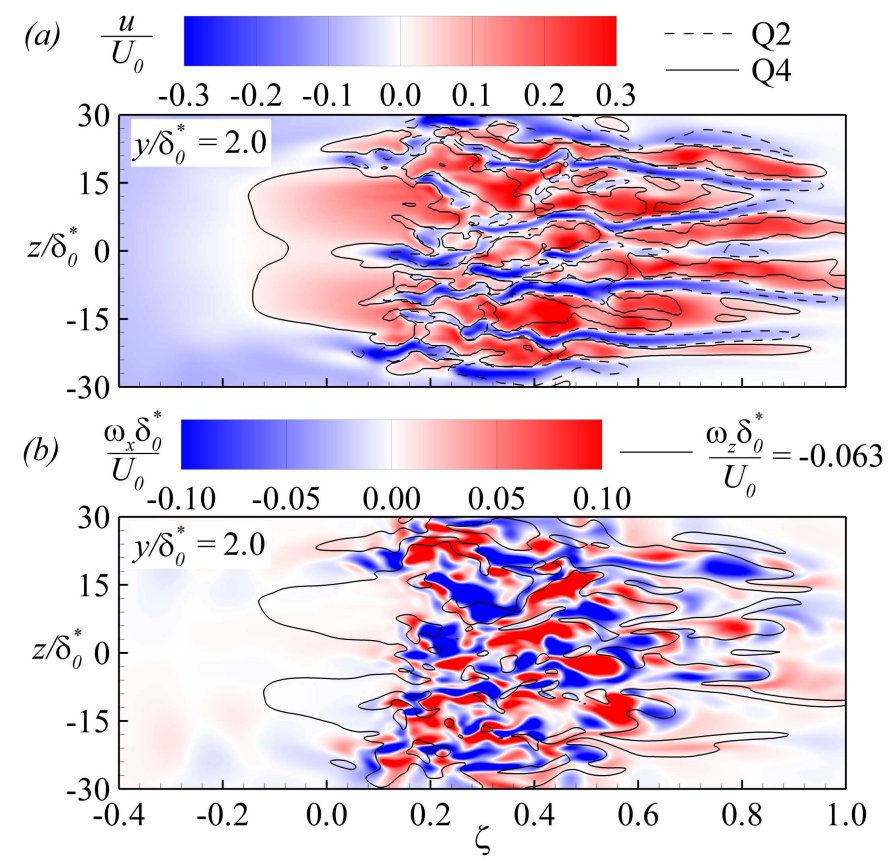

Figure 7.20: Formation of the calmed region upstream of the spot viewed through contours of $(a) u / U_{0}$ and $(b) \omega_{x} \delta_{0}^{*} / U_{0}$ in a $x$-z plane at $y / \delta_{0}^{*}=2.0$ at $\tau=568$. Lines in $(a)$ are of Q2 (dashed) and Q4 (solid) and in $(b)$ are of $\omega_{z} \delta_{0}^{*} / U_{0}=-0.063$. The solid vertical line indicates the spot trailing-edge location. 


\subsubsection{Quantitative description of the spot growth mechanisms}

A quantitative description of the physical mechanisms contributing to the development of new wave packets along the spanwise edges the turbulent spot, which was described qualitatively in Section 7.3.4.3, is obtained by examining the transport of enstrophy, $\xi$, defined as the trace of the perturbation vorticity covariance, $\overline{\omega_{i} \omega_{i}}$. Park et al. (2012) have shown that this property exhibits similar distributions in transitional and fully-developed turbulence, and thus can be used for assessing the non-linear processes that promote the growth of turbulent fluctuations along the spot edge. The transport equation for enstrophy is obtained by the scalar product of the transport equation for the instantaneous vorticity by the instantaneous vorticity vector, time averaging, and then subtracting the scalar product of the transport equation for the time-mean vorticity and the time-mean vorticity vector (e.g. Tennekes and Lumley (1972)), yielding:

$$
\begin{aligned}
& \frac{D}{D t}\left(\frac{\xi}{2}\right)=\underbrace{\overline{\omega_{i}^{\prime} \omega_{j}^{\prime}} \frac{\partial U_{i}}{\partial x_{j}}}_{P 1}+\underbrace{\overline{\omega_{i}^{\prime} \frac{\partial u_{i}^{\prime}}{\partial x_{j}}} \Omega_{j}}_{P 2}-\underbrace{\overline{u_{j}^{\prime} \omega_{i}^{\prime} \frac{\partial \Omega_{i}}{\partial x_{j}}}}_{P 3}+\underbrace{\overline{\omega_{i}^{\prime} \omega_{j}^{\prime} \frac{\partial u_{i}^{\prime}}{\partial x_{j}}}}_{P 4} \\
& -\underbrace{\frac{1}{2} \frac{\partial}{\partial x_{j}}\left(\overline{u_{j}^{\prime} \omega_{i}^{\prime} \omega_{i}^{\prime}}\right)}_{T}+\underbrace{\nu \frac{\partial^{2}}{\partial x_{j} \partial x_{j}}\left(\frac{\xi}{2}\right)}_{D}-\underbrace{\nu \overline{\frac{\partial \omega_{i}^{\prime}}{\partial x_{j}} \frac{\partial \omega_{i}^{\prime}}{\partial x_{j}}}}_{Y}
\end{aligned}
$$

where Einstein summation is implied over the indexed terms. The four production-rate terms can be attributed to the stretching of perturbation vorticity by the local time-mean strain rate $(P 1)$, the stretching of the time-mean vorticity by the fluctuating components of the local strain rate $(P 2)$, exchange of time-mean vorticity into perturbation vorticity by velocity fluctuations occurring in the presence of a local spatial gradient in the time-mean vorticity (P3), and stretching of perturbation vorticity by the fluctuating components of the local strain

rate $(P 4)$. As noted by Tennekes and Lumley (1972), the $\overline{u_{j}^{\prime} \omega_{i}^{\prime}}$ in the $P 3$ term is analogous to the Reynolds stress tensor and it generally has a negative value, hence the negative sign on the $P 3$ term. The physical basis of the $P 3$ term is discussed further in Appendix $\mathrm{B}$. The 
remaining terms represent the rate of transport of enstrophy by turbulent fluctuations $(T)$, viscous diffusion of enstrophy $(D)$, and viscous dissipation of enstrophy $(Y)$. Figure 7.21 plots the distribution of these terms normalized by $\left(U_{0} / \delta_{0}^{*}\right)^{3}$ at time $\tau=568$ in a $y$ - $z$ plane that intersects the spot at $\zeta=0.3$. The vertical line at $z / \delta_{0}^{*}=29.7$ indicates the spanwise edge of the spot as identified in Section 7.3.3. The positive growth rate of enstrophy outboard of the spot seen in Figure $7.21(a)$ is observed to occur primarily via production through the $P 2$ term (Figure 7.21(f)) and to a lesser extent, the $P 1$ and $P 4$ terms (Figure 7.21(e) and (h), respectively). Transport of enstrophy from the interior of the spot by viscous diffusion and convection by the turbulent perturbations also contributes to the growth of turbulence outboard of the spot, as seen in Figures 7.21(b) and $(c)$, respectively, but this effect is much smaller than the production. Similarly, the dissipation of enstrophy, seen in Figure $7.21(d)$ is much smaller than the production terms.

The enstrophy production rates observed in Figure 7.21 can be linked to specific coherent structures developing outboard of the spot edge by examining the components that make up the $P 1, P 2$, and $P 4$ production terms in that location, shown in Figures 7.22 and 7.23. Due to the nature of the laminar baseflow, only three $P 1$ and $P 2$ components are non-zero, of which the $P 1$ term is dominated by the $\overline{\omega_{x}^{\prime} \omega_{y}^{\prime}} \partial U / \partial y$ component (Figure $7.22(b)$ ) and the $P 2$ term is dominated by the $\overline{\omega_{x}^{\prime} \partial u^{\prime} / \partial z} \Omega_{z}$ and $\overline{\omega_{z}^{\prime} \partial w^{\prime} / \partial z} \Omega_{z}$ components, respectively denoted $P 2 a$ and $P 2 b$. Figure 7.23 shows that the $P 4$ term is dominated by the $\overline{\omega_{y}^{\prime} \omega_{z}^{\prime} \partial w^{\prime} / \partial y}$ and $\overline{\omega_{x}^{\prime} \omega_{z}^{\prime} \partial u^{\prime} / \partial z}$ components, respectively denoted $\mathrm{P} 4 \mathrm{a}$ and $\mathrm{P} 4 \mathrm{~b}$. The manner in which the coherent vortical structures developing in the spot generate these enstrophy budget components can be seen in the vorticity and velocity fields plotted in the same $y-z$ plane, shown in Figure 7.24. The lines in Figure 7.24(a) are the projection of the instantaneous vorticity field onto the $y$ - $z$ plane and the contours illustrate the perturbation streamwise vorticity, $\omega_{x}^{\prime} \delta_{0}^{*} / U_{0}$, while the vectors shown in Figure 7.24(b) are the perturbation tangential $\left(v^{\prime} / U_{0}\right.$ and $\left.w^{\prime} / U_{0}\right)$ velocities and the contours illustrate $u^{\prime} / U_{0}$. The vorticity lines labelled 1-4 in Figure 7.24 intersect a hairpin vortex that 

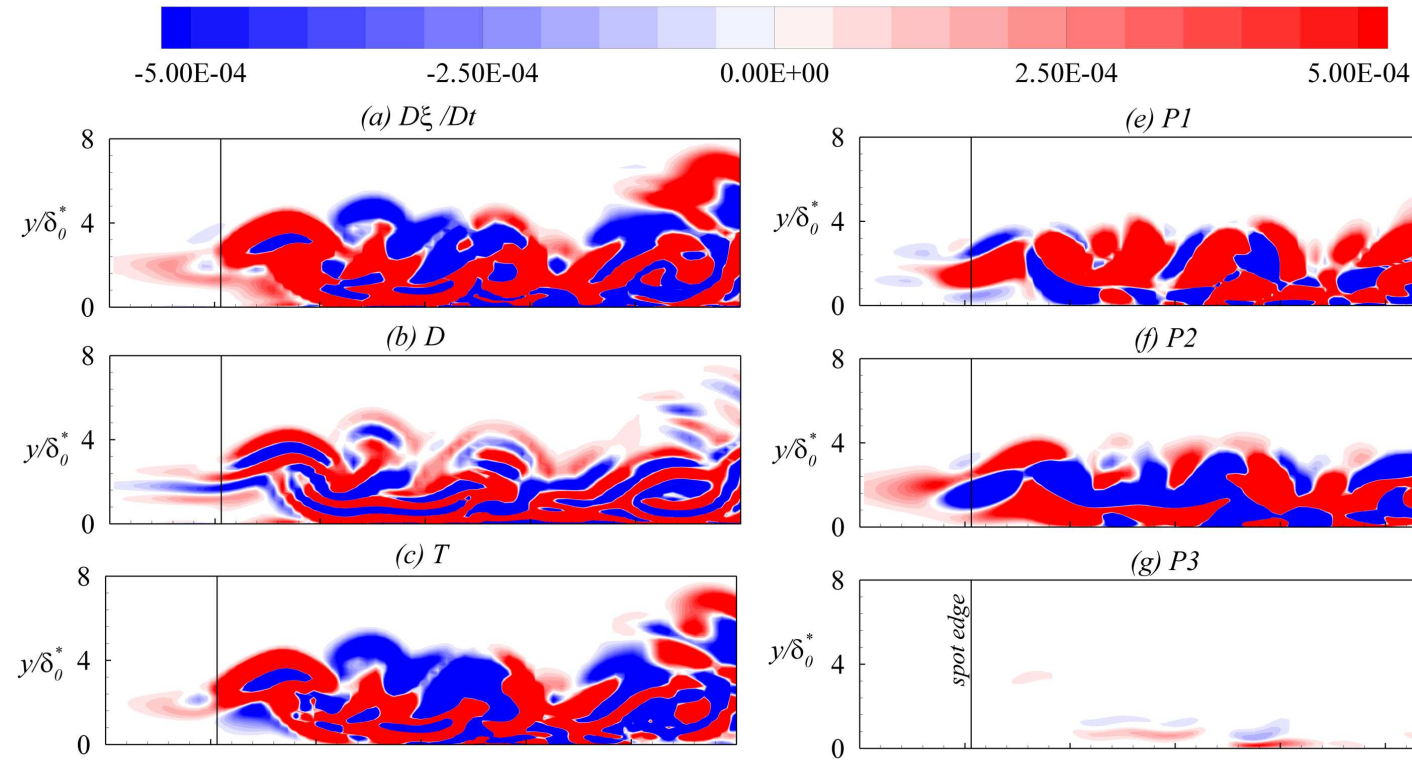

(e) $P 1$
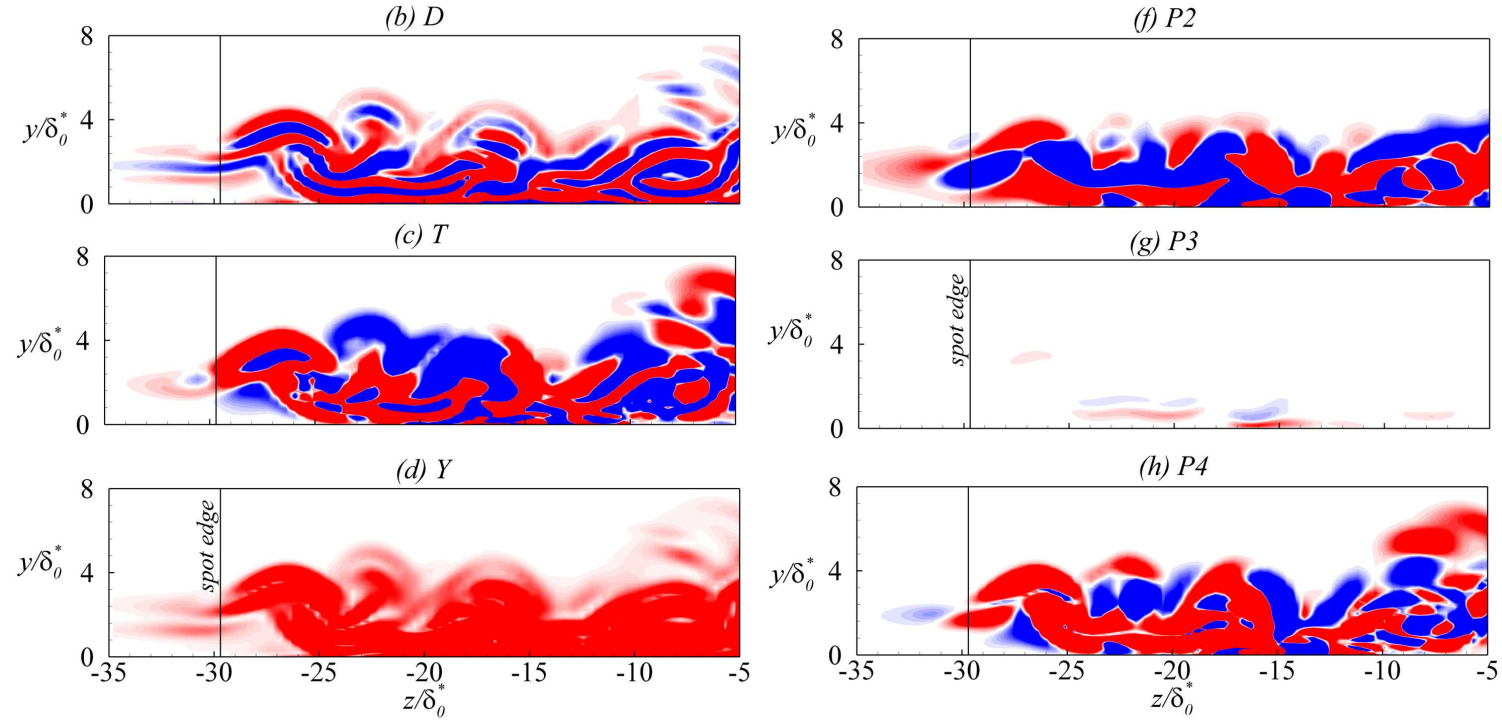

Figure 7.21: Contour levels of the budgets of Equation 7.6 in the $-z$ side of the spot at $\tau=568$ and $x / \delta_{0}^{*}=345$. 

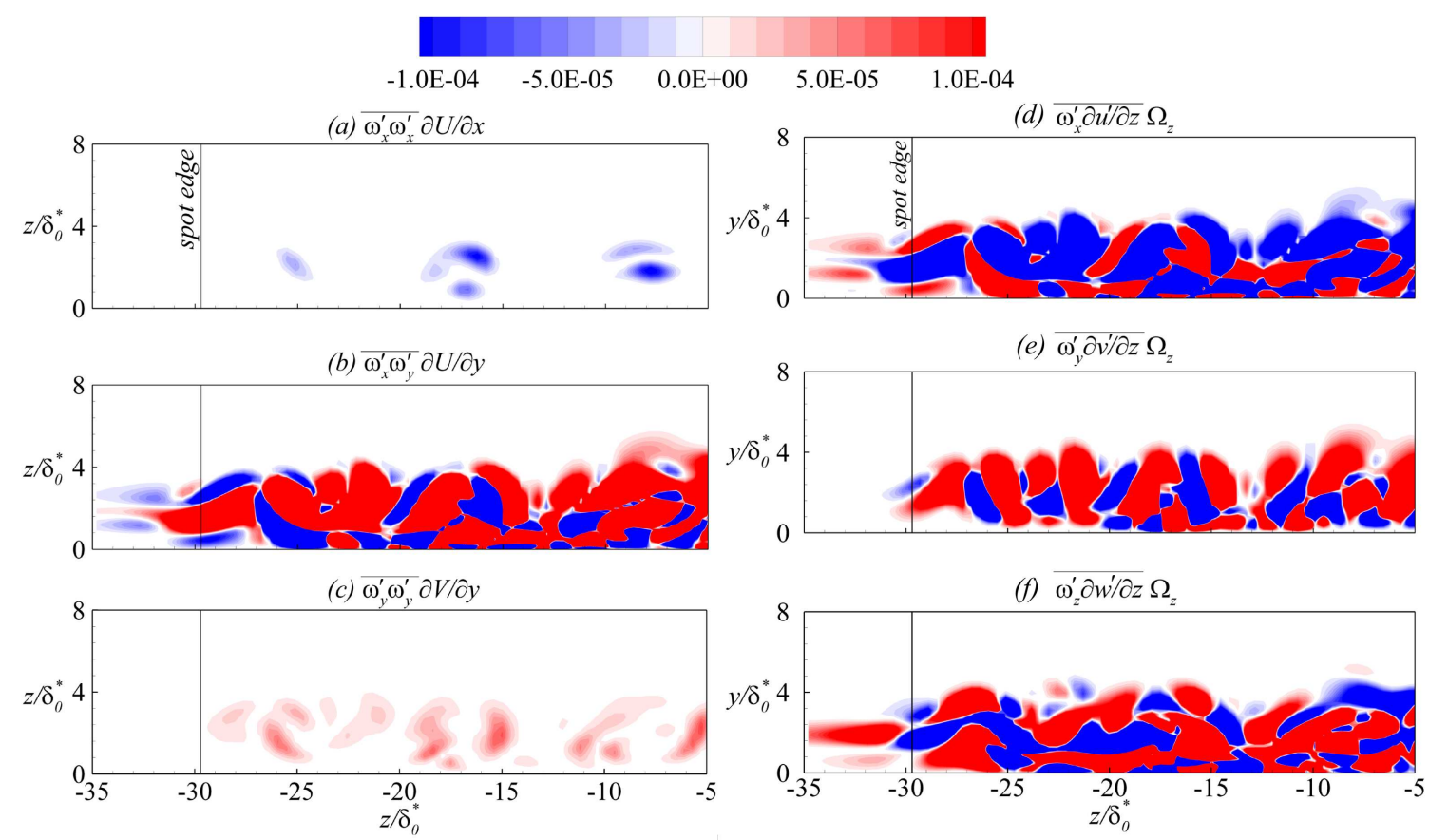

Figure 7.22: Contour levels of the components of the $P 1$ terms $(a)-(c)$ and $P 2$ terms $(d)-(f)$ from Equation 7.6 in the $-z$ side of the spot at $\tau=568$ and $x / \delta_{0}^{*}=345$.

is situated near the edge of the spot; this is the same hairpin vortex that was shown previously in Figure 7.17 encompassing vorticity line 2. The ejection of low-speed fluid between the legs of the hairpin vortex encompassing the vorticity lines labelled 1-4 generates the low-speed streak labelled G in Figure 7.24(b) and stretches and reorients the near-wall spanwise vorticity into the wall-normal direction. This wall-normal vorticity corresponds to the outer segment of the $M$ vortex labelled in Figure 7.17. As the wall-normal vorticity is tilted in the streamwise direction by the background shear, it produces a streamwise vortex labelled L. The L vortex induces strong sweep and ejection motions on its inboard and outboard sides, respectively. The sweep produces the broad high-speed streak seen below the G streak in Figure 7.24(b), while the ejection produces a low-speed streak, labelled I, and due to the non-zero spanwise velocity component of the ejected fluid, also produces weak spanwise motions outboard of the spot directed away from the spot. The near-wall spanwise motions outboard of the spot that are directed inwards are created as the low-momentum fluid convects towards the spot to replace 

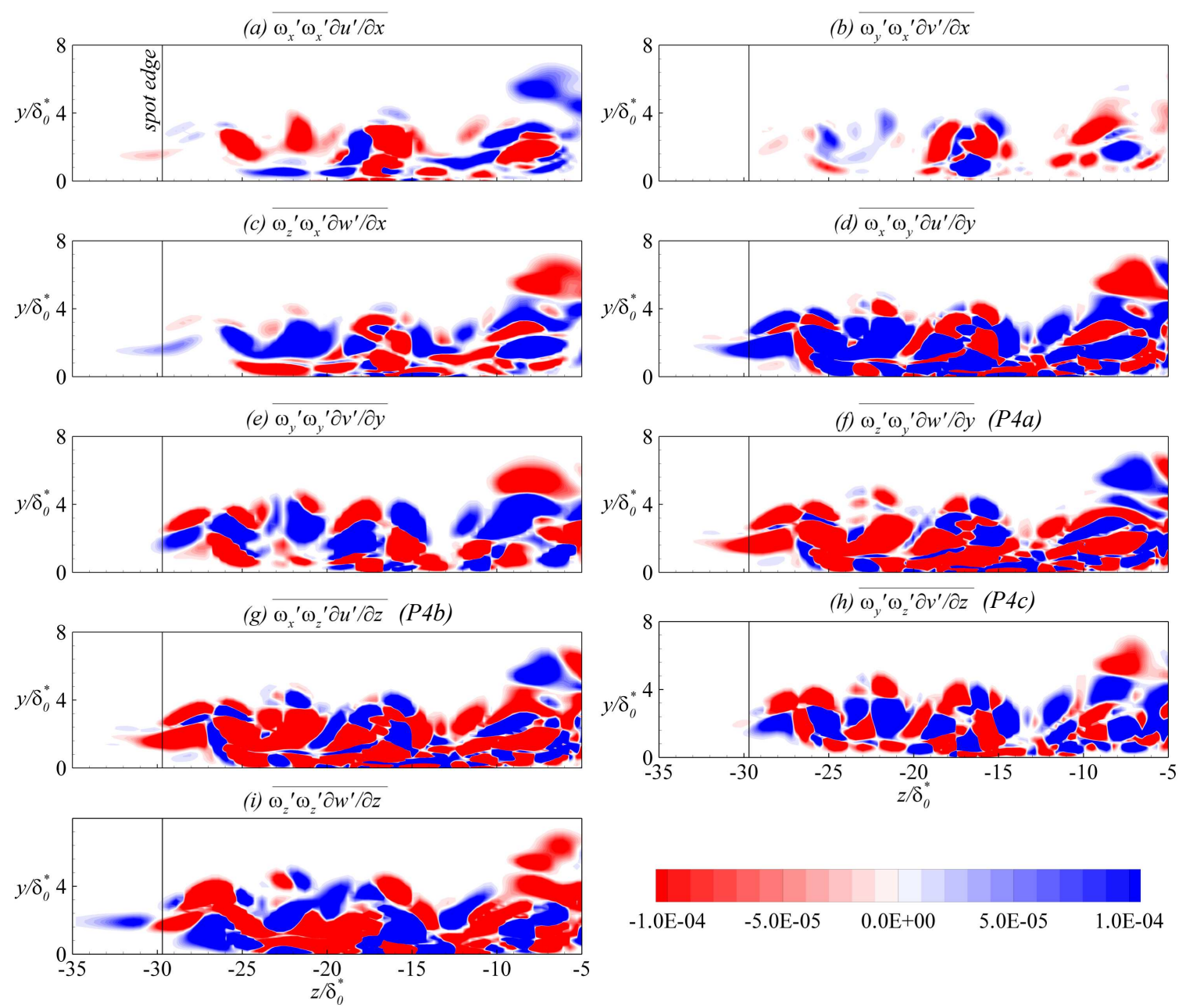

Figure 7.23: Contour levels of the components of the $P 4$ terms from Equation 7.6 in the $-z$ side of the spot at $\tau=568$ and $x / \delta_{0}^{*}=345$.

the near-wall fluid ejected by the L vortex.

The connection between the velocity and vorticity fields plotted in Figure 7.24 and the enstrophy production terms shown in Figures 7.22 and 7.23 is illustrated with the aid of the simplified schematics shown in Figure 7.25. The $P 1$ term is largely created as the wall-normal perturbation vorticity, $\omega_{y}^{\prime}$, is tilted and stretched by the mean strain rate, $\partial U / \partial y$, to produce streamwise vorticity perturbations, $\omega_{x}^{\prime}$, generating enstrophy through the $P 1$ term, as sketched in Figure 7.25(a). This process forms, e.g., the L streamwise vortex in Figure 7.24(a). The 
(a)

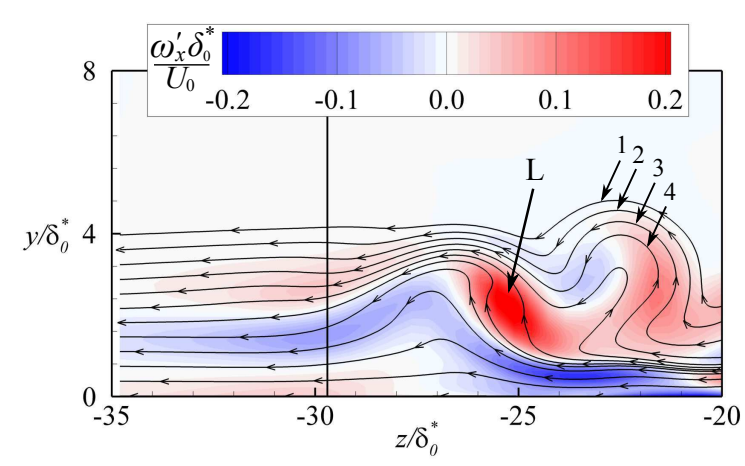

(b)

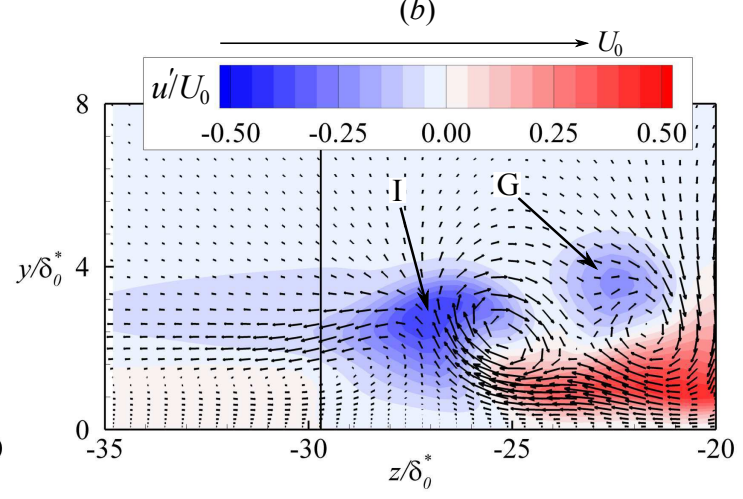

Figure 7.24: Vorticity and velocity fields in the $-z$ side of the spot at $\tau=568$ and $x / \delta_{0}^{*}=345$. In $(a)$, lines are instantaneous vorticity projected onto the $y$ - $z$ plane and flood-contours are streamwise perturbation vorticity. In $(b)$, vectors are velocity perturbation in the $y$ - $z$ plane and flood-contours are streamwise velocity perturbation.

spanwise motions outboard of the spot form regions of $\partial u^{\prime} / \partial z$ and $\partial w^{\prime} / \partial z$, as sketched in Figure $7.25(b)$ and $(c)$. The $\partial u^{\prime} / \partial z$ strain rate tilts the local mean spanwise vorticity in the streamwise direction to produce fluctuating streamwise vorticity, $\omega_{x}^{\prime}$, generating enstrophy through the $P 2 a$ term, as sketched in Figure 7.25(b), while the $\partial w^{\prime} / \partial z$ strain rate stretches or squeezes the local spanwise vorticity so that its amplitude increases or decreases relative to the background mean vorticity, $\Omega_{z}$. The resulting fluctuating spanwise vorticity, $\omega_{z}^{\prime}$, has a sign opposite of the local value of $\partial w^{\prime} / \partial z$ and thus the enstrophy growth rate associated with the $P 2 b$ term is positive outboard of the spot, which agrees with the contours shown in Figure 7.22(f). The spanwise motions outboard of the spot also generate significant regions of $\partial w^{\prime} / \partial y$, which Figure $7.25(d)$ shows tilts the $\omega_{y}^{\prime}$ vorticity associated with the L vortex in the $y$ - $z$ plane to generate $\omega_{z}^{\prime}$ and produce enstrophy through the $P 4 a$ term. Figure $7.25(e)$ shows that the $\partial u^{\prime} / \partial z$ strain rate that is induced by the sweep/ejection motions associated with the L vortex tilts the $\omega_{z}^{\prime}$ generated by the $P 2$ and $P 4 a$ terms in the $x-z$ plane to generate $\omega_{x}^{\prime}$, thus producing enstrophy through the $P 4 b$ term. Similarly, Figure $7.25(f)$ shows that the $\partial v^{\prime} / \partial z$ strain rate induced by the $\mathrm{L}$ vortex tilts $\omega_{z}^{\prime}$ in the $y-z$ plane to generate $\omega_{y}^{\prime}$. 

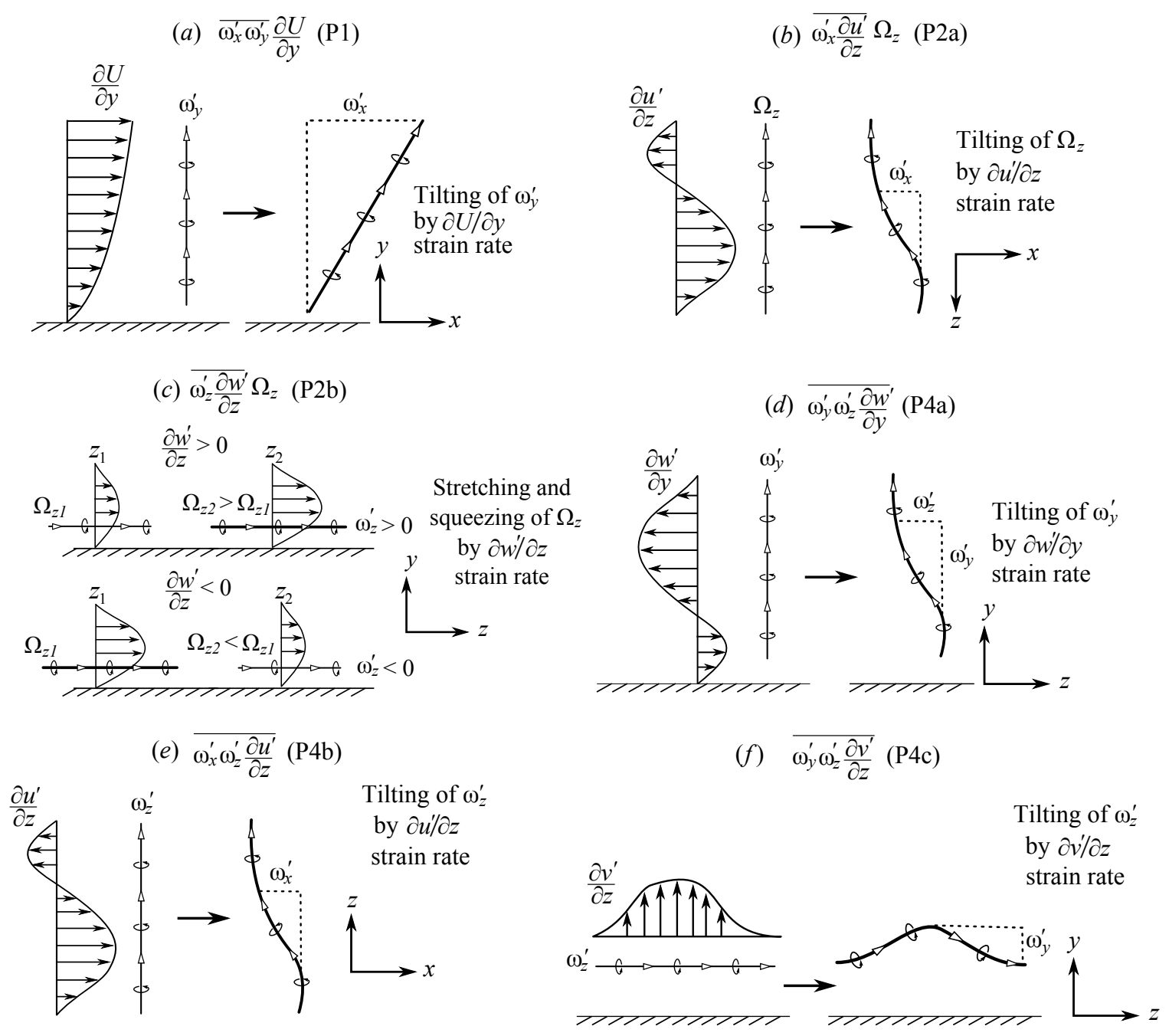

Figure 7.25: Schematic illustrating the enstrophy production mechanisms embodied in $(a)$ the $P 1$ term, $(b)-(c)$ the $P 2$ terms, and $(d)-(f)$ the $P 4$ terms. Filled arrows denote velocity vectors, hollow arrows denote vorticity vectors. Thicker vorticity lines indicate an increase in the contained vorticity. 
The relationship between the above processes and the regeneration mechanisms leading to growth of turbulence along the lateral edge of the spot is illustrated diagrammatically in Figure 7.26. Once the hairpin vortex corresponding to the vorticity lines labelled 1-4 in Figure 7.24 induces a wall-normal component in the fluctuating vorticity field on its outboard side, the $P 1$ process produces $\omega_{x}^{\prime}$ (vortex L) that induces sweep and ejection motions on either side. These sweep/ejection motions initiate three distinct regeneration cycles: in the $P 2 b$ cycle, the $\partial w^{\prime} / \partial z$ strain rate forms $\omega_{z}^{\prime}$ via the $P 2 b$ process, and due to the $P 4 c$ process, this $\omega_{z}^{\prime}$ is reoriented in the wall-normal direction to yield new $\omega_{y}^{\prime}$, which is fed back into the $P 1$ process to close the cycle. In the $P 4 a$ cycle, the $\partial w^{\prime} / \partial y$ strain rate tilts the existing $\omega_{y}^{\prime}$ to form $\omega_{z}^{\prime}$ via the $P 4 a$ process, which then forms new $\omega_{y}^{\prime}$ via the $P 4 c$ process and is fed back into the $P 1$ and $P 4 a$ processes to close the cycle. Finally, in the $P 4 b$ cycle, the $\partial u^{\prime} / \partial z$ strain rate tilts the mean spanwise vorticity into $\omega_{x}^{\prime}$ via the $P 2 a$ process and also tilts the $\omega_{z}^{\prime}$ created by the $P 2$ and $P 4 a$ cycles into $\omega_{x}^{\prime}$ via the $P 4 b$ process; this $\omega_{x}^{\prime}$ created in the $P 4 b$ cycle strengthens the sweep/ejection motions that drives the other two cycles. This explanation of the physical processes indicated in Figure 7.26 suggests that the generation of enstrophy and turbulence along the edge of the spot appears to be more complex than the mechanisms proposed by Singer and Joslin (1994) and Schröder and Kompenhans (2004) depicted in Figures 7.1 and 7.2 , and involves a tightly-coupled interaction between the enstrophy production processes and sweep and ejection processes.

To visualize the contribution of the hairpin-vortex regeneration cycles to the turbulence kinetic energy of the fluid in the spot, iso-contours of the turbulence kinetic energy production rate, denoted $P_{k}^{+}$, are plotted in Figure 7.27 . The production rate is normalized by $u_{\tau}^{4} / \nu$. The plotted iso-contour level of $P_{k}^{+}=0.5$ is about half of the maximum value observed within the spot and was chosen on the basis that it likely shows the contribution of the most-energetic structures within the spot. The region labelled J corresponds to the hairpin vortex intersecting the vorticity lines labelled 1-4 in Figure 7.24. The production due to this structure changes 


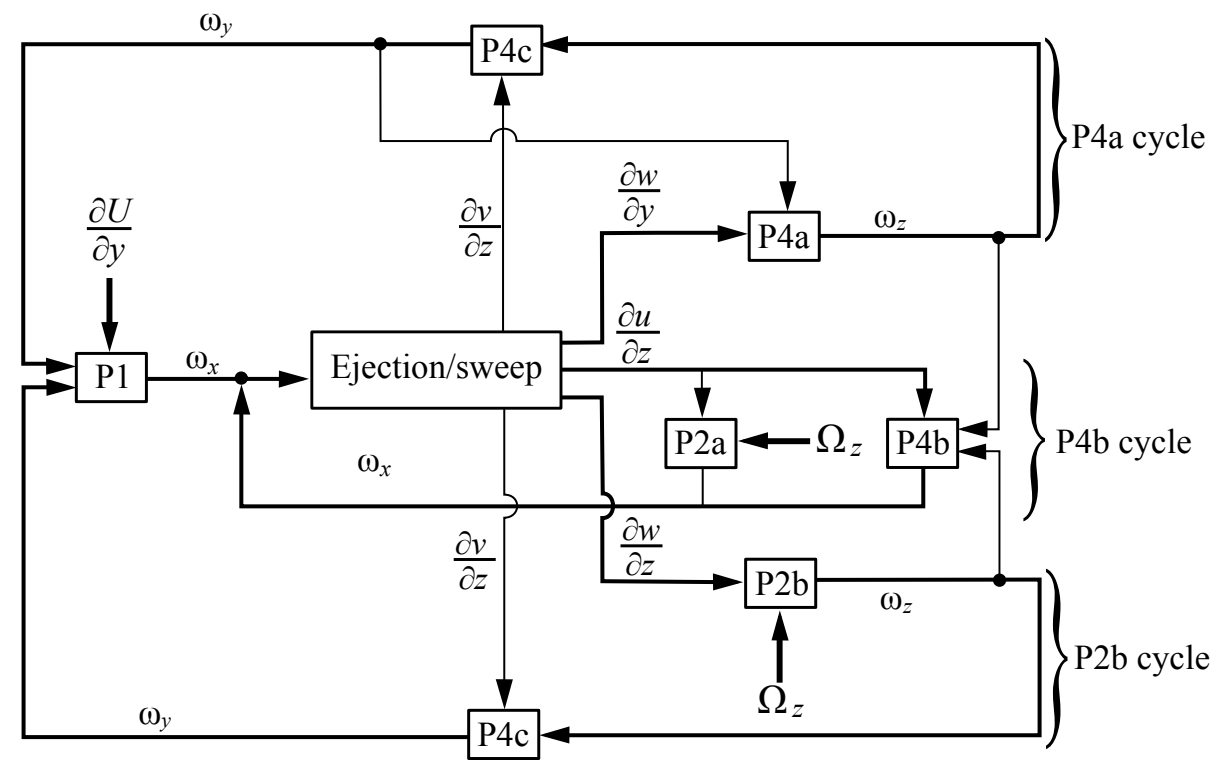

Figure 7.26: Block diagram illustrating the regeneration cycles of turbulent enstrophy observed within the turbulent spot.

only slightly from $\tau=480$ to 524 , suggesting that it is a mature coherent structure and its contribution to the turbulence does not vary significantly in time until $\tau=568$, at which time Figure 7.27 shows that turbulence production moves to smaller scales. The region labelled $\mathrm{T}$ corresponds to the small-scale vortical structures that intersect the vorticity line labelled 4 in Figure 7.17. The regeneration cycles illustrated in Figure 7.26 are activated by $\omega_{y}^{\prime}$ in these regions, as seen in Figure 7.17, and result in turbulence production that is relatively uniform at $\tau=480$ and aligned with the regions of strong $\omega_{y}^{\prime}$ shown in Figure 7.17. As the regeneration mechanisms amplify, the production shifts to smaller scales, seen at $\tau=524$. At $\tau=568$, the young hairpin vortices that form from the regeneration cycles activated near structure $\mathrm{T}$ have enlarged to become regions of notable turbulence production. It is likely that if the simulation were continued further in time, these structures would continue to grow and stretch downstream until the production iso-contours resemble those of structure R. Figure 7.27 suggests that the formation and growth of the streamwise-aligned $\omega_{y}^{\prime}$ shear layers are a major factor in the growth of regions of high turbulence kinetic energy production due to their role in activating the regeneration cycles of Figure 7.26 to form new coherent structures, and 


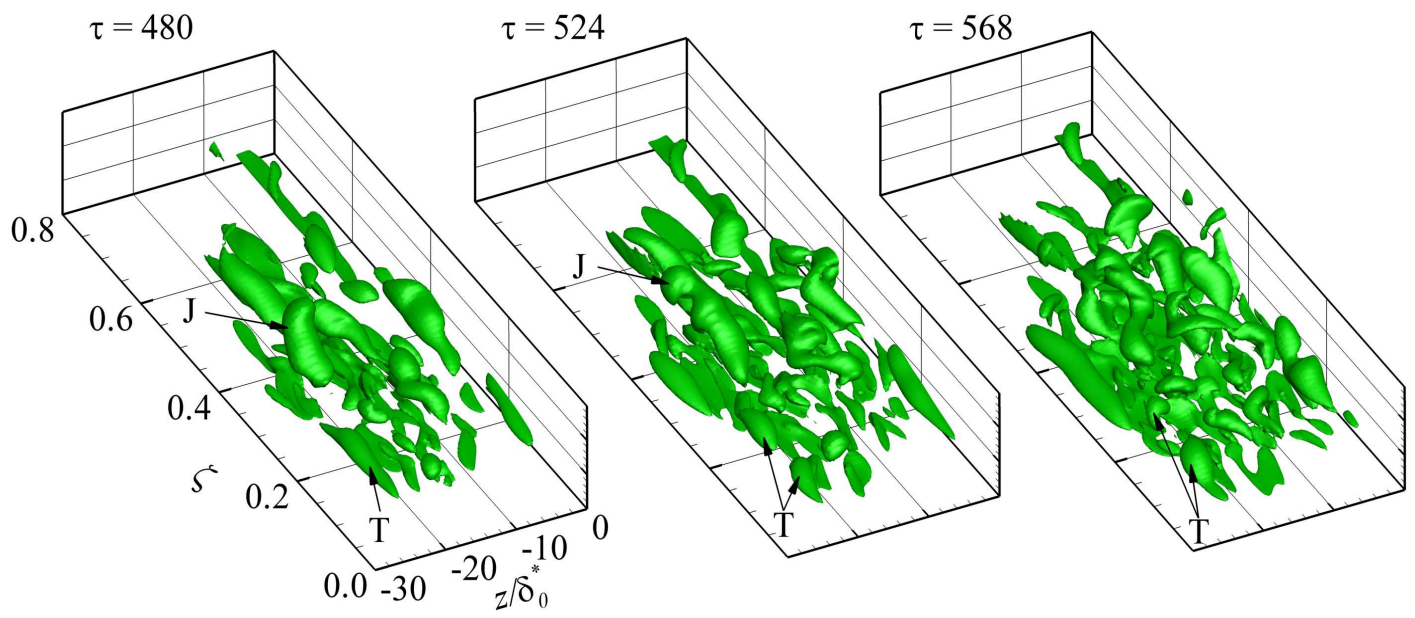

Figure 7.27: Iso-contours of normalized turbulence kinetic energy production, $P^{+}=0.5$, at $\tau=480,524$, and 568 in the $-z$ half of the spot.

hence have a significant impact on the growth of the turbulent spot. 


\section{Chapter 8}

\section{Numerical Investigation of}

\section{Transition in a Boundary Layer}

Subjected to Favourable and

Adverse Streamwise Pressure

Gradients and Elevated Free-Stream

\section{Turbulence}

Citation: $\quad$ Brinkerhoff, J.R. and Yaras, M.I. (2013), "Numerical investigation of transition in a boundary layer subjected to favourable and adverse streamwise pressure gradients and elevated free-stream turbulence," submitted to the Journal of Fluid Mechanics. 


\subsection{Introduction}

Transition to turbulence in boundary layers continues to be actively investigated due to its significant impact on numerous engineering processes, such as affecting the drag of aircraft wings or altering the losses and heat transfer in turbomachinery blade rows. Early theoretical investigations by Orr (1907) and experiments by Schubauer and Skramstad (1947) identified that for flow environments with low disturbance levels, transition occurs through the linear amplification of two-dimensional Tollmien-Schlichting waves within the boundary layer, followed by a non-linear growth phase where the Tollmien-Schlichting waves develop a three-dimensional character before finally breaking down into turbulence (e.g. Klebanoff et al., 1962; Nishioka et al., 1981). In flow environments with elevated free-stream turbulence, instability modes developing on small time scales have been observed and result in the Tollmien-Schlichting route being bypassed, which Morkovin (1969) termed "bypass" transition. Considering that a large percentage of industrial flows occur under high free-stream turbulence conditions (Mayle et al., 1998), an understanding of bypass transition is important for improving the models used for predicting transition.

Early measurements by Dryden (1936) established that a ubiquitous feature of transition under elevated free-stream turbulence is the presence of low-frequency fluctuations in the streamwise velocity within the boundary layer. Klebanoff (1971) observed that the fluctuations take the form of longitudinal streaks of high and low streamwise velocity near the wall and highlighted that increasing the level of free-stream turbulence increases the amplitude of the streaks. The streaks are commonly referred to as Klebanoff modes, although they are not represented by eigenfunctions of the linearized stability equations. When the spanwise streak spacing is normalized by $\nu / u_{\tau}$ (the kinematic viscosity and friction velocity $u_{\tau}=\left(\tau_{w} / \rho\right)^{0.5}$, respectively, where $\tau_{w}$ is the wall shear stress), a value of approximately 100 wall units was

found for a momentum-thickness Reynolds number range of $740 \leq R e_{\theta} \leq 5830$, with a minimal 
variation in the probability distribution of the streak spacing within this Reynolds number range. A linear increase in the streak spacing in the wall-normal direction was also observed by Smith and Metzler (1983) due to spanwise merging of the streaks in the outer region of the boundary layer.

The creation of streamwise streaks in boundary layers subjected to elevated free-stream turbulence is accomplished by two simultaneous processes: the penetration of free-stream disturbances into the boundary layer and the growth of these disturbances within the boundary layer. The former process is severely hampered by the mean boundary-layer shear, which shelters the boundary layer from high-frequency vortical disturbances in the free-stream fluid and allows only low-frequency disturbances to penetrate into the slower boundary layer fluid, a phenomenon referred to as "shear sheltering" (e.g. Hunt and Durbin, 1999). To explain the shear-sheltering process, Jacobs and Durbin (1998) and Zaki and Durbin (2005) note that a complete eigenfunction basis of the Orr-Sommerfeld equations consists of a finite set of discrete modes and a continuous spectrum of modes. Because the eigenfunctions of the discrete modes tend exponentially to zero in the free-stream, free-stream disturbances are properly characterised by a superposition of continuous modes. Numerical solution of the Orr-Sommerfeld equation by Jacobs and Durbin (1998) shows that the eigenfunctions of the continuous modes oscillate sinusoidally in the free-stream but are rapidly damped near the top of the boundary layer, and that their penetration depth into the boundary layer varies inversely with their frequency so that nearly isotropic turbulence in the free-stream produces low-frequency, highly-elongated streaks within the laminar boundary layer (e.g. Jacobs and Durbin, 2001).

Once a disturbance has penetrated into the boundary layer, the form of the disturbance determines whether it will be amplified or decay. A linear theory to predict the form of the most unstable disturbance - that is, the disturbance with maximal growth in a given time period, referred to as an optimal disturbance - via solution of the linearized Navier-Stokes 
equation is presented by Butler and Farrell (1992), Butler and Farrell (1993), Reddy et al. (1998), Andersson et al. (1999), and Luchini (2000). These studies demonstrate that the optimal disturbance in a boundary layer has the form of a pair of streamwise-independent, counter-rotating streamwise vortices. The velocities induced by the optimal streamwise vortices promote wall-normal momentum exchange and thus generate low- and high-speed streamwise streaks (e.g. Kline et al., 1967; Kim et al., 1971). As the streaks amplify, secondary instabilities of the streaks can occur and provide a route for break down to turbulence. Swearingen and Blackwelder (1987) noticed that the highly-inflectional streamwise velocity profiles in the spanwise and wall-normal directions may result in localized inviscid secondary instabilities, of which there are two modes: an asymmetric sinuous instability occurs as oscillations of the streamwise streaks in the streamwise/spanwise plane, while a symmetric varicose instability occurs as a periodic widening and narrowing of the streak (Andersson et al., 2001). Simulations of Elofsson et al. (1999) and Brandt and Henningson (2002) indicate that the streak amplitude determines whether secondary instabilities will occur. For flow over a flat plate with zero streamwise pressure gradient, they observed the onset of a sinuous instability when the streak amplitude reached about $26 \%$ of the free-stream velocity. Varicose instability waves were observed to be more stable, with a critical streak amplitude of about $37 \%$ of the free-stream velocity. In addition to the streak amplitude, the streak width also affects the development of secondary instabilities; when the streak width is narrow, comparable to the shear-layer thickness, the streak is unstable to the asymmetric mode, while for wider streaks, the symmetric mode is preferred (Asai et al., 2002). Konishi and Asai (2004) observed that when the spanwise streak spacing is greater than about 2.5 streak widths, both secondary modes are suppressed.

Brandt and de Lange (2008) showed that the interaction of high- and low-speed streaks determines how streaks affected by symmetric and asymmetric secondary instabilities break down. When break down followed the growth of a symmetric secondary instability, a frontal collision was observed between an incoming high-speed streak and a low-speed streak, while 
in asymmetric break down, the high- and low-speed streaks were observed to slide along each other's spanwise edges. In both cases, a strong shear layer forms between the streaks, the instability of which produces coherent vortical flow structures that initiate the break-down to irregular, small-scale motion. For the symmetric and asymmetric modes, the vortical flow structures consist of $\Lambda$-shaped vortices and a train of quasi-streamwise vortices with alternating sense of rotation, respectively. In a DNS of asymmetric break down, Schlatter et al. (2008) observe that the coherent vortical flow structures occur not as isolated vortices, but in a wave packet consisting of 3-5 vortices straddling the low-speed streak, staggered in the streamwise direction. When the wave packet is viewed in streamwise/wall-normal plane, as in the DNS results presented by Wu et al. (1999), their cross-section viewed in the cutting plane resembles the "cat-eyes" that characterise a Kelvin-Helmholtz-type instability. It is suggested by Schlatter et al. (2008) that the transition scenario proposed by Wu et al. (1999) and Durbin and $\mathrm{Wu}(2007)$ - that streaks break down owing to a Kelvin-Helmholtz instability arising from the inflectional velocity profile of the lifted low-speed streaks - may originate from viewing the flow only in two-dimensional planes and thus may be incorrectly attributing the cat-eye pattern formed by the spanwise waviness of the streak to a Kelvin-Helmholtz-type instability of the low-speed streak.

The final stage of bypass transition occurs as the coherent vortical flow structures generated by the streak instabilities break down into small-scale turbulence. In cases where the streaks are unstable through a symmetric secondary mode, as in the DNS of Sandham and Kleiser (1992), the high-shear layers surrounding the legs of $\Lambda$-shaped vortices roll-up into hairpinshaped vortices, which have been shown to be fundamental structures involved in the selfsustaining mechanism of turbulence in wall-bounded flows (e.g. Adrian et al., 2000; Wu, 2010; Park et al., 2012). In cases where the streaks are unstable through an asymmetric secondary mode, as in the DNS of Schlatter et al. (2008), the amplitude of the spanwise oscillations of the quasi-streamwise vortices straddling the low-speed streaks increases to the point where 
the vortices straddling adjacent low-speed streaks actually connect, forming hairpin-shaped vortices that subsequently lead to the generation of smaller scales. In cases where secondary streak instabilities are not present due to low streak amplitudes or large streak spacing, such as the DNS results of Wu et al. (1999) and Jacobs and Durbin (2001), streak break down occurs through a bursting process that occurs as low-speed streaks move into the outer region of the boundary layer and interact with free-stream eddies, resulting in the creation of patches of irregular motion on the lifted low-speed streaks. The irregular patches differ from classic turbulent spots observed by Emmons (1951) in that they originate high in the boundary layer rather than near the wall. Linear stability analysis and DNS by Zaki and Durbin (2005) found that the coupling of free-stream turbulence to the lifted low-speed streaks is strongly influenced by the frequency of disturbances in the free-stream, and that a high-frequency free-stream mode is required to trigger transition of the low-speed streak.

Whereas the preceding studies are largely limited to bypass transition in flat-plate boundary layers, the flow in a typical engineering device, such as a turbomachinery blade row, will include strong streamwise pressure gradients. In addition to affecting the boundary-layer development, streamwise pressure gradients alter the characteristics of the free-stream turbulence by creating streamwise variations in the free-stream turbulence intensity and integral length scales that produce anisotropy of the free-stream turbulence. A complete picture of bypass transition should therefore include the effects of streamwise pressure gradients and the accompanying streamwise variations in the free-stream turbulence conditions. The effect of a favourable streamwise pressure gradient on the spanwise streak spacing was studied experimentally by Talamelli et al. (2002) and Blair (1992). These authors showed that when the boundary layer is subjected to streamwise acceleration, the streak spacing normalized by the local viscous length scale increases, implying enhanced stability of the boundary layer that results in a downstream shift in the transition-onset location. A DNS by McAuliffe and Yaras (2010) of a boundary layer subjected to a favourable followed by an adverse streamwise pressure gradient typical of 
the low-pressure turbine in a gas-turbine engine found a preponderance for high-speed streaks in the accelerated region, which resulted in an overall increase in the streak width to the point where secondary streak instabilities were prevented despite observing sufficiently-high streak amplitudes. Litvinenko et al. (2005) performed experiments of symmetric secondary streak instabilities in zero and adverse pressure-gradient boundary layers, observing that the adverse pressure gradient amplifies the creation of $\Lambda$-vortices through the symmetric mode. Zaki and Durbin (2006) used linear stability calculations and DNS to highlight that adverse pressure gradients increases the receptivity of the boundary layer to free-stream vortical disturbances, thus enhancing the coupling of low-frequency disturbances to the boundary-layer fluid and strengthening the streamwise streaks.

To assess the sensitivity of bypass transition to the turbulence length scales in the freestream, Brandt et al. (2004) performed a DNS of a transitional flat-plate boundary layer with synthetic turbulent inflow conditions consisting of a superposition of modes of the continuous spectrum of the Orr-Sommerfeld and Squire operators. By varying the energy spectrum of the free-stream turbulence, they observed that the transition-onset location shifts to lower Reynolds numbers as the integral length scale is increased from $2.5 \delta_{0}^{*}$ to $7.5 \delta_{0}^{*}$, where $\delta_{0}^{*}$ is the initial boundary-layer displacement thickness. Jonás et al. (2000) observed a similar trend through measurements of the intermittency, defined as the percentage of time a boundary layer is locally turbulent, and also noted that increasing the integral length scale over a similar range as Brandt et al. (2004) increases the length of the transition region. However, these studies do not discuss how the turbulence integral length scale physically impacts the transition process. More recently, Nagarajan et al. (2007) and Ovchinnikov et al. (2008) demonstrate that the effect of the free-stream turbulence length scale on transition is strongly affected by the flow development along the flat-plate leading edge. Their results demonstrate a complicated interplay between the free-stream turbulence integral length scale and the receptivity of the flow at the plate leading-edge that is qualitatively captured by the ratio of the integral length 
scale to the boundary-layer thickness at transition onset. For the lower value of this ratio, transition occurs through the formation, instability, and break down of streamwise streaks, as described previously. However, for the higher value of this ratio, large, localized wavepackets formed far upstream of the earliest streamwise streaks. These wavepackets were shown to have significantly different properties than those formed by streak instability, which suggests that a threshold free-stream turbulence integral length scale may exist beyond which the sheltering effects of the mean shear are overcome and turbulent spots may be created by free-stream turbulence eddies directly without the development of streamwise streaks.

The review presented in the preceding sections highlights that the current understanding of the mechanisms of bypass transition are not complete, particularly in cases where streamwise pressure gradients alter the boundary-layer stability and modify the length scales, intensity, and isotropy of the free-stream turbulence. Therefore, in this study a high-resolution DNS is performed of a flat-plate boundary layer subjected to elevated free-stream turbulence of large amplitude and length scale and a favourable followed by an adverse streamwise pressure gradient that is representative of the suction side of a low-pressure turbine blade in a gas-turbine engine. The aim is to improve the understanding of the stability of streamwise streaks in accelerating boundary layers, analyze how free-stream turbulence influences the break-down of streamwise streaks, and identify how free-stream turbulence affects the growth of the coherent vortical flow structures resulting from the break-down of the streamwise streaks into turbulent spots to sustain the upstream edge of the turbulent boundary layer.

\subsection{Numerical Method}

A DNS is performed of a laminar boundary layer that transitions to turbulence under elevated free-stream turbulence and a streamwise pressure distribution typical of the suction side of a low-pressure gas-turbine blade. ANSYS CFX ${ }^{\circledR}$ (Version 13), a commercial computational 
fluid dynamics software package, is used to solve the time-varying governing equations using a finite-volume spatial discretization approach. The working fluid is air at $1 \mathrm{~atm}$ and $25^{\circ} \mathrm{C}$. The authors' research group has previously investigated transitional and fully-developed turbulent boundary layers by numerically solving the unsteady mass- and momentum-conservation equations using this commercial solver (e.g. McAuliffe and Yaras, 2010; Brinkerhoff and Yaras, 2011; Azih et al., 2012). The results of these computations show good agreement with experiments in terms of the turbulent mean velocity profile, the streamwise velocity fluctuation spectra, the location of transition onset, the streamwise rate of transition, and the turbulence kinetic energy budgets.

\subsubsection{Computational domain and boundary conditions}

The computational domain used in the present study, shown in Figure 8.1, consists of a contoured ceiling above a flat, no-slip test surface of length $L=800 \mathrm{~mm}$. The coordinate system is oriented as shown in Figure 8.1 with the origin centered along the leading edge of the test surface. The streamwise contour of the ceiling produces a converging and diverging flow path over the test surface, thus imposing a streamwise pressure distribution on the test surface that resembles the pressure distribution on the suction side of an airfoil, specifically that of a low-pressure axial turbine blade. The height of the ceiling above the test-surface is $0.230 \mathrm{~L}$ at the inlet, $0.168 \mathrm{~L}$ at the outlet, and $0.114 \mathrm{~L}$ at the suction-peak location, which results in the ceiling height being always more than 50 times the local value of the boundarylayer displacement thickness, $\delta^{*}$, above the test-surface. The inflow boundary consists of a $9 \times 8$ array of square-shaped jets of $9.3 \mathrm{~mm}$ width spaced $13.1 \mathrm{~mm}$ apart (center to center), which approximates the configuration of a turbulence-generating grid. A uniform streamwise velocity of $11.5 \mathrm{~m} / \mathrm{s}$ is defined at each square-shaped jet. Free-slip walls are specified between the square-shaped jets owing to the difficulty in resolving the wall shear layers on the bars of the turbulence grid. A free-slip region of $0.566 \mathrm{~L}$ length is located between the inflow boundary 


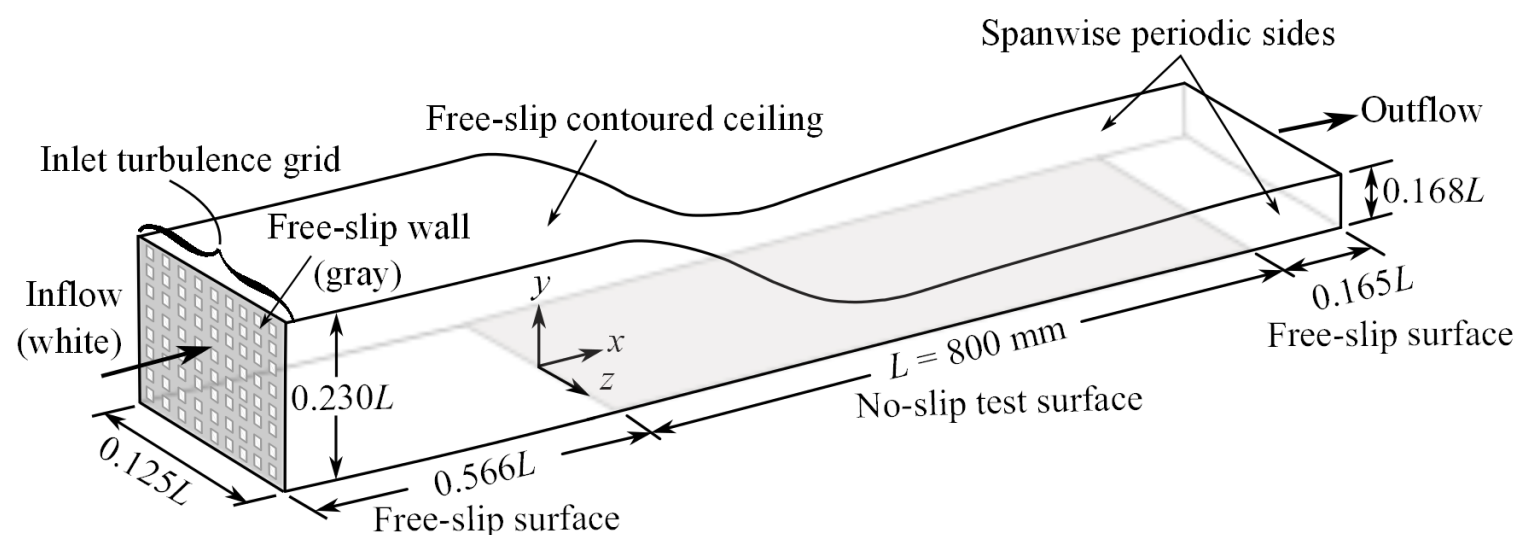

Figure 8.1: Schematic of the computational domain (not to scale).

and the leading edge of the test surface to ensure that the free-stream turbulence recovers from the limited resolution of the shear layers surrounding the square-shaped jets and to allow the grid-generated free-stream turbulence to decay to the desired intensity and length scale. As detailed in Section 8.3.1, this length also ensures that the free-stream turbulence entering the test-surface region is adequately isotropic and homogeneous. A free-slip surface of $0.165 \mathrm{~L}$ length is located downstream of the test surface to place the outflow boundary a conservative distance from the region of interest, as the discretization method employed by ANSYS CFX ${ }^{\circledR}$ at the outflow boundary condition distorts convective flow structures such as turbulent eddies as they exit the domain. Static pressure that remains fixed in an area-averaged sense at $0 \mathrm{~Pa}$ (gauge) is specified at the outflow boundary. Spanwise periodicity is imposed along the sides of the computational domain, and a spanwise width of $S=0.125 \mathrm{~L}$ is chosen to place the sides of the domain a conservative distance away from the region of interest, which is located near the midspan.

\subsubsection{Spatial grid}

A structured grid consisting of hexahedral finite volumes is mapped to the computational domain shown in Figure 8.1. To accurately perform a DNS of the flow in the current study, the 
spatial and temporal grids must be sufficiently refined to capture the smallest scales of the flow, which are on the order of the Kolmogorov length and time scales. For wall-bounded turbulent shear layers, the Kolmogorov length scale is on the order of the viscous length scale, typically reaching a minimum value of $\eta^{+} \approx 2$ close to the no-slip boundary (e.g. Pope, 2000; Kim et al., 1971; Stanislas et al., 2008). In the following, normalization by wall units is indicated with a + superscript, calculated using the maximum local wall shear stress observed in the time-averaged simulation results, which occurs at $x / L=0.785$. The nodes are distributed such that the node spacing yields values of $\Delta x^{+}=11$ and $\Delta z^{+}=8$, corresponding to approximately 4 to 5 times the Kolmogorov length scale at the target Reynolds number. The $y^{+}$value of the nodes adjacent to the no-slip wall are everywhere less than 0.8 . In the range $0.8<y^{+}<30$, the wall-normal node spacing is increased linearly from the value adjacent to the wall to a value that corresponds to the Kolmogorov length scale in the log-law region, which is approximated by:

$$
\eta^{+}=\left(\kappa y^{+}\right)^{1 / 4}
$$

where $\kappa=0.41$ is the von-Karman constant (Pope, 2000; Stanislas et al., 2008). Beyond $y^{+}=30$, the node spacing is increased at a rate of $4 \%$ until $y^{+}=70$. The grid spacing remains constant in the range $70<y^{+}<140$, and is then increased at a rate of $10 \%$ until the free-slip ceiling of the domain is reached. This wall-normal node distribution is shown in Figure 8.2.

To resolve the free-stream turbulence generated upstream of the test surface, a uniform streamwise node spacing of $\Delta x^{+}=11$ is imposed between the inflow plane and the testsurface leading edge. Uniform wall-normal and spanwise node distributions are imposed at the inflow plane such that 32 nodes occur in the $y$ and $z$ directions within each square-shaped jet. The rectangular free-slip regions between the square-shaped jets are resolved with 13 nodes along the short axis and 32 nodes along the long axis. Between the inflow plane and the testsurface leading edge, linear interpolation with respect to the streamwise coordinate is used to modify the wall-normal node distribution in the downstream direction so that the distribution 


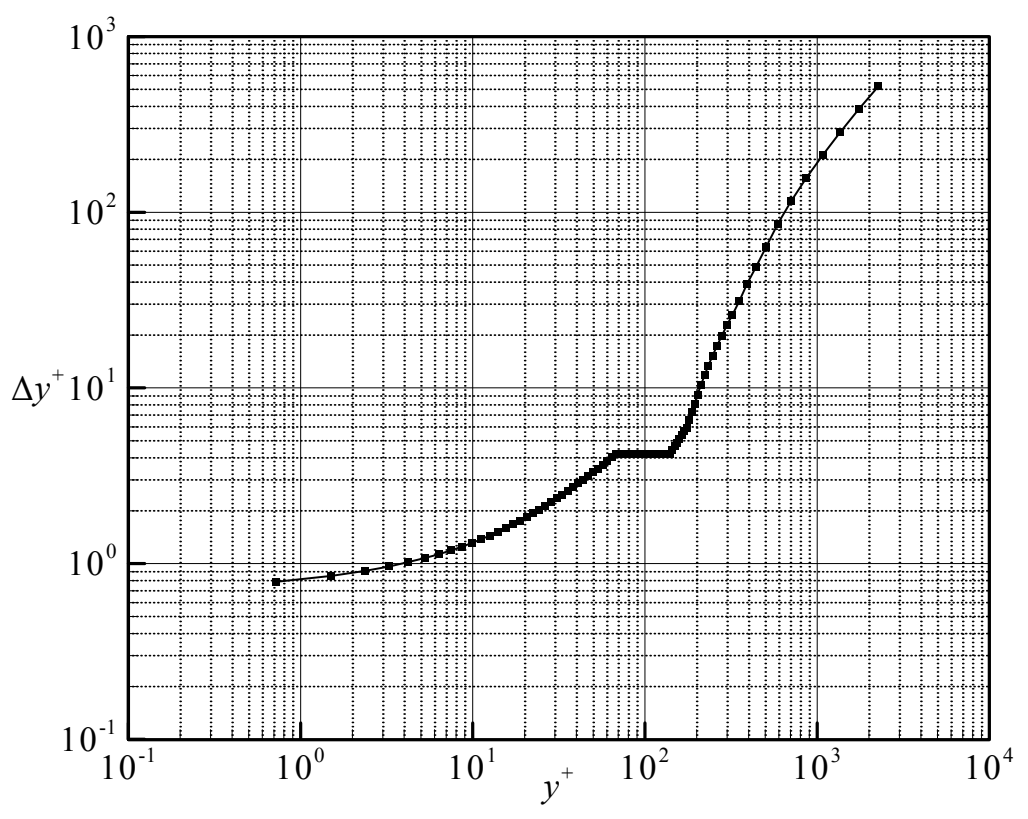

Figure 8.2: Wall-normal node distribution.

at the leading-edge of the test surface matches that shown in Figure 8.2. To ensure that the spatial grid does not affect the characteristics of the simulated free-stream turbulence, the simulations were repeated with twice the number of nodes in the $y$ and $z$ directions. Negligible differences were observed between the refined-grid results and the initial resolution results that are presented in Section 8.3.1.

\subsubsection{Solution method}

Discretization of the governing equations is based on central differencing and second-order Euler backward differencing for the spatial and temporal derivatives, respectively. A timestep size of $\Delta t=3.5 \times 10^{-5} \mathrm{~s}$ or $0.19 t^{+}$, where $t^{+}=\nu / u_{\tau}^{2}$ is the viscous timescale at the timeaveraged location of maximum wall shear stress, which is close to the timestep size of $0.20 t^{+}$ recommended by Choi and Moin (1994) for accurately computing turbulent flows. As an $a$ posteriori check that the time scales of the generated free-stream turbulence are sufficiently resolved, the timestep size is compared to the Kolmogorov time scale of the free-stream 
turbulence occurring just upstream of the test-surface leading edge:

$$
\tau_{\eta}=(\nu / \epsilon)^{0.5}
$$

where $\epsilon$ is the local turbulent dissipation rate computed from the simulation. The selected timestep size is approximately $0.008 \tau_{\eta}$, confirming that the time scales of the free-stream turbulence are properly resolved in the simulation.

Starting from a uniform static pressure of $0 \mathrm{~Pa}$ gauge and streamwise velocity of $4 \mathrm{~m} / \mathrm{s}$, solution convergence of the discretized equations at each timestep is obtained with outer iteration loops serving to converge the coefficients of the linearized governing equations and inner iteration loops facilitating the solution of these equations through W-type algebraic multigrid cycles. Six grid levels are used in the multigrid cycle with one solution sweep performed at each grid level during the restriction passes and three solution sweeps performed at each grid level during the prolongation passes. The transient solution is obtained with one inner-loop iteration per outer-loop iteration, and multiple outer-loop iterations per timestep. For each timestep, the solution is declared as converged when the root-mean-square residuals

of the governing equations are reduced by five orders of magnitude to less than $10^{-6}$. This is realized with up to eight outer-loop iterations per timestep. Approximately 40,000 timesteps were required for the flow to reach a statistically steady state, after which 4096 timesteps were computed for analysis. The simulation was partitioned and executed in parallel on 24 cores of AMD Opteron 6128 processors. Details of the simulation are given in Table 8.1.

\subsubsection{Validation of numerical approach}

Validation of the numerical algorithm and the spatial and temporal resolutions is accomplished by performing a series of companion DNS studies of a temporally-developing zeropressure-gradient turbulent boundary layer and comparing the results of these simulations 
Table 8.1: Flow conditions and numerical details of the current simulation

Test-surface length, $L$ $800 \mathrm{~mm}$

Reference velocity, $U_{0}(\mathrm{~m} / \mathrm{s})$

4.848

Test-surface Reynolds number, $R e_{L}=U_{0} L / \nu$

253,500

Timestep size, $\Delta t(\mathrm{~s})$

$3.5 \times 10^{-5}$

Free-stream turbulence intensity at test-surface leading edge, $T u_{0}$

$5.33 \%$

Streamwise turbulence integral length scale at test-surface leading edge, $\Lambda_{x 0} / \mathrm{L}$

0.017

Momentum-thickness Reynolds number at transition-completion location, $R e_{\theta}$

680

Number of grid nodes

$43.7 \times 10^{6}$

Kinematic viscosity, $\nu\left(\mathrm{m}^{2} / \mathrm{s}\right)$

$1.53 \times 10^{-5}$

Density, $\rho\left(\mathrm{kg} / \mathrm{m}^{3}\right)$

to statistical turbulence properties of corresponding turbulent boundary layers in literature.

The dimensions of the computational domain for the companion simulations are 670, 1820,

and 650 viscous units in the $x, y$, and $z$ directions, respectively, and is spatially-periodic in the $x$ and $z$ directions. The companion simulations are initialized with a spatially-uniform velocity of $1.6 \mathrm{~m} / \mathrm{s}$ and the boundary layer is developed in time until a momentum-thickness Reynolds number of $R e_{\theta}=700$ is reached. Transition is triggered at $R e_{\theta}=450$ through a mass source/sink pair lasting $4.5 t^{+}$that is located near the wall in the center of the domain that injects fluid into the domain at a wall-normal velocity of $0.16 \mathrm{~m} / \mathrm{s}$. The spatial resolutions of the complementary DNS studies are listed in Table 8.2. The Reynolds number was chosen to match the momentum-thickness Reynolds number at the transition-completion location of the present bypass-transition simulation, as listed in Table 8.1. Considering that the transient flow structures in a fully-turbulent boundary layer have a larger range of spatial and temporal scales than a transitional boundary layer at a comparable Reynolds number, the companion studies provide a rigorous case for validating the numerical approach of the present bypass-transition simulations.

The first validation simulation (Grid 1) was performed with a spatial resolution in the $x-z$ plane of $\Delta x^{+}=\Delta z^{+}=3$, which corresponds to about 1.5 times the Kolmogorov length scale at this Reynolds number. The $y^{+}$value of the first node from the no-slip boundary is 0.85 and 
Table 8.2: Computational grids for the validation studies.

\begin{tabular}{lccc}
\hline Simulation & $\Delta x^{+}$ & $\Delta z^{+}$ & $R e_{\theta}$ \\
\hline Grid 1 & 3 & 3 & 700 \\
Grid 2 & 6 & 6 & 700 \\
Spalart (1988) & 20 & 7 & 670 \\
\hline
\end{tabular}

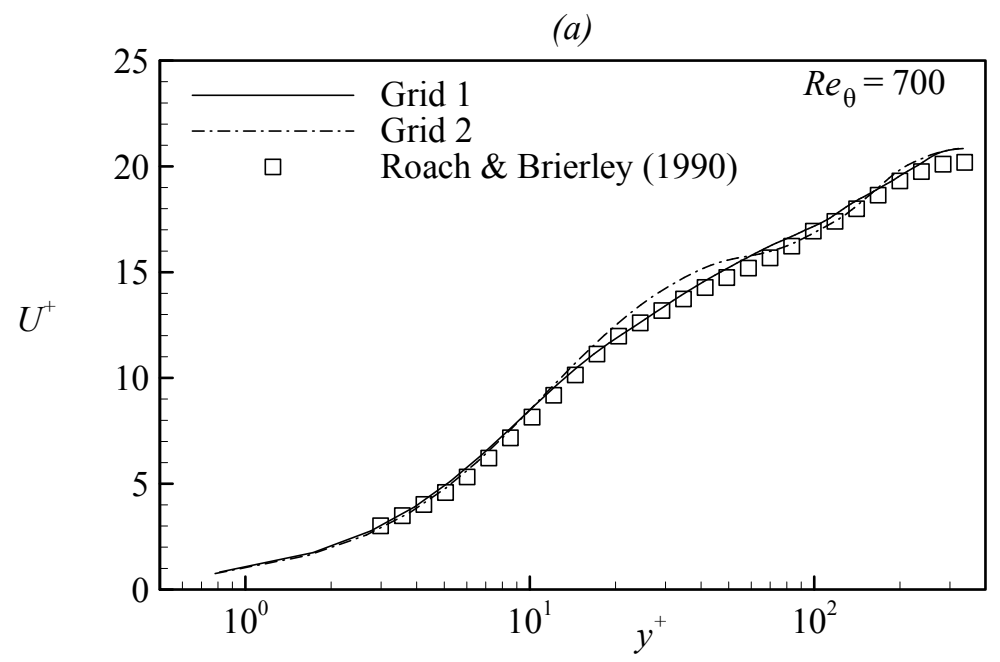

(b)

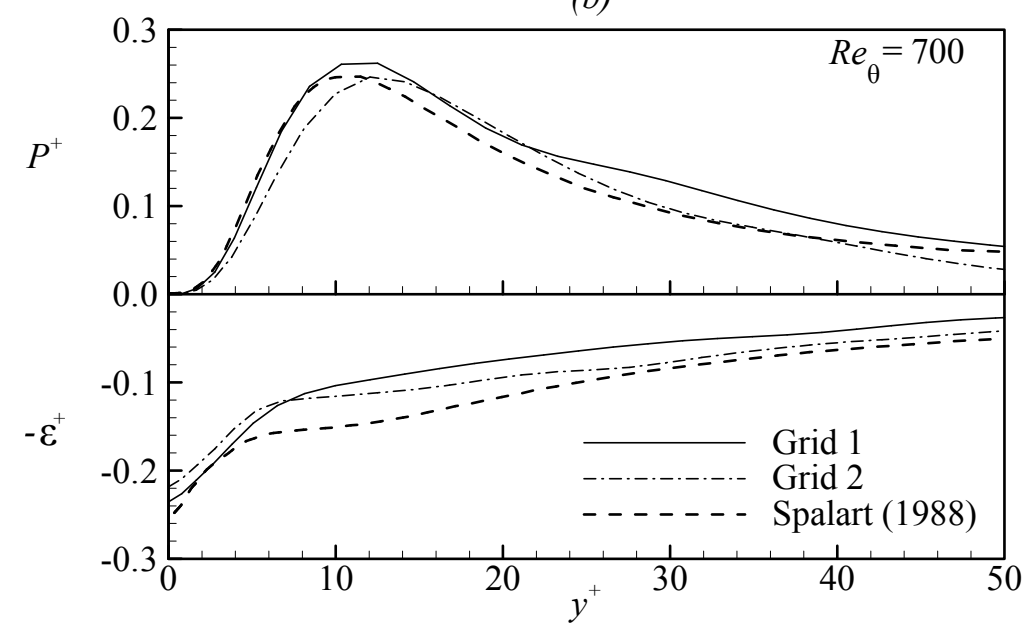

Figure 8.3: Validation DNS study of a zero-pressure-gradient turbulent boundary layer at $R e_{\theta}=700$. (a) Mean velocity profile. (b) Turbulence production and dissipation rate budgets. 
the wall-normal node distribution follows closely the distribution shown in Figure 8.2. Figure 8.3 shows that the results achieve very good agreement compared with the turbulent mean velocity profile measured experimentally by Roach and Brierley (1990) and the turbulence kinetic energy production and dissipation rate budgets (denoted $P^{+}$and $\epsilon^{+}$, respectively) computed by Spalart (1988), who used an algorithm utilizing a spectral method for spatial discretization and a second-order-accurate hybrid Runge-Kutta/Crank-Nicholson scheme for temporal discretization to simulate the boundary layer at a Reynolds number of $R e_{\theta}=670$. The slight disagreement that is observed in the dissipation rate budget is comparable to variation that exists in published literature at $R e_{\theta}=700$ due to differences in the way each study triggers transition to turbulence. To ascertain the sensitivity of the results to the spatial resolution, a second validation DNS (Grid 2) with a grid that closely matches the spatial resolution of the present study was performed at $R e_{\theta}=700$. In comparison to the Grid 1 results shown in Figure 8.3, the coarser grid achieves a similar level of agreement with published results. Therefore, these validation studies provide strong evidence that the present study should accurately capture the flow development in a bypass-transition scenario. As per Figure 8.3, although the Grid-1 results are in slightly better agreement with the simulation data of Spalart (1988) and the experimental data of Roach and Brierley (1990) than the Grid-2 results, the resolution of Grid-2 was adopted for the present simulations to achieve reasonable computing times while still ensuring that the flow physics are accurately captured.

\subsection{Results and Discussion}

The analysis of the results begins by studying the development of the free-stream meanflow and turbulence upstream of the test-surface leading edge. In discussing the upstream development of free-stream turbulence, length scales are normalized by the width of the freeslip bars separating the square-shaped inflow boundaries, $d=3.82 \mathrm{~mm}$, which is analogous 
to the bar width of a turbulence-generating grid consisting of horizontal and vertical bars. Elsewhere in the discussion of the simulation results, the test-surface length, $L=800 \mathrm{~mm}$, is used to normalize length scales. In all sections, velocities are normalized by the temporallyand spatially-averaged free-stream velocity at the test-surface leading edge, $U_{0}=4.848 \mathrm{~m} / \mathrm{s}$, and time is normalized by $L / U_{0}$, with the normalized time denoted $\tau$.

\subsubsection{Free-stream flow development}

As described in Section 8.2.1, the free-stream flow is generated through a periodic array of square-shaped inflow boundaries in an $y$-z plane located $0.566 \mathrm{~L}$ upstream of the test-surface leading edge, which resembles the turbulence-generating grids studied by Roach (1986). The streamwise distribution of the mean streamwise velocity of the free-stream flow is shown in Figure 8.4 by plotting the streamwise velocity averaged in time, denoted as $U$. The flow development upstream of $x / L=-0.25$ is not shown because the flow has not mixed-out to a sufficient extent following the turbulence-generating inflow condition to yield a satisfactorily steady flow. The streamwise acceleration parameter, $\eta=\nu / U^{2}(d U / d x)$, is also shown to illustrate the degree of streamwise acceleration present in the free-stream flow. The two dashed horizontal lines in Figure 8.4 identify the critical range of the acceleration parameter, beyond which several researchers have observed that a turbulent boundary layer will begin to relaminarize (e.g. Moretti and Kays, 1965; Jones and Launder, 1972; Escudier et al., 1998). Figure 8.4 shows that the majority of the flow between the test-surface leading edge and the suction peak location $\left(x_{s p} / L=0.525\right)$ is accelerated at a level well above the critical value for relaminarization, which implies that any disturbances penetrating into or developing in the boundary layer via viscous instability modes are expected to be damped in this region.

The degree of spatial uniformity of the free-stream velocity is shown in Figure 8.5 by plotting contours of the temporally-averaged local velocity, $U$, in several $y$ - $z$ planes, where $U$ 


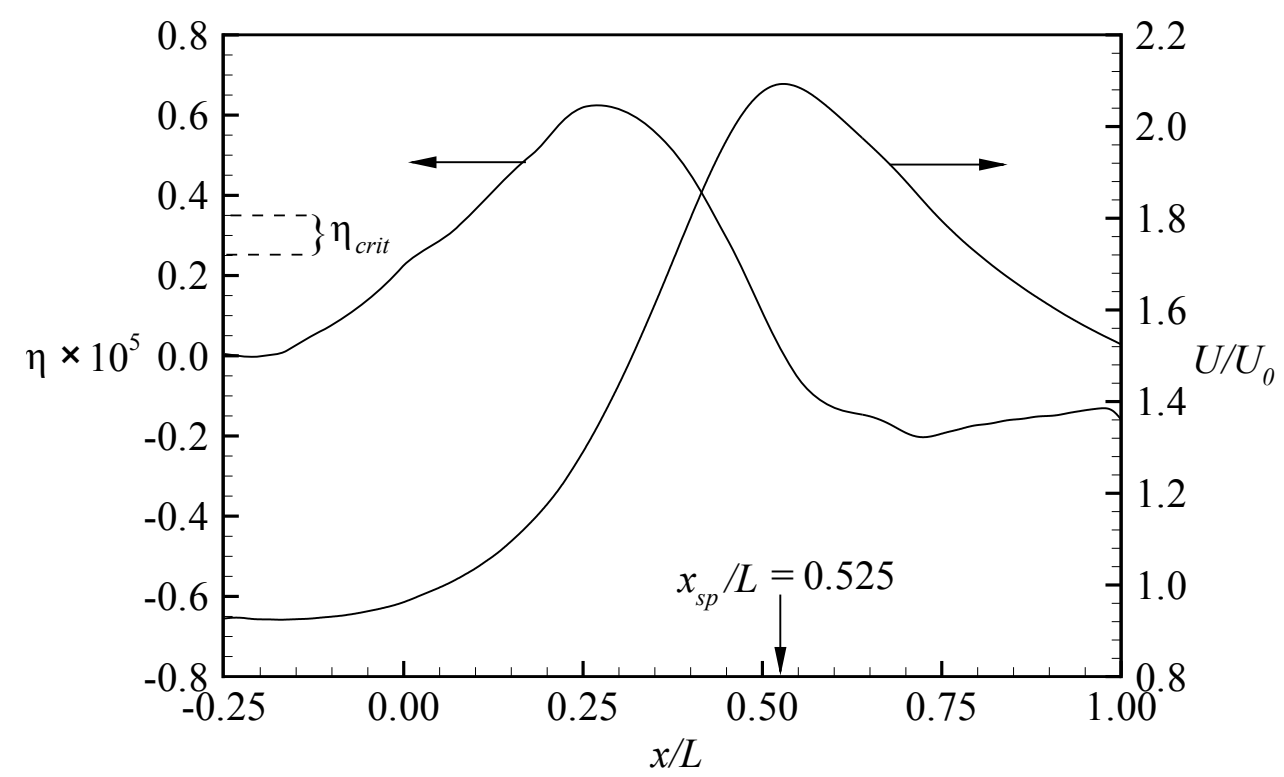

Figure 8.4: Streamwise distributions of free-stream velocity, $U / U_{0}$, and acceleration parameter, $\eta$.

is normalized by the free-stream velocity averaged over the local $y$ - $z$ plane, which is denoted $U_{y z}$. Just upstream of the test-surface leading edge, at $x / L=-0.004$, the non-uniformity is about $0.08 U_{e}$, and it is attributed to the incomplete mixing of the free-stream flow downstream of the turbulence-generating grid. The incomplete mixing is partially due to the spanwiseperiodic boundary conditions that are applied on the side walls of the sub-domains upstream of the test-surface leading edge. As ANSYS CFX does not preserve continuity in the firstderivatives of flow variables across a periodic boundary, eddies passing through the periodic boundaries are deformed and thus the turbulence-grid wakes located near the periodic side walls have an altered mixing rate compared to those located near the center of the domain. Downstream of the test-surface leading edge, at $x / L=0.121$, the free-stream non-uniformity begins to decrease as the free-stream is accelerated by the pressure distribution imposed by the contoured ceiling. By $x / L=0.371$, the non-uniformity is less than $0.04 U_{e}$ and occurs far away from the boundary-layer edge so that the flow near the wall shear layer (below $y / L=0.02$ ) is spatially-uniform to within $0.004 U_{e}$. The free-stream non-uniformity observed in Figure 8.5 is taken into account in the remaining discussion by defining the mean flow field as the spanwise 
average of the temporally-averaged flow field, and the spanwise averaging is performed only on the free-stream flow below $y / L=0.02$ and between $z / L=-0.03$ and 0.03 .

The decay of the free-stream turbulence downstream of the simulated turbulence grid is shown in Figure 8.6. In this figure, $x^{\prime}$ is the streamwise coordinate referenced to the inflow plane. The quantities in Figure 8.6 are spatially-averaged as described above at each streamwise location, and the uncertainty in the spatial mean values is shown by the error bars. For visibility, the error bars are plotted at only selected $x / d$ locations such that they appear uniformly spaced in Figure 8.6. The error bars qualitatively indicate that the degree of spatial non-homogeneity of the free-stream turbulence is small near the leading edge of the test surface; when estimated as the standard error of the value of $T u$ in a plane located at $x / L=-0.004$ $\left(x^{\prime} / d=117.8\right)$, the spatial non-homogeneity is less than $1 \%$ of the spatially-averaged value of turbulence intensity in that plane.

The validity of the present method for generating free-stream turbulence is further assessed by comparing the properties shown in Figure 8.6 with semi-empirical correlations developed by Roach (1986) for grid-generated free-stream turbulence. The grid Reynolds number is $R e_{d}=1211$, which is within the range considered by Roach (1986). The turbulence intensity is plotted in Figure 8.6 $(a)$, defined as $T u=\sqrt{1 / 3\left(\overline{u^{\prime 2}}+\overline{v^{\prime 2}}+\overline{w^{\prime 2}}\right)} / U_{0}$, where the prime indicates velocity fluctuation. The Reynolds normal stresses and turbulence integral length scales are shown in Figure 8.6(b)- $(d)$. Trendlines in Figure 8.6 are determined through a least-squares fit to the data that lies between $x^{\prime} / d=30-118$, where the turbulence is most fully developed. The correlations proposed by Roach (1986) are also plotted to facilitate comparison with the present results. Figure 8.6(a) shows that after an initial growth period immediately downstream of the simulated grid, Tu decays exponentially in agreement with the correlation proposed by Roach (1986). The turbulence integral length scales in Figure 8.6(c)-(d) are calculated by integrating the normalized autocovariance function of the fluctuating streamwise velocity component in 

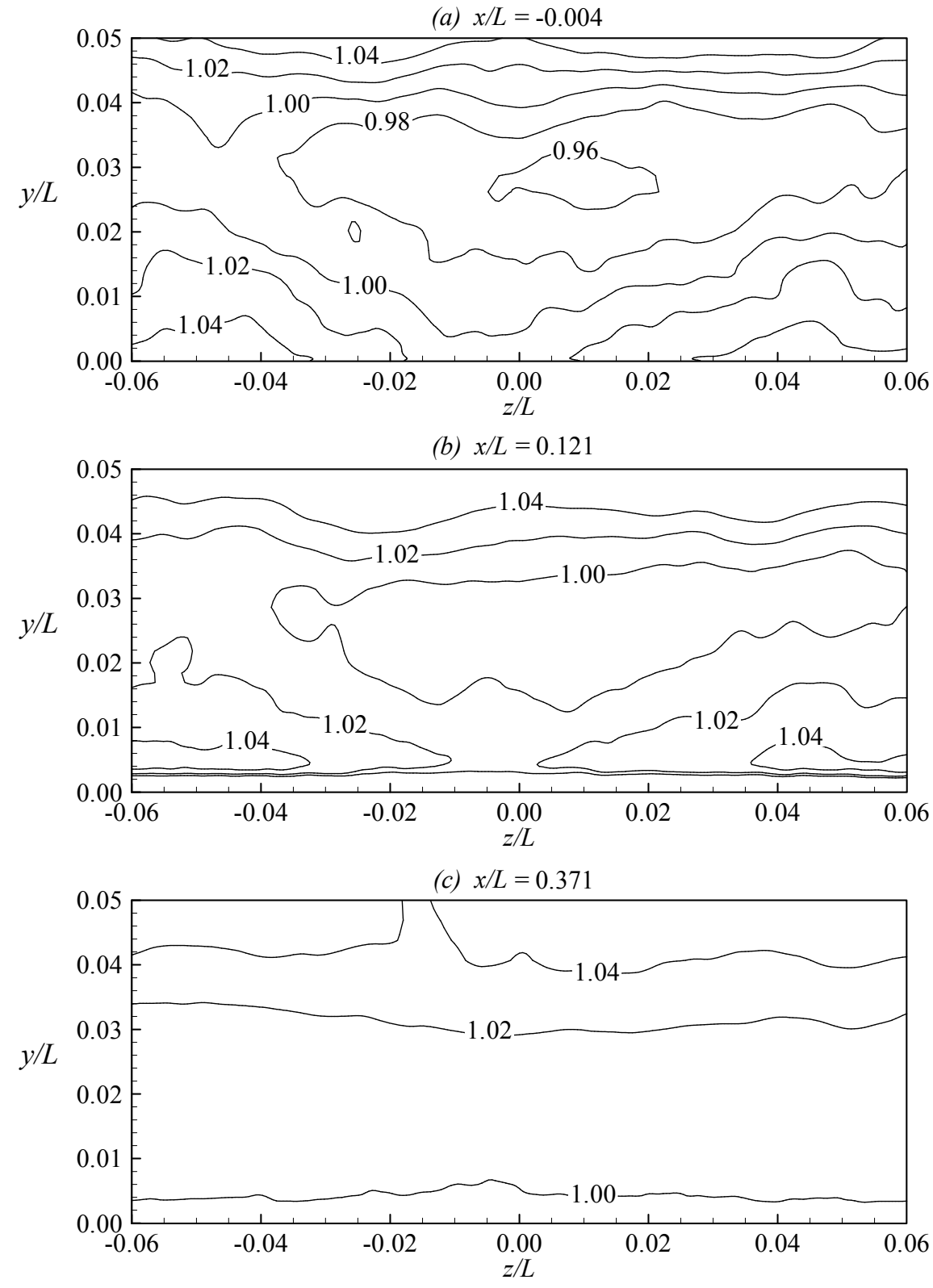

Figure 8.5: Contours of $U / U_{y z}$ in $y$ - $z$ planes at (a) $x / L=-0.004$, (b) $x / L=0.121$, and $(c) x / L=0.371$. 

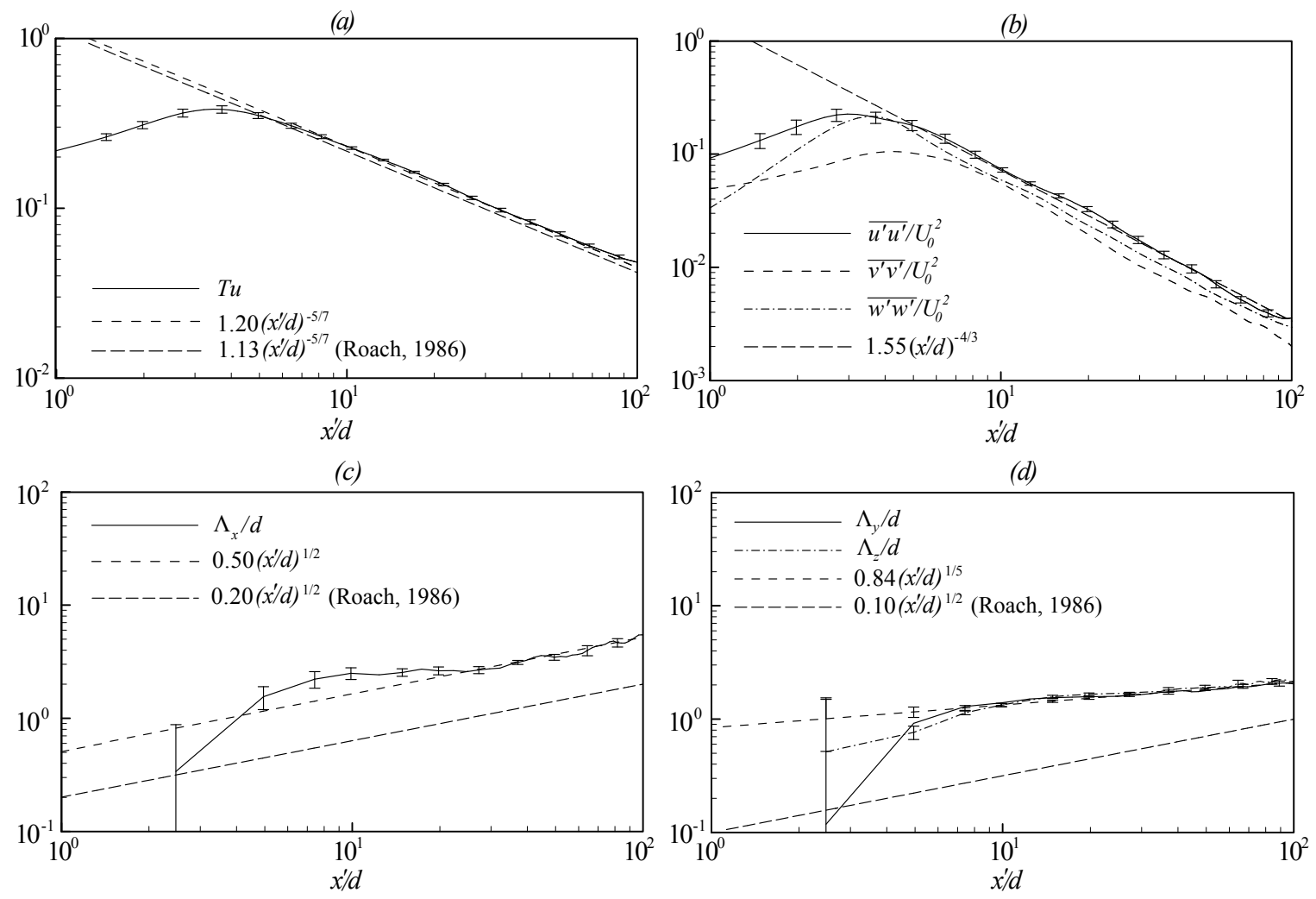

Figure 8.6: Streamwise decay of free-stream turbulence upstream of the test surface. Areaaveraged distribution of $(a)$ turbulence intensity, $T u,(b)$ normal Reynolds stresses, and $(c-d)$ integral length scales. Error bars indicate uncertainty in the spatial mean values. 
the three coordinate directions:

$$
\Lambda_{x_{i}}=\frac{1}{\overline{u^{\prime}\left(x_{i}, t\right)^{2}}} \int_{0}^{\infty} \overline{u^{\prime}\left(x_{i}, t\right) u^{\prime}\left(x_{i}+r, t\right)} d r, \quad i=1,2,3
$$

where $r$ is oriented in the $x_{i}$ direction. By about $30 d$ downstream of the inflow plane, the streamwise integral length scale of the free-stream turbulence (Figure 8.6(c)) grows according to $0.50(x / d)^{1 / 2}$, which agrees favourably with the correlation of Roach (1986) in terms of the streamwise growth rate but is offset vertically. The offset is attributed to differences in the initial state of the generated turbulence; due to computational limitations, the boundary layers around the bars of the turbulence-generating grid are not resolved in the present study, which results in the openings of the numerical turbulence grid being slightly larger than in an experimental turbulence grid of the same geometry. While this does not alter the mechanisms of the free-stream turbulence decay, demonstrated particularly by the agreement of the streamwise growth rates in the length scales, it does alter the length scales of the generated free-stream turbulence and results in the noted differences in the correlation constants. The large uncertainty in the integral length scales at low $x / d$ values is due to wakes behind the closed portions of the simulated turbulence grid, which create large spatial non-uniformities in the turbulence scales that diminish as the wakes dissipate. The integral length scales in the $y$ and $z$ coordinate directions (Figure 8.6(d)) grow at a lower rate than the streamwise length scales. This is likely due to the upstream effect of the manner by which the freestream turbulence is generated in the present study, which as per Figure 8.4 influences the free-stream flow up to about $x / L=-0.25$ or $x^{\prime} / d=60$. The Reynolds normal stresses plotted in Figure 8.6(b) illustrate a slight anisotropy in the free-stream turbulence. The streamwise fluctuation levels are approximately $10 \%$ and $20 \%$ higher than fluctuations in the $y$ and $z$ coordinate directions, respectively, which is consistent with the observations of Roach (1986) in similar configurations. 


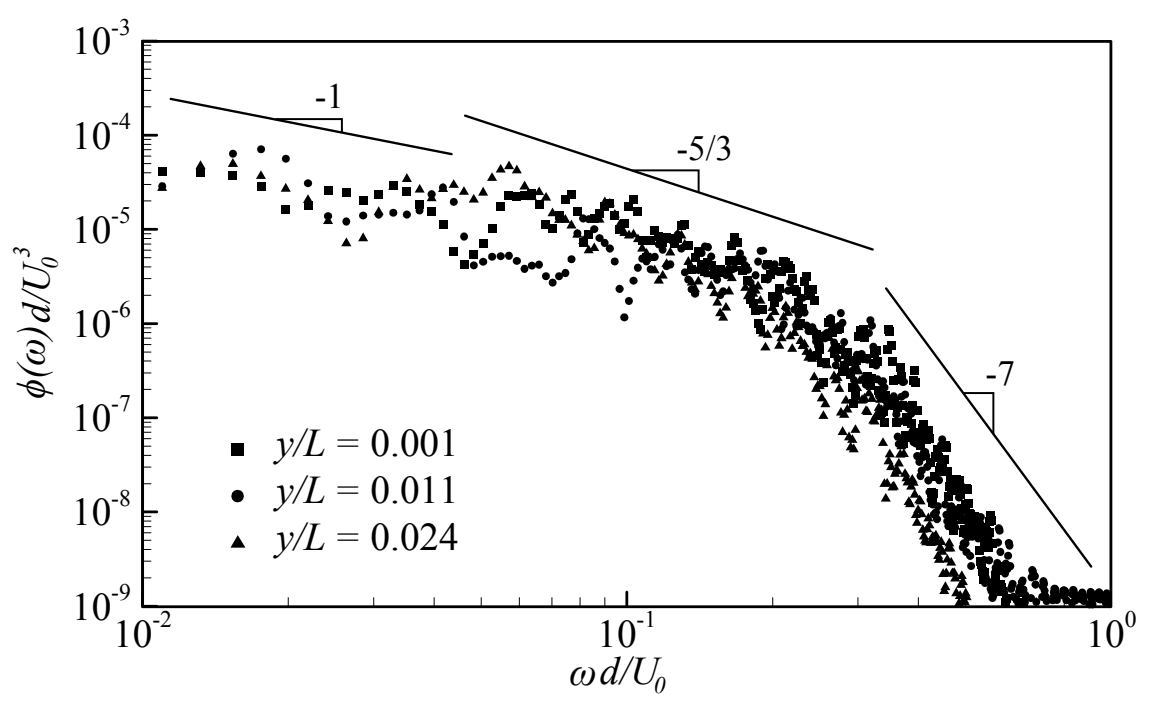

Figure 8.7: Frequency spectrum of the streamwise velocity fluctuations at $x / L=-0.035$.

Figure 8.7 plots the frequency spectrum of streamwise velocity fluctuations in the freestream at $x / L=-0.035$, just upstream of the leading-edge of the test surface. The turbulence at this location is representative of the free-stream turbulence to which the test-surface boundary layer is exposed. The power spectral density, $\phi(\omega)$, and frequency, $\omega$, in Figure 8.7 are normalized by the turbulence bar width, $d$, and reference velocity, $U_{0}$. The power spectra at three wall-normal locations are plotted to illustrate the variation in the turbulence spectrum at a range of wall-normal heights, which Figure 8.7 shows is minimal. Three distinct ranges are observed in the power spectra: a $\omega^{-1}$ range at low frequencies followed by a $\omega^{-5 / 3}$ range at intermediate frequencies, which matches the experiments of Klebanoff (1954), and a $\omega^{-7}$ range at high frequencies that is also observed in a recent DNS of Hickey et al. (2010).

The development of free-stream turbulence downstream of the test-surface leading edge is illustrated in Figure 8.8. The decrease in the turbulence intensity and the increase in the $\Lambda_{x}$ length scale upstream of the suction peak location is due to the strong free-stream acceleration, which dampens the free-stream eddies while stretching them in the $x$ direction. The maximum in the $\Lambda_{x}$ distribution at approximately $x / L=0.3$ coincides with the location of maximum freestream acceleration as per Figure 8.4. Downstream of this location, the streamwise-elongated 

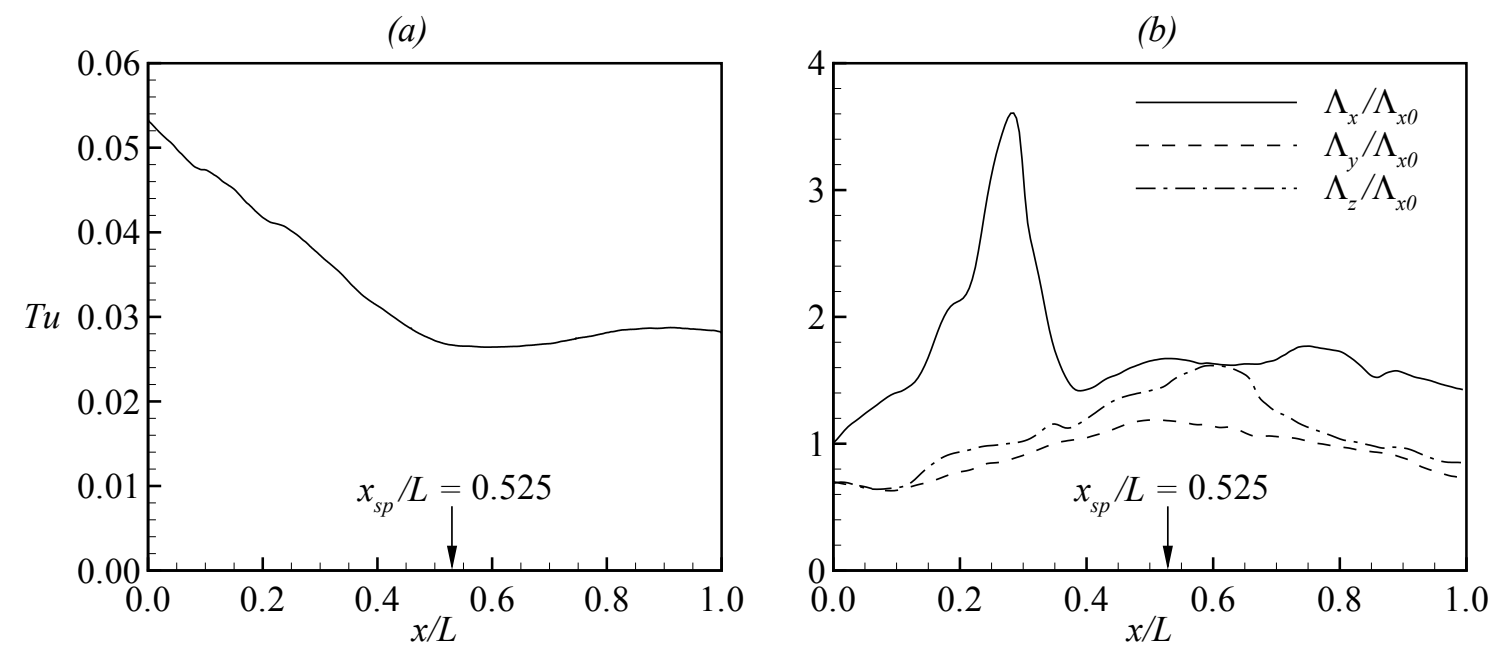

Figure 8.8: Streamwise decay of free-stream turbulence downstream of the test-surface leading edge. Area-averaged distribution of $(a)$ turbulence intensity, Tu, $(b)$ integral length scales normalized by $\Lambda_{x 0}$, the streamwise integral length scale at the test-surface leading edge.

eddies experience a lesser degree of streamwise acceleration on their downstream sides than on their upstream sides so that the eddy is "squeezed" and $\Lambda_{x}$ decreases. Downstream of the suction peak, the streamwise variation in the turbulence intensity and length scales is greatly reduced. As a result, the majority of the transitional region of the test surface occurs under relatively uniform free-stream turbulence conditions with an intensity of $0.026-0.028 U_{0}$ and integral length scales of $0.013 L-0.027 L$.

\subsubsection{Test-surface boundary-layer development}

The development of the test-surface boundary layer is plotted in Figure 8.9(a) through streamwise distributions of the boundary-layer integral parameters. The time-averaged locations of transition onset $\left(x_{t o} / L=0.436\right)$ and completion $\left(x_{t c} / L=0.785\right)$ are also shown. From Figure 8.9(b), the transition onset location is determined through the intermittency parameter, $\gamma$, defined as the percentage of time the boundary layer is turbulent. The intermittency parameter distribution is computed using the algorithm of Volino et al. (2001). 

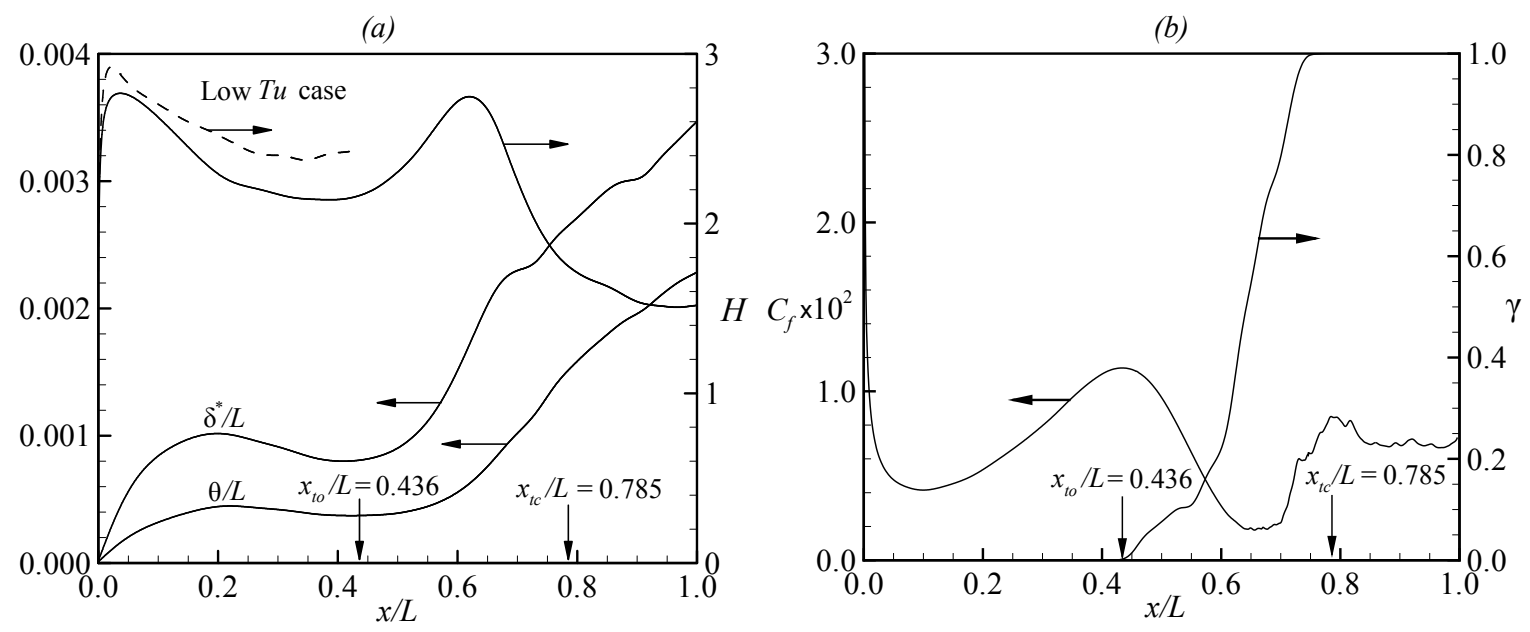

Figure 8.9: Streamwise distributions of $(a)$ displacement thickness $\left(\delta^{*} / L\right)$, momentum thickness $(\theta / L)$, and shape factor $(H) ;(b)$ friction factor $\left(C_{f} \times 10^{2}\right)$ and intermittency $(\gamma)$. The $H$ distribution for a complementary low free-stream turbulence simulation is shown in $(a)$ with dashed lines.

The transition completion location is determined by the local maximum in the friction factor, $C_{f}=2 \tau_{w} / \rho U_{0}^{2}$, which also agrees well with $\gamma=1$.

The effect of the free-stream turbulence on the boundary-layer integral parameters is deduced by comparing the shape factor $(H)$ distribution in Figure 8.9 $(a)$ to the $H$ distribution that would occur under low free-stream turbulence levels. To obtain such a low-turbulence $H$ distribution, a complementary simulation was performed in which the turbulence-generating grid of the present study is replaced with a uniform inlet velocity equal to $U_{0}$. The streamwise velocity distribution in the low-turbulence simulation is identical to the present high-turbulence case in the region upstream of the transition onset location. However, Figure 8.9(a) shows that the $H$ distribution that occurs under low free-stream turbulence is consistently larger than in the present high-turbulence case, which is consistent with previous experimental data of the authors' research group (e.g. Roberts and Yaras, 2005a). The difference in the $H$ distribution under low and high free-stream turbulence levels suggests that free-stream turbulence introduces small, localized perturbations into the laminar boundary layer that yield Reynolds stresses within the boundary layer, described in more detail in Section 8.3.3. The 
mixing associated with the Reynolds stresses fills out the velocity profile of the boundary layer under high free-stream turbulence relative to the boundary layer under low free-stream turbulence, producing lower values of $H$. Downstream of the suction peak $\left(x_{s p} / L=0.525\right)$, the transition rate increases - indicated by the "kink" near this location in the $\gamma$ distribution - and $H$ eventually reaches a steady value of about 1.5 in the fully-turbulent boundary layer.

\subsubsection{Formation of boundary-layer streaks}

Longitudinal low-speed streaks are created in the laminar boundary layer through the selective inclusion of disturbances from the free-stream beginning near the test-surface leading edge (Jacobs and Durbin, 2001). This is seen in Figure $8.10(a)$ by plotting the streamwise distributions of root-mean-square streamwise fluctuation velocity, $u_{r m s}^{\prime} / U_{0}$, at wall-normal locations of $y / L=10.77 \times 10^{-3}$ and $0.850 \times 10^{-3}$. Within the laminar-flow region between $x / L \approx 0.1$ and 0.5 , these locations roughly correspond to $y \approx 10 \delta^{*}$ and $y \approx 1 \delta^{*}$, respectively,

and so are representative of the conditions in the free-stream and well within the boundary layer. The distribution in the free-stream initially shows a slight decreasing trend in $u_{r m s}^{\prime}$ due to the enhanced decay of free-stream turbulence and flow stabilization by the imposed favourable pressure gradient. Past the transition-completion location, the free-stream flow experiences an increase in $u_{r m s}^{\prime}$ due to the influence of disturbances from the transitioned turbulent boundary layer. In contrast, the boundary layer $u_{r m s}^{\prime}$ distribution experiences an abrupt increase in fluctuation strength near $x / L=0.03$ as free-stream disturbances penetrate the boundary layer and are amplified via a transient growth mechanism. The fluctuations reach a steady amplitude by around $x / L=0.1$ and do not grow significantly until the transition onset location is reached. Although not shown, the root-mean-square of the wall-normal velocity fluctuations within the boundary layer is the same order of magnitude as the streamwise fluctuations. The associated Reynolds stresses produce the lower values of $H$ in the laminar boundary layer relative to the low free-stream turbulence case that was noted in Section 8.3.2. The fluctuation 

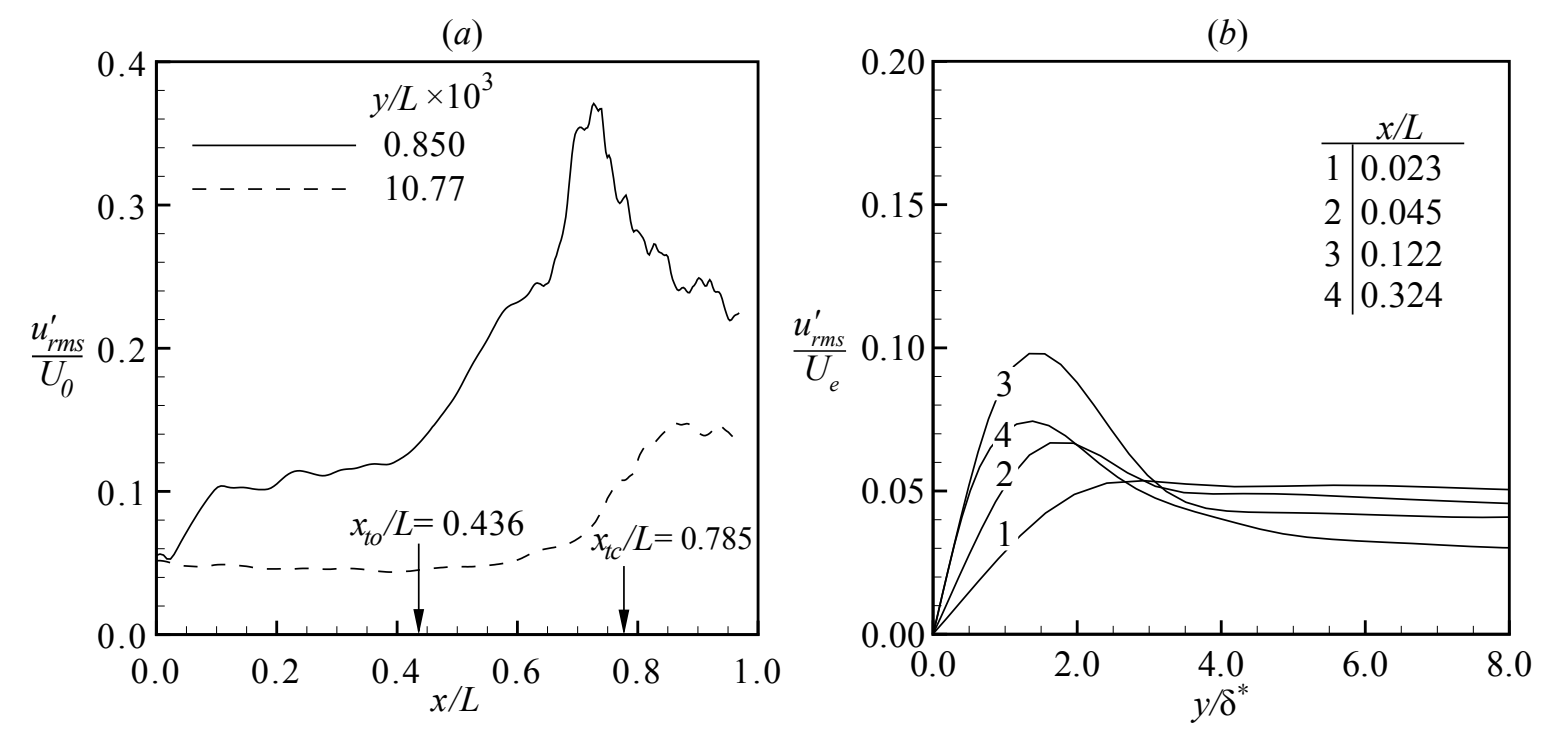

Figure 8.10: (a) Streamwise distribution of $u_{r m s}^{\prime}$ at $y / L=0.850 \times 10^{-3}$ and $y / L=10.77 \times 10^{-3}$. (b) Wall-normal profiles of $u_{r m s}^{\prime}$ in the laminar region.

amplitude increases rapidly within the transition zone due to the non-linear processes described in Section 8.3.4, reaching a peak amplitude near the transition completion location, and then decreases as the flow becomes fully turbulent.

The initial fluctuation growth in the laminar boundary layer is examined in Figure 8.10(b) through wall-normal profiles of $u_{r m s}^{\prime}$ normalized by the local boundary-layer edge velocity, $U_{e}$. The wall-normal coordinate is normalized by the local displacement thickness, $\delta^{*}$, which is between $40 \%-80 \%$ of the local boundary-layer thickness. Profile 1 shows that near the leading edge $(x / L=0.023)$, the fluctuation strength monotonically decreases from the turbulent freestream towards the wall with negligible amplification of disturbances in the boundary layer. Profile 2 shows that by $x / L=0.045$, free-stream disturbances have penetrated the boundary layer and have begun to be amplified, seen through the increase in peak $u_{r m s}^{\prime}$ at $y / \delta^{*}=2$. The location of the disturbance growth relative to the boundary-layer edge indicates that the disturbances are not amplified through a viscous Tollmien-Schlichting (T-S) instability mode, in which the maximum amplification is located near to the wall (e.g. Reynolds and Saric, 1986), but through a transient growth mechanism of the free-stream disturbances. Figure 8.10(a) 
indicates that the disturbance amplitude grows linearly with downstream distance between $x / L=0.1$ and 0.4 , in contrast to the exponential growth rate that occurs via the T-S mode. Downstream of profile 3 , the acceleration imposed by the favourable pressure gradient causes the fluctuation amplitude to decrease slightly relative to the local edge velocity, seen by profile 4 at $x / L=0.324$, although Figure 8.10(a) shows that the disturbance amplitude in an absolute sense remains relatively constant.

Figure 8.11 illustrates the form of the disturbances that penetrate into the boundary layer near the test-surface leading edge. Contours of $u$-velocity fluctuations are plotted in the same wall-normal planes as Figure 8.10(a) plus an intermediate location at $y / L=2.950 \times 10^{-3}$ that is situated above the edge of the boundary layer $\left(y \approx 3 \delta^{*}\right)$ within the plotted streamwise region. Figure 8.11(a) shows that the free-stream turbulence consists of small-scale disturbances that are roughly isotropic. As these disturbances penetrate into the boundary layer, they form streamwise-elongated streaks that primarily consist of narrow $u<0$ fluctuations compensated by broader regions of $u>0$ fluctuations. The shear-sheltering phenomenon observed by Jacobs and Durbin (2001) is clearly distinguished by comparing the three wall-normal planes; the highfrequency free-stream modes are visible near the leading edge in all three planes, but these are quickly damped within the boundary layer such that only the low-frequency streamwise streaks are amplified.

Figure 8.12 portrays a longer portion of the domain than Figure 8.11 at several instances in time to highlight the temporal evolution of the streamwise streaks. The time interval is $0.042 L / U_{0}$, starting from an arbitrary initial time of $\tau=0.000$ that corresponds to the data in Figure 8.11. The behaviour of the streaks observed in Figure 8.12 involves their convection, streamwise lengthening, merging, and meandering within the boundary layer. The convection of a typical low-speed streak is shown in Figure 8.12 by the white line that traces the location of a given point on the streak in time. The highlighted streak, labelled A, is created near $x / L=0.05$ at $\tau=0.000$. As it convects downstream, it lengthens in the streamwise direction 

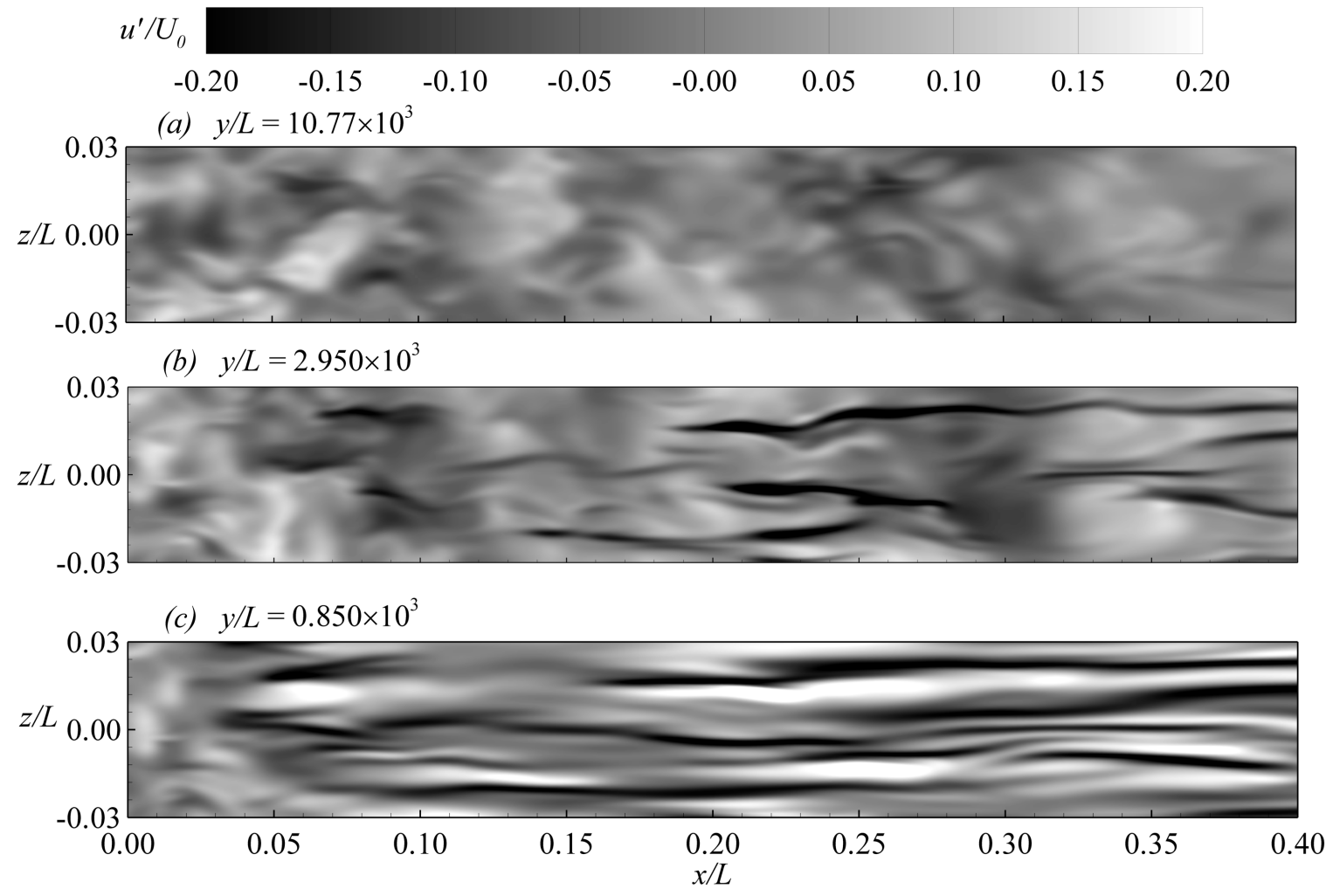

Figure 8.11: Streamwise velocity fluctuation contours at $(a) y \approx 10 \delta^{*},(b) y \approx 3 \delta^{*}$, and $(c) y \approx$ $1 \delta^{*}$. 
while developing stronger $u^{\prime}<0$ fluctuations. For certain streaks, this lengthening results in a joining of axially-aligned streaks, as illustrated by the streaks labelled B and C, which are created near $\tau=0.000$ and $\tau=0.042$, respectively. As both lengthen in the streamwise direction, they join at around $\tau=0.170$ to produce a large-scale streak with an axial length of about $0.5 \mathrm{~L}$. The convection of such large-scale streaks through the boundary layer produces disturbances within the boundary layer at frequencies that are on the order of $U_{0} / L$, which is notably lower than the frequency of the free-stream disturbances. Figure 8.12 also shows that the convecting streamwise streaks are not perfectly aligned in the axial direction but the spanwise location of the streak varies along its length with an amplitude that is on the order of the streak width. When the convecting streaks are viewed in a stationary reference frame, such variation of the streak's location appears as a spanwise meandering of the steaks. The amplitude of meandering has been shown to be a function of the streamwise pressure gradient, where favourable pressure gradients tend to decrease the amplitude of meandering and viceversa (Piomelli et al., 2000). While the meandering resembles the periodic waviness that would result from the development of an asymmetric secondary instability mode on the streak, it is distinct in that it occurs at a very low frequency - on the order of the frequency at which distinct streaks convect past a stationary observer - while the sinuous secondary instability mode occurs at a higher frequency near that of the free-stream disturbances (Schlatter et al., 2008).

The statistical properties of the low-speed streamwise streaks are highlighted by analyzing their spanwise spacing and amplitude. These properties have been shown to be closely connected to the development of secondary streak instabilities, as reviewed in Section 8.1. Figure 8.13(a) plots the mean spanwise spacing of the low-speed streaks at several streamwise stations. The mean spanwise spacing, denoted $\bar{\lambda}$, is calculated by averaging the center-to-center distance between streaks in a $y-z$ plane at a particular axial location at four uniformly-spaced instances in time, resulting in 50 to 150 streaks being included in the average at each streamwise station. The temporal spacing was large enough to ensure that unique streaks are used in the 


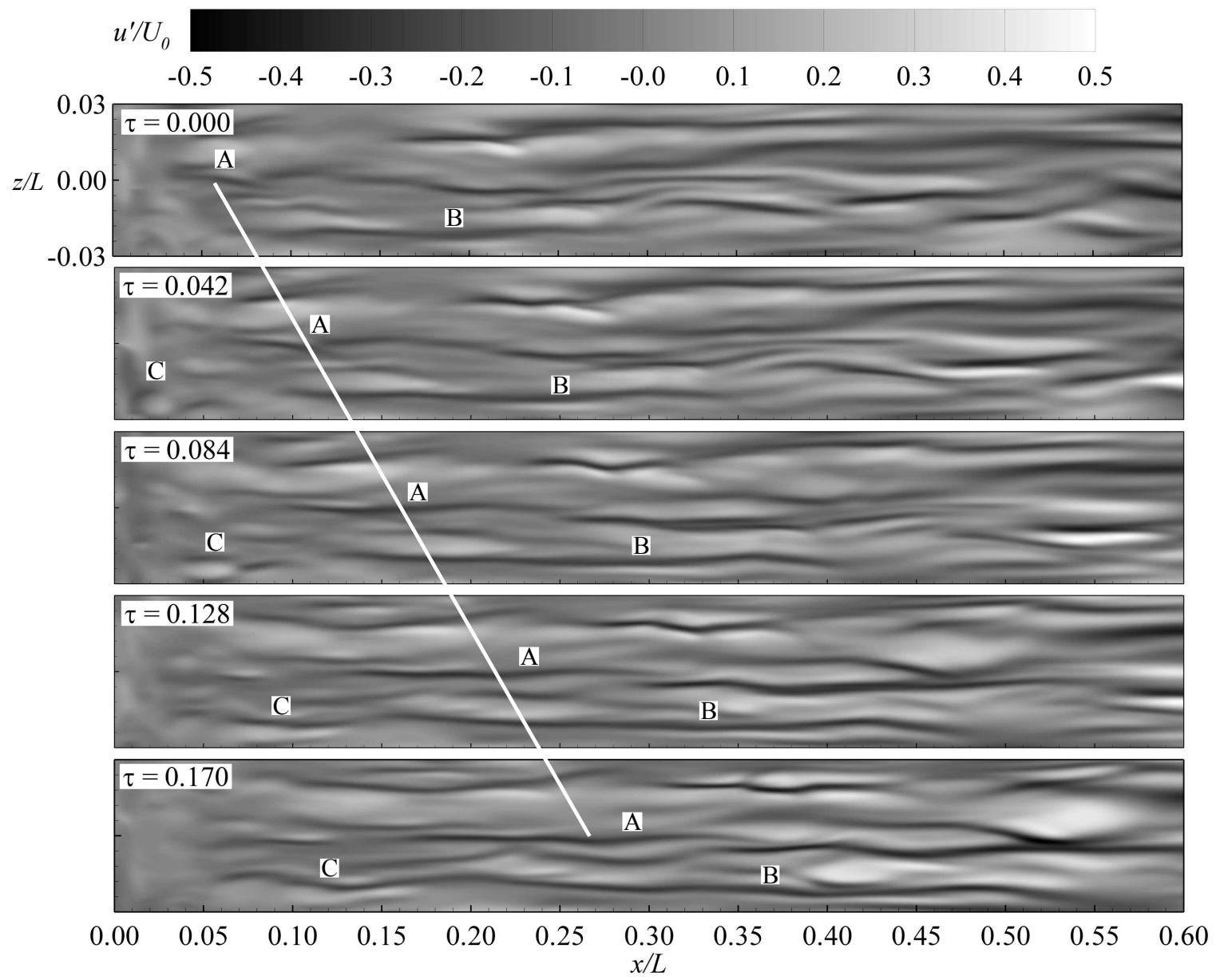

Figure 8.12: Temporal evolution of $u^{\prime}$ contours at $y / L=0.850 \times 10^{-3}$. The contours at $\tau=0.000$ corresponds to the data in Figure 8.11. 
average at each instance in time. The error bars in Figure 8.13(a) indicates the uncertainty in $\bar{\lambda}$ based on the standard error of the mean spacing. A larger uncertainty occurs near the leading edge due to an increased variance in $\lambda$ near the leading edge, primarily due to the freestream turbulence that triggers the streaks. Because the free-stream turbulence is composed of disturbances with a broad spectrum of wavelengths, it is expected that the streaks will initially have a broad range of spacings. Once the free-stream disturbances permeate into the boundary layer, the local conditions within the boundary layer determine what wavelengths will be amplified, and disturbances with non-optimum wavelengths are damped out. The variance in $\lambda$ near the leading edge is also partially due to the irregular meandering of streaks within the boundary layer noted earlier that causes an unsteadiness in the relative spanwise locations of the streaks. The amplitude of the meandering motion steadily decreases as the flow accelerates towards the suction peak so that the variance in $\lambda$ decreases with downstream distance, as shown by the diminishing uncertainty bounds.

Figure 8.13(a) shows that the absolute spanwise spacing of the low-speed streaks $(\bar{\lambda} / L)$ achieves an approximately-constant value upstream of the transition completion location, followed by a lower value in the turbulent region. When normalized by the viscous length based on the local wall shear stress, the streak spacing increases until $x / L=0.371$, which corresponds to the station with the strongest free-stream acceleration and largest wall shear stress (Figures 8.4 and 8.9), where $\overline{\lambda^{+}}=198 \pm 26$ is much larger than the typical spacing of $100 \pm 20$ viscous lengths in zero-pressure-gradient turbulent boundary layers (e.g. Smith and Metzler, 1983; Rajaee et al., 1995), implying enhanced stability of the boundary layer in the accelerated region (e.g. Yaras, 2007). Downstream of $x / L=0.371$, the normalized streak spacing decreases quite rapidly as the boundary layer reacts to the reduction in free-stream acceleration, settling to values in the fully-turbulent region that are in closer agreement to those in zero-pressure-gradient turbulent boundary layers. The increase in $\overline{\lambda^{+}}$downstream of $x / L=0.620$ reflects the decrease in the wall shear stress in the turbulent region (as shown in 

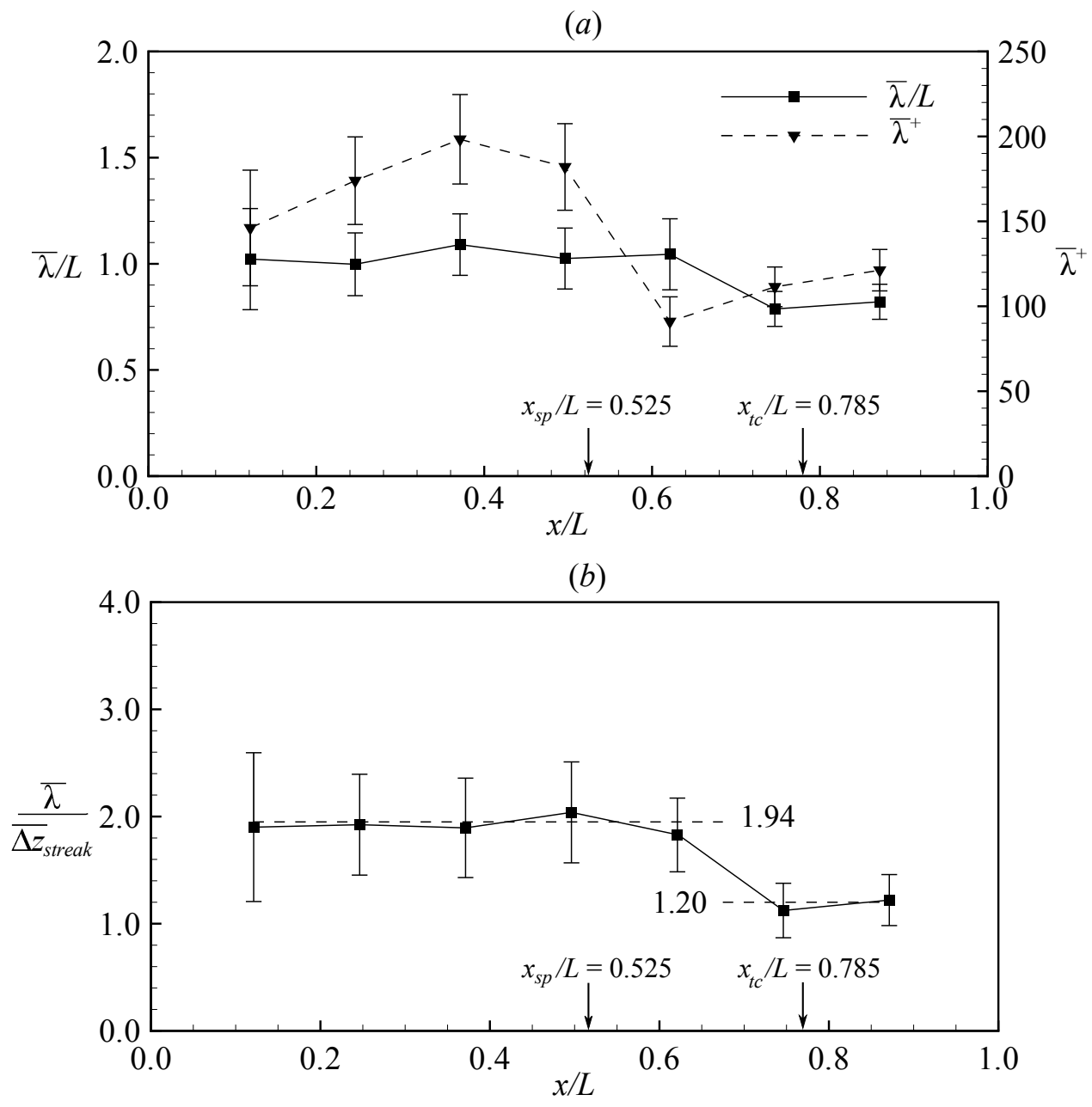

Figure 8.13: Streamwise distribution of the spanwise spacing of the low-speed $u$-velocity streaks. Normalization is by $(a) L$ and $u_{\tau} / \nu$ and $(b) \overline{\Delta z}_{\text {streak }}$. 
Figure 8.9$)$, as $\bar{\lambda} / L$ changes very little.

Figure $8.13(b)$ normalizes $\bar{\lambda}$ by the local width of the low-speed streaks, denoted $\overline{\Delta z}_{\text {streak }}$. The ratio $\bar{\lambda} / \overline{\Delta z}_{\text {streak }}$ has been shown to be relevant in determining whether secondary instabilities of the streamwise streaks will develop (e.g. Konishi and Asai, 2004). Mean streak widths are determined by individually examining each streak in the same axial planes and instances in time as those used in evaluating the streak spacing. For a given streak under examination, its width is determined based on the spanwise locations of the left and right spanwise edges of the streak, which are determined based on threshold values of $u^{\prime} / U_{e}$. Three preliminary widths are calculated using threshold values of $u^{\prime} / U_{e}=0.03,0.05$, and 0.07 , and these preliminary widths are averaged to give an individual mean streak width. The mean of all such individual streak widths over the four simulation times corresponds to the local mean streak width, $\overline{\Delta z}_{\text {streak }}$. To ascertain the sensitivity of $\overline{\Delta z}_{\text {streak }}$ to the threshold values of $u^{\prime} / U_{e}$ and the number of preliminary streak-widths included in the averaging process, the above described method was repeated at selected axial locations using 2, 4, and 6 uniformly-spaced threshold values between $u^{\prime} / U_{e}=0.02$ and 0.08 . The resulting uncertainty in the width of an individual streak due to the choice of threshold values was found to be much smaller than the uncertainty in $\overline{\Delta z}_{\text {streak }}$ due to the varied widths of the all streaks examined at a given axial location. This latter uncertainty is shown by the error bars in Figure 8.13(b).

The trends visible in Figure 8.13(b) indicate that $\bar{\lambda} / \overline{\Delta z}_{\text {streak }}$ achieves distinct but approximately constant values of 1.94 and 1.20 in the laminar and turbulent regions, respectively, despite the range of shear-layer thicknesses and streamwise pressure gradients present in both regions. The low-speed streamwise streaks are, on average, spaced less than 2.5 streak widths apart, which Konishi and Asai (2004) observed is the threshold spacing beyond which secondary instability modes are suppressed. This implies that, at least in terms of the streak spacing, the conditions in the present results are suitable for the development of secondary streak instabilities. However, time histories of the convecting streaks, such as shown in Figure 8.12, 
show no evidence of either symmetric or asymmetric secondary instabilities developing prior to the completion of transition. Instead, the streaks persist until well past the suction peak location and then rapidly break-down into turbulence, which is discussed further in Section 8.3.4. To explain the persistence of the streaks and absence of secondary modes, Figure 8.14 plots the amplitude and disturbance energy of the streaks at the streamwise stations corresponding to Figure 8.13 . The mean streak amplitude, ${\overline{u^{\prime}}}_{\text {streak }}$, is defined as the local maximum of $\left|u^{\prime}\right|$ occurring in a low-speed streak, averaged over multiple streaks using the same method as was used for $\bar{\lambda}$. The disturbance energy of the streaks $\left({\overline{u^{\prime}}}_{\text {streak }} / U_{0}\right)^{2}$ in the laminar-flow region upstream of the suction peak increases linearly, and can be approximated by $\left({\overline{u^{\prime}}}_{\text {streak }} / U_{0}\right)^{2} \approx 3.50 \times 10^{-7}\left(x U_{0} / \nu\right)$. The coefficient $3.50 \times 10^{-7}$ is a measure of the algebraic growth rate of the disturbance energy associated with the streaks. Asai et al. (2007) showed that the algebraic growth rate depends on the initial energy of the free-stream turbulence, and this is confirmed by scaling the algebraic growth rate in the present study by the ratio of $u_{r m s}^{\prime}$ at $x / L=0$ between the present study and Asai et al. (2007). The resulting scaled growth rate in the present study is $2.02 \times 10^{-7}$, which agrees very closely with the value of $2 \times 10^{-7}$ found by Asai et al. (2007) for a zero-pressure-gradient boundary layer. Such a close agreement despite the strongly accelerating conditions in the present results indicates that the favourable pressure gradient upstream of the suction peak does not significantly alter the amplification rate of streamwise streaks in the laminar portion of the boundary layer. This is intuitive considering that the growth of the streaks is the result of the selective inclusion of free-stream disturbances that are developed in the boundary layer through a transient-growth mechanism rather than a spatially-developing instability mode, and so is less sensitive to boundary-layer stabilization by the favourable pressure gradient. Instead, Figure 8.14(b) shows that upstream of the suction peak, the favourable pressure gradient decreases the relative streak amplitudes normalized by the edge velocity of the boundary layer, $U_{e}$, to well below the values of $26 \%$ and $37 \%$ that are respectively necessary for the development of sinuous or varicose secondary modes (e.g. Brandt and Henningson, 2002). Although the streaks reach sufficient amplitudes 
downstream of the suction peak, break-down to turbulence occurs too soon for any secondary streak instabilities to occur.

\subsubsection{Visualisation of streak break-down and transition}

The break-down of the boundary layer to irregular motion is visualized in Figure 8.15 by examining $u^{\prime}$ and $v^{\prime}$ contours in a $y$ - $z$ plane at $y / L=0.850 \times 10^{-3}$, which is about $0.5 \delta^{*}$ above the test surface within the region shown, at time instances in the range $\tau=0.042-0.126$. The $u^{\prime}$ contours in Figure 8.15(a) show that the flow transitions to turbulence approximately between $x / L=0.70$ and $x / L=0.75$, and that there is a notable demarcation visible in the $u^{\prime}$ contours between the turbulent and non-turbulent zones. Upstream of the turbulent zone, orderly arrays of high- and low-speed streamwise streaks are observed with little evidence of secondary streak instabilities owing to the sub-critical streak amplitudes described previously. Instead, transition occurs as individual low-speed streaks break-down into irregular turbulent motion, which is consistent with the observations of Jacobs and Durbin (2001) in a bypass transition of a zero-pressure-gradient boundary layer. To analyze the present transition processes in detail, the break-down of a representative low-speed streak is traced in time in Figure 8.15. Beginning at $\tau=0.042$, a localized disturbance is observed surrounding a low-speed streak located near $z / L=0.00$. By $\tau=0.063$, this disturbance results in the break-down of the low-speed streak into smaller-scale motions that grow in time and form a distinct and finite region of irregular motion that resembles a turbulent spot. By $\tau=0.084$, the spot has enlarged and convected towards the turbulent zone, merging with it by about $\tau=0.105$. The flow at $\tau=0.128$ shows that when the spot merges with the turbulent zone, the interface between the turbulent and non-turbulent zones abruptly shifts to the location of the upstream (trailing) edge of the spot, causing the interface between the turbulent and non-turbulent region to vary in time and in the spanwise direction with an amplitude of up to $0.1 \mathrm{~L}$. 
(a)

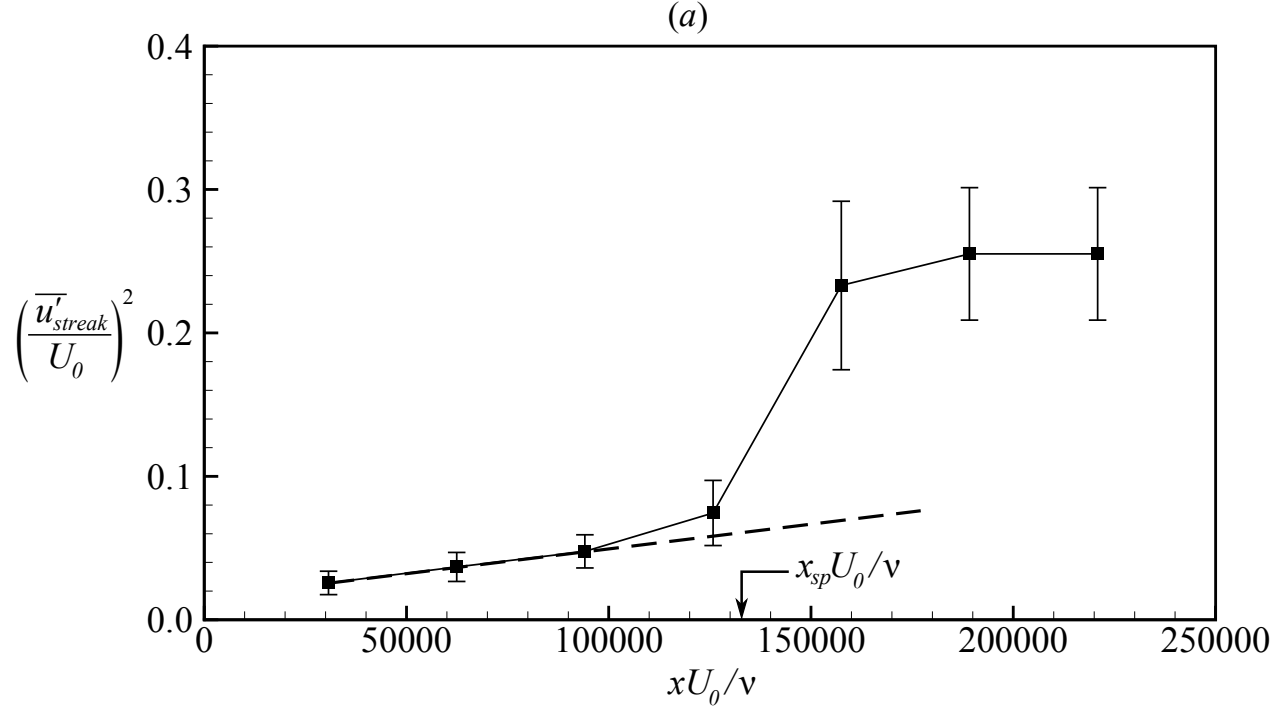

(b)

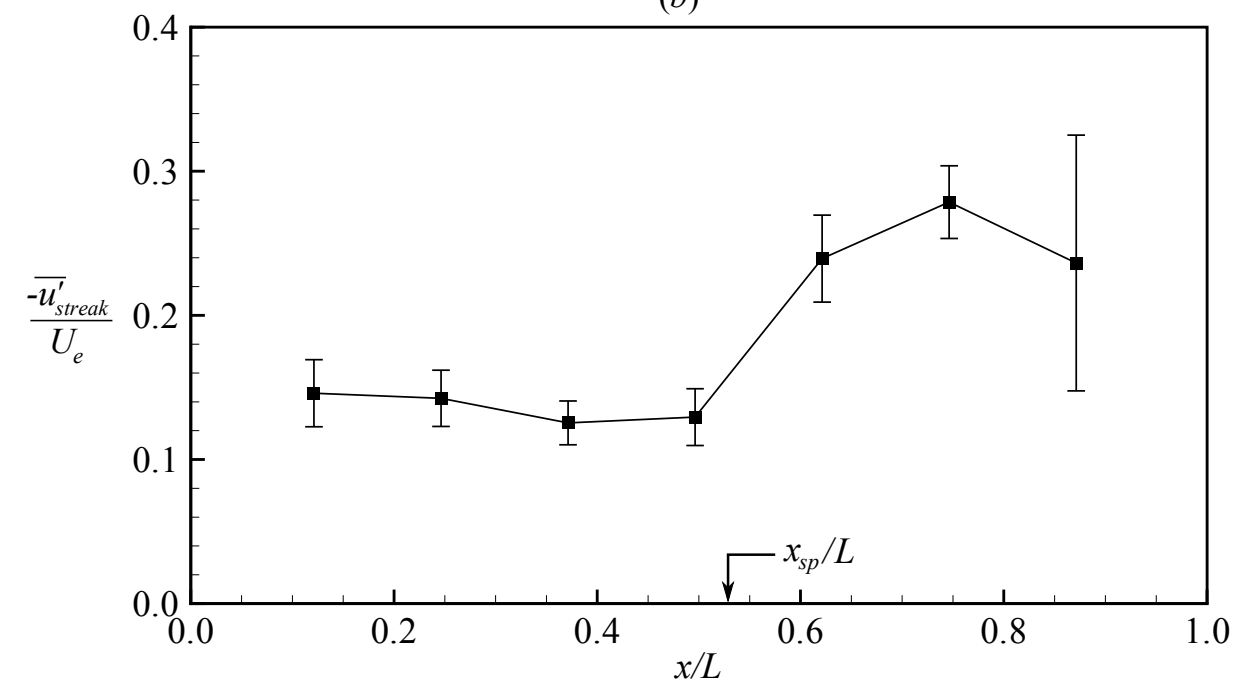

Figure 8.14: Streamwise development of the amplitude of the low-speed $u$-velocity streaks. (a) Relative streak amplitude $\overline{u^{\prime}}{ }_{\text {streak }} / U_{e}$. (b) Disturbance energy $\left(\overline{u^{\prime}} \text { streak } / U_{0}\right)^{2}$; dashed line, $\left({\overline{u^{\prime}}}_{\text {streak }} / U_{0}\right)^{2}=3.50 \times 10^{-7}\left(x U_{0} / \nu\right)$. 

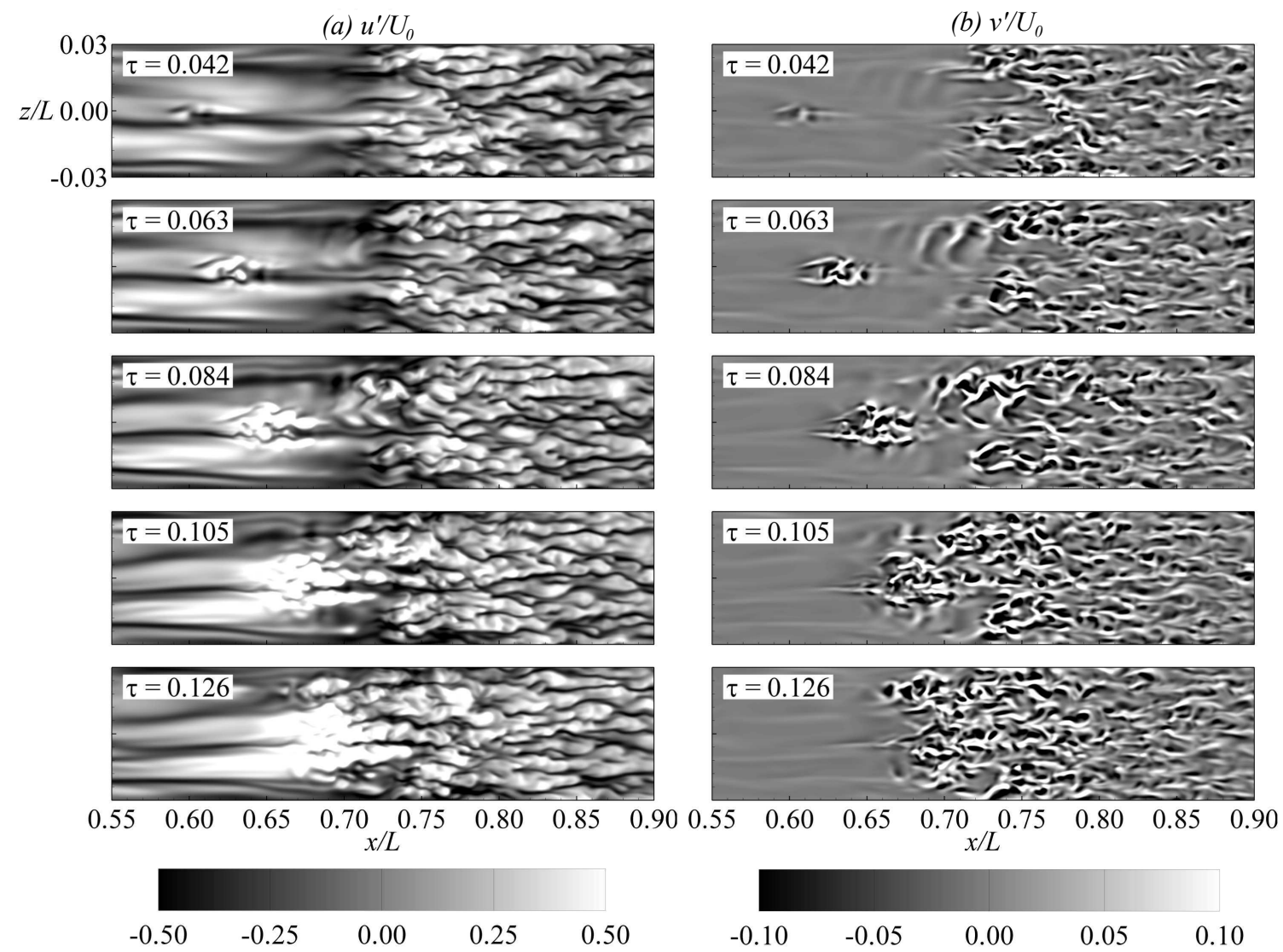

Figure 8.15: Temporal evolution of $(a) u^{\prime}$ and $(b) v^{\prime}$ contours at $y / L=0.850 \times 10^{-3}$. 
The $v$-velocity fluctuations in Figure $8.15(b)$ give a clearer impression of the localized disturbances that cause the break-down of the low-speed streak and the small-scale motions that form within the resulting turbulent spot. The $v^{\prime}$ contours at $\tau=0.042$ shows that the disturbance consists primarily of a strong localized $-v^{\prime}$ motion (sweep) surrounded by weaker and more dispersed $+v^{\prime}$ motions (ejections). At subsequent time instances, the $v^{\prime}$ contours amplify and elongate in sync with the growing turbulent spot, and the spanwise edges of the spot appear to be terminated by ejections. These appear to correspond to an ejection of lowmomentum fluid along the sides of the spot, which numerous studies of isolated turbulent spots in laminar boundary layers have shown to contribute to the lateral growth of the spot (e.g. Schröder and Kompenhans, 2004).

Before the origin of the initial localized disturbance that triggers streak break-down is discussed, it is worthwhile to clarify the connection between the streak break-down in Figure 8.15 and the formation of coherent vortical structures in the transitional boundary layer. To do so, Figure 8.16 re-plots the time instances of Figure 8.15 using three-dimensional iso-contours of negative $u^{\prime}$ and the second invariant of the velocity gradient tensor, denoted $Q$, defined as

$$
Q=\frac{1}{2}\left({\frac{\partial u_{i}}{\partial x_{j}}}^{2}-\frac{\partial u_{i}}{\partial x_{j}} \frac{\partial u_{j}}{\partial x_{i}}\right)
$$

where Einstein summation is implied over the indexed terms. The $Q$ property has been shown to improve the prominence of coherent vortices over the background vorticity (e.g. Jeong and Hussain, 1995). Instantaneous vorticity lines are also plotted to illustrate the correlation of the vorticity field with $Q$; for improved visibility of these lines, the $Q$ iso-contours are rendered semi-translucent. The $-u^{\prime}$ iso-contours at $\tau=0.042$ and 0.063 show that the perturbation of the low-speed streak under consideration (the one located near $z / L=0.00$ ) occurs near $x / L=0.625$ at a height of about $y / L=0.003-0.006\left(2-4 \delta^{*}\right)$. The $Q$ iso-contours indicate that the perturbations correspond to small hairpin vortices that extend from near the wall to about $0.003 L$ or about $2 \delta^{*}$, the initial formation of which is discussed below. By $\tau=0.063$, 

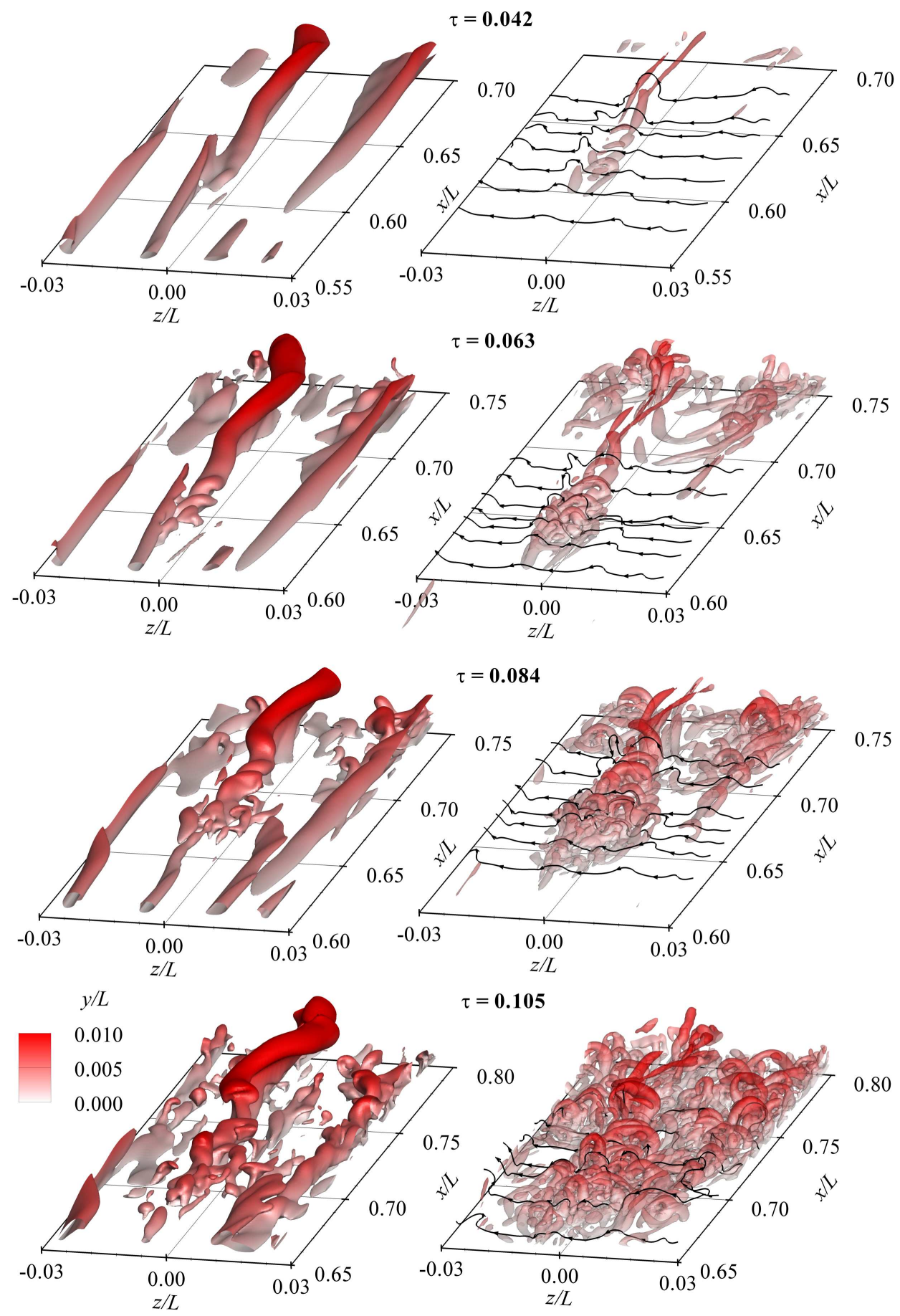

Figure 8.16: Transient break-down of a low-speed streak illustrated through iso-contours of (left) $u^{\prime} / U_{0}=-0.20$ and (right) $Q\left(L / U_{0}\right)^{2}=5000$. Iso-contours are coloured by $y / L$ and lines show selected samples of instantaneous vorticity lines. 
the hairpin vortices have spawned subsequent generations of vortices, both in the streamwise direction and along the spanwise edges of the spot so that by $\tau=0.084,4-5$ generations of hairpin vortices occur across the span of the spot and 6-7 generations of hairpin vortices occur between the spot's leading and trailing edges. The "kinks" in the vorticity lines outboard of the spot's spanwise edges at $\tau=0.084$ and earlier instances indicate regions where ejection of fluid induced by the vortices within the spot have lifted the vorticity line outboard of the spot. As the up-lifted portions of the vorticity lines are stretched by the mean shear, they strengthen and form new hairpin vortices along the spanwise edges of the spot, resulting in the noted increase in the number of hairpin vortices within the spot. By $\tau=0.105$, the spot has convected close enough to the downstream turbulent zone that it becomes difficult to differentiate the vortical structures belonging to the spot from those that make up the turbulent zone.

Returning to the $u^{\prime}$ iso-contours at $\tau=0.063$ in Figure 8.16, it is evident that the wallnormal motions induced by the hairpin vortices within the growing turbulent spot correspond to a roll-up of the low-speed streak into discrete packets of low-speed fluid. The rollup resembles the streamwise-periodic roll-up of a shear layer driven by an inviscid KelvinHelmholtz $(\mathrm{K}-\mathrm{H})$ instability. If $u^{\prime}$ contours are analyzed in an $x-y$ plane that intersects the rolled-up streak, the familiar "cat-eye" pattern associated with the K-H instability is observed, leading Durbin and Wu (2007), McAuliffe and Yaras (2010), and Zaki and Durbin (2005) to suggest that the streak roll-up occurs due to the K-H instability of the shear layer surrounding the streak. The dominant frequency of the K-H instability can be estimated using the streamwise wavelength and convection velocities of the rolled-up segments of the low-speed streak. When this frequency is expressed as a Strouhal number based on the local momentum thickness $\left(\theta / L=0.795 \times 10^{-3}\right)$ and boundary-layer edge velocity $\left(U_{e}=1.949 U_{0}\right)$, the result is $S r_{\theta}=f \theta / U_{e}=0.050$. This value is much larger than the Strouhal number associated with K-H instability in plane shear layers, for which $S r_{\theta}=0.016$ (Ho and Huerre, 1984), and in transitional separation bubbles under low and elevated free-stream turbulence conditions, 
for which $S r_{\theta}=0.011$ and 0.014-0.016, respectively (e.g. McAuliffe and Yaras, 2010). The much higher roll-up frequency in the present results suggests that the $\mathrm{K}-\mathrm{H}$ instability of the shear layer surrounding the streak is modified due to interactions with disturbances from the free-stream turbulence.

To illustrate the nature of this interaction, Figure 8.17 plots $u^{\prime}$ contours and $v^{\prime} / U_{0}= \pm 0.1$ lines in an $x$ - $y$ plane at $z / L=-0.002$, which roughly intersects the middle of the low-speed streak. The time instances in Figure 8.17 are chosen to illustrate the features in the free-stream turbulence that catalyze the formation of the hairpin vortices and accelerate the roll-up of the low-speed streak. The dashed $-v^{\prime}$ contours prevailing in the free-stream upstream of the low-speed streak at $\tau=0.021$ indicate the presence of a discrete vortical disturbance (eddy) in the free-stream turbulence. The anisotropic shape of the eddy is due to the strong freestream acceleration, which stretches it in the streamwise direction as it convects through the favourable-pressure-gradient region of the flow. As the eddy convects through the adversepressure-gradient region (i.e. the region plotted in Figure 8.17), the flow deceleration squeezes it in the streamwise direction so that it gradually regains an approximately-isotropic shape. The $-v^{\prime}$ motions induced by the free-stream eddy create a corresponding region of $+u^{\prime}$ due to the sweep of high-momentum fluid from the free-stream into boundary layer, which results in the formation of a high-speed streak beneath the eddy in the wake of the low-speed streak. Because the low-speed streak moves slower than the local mean flow, the eddy and the highspeed streak it creates gradually overtake the upstream edge of the low-speed streak. This is evident at $\tau=0.032$ where the high-speed streak collides with and begins to convect above the upstream edge of the low-speed streak. As the sweep events caused by the free-stream eddy continually energize the high-speed streak, its amplitude increases and a thin region of very high shear stress develops at the interface between the high- and low-speed streaks. This shear layer is unstable to high-frequency disturbances via the $\mathrm{K}-\mathrm{H}$ instability mode due to its inflectional wall-normal velocity profile so that by $\tau=0.042$, it begins to roll-up into discrete, 


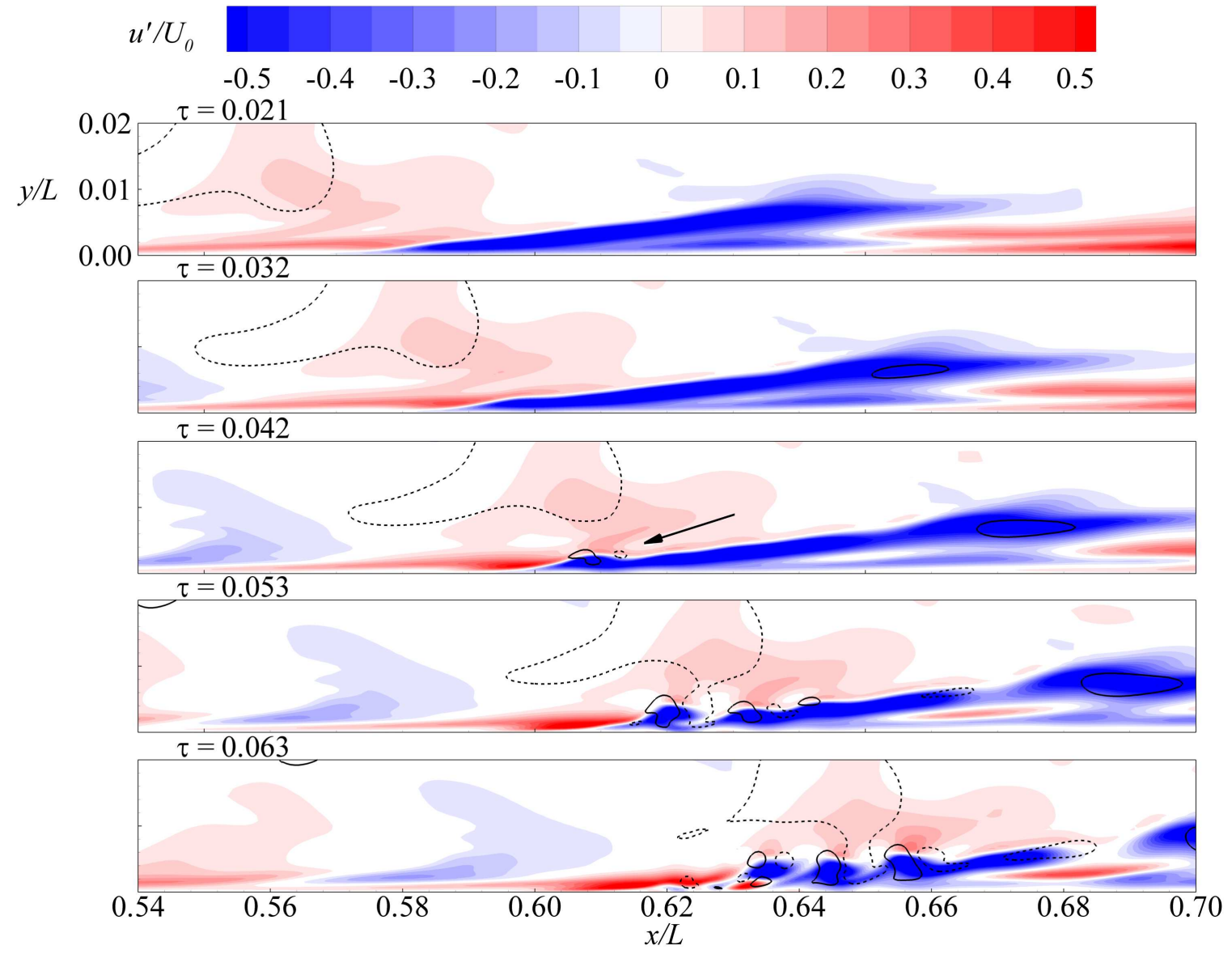

Figure 8.17: Interaction of a free-stream vortical disturbance and a streamwise streak in the $z / L=-0.002$ plane. Contours are of $u^{\prime} / U_{0}$ and lines are $v^{\prime} / U_{0}=0.10$ (solid) and $v^{\prime} / U_{0}=$ -0.10 (dashed). 
spanwise-oriented vortices. According to Helmholtz's vorticity laws, the rolled-up spanwise vortices must remain continuously connected to the spanwise vorticity that exists along the test-surface wall, and because the interfacial shear layer lies relatively far from the wall, the rolled-up segments are convected faster than the ends that remain connected to the near-wall vorticity. The resulting stretching gives the rolled-up vortex its hairpin shape seen at $\tau=0.042$ in Figure 8.16. Small-scale wall-normal motions induced by the hairpin vortices, indicated by the arrow at $\tau=0.042$, create a streamwise-grouping of low-speed fluid, visible at $\tau=0.053$ and 0.063 , and ultimately result in the break-down of the low-speed streak into a turbulent spot.

The flow development shown in Figure 8.17 strongly suggests that the roll-up and breakdown of the low-speed streaks in the present study is accelerated by strong $-v^{\prime}$ motions caused by eddies in the free-stream turbulence above the streaks. The collision of the high-speed streak generated by such an eddy with a downstream low-speed streak produces such high levels of interfacial shear that the roll-up of the low-speed streak via K-H instability occurs at a frequency that is much higher than would occur based on the conditions of the lowspeed streak in isolation. When streaks at other times and spatial locations are analyzed, it is consistently observed that low-speed streaks that are not below a large $-v^{\prime}$ eddy in the free-stream turbulence do not break down into a turbulent spot as in Figure 8.17. Instead, they maintain their coherence until they are eventually entrained into a neighbouring turbulent spot. This implies that a discrete free-stream disturbance is required for streak break-down to occur. Such a requirement is also observed by Liu et al. (2008) in a DNS in which discrete and continuous modes of the Orr-Sommerfeld equation are forced in a transitional zero-pressuregradient boundary layer. The discrete modes correspond to vortical disturbances within the flow and the continuous modes correspond to high- and low-speed streaks. Neither mode is sufficient to cause transition alone, as the discrete modes in isolation tend to be damped, but their mutual interaction results in the formation of a pattern of $\Lambda$-shaped structures with 
a wavelength that is smaller than the secondary instability of the discrete Orr-Sommerfeld modes, which is qualitatively similar to the closely-spaced hairpin vortices observed in the present study. Furthermore, the DNS study Zaki and Durbin (2006) indicates that the local adverse pressure gradient in the present study enhances the coupling between the free-stream vortical disturbances and the boundary-layer streaks. Simulations of Brandt and de Lange (2008) and Schlatter et al. (2008) and particle image velocimetry measurements of Nolan and Walsh (2012) also corroborate the present observations that streak break-down originates in the instability of the interfacial shear layer between the head of an oncoming high-speed streak and the tail of a downstream low-speed streak.

It was noted earlier that the mean shear shelters the boundary layer from high-frequency free-stream disturbances so that only low-frequency disturbances are able to penetrate deep into the boundary layer. However, once the shear layer rolls-up, the instantaneous shear layer becomes spatially discontiguous so that high-frequency disturbances can now penetrate from the free-stream into the boundary layer. This is illustrated in Figure 8.18 by plotting $v^{\prime}$ lines and the instantaneous spanwise vorticity $\left(\omega_{z}\right)$ contours in the rolled-up portions of the shear layer from Figure 8.17. To highlight the temporal development of the shear layer, the axial coordinate is shifted to keep the convecting low-speed streak in the center of the plotted data, and the wall-normal coordinate is stretched by a factor of two. The contiguous shear layer located away from the wall at $\tau=0.032$ corresponds to the strong interfacial shear layer created between the high- and low-speed streaks. Because of the shear-sheltering effect, freestream vortical disturbances are not able to penetrate through this shear layer, as indicated by the $v^{\prime}$ contour lines. However, as the interfacial shear layer rolls-up, as at $\tau=0.053$, it is no longer contiguous and free-stream vortical disturbances are able to penetrate through the shear layer towards the wall, coupling with the small-scale $v^{\prime}$ motions that are induced by the hairpin vortices. In three dimensions, this corresponds to sweep motions from the free-stream disturbances penetrating between the hairpin vortices surrounding the low-speed streak. At 
$\tau=0.074$ it is evident that the sweep motions penetrate far into the boundary layer, reaching very near the wall, where they energize the small-scale vortical structures and accelerate the growth of the turbulent spot.

The physical role of the sweep motions induced by the free-stream vortical disturbances in bypass transition can be investigated quantitatively by examining the transport of enstrophy, $\xi$, defined as the trace of the fluctuating vorticity covariance, $\overline{\omega_{i}^{\prime} \omega_{i}^{\prime}}$. Park et al. (2012) have shown that this property exhibits similar distributions in transitional and fully-developed turbulence, and thus is appropriate for assessing the development of non-linear interactions between the free-stream vortical disturbances and the streamwise streaks during transition. The transport equation for enstrophy is obtained by the scalar product of the transport equation for the instantaneous vorticity by the instantaneous vorticity vector, time averaging, and then subtracting the scalar product of the transport equation for the time-mean vorticity and the time-mean vorticity vector (e.g. Tennekes and Lumley (1972)), yielding:

$$
\begin{aligned}
\frac{D}{D t}\left(\frac{\xi}{2}\right) & =\underbrace{\overline{\omega_{i}^{\prime} \omega_{j}^{\prime} \frac{\partial U_{i}}{\partial x_{j}}}}_{P 1}+\underbrace{\overline{\omega_{i}^{\prime} \frac{\partial u_{i}^{\prime}}{\partial x_{j}}} \Omega_{j}}_{P 2}-\underbrace{\overline{u_{j}^{\prime} \omega_{i}^{\prime}} \frac{\partial \Omega_{i}}{\partial x_{j}}}_{P 3}+\underbrace{\overline{\omega_{i}^{\prime} \omega_{j}^{\prime} \frac{\partial u_{i}^{\prime}}{\partial x_{j}}}}_{P 4} \\
& -\underbrace{\frac{1}{2} \frac{\partial}{\partial x_{j}}\left(\overline{u_{j}^{\prime} \omega_{i}^{\prime} \omega_{i}^{\prime}}\right)}_{T}+\underbrace{\nu \frac{\partial^{2}}{\partial x_{j} \partial x_{j}}\left(\frac{\xi}{2}\right)}_{D}-\underbrace{\nu \frac{\partial \omega_{i}^{\prime} \frac{\partial \omega_{i}^{\prime}}{\partial x_{j}} \frac{\partial x_{j}}{\partial}}{2}}_{Y}
\end{aligned}
$$

where Einstein summation is implied over the indexed terms. The four production-rate terms can be attributed to the stretching of instantaneous vortices by the mean strain rate $(P 1)$; the stretching of the time-mean vorticity, $\Omega$, by the fluctuating components of the local strain rate $(P 2)$; exchange of time-mean vorticity, $\Omega$, into fluctuating vorticity, $\omega^{\prime}$, by velocity fluctuations occurring in the presence of a local spatial gradient in the time-mean vorticity $(P 3)$; and stretching of instantaneous vortices by the fluctuating components of the local strain rate (P4). As noted by Tennekes and Lumley (1972), the $\overline{u_{j} \omega_{i}}$ in the P3 term is analogous to the Reynolds stress tensor and it generally has a negative value, hence the negative sign on 

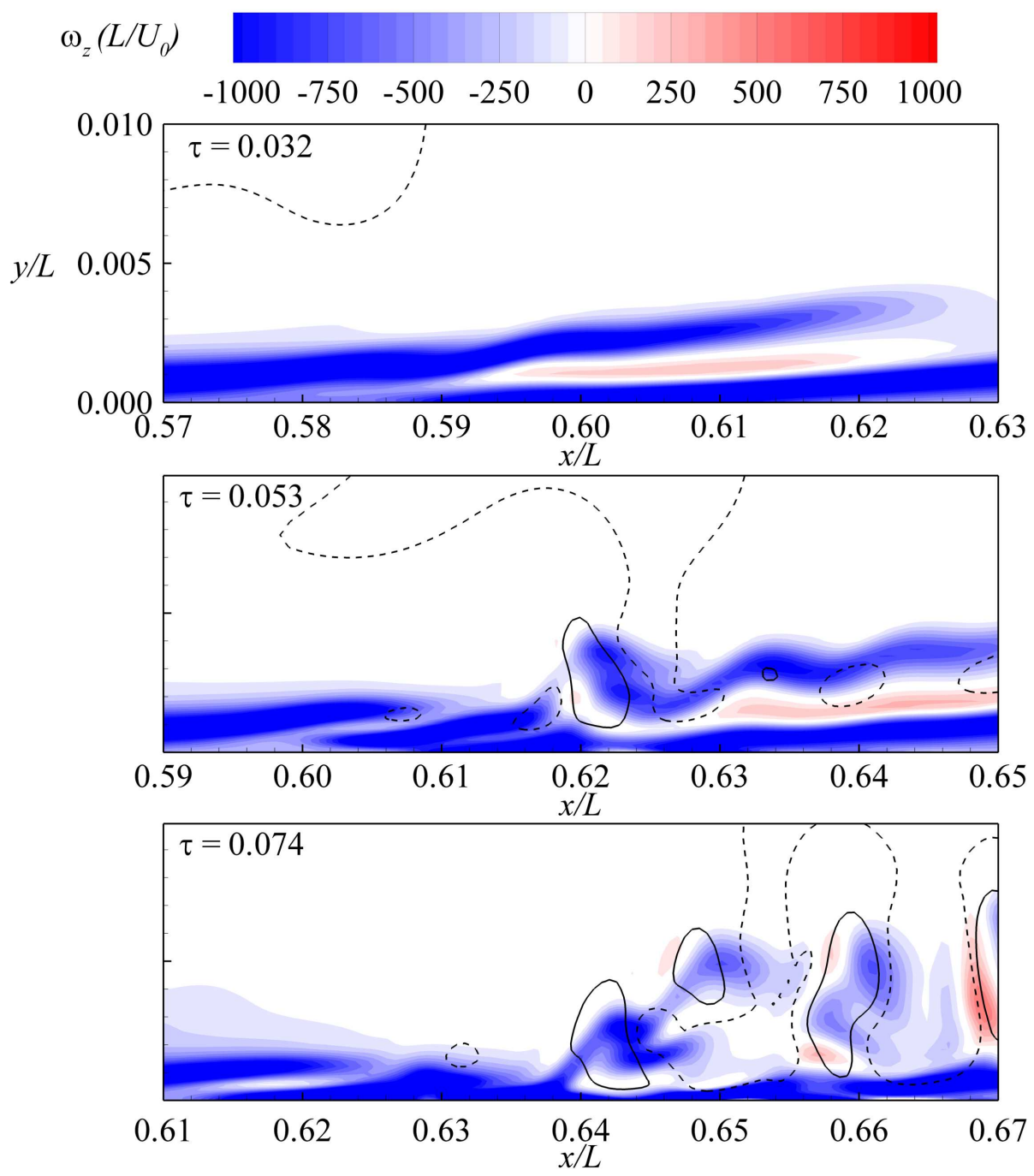

Figure 8.18: Temporal evolution of instantaneous spanwise shear through $\omega_{z}\left(L / U_{0}\right)$ contours in the $z / L=-0.002$ plane. 
the $P 3$ term. The remaining terms represent the rate of transport of enstrophy by turbulent fluctuations $(T)$, viscous diffusion of enstrophy $(D)$, and viscous dissipation of enstrophy $(Y)$. Figure 8.19 plots the enstrophy distribution normalized by $\left(U_{0} / L\right)^{2}$ and the enstrophy equation budgets normalized by $\left(U_{0} / L\right)^{3}$ at time $\tau=0.042$ in a $x$-y plane at $z / L=-0.002$. The positive growth rate of enstrophy seen in Figure 8.19(a) is observed to occur primarily in the interfacial shear-layer region between the low- and high-speed streaks and, to a lesser extent, in the nearwall region beneath the low-speed streak. The enstrophy budgets indicate that production occurs primarily through the $P 2$ and $P 4$ terms (Figures $8.19(f)$ and $(h)$, respectively) and to a lesser extent, the $P 1$ term (Figure $8.19(e)$ ). These enstrophy production rates can be linked to specific processes by examining the components that make up these production terms in their respective locations, shown in Figures 8.20 and 8.21 . The $P 1$ term is comprised almost entirely of the $\overline{\omega_{x}^{\prime} \omega_{y}^{\prime}} \partial U / \partial y$ component (Figure $8.20(b)$ ) and the P2 term is dominated by the $\overline{\omega_{x}^{\prime} \partial u^{\prime} / \partial z} \Omega_{z}$ and $\overline{\omega_{z}^{\prime} \partial w^{\prime} / \partial z} \Omega_{z}$ components (Figure 8.20(e) and $(g)$ ). Figure 8.21 shows that in the region below the interfacial shear layer, $P 4$ is dominated by the $\overline{\omega_{z}^{\prime} \omega_{y}^{\prime} \partial w^{\prime} / \partial y}$ and $\overline{\omega_{x}^{\prime} \omega_{z}^{\prime} \partial u^{\prime} / \partial z}$ components, respectively denoted $P 4 a$ and $P 4 b$. Within the interfacial shear layer, $P 4$ is dominated by the $\overline{\omega_{y}^{\prime} \omega_{z}^{\prime} \partial v^{\prime} / \partial z}, \overline{\omega_{x}^{\prime} \omega_{y}^{\prime} \partial u^{\prime} / \partial y}$, and $\overline{\omega_{z}^{\prime} \omega_{z}^{\prime} \partial w^{\prime} / \partial z}$ components, respectively denoted $P 4 c, P 4 d$, and $P 4 e$.

The connection between the above-noted enstrophy production components and the fluctuating velocity and vorticity fields that exist within the low-speed streak is shown in Figure 8.22. The fluctuating streamwise velocity $\left(u^{\prime}\right)$ and wall-normal vorticity $\left(\omega_{y}^{\prime}\right)$ are plotted in the $y$ - $z$ plane at $x / L=0.625$, which intersects the rolled-up portion of the low-speed streak at time $\tau=0.053$. The vectors and $+u^{\prime}$ contours near the wall at around $z / L \approx-0.003$ illustrate strong sweep motions that are induced by the free-stream vortical disturbances as they penetrate through the shear layer towards the wall. The vectors show that as the sweep motions approach the wall, they are turned in the spanwise direction by the solid surface, generating strong local $w^{\prime}$ motions and hence large $\partial w^{\prime} / \partial z$ strain-rates. Other strain-rate 


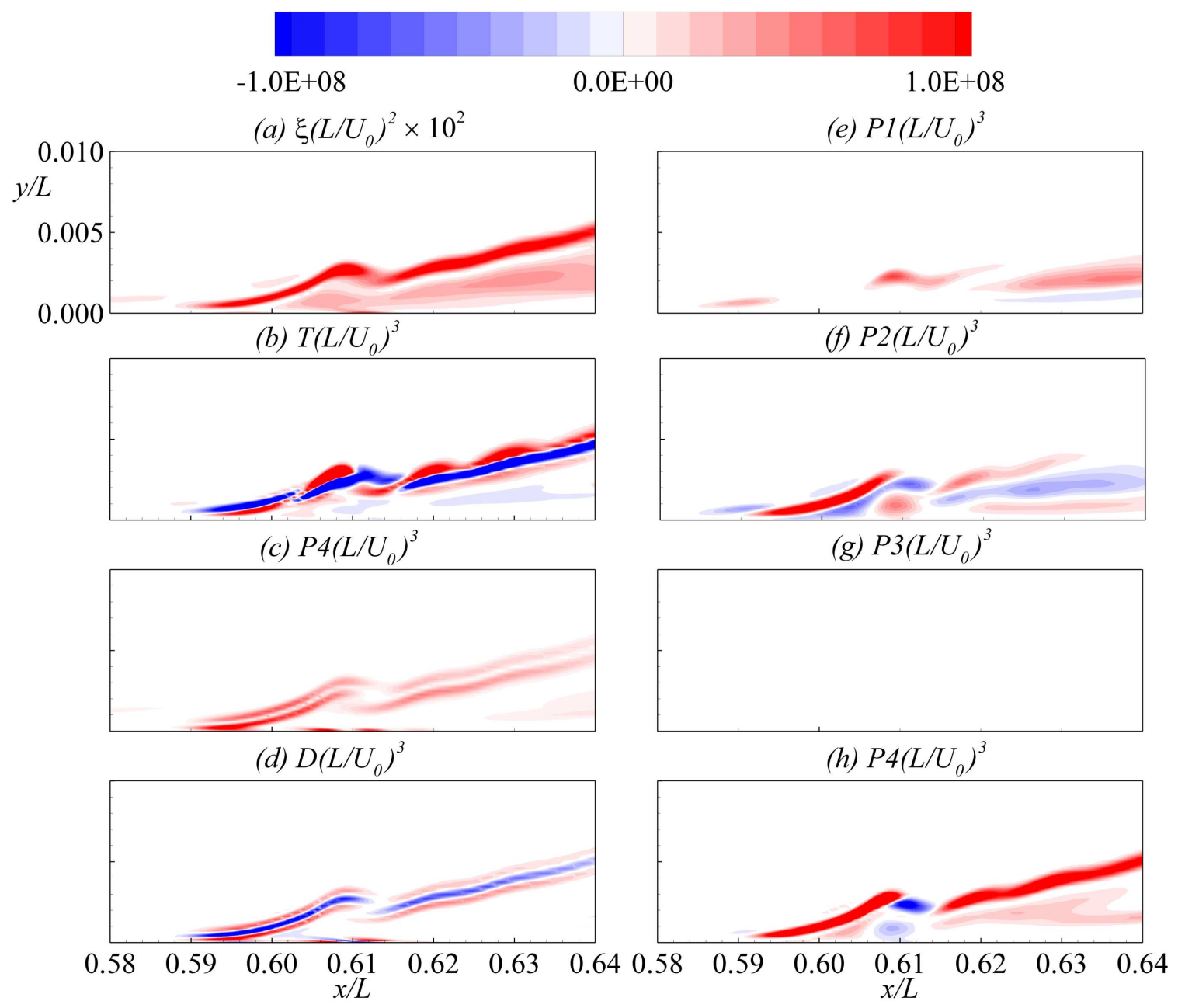

Figure 8.19: Enstrophy equation budgets in the $z / L=-0.002$ plane at $\tau=0.042$. 


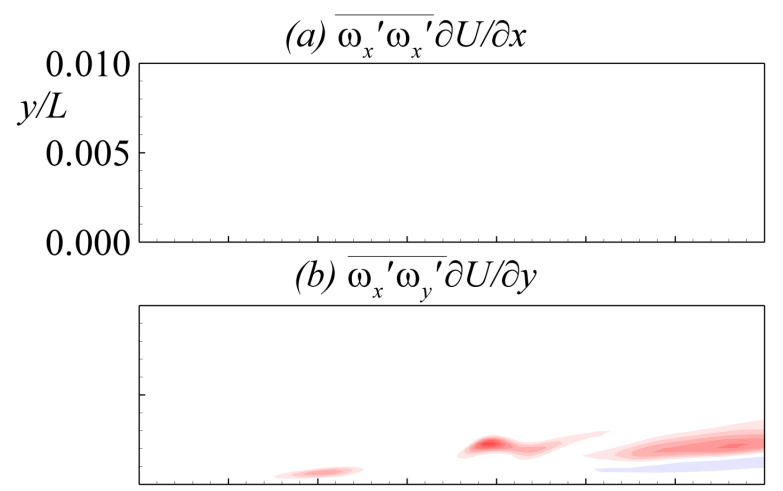

(c) $\overline{\omega_{x}{ }^{\prime} \omega_{y}{ }^{\prime}} \partial V / \partial x$

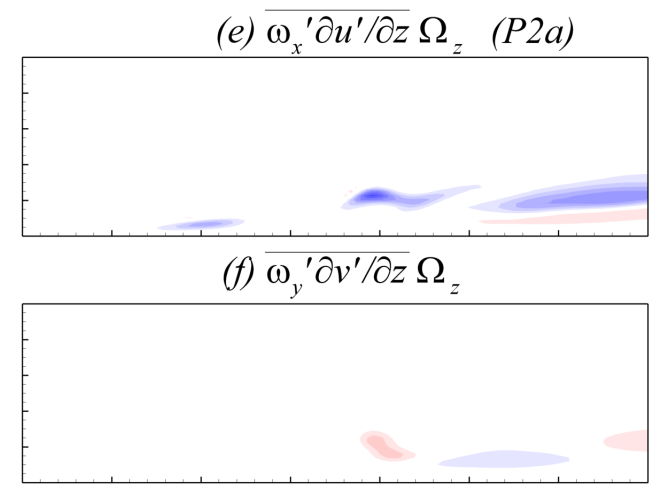

(g) $\overline{\omega_{z}{ }^{\prime} \partial w^{\prime} / \partial z} \Omega_{z}(P 2 b)$

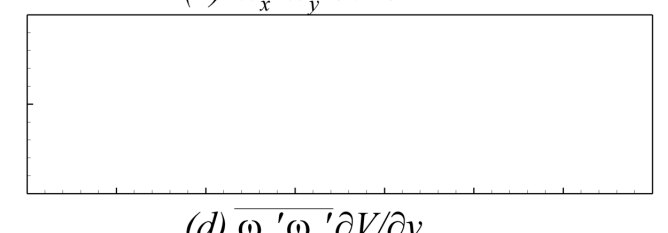

(d) $\overline{\omega_{y}{ }^{\prime} \omega_{y}{ }^{\prime}} \partial V / \partial y$
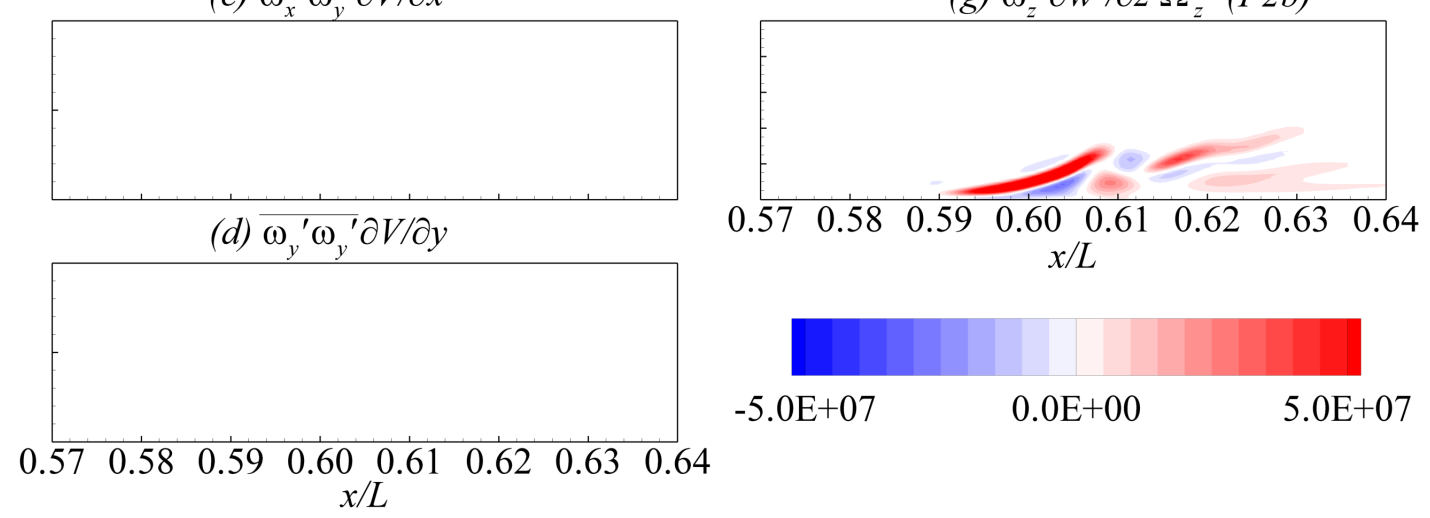

Figure 8.20: Components of the $P 1$ terms $(a)-(d)$ and $P 2$ terms $(e)-(g)$ from Equation 8.5 in the $z / L=-0.002$ plane at $\tau=0.042$. 

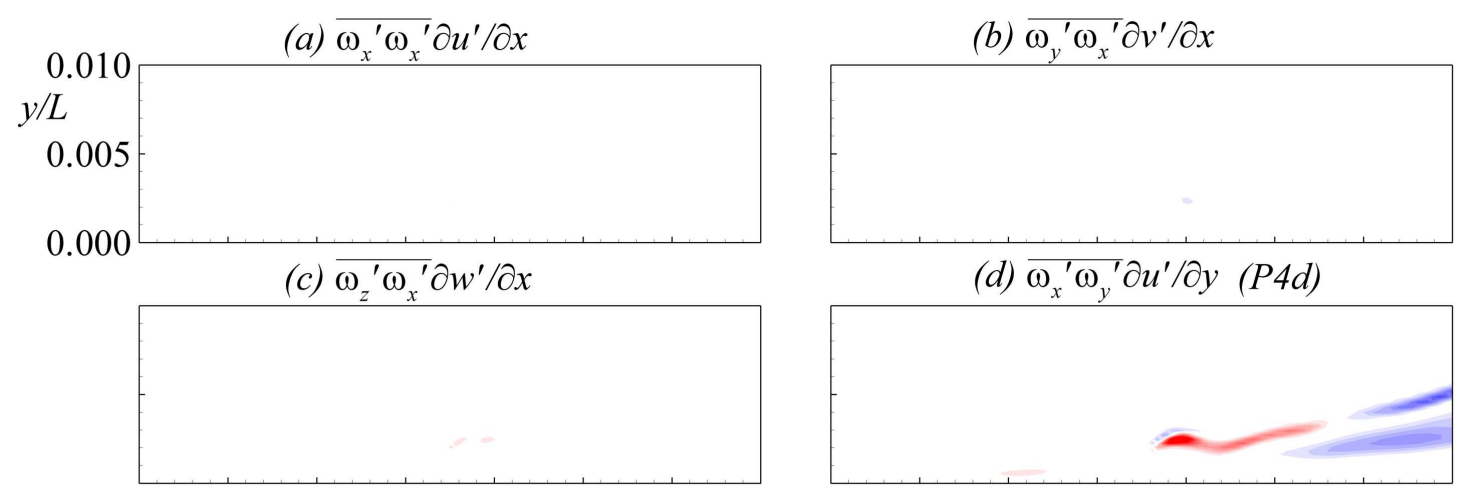

(e) $\overline{\omega_{y}{ }^{\prime} \omega_{y}{ }^{\prime}} \partial v^{\prime} / \partial y$

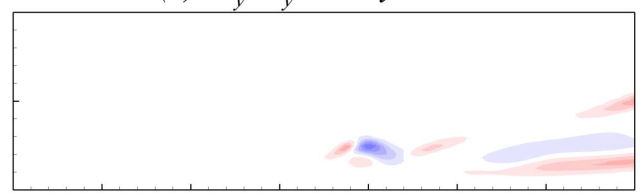

(g) $\overline{\omega_{x}{ }^{\prime} \omega_{z}{ }^{\prime}} \partial u^{\prime} / \partial z \quad(P 4 b)$

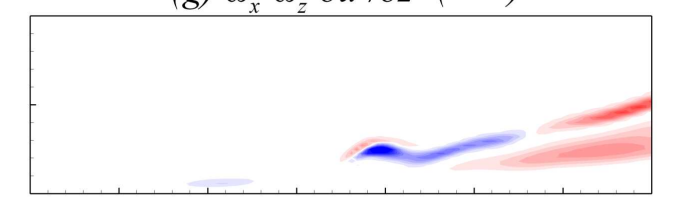

(i) $\overline{\omega_{z}{ }^{\prime} \omega_{z}{ }^{\prime}} \partial w^{\prime} / \partial z(P 4 e)$
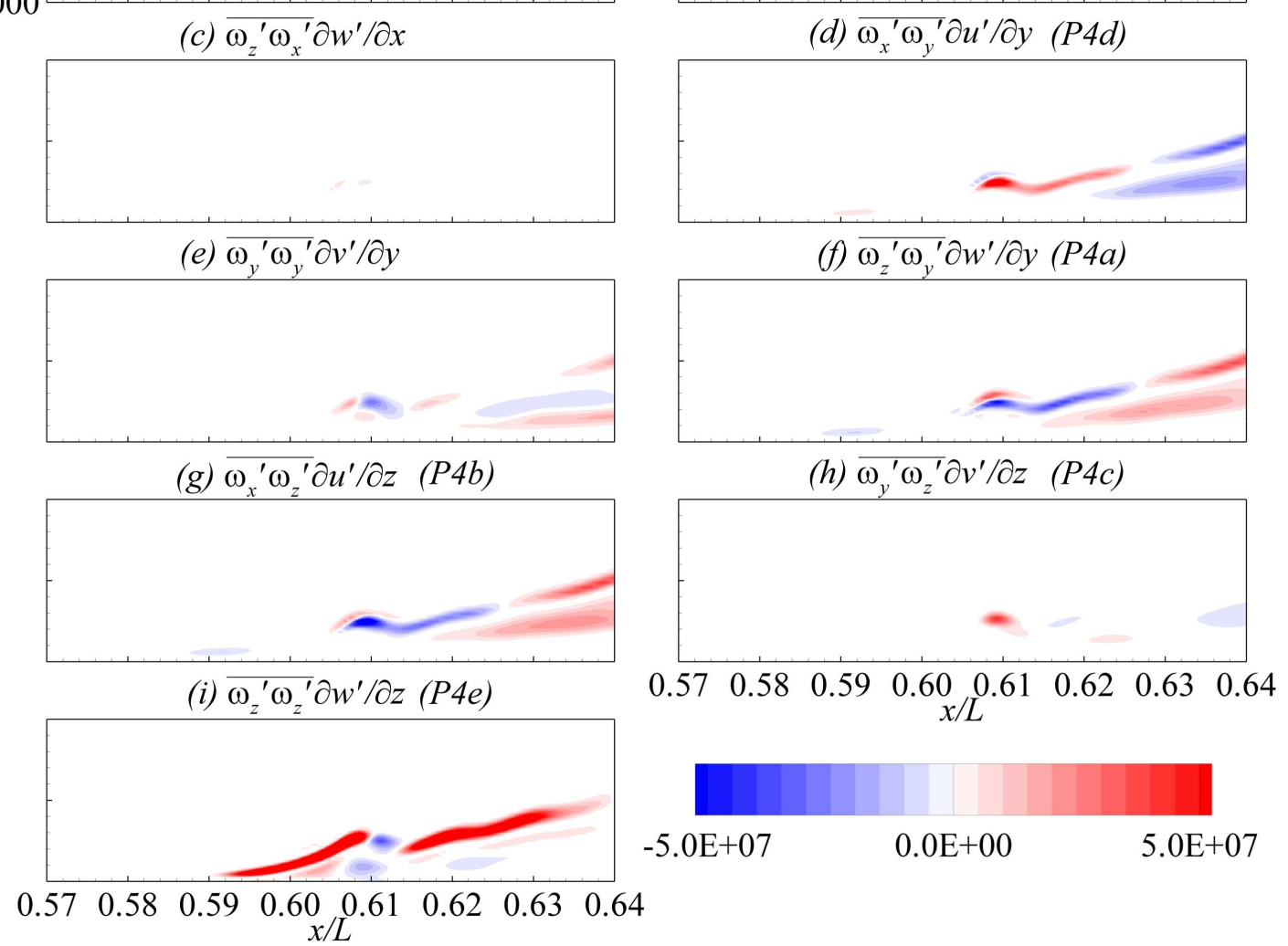

Figure 8.21: Components of the $P 4$ terms from Equation 8.5 in the $z / L=-0.002$ plane at $\tau=0.042$. 
(a)

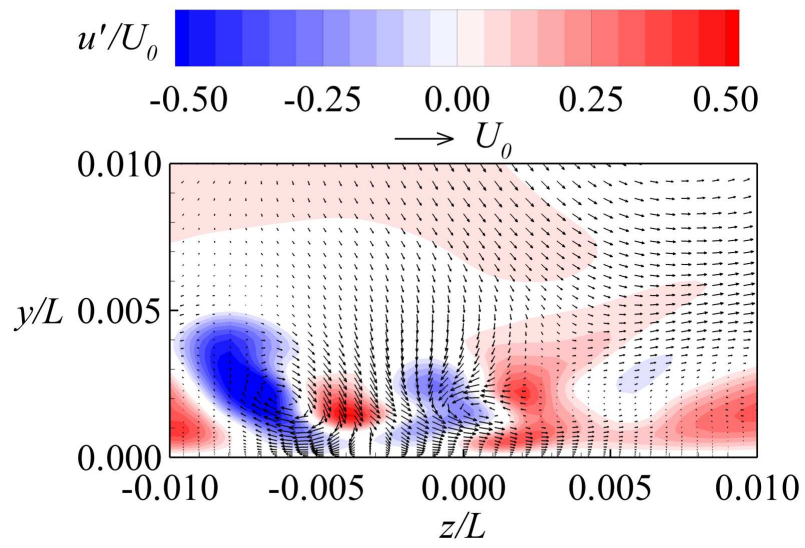

(b)
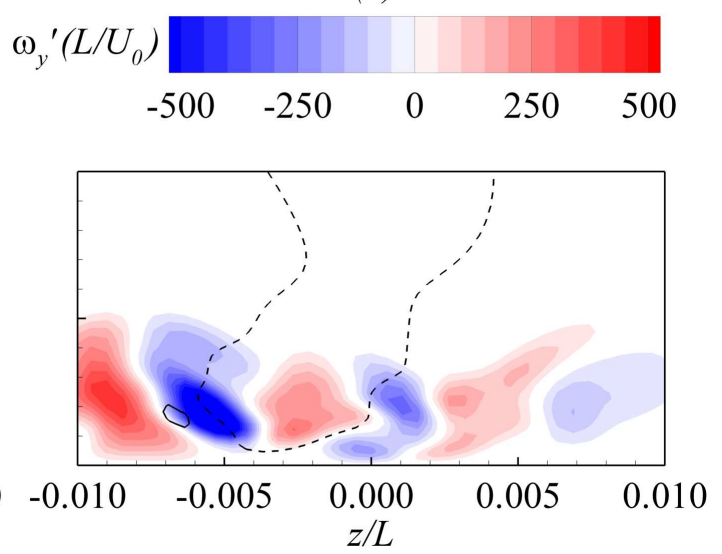

Figure 8.22: Contours of $(a) u^{\prime} / U_{0}$ and $(b) \omega_{y}^{\prime}\left(L / U_{0}\right)$ in the $x / L=0.625$ plane at $\tau=0.053$. Vectors in $(a)$ illustrate the in-plane $v^{\prime}$ and $w^{\prime}$ motions, and lines in $(b)$ are $v^{\prime} / U_{0}=0.10$ (solid) and $v^{\prime} / U_{0}=-0.10$ (dashed).

terms are generated by the legs of the hairpin vortices. Because the hairpin-vortex legs are angled upwards in the $x-y$ plane (e.g., Figure 8.16), they contain distinct regions of positive and negative $\omega_{y}^{\prime}$, as shown in Figure 8.22(b).

The connection between the velocity and vorticity fields shown in Figure 8.22 and the enstrophy production terms is illustrated with the aid of the simplified schematics shown in Figure 8.23. The $P 1$ term is largely created as the $\omega_{y}^{\prime}$ associated with the legs of the hairpin vortices is tilted by the mean strain rate, $\partial U / \partial y$, to produce streamwise vorticity fluctuations, $\omega_{x}^{\prime}$, as sketched in Figure 8.23(a). Regions of $\partial u^{\prime} / \partial z$ and $\partial w^{\prime} / \partial z$ are also induced by the legs of the hairpin vortices. Figure $8.23(b)$ shows that the $\partial u^{\prime} / \partial z$ strain rate tilts the local mean spanwise vorticity in the streamwise direction to produce fluctuating streamwise vorticity, $\omega_{x}^{\prime}$, generating enstrophy through the $P 2 a$ term. Figure $8.23(c)$ shows that the $\partial w^{\prime} / \partial z$ strain rate stretches or squeezes the local spanwise vorticity, $\Omega_{z}$. The spanwise motions are not uniformly distributed in the wall-normal direction, thus they also generate significant regions of $\partial w^{\prime} / \partial y$, which Figure $8.23(d)$ shows tilts the $\omega_{y}^{\prime}$ vorticity associated with the upright legs of the hairpin vortices in the $y-z$ plane to generate $\omega_{z}^{\prime}$ and produce enstrophy through the $P 4 a$ term. Figure 8.23(e) shows that the $\partial u^{\prime} / \partial z$ strain rate tilts the $\omega_{z}^{\prime}$ generated by the 
$P 2 b$ and $P 4 a$ terms in the $x$ - $z$ plane to generate $\omega_{x}^{\prime}$, thus producing enstrophy through the $P 4 b$ term. Similarly, Figure $8.23(f)$ shows that the $\partial v^{\prime} / \partial z$ strain rate induced by the hairpin vortex tilts $\omega_{z}^{\prime}$ in the $y-z$ plane to generate $\omega_{y}^{\prime}$ and produce enstrophy through the $P 4 c$ term. Figure $8.23(g)$ shows that the $\partial u^{\prime} / \partial y$ strain rate that is associated with the low- and highspeed streamwise streaks tilts the $\omega_{y}^{\prime}$ vorticity within the legs of the hairpin vortices in the $x-y$ plane to generate $\omega_{x}^{\prime}$ and produce enstrophy through the $P 4 d$ term. Finally, Figure 8.23( $h$ ) shows that the $\partial w^{\prime} / \partial z$ strain rate stretches or squeezes the fluctuating spanwise vorticity, $\omega_{z 2}^{\prime}$, so that its amplitude increases or decreases relative to the unstrained value, $\omega_{z 1}^{\prime}$, producing enstrophy through the $P 4 e$ term.

The relationship between the $P 1, P 2, P 4 a, P 4 b$, and $P 4 c$ terms during transition has already been demonstrated by Brinkerhoff and Yaras (2013) based on a DNS of a laminar boundary layer that transitions under low free-stream turbulence conditions. The block diagram they used to illustrate the mechanisms leading to the growth of turbulence is shown in Figure 8.24. Brinkerhoff and Yaras triggered a disturbance that initiated a wall-normal component in the fluctuating vorticity field $\left(\omega_{y}^{\prime}\right)$, which activates the $P 1$ term to produce $\omega_{x}^{\prime}$. The sweep/ejection motions induced by the $\omega_{x}^{\prime}$ initiate three distinct regeneration cycles: in the $P 2 b$ cycle, the $\partial w^{\prime} / \partial z$ strain rate forms $\omega_{z}^{\prime}$ via the $P 2 b$ term, and due to the $P 4 c$ term, this $\omega_{z}^{\prime}$ is reoriented in the wall-normal direction to yield new $\omega_{y}^{\prime}$, which is fed back into the $P 1$ term to close the $P 2$ cycle. In the $P 4 a$ cycle, the $\partial w^{\prime} / \partial y$ strain rate tilts the existing $\omega_{y}^{\prime}$ to form $\omega_{z}^{\prime}$ via the $P 4 a$ term, which then forms new $\omega_{y}^{\prime}$ via the $P 4 c$ term that is fed back into the $P 1$ and $P 4 a$ terms to close the $P 4 a$ cycle. Finally, in the $P 4 b$ cycle, the $\partial u^{\prime} / \partial z$ strain rate tilts the $\omega_{z}^{\prime}$ created by the $P 2$ and $P 4 a$ cycles into $\omega_{x}^{\prime}$ via the $P 4 b$ term, which strengthens the sweep/ejection motions that drives the other two cycles.

In the present study, the three regeneration cycles proposed by Brinkerhoff and Yaras (2013) are present within the turbulent spot that develops following the roll-up of the low-speed streak. In addition, the elevated free-stream turbulence conditions introduce the additional 

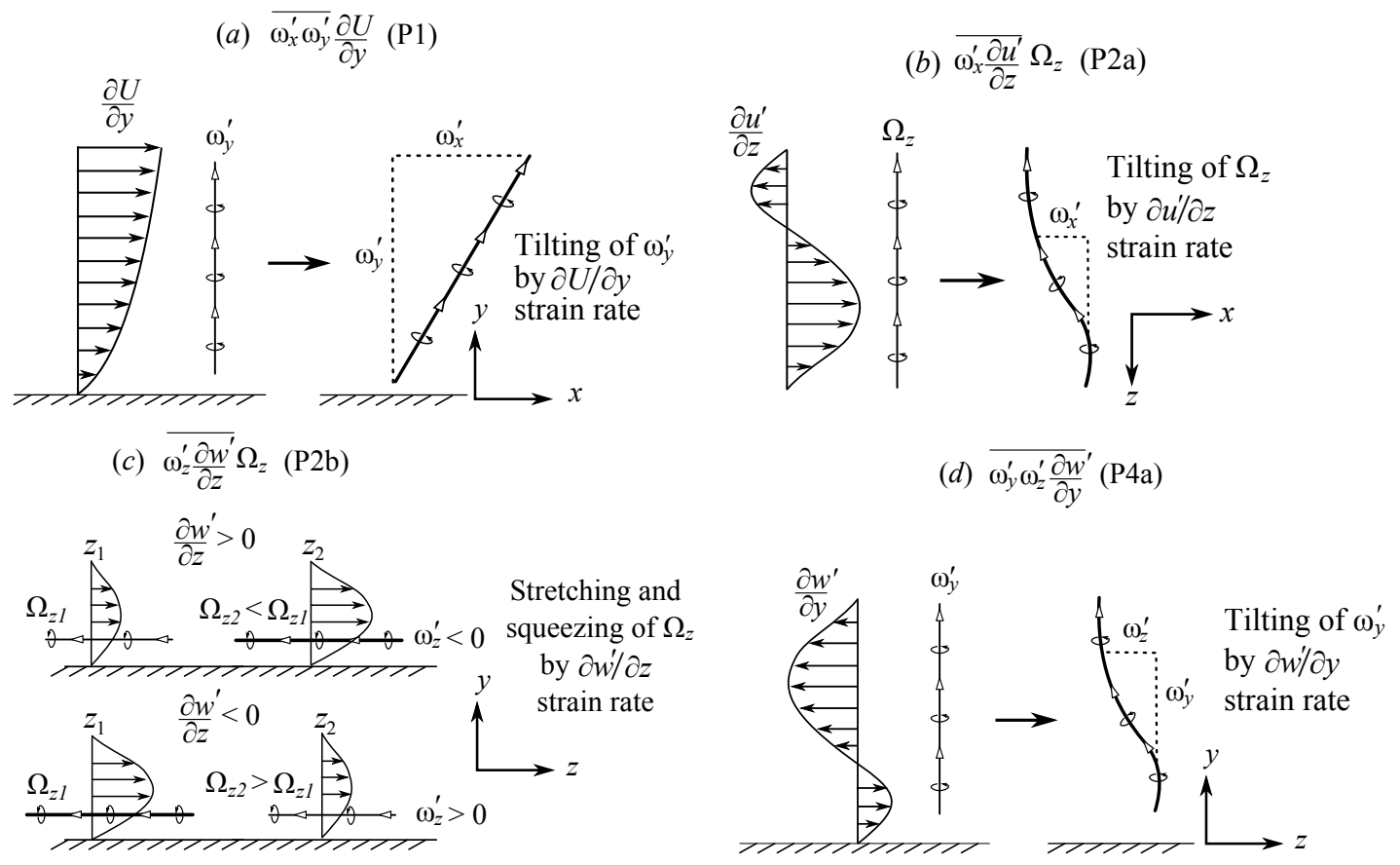

(e) $\overline{\omega_{x}^{\prime} \omega_{z}^{\prime} \frac{\partial u^{\prime}}{\partial z}}(\mathrm{P} 4 \mathrm{~b})$

(f) $\overline{\omega_{y}^{\prime} \omega_{z}^{\prime} \frac{\partial v^{\prime}}{\partial z}}(\mathrm{P} 4 \mathrm{c})$
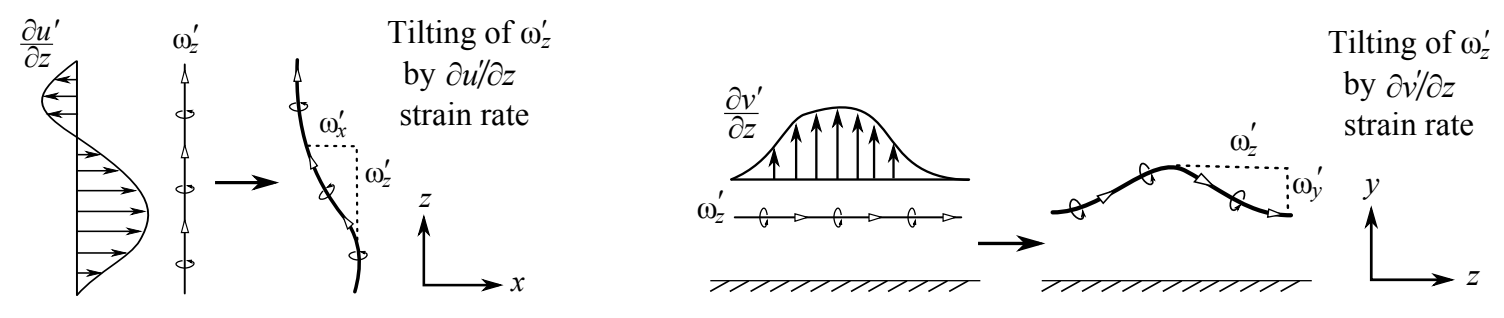

(g) $\overline{\omega_{x}^{\prime} \omega_{y}^{\prime} \frac{\partial u^{\prime}}{\partial y}}(\mathrm{P} 4 \mathrm{~d})$

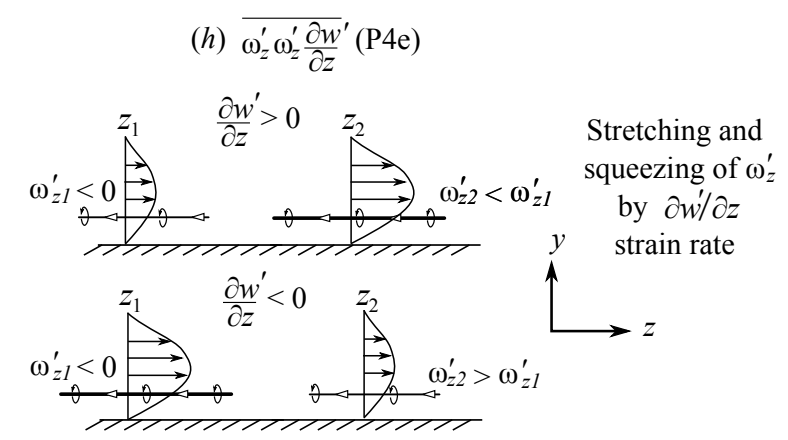

Figure 8.23: Schematic illustrating the enstrophy production mechanisms embodied in $(a)$ the $P 1$ term, $(b-c)$ the $P 2$ terms, and $(d)-(h)$ the $P 4$ terms. Filled arrows denote velocity vectors, hollow arrows denote vorticity vectors. Thicker vortex lines indicate an increase in the contained vorticity. 


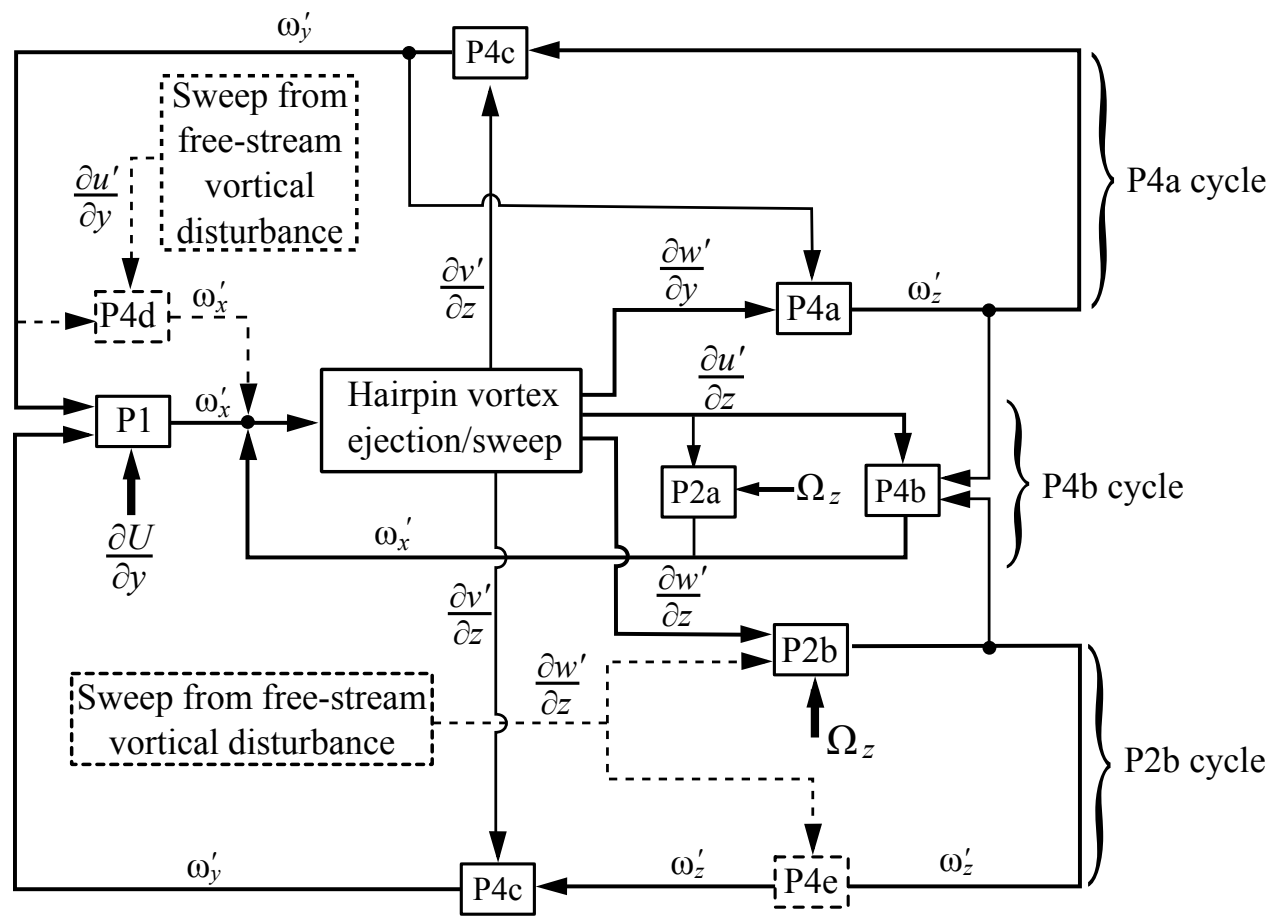

Figure 8.24: The block diagram of Brinkerhoff and Yaras (2013) illustrating the regeneration cycles of turbulent enstrophy within a turbulent spot, modified to include the effect of sweep motions induced by free-stream vortical disturbances. 
contributions of the $P 4 d$ and $P 4 e$ terms. As shown in Figure 8.22, the additional two terms originate in the wall-normal sweep sweep motions that are generated by the free-stream vortical disturbances as they penetrate between the rolled-up segments of the interfacial shear layer. The added terms are included in the block diagram of Brinkerhoff and Yaras (2013) in Figure 8.24 using dashed lines. The enhanced $\partial u^{\prime} / \partial y$ strain rate that results from the sweep motions generated by the free-stream turbulence enhances the formation of $\omega_{x}^{\prime}$ through the $P 4 d$ terms, which strengthens the $P 2 b$ and $P 4 a$ cycles. Similarly, the enhanced $\partial w^{\prime} / \partial z$ strain rate increases the formation of $\omega_{z}^{\prime}$ through the $P 2 b$ and $P 4 e$ terms, which strengthens the $P 2 b$ cycle. The strengthening of the $P 2 b$ and $P 4 a$ cycles implies a much higher enstrophy-production rate under elevated free-stream turbulence conditions, and hence more rapid transition to turbulence. This explanation of the physical processes indicated in Figure 8.24 suggests that elevated free-stream turbulence does not fundamentally alter the transition mechanisms that occur under low free-stream turbulence, but contributes sweep motions that result in extra enstrophy-production processes that accelerate the existing mechanisms, ultimately resulting in a much higher production rate of turbulence under the present elevated free-stream turbulence conditions. 


\section{Chapter 9}

\section{Summary, Contributions and Recommendations}

This chapter summarizes the individual contributions of the preceding chapters to illustrate how they cumulatively address the overall objectives of the thesis. The overall contributions of the thesis and recommendations for future work are also presented.

\subsection{Summary of Investigations}

\subsubsection{Effect of interacting viscous/inviscid instability modes in shear-layer transition}

In Chapter 4, shear-layer transition of a boundary layer that develops on a flat plate under low free-stream turbulence was examined numerically using direct numerical simulation (DNS). The flow Reynolds number and streamwise pressure distribution are representative of the suction side of a typical low-pressure axial turbine blade. The growth of disturbances that 
lead to transition of the boundary layer occurs in two regions by two unique instability modes: ( $i$ ) a viscous mode in the attached shear layer upstream of the flow separation location and (ii) an inviscid mode in the separated shear layer downstream of the flow separation location.

In the attached boundary layer upstream of separation, disturbance growth occurs through the viscous instability mode. The disturbances are identified as Tollmien-Schlichting (T-S) waves, which in a physical sense correspond to a streamwise grouping of spanwise vorticity within the boundary layer. Their growth rate is compared with predictions based on linear stability theory (LST) and qualitative agreement is achieved between the computed results and LST in terms of the streamwise growth of the T-S waves. As the T-S waves are amplified in the attached boundary layer, segments of the grouped spanwise vorticity are reoriented to establish a component in the streamwise direction, which then undergo lift-up and stretching to produce $\Lambda$-shaped vortices within the attached boundary layer. As the flow separates from the flat plate, the $\Lambda$-vortices remain embedded in the shear layer and are amplified by the high local shear stress to form coherent hairpin vortices that resemble those observed in turbulent boundary layers.

In the separated shear layer downstream of flow separation, an inviscid Kelvin-Helmholtz $(\mathrm{K}-\mathrm{H})$ instability mechanism results in the formation and periodic shedding of spanwise vortices from the shear layer. A spanwise non-uniformity of the shed spanwise vortices occurs at a wavelength approximately equal to the spanwise wavelength of the $\Lambda$-vortices in the attached shear layer, indicative of an interaction between the spanwise vortices and $\Lambda$-vortices. The interaction begins when the $\Lambda$-vortices developed through the viscous instability mode convect into the separated shear layer and are stretched by the high local shear stress into coherent hairpin vortices. The vorticity in the legs and head of a hairpin vortex eject low-momentum fluid from the wall upwards between the legs of the hairpin vortex. As the shedding spanwise vortices encounter the column of low-momentum fluid, portions are retarded relative to the rest of the spanwise vortex, producing a spanwise-wavy pattern that amplifies and eventually 
results in the break-down of the spanwise vortex into small-scale wave packets. The region where the interaction between the viscous and inviscid instability modes occurs coincides with the time-averaged locations of transition onset and completion, indicating that the interaction has a significant impact on the transition process.

The interaction between the viscous and inviscid instability modes in the separated shear layer accelerates the three-dimensional break-up of the shear layer into discrete wave packets. The wave packets consist of one or more hairpin-shaped vortex loops that point in the upstream

direction. As a wave packet convects downstream, the primary vortex loop induces two to three additional loops downstream of the primary loop, while the primary loop is lifted away from the wall and persists well into the reattached turbulent boundary layer.

\subsubsection{Effect of swept-blade conditions in shear-layer transition}

In Chapter 5, the effect of aeroengine-realistic blade sweep on shear-layer transition was studied numerically using DNS under free-stream turbulence levels, Reynolds numbers, and streamwise pressure distributions similar to those considered in Chapter 4. To introduce the effect of blade sweep, the leading edge of the test surface in one of the simulations is swept by an angle of $45^{\circ}$. The effect of blade sweep on the instability mechanisms discussed in Chapter 4 is assessed through a comparison against the results of an unswept configuration.

In the swept configuration, the 3D pressure field imparts a spanwise pressure force on the flow that causes the free-stream to turn in the spanwise plane. Within the boundary layer, the lower flow velocity causes the spanwise pressure force to be unbalanced, which leads to the formation of a crossflow velocity profile in the attached laminar boundary layer directed perpendicular to the local inviscid streamline at the boundary-layer edge. This crossflow motion results in a spanwise redistribution of mass and tangential momentum within the boundary layer in the swept configuration that results in a $4 \%$ increase in the value of the 
momentum-thickness Reynolds number at the time-averaged separation location compared to the unswept configuration.

The inflectional shape of the crossflow profiles favours the development of an inviscidunstable crossflow instability. Under the low free-stream turbulence conditions of this study, the crossflow instability is expected to form stationary vortices within the boundary layer that are closely aligned with the local free-stream streamline. However, due to the stabilizing effect of the strong favourable streamwise pressure gradient that is applied to the test surface upstream of the suction peak, such stationary vortices are not observed in the flow. Furthermore, it is observed that the growth rate of T-S waves within the attached boundary layer upstream of separation is not substantially different in the swept configuration compared with the unswept configuration.

The absence of a stationary crossflow mode and the comparable growth rates of T-S waves in the attached shear layer suggests that the crossflow instability mode in the swept configuration does not play a significant role in the growth of disturbances leading to transition. Instead, the transition process is dominated by the development and interaction of the $2 \mathrm{D}$ viscous and inviscid instability modes that respectively develop in the attached and separated regions. While the growth of disturbances in the attached region due to viscous instability occurs at a similar rate in the swept and unswept configurations, the amplification rate and most amplified frequency of the K-H instability mode in the separated region, is notably higher in the swept configuration. As a result, the location where the shear layer breaks down to small-scale turbulence shifts upstream in the swept configuration and the turbulent mixing of the separated shear layer is also modified, yielding a downstream shift in the time-averaged reattachment location.

The increased amplification rate of the K-H instability mode in the separated shear layer of the swept configuration is attributed to the higher value of the momentum-thickness Reynolds 
number at the time-averaged separation location. The sensitivity of the K-H instability to this parameter is documented in unswept cases. Chapter 5 therefore concludes that although crossflow motion in the upstream boundary layer and within the separation bubble does not appear to have a noticeable effect on the instability development of the transitional separation bubble, the 3D pressure field does alter the separation-bubble development by slightly modifying the boundary-layer integral parameters at separation.

\subsubsection{Effect of free-stream acceleration on the development of a transverse jet and the resulting formation of coherent vortical flow structures}

In Chapter 6, the effect of strong free-stream acceleration on the formation of coherent vortical flow structures during transition was studied. Direct numerical simulation was used to study the flowfield created by a square jet ejected transversely into a laminar, accelerating main flow. A jet with a velocity ratio of $r=9.78$ was pulsed using a single period of a square wave with a pulse duration equivalent to the time required for the jet fluid to travel 173 jet widths at its core velocity. A strongly-favourable streamwise pressure gradient was applied to the main flow with the expectation that $(i)$ it would stabilize the vortical structures created by the transverse jet for clearer analysis and (ii) allow an investigation into the effect of freestream acceleration on the formation of coherent vortical structures in the boundary layer. The simulation results demonstrate that the jet trajectory evolved throughout the lifetime of the jet, and consequently coefficients of a scaling law that was empirically fitted to the time-averaged jet trajectory were sensitive to the time-averaging period. The coefficients corresponding to the time-averaging period that most-closely approximated the statistically steady-state were somewhat larger than the ranges of published values. This suggests that the level of freestream acceleration present in the main flow-which exceeds the level typically required for a turbulent flow to relaminarize - affects the flowfield produced by the transverse jet. 
Further clues to the nature of this effect were obtained by analyzing the transient development of vortical structures after the activation of the jet. The initial ejection of vorticity from the jet produced a vortex loop, the induced velocity field of which was important in initiating the separation of the main-flow boundary layer upwind of the jet, which then quickly rolled-up into the horseshoe vortex. The spatial location and strength of the horseshoe vortex remained quite steady in time, and spectral analysis of the fluctuations in the jet boundarylayer revealed similar fluctuation amplitudes on the upwind and downwind sides of the jet, indicating that the horseshoe vortex did not induce substantial fluctuations in the near-wall fluid in the jet-pipe. This observation is a strong indicator that, by delaying separation of the main-flow boundary layer, the favourable streamwise pressure gradient weakens the horseshoe vortex and removes or substantially reduces the oscillations typically associated with the horseshoe vortex system at the high Reynolds number of the simulated jet (e.g. Kelso et al., 1996; Krothapalli et al., 1990).

Shortly after the jet was activated, a Kelvin-Helmholtz-like instability of the upwind and downwind jet shear-layers resulted in a roll-up of the shear layers at a characteristic Strouhal number of $S t=f D / v_{\text {jet }}=0.55$, leading to the formation of shear-layer vortices. Other low- and high-frequency disturbances were observed in the shear layer, attributed to the early development of a sub-harmonic pairing instability of the shear-layer vortices and the transient evolution of structures downwind of the jet, respectively. The shape of the shed vortices was consistent with the mechanism of folding of the shear-layer described in several studies (Kelso et al., 1996; Lim et al., 2001; Cortelezzi and Karagozian, 2001). The jet vorticity accumulated at the upwind and downwind corners of the jet to form corner vortex pairs. The pair originating in the upwind corner were fed primarily by the main-flow fluid that was entrained from the sides and rear of the jet. This pair remained relatively stationary in time and eventually combined with the shear-layer vortices to create large, counter-rotating streamwise vortices, identified as the counter-rotating vortex pair (CVP). In contrast, the vortex pair originating in 
the downwind corner was fed primarily by vorticity that was reoriented and entrained from the main-flow boundary layer as it separated downwind of the jet in response to the local adverse pressure gradient set-up behind the jet. This pair was highly unsteady: its induced velocity field led to an instability that produced hairpin-shaped vortex loops in the main-flow boundary layer downwind of the jet and highly-elongated, downwards-angled vortices attached to the jet trajectory. The development of these structures in the wake of the jet, rather than the upright wake vortices typical of transverse-jet wakes, is attributed primarily to the stabilization of the main-flow boundary layer by the favourable streamwise pressure gradient.

\subsubsection{Generation and growth of coherent flow structures in a turbulent spot}

In Chapter 7, the wave packets that were identified in the reattached turbulent boundary layer in Chapter 4 were revisited to investigate the mechanism leading to the growth and regeneration of the hairpin-like vortices within the wave packets. Two DNS studies were performed that simulate an artificially-triggered turbulent spot in a laminar, zero-pressuregradient boundary layer. The first simulation in Chapter 7 closely resembles the study from Chapter 6 in that it captures the initial formation of hairpin vortices triggered by a pulsed jet and their subsequent development into a young turbulent spot, although the simulations in Chapter 7 have a zero streamwise pressure gradient in contrast to the strongly-favourable pressure gradient studied in Chapter 6. The second simulation in Chapter 7 captures the growth of the young turbulent spot into a mature turbulent region containing a large number of hairpin vortices. Mapping of transient flow information from the first to the second simulation domain allowed for the full history of the turbulent spot to be accurately simulated, from creation to maturity.

The formation of coherent flow structures within the triggered spot begins as the triggering jet interacts with the test-surface boundary layer to form a pair of coherent, counter-rotating 
streamwise vortices within the boundary layer immediately downstream of the jet orifice. These vortices activate a hairpin-vortex regeneration process that produces new sets of hairpin vortices straddling the streamwise vortices. As the regeneration of new hairpin vortices continues, they form into wave packets consisting of two to three closely-spaced, streamwisealigned hairpin vortices. New wave packets are repeatedly generated along the spanwise edges of the spot, resulting in lateral growth of the spot at a rate that is in excellent agreement with published literature. More wave packets are observed near the trailing edge of the spot than at the leading edge, attributed to the fact that the hairpin vortices near the leading edge have much larger spatial scales and hence extend farther from the wall. As a result, the wallnormal motions induced by these large-scale hairpin vortices cannot form streamwise streaks and thus cannot activate the hairpin-vortex regeneration process. Instead, the non-spanwiseuniform downwash motions induced by the large-scale hairpin vortices near the spot leading edge reorient the downstream spanwise vorticity to form streamwise vortices that extend above the laminar overhang region below the spot leading edge. The wall-normal motions induced by these streamwise vortices convect fluid from the laminar region into the leading edge of the spot and reorient spanwise vorticity in the laminar region such that it strengthens the coherent vortical structures present near the leading edge, affecting an increase in their spatial scale. The convection and reorientation of vorticity accomplished by the leading-edge streamwise vortices result in the longitudinal growth of the spot at a rate that is in excellent agreement with published literature.

The lateral growth of the turbulent spot is found to occur through two complementary mechanisms: $(i)$ the regeneration of hairpin vortices within a wave packet and $(i i)$ the formation of new wave packets along the spanwise edges of the spot. The regeneration of hairpin vortices within a wave packet is found to be activated by the streamwise vorticity in the legs of a parent hairpin vortex. The streamwise vorticity induces upwash between the legs of the hairpin vortex and downwash outboard of the legs of the hairpin vortex. These 
wall-normal motions perturb the spanwise vorticity to form small wall-normal-oriented loops in the boundary-layer vorticity surrounding the two legs of the parent hairpin vortex. The shear layers formed by the wall-normal component of the reoriented vorticity roll-up due to an inviscid instability in the $x-z$ plane, leading to a streamwise grouping of wall-normal vorticity into pairs of secondary hairpin vortices that each straddle a single leg of the parent hairpin vortex. When the parent hairpin vortex has a small spatial scale so that its legs are located in close proximity to each other, the secondary hairpin vortices join to form a single hairpin vortex that straddles both legs of the parent hairpin, although this topology is observed less frequently.

The formation of new wave packets along the spanwise edges of the spot follows a similar mechanism as the regeneration of hairpin vortices within the spot. However, because the parent hairpin vortex is located at the laminar/turbulent interface, the downwash of high-momentum fluid on its outboard side stretches the background spanwise vorticity into the streamwise direction, introducing a streamwise component into the boundary-layer vorticity. Self-induced lift-up of the stretched vorticity reorients it in the wall-normal direction and allows the inviscid instability of the wall-normal vorticity shear layers to activate as before to produce one or more discrete hairpin vortices outboard of the parent hairpin vortex. The formation of new wave packets along the spanwise edges of the spot thus follows the same fundamental process as the creation of hairpin vortices within the spot interior. These processes generate high levels of perturbation spanwise vorticity within the spot's interior. As the spot convects downstream, the perturbation spanwise vorticity embedded in the slower-moving fluid near-wall fluid extends past the spot's trailing edge into the wake. Downwash motions induced by the perturbation spanwise vorticity brings high-momentum fluid into the wake of the spot, producing a calmed region that grows in sync with the spot's longitudinal growth.

A quantitative description of the hairpin-vortex and wave-packet regeneration mechanisms is obtained through the transport of enstrophy, which illustrates how the mean and pertur- 
bation velocity and vorticity fields interact with the local mean and perturbation strain rates to promote the growth of turbulent perturbations. The production of enstrophy along the spanwise edges of the spot primarily occurs by the following processes:

1. The tilting and stretching of perturbation wall-normal vorticity $\left(\omega_{y}^{\prime}\right)$ in the streamwise direction by the mean $\partial U / \partial y$ strain rate;

2. The stretching and squeezing of the mean spanwise vorticity $\left(\Omega_{z}\right)$ by the fluctuating $\partial w^{\prime} / \partial z$ strain rate;

3. The tilting and stretching of perturbation wall-normal vorticity $\left(\omega_{y}^{\prime}\right)$ in the spanwise direction by the fluctuating $\partial w^{\prime} / \partial y$ strain rate;

4. The tilting and stretching of perturbation spanwise vorticity $\left(\omega_{z}^{\prime}\right)$ in the streamwise direction by the fluctuating $\partial u^{\prime} / \partial z$ strain rate; and

5. The tilting and stretching of perturbation spanwise vorticity $\left(\omega_{z}^{\prime}\right)$ in the wall-normal direction by the fluctuating $\partial v^{\prime} / \partial z$ strain rate.

These fluctuating vorticity components and strain rates are caused by the coherent vortical structures that exist within the spot. Specifically, a hairpin vortex situated at the edge of the spot induces an upwash of low-speed fluid between its legs and downwash of high-speed fluid to the outside that reorients the mean spanwise vorticity into the wall-normal direction (i.e. generates $\left.\omega_{y}\right)$. Inviscid instability of the reoriented wall-normal vorticity results in a streamwise grouping of wall-normal vorticity leading to an eventual roll-up of the wall-normal vorticity shear layer into a coherent hairpin vortex outboard of the original hairpin. The strong wall-normal (ejection/sweep) and spanwise motions induced by this hairpin vortex create the fluctuating strain rates required for the enstrophy-production processes. A diagrammatic representation of the wave-packet regeneration process is presented, which highlights how the wall-normal motions induced by a parent hairpin vortex triggers the reorientation of the 
background vorticity that yields, after a sequence of steps, new generations of hairpin vortices. It is thus demonstrated that the formation of new wave packets involves a tightly-coupled interaction between the enstrophy production processes and the sweep and ejection motions induced by hairpin vortices within the interior and along the spanwise edge of the spot.

\subsubsection{Effect of elevated free-stream turbulence in shear-layer transition}

In Chapter 8, the DNS of Chapter 4 was repeated with elevated free-stream turbulence conditions with the aim of understanding how elevated free-stream turbulence affects $(i)$ the growth of disturbances in the laminar attached boundary layer and (ii) the development of coherent structures in a turbulent spot once transition has initiated. Elevated free-stream turbulence conditions are created upstream of the test-surface through a periodic array of square inlets that mimic a physical turbulence-generating grid. The streamwise development of the free-stream turbulence upstream of the leading edge of the test surface closely matches published literature in terms of the decay rate of turbulence intensity, the growth rates of the turbulence integral length scales, and the spectral content of the free-stream turbulence.

A favourable followed by an adverse streamwise pressure gradient are imposed on the boundary layer that develops on the test surface. Longitudinal streamwise streaks are created early in the laminar boundary layer through the selective inclusion of low-frequency disturbances from the free-stream turbulence. The arithmetic mean of the dimensional spanwise streak spacing achieves approximately-constant but unique values in the laminar and turbulent regions and does not show a particular sensitivity to the level of streamwise pressure gradient. When normalized by local inner variables, however, the streak spacing increases in the accelerating region, indicating stabilization by the favourable pressure gradient, and then

decreases in the decelerating region to a value that matches the typical spacing observed in zero-pressure-gradient turbulent boundary layers. The streak amplitudes remain below $20 \%$ 
upstream of the transition location and hence secondary instabilities of the streaks are not observed.

Break-down of the streaks occurs as the shear layer surrounding individual low-speed streaks roll-up into coherent spanwise-oriented vortices. Streaks that roll-up and break down are found to be consistently accompanied by a discrete vortical disturbance in the free-stream above the streak that induces strong sweep motions of high-momentum fluid towards the wall. The sweep creates a high-speed streak in the boundary layer upstream of the low-speed streak. As the head of the high-speed streak flows above the tail of the low-speed streak, a thin region of very high shear stress forms along the interface of the two streaks. Because of the high shear stress, the interfacial shear layer rolls-up via a Kelvin-Helmholtz instability at a frequency that is much higher than would occur based on the conditions of the low-speed streak in isolation. The rolled-up shear layer rapidly forms hairpin-like vortices that subsequently spawn new hairpins and ultimately a small turbulent spot.

After the roll-up of the low-speed streak, the shear layer becomes spatially discontiguous and high-frequency free-stream disturbances can penetrate far into the boundary layer, where they energize the small-scale vortical structures and accelerate the growth of the turbulent spot. Their effect on the non-linear processes within the growing turbulent spot were analyzed quantitatively as in Chapter 7 through the transport of enstrophy. In addition to the processes identified in Chapter 7 for a spot growing under low free-stream turbulence, two new enstrophyproduction terms were observed within the spot due to fluctuating strain rates created by highfrequency sweep motions induced by the elevated free-stream turbulence. The first new term (denoted $P 4 d$ ) relates the $\partial u^{\prime} / \partial y$ strain rate induced by the high-frequency sweep motions to enhanced streamwise tilting of fluctuating wall-normal vorticity in the legs of hairpin vortices. The second new term relates the $\partial w^{\prime} / \partial z$ strain rate created as the sweep motions encounter the wall and turn in the spanwise direction to enhanced stretching or squeezing of fluctuating spanwise vorticity in the boundary layer. These two new terms strengthen the enstrophy- 
production cycles identified in Chapter 7, resulting in an increased production rate of enstrophy and accelerated growth of the turbulent spot. Chapter 8 concludes that elevated free-stream turbulence does not fundamentally alter the spot-growth mechanisms that exist under low freestream turbulence conditions but merely accelerates the existing mechanisms by contributing additional enstrophy-generation processes.

\subsection{Contributions}

The main contributions of the present research are:

- The demonstration of an interaction between disturbances developed in the attached boundary layer upstream of separation via a viscous instability mode and disturbances developed in the downstream separated shear layer via an inviscid instability mode.

- The demonstration that such an interaction results in spanwise non-uniformity of the vortical flow structures that periodically shed from the downstream end of the separated shear layer and promotes the break-down of the shear layer into three-dimensional turbulent wave packets.

- The demonstration that aeroengine-realistic blade sweep does not fundamentally alter the interaction of inviscid and viscous instability modes observed under unswept conditions.

- The demonstration that a swept-blade configuration increases the amplification rate and most-amplified frequency of the inviscid instability mode primarily by modifying the boundary-layer integral parameters at separation, leading to an earlier break-down of the shear layer to small-scale turbulence, and not due to the presence of crossflow instability.

- The identification of the effect of a highly-favourable streamwise pressure gradient on the development of the flow field surrounding a pulsed transverse jet and the coherent vortical flow structures that are triggered by the jet in the main-flow boundary layer. 
- The identification of the mechanisms by which hairpin vortices regenerate within an existing wave packet and by which new wave packets are created along the spanwise edges of a turbulent spot.

- The identification of the mechanism for the longitudinal growth of a turbulent spot through the spatial enlargement of the coherent vortical structures in the leading-edge region of the turbulent spot

- The identification of the mechanism for the formation of the calmed region in the wake of a turbulent spot.

- The identification of the role of free-stream turbulence in precipitating the break-down of streamwise streaks under elevated turbulence conditions, and the identification of the free-stream and boundary-layer conditions required for such a break-down to occur.

- The demonstration of the mechanism by which free-stream turbulence accelerates the formation of new packets of hairpin vortices within a turbulent spot that forms following the inviscid roll-up of a low-speed streamwise streak.

\subsection{Recommendations for Future Work}

In addition to the contributions listed in Section 9.2, this thesis has also identified several areas that remain to be addressed to provide a more complete understanding of shear-layer transition.

In Chapter 4, the development of wave packets downstream of the reattachment location is analyzed over a streamwise distance that corresponds to about 1.5 times the time-averaged length of the separation bubble. However, Alam and Sandham (2000) and McAuliffe and Yaras (2010) identified that the recovery of an equilibrium turbulent boundary layer after reattachment requires up to seven time-averaged bubble lengths. The slow decay of turbulence 
kinetic energy suggests the presence of coherent structures with large spatial scales and long life times. Due to computational constraints, the nature of such structures was not examined and the mechanism whereby very-large-scale coherent motions remain in the reattached turbulent boundary layer remains incomplete. Such a study can now be attempted through DNS using the larger computational resources that have subsequently become available, or by passing the transient flowfield presented in Chapter 4 into a second simulation as a transient inflow boundary, as was done in Chapter 7, to continue analyzing the flow's streamwise development into an equilibrium turbulent boundary layer.

Regarding the effect of a swept-blade configuration on shear-layer transition discussed in Chapter 5, the analysis was restricted to conditions with low levels of free-stream turbulence in which stationary crossflow waves were expected but not observed. If the study was repeated with elevated free-stream turbulence conditions, the crossflow instability would change to favour the production of travelling waves in the boundary layer. Because the travelling crossflow waves convect through the boundary layer in a manner similar to Tollmien-Schlichting waves, the travelling waves may convect into the separated shear layer and interact with the inviscid instability modes in a manner resembling the interaction of instabilities in Chapter 4 . If such an interaction were to occur, it could potentially affect the transition process in a more substantial manner than was observed in Chapter 5. Such a study was not attempted during the present research because of the high computational expense and time required to generate the free-stream turbulence conditions. Now that suitable free-stream turbulence has been generated in Chapter 8, future work could introduce it as a transient inflow boundary to assess its effect in swept-blade configurations.

Further research is also needed to understand the effect of a finite leading-edge radius on the instability development in a swept-blade configuration. On realistic LPT blades, the leading edge has a finite radius, and as flow streamlines turn to flow over the leading edge, a local adverse streamwise pressure gradient is created in which the spanwise pressure force 
can be strongly amplified and trigger a much-stronger initial stationary crossflow instability wave. Future studies need to investigate what effect such a configuration would have on the subsequent development of instability and transition.

The hairpin-vortex and wave-packet regeneration models presented in this thesis are derived from flows developing under low and elevated free-stream turbulence. However, other environmental factors may accelerate or dampen the growth of coherent vortical structures in turbulent spots. For instance, the periodic impingement of the turbulent wakes generated by upstream blade rows has been shown by Wu and Durbin (2001) to result in the formation of small-scale vortices near the wall; a DNS investigating how the turbulent wakes and the resulting vortices affect the regeneration mechanisms described herein is another avenue for future research. Also, the effect of surface roughness needs to be investigated to shed light on how it promotes transition in the boundary layer. A DNS study using a spatial grid that resolves the surface roughness distributions may be feasible with the current computational resources of the authors' research group. 


\section{References}

Abdalla, I. E. and Yang, Z. (2004), "Numerical study of the instability mechanism in transitional separating-reattaching flow," International Journal of Heat and Fluid Flow, 25, pp. 593-605.

Acarlar, M. S. and Smith, C. R. (1987), "A study of hairpin vortices in a laminar boundary layer. Part 2. Hairpin vortices generated by fluid injection," Journal of Fluid Mechanics, 175, pp. 43-83.

Adrian, R., Meinhart, C. and Tomkins, C. (2000), "Vortex organization in the outer region of the turbulent boundary layer," Journal of Fluid Mechanics, 422, pp. 1-54.

Alam, M. and Sandham, N. D. (2000), "Direct numerical simulation of 'short' laminar separation bubbles with turbulent reattachment," Journal of Fluid Mechanics, 410, pp. 128.

Andersson, P., Berggren, M. and Henningson, D. (1999), "Optimal disturbances and bypass transition in boundary layers," Physics of Fluids, 11, pp. 134-150.

Andersson, P., Brandt, L., Bottaro, A. and Henningson, D. (2001), "On the breakdown of boundary layer streaks," Journal of Fluid Mechanics, 428, pp. 29-60.

ANSYS (2006), ANSYS CFX-Solver Theory Guide, 11 ed., ANSYS, Inc., 275 Technology Drive, Canonsburg, PA. 
Arnal, D., Casalis, G., Reneaux, J. and Cousteix, J. (1997), "Laminar-turbulent transition in subsonic boundary-layers: research and applications in France," AIAA Paper No. 19971905.

Asai, M., Konishi, Y., Oizumi, Y. and Nishioka, M. (2007), "Growth and breakdown of lowspeed streaks leading to wall turbulence," Journal of Fluid Mechanics, 586, pp. 371-396.

Asai, M., Minagawa, M. and Nishioka, M. (2002), "The instability and breakdown of a nearwall low-speed streak," Journal of Fluid Mechanics, 455, pp. 289-314.

Asai, M. and Nishioka, M. (1989), "Origin of the peak-valley wave structure leading to wall turbulence," Journal of Fluid Mechanics, 208, pp. 1-23.

Azih, C., Brinkerhoff, J. R. and Yaras, M. I. (2012), "Direct numerical simulation of convective heat transfer in a zero-pressure-gradient boundary layer with supercritical water," Journal of Thermal Science, 21, pp. 49-59.

Baker, C. J. (1979), "The laminar horseshoe vortex," Journal of Fluid Mechanics, 95, pp. 347367.

Bao, F. and Dallmann, U. C. (2004), "Some physical aspects of separation bubble on a rounded backward-facing step," Aerospace Science and Technology, 8, pp. 83 - 91.

Bertolotti, F. P., Herbert, T. and Spalart, P. R. (1992), "Linear and nonlinear stability of the Blasius boundary layer," Journal of Fluid Mechanics, 242, pp. 441-474.

Bippes, H. (1999), "Basic experiments on transition in three-dimensional boundary layers dominated by crossflow instability," Progress in Aerospace Sciences, 35, pp. 363-412.

Bippes, H. and Lerche, T. (1997), "Transition prediction in three-dimensional boundary-layer flows unstable to crossflow instability," AIAA Paper No. 97-1906. 
Blair, M. F. (1992), "Boundary-layer transition in accelerating flows with intense freestream turbulence: Part 1-Disturbances upstream of transition onset," Journal of Fluids Engineering, 114, pp. 313-321.

Bowman, C. T. (1992), "Control of combustion-generated nitrogen oxide emissions: technology driven by regulation," Twenty-Fourth Symposium on Combustion, 24, pp. 859 - 878.

Brandt, L. and de Lange, H. (2008), "Streak interactions and breakdown in boundary layer flows," Physics of Fluids, 20, pp. 024107.

Brandt, L. and Henningson, D. (2002), "Transition of streamwise streaks in zero-pressuregradient boundary layers," Journal of Fluid Mechanics, 472, pp. 229-261.

Brandt, L., Schlatter, P. and Henningson, D. (2004), "Transition in boundary layers subject to free-stream turbulence," Journal of Fluid Mechanics, 517, pp. 167-198.

Brinkerhoff, J. R. and Yaras, M. I. (2011), "Interaction of viscous and inviscid instability modes in separation-bubble transition," Physics of Fluids, 23, pp. 124102.

Brinkerhoff, J. R. and Yaras, M. I. (2012), "Direct numerical simulation of a square jet ejected transversely into an accelerating, laminar main flow," Flow, Turbulence and Combustion, 89, pp. 519-546.

Brinkerhoff, J. R. and Yaras, M. I. (2013), "Numerical investigation of the generation and growth of coherent flow structures in a triggered turbulent spot," Journal of Fluid Mechanics, pp. under review.

Broadwell, J. E. and Breidenthal, R. E. (1984), "Structure and mixing of a transverse jet in incompressible flow," Journal of Fluid Mechanics, 148, pp. 405-412.

Butler, K. M. and Farrell, B. F. (1992), "Three-dimensional optimal perturbations in viscous shear flow," Physics of Fluids, 4, pp. 1637-1650. 
Butler, K. M. and Farrell, B. F. (1993), "Optimal perturbations and streak spacing in wallbounded turbulent shear flow," Physics of Fluids, 5, pp. 774-777.

Cantwell, B., Coles, D. and Dimotakis, P. (1978), "Structure and entrainment in the plane of symmetry of a turbulent spot," Journal of Fluid Mechanics, 87, pp. 641-672.

Chernyshenko, S. and Baig, M. (2005), "The mechanism of streak formation in near-wall turbulence," Journal of Fluid Mechanics, 544, pp. 99-131.

Cherubini, S., Robinet, J.-C., Bottaro, A. and De Palma, P. (2010), "Optimal wave packets in a boundary layer and initial phases of a turbulent spot," Journal of Fluid Mechanics, 656, pp. 231-259.

Choi, H. and Moin, P. (1994), "Effects of the computational time step on numerical solutions of turbulent flow," Journal of Computational Physics, 113, pp. $1-4$.

Chong, M. S., Perry, A. E. and Cantwell, B. J. (1990), "A general classification of threedimensional flow fields," Physics of Fluids A, 2, pp. 765-777.

Chong, T. P. and Zhong, S. (2003), "Development of turbulent wedges in favourable pressure gradients," AIAA Paper no. 2003-4245.

Chong, T. P. and Zhong, S. (2005), "On the three-dimensional structure of turbulent spots," Journal of Turbomachinery, 127, pp. 545-552.

Corke, T. C. and Mangano, R. A. (1989), "Resonant growth of three-dimensional modes in transitioning Blasius boundary layers," Journal of Fluid Mechanics, 209, pp. 93-150.

Cortelezzi, L. and Karagozian, A. R. (2001), "On the formation of the counter-rotating vortex pair in transverse jets," Journal of Fluid Mechanics, 446, pp. 347-373.

Craik, A. D. D. (1971), "Non-linear resonant instability in boundary layers," Journal of Fluid Mechanics, 50, pp. 393-413. 
Dagenhart, J. R. and Saric, W. S. (1999), "Crossflow instability and transition experiments in swept-wing Flow," NASA Report TP-1999-209344.

Davis, S. S. (1996), "The structure of stable and unstable waves in a Blasius boundary layer," AIAA Paper no. 96-0187.

de B. Alves, L. S., Kelly, R. E. and Karagozian, A. R. (2008), "Transverse-jet shear-layer instabilities. Part 2. Linear analysis for large jet-to-crossflow velocity ratio," Journal of Fluid Mechanics, 602, pp. 383-401.

Deyhle, H. and Bippes, H. (1996), "Disturbance growth in an unstable three-dimensional boundary layer and its dependence on environmental conditions," Journal of Fluid Mechanics, 316, pp. 73-113.

Diwan, S. S. and Ramesh, O. N. (2009), "On the origin of the inflectional instability of a laminar separation bubble," Journal of Fluid Mechanics, 629, pp. 263-298.

Djenidi, L. and Antonia, R. A. (1993), "LDA measurements in low Reynolds number turbulent boundary layer," Experiments in Fluids, 14, pp. 280-288.

Dovgal, A., Kozlov, V. and Michalke, A. (1994), "Laminar boundary layer separation: instability and associated phenomena," Progress in Aerospace Sciences, 30, pp. 61 94.

D'Ovidio, A., Harkins, J. A. and Gostelow, J. P. (2001a), "Turbulent spots in strong adverse pressure gradients Part 1-Spot behavior," ASME TURBO EXPO 2001, number 2001GT-0194.

D'Ovidio, A., Harkins, J. A. and Gostelow, J. P. (2001b), "Turbulent spots in strong adverse pressure gradients Part 2-Spot propagation and spreading rates," ASME TURBO EXPO 2001, number 2001-GT-0406. 
D'Ovidio, A., Thomas, R. L. and Gostelow, J. P. (2002), "Development of viscous instability in wake-disturbed flows over a laminar separation bubble," AIAA Paper no. 2002-0295.

Drazin, P. G. (2002), Introduction to Hydrodynamic Stability, Cambridge University Press, New York.

Dryden, H. L. (1936), "Air flow in the boundary layer near a plate," , NACA Report No. 562.

Durbin, P. and Wu, X. (2007), "Transition beneath vortical disturbances," Annual Review of Fluid Mechanics, 39, pp. 107-128.

Elofsson, P. A., Kawakami, M. and Alfredsson, P. H. (1999), "Experiments of the stability of streamwise streaks in plane Poiseuille flow," Physics of Fluids, 11, pp. 915-930.

Emmons, H. W. (1951), "The laminar-turbulent transition in a boundary layer-Part 1," Journal of Aeronautical Sciences, 18, pp. 490-498.

Eroglu, A. and Breidenthal, R. E. (2001), "Structure, penetration, and mixing of pulsed jets in crossflow," AIAA Journal, 39, pp. 417-423.

Escudier, M., Abdel-Hameed, A., Johnson, M. and Sutcliffe, C. (1998), "Laminarisation and re-transition of a turbulent boundary Layer subjected to favourable pressure gradient," Experiments in Fluids, 26, pp. 491-502.

Fiedler, H. (1988), "Coherent structures in turbulent flows," Progress in Aerospace Sciences, 25, pp. $231-269$.

Fric, T. F. and Roshko, A. (1994), "Vortical structure in the wake of a transverse jet," Journal of Fluid Mechanics, 279, pp. 1-47.

Frigo, M. and Johnson, S. G. (2005), "The Design and Implementation of FFTW3," Proceedings of the IEEE, 93, pp. 216-231. 
Gad-El-Hak, M., Blackwelder, R. F. and Riley, J. J. (1981), "On the growth of turbulent regions in laminar boundary layers," Journal of Fluid Mechanics, 110, pp. 73-95.

Gaster, M. (1974), "On the effects of boundary-layer growth on flow stability," Journal of Fluid Mechanics, 66, pp. 465-480.

Goldstein, M. E. (1983), "The evolution of Tollmien-Sclichting waves near a leading edge," Journal of Fluid Mechanic, 127, pp. 59-81.

Gostelow, J. P., Melwani, N. and Walker, G. J. (1996), "Effects of streamwise pressure gradients on turbulent spot development," ASME Journal of Turbomachinery, 118, pp. 737-743.

Gregory, N., Stuart, J. T. and Walker, W. S. (1955), "On the stability of three-dimensional boundary layers with application to the flow due to a rotating disk," Philosophical Transactions of the Royal Society of London. Series A, Mathematical and Physical Sciences, 248, pp. 155-199.

Guo, H., Lian, Q. X., Li, Y. and Wang, H. W. (2004), "A visual study on complex flow structures and flow breakdown in a boundary layer transition," Experiments in Fluids, 37, pp. 311-322.

Gutmark, E. J. and Grinstein, F. F. (1999), "Flow control with noncircular jets," Annual Review of Fluid Mechanics, 31, pp. 239-272.

Hain, R., Kähler, C. J. and Radespiel, R. (2009), "Dynamics of laminar separation bubbles at low-Reynolds-number aerofoils," Journal of Fluid Mechanics, 630, pp. 129-153.

Hall, P. (1985), "The Görtler-vortex intstability mechanism in three-dimensional boundary layers," Proceedings of the Royal Society of London, Series A, Mathematical and Physical Sciences, 399, pp. 135-152.

Hama, F. R., Long, J. D. and Hegarty, J. C. (1957), "On transition from laminar to turbulent flow," Journal of Applied Physics, 28, pp. 388-394. 
Hammond, D. A. and Redekopp, L. G. (1998), "Local and global instability properties of separation bubbles," European Journal of Mechanics - B/Fluids, 17, pp. 145 - 164.

Hatman, A. and Wang, T. (1998), "Separated-flow transition. Part 1-Experimental methodology and mode classification," ASME Paper No. 98-GT-461.

Haven, B. A. and Kurosaka, M. (1997), "Kidney and anti-kidney vortices in crossflow jets," Journal of Fluid Mechanics, 352, pp. 27-64.

Henningson, D. S. and Alfredsson, P. H. (1987), "The wave structure of turbulent spots in plane Poiseuille flow," Journal of Fluid Mechanics, 178, pp. 405-421.

Henningson, D., Spalart, P. and Kim, J. (1987), "Numerical simulations of turbulent spots in plane Poiseuille and boundary-layer flow," Physics of Fluids, 30, pp. 2914-2917.

Hernon, D., Walsh, E. and McEligot, D. (2007), "Experimental investigation into the routes to bypass transition and the shear-sheltering phenomenon," Journal of Fluid Mechanics, 591, pp. 461-479.

Hetsch, T. and Rist, U. (2009a), "An analysis of the structure of laminar separation bubbles in swept infinite geometries," European Journal of Mechanics - B/Fluids, 28, pp. 486 493.

Hetsch, T. and Rist, U. (2009b), "The influence of sweep on the linear stability of a series of swept laminar separation bubbles," European Journal of Mechanics - B/Fluids, 28, pp. $494-505$.

Hickey, J.-P., Blakie, S., Gray, C. and Wu, X. (2010), "Further studies on the statistics and structures of flat-plate boundary layer with passing wakes," International Journal of Heat and Fluid Flow, 31, pp. $315-326$.

Hidalgo, P., Lang, A. and Thacker, W. (2006), "POD study of the coherent structures within a turbulent Spot," AIAA Paper no. 2006-1099. 
Ho, C. M. and Huerre, P. (1984), "Perturbed free shear layers," Annual Review of Fluid Mechanics, 16, pp. 365-424.

Hodson, H. P. and Howell, R. J. (2005), "The role of transition in high-lift low-pressure turbines for aeroengines," Progress in Aerospace Sciences, 41, pp. 419 - 454.

Horton, H. P. (1968), "Laminar Separation Bubbles in Two and Three Dimensional Incompressible Flow," Ph.D. thesis, University of London.

Huang, L. S. and Ho, C. M. (1990), "Small-scale transition in a plane mixing layer," Journal of Fluid Mechanics, 310, pp. 475-500.

Hultgren, L. S. and Gustavsson, L. H. (1981), "Algebraic growth of disturbances in a laminar boundary layer," Physics of Fluids, 24, pp. 1000-1004.

Hunt, J. C. R. and Durbin, P. A. (1999), "Perturbed vortical layers and shear sheltering," Fluid Dynamics Research, 24, pp. 375.

Hutchinson, B. R. and Raithby, G. D. (1986), "A multigrid method based on the additive correction strategy," Numerical Heat Transfer, 9, pp. 511-537.

Inasawa, A., Izawa, S., kui Xiong, A. and Fukunishi, Y. (2007), "Study on the flow structure at the spanwise edge of spreading turbulent region and its control," Fluid Dynamics Research, 39, pp. 475-492.

Jacobs, R. G. and Durbin, P. A. (1998), "Shear sheltering and the continuous spectrum of the Orr-Sommerfeld equation," Physics of Fluids, 10, pp. 2006-2011.

Jacobs, R. G. and Durbin, P. A. (2001), "Simulations of bypass transition," Journal of Fluid Mechanics, 428, pp. 185-221.

Jeong, J. and Hussain, F. (1995), "On the identification of a vortex," Journal of Fluid Mechanics, 285, pp. 69-94. 
Johari, H. (2006), "Scaling of fully pulsed jets in crossflow," AIAA Journal, 44, pp. 2719-2725.

Johnson, M. W. (2001), "On the flow structure within a turbulent spot," International Journal of Heat and Fluid Flow, 22, pp. $409-416$.

Jonás, P., Mazur, O. and Uruba, V. (2000), "On the receptivity of the by-pass transition to the length scale of the outer stream turbulence," European Journal of Mechanics - B/Fluids, 19, pp. $707-722$.

Jones, L., Sandberg, R. and Sandham, N. (2008), "Direct numerical simulations of forced and unforced separation bubbles on an airfoil at incidence," Journal of Fluid Mechanics, 602, pp. $175-207$.

Jones, V. P. and Launder, B. E. (1972), "Some properties of sink-flow turbulent boundary layers," Journal of Fluid Mechanics, 56, pp. 337-351.

Kachanov, Y. S. (1994), "Physical mechanisms of laminar boundary-layer transition," Annual Review of Fluid Mechanics, 26, pp. 411-482.

Kamotani, Y. and Greber, I. (1972), "Experiments on a turbulent jet in a cross flow," AIAA Journal, 10, pp. 1425-1429.

Karagozian, A. R. (2010), "Transverse jets and their control," Progress in Energy and Combustion Science, 36, pp. 531-553.

Katz, Y., Seifert, A. and Wygnanski, I. (1990), "On the evolution of the turbulent spot in a laminar boundary layer with a favourable pressure gradient," Journal of Fluid Mechanics, 221, pp. 1-22.

Keffer, J. F. and Baines, W. D. (1963), "The round turbulent jet in a cross-wind," Journal of Fluid Mechanics, 15, pp. 481-496.

Kelso, R. M., Lim, T. T. and Perry, A. E. (1996), "An experimental study of round jets in cross-flow," Journal of Fluid Mechanics, 306, pp. 111-144. 
Kelso, R. M. and Smits, A. J. (1995), "Horseshoe vortex systems resulting from the interaction between a laminar boundary layer and a transverse jet," Physics of Fluids, 7, pp. 153-158.

Kim, H., Kline, S. and Reynolds, W. (1971), "The production of turbulence near a smooth wall in a turbulent boundary layer," Journal of Fluid Mechanics, 50, pp. 133-160.

Klebanoff, P. (1954), "Characteristics of turbulence in a boundary layer with zero pressure gradient," No. TN-3178, NACA.

Klebanoff, P. (1971), "Effect of freestream turbulence on the laminar boundary layer," Bulletin of the American Physical Society, 10, pp. 1323.

Klebanoff, P. and Tidstrom, K. (1959), "Evolution of amplified waves leading to transition in a boundary layer with zero pressure gradient," National Advisory Committee for Aeronautics Report No. D195.

Klebanoff, P., Tidstrom, K. and Sargent, L. (1962), "The three-dimensional nature of boundary-layer instability," Journal of Fluid Mechanics, 12, pp. 1-34.

Kline, S. J., Reynolds, W. C., Schraub, F. A. and Runstadler, P. W. (1967), "The structure of turbulent boundary layers," Journal of Fluid Mechanics, 30, pp. 741-773.

Knapp, C. F. and Roache, P. J. (1968), "A combined visual and hot-wire anemometer investigation of boundary-layer transition," AIAA Journal, 6, pp. 29-36.

Konishi, Y. and Asai, M. (2004), "Experimental investigation of the instability of spanwiseperiodic low-speed streaks," Fluid Dynamics Research, 34, pp. 299-315.

Krishnan, L. and Sandham, N. D. (2007), "Strong interaction of a turbulent spot with a shock-induced separation bubble," Physics of Fluids, 19, pp. 016102.

Krothapalli, A., Lourenco, L. and Buchlin, J. (1990), "Separated flow upstream of a jet in crossflow," AIAA Journal, 28, pp. 414-420. 
Landahl, M. (1980), "A note on an algebraic instability of inviscid parallel shear flow," Journal of Fluid Mechanics, 98, pp. 243-251.

Lim, T. T., New, T. H. and Luo, S. C. (2001), "On the development of large-scale structures of a jet normal to a cross flow," Physics of Fluids, 13, pp. 770-775.

Liscinsky, D. S., True, B. and Holdeman, J. D. (1996), "Crossflow mixing of noncircular jets," Journal of Propulsion and Power, 12, pp. 225-236.

Litvinenko, Y., Chernoray, V., Kozlov, V., Grek, G., Löfdahl, L. and Chun, H. (2005), “Adverse pressure gradient effect on nonlinear varicose instability of a streaky structure in an unswept wing boundary layer," Physics of Fluids, 17, pp. 118106.

Liu, Y., Zaki, T. and Durbin, P. (2008), "Boundary-layer transition by interaction of discrete and continuous modes," Journal of Fluid Mechanics, 604, pp. 199-233.

Luchini, P. (2000), "Reynolds-number independent stability of the boundary layer over a flat surface: optimal perturbations," Journal of Fluid Mechanics, 404, pp. 289-309.

Mack, L. M. (1984), "Transition prediction and linear stability theory," AGARD Report No. 709.

Makita, H. and Nishizawa, A. (2001), "Characteristics of internal vortical structures in a merged turbulent spot," Journal of Turbulence, 2.

Malik, M. R., Li, F., Choudhari, M. M. and Chang, C. L. (1999), "Secondary instability of crossflow vortices and swept-wing boundary-layer transition," Journal of Fluid Mechanics, 399, pp. 85-115.

Malkiel, E. and Mayle, R. E. (1996), "Transition in a separation bubble," ASME Journal of Turbomachinery, 118, pp. 752-759.

Margason, R. J. (1993), "Fifty years of jet in crossflow research," Computational and Experimental Assessment of Jets in Cross Flow, number CP-539, AGARD Rept CP-539. 
Marxen, O., Rist, U. and Wagner, S. (2004), "Effect of spanwise-modulated disturbances on transition in a separated boundary layer," AIAA Journal, 42, pp. 937-944.

Mayle, R. E. (1991), "The role of laminar-turbulent transition in gas turbine engines," Journal of Turbomachinery, 113, pp. 509-536.

Mayle, R. E., Dullenkopf, K. and Schulz, A. (1998), "The turbulence that matters," Journal of Turbomachinery, 120, pp. 402-409.

McAuliffe, B. R. (2007), "Transition In Separation Bubbles: Physical Mechanisms and Passive Control Techniques," Ph.D. thesis, Carleton University, Ottawa, Canada.

McAuliffe, B. R. and Yaras, M. I. (2005), "Separation bubble transition measurements on a low-Re airfoil using particle image velocimetry," ASME Paper No. 2005-GT-68663.

McAuliffe, B. R. and Yaras, M. I. (2006), "Numerical study of instability mechanisms leading to transition in separation bubbles," Proceedings of ASME Turbo Expo 2006: Power for Land, Sea and Air, number GT2006-91018, American Society of Mechanical Engineers.

McAuliffe, B. R. and Yaras, M. I. (2007a), "Numerical study of turbulent spot development in a separated shear layer," Proceedings of ASME Turbo Expo 200\%: Power for Land, Sea and Air, number GT2007-27604, American Society of Mechanical Engineers.

McAuliffe, B. and Yaras, M. (2007b), "Transition mechanisms in separation bubbles under low and elevated freestream turbulence," Proceedings of ASME Turbo Expo 200\%: Power for Land, Sea and Air, number GT2007-27605, American Society of Mechanical Engineers.

McAuliffe, B. and Yaras, M. (2010), "Transition mechanisms in separation bubbles under lowand elevated-freestream turbulence," Journal of Turbomachinery, 132, pp. 011004.

McMahon, H. M., Hester, D. D. and Palfery, J. G. (1971), "Vortex shedding from a turbulent jet in a cross-wind," Journal of Fluid Mechanics, 48, pp. 73-80. 
Megerian, S., Davitian, J., de B. Alves, L. S. and Karagozian, A. R. (2007), "Transverse-jet shear-layer instabilities. Part 1. experimental studies," Journal of Fluid Mechanics, 593, pp. 93-129.

Michelassi, V., Wissink, J. and Rodi, W. (2002), "Analysis of DNS and LES of flow in a low pressure turbine cascade with incoming wakes and comparison with experiments," Flow, Turbulence, and Combustion, 69, pp. 295-330.

Moretti, P. M. and Kays, W. M. (1965), "Heat transfer to turbulent boundary layer with varying free-stream velocity and varying surface temperature - an experimental study," International Journal of Heat and Mass Transfer, 9, pp. 1187-1202.

Morkovin, M. (1969), On the Many Faces of Transition, pp. 1-31, Viscous Drag Reduction, Plenum.

Morkovin, M. V. (1993), "Bypass-transition research: issues and philosophy," Instabilities and Turbulence in Engineering Flows, edited by D. E. Ashpis, T. B. Gatski, and R. Hirsh, pp. 3-30, Kluwer Academic Publishers, Boston, pp. 3-30.

Morton, B. R. and Ibbetson, A. (1996), "Jets deflected in a crossflow," Experimental Thermal and Fluid Science, 12, pp. 112-133.

Muppidi, S. and Mahesh, K. (2005), "Study of trajectories of jets in crossflow using direct numerical simulations," Journal of Fluid Mechanics, 530, pp. 81-100.

Muti-Lin, J. C. and Pauley, L. L. (1996), "Low-Reynolds number separation on an airfoil," AIAA Journal, 34, pp. 1570-1577.

Na, Y. and Moin, P. (1998), "Direct numerical simulation of a separated turbulent boundarylayer," Journal of Fluid Mechanics, 374, pp. 379-405.

Nagarajan, S., Lele, S. K. and Ferziger, J. H. (2007), "Leading-edge effects in bypass transition," Journal of Fluid Mechanics, 572, pp. 471-504. 
Narasimha, R. (1985), "The laminar-turbulent transition zone in the boundary layer," Progress in Aerospace Sciences, 22, pp. 29-80.

Narasimha, R. and Sreenivasan, K. R. (1979), "Relaminarization of fluid flows," Advances in Applied Mechanics, 19, pp. 221-309.

Narayanan, S., Barooah, P. and Cohen, J. M. (2003), "Dynamics and control of an isolated jet in crossflow," AIAA Journal, 41, pp. 2316-2330.

Nishioka, M., Asai, M. and Iida, S. (1981), "Wall phenomena in the final stage of transition to turbulence," Transition and Turbulence: Proceedings of the Symposium on Transition and Turbulence in Fluids, edited by R. Meyer, Academic Press, pp. 113-126.

Nitschke-Kowsky, P. and Bippes, H. (1988), "Instability and transition of a three-dimensional boundary layer on a swept flat plate," Physics of Fluids, 31, pp. 786-795.

Nolan, K. P. and Walsh, E. J. (2012), "Particle image velocimetry measurements of a transitional boundary layer under free stream turbulence," Journal of Fluid Mechanics, 702, pp. $215-238$.

Orr, W. (1907), "The stability of instability of the steady motions of a perfect liquid and of a viscous liquid. Part I. a perfect liquid." Proceedings of the Royal Irish Academy, 27, pp. 9-68.

Ovchinnikov, V., Choudhari, M. M. and Piomelli, U. (2008), "Numerical simulations of boundary-layer bypass transition due to high-amplitude free-stream turbulence," Journal of Fluid Mechanics, 613, pp. 135-169.

Park, G., Wallace, J., Wu, X. and Moin, P. (2012), "Boundary layer turbulence in transitional and developed states," Physics of Fluids, 24, pp. 035105.

Perry, A. E., Lim, T. T. and Teh, E. W. (1981), "A visual study of turbulent spots," Journal of Fluid Mechanics, 104, pp. 387-405. 
Pierrehumbert, R. T. and Widnall, S. E. (1982), "The two- and three-dimensional instabilities of a spatially periodic shear layer," Journal of Fluid Mechanics, 114, pp. 59-82.

Piomelli, U., Balaras, E. and Pascarelli, A. (2000), "Turbulent structures in accelerating boundary layers," Journal of Turbulence, 1.

Pope, S. (2000), Turbulent Flows, Cambridge University Press, New York.

Pullan, G. and Harvey, N. W. (2007), "Influence of sweep on axial flow turbine aerodynamics at midspan," Journal of Turbomachinery, 129, pp. 591-598.

Radeztsky, R. H., Reibert, M. S. and Saric, W. S. (1999), "Effect of micron-sized roughess on transition in swept-wing flows," AIAA Journal, 37, pp. 1371-1377.

Rajaee, M., Karlsson, S. and Sirovich, L. (1995), "On the streak spacing and vortex roll size in a turbulent channel flow," Physics of Fluids, 7, pp. 2439-2443.

Rayleigh, J. (1887), "On the stability of certain fluid motions," Proceedings of the London Mathematical Society, 19, pp. 67.

Reddy, S., Schmid, P., Baggett, J. and Henningson, D. (1998), "On stability of streamwise streaks and transition thresholds in plane channel flows," Journal of Fluid Mechanics, 365, pp. 269-303.

Reed, H. L. and Saric, W. S. (1989), "Stability of three-dimensional boundary layers," Annual Review of Fluid Mechanics, 21, pp. 235-284.

Reed, H., Saric, W. S. and Arnal, D. (1996), "Linear stability theory applied to boundary layers," Annual Review of Fluid Mechanics, 28, pp. 389-428.

Reynolds, G. A. and Saric, W. S. (1986), "Experiments on the stability of the flat-plate boundary layer with suction," AIAA Journal, 24, pp. 202-207. 
Ripley, M. D. and Pauley, L. L. (1993), "The unsteady structure of two-dimensional steady laminar separation," Physics of Fluids A: Fluid Dynamics, 5, pp. 3099-3106.

Rist, U. and Augustin, K. (2006), "Control of laminar separation bubbles using instability waves," AIAA Journal, 44, pp. 2217-2223.

Rist, U. and Maucher, U. (2002), "Investigations of time-growing instabilities in laminar separation bubbles," European Journal of Mechanics B/Fluids, 21, pp. 495-509.

Rivero, A., Feré, J. A. and Giralt, F. (2001), "Organized motions in a jet in crossflow," Journal of Fluid Mechanics, 444, pp. 117-149.

Roach, P. (1986), "The generation of nearly isotropic turbulence by means of grids," Heat and Fluid Flow, 8, pp. 82-92.

Roach, P. and Brierley, D. (1990), "The influence of a turbulent free stream on zero pressure gradient transitional boundary layer development. Part 1: Test cases T3A and T3B," Numerical simulation of unsteady flows and transition to turbulence, edited by D. Pironneau, W. Rode, and I. Ryhming, Cambridge University Press.

Roberts, S. K. (2005), "Boundary layer transition in attached and separated flows at low Reynolds number," Ph.D. thesis, Carleton University, Ottawa, Canada.

Roberts, S. K. and Yaras, M. I. (2005a), "Boundary layer transition affected by surface roughness and freestream turbulence," Journal of Fluids Engineering, 127, pp. 449-457.

Roberts, S. K. and Yaras, M. I. (2005b), "Modeling transition in separated and attached boundary layers," Journal of Turbomachinery, 127, pp. 402-411.

Roberts, S. K. and Yaras, M. I. (2006), "Large-eddy simulation of transition in a separation bubble," Journal of Fluids Engineering, 128, pp. 232-238.

Robinson, S. K. (1991), "Coherent motions in the turbulent boundary layer," Annual Review of Fluid Mechanics, 23, pp. 601-639. 
Rudy, D. H. and Strikwerda, J. C. (1980), "A nonreflecting outflow boundary condition for subsonic Navier-Stokes calculations," Journal of Computational Physics, 36, pp. 55-70.

Ruggeri, R. S., Callaghan, E. E. and Bowden, D. T. (1950), "Penetration of air jets issued from circular, square and elliptical orifices directed perpendicular to an air Stream," No. TN-2019, NACA.

Sabatino, D. R. and Smith, C. R. (2008), "Turbulent spot flow topology and mechanisms for surface heat transfer," Journal of Fluid Mechanics, 612, pp. 81-105.

Sandham, N. and Kleiser, L. (1992), "The late stages of transition to turbulence in channel flow," Journal of Fluid Mechanics, 245, pp. 319-348.

Sankaran, R. and Antonia, R. A. (1988), "Influence of a favourable pressure gradient on the growth of a turbulent spot," AIAA Journal, 26, pp. 885-887.

Saric, W. S., Carrillo, R. B. and Reibert, M. S. (1998), "Nonlinear stability and transition in 3-D boundary layers," Meccanica, 33, pp. 469-487.

Saric, W. S. and Nayfeh, A. H. (1975), "Non-parallel stability of boundary-layer flows," Physics of Fluids, 18, pp. 945-950.

Saric, W. S., Reed, H. L. and Kerschen, E. J. (2002), "Boundary-layer receptivity to freestream disturbances," Annual Review of Fluid Mechanics, 34, pp. 291-319.

Saric, W. S., Reed, H. L. and White, E. B. (2003), "Stability and transition of three-dimensional boundary layers," Annual Review of Fluid Mechanics, 35, pp. 413-440.

Saric, W. S. and Thomas, A. S. W. (1983), "Experiments on the subharmonic route to turbulence in boundary layers," Turbulence and chaotic phenomena in fluids; Proceedings of the International Symposium, Kyoto, Japan, September 5-10, 1983. 
Sau, A., Sheu, T. W. H., Tsai, S. F., Hwang, R. R. and Chiang, T. P. (2004), "Structural development of vortical flows around a square jet in cross-flow," Proceedings: Mathematical, Physical and Engineering Sciences, 460, pp. 3339-3368.

Sau, R. and Mahesh, K. (2008), "Dynamics and mixing of vortex rings in crossflow," Journal of Fluid Mechanics, 604, pp. 389-409.

Sau, R. and Mahesh, K. (2010), "Optimization of pulsed jets in crossflow," Journal of Fluid Mechanics Digital Archive, 653, pp. 365-390.

Schlatter, P., Brandt, L., de Lange, H. C. and Henningson, D. S. (2008), "On streak breakdown in bypass transition," Physics of Fluids, 20, pp. 101505.

Schlichting, H. (1968), Boundary Layer Theory, McGraw-Hill, New York.

Schmid, P. and Henningson, D. (2000), Stability and Transition in Shear Flows, SpringerVerlag.

Schröder, A., Geisler, R., Elsinga, G. E., Scarano, F. and Dierksheide, U. (2008), "Investigation of a turbulent spot and a tripped turbulent boundary layer flow using time-resolved tomographic PIV," Experiments in Fluids, 44, pp. 305-316.

Schröder, A. and Kompenhans, J. (2004), "Investigation of a turbulent spot using multi-plane stereo particle image velocimetry," Experiments in Fluids, 36, pp. 82-90.

Schubauer, G. B. and Klebanoff, P. S. (1956), "Contributions on the mechanics of boundary layer transition," National Advisory Committee for Aeronautics Report No. 1289.

Schubauer, G. B. and Skramstad, H. (1947), "Laminary boundary layer oscillations and transition on a flat plate," Journal of the Research of the National Bureau of Standards, 38, pp. 251-292.

Schumacher, J. and Eckhardt, B. (2001), "Evolution of turbulent spots in a parallel shear flow," Physical Review E, 63, pp. 046307. 
Shapiro, S. R., King, J. M., M'Closkey, R. and Karagozian, A. (2006), "Optimization of controlled jets in crossflow," AIAA Journal, 44, pp. 1292-1298.

Singer, B. A. (1996), "Characteristics of a young turbulent spot," Physics of Fluids, 8, pp. 509521.

Singer, B. A. and Joslin, R. D. (1994), "Metamorphosis of a hairpin vortex into a young turbulent spot," Physics of Fluids, 6, pp. 3724-3736.

Smith, C. R. and Metzler, S. P. (1983), "The characteristics of low-speed streaks in the nearwall region of a turbulent boundary layer," Journal of Fluid Mechanics, 129, pp. 27-54.

Smith, C. R., Walker, J. D. A., Haidari, A. H. and Sobrun, U. (1991), "On the dynamics of near-wall turbulence," Philosophical Transactions: Physical Sciences and Engineering, 336, pp. 131-175.

Spalart, P. R. (1988), "Direct simulation of a turbulent boundary layer up to $R e_{\theta}=1410$," Journal of Fluid Mechanics, 187, pp. 61-98.

Spalart, P. R. (1993), "Numerical study of transition induced by suction devices," Int. Conference on Near-Wall Turbulent Flows, pp. 849-858.

Spalart, P. R. and Strelets, M. K. (2000), "Mechanism of transition and heat transfer in a separation bubble," Journal of Fluid Mechanics, 403, pp. 329-349.

Stanislas, M., Perret, L. and Foucaut, J.-M. (2008), "Vortical structures in the turbulent boundary layer: a possible route to a universal representation," Journal of Fluid Mechanics, 602, pp. 327-382.

Strand, J. S. and Goldstein, D. B. (2011), "Direct numerical simulations of riblets to constrain the growth of turbulent spots," Journal of Fluid Mechanics, 668, pp. 267-292.

Suder, K. L., O’Brien, J. E. and Reshotko, E. (1988), "Experimental study of bypass transition in a boundary layer," NASA Technical Memorandum no. 100913. 
Suponitsky, V., Cohen, J. and Bar-Yoseph, P. Z. (2005), "The generation of streaks and hairpin vortices from a localized vortex disturbance embedded in unbounded uniform shear flow," Journal of Fluid Mechanics, 535, pp. 65-100.

Swearingen, J. and Blackwelder, R. (1987), "The growth and breakdown of streamwise vortices in the presence of a wall," Journal of Fluid Mechanics, 182, pp. 255-290.

Talamelli, A., Fornaciari, N., Westin, K. J. A. and Alfredsson, P. H. (2002), "Experimental investigation of streaky structures in a relaminarizing boundary layer," Journal of Turbulence, 3, pp. Art. No. N18.

Talan, M. and Hourmouziadis, J. (2002), "Characteristic regimes of transition separation bubbles in unsteady flow," Flow, Turbulence, and Combustion, 69, pp. 207-227.

Tani, I. (1964), "Low-speed flows involving bubble separations," Progress in Aerospace Sciences, 5, pp. 70-104.

Tani, I. and Hama, F. R. (1953), "Some experiments on the effect of a single roughness element on boundary layer transition," Journal of the Aeronautical Sciences, 20, pp. 289-290.

Tennekes, H. and Lumley, J. (1972), A First Course in Turbulence, MIT Press.

Tillmark, N. (1995), "On the spreading mechanism of a turbulent spot in plane Couette flow," Europhysics Letters, 6, pp. 481-485.

Vermeulen, P. J., Grabinski, P. and Ramesh, V. (1992), "Mixing of an acoustically excited air jet with a confined hot crossflow," Journal of Engineering for Gas Turbines and Power, 114, pp. $46-54$.

Volino, R. J. and Bohl, D. G. (2004), "Separated flow transition mechanism and prediction with high and low freestream turbulence under low pressure turbine conditions," ASME Paper No. 2004-GT-53360. 
Volino, R. J. and Simon, T. W. (1995), "Bypass transition in boundary layers including curvature and favorable pressure gradient effects," Journal of Turbomachinery, 117, pp. 166-174.

Volino, R., Schultz, M. and Pratt, C. (2001), "Conditional sampling in a transitional boundary layer under high freestream turbulence conditions," ASME Paper 2001-GT-0192.

Walker, G. J. (1989), "Transitional flow on axial turbomachine blading," Journal of the American Institute for Aeronautics and Astronautics, 27, pp. 595-602.

Wasserman, P. and Kloker, M. (2002), "Mechanisms and passive control of crossflow-vortexinduced transition in a three-dimensional boundary layer," Journal of Fluid Mechanics, 456, pp. 49-84.

Watmuff, J. H. (1999), "Evolution of a wave packet into vortex loops in a laminar separation bubble," Journal of Fluid Mechanics, 397, pp. 119-169.

White, F. M. (2006), Viscous Fluid Flow, 3 ed., McGraw-Hill.

Winant, C. D. and Browand, F. K. (1974), "Vortex pairing: the mechanism of turbulent mixing-layer growth at moderate Reynolds number," Journal of Fluid Mechanics, 63, pp. $237-255$.

Wissink, J. G. and Rodi, W. (2002), "DNS of transition in a laminar separation bubble," Advances in Turbulence IX, Proceedings of the Ninth European Turbulence Conference, edited by I. P. Casto, P. E. Hancock, and T. G. Thomas, pp. 728-730.

Wissink, J. G. and Rodi, W. (2006), "Direct numerical simulations of transitional flow in turbomachinery," Journal of Turbomachinery, 128, pp. 668-678.

Wu, X. (2010), "Establishing the generality of three phenomena using a boundary layer with free-stream passing wakes," Journal of Fluid Mechanics, 664, pp. 193-219. 
Wu, X. and Durbin, P. A. (2001), "Evidence of longitudinal vortices evolved from distorted wakes in a turbine passage," Journal of Fluid Mechanics, 446, pp. 199-228.

Wu, X., Jacobs, R. G., Hunt, J. C. R. and Durbin, P. A. (1999), "Simulation of boundary layer transition induced by periodically Passing wakes," Journal of Fluid Mechanics, 398, pp. 109-153.

Wu, X. and Moin, P. (2009), "Direct numerical simulation of turbulence in a nominally zeropressure-gradient flat-plate boundary layer," Journal of Fluid Mechanics, 630, pp. 5-41.

Wygnanski, I., Sokolov, M. and Friedman, D. (1976), "On a turbulent 'spot' in a laminar boundary layer," Journal of Fluid Mechanics, 78, pp. 785-819.

Yang, Z. and Voke, P. R. (2001), "Large-eddy simulation of boundary-layer separation and transition at a change of surface curvature," Journal of Fluid Mechanics, 439, pp. 305333.

Yaras, M. I. (2001), "Measurements of the effects of pressure-gradient history on separationbubble transition," ASME Paper No. 2001-GT-0193.

Yaras, M. I. (2002), "Measurements of the effects of freestream turbulence on separation-bubble transition," ASME Paper No. GT-2002-30232.

Yaras, M. I. (2007), "An experimental study of artificially-generated turbulent spots under strong favorable pressure gradients and freestream turbulence," Journal of Fluids Engineering, 129, pp. 563-572.

Yaras, M. I. (2012), "Instability and transition in a separation bubble under a three-dimensional freestream pressure distribution," Journal of Turbomachinery, 134, pp. 031019.

Yuan, L. L., Street, R. L. and Ferziger, J. H. (1999), "Large-eddy simulations of a round jet in crossflow," Journal of Fluid Mechanics, 379, pp. 71-104. 
Zaki, T. and Durbin, P. (2005), "Mode interaction and the bypass route to transition," Journal of Fluid Mechanics, 531, pp. 85-111.

Zaki, T. and Durbin, P. (2006), "Continuous mode transition and the effects of pressure gradient," Journal of Fluid Mechanics, 563, pp. 357-388.

Ziefle, J. and Kleiser, L. (2009), "Large-eddy simulation of a round jet in crossflow," AIAA Journal, 47, pp. 1158-1172. 


\section{Appendix A}

\section{Domain and Grid Coordinates}

This appendix describes the in-house software that is used to generate the computational domains and spatial grids used in this thesis. An overview of the in-house grid generation software is given followed by a detailed description of the spatial grids.

\section{A.1 Description of the Grid Generation Software}

\section{A.1.1 Introduction}

The grid generation software used in the present research is called PatranMeshGenerator. It is an in-house software written in ANSI C to create a structured, Cartesian grid for CFD simulations. The grid created by PatranMeshGenerator is formatted as a Patran Neutral file, which can be imported into ANSYS CFX and other commercial CFD solvers. A number of features are available in the code:

- Multiple wall-normal and spanwise node-spacing distributions can be defined.

- The domain ceiling can by contoured using multiple polynomial expressions. 
- Sinusoidal spanwise-waviness can be created at the trailing edge of the grid surface and/or ceiling.

- The grid can be bent along a circular arc to create a domain with constant-radius streamline curvature.

- The geometry can be offset (translated) in the $y$ and $z$ directions.

- A two-dimensional turbulence-generating grid consisting of an array of square inlets can be defined.

- Two- and three-dimensional Tecplot $囚$-formatted files can be created for previewing the grid.

The code consists of two modules: an input text file and the program executable. To run the program, copy the executable PatranMeshGenerator .exe to the directory containing the input file and double-click on the executable file to execution. The program prompts the user to enter the name of the input file. Once the file name is entered, the program reads the input text file, creates the structured grid, optionally writes two Tecplot@-formatted files (a twodimensional $x-y$ slice and the full three-dimensional grid), and then writes the Patran Neutral file for input to the CFX pre-processor.

\section{A.1.2 Input text file format}

The input text file that is read by PatranMeshGenerator contains information regarding the shape of the computational domain and the placement of the grid nodes. It must be ASCII-formatted (ie. a file extension .txt or equivalent) in order to be properly read by PatranMeshGenerator. The program is very sensitive to the format of the input text file, and it may fail to create the grid if a small error exists in the input file. A sample input file is also provided in the PatranMeshGenerator source directory. 
Table A.1: Limitations of PatranMeshGenerator

\begin{tabular}{lc}
\hline Item & Value \\
\hline Maximum $i$ nodes & 600 \\
Maximum $j$ nodes & 150 \\
Maximum $k$ nodes & 300 \\
Maximum $y$ distributions & 20 \\
Maximum $z$ distributions & 20 \\
Maximum order of polynomial to define ceiling shape & 10 \\
Maximum number of ceiling segments & 10 \\
Maximum number of turbulence grid segments & 200 \\
Maximum length of input file name & 50 characters \\
\hline
\end{tabular}

\section{A.1.3 Software limitations}

PatranMeshGenerator is limited in the number of computational nodes that can be specified, the number of polynomial segments that can define the ceiling shape, the maximum length of the input data file, and other constraints. Table A.1 lists the limitations of PatranMeshGenerator. The maximum $i, j, k$ nodes can be easily modified by changing the $\mathrm{C}$ pre-processor directives at the start of the source code (ie. the lines beginning with \#define) and re-compiling.

Beyond the limitations specified in the source code of PatranMeshGenerator, an additional limitation is present in PatranMeshGenerator due to the formatting of a Patran Neutral file. The element numbers in a Patran Neutral file are only separated by 8 characters, and hence the maximum number of nodes in a single Patran Neutral file is 9,999,999. Computational domains requiring more than this many nodes must be sub-divided into sub-domains, with a separate grid file for each. The most convenient coordinate direction to split a sub-domain is at a specified $j$ node i.e. in the $x$ - $z$ plane. The second-best choice is at a specified $i$ node i.e. in the $y-z$ plane.

It should be noted that sub-dividing a large grid into separate grids can have a detrimental impact on the simulation results if the nodes along the mating faces of the adjacent sub- 
domains do not have a one-to-one correspondence. CFX can handle a mismatch in the node locations across a domain interface by using an interpolation algorithm termed a "general grid interface," (GGI) but the gradients across a GGI interface will not be continuous if the interfacial nodes are mismatched. Therefore, the use of a GGI interface is discouraged.

\section{A.2 Domain and Grid Information}

\section{A.2.1 Paper 1}

The simulation described in Chapter 4 contains 6 sub-domains.

\author{
Domain 1 \\ $x$ length: \\ $z$ length: \\ Sweep angle: \\ Bend angle: \\ $y$ offset: \\ $z$ offset: \\ Number of $i$ nodes: \\ Number of $j$ nodes: \\ Number of $k$ nodes: \\ Number of $j$ node distributions: \\ $i$-indices of $j$ node distributions: \\ Number of $k$ node distributions: \\ $i$-indices of $k$ node distributions: \\ Number of ceiling segments: \\ Segment 1 polynomial order: \\ Segment $1 x_{0}, x_{L}$ locations: \\ Segment 1 polynomial coefficients:
}

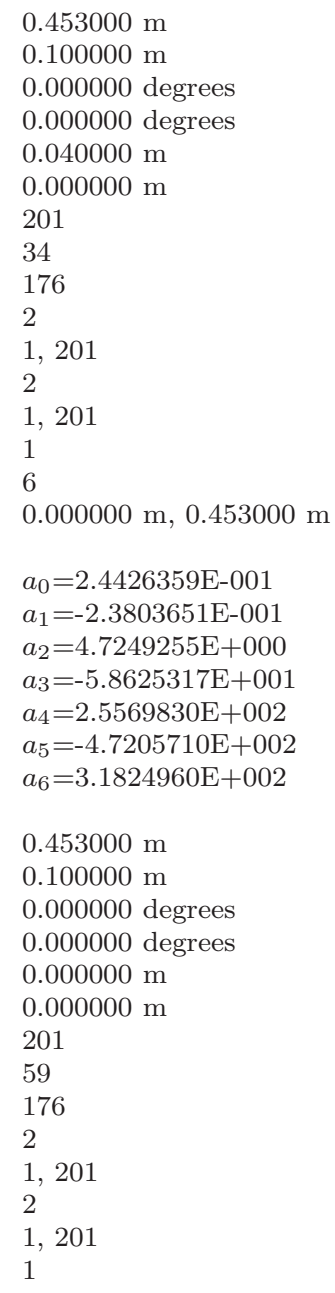


Segment 1 polynomial order:

Segment $1 x_{0}, x_{L}$ locations:

Segment 1 polynomial coefficients:

\section{Domain 3}

$x$ length:

$z$ length:

Sweep angle:

Bend angle:

$y$ offset:

$z$ offset:

Number of $i$ nodes:

Number of $j$ nodes:

Number of $k$ nodes:

Number of $j$ node distributions:

$i$-indices of $j$ node distributions:

Number of $k$ node distributions:

$i$-indices of $k$ node distributions:

Number of ceiling segments:

Segment 1 polynomial order:

Segment $1 x_{0}, x_{L}$ locations:

Segment 1 polynomial coefficients:

Segment 2 polynomial order:

Segment $2 x_{0}, x_{L}$ locations:

Segment 2 polynomial coefficients:

Domain 4

$x$ length:

$z$ length:

Sweep angle:

Bend angle:

$y$ offset:

$z$ offset:

Number of $i$ nodes:

Number of $j$ nodes:

Number of $k$ nodes:

Number of $j$ node distributions:

$i$-indices of $j$ node distributions:

Number of $k$ node distributions:

$i$-indices of $k$ node distributions:

Number of ceiling segments:

Segment 1 polynomial order:

Segment $1 x_{0}, x_{L}$ locations:

Segment 1 polynomial coefficients:

\section{Domain 5}

$x$ length:

$z$ length:

Sweep angle:

Bend angle:

$y$ offset:

$z$ offset:

Number of $i$ nodes:

Number of $j$ nodes:

Number of $k$ nodes:

Number of $j$ node distributions:

$i$-indices of $j$ node distributions:
0

$0.000000 \mathrm{~m}, 0.453000 \mathrm{~m}$

$a_{0}=3.9999999 \mathrm{E}-002$

$0.800000 \mathrm{~m}$
$0.100000 \mathrm{~m}$
0.000000 degrees
0.000000 degrees
$0.040000 \mathrm{~m}$
$0.000000 \mathrm{~m}$
491
15
176
2
1,491
2
1,491
2
5
$0.453000 \mathrm{~m}, 0.867500 \mathrm{~m}$

$a_{0}=2.6602383 \mathrm{E}+000$

$a_{1}=-2.0658575 \mathrm{E}+001$

$a_{2}=6.6073723 \mathrm{E}+001$

$a_{3}=-1.0111778 \mathrm{E}+002$

$a_{4}=7.3559174 \mathrm{E}+001$

$a_{5}=-2.0421898 \mathrm{E}+001$

$0.867500,1.253050$

$a_{0}=-2.3777001 \mathrm{E}-002$

$a_{1}=1.1430500 \mathrm{E}-001$

$0.800000 \mathrm{~m}$

$0.100000 \mathrm{~m}$

0.000000 degrees

0.000000 degrees

$0.000000 \mathrm{~m}$

$0.000000 \mathrm{~m}$

491

59

176

2

1,491

2

1,491

1

$0.453000 \mathrm{~m}, 1.253050 \mathrm{~m}$

$a_{0}=3.9999999 \mathrm{E}-002$

$0.100000 \mathrm{~m}$

$0.100000 \mathrm{~m}$

0.000000 degrees

0.000000 degrees

$0.040000 \mathrm{~m}$

$0.000000 \mathrm{~m}$

28

34

176

2

1, 28 
Number of $k$ node distributions: $i$-indices of $k$ node distributions:

Number of ceiling segments:

Segment 1 polynomial order:

Segment $1 x_{0}, x_{L}$ locations:

Segment 1 polynomial coefficients:

\section{Domain 6}

$x$ length:

$z$ length:

Sweep angle:

Bend angle:

$y$ offset:

$z$ offset:

Number of $i$ nodes:

Number of $j$ nodes:

Number of $k$ nodes:

Number of $j$ node distributions:

$i$-indices of $j$ node distributions:

Number of $k$ node distributions:

$i$-indices of $k$ node distributions:

Number of ceiling segments:

Segment 1 polynomial order:

Segment $1 x_{0}, x_{L}$ locations:

Segment 1 polynomial coefficients:
2

1,28

1

$1.253000 \mathrm{~m}, 1.353000 \mathrm{~m}$

$a_{0}=2.2693940 \mathrm{E}-001$

$a_{1}=-8.5787997 \mathrm{E}-002$

$0.100000 \mathrm{~m}$

$0.100000 \mathrm{~m}$

0.000000 degrees

0.000000 degrees

$0.000000 \mathrm{~m}$

$0.000000 \mathrm{~m}$

28

59

176

2

1,28

2

1,28

1

0

$1.253000 \mathrm{~m}, 1.353000 \mathrm{~m}$

$a_{0}=3.9999999 \mathrm{E}-002$

\section{A.2.2 Paper 2}

As described in Chapter 5, two simulations are performed in Paper 2 with swept and unswept conditions. Both contain 6 sub-domains.

\section{Swept Domain 1}

$x$ length:

$z$ length:

Sweep angle:

Bend angle:

$y$ offset:

$z$ offset:

Number of $i$ nodes:

Number of $j$ nodes:

Number of $k$ nodes:

Number of $j$ node distributions:

$i$-indices of $j$ node distributions:

Number of $k$ node distributions:

$i$-indices of $k$ node distributions:

Number of ceiling segments:

Segment 1 polynomial order:

Segment $1 x_{0}, x_{L}$ locations:

Segment 1 polynomial coefficients:

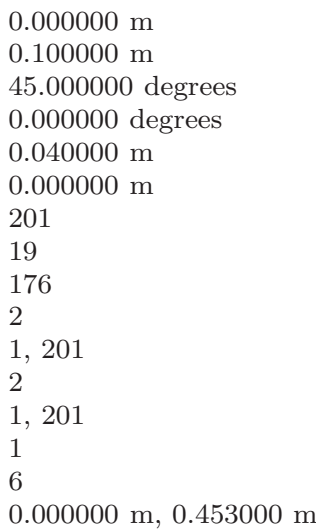

$0.000000 \mathrm{~m}$ 
$z$ length:

Sweep angle:

Bend angle:

$y$ offset:

$z$ offset:

Number of $i$ nodes:

Number of $j$ nodes:

Number of $k$ nodes:

Number of $j$ node distributions:

$i$-indices of $j$ node distributions:

Number of $k$ node distributions:

$i$-indices of $k$ node distributions:

Number of ceiling segments:

Segment 1 polynomial order:

Segment $1 x_{0}, x_{L}$ locations:

Segment 1 polynomial coefficients:

\section{Swept Domain 3}

$x$ length:

$z$ length:

Sweep angle:

Bend angle:

$y$ offset:

$z$ offset:

Number of $i$ nodes:

Number of $j$ nodes:

Number of $k$ nodes:

Number of $j$ node distributions:

$i$-indices of $j$ node distributions:

Number of $k$ node distributions:

$i$-indices of $k$ node distributions:

Number of ceiling segments:

Segment 1 polynomial order:

Segment $1 x_{0}, x_{L}$ locations:

Segment 1 polynomial coefficients:

Segment 2 polynomial order:

Segment $2 x_{0}, x_{L}$ locations:

Segment 2 polynomial coefficients:

\section{Swept Domain 4}

$x$ length:

$z$ length:

Sweep angle:

Bend angle:

$y$ offset:

$z$ offset:

Number of $i$ nodes:

Number of $j$ nodes:

Number of $k$ nodes:

Number of $j$ node distributions:

$i$-indices of $j$ node distributions:

Number of $k$ node distributions:

$i$-indices of $k$ node distributions:

Number of ceiling segments:

Segment 1 polynomial order:

Segment $1 x_{0}, x_{L}$ locations:

Segment 1 polynomial coefficients:

\author{
$0.100000 \mathrm{~m}$ \\ 45.000000 degrees \\ 0.000000 degrees \\ $0.000000 \mathrm{~m}$ \\ $0.000000 \mathrm{~m}$ \\ 201 \\ 59 \\ 176 \\ 2 \\ 1,201 \\ 2 \\ 1,201 \\ 1 \\ $0.000000 \mathrm{~m}, 0.453000 \mathrm{~m}$
}

$a_{0}=3.9999999 \mathrm{E}-002$

$0.000000 \mathrm{~m}$

$0.100000 \mathrm{~m}$

45.000000 degrees

0.000000 degrees

$0.040000 \mathrm{~m}$

$0.000000 \mathrm{~m}$

580

19

176

2

1,580

2

1,580

2

$0.453000 \mathrm{~m}, 0.867500 \mathrm{~m}$

$a_{0}=2.6602383 \mathrm{E}+000$

$a_{1}=-2.0658575 \mathrm{E}+001$

$a_{2}=6.6073723 \mathrm{E}+001$

$a_{3}=-1.0111778 \mathrm{E}+002$

$a_{4}=7.3559174 \mathrm{E}+001$

$a_{5}=-2.0421898 \mathrm{E}+001$

1

$0.867500 \mathrm{~m}, 1.353050 \mathrm{~m}$

$a_{0}=-2.3777001 \mathrm{E}-002$

$a_{1}=1.1430500 \mathrm{E}-001$

$0.000000 \mathrm{~m}$

$0.100000 \mathrm{~m}$

45.000000 degrees

0.000000 degrees

$0.000000 \mathrm{~m}$

$0.000000 \mathrm{~m}$

580

59

176

2

1,580

2

1,580

1

$0.453000 \mathrm{~m}, 1.353050 \mathrm{~m}$ 


\author{
Swept Domain 5 \\ $x$ length: \\ $z$ length: \\ Sweep angle: \\ Bend angle: \\ $y$ offset: \\ $z$ offset: \\ Number of $i$ nodes: \\ Number of $j$ nodes: \\ Number of $k$ nodes: \\ Number of $j$ node distributions: \\ $i$-indices of $j$ node distributions: \\ Number of $k$ node distributions: \\ $i$-indices of $k$ node distributions: \\ Number of ceiling segments: \\ Segment 1 polynomial order: \\ Segment $1 x_{0}, x_{L}$ locations: \\ Segment 1 polynomial coefficients:
}

\section{Swept Domain 6}

$x$ length:

$z$ length:

Sweep angle:

Bend angle:

$y$ offset:

$z$ offset:

Number of $i$ nodes:

Number of $j$ nodes:

Number of $k$ nodes:

Number of $j$ node distributions:

$i$-indices of $j$ node distributions:

Number of $k$ node distributions:

$i$-indices of $k$ node distributions:

Number of ceiling segments:

Segment 1 polynomial order:

Segment $1 x_{0}, x_{L}$ locations:

Segment 1 polynomial coefficients:

\section{Unswept Domain 1}

$x$ length:

$z$ length:

Sweep angle:

Bend angle:

$y$ offset:

$z$ offset:

Number of $i$ nodes:

Number of $j$ nodes:

Number of $k$ nodes:

Number of $j$ node distributions:

$i$-indices of $j$ node distributions:

Number of $k$ node distributions:

$i$-indices of $k$ node distributions:

Number of ceiling segments:

Segment 1 polynomial order:

Segment $1 x_{0}, x_{L}$ locations:

Segment 1 polynomial coefficients: $a_{0}=3.9999999 \mathrm{E}-002$

$0.000000 \mathrm{~m}$
$0.100000 \mathrm{~m}$
45.000000 degrees
$0.000000 \mathrm{degrees}$
$0.040000 \mathrm{~m}$
$0.000000 \mathrm{~m}$
28
19
176
2
1,28
2
1,28
1
1
$1.353000 \mathrm{~m}, 1.453050 \mathrm{~m}$
$a_{0}=2.4694940 \mathrm{E}-001$
$a_{1}=-8.5787997 \mathrm{E}-002$
$0.000000 \mathrm{~m}$
$0.100000 \mathrm{~m}$
$45.000000 \mathrm{degrees}$
$0.000000 \mathrm{degrees}$
$0.000000 \mathrm{~m}$
$0.000000 \mathrm{~m}$
28
59
176
2
1,28
2
1,28
1
0
$1.353000 \mathrm{~m}, 1.453050 \mathrm{~m}$

$a_{0}=3.9999999 \mathrm{E}-002$

$0.000000 \mathrm{~m}$
$0.100000 \mathrm{~m}$
0.000000 degrees
0.000000 degrees
$0.040000 \mathrm{~m}$
$0.000000 \mathrm{~m}$
201
19
176
2
1,201
2
1,201
1
5
$0.000000 \mathrm{~m}, 0.453000 \mathrm{~m}$
$a_{0}=2.4424638 \mathrm{E}-001$
$a_{1}=1.0036357 \mathrm{E}-003$
$a_{2}=-5.1358473 \mathrm{E}-001$
$a_{3}=-2.7097657 \mathrm{E}-001$
$a_{4}=5.1298285 \mathrm{E}+000$
$a_{5}=-6.4607840 \mathrm{E}+000$


Unswept Domain 2

$x$ length:

$z$ length:

Sweep angle:

Bend angle:

$y$ offset:

$z$ offset:

Number of $i$ nodes:

Number of $j$ nodes:

Number of $k$ nodes:

Number of $j$ node distributions:

$i$-indices of $j$ node distributions:

Number of $k$ node distributions:

$i$-indices of $k$ node distributions:

Number of ceiling segments:

Segment 1 polynomial order:

Segment $1 x_{0}, x_{L}$ locations:

Segment 1 polynomial coefficients:

\section{Unswept Domain 3}

$x$ length:

$z$ length:

Sweep angle:

Bend angle:

$y$ offset:

$z$ offset:

Number of $i$ nodes:

Number of $j$ nodes:

Number of $k$ nodes:

Number of $j$ node distributions:

$i$-indices of $j$ node distributions:

Number of $k$ node distributions:

$i$-indices of $k$ node distributions:

Number of ceiling segments:

Segment 1 polynomial order:

Segment $1 x_{0}, x_{L}$ locations:

Segment 1 polynomial coefficients:

Segment 2 polynomial order:

Segment $2 x_{0}, x_{L}$ locations:

Segment 2 polynomial coefficients:

Segment 3 polynomial order:

Segment $3 x_{0}, x_{L}$ locations:

Segment 3 polynomial coefficients:

\section{Unswept Domain 4}

$x$ length:

$z$ length:

Sweep angle:

Bend angle:

$y$ offset:

$z$ offset:

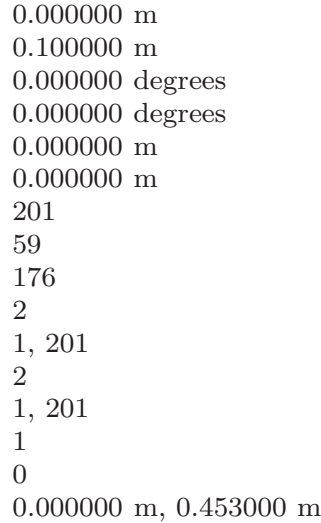

$a_{0}=1.7636266 \mathrm{E}-002$

$a_{1}=1.1186940 \mathrm{E}-001$

$0.000000 \mathrm{~m}$ $0.100000 \mathrm{~m}$ 0.000000 degrees 0.000000 degrees $0.000000 \mathrm{~m}$ $0.000000 \mathrm{~m}$ 
Number of $i$ nodes:

Number of $j$ nodes:

59

Number of $k$ nodes:

176

Number of $j$ node distributions:

$i$-indices of $j$ node distributions:

1,580

Number of $k$ node distributions:

$i$-indices of $k$ node distributions:

1,580

Number of ceiling segments:

Segment 1 polynomial order:

Segment $1 x_{0}, x_{L}$ locations:

0

Unswept Domain 5

$x$ length:

$z$ length:

Sweep angle:

Bend angle:

$y$ offset:

$z$ offset:

Number of $i$ nodes:

Number of $j$ nodes:

Number of $k$ nodes:

Number of $j$ node distributions:

$i$-indices of $j$ node distributions:

Number of $k$ node distributions:

$i$-indices of $k$ node distributions:

Number of ceiling segments:

Segment 1 polynomial order:

Segment $1 x_{0}, x_{L}$ locations:

Segment 1 polynomial coefficients:

$0.453000 \mathrm{~m}, 1.353000 \mathrm{~m}$

$a_{0}=3.9999999 \mathrm{E}-002$

$0.000000 \mathrm{~m}$

$0.100000 \mathrm{~m}$

0.000000 degrees

0.000000 degrees

$0.040000 \mathrm{~m}$

$0.000000 \mathrm{~m}$

28

19

176

2

1,28

1,28

1

1

$1.353000 \mathrm{~m}, 1.503000 \mathrm{~m}$

$a_{0}=2.8506741 \mathrm{E}-001$

$a_{1}=-8.5787997 \mathrm{E}-002$

\section{Unswept Domain 6}

$x$ length:

$z$ length:

Sweep angle:

Bend angle:

$y$ offset:

$z$ offset:

Number of $i$ nodes:

Number of $j$ nodes:

Number of $k$ nodes:

Number of $j$ node distributions:

$i$-indices of $j$ node distributions:

Number of $k$ node distributions:

$i$-indices of $k$ node distributions:

Number of ceiling segments:

Segment 1 polynomial order:

Segment $1 x_{0}, x_{L}$ locations:

$0.000000 \mathrm{~m}$

$0.100000 \mathrm{~m}$

0.000000 degrees

0.000000 degrees

$0.000000 \mathrm{~m}$

$0.000000 \mathrm{~m}$

28

59

176

2

1,28

2

1,28

1

0

$1.353000 \mathrm{~m}, 1.453050 \mathrm{~m}$

Segment 1 polynomial coefficients:

$a_{0}=3.9999999 \mathrm{E}-002$

\section{A.2.3 Paper 3}

The simulation described in Chapter 6 contains 5 sub-domains.

\section{Domain 1}

$x$ length:

$z$ length:

Sweep angle:
$0.380000 \mathrm{~m}$

$0.109820 \mathrm{~m}$

0.000000 degrees 
Bend angle:

$y$ offset:

$z$ offset:

Number of $i$ nodes:

Number of $j$ nodes:

Number of $k$ nodes:

Number of $j$ node distributions:

$i$-indices of $j$ node distributions:

Number of $k$ node distributions:

$i$-indices of $k$ node distributions:

Number of ceiling segments:

Segment 1 polynomial order:

Segment $1 x_{0}, x_{L}$ locations:

Segment 1 polynomial coefficients:

\section{Domain 2}

$x$ length:

$z$ length:

Sweep angle:

Bend angle:

$y$ offset:

$z$ offset:

Number of $i$ nodes:

Number of $j$ nodes:

Number of $k$ nodes:

Number of $j$ node distributions:

$i$-indices of $j$ node distributions:

Number of $k$ node distributions:

$i$-indices of $k$ node distributions:

Number of ceiling segments:

Segment 1 polynomial order:

Segment $1 x_{0}, x_{L}$ locations:

Segment 1 polynomial coefficients:

\section{Jet Orifice Information}

Number of sub-regions:

Location:

Sub-region $1 i_{\min }, i_{\max }, k_{\min }, k_{\max }$ :

Domain 3

$x$ length:

$z$ length:

Sweep angle:

Bend angle:

$y$ offset:

$z$ offset:

Number of $i$ nodes:

Number of $j$ nodes:

Number of $k$ nodes:

Number of $j$ node distributions:

$i$-indices of $j$ node distributions:

Number of $k$ node distributions:

$i$-indices of $k$ node distributions:

Number of ceiling segments:

Segment 1 polynomial order:

Segment $1 x_{0}, x_{L}$ locations:

Segment 1 polynomial coefficients:

\section{Domain 4}

$x$ length:

$z$ length:

Sweep angle:

Bend angle:

\author{
0.000000 degrees \\ $0.051840 \mathrm{~m}$ \\ $0.000000 \mathrm{~m}$ \\ 345 \\ 15 \\ 266 \\ 2 \\ 1,345 \\ 2 \\ 1,345 \\ 1 \\ $0.000000 \mathrm{~m}, 0.380000 \mathrm{~m}$
}

$a_{0}=2.5184000 \mathrm{E}-001$

$a_{1}=-2.00000 \mathrm{E}-001$

$0.380000 \mathrm{~m}$

$0.109820 \mathrm{~m}$

0.000000 degrees

0.000000 degrees

$0.021840 \mathrm{~m}$

$0.000000 \mathrm{~m}$

345

74

266

2

1, 345

1,345

0

$0.000000 \mathrm{~m}, 0.380000 \mathrm{~m}$

$a_{0}=2.9999999 \mathrm{E}-002$

$j$-plane

$116,175,102,161$

$0.170000 \mathrm{~m}$

$0.109820 \mathrm{~m}$

0.000000 degrees

0.000000 degrees

$0.051840 \mathrm{~m}$

$0.000000 \mathrm{~m}$

23

15

266

2

1,23

2

1,23

1

1

$0.380000 \mathrm{~m}, 0.550000 \mathrm{~m}$

$a_{0}=2.5184000 \mathrm{E}-001$

$a_{1}=4.3173656 \mathrm{E}-003$

0.170000

0.109820

0.000000 degrees

0.000000 degrees 
$y$ offset:

$z$ offset:

Number of $i$ nodes:

Number of $j$ nodes:

Number of $k$ nodes:

Number of $j$ node distributions:

$i$-indices of $j$ node distributions:

Number of $k$ node distributions:

$i$-indices of $k$ node distributions:

Number of ceiling segments:

Segment 1 polynomial order:

Segment $1 x_{0}, x_{L}$ locations:

Segment 1 polynomial coefficients:

\section{Jet Domain}

$x$ length:

$z$ length:

Sweep angle:

Bend angle:

$y$ offset:

$z$ offset:

Number of $i$ nodes:

Number of $j$ nodes:

Number of $k$ nodes:

Number of $j$ node distributions:

$i$-indices of $j$ node distributions:

Number of $k$ node distributions:

$i$-indices of $k$ node distributions:

Number of ceiling segments:

Segment 1 polynomial order:

Segment $1 x_{0}, x_{L}$ locations:

Segment 1 polynomial coefficients:

\author{
0.021840 \\ 0.000000 \\ 23 \\ 74 \\ 266 \\ 1,23 \\ 1,23 \\ 1 \\ $0.380000,0.550000$ \\ $a_{0}=2.9999999 \mathrm{E}-002$ \\ $0.002200 \mathrm{~m}$ \\ $0.002200 \mathrm{~m}$ \\ 0.000000 degrees \\ 0.000000 degrees \\ $0.000000 \mathrm{~m}$ \\ $0.052877 \mathrm{~m}$ \\ 60 \\ 88 \\ 60 \\ 1,60 \\ 1,60 \\ 1 \\ 0 \\ $0.177540 \mathrm{~m}, 0.179740 \mathrm{~m}$
}

$a_{0}=2.1840001 \mathrm{E}-002$

\section{A.2.4 Paper 4}

As described in Chapter 7, two separate simulations are performed in Paper 3 with different sub-domain sizes and spatial grids. Simulation-1 contains 7 sub-domains and Simulation-2 contains 2 sub-domains.

\section{Simulation-1, Domain 1}

$x$ length:

$z$ length:

Sweep angle:

Bend angle:

$y$ offset:

$z$ offset:

Number of $i$ nodes:

Number of $j$ nodes:

Number of $k$ nodes:

Number of $j$ node distributions:

$i$-indices of $j$ node distributions:

Number of $k$ node distributions:

$i$-indices of $k$ node distributions:

Number of ceiling segments:

Segment 1 polynomial order:

Segment $1 x_{0}, x_{L}$ locations:

Segment 1 polynomial coefficients:

\section{Simulation-1, Domain 2}

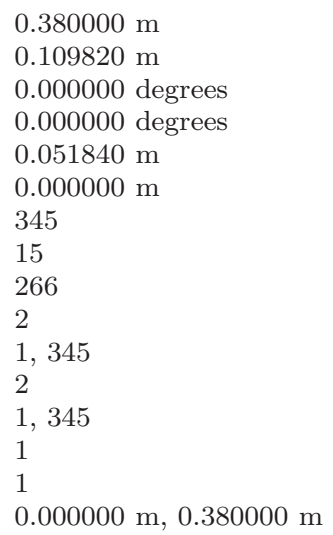

$a_{0}=2.5184000 \mathrm{E}-001$

$a_{1}=4.3173656 \mathrm{E}-003$ 
$x$ length:
$z$ length:
Sweep angle:
Bend angle:
$y$ offset:
$z$ offset:
Number of $i$ nodes:
Number of $j$ nodes:
Number of $k$ nodes:
Number of $j$ node distributions:
$i$-indices of $j$ node distributions:
Number of $k$ node distributions:
$i$-indices of $k$ node distributions:
Number of ceiling segments:
Segment 1 polynomial order:
Segment $1 x_{0}, x_{L}$ locations:
Segment 1 polynomial coefficients:

Jet Orifice Information

Number of sub-regions:

Location:

Sub-region $1 i_{\min }, i_{\max }, k_{\min }, k_{\max }$ :

Simulation-1, Domain 3

$x$ length:

$z$ length:

Sweep angle:

Bend angle:

$y$ offset:

$z$ offset:

Number of $i$ nodes:

Number of $j$ nodes:

Number of $k$ nodes:

Number of $j$ node distributions:

$i$-indices of $j$ node distributions:

Number of $k$ node distributions:

$i$-indices of $k$ node distributions:

Number of ceiling segments:

Segment 1 polynomial order:

Segment $1 x_{0}, x_{L}$ locations:

Segment 1 polynomial coefficients:

\section{Simulation-1, Domain 4}

$x$ length:

$z$ length:

Sweep angle:

Bend angle:

$y$ offset:

$z$ offset:

Number of $i$ nodes:

Number of $j$ nodes:

Number of $k$ nodes:

Number of $j$ node distributions:

$i$-indices of $j$ node distributions:

Number of $k$ node distributions:

$i$-indices of $k$ node distributions:

Number of ceiling segments:

Segment 1 polynomial order:

Segment $1 x_{0}, x_{L}$ locations:

Segment 1 polynomial coefficients:

\section{Simulation-1, Jet Domain}

$x$ length:

$z$ length:

\author{
$0.380000 \mathrm{~m}$ \\ $0.109820 \mathrm{~m}$ \\ 0.000000 degrees \\ 0.000000 degrees \\ $0.021840 \mathrm{~m}$ \\ $0.000000 \mathrm{~m}$ \\ 345 \\ 74 \\ 266 \\ 1, 345 \\ 2 \\ 1, 345 \\ 1 \\ $0.000000 \mathrm{~m}, 0.380000 \mathrm{~m}$
}

$a_{0}=2.9999999 \mathrm{E}-002$

1

$j$-plane

$116,175,102,161$

$0.170000 \mathrm{~m}$

$0.109820 \mathrm{~m}$

0.000000 degrees

0.000000 degrees

$0.051840 \mathrm{~m}$

$0.000000 \mathrm{~m}$

23

15

266

1,23

1,23

1

$0.380000 \mathrm{~m}, 0.550000 \mathrm{~m}$

$a_{0}=2.5184000 \mathrm{E}-001$

$a_{1}=4.3173656 \mathrm{E}-003$

0.170000

0.109820

0.000000 degrees

0.000000 degrees

0.021840

0.000000

23

74

266

2

1,23

2

1,23

1

$0.380000,0.550000$

$a_{0}=2.9999999 \mathrm{E}-002$

$0.002200 \mathrm{~m}$

$0.002200 \mathrm{~m}$ 
Sweep angle:

Bend angle:

$y$ offset:

$z$ offset:

Number of $i$ nodes:

Number of $j$ nodes:

Number of $k$ nodes:

Number of $j$ node distributions:

$i$-indices of $j$ node distributions:

Number of $k$ node distributions:

$i$-indices of $k$ node distributions:

Number of ceiling segments:

Segment 1 polynomial order:

Segment $1 x_{0}, x_{L}$ locations:

Segment 1 polynomial coefficients:

Simulation-1, Upstream Domain 1

$x$ length:

$z$ length:

Sweep angle:

Bend angle:

$y$ offset:

$z$ offset:

Number of $i$ nodes:

Number of $j$ nodes:

Number of $k$ nodes:

Number of $j$ node distributions:

$i$-indices of $j$ node distributions:

Number of $k$ node distributions:

$i$-indices of $k$ node distributions:

Number of ceiling segments:

Segment 1 polynomial order:

Segment $1 x_{0}, x_{L}$ locations:

Segment 1 polynomial coefficients:

Simulation-1, Upstream Domain 2

$x$ length:

$z$ length:

Sweep angle:

Bend angle:

$y$ offset:

$z$ offset:

Number of $i$ nodes:

Number of $j$ nodes:

Number of $k$ nodes:

Number of $j$ node distributions:

$i$-indices of $j$ node distributions:

Number of $k$ node distributions:

$i$-indices of $k$ node distributions:

Number of ceiling segments:

Segment 1 polynomial order:

Segment $1 x_{0}, x_{L}$ locations:

Segment 1 polynomial coefficients:

\section{Simulation-2, Domain 1}

$x$ length:

$z$ length:

Sweep angle:

Bend angle:

$y$ offset:

$z$ offset:

Number of $i$ nodes:

Number of $j$ nodes:

Number of $k$ nodes:

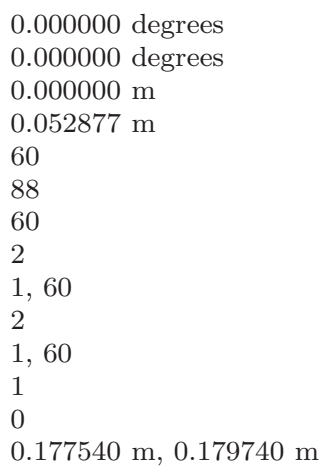

$a_{0}=2.1840001 \mathrm{E}-002$

$0.180000 \mathrm{~m}$

$0.109820 \mathrm{~m}$

0.000000 degrees

0.000000 degrees

$0.051840 \mathrm{~m}$

$0.000000 \mathrm{~m}$

24

15

266

2

1, 24

1,24

1

0

$0.000000 \mathrm{~m}, 0.180000 \mathrm{~m}$

$a_{0}=2.5184000 \mathrm{E}-001$

$0.180000 \mathrm{~m}$

$0.109820 \mathrm{~m}$

0.000000 degrees

0.000000 degrees

$0.021840 \mathrm{~m}$

$0.000000 \mathrm{~m}$

24

74

266

2

1,24

2

1,24

1

0

$0.000000 \mathrm{~m}, 0.180000 \mathrm{~m}$

$a_{0}=2.9999999 \mathrm{E}-002$

$0.700000 \mathrm{~m}$

$0.160960 \mathrm{~m}$

0.000000 degrees

0.000000 degrees

$0.021840 \mathrm{~m}$

$0.000000 \mathrm{~m}$

456

82

266 
Number of $j$ node distributions: $i$-indices of $j$ node distributions:

Number of $k$ node distributions: $i$-indices of $k$ node distributions:

Number of ceiling segments:

Segment 1 polynomial order:

Segment $1 x_{0}, x_{L}$ locations:

Segment 1 polynomial coefficients:

\section{Simulation-2, Domain 2}

Domain name:

Patran flag:

Tecplot flag:

Inlet grid flag:

Lobed geometry flag:

Bend in circular arc flag:

$x$ length:

$z$ length:

Sweep angle:

Bend angle:

$y$ offset:

$z$ offset:

Number of $i$ nodes:

Number of $j$ nodes:

Number of $k$ nodes:

Number of $j$ node distributions:

$i$-indices of $j$ node distributions:

Number of $k$ node distributions:

$i$-indices of $k$ node distributions:

Number of ceiling segments:

Segment 1 polynomial order:

Segment $1 x_{0}, x_{L}$ locations:

Segment 1 polynomial coefficients:
2

1,456

2

1,456

1

$0.340401 \mathrm{~m}, 1.040401 \mathrm{~m}$

$a_{0}=1.5721603 \mathrm{E}-001$

$a_{1}=4.3916348 \mathrm{E}-003$

$a_{2}=-3.4603397 \mathrm{E}-003$

$a_{3}=2.1097052 \mathrm{E}-003$

$a_{4}=-5.5677391 \mathrm{E}-004$

domain3

1

0

0.120000

0.160960

0.000000 degrees

0.000000 degrees

0.021840

0.000000

17

82

266

2

1,17

2

1,17

1

$1.040401,1.160401$

$a_{0}=1.5976335 \mathrm{E}-001$

\section{A.2.5 Paper 5}

The simulation described in Chapter 8 contains 10 sub-domains.

\author{
Domain 1 \\ $x$ length: \\ $z$ length: \\ Sweep angle: \\ Bend angle: \\ $y$ offset: \\ $z$ offset: \\ Number of $i$ nodes: \\ Number of $j$ nodes: \\ Number of $k$ nodes: \\ Number of $j$ node distributions: \\ $i$-indices of $j$ node distributions: \\ Number of $k$ node distributions: \\ $i$-indices of $k$ node distributions: \\ Number of ceiling segments: \\ Segment 1 polynomial order: \\ Segment $1 x_{0}, x_{L}$ locations:
}

$0.000000 \mathrm{~m}$
$0.100000 \mathrm{~m}$
0.000000 degrees
0.000000 degrees
$0.040000 \mathrm{~m}$
$0.000000 \mathrm{~m}$
480
34
237
2
1,480
2
1,480
1
0
$0.000000 \mathrm{~m}, 0.453000 \mathrm{~m}$


Segment 1 polynomial coefficients:

Domain 1 Turbulence Grid Information Number of sub-regions:

Location:

Sub-region 1

Sub-region 2

Sub-region 3

Sub-region 4

Sub-region 5

Sub-region 6

Sub-region 7

Sub-region 8

Sub-region 9

Sub-region 10

Sub-region 11

Sub-region 12

Sub-region 13

Sub-region 14

Sub-region 15

Sub-region 16

Sub-region 17

Sub-region 18

Sub-region 19

Sub-region 20

Sub-region 21

Sub-region 22

Sub-region 23

Sub-region 24

Sub-region 25

Sub-region 26

Sub-region 27

Sub-region 28

Sub-region 29

Sub-region 30

Sub-region 31

Sub-region 32

Sub-region 33

Sub-region 34

Sub-region 35

Sub-region 36

Sub-region 37

Sub-region 38

Sub-region 39

Sub-region 40

Sub-region 41

Sub-region 42

Sub-region 43

Sub-region 44

Sub-region 45

Sub-region 46

Sub-region 47

Sub-region 48

Domain 2

$x$ length:

$z$ length:

Sweep angle:

Bend angle:

$y$ offset:

$z$ offset:

Number of $i$ nodes:

Number of $j$ nodes: $a_{0}=1.4450000 \mathrm{E}-001$

48

$i$-plane

$j_{\min }, j_{\max }, k_{\min }, k_{\max }$

$1,8,1,23$

$10,13,1,23$

$15,18,1,23$

$20,23,1,23$

$25,28,1,23$

$30,33,1,23$

$1,8,32,54$

$10,13,32,54$

$15,18,32,54$

$20,23,32,54$

$25,28,32,54$

$30,33,32,54$

$1,8,63,85$

$10,13,63,85$

$15,18,63,85$

$20,23,63,85$

$25,28,63,85$

$30,33,63,85$

$1,8,94,116$

$10,13,94,116$

$15,18,94,116$

$20,23,94,116$

$25,28,94,116$

$30,33,94,116$

$1,8,125,147$

$10,13,125,147$

$15,18,125,147$

$20,23,125,147$

$25,28,125,147$

$30,33,125,147$

$1,8,156,178$

$10,13,156,178$

$15,18,156,178$

$20,23,156,178$

$25,28,156,178$

$30,33,156,178$

1, 8, 187, 209

10, 13, 187, 209

15, 18, 187, 209

$20,23,187,209$

25, 28, 187, 209

30, 33, 187, 209

$1,8,218,237$

$10,13,218,237$

15, 18, 218, 237

$20,23,218,237$

$25,28,218,237$

$30,33,218,237$

$0.000000 \mathrm{~m}$

$0.100000 \mathrm{~m}$

0.000000 degrees

0.000000 degrees

$0.000000 \mathrm{~m}$

$0.000000 \mathrm{~m}$

480

59 
Number of $k$ nodes:

Number of $j$ node distributions:

$i$-indices of $j$ node distributions:

Number of $k$ node distributions:

$i$-indices of $k$ node distributions:

Number of ceiling segments:

Segment 1 polynomial order:

Segment $1 x_{0}, x_{L}$ locations:

Segment 1 polynomial coefficients:

Domain 2 Turbulence Grid Information

Number of sub-regions:

Location:

Sub-region 1

Sub-region 2

Sub-region 3

Sub-region 4

Sub-region 5

Sub-region 6

Sub-region 7

Sub-region 8

Sub-region 9

Sub-region 10

Sub-region 11

Sub-region 12

Sub-region 13

Sub-region 14

Sub-region 15

Sub-region 16

Sub-region 17

Sub-region 18

Sub-region 19

Sub-region 20

Sub-region 21

Sub-region 22

Sub-region 23

Sub-region 24

Domain 3

$x$ length:

$z$ length:

Sweep angle:

Bend angle:

$y$ offset:

$z$ offset:

Number of $i$ nodes:

Number of $j$ nodes:

Number of $k$ nodes:

Number of $j$ node distributions:

$i$-indices of $j$ node distributions:

Number of $k$ node distributions:

$i$-indices of $k$ node distributions:

Number of ceiling segments:

Segment 1 polynomial order:

Segment $1 x_{0}, x_{L}$ locations:

Segment 1 polynomial coefficients:
237

1,480

1,480

1

$0.000000 \mathrm{~m}, 0.453000 \mathrm{~m}$

$a_{0}=3.9999999 \mathrm{E}-002$

24

$i$-plane

$j_{\min }, j_{\max }, k_{\min }, k_{\max }$

$3,15,1,23$

$22,34,1,23$

$41,53,1,23$

$3,15,32,54$

$22,34,32,54$

$41,53,32,54$

$3,15,63,85$

$22,34,63,85$

$41,53,63,85$

$3,15,94,116$

$22,34,94,116$

$41,53,94,116$

$3,15,125,147$

$22,34,125,147$

$41,53,125,147$

$3,15,156,178$

$22,34,156,178$

$41,53,156,178$

3, 15, 187, 209

22, 34, 187, 209

41, 53, 187, 209

$3,15,218,237$

$22,34,218,237$

$41,53,218,237$

$0.000000 \mathrm{~m}$
$0.100000 \mathrm{~m}$
0.000000 degrees
0.000000 degrees
$0.040000 \mathrm{~m}$
$0.000000 \mathrm{~m}$
492
34
237
2
1,492
2
1,492
1
5
$0.453000 \mathrm{~m}, 0.820680 \mathrm{~m}$
$a_{0}=2.6357293 \mathrm{E}+000$
$a_{1}=-2.0658575 \mathrm{E}+001$
$a_{2}=6.6073723 \mathrm{E}+001$
$a_{3}=-1.011778 \mathrm{E}+002$
$a_{4}=7.3559174 \mathrm{E}+001$
$a_{5}=-2.0421898 \mathrm{E}+001$

Domain 4 
$x$ length:

$z$ length:

Sweep angle:

Bend angle:

$y$ offset:

$z$ offset:

Number of $i$ nodes:

Number of $j$ nodes:

Number of $k$ nodes:

Number of $j$ node distributions:

$i$-indices of $j$ node distributions:

Number of $k$ node distributions:

$i$-indices of $k$ node distributions:

Number of ceiling segments:

Segment 1 polynomial order:

Segment $1 x_{0}, x_{L}$ locations:

Segment 1 polynomial coefficients:

\section{Domain 5}

$x$ length:

$z$ length:

Sweep angle:

Bend angle:

$y$ offset:

$z$ offset:

Number of $i$ nodes:

Number of $j$ nodes:

Number of $k$ nodes:

Number of $j$ node distributions:

$i$-indices of $j$ node distributions:

Number of $k$ node distributions:

$i$-indices of $k$ node distributions:

Number of ceiling segments:

Segment 1 polynomial order:

Segment $1 x_{0}, x_{L}$ locations:

Segment 1 polynomial coefficients:

Segment 2 polynomial order:

Segment $2 x_{0}, x_{L}$ locations:

Segment 2 polynomial coefficients:

\section{Domain 6}

$x$ length:

$z$ length:

Sweep angle:

Bend angle:

$y$ offset:

$z$ offset:

Number of $i$ nodes:

Number of $j$ nodes:

Number of $k$ nodes:

Number of $j$ node distributions:

$i$-indices of $j$ node distributions:

Number of $k$ node distributions:

$i$-indices of $k$ node distributions:

Number of ceiling segments:

Segment 1 polynomial order:

Segment $1 x_{0}, x_{L}$ locations:

\author{
$0.000000 \mathrm{~m}$ \\ $0.100000 \mathrm{~m}$ \\ 0.000000 degrees \\ 0.000000 degrees \\ $0.000000 \mathrm{~m}$ \\ $0.000000 \mathrm{~m}$ \\ 492 \\ 59 \\ 237 \\ 2 \\ 1, 492 \\ 2 \\ 1,492 \\ 1 \\ 0 \\ $0.453000 \mathrm{~m}, 0.820690 \mathrm{~m}$
}

$a_{0}=3.9999999 \mathrm{E}-002$

$0.000000 \mathrm{~m}$

$0.100000 \mathrm{~m}$

0.000000 degrees

0.000000 degrees

$0.040000 \mathrm{~m}$

$0.000000 \mathrm{~m}$

492

34

237

2

1, 492

2

1,492

2

5

$0.820680 \mathrm{~m}, 0.867500 \mathrm{~m}$

$a_{0}=2.6357293 \mathrm{E}+000$

$a_{1}=-2.0658575 \mathrm{E}+001$

$a_{2}=6.6073723 \mathrm{E}+001$

$a_{3}=-1.0111778 \mathrm{E}+002$

$a_{4}=7.3559174 \mathrm{E}+001$

$a_{5}=-2.0421898 \mathrm{E}+001$

1

$0.867500 \mathrm{~m}, 1.050150 \mathrm{~m}$

$a_{0}=-4.7791999 \mathrm{E}-002$

$a_{1}=1.1371000 \mathrm{E}-001$

$0.000000 \mathrm{~m}$

$0.100000 \mathrm{~m}$

0.000000 degrees

0.000000 degrees

$0.000000 \mathrm{~m}$

$0.000000 \mathrm{~m}$

492

59

237

2

1, 492

2

1, 492

1

0

$0.820680 \mathrm{~m}, 1.050150 \mathrm{~m}$ 
Segment 1 polynomial coefficients:

\section{Domain 7 \\ $x$ length: \\ $z$ length: \\ Sweep angle: \\ Bend angle: \\ $y$ offset: \\ $z$ offset:}

Number of $i$ nodes:

Number of $j$ nodes:

Number of $k$ nodes:

Number of $j$ node distributions:

$i$-indices of $j$ node distributions:

Number of $k$ node distributions:

$i$-indices of $k$ node distributions:

Number of ceiling segments:

Segment 1 polynomial order:

Segment $1 x_{0}, x_{L}$ locations:

Segment 1 polynomial coefficients:

\section{Domain 8}

$x$ length:

$z$ length:

Sweep angle:

Bend angle:

$y$ offset:

$z$ offset:

Number of $i$ nodes:

Number of $j$ nodes:

Number of $k$ nodes:

Number of $j$ node distributions:

$i$-indices of $j$ node distributions:

Number of $k$ node distributions:

$i$-indices of $k$ node distributions:

Number of ceiling segments:

Segment 1 polynomial order:

Segment $1 x_{0}, x_{L}$ locations:

Segment 1 polynomial coefficients:

\section{Domain 9}

$x$ length:

$z$ length:

Sweep angle:

Bend angle:

$y$ offset:

$z$ offset:

Number of $i$ nodes:

Number of $j$ nodes:

Number of $k$ nodes:

Number of $j$ node distributions:

$i$-indices of $j$ node distributions:

Number of $k$ node distributions:

$i$-indices of $k$ node distributions:

Number of ceiling segments:

Segment 1 polynomial order:

Segment $1 x_{0}, x_{L}$ locations:

Segment 1 polynomial coefficients:

\section{Domain 10}

$x$ length:

$z$ length:

Sweep angle:

\author{
$a_{0}=3.9999999 \mathrm{E}-002$ \\ $0.000000 \mathrm{~m}$ \\ $0.100000 \mathrm{~m}$ \\ 0.000000 degrees \\ 0.000000 degrees \\ $0.040000 \mathrm{~m}$ \\ $0.000000 \mathrm{~m}$ \\ 493 \\ 34 \\ 237 \\ 2 \\ 1,493 \\ 2 \\ 1,493 \\ 1 \\ 1 \\ $1.050150 \mathrm{~m}, 1.253000 \mathrm{~m}$
}

$a_{0}=-4.7791999 \mathrm{E}-002$

$a_{1}=1.1371000 \mathrm{E}-001$

$0.000000 \mathrm{~m}$

$0.100000 \mathrm{~m}$

0.000000 degrees

0.000000 degrees

$0.000000 \mathrm{~m}$

$0.000000 \mathrm{~m}$

493

59

237

1, 493

1, 493

1

0

$1.050150 \mathrm{~m}, 1.253000 \mathrm{~m}$

$a_{0}=3.9999999 \mathrm{E}-002$

$0.000000 \mathrm{~m}$

$0.100000 \mathrm{~m}$

0.000000 degrees

0.000000 degrees

$0.040000 \mathrm{~m}$

$0.000000 \mathrm{~m}$

25

34

237

2

1, 25

2

1,25

0

$1.253000 \mathrm{~m}, 1.375000 \mathrm{~m}$

$a_{0}=9.4687000 \mathrm{E}-002$

$0.000000 \mathrm{~m}$

$0.100000 \mathrm{~m}$

0.000000 degrees 
Bend angle:

$y$ offset:

$z$ offset:

Number of $i$ nodes:

Number of $j$ nodes:

Number of $k$ nodes:

Number of $j$ node distributions:

$i$-indices of $j$ node distributions:

Number of $k$ node distributions:

$i$-indices of $k$ node distributions:

Number of ceiling segments:

Segment 1 polynomial order:

Segment $1 x_{0}, x_{L}$ locations:

Segment 1 polynomial coefficients:
0.000000 degrees

$0.000000 \mathrm{~m}$

$0.000000 \mathrm{~m}$

25

59

237

2

1,25

2

1,25

1

$1.253000 \mathrm{~m}, 1.375000 \mathrm{~m}$

$a_{0}=3.9999999 \mathrm{E}-002$ 


\section{Appendix B}

\section{Description of the P3 Enstrophy Production Term}

This appendix provides additional detail on the physical mechanisms of the $P 3$ production term in the enstrophy transport equation (Equations 7.6 and 8.5):

$$
-\overline{u_{j}^{\prime} \omega_{i}^{\prime}} \frac{\partial \Omega_{i}}{\partial x_{j}}
$$

Consider a boundary layer with the velocity profile sketched in Figure B.1(a). The wallnormal vorticity distribution, $\Omega(y)$, shown in Figure B.1 is plotted to the left of the velocity profile. The length of the arrows in the $\Omega(y)$ distribution indicate the magnitude of the timemean spanwise vorticity within the boundary layer and they point in the $-x$ direction to indicate that the spanwise vorticity has a negative sign (clockwise rotation). The signed value of $\Omega_{z}$ is becoming larger (i.e. less negative) with increasing $y$, and hence $\partial \Omega_{z} / \partial y>0$ in the boundary layer. In a steady, fully-developed boundary layer, the other components of the mean vorticity gradient are much smaller than $\partial \Omega_{z} / \partial y$ and so are presently neglected.

At time $t_{1}$, a vorticity line is located at a height of $y_{1}$ with instantaneous vorticity $\omega_{z 1}$. 
Between time $t_{1}$ and $t_{2}=t_{1}+\Delta t$, the vorticity line is perturbed in the $-y$ direction by a wall-normal velocity fluctuation $v^{\prime}<0$ into a new position $y_{2}$. The vorticity line keeps its original vorticity for the instant after it is perturbed so that its instantaneous vorticity at $t_{2}$ is the same as $t_{1}$ :

$$
\omega_{z 1}=\omega_{z 2}
$$

Decomposing the instantaneous vorticity into the mean and fluctuating components yields

$$
\Omega_{z 1}+\omega_{z 1}^{\prime}=\Omega_{z 2}+\omega_{z 2}^{\prime}
$$

Assuming that the fluctuating vorticity at time $t_{1}$ is zero, which implies that the vorticity line at time $t_{1}$ is undisturbed, the fluctuating vorticity at time $t_{2}$ is

$$
\omega_{z 2}^{\prime}=\Omega_{z 1}-\Omega_{z 2}>0
$$

The right-hand-side of Equation B.3 is positive due to the mean vorticity gradient $\partial \Omega_{z} / \partial y>0$. This indicates that in a boundary layer with $\partial \Omega / \partial y>0$, a negative wall-normal velocity fluctuation, $v^{\prime}<0$, produces a positive fluctuating vorticity component, $\omega_{z}^{\prime}>0$, and hence $\overline{u_{j}^{\prime} \omega_{i}^{\prime}}<0$. The negative sign in the $P 3$ term is therefore required to make the overall production

$$
\text { (a) } t_{1} \quad \text { (b) } t_{2}=t_{1}+\Delta t
$$

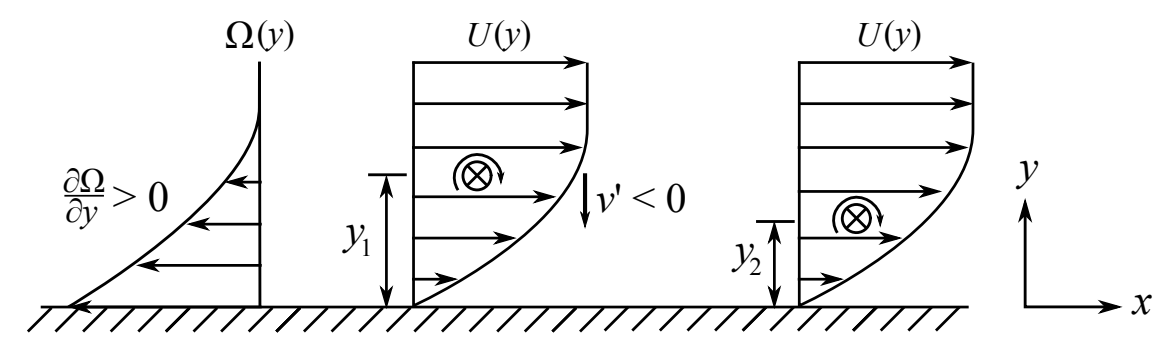

Figure B.1: Schematic illustrating the production of enstrophy via the P3 term in a boundary layer 
rate positive:

$$
-\underbrace{\overline{u_{j}^{\prime} \omega_{i}^{\prime}}}_{<0} \underbrace{\frac{\partial \Omega_{i}}{\partial x_{j}}}_{>0}
$$

From the above discussion, it is clear that the $P 3$ term generates enstrophy by displacing vorticity from the mean vorticity field into a new position within a mean vorticity gradient, thereby contributing to the fluctuating component of vorticity. Tennekes and Lumley (1972) refer to this as an "exchange" of vorticity from the mean component to the fluctuating component, and they note that the process is analogous to the production-rate term of the transport equation for turbulence kinetic energy, $-\overline{u_{i}^{\prime} u_{j}^{\prime}} S_{i j}$, in which the Reynolds stress tensor $\left(\overline{u_{i}^{\prime} u_{j}^{\prime}}\right)$ is an analogue to the $\overline{u_{j}^{\prime} \omega_{i}^{\prime}}$ tensor in the $P 3$ term, and the mean strain rate tensor $\left(S_{i j}\right)$ is an analogue to the $\partial \Omega_{i} / \partial x_{j}$ tensor in the $P 3$ term. 


\section{Appendix C}

\section{ComPost Operator's Manual}

ComPost (Computational Post-processor) is a software that was written to process the results of the computational simulations presented in this thesis. This manual provides the details of the structure, input files, operating parameters, and capabilities of the program. This manual is included in this thesis to allow the figures presented in the thesis manuscript to be reproduced and to facilitate the further use of ComPost as a tool for future members of the author's research group.

\section{C.1 Program structure}

ComPost operates according to the flowchart presented in three parts in Figures C.1-C.3. The details of ComPost are as follows:

- Written in ANSI C using the Dev C++ IDE development with the Mingw port of the GCC compiler.

- Compiled in Windows 7 and Linux environments.

- Not parallelized for multi-processor environments, but supports multiple simultaneous 
runs on sequential portions of the CFD dataset.

- Uniform treatment of $2 \mathrm{D}$ and $3 \mathrm{D}$ datasets.

- Writes files to ASCII or binary Tecplotß formats.

- Capabilities:

- Computing temporal and spanwise averages and perturbation quantities

- Computing integral quantities and velocity profiles for instantaneous or averaged data

- Extracting time traces

- Calculating statistical turbulence quantities, such as turbulence kinetic energy budgets, enstrophy transport terms, RMS-disturbance profiles, and Reynolds stresses

- Calculating quantities suitable for visualizing vortical flows, including $Q$-parameter, vorticity vector.

ComPost does not have a graphical user interface. Instead, a user supplies a text input file with directives that control how ComPost will run. ComPost is sensitive to the formatting of the input file; the format presented in the sample input file must be maintained.

\section{C.2 File structure}

The executable simulation file ComPost_main.c is compiled with the following header files:

ComPost_main.c: Contains the bulk of the ComPost code, structured as in Figures C.1-C.3

computeEns.h: Calculates terms of the enstrophy transport equation from fluctuating quantities determined from subtracting the time-averaged flow field from the instantaneous flow 


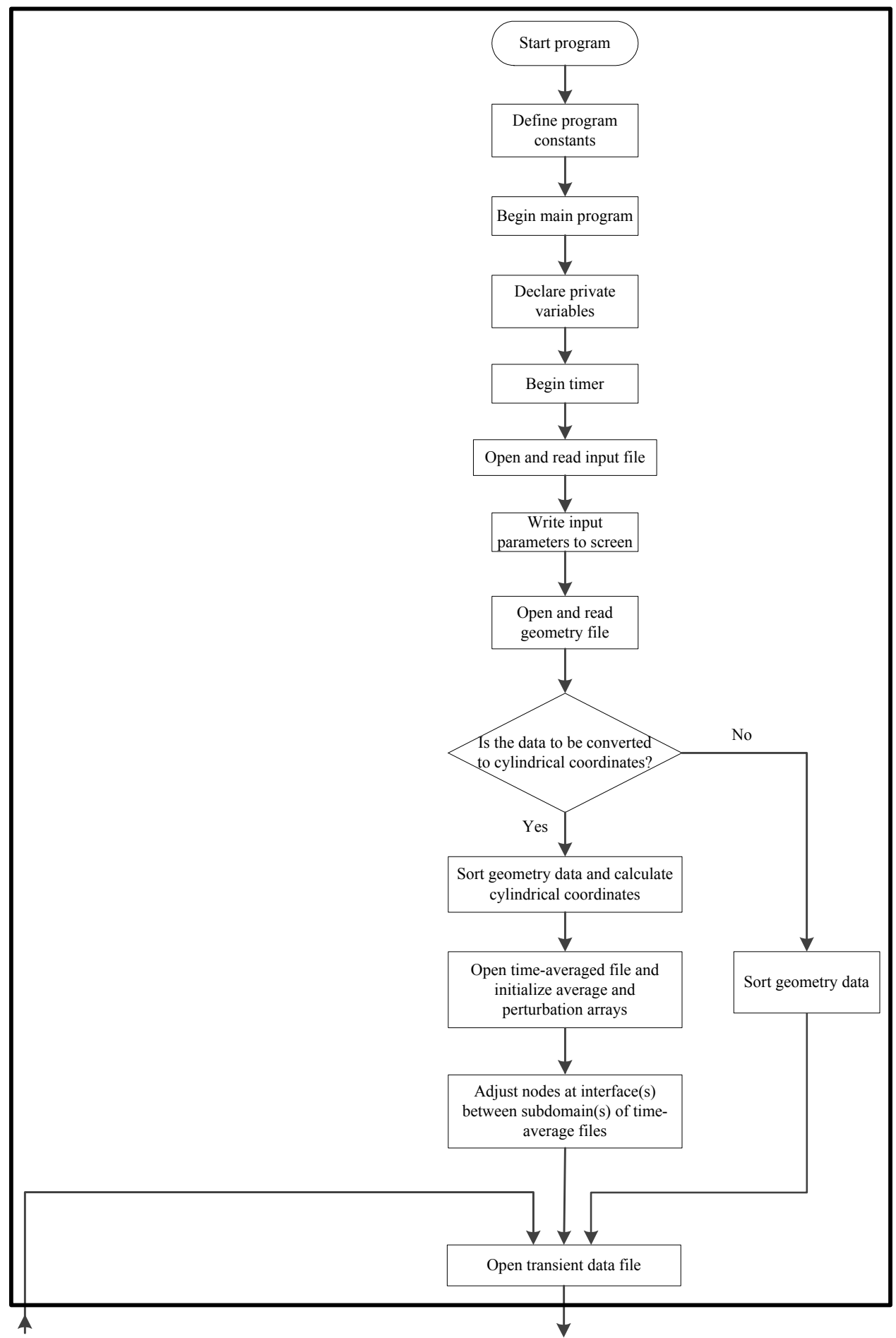

Figure C.1: Flowchart of ComPost (Part 1 of 3) 


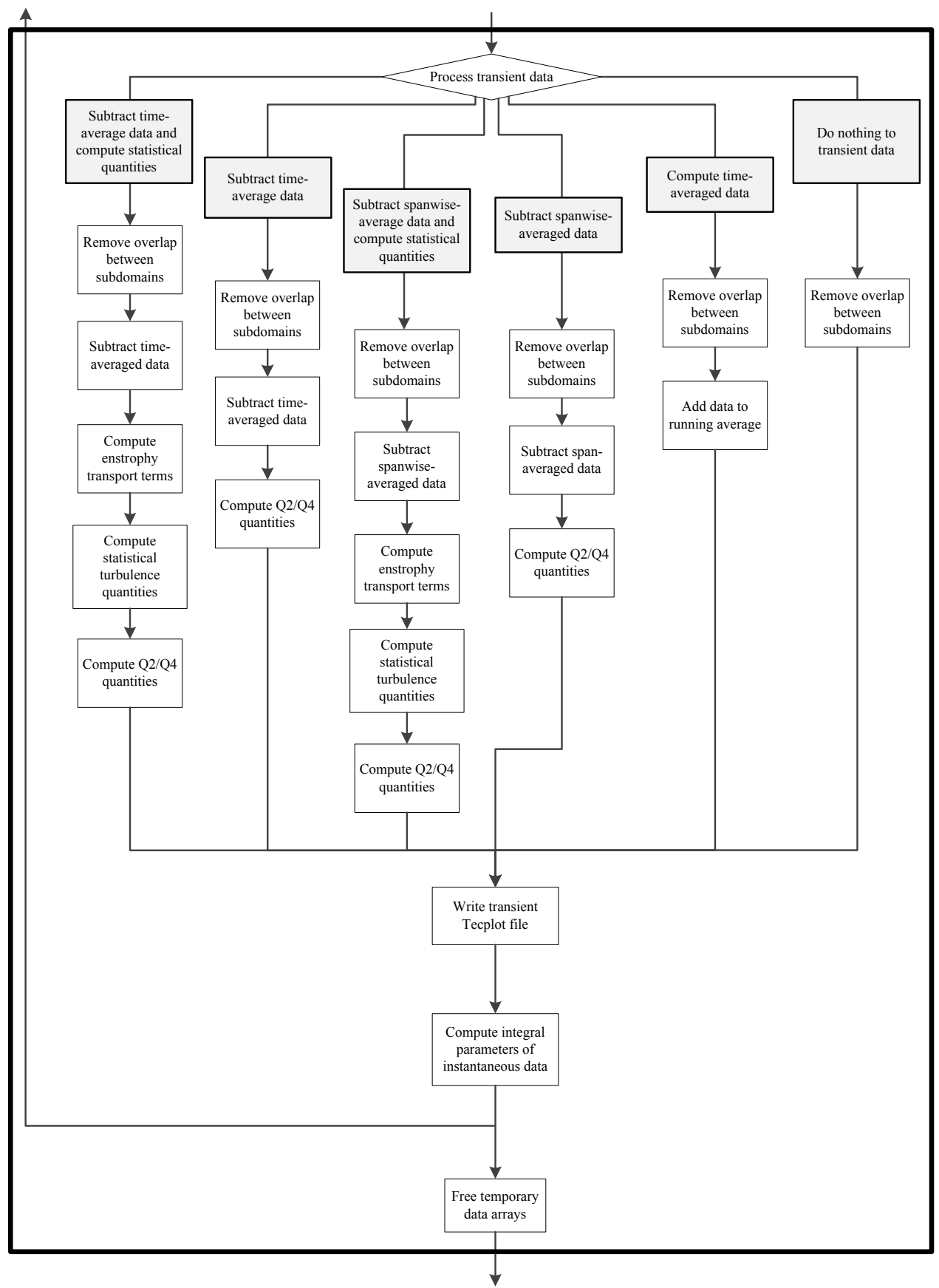

Figure C.2: Flowchart of ComPost (Part 2 of 3) 


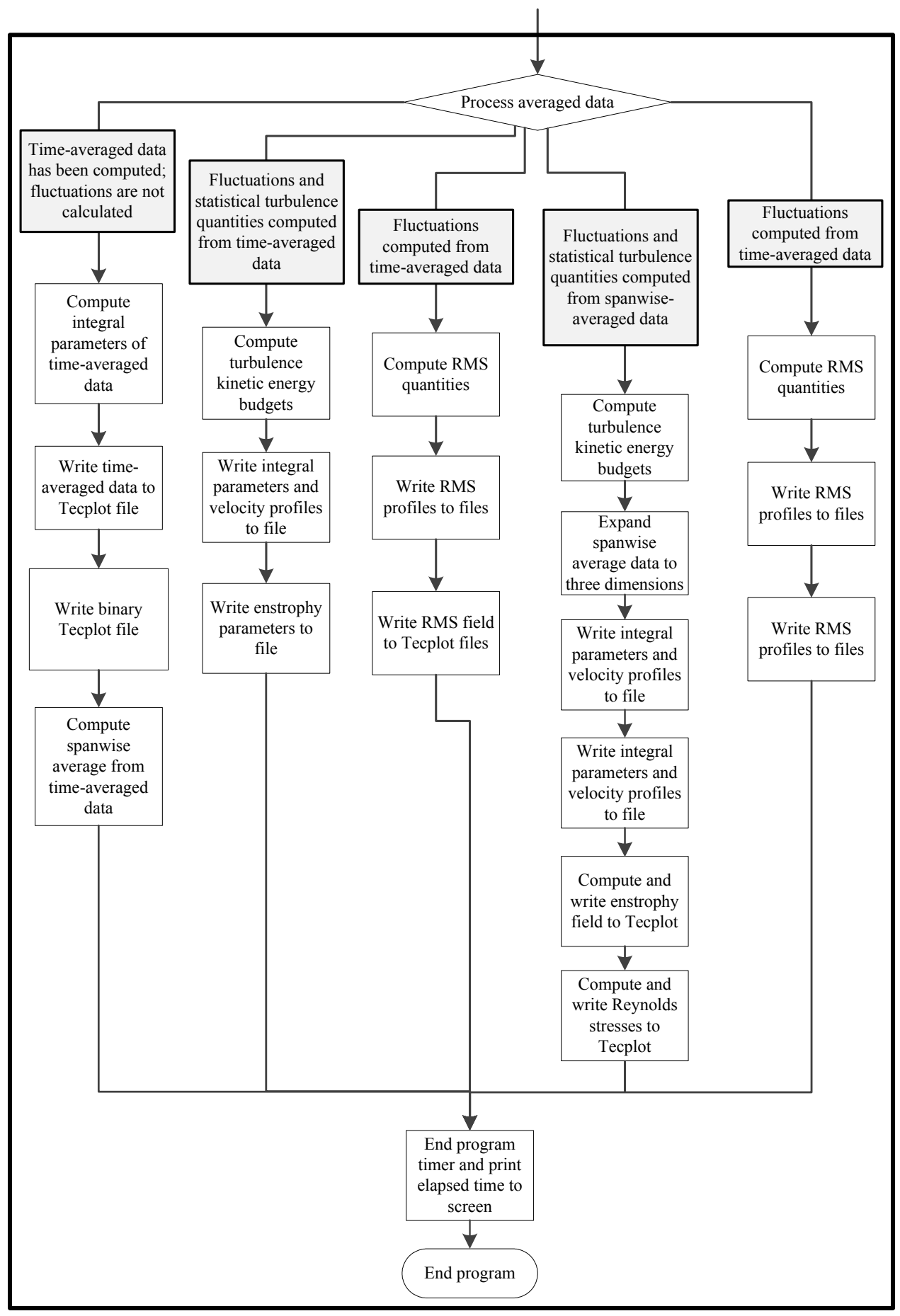

Figure C.3: Flowchart of ComPost (Part 3 of 3) 
computeEnsSpan.h: Calculates terms of the enstrophy transport equation from fluctuating quantities determined by subtracting the spanwise-averaged flow field from the instantaneous flow

computeEnstrophy.h: Defines the function protocols calling the computeEns.h and computeEnsSpan.h routines

computeIntQnt.h: Calculates the boundary-layer integral parameters from the instantaneous and/or time-averaged flow field

computeQ1234.h: Calculates the quadrants corresponding to the Reynolds stress tensor (Figure 2.10)

computeSpanAvg.h: Computes the spanwise-averaged flow field from a three-dimensional flow field

computeTKE.h: Calculates budgets of the turbulence kinetic energy equation from fluctuating velocity field

declareVars.h: Defines the variables used by ComPost

differentiate.h: Computes the first and second derivatives from input arrays of data

expandSpanAvgData.h: Expands the spanwise-averaged data to three dimensions

extractTimeTrace.h: Extracts time-traces of transient data

initAvgVars.h: Initialize average variable arrays

initEns Vars.h: Initialize enstrophy variable arrays

initPerturbVars.h: Initialize perturbation variable arrays 
initQVars.h: Initialize variable arrays for calculating the second-invariant of the velocitygradient tensor

initReStressVars.h: Initialize arrays for calculating the Reynolds stress tensor

initSimVars.h: Initialize simulation variable arrays

initSortGeo.h: Initialize geometry sorting variable arrays

initTKEVars.h: Initialize variable arrays for calculating budgets of turbulence kinetic energy

initVortVars.h: Initialize variable arrays for calculating vorticity

initVars.h: Calling function for previous initialize variable arrays header files

openFiles.h: Contains commands to open transient data files, geometry files, and averaged data files

readInputFile.h: Commands to read the ComPost input file

reorder.h: Reorders the transient data according to the $x, y, z$ ordering of the sorted geometry file

reorderTavg.h: Reorders the time-averaged data according to the $x, y, z$ ordering of the sorted geometry file

shiftNodes.h: Shifts nodes in order to remove the overlap between mating subdomains

sort3Ddata.h: Sorts 3D data such that in the ordered data, the $x$ array changes fastest, followed by $y$, and $z$ changes the slowest

trapz.h: Performs integration using trapezoidal law

vorticity3D.h: Calculates the $3 \mathrm{D}$ vorticity vector 
write_timetrace.h: Write time-trace data to ASCII files

writeDat.h: Writes data to ASCII-formatted files

writeInputParams.h: Writes input-file parameters to the screen

writePlt.h: Converts ASCII-formatted Tecplot@ files to binary format

writeReStress.h: Writes the Reynolds-stress tensor parameters to files

writeRMS.h: Writes root-mean-square fluctuation quantities to files

writeTrans.h: Writes transient data to files

\section{C.3 Input files}

The input files that are required for running ComPost are described below:

input_xyz.txt: Input file for the get_xyz geometry input program

input_compost.txt: Input file for ComPost

The input_xyz.txt file is used in a program named get_xyz. This program extracts the geometry information from the computational results. It is run prior to running ComPost. The contents of these input files are described in Sections C.3.1 and C.3.2.

\section{C.3.1 Input file for the get_xyz program}

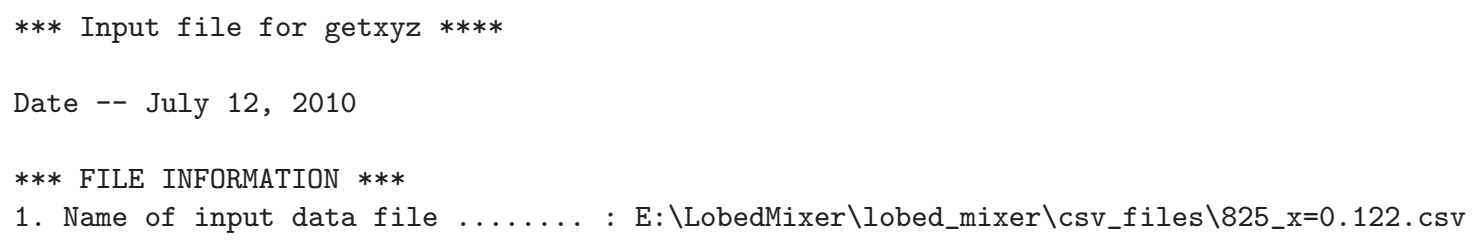


2. Name of output geometry file ...: E: \LobedMixer \lobed_mixer \csv_files $\backslash x y z \_x=0.122 . c S v$

3. Number of lines to skip .....: 6

4. Number of lines to read ......: 31711

\section{Instructions for use:}

- Modify only the arguments shown in Section C.3.1, and do not remove any lines.

- The program searches for the colon $(:)$ at the end of the description of each argument to precede the argument itself, and it expects the arguments to occur in a specific order. Note that the program searches for the string ":", rather than the character, so a blank space or tab must appear both before and after the colon.

- If an argument is not applicable, an appropriate argument must still appear. Null arguments will cause the program to crash.

\section{C.3.2 Input file for the ComPost program}

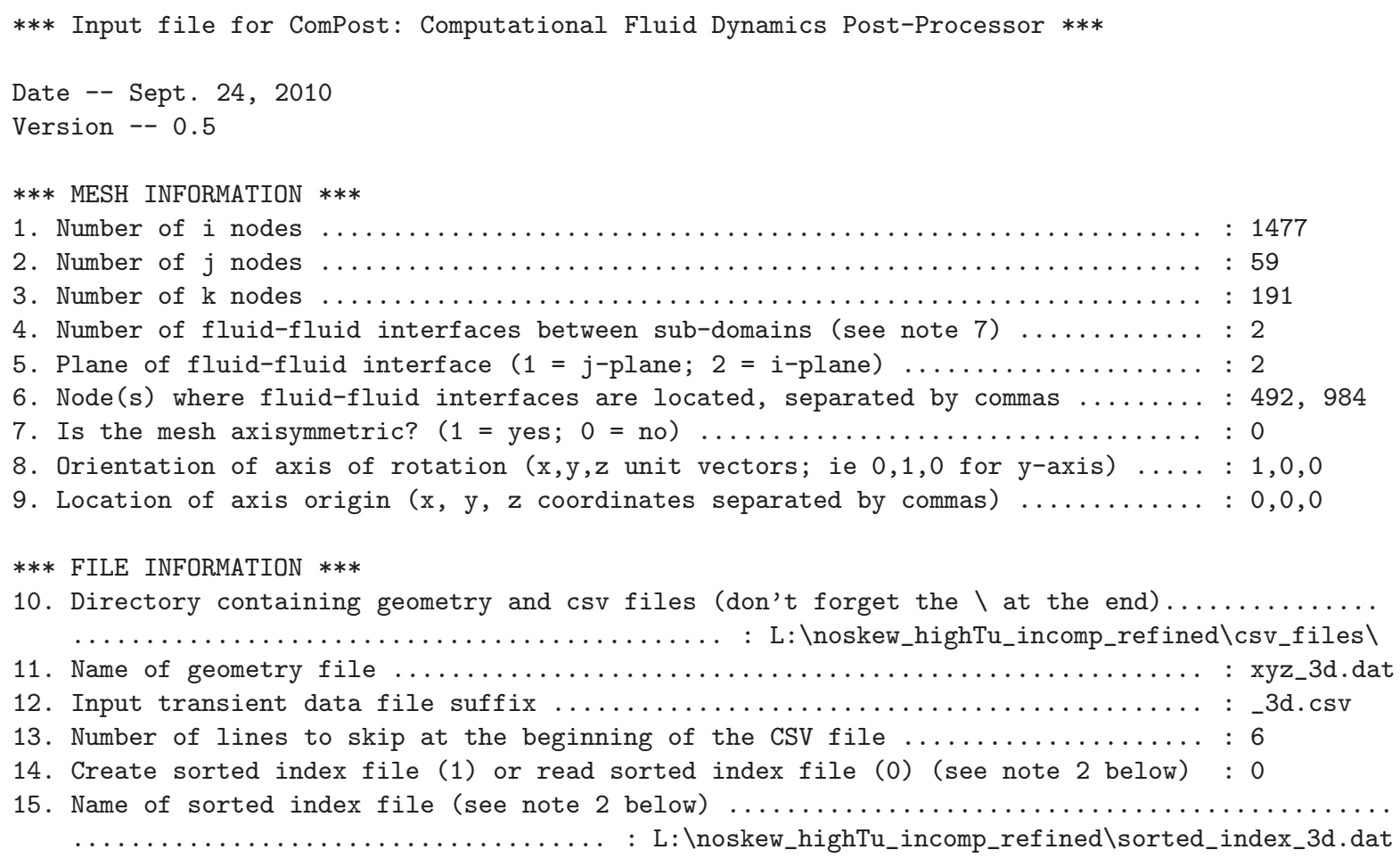




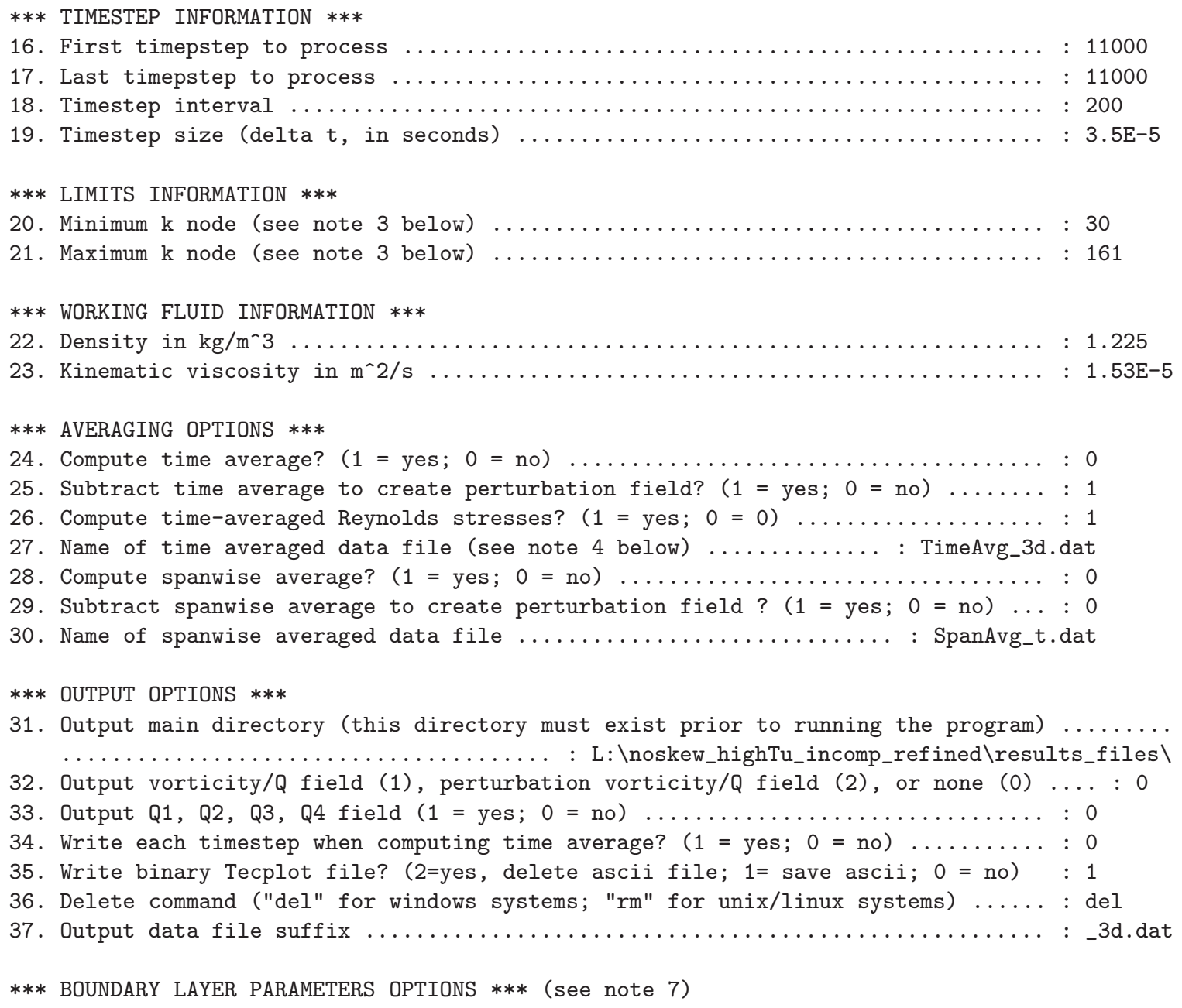


The following points should be noted when preparing the input files to ComPost:

1. Modify only the arguments shown in Section C.3.2, and do not remove any lines. The program searches for the colon (:) at the end of the description of each argument to precede the argument itself, and it expects the arguments to occur in a specific order. Note that the program searches for the string ":", rather than the character, so a blank space or tab must appear both before and after the colon. If an argument is not applicable, an appropriate argument must still appear. Null arguments will cause the program to crash.

2. The data that is written by CFX-Post is written as unstructured elements. To sort the data in a structured $\mathrm{i}-\mathrm{j}-\mathrm{k}$ format that is readable by Tecplot, the geometry file has to be sorted. The sorting algorithm sorts the indices of the geometry. If the argument of line 8 is 1 , the sorting is performed once and the sorted indices are saved to the file named on line 9 . If the argument of line 8 is 0 , the index file named on line 9 is read and the sorting is not performed; this argument should be used if the index file already exists.

3. These values are the minimum and maximum $\mathrm{k}$ node values that will be processed. Values with $\mathrm{k}$ nodes less than the minimum or greater than the maximum will not be processed. This allows for smaller data files.

4. If the time-averaged perturbation field is desired, the argument for line 17 should be 1 . The program will read the time-averaged data file named on line 18 and subtract the time-averaged results from the transient results at each time step. If the time-averaged data file doesn't exist, the user must first create it by putting 1 for the argument at line 16 and 0 for the argument at line 17.

5. If this argument is one, data files for each timestep will be written when the time average is being calculated. If the argument is zero, only the time-averaged data file will be written. If the compute time average flag (line 16) equals 1, only primitive variables 
$(P, u, v, w)$ will be written, not vorticity. This is because when the time average is being computed, the $(u, v, w)$ arrays contain results for more than one timestep.

6. If the dataset consists of multiple sub-domains, they must be stacked vertically on top of each other such that the interface occurs at a constant j-node. Line 4 contains the number of fluid-fluid interface planes that occur within the data. For example, if there are two sub-domains, there will be one fluid-fluid interface, if there are three sub-domains, there will be two interfaces, and so on. Line 5 lists the j-node location(s) of each interface separated by a space or tab. If there is no interface, put a zero on line 4 and a zero on line 5. Maximum number of interfaces is 10 .

7. Maximum number of planes $=50$

8. Exporting the time trace appends the values of $P, u, v, w$ at each timestep to a file at the specified $i, j, k$ node intervals. After all timesteps have been process, these files will contain the time history of that signal. The duration and timestep of the time signals are determined by the arguments on lines 12-15.

9. In order to export enstrophy transport equation terms, the "Compute time-averaged Reynolds stresses" flag must equal 1, the "Compute time average" flag must equal 0, and "Subtract time average to create perturbation field" flag must equal 1.

- NOTE: If the streamwise and spanwise intervals (lines 41 and 42) are small, many files will be created. This will cause ComPost to run slowly and may freeze your computer as the hard disk tries to open and write to thousands of files.

- NOTE: At each timestep, ComPost appends the values of $P, u, v$, and $w$ to the time trace files. If the files don't exist, ComPost will create them. If files with the same name already exist, ComPost will append to the end of these files. Therefore, the user must make sure that the time trace directory is empty or filenames are suitably changed to prevent ComPost from writing a time trace on the end of a different time trace. 


\section{C.4 Procedures for using ComPost}

To run ComPost, follow the sequence shown below:

1. Open the input text file input_compost.txt in a text editor and modify the command arguments as desired. An example of the input file is given in Section C.3.2. An in-depth description of the various commands and options are given in the following sub-sections.

2. Move the ComPost executable file into the working directory and run it by double-clicking on it. Enter the name of the input text file when prompted. Alternatively, ComPost can be run from the command line with the input text file given as an argument by typing:

\$ ComPost input_compost.txt

from within the working directory.

At this point, ComPost is finished receiving user input and begins to process the data. The following sub-sections describes the operation of ComPost in more detail.

\section{C.4.1 Sorting structured-grid results}

The sorting routine in ComPost is activated as follows:

1. Generate the input file input_getxyz.txt for the get_xyz program, as described in Section C.3.1.

2. Open the input text file input_compost.txt and make the following changes:

(a) On lines 1-3, input the number of $i, j, k$ nodes

(b) On line 4, enter the number of fluid-fluid interfaces between sub-domains that occur within the exported region. 
(c) On line 5, input the direction flag for the plane where fluid-fluid interfaces are located, 1 for $j$ and 2 for $i$. Note that ComPost can only hand fluid-fluid interfaces in one coordinate direction at once.

(d) On line 5, input the node numbers where the fluid-fluid interfaces are located, separated by commas.

(e) On line 10, input the directory containing the mesh information and data .csv files

(f) On line 11, input the name of the mesh information file

(g) On line 14, set the sorted index file flag to 1, which means that the mesh geometry will be sorted and the sorted index list will be written to a file.

(h) On line 15, input the name of the file for saving the sorted index list.

3. Run ComPost once.

The sorting step uses the quicksort algorithm and takes a substantial amount of wall-clock time for 3D datasets. Therefore, user can choose to read this sorted index file into memory the next time they run ComPost instead of performing the sort again.

\section{C.4.2 Calculating temporal averages}

To compute a temporal average, ComPost reads data files from successive timesteps and performs an arithmetic average. This is activated as follows:

1. On line 24 , set the compute time average flag to 1 .

2. On lines 25-26, set the flags to 0.

3. On line 27 , input the name of the time-averaged data file. This file will be created in the directory specified on line 31 .

4. On lines 28-30, set the flags to 0 .

5. Run ComPost once. 


\section{C.4.3 Calculating spatial averages}

To compute a spatial average, ComPost performs an arithmetic average across the spanwise (z) coordinate direction. This is activated as follows:

1. On lines 24-26, set the flags to 0.

2. On lines 28 , set the compute spatial average flag to 1 .

3. On line 29 , set the flag to 0 .

4. On line 30, input the name of the spanwise-averaged data file. This file will be created in the directory specified on line 31 .

5. Run ComPost once.

To compute a temporal average followed by a spanwise average, change the text input file on lines 24-27 as indicated in Section C.4.2 and on lines 28-30 as indicated in Section C.4.3, and then run ComPost once. The temporally-/spatially-averaged data is written to the file specified on line 30 .

\section{C.4.4 Calculating integral parameters}

To calculate the integral parameters based on the time-averaged data, follow the steps described in Section C.4.2, but with the following additional changes to the input text file:

1. On line 31, input the directory where the results will be written.

2. Create a folder called bl_params in the directory specified on line 31.

3. On line 38, set the compute integral parameters flag to 1. 
4. On line 39, set the streamwise or spanwise distribution flag to 1 if the streamwise distribution of integral parameters is desired or 2 if the spanwise distribution of integral parameters is desired.

5. On line 40, set the number of nodes flag to the number of locations where the integral parameters will be calculated. If the flag on line 39 is 1, this corresponds to the number of spanwise stations where the streamwise distributions of the integral parameters will be calculated. If the flag on line 39 is 2 , this corresponds to the number of streamwise stations where the spanwise distributions of the integral parameters will be calculated.

6. On line 41, enter the node indices where the integral parameters will be calculated. For example, if the number on line 40 is 1 , there are $50 k$ nodes, and the integral parameters are desired at the mid-span, then enter 24.

7. On lines 42 and 43 , enter the maximum and minimum $j$ nodes, respectively, to include in the integration of the integral parameters.

8. Run ComPost once. After the time-averaged data file is computed, the integral parameters will be written to the bl_params folder. Because these integral parameters correspond to the time-averaged data, the files are given a prefix of $0-$.

To calculate the integral parameters based on instantaneous data, follow the steps described above but with the flag on line 38 set to 2 . The integral parameters will be computed to the bl_params folder after each timestep is read. The files at each timestep are given a prefix of $t$-, where the $t$ is replaced with the timestep value.

\section{C.4.5 Outputting velocity profiles}

To calculate the velocity profiles based on the time-averaged data, follow the steps described in Section C.4.4, but with the following additional changes to the input text file:

1. Create a folder called vel_profile in the directory specified on line 31 . 
2. On line 44, set the export velocity profile flag to 1.

3. On line 45, input the streamwise interval for exporting velocity profiles. A value of 10 indicates every tenth node in the coordinate direction specified on line 39.

4. Run ComPost once. After the integral parameters have been exported, the velocity profiles will be written to the vel_profile folder. Because these velocity profiles correspond to the time-averaged data, the files are given a prefix of $0-$.

To output the velocity profiles of the instantaneous data, follow the steps described above but with the flag on line 38 set to 2 . The velocity profiles will be written to the vel_profile folder after each timestep is read. The files at each timestep are given a prefix of $t$-, where the $t$ is replaced with the timestep value.

\section{C.4.6 Calculating fluctuation quantities}

To calculate the fluctuation quantities by subtracting the time-averaged data from the instantaneous flow field, do as follows:

1. Follow the steps described in Section C.4.2 and run ComPost once.

2. Switch the flag on line 24 to 0 and on line 25 to 1.

3. Run ComPost again. The time-averaged results file listed on line 27 will be read prior to the timestep files, and the averaged values will be subtracted from the instantaneous results.

To calculate the fluctuation quantities by subtracting the spanwise-averaged data from the instantaneous flow field, do as follows:

1. Follow the steps described in Section C.4.3 and run ComPost once. 
2. Switch the flag on line 28 to 0 and on line 29 to 1.

3. Run ComPost again. The time-averaged results file listed on line 30 will be read prior to the timestep files, and the averaged values will be subtracted from the instantaneous results.

\section{C.4.7 Outputting time traces}

To output the time traces from the instantaneous simulation data, follow the steps described in Section C.4.4, but with the following additional changes to the input text file:

1. Create a folder called time_trace in the directory specified on line 31.

2. On line 39, set the compute integral parameters flag to 2.

3. On line 46 , set the export time trace flag to 1.

4. On line 47, input the interval for exporting the time trace. If 5 is entered, time traces will be exported every fifth node in the coordinate direction specified on line 39.

5. On line 48, input the wall-normal interval for exporting the time trace. If 2 is entered, time traces will be exported every other node in the wall-normal $(y)$ direction.

6. Run ComPost once. After the first timestep is process, time traces will be written to separate files for $P, u, v, w$ in the time_trace folder. After each timestep, the data will be appended to the end of each time trace file. The files are given a prefix of $\mathrm{p}^{-}, \mathrm{u}^{-}, \mathrm{v}^{-}$, and $\mathrm{w}$ - for the $P, u, v, w$ traces, respectively.

To output a time trace of the fluctuation quantities, follow the steps described in Section C.4.6, run ComPost once, and then perform the above steps. 


\section{C.4.8 Exporting Tecplot-formatted transient results}

To export simulation results in Tecplot-formatted binary files, follow the steps described in Section C.4.2 with the following changes in the input text file:

1. On line 31, specify the directory where the transient results files will be written.

2. On line 34 , set the write timestep flag to 1.

3. On line 35 , set the write Tecplot file flag to 1 or 2 . If the flag is set to 1 , the ASCII file is saved, while if the flag is set to 2, the ASCII file is deleted after the Tecplot-formatted file has been written.

4. On line 36, input the delete command for the local operating system, either del for Windows $($ ) or rm for Linux.

5. On line 37, specify the suffix of the transient results files that will be created.

6. Run ComPost once. After each timestep is processed, a Tecplot-formatted data file (extension .plt) will be created in the directory specified on line 31 .

\section{C.4.9 Calculating the vorticity field}

To calculate the vorticity field at each timestep, follow the steps described in Section C.4.8 with the following changes to the input file:

1. On line 32 , set the output vorticity/Q field flag to 1 or 2 . If the flag is set to 1 , the vorticity is computed from the instantaneous velocity field. If the flag is set to 2 , the vorticity is computed from the fluctuation velocity field, described in Section C.4.6.

If the fluctuation vorticity field is desired, the fluctuation quantities have to be computed by modifying the input text file as described in Section C.4.6 in addition to the above change. 


\section{C.4.10 Calculating statistical turbulence quantities}

The statistical turbulence information includes the Reynolds stresses, turbulence kinetic energy, turbulence intensity, and turbulence kinetic energy production and dissipation budgets. To compute this information, follow the steps in Section C.4.6, but before running ComPost the second time, make the following changes to the input text file:

1. On line 26, set the compute Reynolds stresses flag to 1.

To output the enstrophy transport terms, in addition to the above step, set the flag on line 49 to 1 . To output the Reynolds stress profiles, do as follows:

1. Create a folder called vel_profile in the directory specified on line 31.

2. On line 44, set the export velocity profile flag to 1.

3. On line 45, input the streamwise interval for exporting velocity profiles. A value of 10 indicates every tenth node in the coordinate direction specified on line 39 .

\section{C.5 Spectral analysis}

To perform spectral analysis of the computed data, an in-house program called computeFFT was created. This program computes the spectral power density of the time-traces extracted from the simulation results using ComPost. The discrete Fourier transform is performed using the open-source library FFTW, available from http://www.fftw.org/. To compute the frequency spectra:

1. Output the time traces following the steps in Section C.4.7.

2. Open the text input file input_computeFFT using a text editor. The input file is shown in Section C.5.1. 
3. On line 1, input the directory location where the time traces are located.

4. On line 2, input the file suffix of the time trace files.

5. On line 3, input the directory where the power spectral density files will be written.

6. On line 4, input the file suffix of the power spectral density files.

7. On line 5, input the file suffix of the root-mean-square fluctuation files.

8. On line 6, set the number of samples to include in the Fourier transform computation. This value should be $2^{n}$, where $n$ is a real integer.

9. On line 7, set the number of timesteps to skip at the beginning of the time trace. This allows the window for the FFT to be adjusted forwards in time as required.

10. On line 8, enter the timestep size between samples in the time trace. This is equal to the simulation timestep times the timestep interval in the ComPost run.

11. On line 9 , enter the variable trace that will be computed $(\mathrm{P}, \mathrm{u}, \mathrm{v}, \mathrm{w})$ or a for all four variables.

12. On lines 10 to 15 , enter the nodal information for where the spectral analysis will be conducted.

13. Run computeFFT with the input text file as an argument:

\$ computeFFT input_computeFFT.txt

\section{C.5.1 Input file for the compute_FFT program}

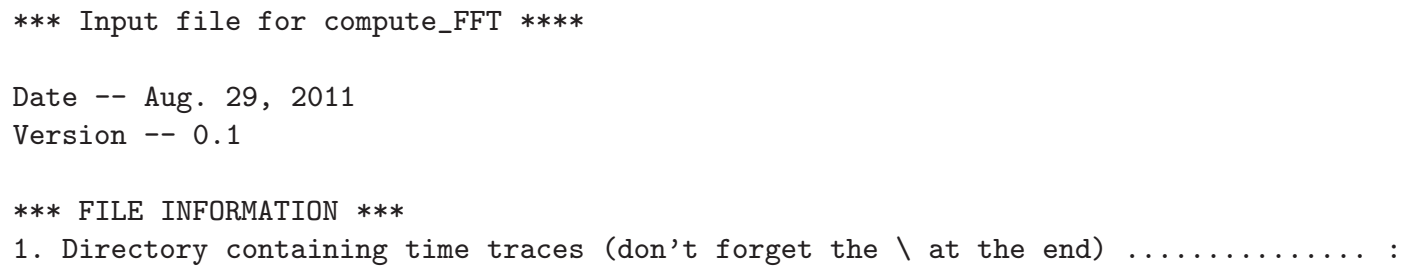




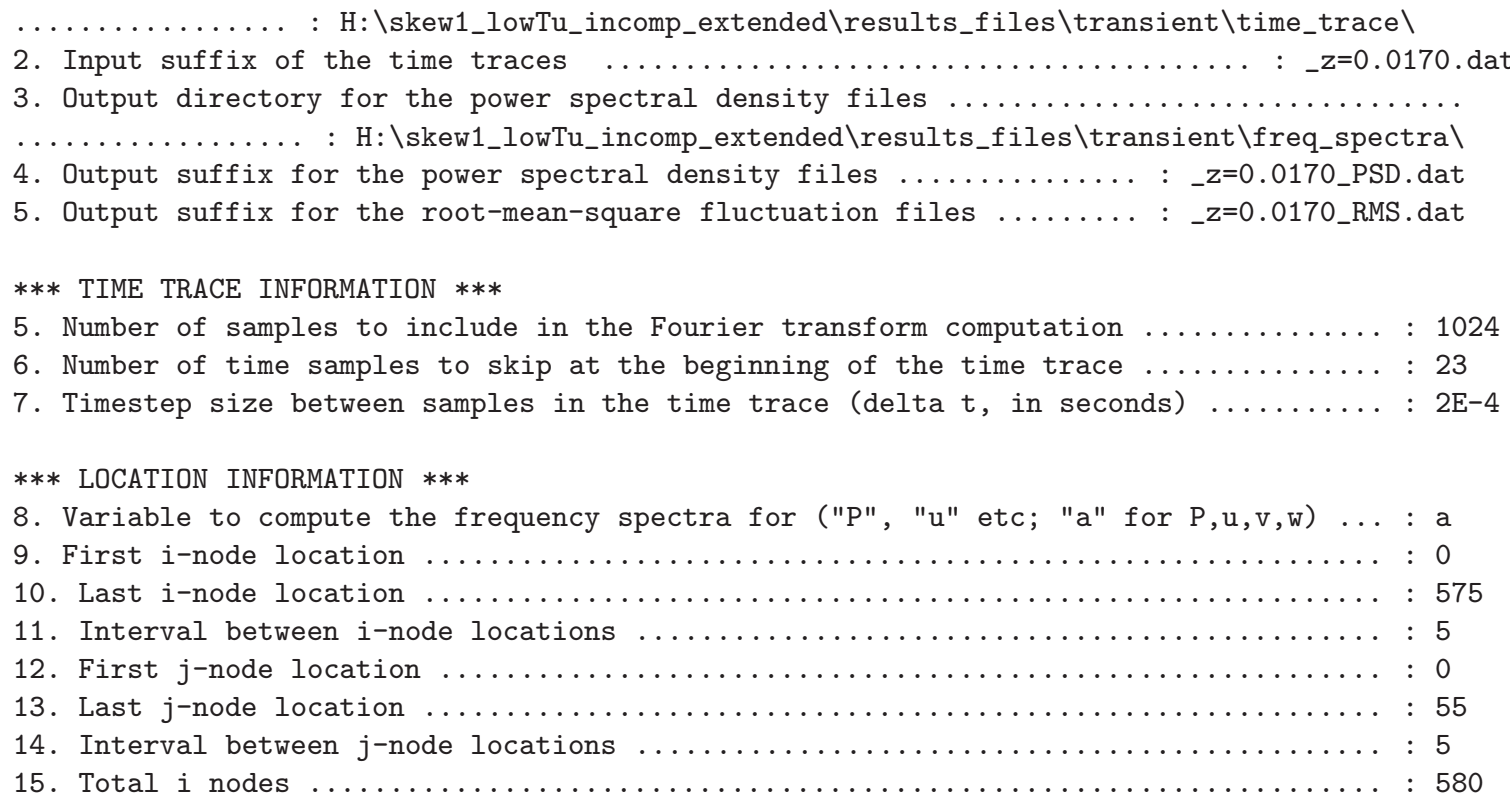

\section{Instructions for use:}

- Modify only the arguments in the list above, and do not remove any lines.

- The program searches for the colon $(:)$ at the end of the description of each argument to precede the argument itself, and it expects the arguments to occur in a specific order. Note that the program searches for the string ":", rather than the character, so a blank space or tab must appear both before and after the colon.

- If an argument is not applicable, an appropriate argument must still appear. Null arguments will cause the program to crash. 
Appendix D

\section{Permissions to Reproduce}

\section{Copyrighted Material}

This appendix contains the permissions obtained from the publishers allowing the reproduction of the copyrighted material presented in Chapters 4-6. 


\title{
AMERICAN INSTITUTE OF PHYSICS LICENSE TERMS AND CONDITIONS
}

Jul 07, 2013

\author{
All payments must be made in full to CCC. For payment instructions, please see \\ information listed at the bottom of this form. \\ License Number 3183770894090 \\ Order Date Jul 07, 2013 \\ Publisher American Institute of Physics \\ Publication Physics of Fluids \\ Article Title Interaction of viscous and inviscid instability modes in separation- \\ Author \\ bubble transition \\ Online Publication Date \\ Joshua R. Brinkerhoff, Metin I. Yaras \\ Volume number 23

Issue number 12 \\ Type of Use Thesis/Dissertation \\ Requestor type Author (original article) \\ Format Print and electronic \\ Portion Excerpt (> 800 words) \\ Will you be translating? No \\ Title of your thesis / Interaction of Instability Modes and Free-Stream Conditions in \\ dissertation Shear-Flow Transition \\ Expected completion date Aug 2013 \\ Estimated size (number of 300 \\ pages) \\ Total $\quad 0.00$ USD \\ Terms and Conditions \\ American Institute of Physics -- Terms and Conditions: Permissions Uses \\ American Institute of Physics ("AIP") hereby grants to you the non-exclusive right and license to \\ use and/or distribute the Material according to the use specified in your order, on a one-time \\ basis, for the specified term, with a maximum distribution equal to the number that you have \\ ordered. Any links or other content accompanying the Material are not the subject of this license.
}

1. You agree to include the following copyright and permission notice with the reproduction of the Material:"Reprinted with permission from [FULL CITATION]. Copyright [PUBLICATION YEAR], American Institute of Physics." For an article, the copyright and permission notice must be printed on the first page of the article or book chapter. For photographs, covers, or tables, the copyright and permission notice may appear with the Material, in a footnote, or in the reference list.

2. If you have licensed reuse of a figure, photograph, cover, or table, it is your responsibility to ensure that the material is original to AIP and does not contain the copyright of another entity, and that the copyright notice of the figure, photograph, cover, or table does not indicate that it was reprinted by AIP, with permission, from another source. Under no circumstances does AIP, purport or intend to grant permission to reuse material to which it does not hold copyright.

3. You may not alter or modify the Material in any manner. You may translate the 
RE: Permission request: J. of Turbomach. vol. 135(4) no. 041006

Subject: RE: Permission request: J. of Turbomach. vol. 135(4) no. 041006 From: Beth Darchi <DarchiB@asme.org>

Date: 10/07/2013 4:14 PM

To: 'Joshua Brinkerhoff' <joshuabrinkerhoff@connect.carleton.ca>

Dear Mr. Brinkerhoff:

It is our pleasure to grant you permission to use ASME paper "Direct Numerical Simulations of Transitional Separation-Bubble Development in Swept-Blade Flow Conditions," by Joshua R. Brinkerhoff and Metin I. Yaras, Journal of Turbomachinery, Volume 135, Issue 4, 2013, as cited in your letter for inclusion in a Ph.D. thesis entitled Interaction of Instability Modes and Free-Stream Conditions in Shear-Flow Transition to be published by Carleton University.

Permission is granted for the specific use as stated herein and does not permit further use of the materials without proper authorization. Proper attribution must be made to the author(s) of the materials. Please note: if any or all of the figures and/or Tables are of another source, permission should be granted from that outside source or include the reference of the original source. ASME does not grant permission for outside source material that may be referenced in the ASME works.

As is customary, we request that you ensure full acknowledgment of this material, the author(s), source and ASME as original publisher. Acknowledgment must be retained on all pages printed and distributed.

Many thanks for your interest in ASME publications.

Sincerely,

Beth Darchi

Permissions \& Copyright

ASME, 2 Park Aven

New York, NY 10016

$\mathrm{F}: 212-591-7841$

E: darchib@asme.org

From: Joshua Brinkerhoff [mailto:joshuabrinkerhoff@connect.carleton.ca]

Sent: Sunday, July 07, 2013 2:55 PM

To: permissions@asme.org

Dear ASME,

This email is a request for permission to use the paper "Direct Numerical Simulations of Transitional Separation-Bubble Development in Swept-Blade Flow Conditions" published in 2013 in the Journal of Turbomachinery in my PhD thesis. The citation information is recorded below and in the attached permission request form. I am the first author of the paper and intend on reproducing the text, figures, and tables in a chapter of my thesis.

Thank you,

Joshua Brinkerhoff 
SPRINGER LICENSE TERMS AND CONDITIONS

This is a License Agreement between Joshua R Brinkerhoff ("You") and Springer ("Springer") provided by Copyright Clearance Center ("CCC"). The license consists of your order details, the terms and conditions provided by Springer, and the payment terms and conditions.

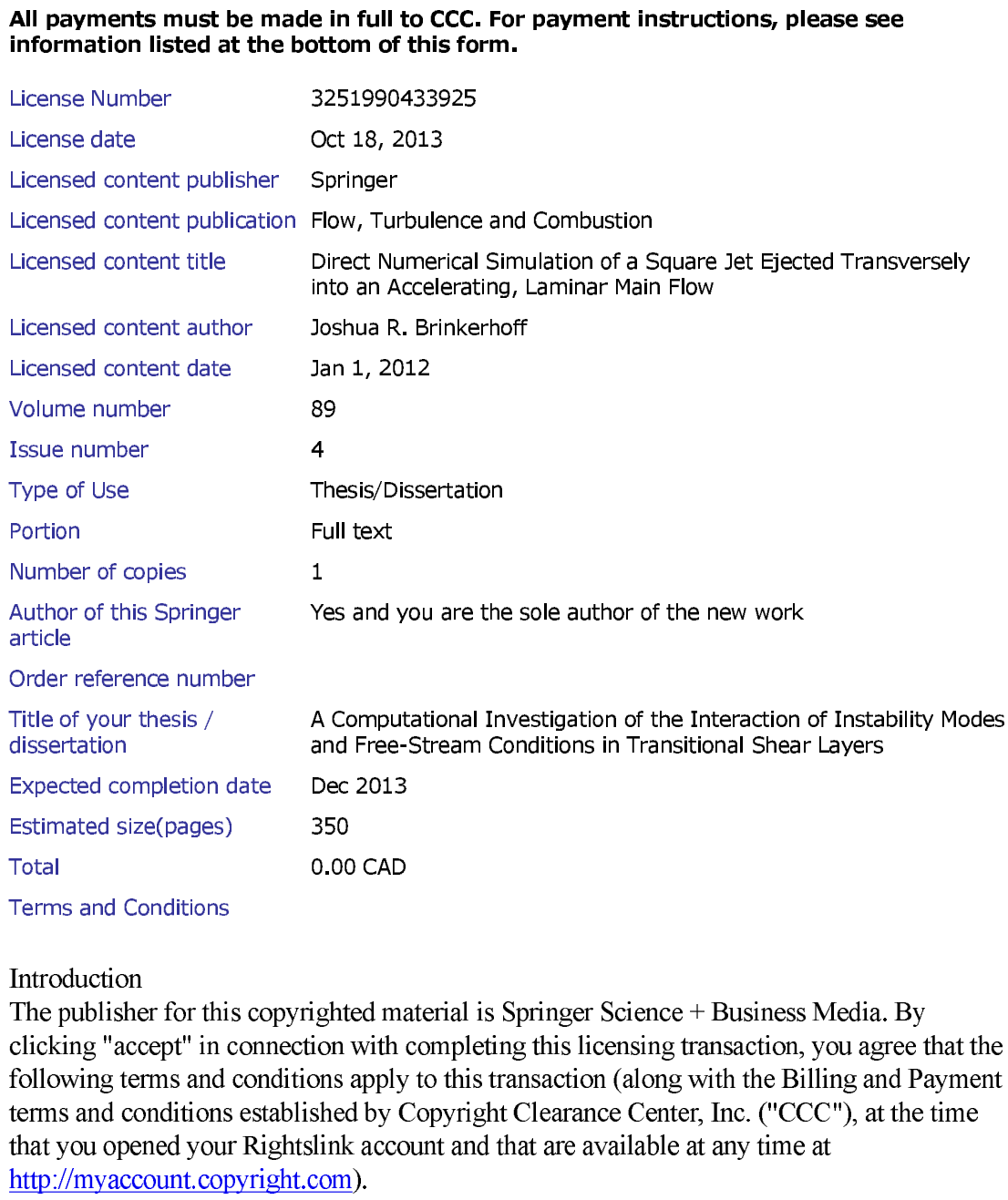

Introduction

The publisher for this copyrighted material is Springer Science + Business Media. By clicking "accept" in connection with completing this licensing transaction, you agree that the following terms and conditions apply to this transaction (along with the Billing and Payment terms and conditions established by Copyright Clearance Center, Inc. ("CCC"), at the time that you opened your Rightslink account and that are available at any time at http://myaccount.copyright.com). 